\title{
PATRONES DE DISTRIBUCIÓN ESPACIAL EN PLANTAS CLONALES
}

\author{
Israel Gustavo Carrillo-Angeles y María del Carmen Mandujano ${ }^{1}$ \\ Instituto de Ecología, Universidad Nacional Autónoma de México \\ ${ }^{1}$ Autor para la correspondencia: mcmandu@ecologia.unam.mx
}

\begin{abstract}
Resumen: En las especies clonales predomina la distribución agregada de los ramets, pero se plantea que la propagación por diásporas móviles o no ligadas, (por ejemplo, bulbilos y semillas agamospérmicas) forma arreglos espaciales de genets más entremezclados, que la propagación por estructuras ligadas (rizomas y estolones), y se sugiere que los arreglos entremezclados de genets favorecen la entrecruza. En este trabajo se presenta una revisión de 53 estudios en plantas clonales, para investigar la influencia de la producción de propágulos clonales, por diferentes formas de crecimiento clonal, en la formación de arreglos espaciales de genets y ramets. La hipótesis es que los arreglos entremezclados promueven la entrecruza, suponiendo que las poblaciones donde la entrecruza es más frecuente tenderán a presentar niveles más altos de diversidad genotípica. Los estudios revisados muestran un sesgo hacia herbáceas perennes rizomatosas. Predominan los patrones de distribución agregados de parches de ramets de distintos genets no entremezclados. Las poblaciones de especies que tienen ambos tipos de crecimiento de propágulos clonales (ligados y no ligados) y de especies que sólo presentan crecimiento ligado presentan patrones de distribución espacial de genets y ramets más entremezclados que las especies que sólo presentan crecimiento ligado. Encontramos que existe variación intraespecífica tanto en los patrones de distribución espacial como en los patrones reproductivos en las diferentes poblaciones. Sin embargo, utilizando índices de diversidad genotípica como estimadores, solamente se encontró un patrón congruente con el planteamiento sobre el efecto del arreglo espacial de los genets y los ramets en la proporción de genotipos distinguibles. Generalizar las consecuencias reproductivas y genéticas del arreglo espacial de los genets y los ramets continúa siendo complejo, debido a que una reducción del reclutamiento sexual y una dispersión restringida de polen, semillas y propágulos vegetativos pueden generar estructura genética local en las poblaciones. La estructura genética local tanto a nivel de genets (debida a la dispersión restringida de polen, semillas o ambos) como de ramets (indicando dispersión restringida de propágulos vegetativos) fue detectada en 46 poblaciones distribuidas en 16 especies. Estos factores aunados a la variedad de métodos de muestreo empleados en los trabajos publicados para detectar genets y ramets, la falta de estimadores numéricos comparables para describir la distribución espacial y la carencia de estudios en especies que se propagan por diásporas móviles o no ligadas reducen la resolución del efecto que tiene el tipo de crecimiento clonal en la formación de arreglos entremezclados de genets distintos sobre la diversidad genotípica.
\end{abstract}

Palabras clave: clonalidad, crecimiento, distribución, diversidad, entrecruza

\begin{abstract}
Clonal plants are predominantly associated with a clumped distribution of ramets. However, propagation by unlinked means generates a mixed arrangement instead of a clumped one found by linked propagules and intermingled arrangements of genets favor outcrossing. This paper reviews 53 studies on clonal plants to assess the influence of the production of clonal offspring by different means on the spatial arrangement of genets and ramets. We also evaluate the assumption that mixed arrangements promote outcrossing, under the hypothesis that populations where outcrossing is common would have higher levels of genotypic diversity. The studies that were consulted showed a strong bias towards rhizomatous perennial herbs. Clumped distribution patterns predominate in which patches consist of discrete groups of ramets from one or more genets that do not spatially mix. Populations having both types of propagation (linked and unlinked) and species that only have linked propagation have a higher spatially mixed genet and ramet distribution pattern than species that only have unlinked propagation. However, using genotypic diversity indices, we did not find a consistent pattern between the spatial arrangement and outcorssing rates. The reproductive and genetic consequences of the spatial arrangement of genets and ramets continues to be a difficult task, due to a range of factors such as the variety of methods, the lack of good comparative numeric estimations to describe the spatial arrangements and the lack of studies in species that propagate through unlinked propagules.
\end{abstract}

Key words: clonality, distribution, diversity, growth, outcrossing

a clonalidad da lugar a la producción de ramets, es decir, Lunidades potencialmente independientes que son genéticamente idénticas a la planta progenitora (Widén et al., 1994; van Groenendael et al., 1996; de Kroon y van Groenendael,
1997; Klims et al., 1997). En consecuencia, en las poblaciones de organismos clonales el concepto de "individuo" puede definir a los individuos genéticos formados a partir de un cigoto (genets) y a las copias física y fisiológicamente 
independientes de cada genotipo o ramets independientes (Figura 1; Kays y Harper, 1974; Harper, 1985; Jackson et al., 1985). La clonalidad está ampliamente distribuida en plantas, algunas estimaciones para angiospermas varían entre 30\% y más del 50\% (Tiffney y Niklas, 1985; Klim s et al., 1997; Aarssen, 2008), entre el 40\% y $80 \%$ en especies de taiga, tundra y de zonas templadas (van Groenendael y de Kroon, 1990 Klim s et al., 1997) y el 44\% de plantas invasoras en China (Liu et al., 2006), está ampliamente representada en monocotiledóneas y se presenta con baja frecuencia en las gimnospermas (van Groenendael et al., 1996; Peterson y Jones, 1997). Respecto a las condiciones ambientales, se sugiere que la clonalidad predomina en condiciones estresantes como suelos pobres en nitrógeno, suelos húmedos, cuerpos de agua, hábitats sombreados y ambientes fríos en altitudes y latitudes elevadas (Tiffney y Niklas, 1985; van Groenendael et al., 1996; Klim s et al., 1997).

La clonalidad en plantas se ha estudiado con enfoques muy diversos. Por ejemplo, se ha analizado la frecuencia de especies clonales y la variación en las formas de crecimiento clonal en distintos ambientes (p. ej., Tiffney y Niklas, 1985; van Groenendael et al., 1996; Klim s et al., 1997), las implicaciones de la integración fisiológica de los ramets y la plasticidad morfológica del crecimiento clonal en ambientes heterogéneos y en condiciones de estrés competitivo (p. ej., Bell, 1984; Hartnett y Bazzaz, 1985; de Kroon y Hutchings, 1995; Price y Marshall, 1999), el efecto de la clonalidad en la demografía (p. ej., Caswell, 1985; McFadden, 1991; Damman y Cain, 1998; Mandujano et al., 2001), la comparación de la diversidad genética entre especies de plantas clonales y no clonales (p. ej., Ellstrand y Roose, 1987; Widén et al., 1994) y las consecuencias reproductivas y evolutivas de la geitonogamia asociada con la clonalidad (p. ej., Handel, 1985; de Jong et al., 1992; Eckert, 2000).

Las implicaciones reproductivas y genéticas de reducir la entrecruza incrementando la geitonogamia han aumentado el interés en el estudio de los patrones de distribución espacial de genets y ramets en poblaciones de plantas clonales. Debido a que los propágulos vegetativos suelen tener una capacidad de dispersión restringida, el reclutamiento clonal frecuente puede reducir la entrecruza e incrementar la autocruza (p. ej., autogamia y geitonogamia), determinando los niveles de variación y la estructuración genética en las poblaciones (Handel, 1985; Eckert, 2002; Ruggiero et al., 2005; Honnay et al., 2006). En especies autocompatibles, los costos reproductivos de la geitonogamia están asociados al incremento de la autogamia y a las probabilidades de expresar depresión endogámica, a la vez que disminuye la adecuación paterna reduciendo la cantidad de polen disponible para la entrecruza; mientras que en las especies autoincompatibles, la geitonogamia afecta la producción de semillas, debido a la saturación de los estigmas con polen incompatible y al aborto de óvulos, y puede resultar en el desperdicio de grandes cantidades de polen (de Jong et al.,
1992; Charpentier, 2002).

En plantas clonales los patrones de distribución espacial pueden ser afectados por la manera en que se generan los ramets. Las formas de crecimiento clonal son muy diversas, pero existen algunas clasificaciones que facilitan el entendimiento de esta variación. Tiffney y Niklas (1985) propusieron cuatro tipos principales de crecimiento: (1) Crecimiento establecido, dado por el inicio del desarrollo de un organismo individual a partir de un propágulo (puede considerarse como crecimento clonal si el propágulo es vegetativo, ya que el propágulo también puede ser una semilla producida sexualmente). (2) Crecimiento restaurativo, que se refiere a la reparación de un daño local en el individuo mediante la formación de tejido calloso, pero no hay regeneración de los órganos completos cuando estos se pierden. (3) Crecimiento regenerativo, que se refiere a la reparación y el reemplazo de órganos dañados (p. ej., crecimiento de una rama desprendida). (4) Crecimiento reproductivo, dado por la multiplicación del individuo fisiológico (p. ej., clonalidad). Estos autores también ordenan los tipos de crecimiento clonal en ligado y no ligado, considerando las estructuras de conexión (Figura 1). En el crecimiento ligado los propágulos vegetativos pueden desarrollarse hasta formar individuos completos (con raíces, tallos y hojas) manteniéndose unidos a la planta madre y su dispersión depende del crecimiento de ramificaciones de tallos y raíces, mientras que en el no ligado, los propágulos vegetativos se desprenden de la planta madre y se desarrollan hasta individuos completos separados de la planta madre. La dispersión de los propágulos en este caso es más dependiente de factores bióticos (p. ej., animales) o abióticos (p. ej., corrientes de agua, gravedad, viento) que en el crecimiento ligado y permite un mayor desplazamiento de los propágulos. El crecimiento reproductivo o clonalidad, que es la que interesa en esta revisión por su influencia en el arreglo espacial de genets y ramets, puede ser de dos tipos: (i) reiteración traumática, definida como la producción de un nuevo individuo inducida por estrés, ya sea por daño o por condiciones ambientales que limitan la reproducción sexual (por ejemplo, la producción de bulbilos en los agaves por daño al escapo floral o por deficiencia de polen) y (ii) la reiteración programada, como la producción de un nuevo individuo por un meristemo como parte normal del desarrollo (Tiffney y Niklas, 1985; Arizaga y Ezcurra, 2002).

La distribución espacial de los individuos en las poblaciones se ha clasificado en tres patrones básicos: aleatorio, uniforme y agregado (Figura 2; Gleason, 1920; Clark y Evans, 1954; Barbour et al., 1987). En poblaciones naturales de plantas, los individuos suelen distribuirse en forma más o menos agregada, debido a la distribución heterogénea de los recursos (Couteron y Kokou, 1997; Chen y Bradshaw, 1999), a la interacción con otras especies (Shreve, 1917; Mandujano et al., 1998) y a la dispersión restringida de semillas y propágulos vegetativos (Gibson y Menges, 1994; Okuda et al., 1997; Clark-Tapia et al., 2005). En plantas 
clonales además, el arreglo espacial de los genets y ramets puede ordenarse en dos patrones básicos, uno caracterizado por la agregación de ramets del mismo genet y el otro por una distribución entremezclada de ramets de distintos genets (Figura 2; de Kroon y Hutchings, 1995; Charpentier, 2002; Clark-Tapia et al., 2005). Estos arreglos espaciales de genets y ramets pueden estar asociados a la manera en que se producen los ramets (esto es por crecimiento clonal ligado o no ligado) y se ha propuesto que en las plantas que se propagan mediante crecimiento no ligado forman arreglos más entremezclados que las especies con crecimiento ligado (Gabrielsen y Brochmann, 1998), pero esto no ha sido demostrado. Por otro lado, debido a que la clonalidad puede incrementar la tasa de geitonogamia entre los ramets del mismo genet, puede esperarse que los arreglos entremezclados reduzcan la geitonogamia y promuevan la entrecruza como resultado del incremento local en el número de genets (Charpentier, 2002).

En algunas revisiones sobre el tema de la clonalidad se han planteado hipótesis sobre las implicaciones reproduc-

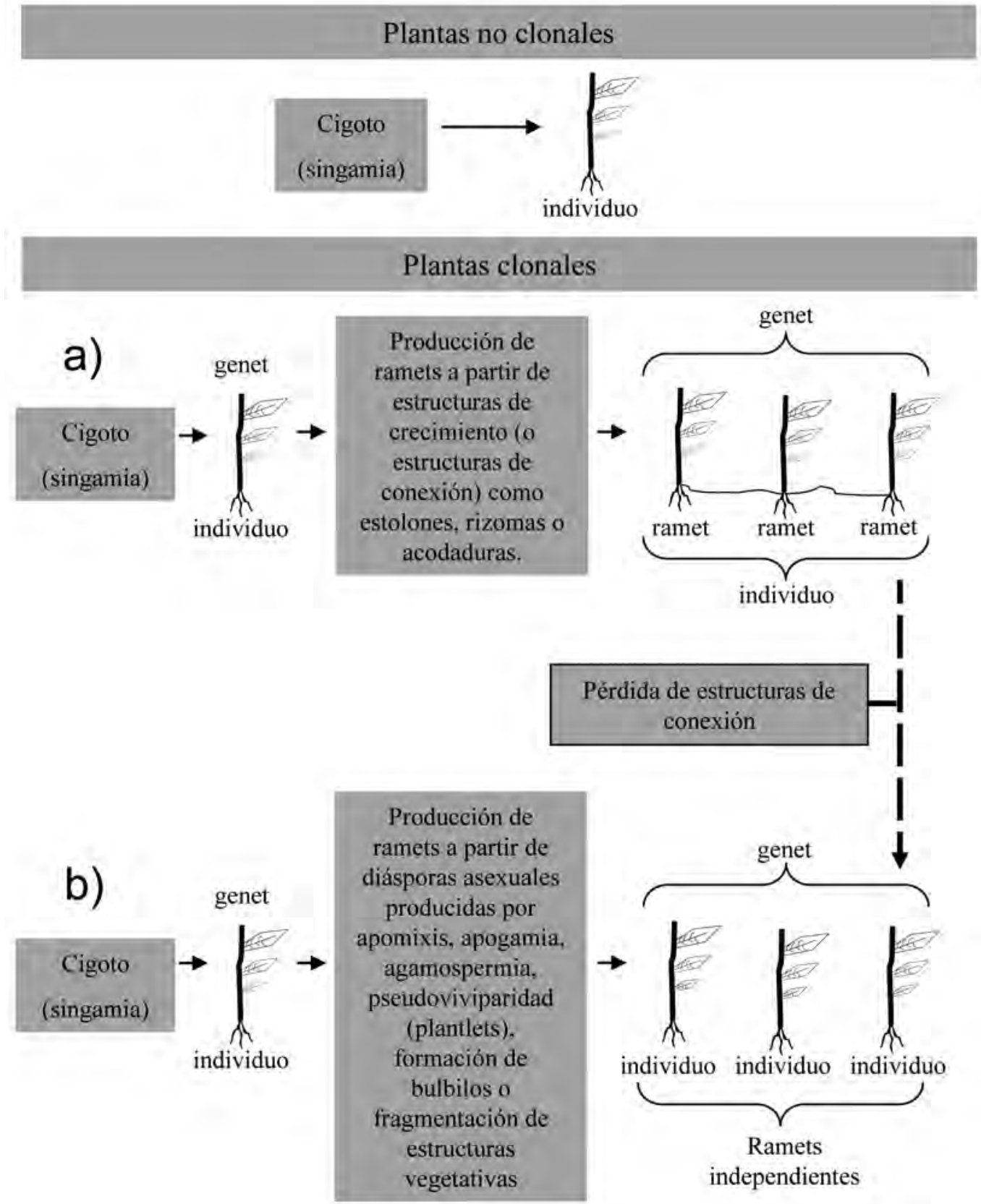

Figura 1. Formación de individuos en plantas no clonales y en plantas clonales. a) crecimiento clonal ligado y b) crecimiento clonal no ligado en la clasificación de Tiffney y Niklas (1985). 
$\mathrm{PDE}=$ Patrones de distribución espacial de los individuos y ramets en las poblaciones

(clonales y no clonales)
AER $=$ Arreglos espaciales de los ramets de distintos genets (clonales)

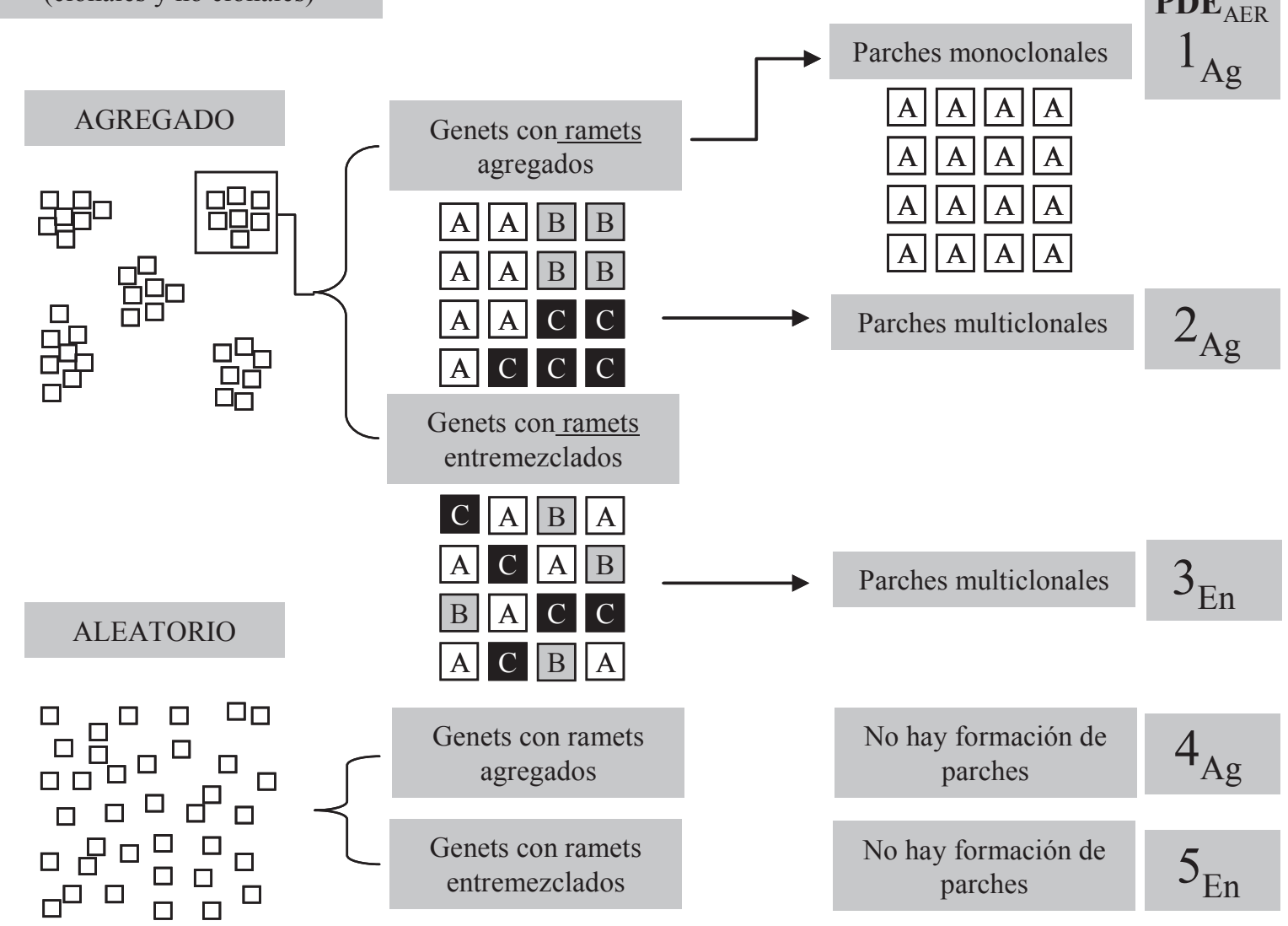

\section{UNIFORME}
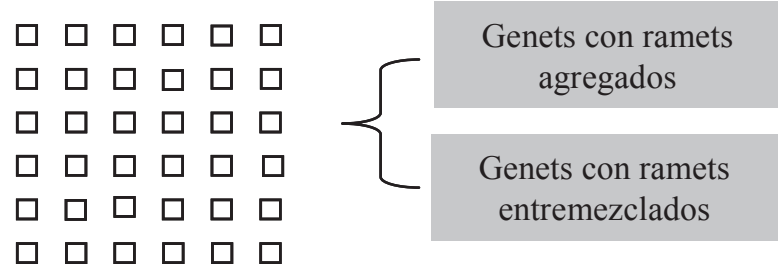

\begin{tabular}{|c|c|c|c|}
\hline $\begin{array}{c}\text { No hay formación de } \\
\text { parches }\end{array}$ & $6_{\mathrm{Ag}}$ \\
\hline $\begin{array}{c}\text { No hay formación de } \\
\text { parches }\end{array}$ & $7_{\mathrm{En}}$ \\
\hline
\end{tabular}

Figura 2. Patrones de distribución espacial de los individuos en las poblaciones de plantas no clonales y de plantas clonales. El número en el extremo derecho representa la clave del patrón de distribución espacial y el arreglo espacial de los ramets de distintos genets (PDE y AER, Apéndice). El subíndice Ag indica que los ramets de cada genet no se entremezclan con los ramets de genets distintos y el subíndice En indica que los ramets de diferentes genets se entremezclan.

tivas y genéticas de los arreglos espaciales de los genets y ramets, asociados con las distintas formas de producir descendientes clonales (Widén et al., 1994; Charpentier, 2002; Mandujano-Sánchez, 2007; Honnay y Jaquemyn, 2008). No obstante, los intentos para probar estas hipótesis son muy escasos y las posibilidades de realizar algún tipo de análisis numérico son restringidas por la baja disponibilidad de publicaciones (p. ej., Widén et al., 1994), o en los análisis sólo se incluyen especies con crecimiento ligado (p. ej., Honnay y Jaquemyn, 2008). En este trabajo presentamos una revisión de varios estudios que evalúan los patrones de distribución espacial en plantas clonales y a partir de la información 
proporcionada en dichos estudios, analizamos el planteamiento de que en poblaciones de plantas con crecimiento clonal ligado el arreglo espacial de los ramets de distintos genets es agregado, mientras que en poblaciones de plantas con crecimiento clonal no ligado predominan los arreglos espaciales entremezclados. Adicionalmente, intentamos poner a prueba la hipótesis de que los arreglos entremezclados promueven la entrecruza suponiendo que las poblaciones donde la entrecruza es más frecuente tenderán a presentar niveles más altos de diversidad genotípica.

\section{Material y métodos}

La revisión de los trabajos publicados sobre la distribución espacial clonal en plantas se llevó a cabo mediante una búsqueda de trabajos en bases de datos especializadas (CSA Cambridge, Ebsco HOST, ISI Web of Knowledge, JSTOR y Scopus) y no especializadas (Google, Google scholar y Yahoo). La búsqueda se hizo empleando los siguientes términos: clonal structure, spatial distribution of genets (andl or ramets), clonal architecture y clonal diversity y abarcó de 1990 a 2011, debido a que la mayoría de los estudios en plantas clonales con este enfoque se han realizado después de 1990.

Los criterios principales para incluir una especie en la revisión fue que se presentara una descripción de la distribución espacial de las plantas individuales (esto es sin importar el genotipo) en las poblaciones naturales y que se evaluara el arreglo espacial de los genets y los ramets (es decir agregado o entremezclado) mediante planos de distribución de los genotipos, análisis de autocorrelación espacial o compatibilidad de las cruzas entre ramets contiguos. Para cada especie se obtuvo el tipo de estructuras vegetativas a mediante las cuales se lleva a cabo la propagación clonal a partir de la información proporcionada en los estudios correspondientes o en estudios previos realizados a menudo por los mismos autores. El segundo criterio en orden de importancia fue que se reportaran valores de diversidad genotípica ( $D=$ índice de diversidad de Simpson y proporción de genotipos distinguibles $G / N$ ) para las poblaciones de las diferentes especies. Otros criterios fueron que se proporcionara información sobre las características reproductivas como la presencia de sistemas de incompatibilidad, la expresión sexual, si se ha observado reclutamiento sexual en las poblaciones y el tipo de polinización. Con base en esta información, se asignó a las especies el tipo de crecimiento clonal (i. e. ligado y no ligado, Figura 1) de acuerdo con la clasificación de Tiffney y Niklas (1985) y el patrón de distribución espacial y el tipo de arreglo espacial de los ramets (agregado o entremezclado) según el esquema presentado en la Figura 2. La información obtenida se presenta en el apéndice.

Para analizar el planteamiento de que en poblaciones de plantas con crecimiento clonal ligado el arreglo espacial de los ramets es agregado, mientras que en poblaciones de plantas con crecimiento clonal no ligado predomina el arreglo espacial entremezclado se construyó una tabla de contingencia con tres categorías de tipo de crecimiento clonal como columnas (ligado, combinación ligado-no ligado y no ligado) y tres categorías del arreglo espacial de los ramets como renglones (agregado, combinación agregado-entremezclado y entremezclado) y se analizó con una prueba de ${ }^{2}$ con el paquete estadístico JMP versión 7.0 (SAS Institute Inc). Para evaluar las celdas que contribuyen significativamente al valor de ${ }^{2}$ se hizo un análisis de residuales ajustados (Everitt, 1977).

Para probar la hipótesis de que las poblaciones con arreglos entremezclados de ramets presentan niveles más altos de diversidad genotípica, se compararon los valores del índice de diversidad de Simpson $(D)$ y la proporción de genotipos distinguibles $(G / N)$ entre las tres categorías de patrón de distribución clonal y entre las tres categorías de tipo de crecimiento clonal con una prueba de Kruskall-Wallis con el paquete estadístico JMP versión 7.0 (SAS Institute Inc).

\section{Resultados}

En la revisión se incluyeron 53 estudios que comprenden 122 poblaciones distribuidas en 44 especies de plantas clonales y en 27 familias (Apéndice). Las familias mejor representadas son Liliaceae y Rosaceae con cuatro especies cada una; Cactaceae, Cyperaceae, Ericaceae, Poaceae y Salicaceae con tres especies cada una y Zosteraceae con dos especies. El resto de las familias estuvieron representadas por una sola especie. Las especies que predominan son herbáceas perennes $(60 \%)$, y las plantas con hábito arbustivo y arbóreo se encuentran poco representadas (20\% en ambos casos).

La mayoría de las especies presentan crecimiento clonal ligado (70\%), dominando la propagación a través de rizomas (45\% de las especies). Las especies con crecimiento clonal ligado y no ligado representan el 14\% propagándose clonalmente por crecimiento ligado a través de rizomas y acodaduras, y por crecimiento no ligado a través de bulbilos, plantlets y desprendimiento de ramas; las especies sólo con crecimiento no ligado representan el 16\%, propagándose por bulbilos, semillas producidas por agamospermia, desprendimiento de ramas y gémulas (ver glosario para la definición de las estructuras implicadas en el crecimiento ligado y no ligado, Apéndice). Respecto a la distribución espacial, $50 \%$ del total de las especies presentan genets con ramets agregados, $30 \%$ presentan una combinación de arreglos agregados-entremezclados y $20 \%$ presentan arreglos entremezclados (Apéndice).

$\mathrm{Al}$ comparar las poblaciones con diferentes arreglos espaciales de genets y ramets [agregados (Ag), En (entremezcaldos) y $\mathrm{Ag}+\mathrm{En}$ (combinados)] encontramos que las poblaciones de especies con crecimiento ligado y no ligado y 
Cuadro 1. Número (observado y esperado) de poblaciones con diferentes tipos de crecimiento clonal de acuerdo con la clasificación de Tiffney y Niklas (1985) ( $\mathrm{Lg}=$ ligado, Lg+NI = ligado y no ligado, $\mathrm{Nl}=$ no ligado), que mostraron arreglos espaciales de los ramets de distintos genets: Ag = agregados, Ag+En = combinación de agregados y entremezclados, y En = entremezclados (figura 2). Los valores entre paréntesis indican el número de especies y familias (especies/familias) representadas en cada celda. Los valores de los residuales ajustados en negritas indican las celdas que contribuyen significativamente al valor de $\chi^{2}$, el signo indica mayor (sin signo) o menor número (-) de poblaciones de las esperadas por azar.

\begin{tabular}{|c|c|c|c|}
\hline Observados & Ligado & Ligado+No ligado & No ligado \\
\hline Agregado & $49(21 / 13)$ & $7(2 / 2)$ & $4(2 / 2)$ \\
\hline Agregado+entremezclado & $11(5 / 5)$ & $25(5 / 5)$ & $7(3 / 3)$ \\
\hline Entremezclado & $10(5 / 5)$ & $2(2 / 1)$ & $7(2 / 2)$ \\
\hline \multicolumn{4}{|l|}{ Esperados } \\
\hline & $\operatorname{Lg}$ & $\mathrm{Lg}+\mathrm{NI}$ & $\mathrm{Nl}$ \\
\hline Agregado & 34 & 17 & 9 \\
\hline Agregado+entremezclado & 25 & 12 & 6 \\
\hline Entremezclado & 11 & 5 & 3 \\
\hline \multicolumn{4}{|l|}{ Residuales ajustados } \\
\hline & $\operatorname{Lg}$ & $\mathrm{Lg}+\mathrm{NI}$ & $\mathrm{Nl}$ \\
\hline Agregado & 5.34 & -3.93 & -2.48 \\
\hline Agregado+entremezclado & -5.24 & 5.50 & 0.35 \\
\hline Entremezclado & -0.46 & -1.83 & 2.95 \\
\hline
\end{tabular}

de especies que sólo presentan crecimiento no ligado presentaron arreglos espaciales (AER) más entremezclados que las poblaciones de especies que sólo presentan crecimiento ligado ( ${ }^{2}=43.6, P<0.0001,4$ g.l.; Cuadro 1). Predominan los patrones de distribución (PDE) con ramets distribuidos en parches discretos sobre el área ocupada por la población (patrón de distribución espacial agregado, $46 \%$ de las poblaciones); estos parches son multiclonales (con dos o más genets) en la mayoría de los casos y el arreglo espacial de los ramets de distintos genets es agregado (19\% de las poblaciones, $\mathrm{PDE}_{\mathrm{AER}} 2_{\mathrm{Ag}}$, Figura 2), presenta una combinación agregado-entremezclado (10\% de las poblaciones, combinación $\mathrm{PDE}_{\mathrm{AER}} 2_{\mathrm{Ag}}-3_{\mathrm{En}}$, Figura 2) o es entremezclado solamente (3\% de las poblaciones, $\mathrm{PDE}_{\mathrm{AER}} 3_{\mathrm{En}}$, Figura 2). En otras poblaciones donde los ramets se distribuyen formando parches discretos el arreglo espacial de los ramets de distintos genets es agregado, pero los parches son multiclonales y monclonales (9\% de las poblaciones, combinación $\mathrm{PDE}_{\mathrm{AER}}$ $1_{\mathrm{Ag}}-\mathrm{Ag}_{\mathrm{Ag}}$, Figura 2) o sólo son monoclonales (5\% de las poblaciones, $\mathrm{PDE}_{\mathrm{AER}} 1_{\mathrm{Ag}}$, Figura 2).

Los patrones de distribución espacial con ramets distribuidos aleatoriamente sobre el área ocupada por la población (patrón de distribución espacial aleatorio) representaron el $44 \%$ de las poblaciones, predominando el arreglo espacial agregado-entremezclado de los ramets de distintos genets $\left(22 \%\right.$ de las poblaciones, combinación de $\mathrm{PDE}_{\mathrm{AER}}$ $4_{\mathrm{Ag}}-5_{\mathrm{En}}$, Figura 2), el arreglo agregado se presentó en $10 \%$ de las poblaciones ( $\mathrm{PDE}_{\mathrm{AER}} 4_{\mathrm{Ag}}$, Figura 2) y el arreglo entremezclado en $12 \%$ de las poblaciones $\left(\mathrm{PDE}_{\mathrm{AER}} 5_{\mathrm{En}}\right.$, Figura
2). El resto de las poblaciones (10\%) presentaron una combinación de patrones de distribución con ramets distribuidos aleatoriamente sobre una parte del área ocupada por la población y en otra formando parches discretos monoclonales o parches multiclonales con arreglos espaciales agregados y entremezclado de los ramets de distintos genets (combinación de $\mathrm{PDE}_{\mathrm{AER}} 1_{\mathrm{Ag}}-4_{\mathrm{Ag}}, 1_{\mathrm{Ag}}-5_{\mathrm{En}}, 2_{\mathrm{Ag}}-4_{\mathrm{Ag}}, 2_{\mathrm{Ag}}-5_{\mathrm{En}}$, Figura 2).

Las poblaciones de especies con una combinación de tipos de crecimiento clonal ligado y no ligado $(\mathrm{Lg}-\mathrm{Nl})$ presentaron valores más bajos de la proporción de genotipos distinguibles (promedio \pm E.E., $G / N=0.24 \pm 0.04 ; H=$ 6.93, $P=0.031,2$ g.l.) que las poblaciones de especies con crecimiento ligado (promedio \pm E.E., $G / N=0.38 \pm 0.03$ ) y con crecimiento no ligado (promedio \pm E.E., $G / N=0.39 \pm$ 0.06 ), pero las diferencias sólo fueron significativas con respecto a las poblaciones de especies con crecimiento ligado (Figura 3a). Respecto al arreglo espacial de los ramets de distintos genets, las poblaciones con una combinación de arreglos agregado y entremezclado (Ag-En) presentaron valores menores del índice de diversidad de Simpson (promedio \pm E.E., $D=0.57 \pm 0.05 ; H=13.2, P=0.0014,2$ g.l.) que las poblaciones con arreglos agregados (promedio \pm E.E., $D=0.80 \pm 0.04$ ) y con arreglos entremezclados (promedio \pm E.E., $D=0.76 \pm 0.06$; Figura 3). Estas diferencias sugieren que el reclutamiento sexual es más bajo en poblaciones de especies que presentan una combinación de formas de crecimiento clonal ligado-no ligado ( $\mathrm{Lg}-\mathrm{Nl}$ ) y una combinación de arreglos de los ramets de distintos genets agregado-entremezclado (Ag-En), sin embargo estas diferencias 

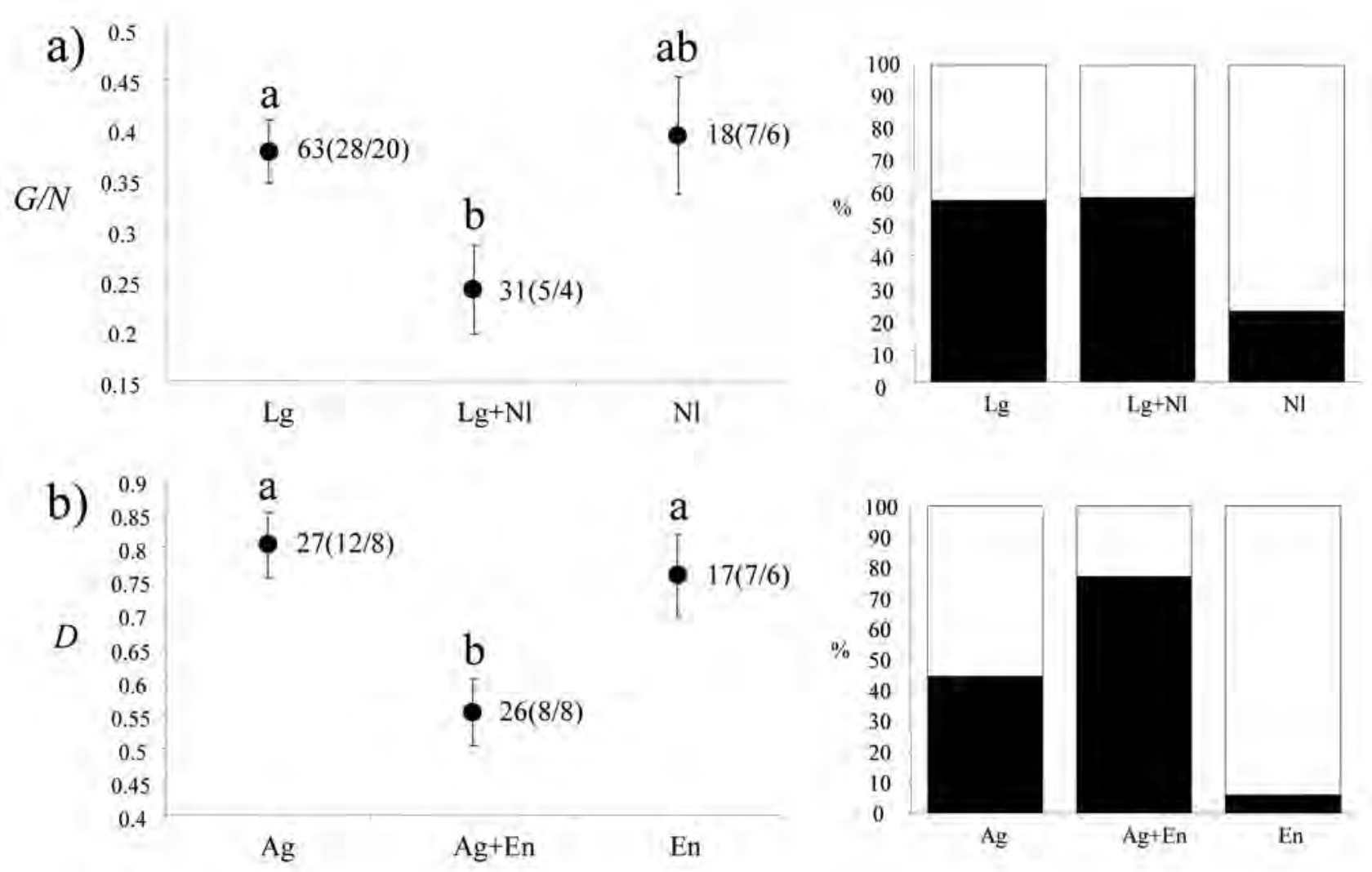

Figura 3. Comparación de la diversidad clonal (promedio \pm EE) entre poblaciones de especies con a) distinto tipo de crecimiento clonal: $\mathrm{Lg}=$ ligado, $\mathrm{Lg}+\mathrm{Nl}$ = con ambos tipos de crecimiento y $\mathrm{Nl}$ = no ligado y b) con distintos arreglos espaciales de los ramets de distintos genets: $\mathrm{Ag}=$ agregados, $\mathrm{Ag}+\mathrm{En}$ = combinación de agregados y entremezclados, y En = entremezclados (Figura 2). En cada grupo se indica el número de poblaciones (número de especies / número de familias) y los grupos que difieren significativamente se indican con una letra distinta arriba de la barra del promedio \pm E.E. de cada grupo. Las gráficas de barras del lado derecho muestran el porcentaje de poblaciones con polinización abiótica (en negro) y con polinización biótica (en blanco).

pueden estar asociadas con otros factores como el tipo de polinización, y no con el tipo de crecimiento y el arreglo espacial de los ramets de distintos genets. La mayoría de las poblaciones con tipo de crecimiento $\mathrm{Lg}-\mathrm{Nl}$ y arreglos de ramets de distintos genets Ag-En corresponden a especies con polinización abiótica (Figura 3).

\section{Discusión}

El efecto que tienen los modos de clonalidad en las plantas en la conformación de distintos arreglos espaciales de genets y ramets es un campo relativamente novedoso, que se ha explorado en los últimos 20 años y la mayoría de los estudios que hay sobre el tema se han desarrollado después de 1990. Widén et al. (1994) reportan que de 40 estudios que comprenden un total de 45 especies de plantas clonales, menos de la tercera parte proporcionan información sobre el arreglo espacial de los genets en las poblaciones y sólo en $12.5 \%$ se construyeron planos de distribución de todos los ramets, dentro de las parcelas o poblaciones, identificando los genotipos multilocus. En esta revisión, en el $98 \%$ de los estudios incluidos se identifican los genotipos multilocus mediante marcadores dominantes, codominantes o ambos siguiendo tres métodos de muestreo: en el 56\% de los estudios se utilizan parcelas, en el $24 \%$ transectos y en el $18 \%$ se muestrean todos o varios individuos de las poblaciones. El 2\% restante está representado por un estudio donde el patrón de distribución es descrito y la distribución de los ramets se sugiere por experimentos de cruzas compatibles entre ramets cercanos (en Scirpus maritimus, Charpentier et al., 2000).

A pesar de que en la actualidad se cuenta con más información sobre los patrones de distribución espacial en poblaciones de especies clonales, hacer comparaciones de dichos patrones considerando especies con distintas formas de crecimiento clonal para analizar las consecuencias reproductivas y genéticas del arreglo espacial de los genets y los ramets continúa siendo un reto. Algunos factores que influyen en este hecho son que la evaluación de la variación en los patrones de distribución espacial en plantas clonales 
enfrenta la dificultad que implica trabajar con la mayoría de los individuos en muchas poblaciones, principalmente a nivel molecular. Aunque en la actualidad las herramientas moleculares se han vuelto más accesibles, trabajar con un gran número de ejemplares sigue siendo difícil y costoso. Otros problemas son la falta de estimadores numéricos comparables para describir la distribución espacial en las poblaciones de diferentes especies y la variación en los métodos de muestreo. Por ejemplo, el muestreo mediante transectos colectando muestras de ramets cada determinada distancia (p. ej., Jonsson et al., 1996; Esselman et al., 1999) permite evaluar la capacidad de expansión longitudinal de los genets, pero no permite tener una descripción de la expansión radial y por lo tanto una evaluación más precisa del nivel de entremezcla de los ramets de distintos genets. En contraste, el muestreo de todos o de un gran número de individuos dentro de parcelas o en las áreas ocupadas por las poblaciones es otro método empleado en estudios con especies de plantas clonales (p. ej., Shimizu et al., 2002; Ruggieiro et al., 2005). Este tipo de muestreo puede ser un método más adecuado para analizar y comparar la estructura espacial clonal, porque permite detectar el arreglo radial de distintos genets. Sin embargo, los tamaños de muestra muy grandes pueden limitar la aplicación de este método, ya que en la mayoría de los casos es necesario emplear marcadores moleculares para identificar a los genets.

En particular, además de los problemas mencionados anteriormente es importante considerar las dificultades adicionales que detectamos en este estudio y que pueden afectar los patrones encontrados a través de nuestra aproximación. La mayoría de los estudios que analizan la distribución espacial de genets y ramets parecen estar sesgados hacia especies de herbáceas perennes con crecimiento clonal ligado (45\% de las especies), predominando las especies rizomatosas (43\% de las especies), mientras que las especies con crecimiento no ligado están poco representadas, impidiendo contar con tamaños de muestra equivalentes para hacer comparaciones más precisas. Por otro lado, la aproximación que decidimos emplear en este trabajo compara a las poblaciones más que a las especies, debido a que hemos detectado que hay una importante variación intraespecífica tanto en los patrones de distribución como en los patrones reproductivos. No obstante, esta variación se omite en algunos estudios o está mejor representada para algunas especies.

Los resultados obtenidos en este trabajo sugieren que las especies que se propagan por crecimento no ligado tienden a formar arreglos espaciales de los ramets de distintos genets más entremezclados como se ha planteado (Gabrielsen y Brochmann, 1998). No obstante, en especies con crecimiento ligado que además se propagan clonalmente por crecimiento no ligado, como el desprendimiento de ramas, el cual consideramos en este estudio como una forma de crecimiento no ligado en los análisis, debido a que los ramets originados por este medio se desarrollan hasta individuos com- pletos separados de la planta madre y a que su dispersión no depende del crecimiento horizontal de tallos y raíces (p.ej., Lophocereus schottii, Parker y Hamrick, 1992; Pteridium aquilinum, Parks y Werth, 1993; Cladium jamaicense, Ivey y Richards, 2001; Ferocactus robustus, Carrillo-Angeles et al., 2011), o que se propagan por la extensión de rizomas en especies con crecimiento en guerrilla (p. ej., Cymodocea nodosa, Ruggieiro et al., 2005 e Ilex leucoclada, Torimaru y Tomaru, 2005) también forman arreglos de genets entremezclados. Algunos factores externos que pueden influir en el arreglo entremezclado de genets son la dispersión por actividades humanas (Parks y Werth, 1993; Brzosko et al., 2002), el acarreo de propágulos vegetativos por animales y corrientes de agua (Parker y Hamrick, 1992; Brzosko et al., 2002), el reclutamiento de nuevos genets en áreas perturbadas donde permanecen fragmentos de clones que ocuparon grandes extensiones (Parks y Werth, 1993) y la dispersión de semillas producidas por autogamia que presentan genotipos multilocus idénticos (Keane et al., 1999; Brzosko et al., 2002).

Respecto a la hipótesis de que las poblaciones con arreglos espaciales entremezclados de los ramets de distintos genets presentan mayor entrecruza, medido indirectamente mediante la diversidad genotípica, nuestros resultados concuerdan parcialmente con el patrón esperado. El G/N es significativamente mayor en los arreglos de especies con producción de vástagos no ligados, en donde predominan los arreglos de genets entremezclados. Para probar esta hipótesis consideramos que si la entrecruza es más alta en poblaciones con arreglos entremezclados, habría mayores niveles de diversidad genotípica. Sin embargo, ésto no se refleja en el caso del índice de Simpson. Las diferencias en los índices de diversidad genotípica entre las poblaciones de especies que combinan el crecimiento clonal ligado-no ligado ( $\mathrm{Lg}$ $\mathrm{Nl})$ y el arreglo espacial agregado-entremezclado (Ag-En), respecto de las poblaciones que presentan uno u otro tipo de crecimiento y de arreglo espacial están asociadas con el tipo de polinización (biótica o abiótica), lo cual apoya el planteamiento de que la polinización biótica puede incrementar la eficiencia de la fertilización (Barret et al.,1993; Richards, 1997; Reusch, 2003). Los análisis para probar este planteamiento están fuera del alcance del presente estudio.

Además de los efectos sobre el reclutamiento sexual y la diversidad genotípica, los patrones de distribución espacial en especies clonales pueden afectar la diversidad y la estructura genética de las poblaciones. Por ejemplo, la formación de parches monoclonales en especies con entrecruza obligada, aunada al desplazamiento de genets por competencia, puede eliminar la capacidad de una población para reproducirse sexualmente en tiempos relativamente cortos (Honnay y Bossuyt, 2005). Adicionalmente, una reducción del reclutamiento sexual y una dispersión restringida de polen, semillas y propágulos vegetativos pueden generar estructura genética local en las poblaciones. Este segundo aspecto se 
evalúa en varios de los estudios revisados (47\%). La estructura genética local tanto a nivel de genets (indicando una dispersión restringida de polen, semillas o ambos) como de ramets (indicando dispersión restringida de propágulos vegetativos) se detectó en 46 poblaciones distribuidas en 16 especies; 28 poblaciones corresponden a cinco especies arbóreas que muestran estructura genética local a distancias menores de $50 \mathrm{~m}$ (Sequoia sempervirens, Douhovnikoff et al., 2004) y a distancias menores de $25 \mathrm{~m}$ (Eurya emarginata, Chung y Epperson, 2000; Cryptomeria japonica, Shimizu et al., 2002; Hirayama y Sakimoto, 2008; Populus tremula, Suvanto y Latva-Karjantvaa, 2005); tres poblaciones corresponden a dos especies arbustivas (Echinosophora koreensis, Chung et al., 2006; Stenocereus eruca, ClarkTapia et al., 2005) que presentan estructura genética local a distancias menores de $20 \mathrm{~m}$. El resto de las poblaciones corresponden a hierbas perennes que muestran estructura genética local desde distancias menores de $30 \mathrm{~m}$, pero más frecuentemente a distancias menores de $15 \mathrm{~m}$.

En promedio, los valores del índice de diversidad de Simpson $(D)$ y la proporción de genotipos distinguibles $(G / N)$ fueron altos en las poblaciones representadas en los estudios incluidos en la presente revisión y son similares a los reportados para 21 especies de plantas clonales $(D=$ 0.62 y $G / N=0.16$ ) por Ellstrand y Roose (1987). Se han planteado algunas hipótesis sobre los escenarios que pueden explicar altos niveles de diversidad genotípica: (i) las poblaciones pudieron ser fundadas por varios propágulos sexuales que se mantienen por clonalidad, (ii) el reclutamiento sexual fue más frecuente en el pasado, aunque en la actualidad la producción de descendientes sexuales no sea evidente (Ellstrand y Roose, 1987; Eriksson, 1989, 1993; Widén et al., 1994); (iii) los genotipos heterócigos se ven favorecidos selectivamente, manteniendo altos niveles de diversidad genética (Widén et al., 1994); (iv) los riesgos de mortalidad entre los ramets de un genet pueden ser distintos si tienen independencia física y fisiológica (Eriksson 1989, 1993, Pan y Price, 2002), lo cual puede reducir las probabilidades de supervivencia / extinción del genet; (v) en especies con ciclos de vida largos los eventos raros de reclutamiento sexual pueden ser suficientes para mantener niveles altos de diversidad genética (Eriksson 1989, 1993, Parker y Hamrick, 1992; Brzosko et al., 2002; Clark-Tapia et al., 2005); (vi) los genotipos pueden mantenerse a través de la supervivencia de algunos ramets el tiempo suficiente para que algunos genotipos se integren a la población de manera esporádica; (vii) la heterogeneidad ambiental puede generar condiciones de selección diversificante que permiten la coexistencia de varios genotipos (Widén et al., 1994). Otra posibilidad para el mantenimiento de diversidad clonal en poblaciones de plantas, que surge al considerar la variación de la distribución espacial de genets y ramets, es que la clonalidad puede mantener altos niveles de entrecruza e incrementar las probabilidades de reclutamiento sexual si los ramets se dispersan hacia vecindarios genéticamente variables, debido a la distribución entremezclada de genets.

Las explicaciones a los niveles relativamente altos de diversidad genotípica en las poblaciones de las especies incluidas en esta revisión comprenden los eventos esporádicos de reclutamiento sexual y el establecimiento inicial de varios genets. En algunos casos se sugiere un reclutamiento sexual frecuente a partir de los altos niveles de diversidad genotípica detectados con los marcadores moleculares (p. ej., Parks y Werth, 1993; Diggle et al., 1998; Misuki et al., 2010). No obstante, el reclutamiento sexual en poblaciones naturales sólo se reporta en Prunus ssiori, Rubus saxatilis (Eriksson y Bremer, 1993), Cypripedium calceolus (Brzosko et al., 2002) y Maianthemum dilatatum (Wilson et al., 2005b). Otros factores que se proponen para explicar la diversidad genotípica son que la integración fisiológica entre los ramets de un genet puede reducir el riesgo de que un genotipo en particular desaparezca, cuando las condiciones ambientales locales son adversas, contribuyendo a mantener la diversidad genética (Hartnett y Bazzaz, 1985), el incremento en la tasa de entrecruza, debido a la entremezcla de genets en especies con crecimiento en guerrilla (Ruggieiro et al., 2005) y en especies que se propagan clonalmente por desprendimiento de ramas y producción de bulbilos (Parker y Hamrick, 1992; Gabrielsen y Brochmann, 1998).

El estudio de los patrones de distribución espacial en especies clonales es importante para analizar las consecuencias reproductivas y genéticas del arreglo espacial de los genets y los ramets en las poblaciones naturales. Estas consecuencias pueden explorarse de una manera más precisa analizando la variación de la distribución de genets y ramets entre las poblaciones de una misma especie. Sin embargo, la evidencia de estos aspectos en organismos clonales aún es escasa y hace falta realizar estudios considerando diferentes tipos de crecimiento clonal. El análisis de los patrones de distribución espacial pueden ser muy útiles para evaluar otros aspectos del ciclo de vida de las especies clonales como el efecto de una variación persistente en la producción de propágulos clonales y sexuales entre hábitats, que puede afectar diferencialmente la dinámica de las poblaciones. Asimismo, es necesario analizar la dinámica temporal de los arreglos espaciales de los genets y ramets en las poblaciones para hacer inferencias adecuadas sobre las consecuencias ecológicas y evolutivas de la clonalidad.

\section{Agradecimientos}

Proyectos: IN IN205007 Efecto de la estructura clonal sobre la dinámica poblacional y la reproducción de una cactácea con ciclo de vida complejo, Opuntia microdasys.

Becas: doctorado Conacyt a I. Carrillo-Angeles y estancia sabática en New Mexico State University, otorgada por DGAPA-PASPA- Universidad Nacional Autónoma de México a M. Mandujano. Este trabajo es parte de la tesis de 
doctorado de Israel Gustavo Carrillo Angeles, Posgrado en Ciencias Biológicas de la UNAM. Agradecemos la revisión, sugerencias en el trabajo así como el apoyo de los miembros del comité tutoral, Doctores Teresa Valverde y Francisco Molina. Asimismo a la Dra. Ana Mendoza, Dr. Jordan Golubov y dos revisores anónimos por sus sugerencias al artículo.

\section{Literatura citada}

Aarssen L.W. 2008. Death without sex-the 'problem of the small' and selection for reproductive economy in flowering plants. Evolutionary Ecology 22:279-298.

Albert T., Raspé O. y Jacquemart A.L. 2003. Clonal structure in Vaccinium myrtillus L. revealed by RAPD and AFLP markers. International Journal of Plant Sciences 164:649-655.

Arens P., Grashof-Bokdam C.J., van der Sluis T. y Smulders M.J.M. 2005. Clonal diversity and genetic differentiation of Maianthemum bifolium among forest fragments of different age. Plant Ecology 179:169-180.

Arizaga S. y Ezcurra E. 2002. Propagation mechanisms in Agave macrovantha (Agavaceae), a tropical arid land succulent rosette. American Journal of Botany 89:632-641.

Barbour M.G., Burk J.H. y Pitts W.D. 1987. Terrestrial Plant Ecology. The Benjamin-Cummings Publishing Company, Menlo Park.

Barret S.C.H., Eckert C.G. y Husband B.C. 1993. Evolutionary processes in aquatic plant populations. Aquatic Botany 44:105-145.

Bell A.D. 1984. Dynamic morphology: A contribution to plant population ecology. En: Dirzo R. y Sarukhán J. Eds. Perspectives on Plant Population Ecology, pp. 49-65, Sinauer Associates Inc. Publisher, Sunderland.

Brzosko E., Wróblewska A. y Ratkiewicz M. 2002. Spatial genetic structure and clonal diversity of island populations of lady's slipper (Cyperidium calceolus) from the Biebrza National Park (northeast Poland). Molecular Ecology 11:2499-2509.

Bushakra J.M., Hodges S.A., Cooper J.B. y Kaska D.D. 1999. The extent of clonality and genetic diversity in the Santa Cruz Island ironwood, Lyonothamnus floribundus. Molecular Ecology 8:471-475.

Camacho F.J. y Liston A. 2001. Population structure and genetic diversity of Botrychium pumicola (Ophioglossaceae) based on inter-simple sequence repeats (ISSR). American Journal of Botany 88:1065-1070.

Carrillo-Angeles I.G., Mandujano M.C. y Golubov J. 2011. Influences of the genetic neighborhood on ramet reproductive success in a clonal desert cactus. Population Ecology 53:449458.

Caswell H. 1985. The evolutionary demography of clonal reproduction. En: Jackson J.B.C., Buss L.W. y Cook R.E. Eds. Population Biology and Evolution of Clonal Organisms, pp. 187224, Yale University Press, New Haven.

Charpentier A. 2002. Consequences of clonal growth for plant mating. Evolutionary Ecology 15:521-530.

Charpentier A., Grillas P. y Thompson J.D. 2000. The effects of population size limitation on fecundity in mosaic populations of the clonal macrophyte Scirpus maritimus (Cyperaceae). American Journal of Botany 87:502-507.

Chen J. y Bradshaw G.A. 1999. Forest structure in space: a case study of an old growth spruce-fir forest in Changbaishan Natural Reserve, PR China. Forest Ecology and Management 120:219-233.

Chung M.G. y Epperson B.K. 2000. Clonal and spatial genetic structure in Eurya emarginata (Theaceae). Heredity 84:170177.

Chung J.M., Lee B.C., Kim J.S., Park C., Chung M.Y. y Chung M.G. 2006. Fine-scale genetic structure among genetic individuals of the clone-forming monotypic genus Echinospora koreensis (Fabaceae). Annals of Botany 98:165-173.

Clark P.J. y Evans F.C. 1954. Distance to nearest neighbor as a measure of spatial relationships in populations. Ecology 35:445-453.

Clark-Tapia R., Alfonso-Corrado C., Eguiarte L.E. y Molina-Freaner F. 2005. Clonal diversity and distribution in Stenocereus eruca (Cactaceae), a narrow endemic cactus of the Sonoran Desert. American Journal of Botany 92:272-278.

Clark-Tapia R., Alfonso-Corrado C., Mandujano M.C. y MolinaFreaner F. 2006. Reproductive consequences of clonal growth in Stenocereus eruca, a rare clonal cactus of the Sonoran desert. Evolutionary Ecology 20:131-142.

Couteron P. y Kokou K. 1997. Woody vegetation spatial patterns in a semi-arid savanna of Burkina Faso, West Africa. Plant Ecology 132:211-227.

Damman H. y Cain M.L. 1998. Population growth and viability analyses of the clonal woodland herb, Asarum canadense. Journal of Ecology 86:13-26.

de Jong T.J., Waser N.M., Price M.V. y Ring R.M. 1992. Plant size, geitonogamy and seed set in Ipomopsis aggregata. Oecologia 89:310-315.

de Kroon H. y van Groenendael J. Eds. 1997. The Ecology and Evolution of Clonal Plants. Backhuys Publishers, Leiden.

de Kroon H. y Hutchings M.J. 1995. Morphological plasticity in clonal plants: the foraging concept reconsidered. Journal of Ecology 83:143-152.

Diggle P.K., Lower S. y Ranker T.A. 1998. Clonal diversity in alpine populations of Polygonum viviparum (Polygonaceae). International Journal of Plant Sciences 159:606-615.

Diggle P.K., Meixner M.A., Carroll A.B. y Aschwanden C.F. 2002. Barriers to sexual reproduction in Polygonum viviparum: A comparative developmental analysis of $P$. viviparum and $P$. bistortoides. Annals of Botany 89:145-156.

Douhovnikoff V. y Dood R.S. 2003. Intra-clonal variation and a similarity threshold for identification of clones: application to $\mathrm{Sa}$ lix exigua using AFLP molecular markers. Theoretical Applied Genetics 106:1307-1315.

Douhovnikoff V., Cheng A.M. y Dodd R.S. 2004. Incidence, size and spatial structure of clones in second-growth stands of coast redwood, Sequoia sempervirens (Cupressaceae). American Journal of Botany 91:1140-1146.

Douhovnikoff V., McBride J.R. y Dodd R.S. 2005. Salix exigua clonal growth and population dynamics in relation to disturbance regime variation. Ecology 86:446-452.

Eckert C.G. 2000. Contributions of autogamy and geitonogamy to self-fertilization in a mass-flowering, clonal plant. Ecology 81:532-542.

Eckert C.G. 2002. The loss of sex in clonal plants. Evolutionary Ecology 15:501-520.

Ellstrand N.C. y Roose M.L. 1987. Patterns of genotypic diversity in clonal plant species. American Journal of Botany 74:123-131. 
Eriksson O. 1989. Seedling dynamics and life histories in clonal plants. Oikos 55:231-238.

Eriksson O. 1993. Dynamics of genets in clonal plants. Trends in Ecology \& Evolution 8:313-316.

Eriksson O. y Bremer B. 1993. Genet dynamics of the clonal plant Rubus saxatilis. Journal of Ecology 81:533-542.

Esselman E.J., Jianqiang L., Crawford D.J., Winduss J.L. y Wolfe A.D. 1999. Clonal diversity in the rare Calamagrostis porteri ssp insperata (Poaceae): comparative results for allozymes and random amplified polymorphic DNA (RAPD) and intersimple sequence repeat (ISSR) markers. Molecular Ecology 8:443451.

Everitt B.S. 1977. The Analysis of Contingency Tables. Chapmann \& Hall, Bury St Edmunds.

Gabrielsen T.M. y Brochmann C. 1998. Sex after all: high levels of diversity detected in the arctic clonal plant Saxifraga cernua using RAPD markers. Molecular Ecology 7:1701-1708.

Garnier L.K.M., Durand J. y Dajoz I. 2002. Limited seed dispersal and microspatial population structure of an agamospermous grass of West African savannahs, Hyparrhenia diplandra (Poaceae). American Journal of Botany 89:1785-1791.

Gibson D.J. y Menges E.S. 1994. Population structure and spatial pattern in the dioecious shrub Ceratiola ericoides. Journal of Vegetation Sciences 5:337-346.

Gleason H.A. 1920. Some applications of the quadrat method. Bulletin of the Torrey Botanical Club 47:21-33.

Hämmerli A. y Reusch T.B.H. 2003a. Flexible mating: cross-pollination affects sex expression in a marine clonal plant. Journal of Evolutionary Biology 18:1096-1105.

Hämmerli A. y Reusch T.B.H. 2003b. Genetic neighbourhood of clone structures in eelgrass meadows quantified by spatial autocorrelation of microsatellite markers. Heredity 91:448-455.

Handel S.N. 1985. The intrusion of clonal growth patterns on plant breeding system. The American Naturalist 125:367-384.

Harper J. 1985. Modules, branches, and the capture of resources. En: Jackson J.B.C., Buss L.W. y Cook R.E. Eds. Population Biology and Evolution of Clonal Organisms, pp. 1-33, Yale University Press, New Haven.

Hartnett D.C. y Bazzaz F.A. 1985. The integration of neighbourhood effects by clonal genets in Solidago canadensis. The Journal of Ecology 73:415-427.

Hirayama K. y Sakimoto M. 2008. Clonal structure and diversity of Cryptomeria japonica along a slope in a cool-temperate, oldgrowth mixed forest in the snowy region of Japan. Canadian Journal of Forest Research 38:2804-2813.

Honnay O. y Bossuyt B. 2005. Prolonged clonal growth: escape route or route to extinction? Oikos 108:427-432.

Honnay O., Jacquemyn H., Roldán-Ruiz I. y Hermy M. 2006. Consequences of prolonged clonal growth on local and regional genetic structure and fruiting success of the forest perennial Maianthemum bifolium. Oikos 112:21-30.

Honnay O. y Jacquemyn H. 2008. A meta-analysis of the relation between mating system, growth form and genotypic diversity in clonal plant species. Evolutionary Ecology 22:299-312.

Ivey C.T. y Richards J.H. 2001. Genotypic diversity and clonal structure of everglades sawgrass, Cladium jamaicense (Cyperaceae). International Journal of Plant Sciences 162:1327-1335.

Jackson J.B.C., Buss L.W. y Cook R.E. Eds. 1985. Population Biology and Evolution of Clonal organisms. Yale University Press, New Haven.
Jacquemyn H., Brys R., Honnay O., Hermy M. y Roldán-Ruiz I. 2005. Local forest environment largely affects below-ground growth, clonal diversity and fine-scale spatial genetic structure in the temperate deciduous forest herb Paris quadrifolia. Molecular Ecology 14:4479-4488.

Jonsson B.O., Jónsdóttir I.S. y Crongberg N. 1996. Clonal diversity and allozyme variation in populations of the arctic sedge Carex bigelowii (Cyperaceae). Journal of Ecology 84:449-459.

Kays S. y Harper J.L. 1974. The regulation of plant and tiller density in a grass sward. Journal of Ecology 62:97-105.

Keane B., Pelikan S., Toth G.P., Smith M.K. y Rogstad S.H. 1999. Genetic diversity of Typha latifolia (Typhaceae) and the impact of pollutants examined with tamdem-repetitive DNA probes. American Journal of Botany 86:1226-1238.

Kjølner S., Såstad S.M. y Brochmann C. 2006. Clonality and recombination in the arctic plant Saxifraga cernua. Botanical Journal of the Linnean Society 152:209-217.

Klimeš L., Klimešová J., Hendriks R.J.J. y van Groenendael J.M. 1997. Clonal plant architecture: a comparative analysis of form and function. En: de Kroon H. y van Groenendael J. Eds. The Ecology and Evolution of Clonal Plants, pp. 1-29, Backhuys Publishers, Leiden.

Korpelainen H. 1994. Sex ratios and resource allocation among sexually reproducing plants of Rubus chamaemorus. Annals of Botany 74:627-632.

Korpelainen H., Antonious-Klemola K. y Werlemark G. 1999. Clonal structure of Rubus chamaemorus populations: comparison of different molecular methods. Plant Ecology 143:123-128.

Kreher S.A., Foré S.A. y Collins B.S. 2000. Genetic variation within and among patches of the clonal species, Vaccinium stamineum L. Molecular Ecology 9:1247-1252.

Kudoh H., Shibaike H., Takasu H., Whigham D.F. y Kawano S. 1999. Genet structure and determinants of clonal structure in a temperate deciduous woodland herb, Uvularia perfoliata. Journal of Ecology 87:244-257.

Liston A., Wilson B.L., Robinson W.A., Doescher P.S., Harris N.R. y Svejcar T. 2003. The relative importance of sexual reproduction versus clonal spread in an arid bunchgrass. Oecologia 137:216-225.

Liu J., Dong M., Miao S.L., Li Z.Y., Song M.H. y Wang R.Q. 2006. Invasive alien plants in China: role of clonality and geographical origin. Biological Invasions 8:1461-1470.

Mandujano M.C., Montaña C., Méndez I. y Golubov J. 1998. The relative contributions of sexual reproduction and clonal propagation in Opuntia rastrera from two habitats in the Chihuahuan desert. Journal of Ecology 86:911-921.

Mandujano M.C., Montaña C., Franco M., Golubov J. y FloresMartínez A. 2001. Integration of demographic annual variability in a clonal desert cactus. Ecology 82:344-359.

Mandujano-Sánchez M.C. 2007. La clonalidad y sus efectos en la biología de poblaciones. En: Eguiarte L.E., Souza V. y Aguirre X. Comp. Ecología Molecular, pp. 215-250, Secretaría de Medio Ambiente y Recursos Naturales-Instituto Nacional de Ecología-Universidad Nacional Autónoma de México-Comosión Nacional para el Conocimiento y Uso de la Biodiversidad, México, D.F.

Mayes S.G., McGinley M.A. y Werth C.R. 1998. Clonal population structure and genetic variation in sand-shinnery oak, Quercus havardii (Fagaceae). American Journal of Botany 85:1609-1617. McFadden C.S. 1991. A comparative demographic analysis of clonal 
reproduction in a temperate soft coral. Ecology 72:1849-1866.

Misuki I., Ishida K., Tani N. y Tsumura Y. 2010. Fine-scale spatial structure of genets and sexes in the dioecious plant Dioscorea japonica, which disperses by both bulbils and seeds. Evolutionary Ecology 24:1399-1415.

Mori Y., Nagamitsu T. y Kubo T. 2009. Clonal growth and its effects on male and female reproductive success in Prunus ssiori (Rosaceae). Population Ecology 51:175-186.

Nagamitsu T., Ogawa M., Ishida K. y Tanouchi H. 2004. Clonal diversity, genetic structure, and mode of recruitment in a Prunus ssiori population established after volcanic eruptions. Plant Ecology 174:1-10.

Okuda T., Kachi N., Yap S.K. y Manokaran N. 1997. Tree distribution pattern and fate of juveniles in a lowland tropical rain forest - implications for regeneration and maintenance of species diversity. Plant Ecology 131:155-171.

Pan J.J. y Price J.S. 2002. Fitness and evolution in clonal plants: the impact of clonal growth. Evolutionary Ecology 15:583-600.

Parker K.C. y Hamrick J.L. 1992. Genetic diversity and clonal structure in a columnar cactus, Lophocereus schottii. American Journal of Botany 79:86-96.

Parks C. y Werth C.R. 1993. A study of spatial features of clones in a population of bracken fern, Pteridium aquilinum (Dennstaedtiaceae). American Journal of Botany 80:537-544.

Peterson C.J. y Jones R.H. 1997. Clonality in woody plants: a review and comparison with clonal herbs. En: de Kroon H. y van Groenendael J. Eds. The Ecology and Evolution of Clonal Plants, pp. 263-289, Backhuys Publishers, Leiden.

Pfeiffer T., Klahr A., Heinrich A. y Schnittler M. 2011. Does sex make a difference? Genetic diversity and spatial genetic structure in two co-occurring species of Gagea (Liliaceae) with contrasting reproductive strategies. Plant Systematics and Evolution 292:189-201.

Pornon A. y Escaravage N. 1999. Genotypic structure in clonal Rhododendron ferrugineum L. (Ericaceae) populations: origin and manteinance. Plant Ecology 141:145-150.

Pornon A., Escaravage N., Thomas P. y Taberlet P. 2000. Dynamics of genotypic structure in clonal Rhododendron ferrugineum (Ericaceae) populations. Molecular Ecology 9:1099-1111.

Pornon A., Escaravage N., Till-Botraud I. y Doche B. 1997. Variation of reproductive traits in Rhododendron ferrugineum $\mathrm{L}$. (Ericaceae) populations along a successional gradient. Plant Ecology 130:1-11.

Price E.A.C. y Marshall C. 1999. Clonal plants and environmental heterogeneity. Plant Ecology 141:3-7.

Reisch C., Schurm S. y Poschlod P. 2007. Spatial genetic structure and clonal diversity in an alpine population of Salix herbacea (Salicaceae). Annals of Botany 99:647-651.

Reusch T.B.H. 2003. Floral neighbourhoods in the sea: how floral density, opportunity for outcrossing and population fragmentation affect seed set in Zostera marina. Journal of Ecology 91:610-615.

Richards A.J. 1997. Plant Breeding Systems. Chapman \& Hall,
Londres.

Rosseto M., Gross C.L., Jones R. y Hunter J. 2004. The impact of clonality on an endangered tree (Elaeocarpus williamsianus) in a fragment rain forest. Biological Conservation 117:33-39.

Ruggieiro M.V., Capone S., Pirozzi P., Reusch T.B.H. y Procaccini G. 2005. Mating system and clonal architecture: a comparative study in two marine angiosperms. Evolutionary Ecology 19:487-499.

Shimizu Y, Ando M. y Sakai F. 2002. Clonal structure of natural populations of Cryptomeria japonica growing at different positions on slopes, detected using RAPD markers. Biochemical Systematics and Ecology 30:733-748.

Shreve F. 1917. The establishment of desert perennials. Journal of Ecology 5:210-216.

Sipes S.D. y Wolf P.G. 1997. Clonal structure and patterns of allozyme diversity in the rare endemic Cycladenia humilis var. jonesii (Apocynaceae). American Journal of Botany 84:401-409.

Stehlik I. y Holderegger R. 2000. Spatial genetic structure and clonal diversity of Anemone nemorosa in late successional deciduous woodlands of Central Europe. Journal of Ecology 88:424-435.

Suvanto L.I. y Latva-Karjantvaa T.B. 2005. Clone identification and clonal structure of the European aspen (Populus tremula). Molecular Ecology 14:2851-2860.

Tiffney B.H. y Niklas K.J. 1985. Clonal growth in land plants: a paleobotanical perspective. En: Jackson J.B.C., Buss L.W. y Cook R.E. Eds. Population Biology and Evolution of Clonal Organisms, pp. 35-66, Yale University Press, New Haven.

Tooke F., Ordidge M., Chiurugwi T. y Battey N. 2005. Mechanisms and function of flower and inflorescence reversion. Journal of Experimental Botany 56:2587-2599.

Torimaru T. y Tomaru N. 2005. Fine-scale clonal structure and diversity within patches of a clone-forming dioecious shrub, Ilex leucoclada (Aquifoliaceae). Annals of Botany 95:295-304.

van Dijk P.J. y Bakx-Schotman J.M.T. 2004. Formation of unreduced megaspores (diplospory) in apomictic dandelions (Taraxacum officinale, s.l.) is controlled by a sex-specific dominant locus. Genetics 166:483-492.

van Groenendael J.M. y de Kroon H. 1990. Clonal Growth in Plants: Regulation and Function. SPB Academic Publishing, La Haya.

van Groenendael J.M., Klimeš L., Klimešová J. y Hendriks R.J.J. 1996. Comparative ecology of clonal plants. Philosophical Transactions of Royal Society of London 351:1331-1339.

Widén B., Cronberg N. y Widén M. 1994. Genotypic diversity, molecular markers and spatial distribution of genets in clonal plants, a literature survey. Folia Geobotanica y Phytotaxonomica 29:245-263.

Wilson A.S.G., van der Kamp B.J. y Ritland C. 2005a. Opportunities for geitonogamy in the clonal herb Maianthemum dilatatum. Canadian Journal of Botany 83:1082-1087.

Wilson A.S.G., van der Kamp B.J. y Ritland C. 2005b. Spatial genetic and clonal structure in Maianthemum dilatatum as defined by AFLP markers. Canadian Journal of Botany 83:1126-1132. 
Apéndice. Características de las especies y poblaciones: $\mathbf{S C R Z}=$ sistema de cruza, $\mathbf{P O L}=$ polinización, $\mathbf{P R O R}=$ estructuras o procesos que dan origen a los ramets, CCT-N = tipo de crecimiento clonal en la clasificación de Tiffney y Niklas (1985), POB/HL = número de población y/o hábitat local, $\mathbf{P D E}=$ clave del patrón de distribución espacial de acuerdo a la figura 2, AER = arreglo espacial de los ramets de distintos genets, $\boldsymbol{D}=$ índice de diversidad clonal de Simpson, $\boldsymbol{G} / \mathbf{N}=$ proporción de genotipos distinguibles, Métodos = métodos empleados para la evaluación de los parámetros. El significado de las abreviaturas empleadas en cada columna se muestra al final del cuadro.

\begin{tabular}{|c|c|c|c|c|c|c|c|c|c|c|c|c|c|}
\hline Especie (Familia) & Hábito & SCRZ & POL & PROR & CCT-N & $\begin{array}{c}\text { POB/ } \\
\mathrm{HL}\end{array}$ & $\begin{array}{c}\text { PDE } \\
\text { (fig. 2) }\end{array}$ & $\begin{array}{c}\text { AER } \\
\text { (fig. 2) }\end{array}$ & $D$ & $G / N$ & $\begin{array}{l}\text { Estructura } \\
\text { genética } \\
\text { local (EGL) }\end{array}$ & $\begin{array}{c}\text { Métodos } \\
\text { PDE, } D, G / N \\
\text { y EGL }\end{array}$ & Referencias \\
\hline $\begin{array}{l}\text { Anemone } \\
\text { nemorosa } \\
\text { (Ranunculaceae) }\end{array}$ & $\mathrm{Hp}$ & A & $\mathrm{Bt}$ & $\begin{array}{c}\mathrm{Rzm+} \\
\mathrm{drm}\end{array}$ & $\mathrm{Lg}+\mathrm{NI}$ & $\begin{array}{l}\text { ppm } \\
(20)\end{array}$ & $(4,5)$ & $\mathrm{Ag}+\mathrm{En}$ & $\mathrm{n} / \mathrm{e}$ & 0.95 & $\begin{array}{l}\operatorname{asp},<1 \mathrm{~m} \\
(\mathrm{rm})\end{array}$ & tra+ISZ+asp & $\begin{array}{l}\text { Stehlik y } \\
\text { Holderegger } \\
\text { 2000* }\end{array}$ \\
\hline $\begin{array}{l}\text { Botrychium } \\
\text { pumicola } \\
\text { (Ophioglossaceae) }\end{array}$ & $\mathrm{Hp}$ & A & $\mathrm{Ab}$ & gmas & $\mathrm{NI}$ & $\begin{array}{l}\text { p } 1 \\
\text { p } 2 \\
\text { p } 3\end{array}$ & $\begin{array}{l}(2,5) \\
(2,5)\end{array}$ & $\begin{array}{l}\mathrm{Ag}+\mathrm{En} \\
\mathrm{Ag}+\mathrm{En} \\
\mathrm{Ag}+\mathrm{En}\end{array}$ & $\begin{array}{c}0.14 \\
0.16 \\
0.1\end{array}$ & $\begin{array}{l}0.93 \\
0.9 \\
0.65\end{array}$ & $\begin{array}{c}\text { mt: no } \\
\text { detectada } \\
\text { mt: no } \\
\text { detectada } \\
\text { mt: no } \\
\text { detectada }\end{array}$ & prc + ISSR $+m t$ & $\begin{array}{c}\text { Camacho y } \\
\text { Liston, 2001* }\end{array}$ \\
\hline $\begin{array}{l}\text { Calamagrostis } \\
\text { porteri ssp. } \\
\text { insperata (Poaceae) }\end{array}$ & $\mathrm{Hp}$ & A & $\mathrm{Ab}$ & rzm & $\operatorname{Lg}$ & $\begin{array}{l}\text { p } 2 \\
\text { p } 3 \\
\text { p } 4\end{array}$ & $\begin{array}{l}2 \\
2 \\
2\end{array}$ & $\begin{array}{l}\mathrm{Ag} \\
\mathrm{Ag} \\
\mathrm{Ag}\end{array}$ & $\begin{array}{l}n / e \\
n / e \\
n / e\end{array}$ & $\begin{array}{c} \\
1 \\
0.82 \\
0.91\end{array}$ & $\begin{array}{l}\mathrm{n} / \mathrm{e} \\
\mathrm{n} / \mathrm{e} \\
\mathrm{n} / \mathrm{e}\end{array}$ & $\begin{array}{c}\text { desc+tra+ } \\
\text { ISRR+ } \\
\text { RAPD }\end{array}$ & $\begin{array}{c}\text { Esselman } \\
\text { et al., } \\
1999\end{array}$ \\
\hline $\begin{array}{l}\text { Carex bigelowii } \\
\text { (Cyperaceae) }\end{array}$ & $\mathrm{Hp}$ & $?$ & $\mathrm{Ab}$ & rzm & $\operatorname{Lg}$ & $\begin{array}{l}\text { p } 1 \\
\text { p } 2 \\
\text { p } 3\end{array}$ & $\begin{array}{l}4 \\
4 \\
4\end{array}$ & $\begin{array}{l}\mathrm{Ag} \\
\mathrm{Ag} \\
\mathrm{Ag}\end{array}$ & $\begin{array}{l}0.98 \\
0.98 \\
0.98\end{array}$ & $\begin{array}{l}0.52 \\
0.63 \\
0.48\end{array}$ & $\begin{array}{l}\text { n/e } \\
\text { n/e } \\
\text { n/e }\end{array}$ & tra+ISZ & $\begin{array}{c}\text { Jonsson } \\
\text { et al., 1996* }\end{array}$ \\
\hline $\begin{array}{l}\text { Cladium jamaicense } \\
\text { (Cyperaceae) }\end{array}$ & $\mathrm{Hp}$ & $?$ & $\mathrm{Ab}$ & $\begin{array}{c}\text { rzm+ } \\
\text { pnt }\end{array}$ & $\mathrm{Lg}+\mathrm{Nl}$ & $\begin{array}{l}p 1 \\
\text { p } 2 \\
\text { p } 3 \\
\text { p } 4 \\
\text { p } 5 \\
\text { p } 6 \\
\text { p } 7 \\
\text { p } 8 \\
\text { p } 9 \\
\text { p } 10 \\
\text { p } 11 \\
\text { p } 12 \\
\text { p } 13 \\
\text { p } 14\end{array}$ & $\begin{array}{l}(4,5) \\
(4,5) \\
(4,5) \\
(4,5) \\
(4,5) \\
1 \\
(4,5) \\
(4,5) \\
(4,5) \\
(4,5) \\
(4,5) \\
(4,5) \\
(4,5) \\
(4,5)\end{array}$ & $\begin{array}{c}A g+E n \\
A g+E n \\
A g+E n \\
A g+E n \\
A g+E n \\
A g \\
A g+E n \\
A g+E n \\
A g+E n \\
A g+E n \\
A g+E n \\
A g+E n \\
A g+E n \\
A g+E n\end{array}$ & $\begin{array}{c}0.236 \\
0.269 \\
0.271 \\
0.682 \\
0.159 \\
0 \\
0.538 \\
0.212 \\
0.771 \\
0.698 \\
0.814 \\
0.682 \\
0.673 \\
0.444\end{array}$ & $\begin{array}{l}0.044 \\
0.044 \\
0.125 \\
0.231 \\
0.043 \\
0.021 \\
0.146 \\
0.068 \\
0.106 \\
0.143 \\
0.261 \\
0.114 \\
0.13 \\
0.043\end{array}$ & $\begin{array}{c}\text { mpic: no } \\
\text { detectada } \\
\text { mpic: no } \\
\text { detectada } \\
\text { mpic: no } \\
\text { detectada } \\
\text { mpic: no } \\
\text { detectada } \\
\text { mpic: no } \\
\text { detectada } \\
\text { n/a } \\
\text { mpic: no } \\
\text { detectada } \\
\text { mpic: no } \\
\text { detectada } \\
\text { mpic: no } \\
\text { detectada } \\
\text { mpic: no } \\
\text { detectada } \\
\text { mpic: no } \\
\text { detectada } \\
\text { mpic: no } \\
\text { detectada } \\
\text { mpic: no } \\
\text { detectada } \\
\text { mpic: no } \\
\text { detectada }\end{array}$ & $\begin{array}{l}\text { tra+lSZ+ } \\
\text { mpic }\end{array}$ & $\begin{array}{l}\text { Ivey y Richards, } \\
\text { 2001* }\end{array}$ \\
\hline
\end{tabular}


Apéndice. Continuación

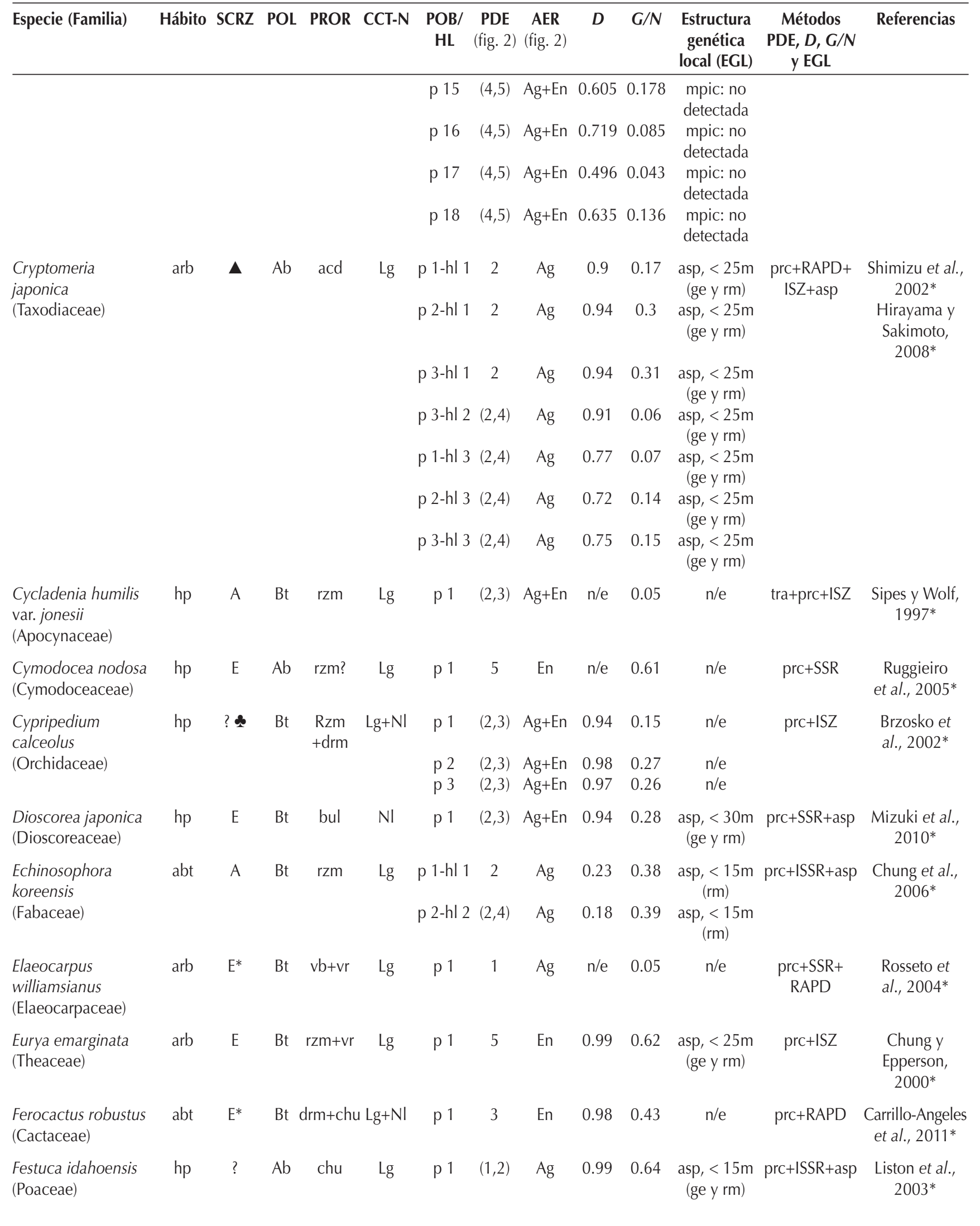


Apéndice. Continuación

\begin{tabular}{|c|c|c|c|c|c|c|c|c|c|c|c|c|c|}
\hline Especie (Familia) & Hábito & SCRZ & POL & PROR & CCT-N & $\begin{array}{c}\mathrm{POB} / \\
\mathrm{HL}\end{array}$ & $\begin{array}{l}\text { PDE } \\
\text { (fig. 2) }\end{array}$ & $\begin{array}{c}\text { AER } \\
\text { (fig. 2) }\end{array}$ & $D$ & $G / N$ & $\begin{array}{l}\text { Estructura } \\
\text { genética } \\
\text { local (EGL) }\end{array}$ & $\begin{array}{c}\text { Métodos } \\
\text { PDE, } D, G / N \\
\text { y EGL } \\
\end{array}$ & Referencias \\
\hline \multirow[t]{3}{*}{$\begin{array}{l}\text { Gagea lutea } \\
\text { (Liliaceae) }\end{array}$} & \multirow[t]{3}{*}{ hg } & \multirow[t]{3}{*}{$?$} & \multirow[t]{3}{*}{$\mathrm{Bt}$} & \multirow[t]{3}{*}{ buls } & \multirow[t]{3}{*}{$\mathrm{Nl}$} & p 1 & $(2,3)$ & $\mathrm{Ag}+\mathrm{En}$ & $\mathrm{n} / \mathrm{e}$ & 0.51 & \multirow{3}{*}{\multicolumn{2}{|c|}{$\begin{array}{l}\text { asp, }<8 \mathrm{~m}, 16 \text { tra+AFLP+asp } \\
\mathrm{a}<24 \mathrm{~m}(\mathrm{rm}) \\
\mathrm{asp},<8 \mathrm{~m}, 16 \\
\mathrm{a}<24 \mathrm{~m}(\mathrm{rm}) \\
\mathrm{asp},<8 \mathrm{~m}, 16 \\
\mathrm{a}<24 \mathrm{~m}(\mathrm{rm})\end{array}$}} & \multirow[t]{3}{*}{$\begin{array}{l}\text { Pfeiffer et al., } \\
2011^{*}\end{array}$} \\
\hline & & & & & & p 2 & $(2,3)$ & $\mathrm{Ag}+\mathrm{En}$ & $\mathrm{n} / \mathrm{e}$ & 0.57 & & & \\
\hline & & & & & & p 3 & $(2,3)$ & $\mathrm{Ag}+\mathrm{En}$ & $\mathrm{n} / \mathrm{e}$ & 0.61 & & & \\
\hline \multirow{3}{*}{$\begin{array}{l}\text { Gagea spathacea } \\
\text { (Liliaceae) }\end{array}$} & \multirow[t]{3}{*}{ hg } & \multirow[t]{3}{*}{$?$} & \multirow[t]{3}{*}{$\mathrm{Bt}$} & \multirow[t]{3}{*}{ buls } & \multirow[t]{3}{*}{$\mathrm{NI}$} & p 1 & 1 & $\mathrm{Ag}$ & $\mathrm{n} / \mathrm{e}$ & 0.001 & $\mathrm{n} / \mathrm{a}$ & \multirow[t]{3}{*}{ tra+AFLP } & \multirow{3}{*}{$\begin{array}{r}\text { Pfeiffer et al., } \\
2011^{*}\end{array}$} \\
\hline & & & & & & p 2 & 1 & $\mathrm{Ag}$ & $\mathrm{n} / \mathrm{e}$ & 0.001 & $\mathrm{n} / \mathrm{a}$ & & \\
\hline & & & & & & p 3 & 1 & $\mathrm{Ag}$ & $\mathrm{n} / \mathrm{e}$ & 0.001 & $\mathrm{n} / \mathrm{a}$ & & \\
\hline $\begin{array}{l}\text { Hyparrhenia } \\
\text { diplandra (Poaceae) }\end{array}$ & hp & $?$ & $\mathrm{Ab}$ & agp & $\mathrm{NI}$ & p 1 & 5 & En & 0.85 & 0.07 & $\begin{array}{l}\text { asp, }<6 \mathrm{~m} \\
\quad(\mathrm{rm})\end{array}$ & $\begin{array}{c}\text { prc+lSZ } \\
+ \text { CLO+asp }\end{array}$ & $\begin{array}{c}\text { Garnier et al., } \\
2002^{*}\end{array}$ \\
\hline $\begin{array}{l}\text { Ilex leucoclada } \\
\text { (Aquifoliaceae) }\end{array}$ & abt & E & $\mathrm{Bt}$ & $\operatorname{acd} \mathbf{\Lambda}$ & $\operatorname{Lg}$ & p 1 & $(2,3)$ & $\mathrm{Ag}+\mathrm{En}$ & 0.67 & 0.09 & $\mathrm{n} / \mathrm{e}$ & prc+RAPD & $\begin{array}{c}\text { Torimaru y } \\
\text { Tomaru, 2005* }\end{array}$ \\
\hline \multirow[t]{8}{*}{$\begin{array}{l}\text { Lophocereus schottii } \\
\text { (Cactaceae) }\end{array}$} & \multirow[t]{8}{*}{ arb } & \multirow[t]{8}{*}{ A } & \multirow[t]{8}{*}{$\mathrm{Bt}$} & \multirow[t]{8}{*}{$\begin{array}{l}\mathrm{drm} \\
+\mathrm{acd}\end{array}$} & \multirow[t]{8}{*}{$\mathrm{Lg}+\mathrm{NI}$} & p 1-hl 1 & 5 & En & 0.95 & 0.64 & $\begin{array}{c}\text { SG: 0-10 } \\
<10-50>\text { más } \\
\text { de } 50 m \text { (ge) }\end{array}$ & \multirow{8}{*}{ prc+ISZ+SG } & \multirow[t]{8}{*}{$\begin{array}{l}\text { Parker y } \\
\text { Hamrick, } \\
1992^{*}\end{array}$} \\
\hline & & & & & & p 2-hl 2 & 2 & $\mathrm{Ag}$ & 0.95 & 0.52 & $\begin{array}{l}\text { SG: } 0-10 \\
<10-50>\text { más } \\
\text { de } 50 \mathrm{~m} \text { (ge) }\end{array}$ & & \\
\hline & & & & & & p 3-hl 3 & 2 & $\mathrm{Ag}$ & 0.93 & 0.65 & $\begin{array}{c}\text { SG: } 0-10 \\
<10-50>\text { más } \\
\text { de } 50 m \text { (ge) }\end{array}$ & & \\
\hline & & & & & & p 4-hl 1 & $(1,2)$ & $\mathrm{Ag}$ & 0.94 & 0.24 & $\begin{array}{l}\text { SG: } 0-10> \\
\text { 10-50>más } \\
\text { de } 50 \mathrm{~m} \text { (ge) }\end{array}$ & & \\
\hline & & & & & & p 5-hl 4 & $(1,2)$ & $\mathrm{Ag}$ & 0.92 & 0.41 & $\begin{array}{l}\text { SG: } 0-10> \\
10-50<\text { más } \\
\text { de } 50 m \text { (ge) }\end{array}$ & & \\
\hline & & & & & & p 6-hl 4 & $(1,2)$ & $\mathrm{Ag}$ & 0.87 & 0.45 & $\begin{array}{c}\text { SG: } 0-10> \\
10-50<\text { más } \\
\text { de } 50 m \text { (ge) }\end{array}$ & & \\
\hline & & & & & & p 7-hl 2 & $(1,2)$ & $\mathrm{Ag}$ & 0.8 & 0.18 & $\begin{array}{l}\text { SG: } 0-10> \\
\text { 10-50>más } \\
\text { de } 50 \mathrm{~m} \text { (ge) }\end{array}$ & & \\
\hline & & & & & & p 8-hl 5 & $(1,5)$ & $\mathrm{Ag}+\mathrm{En}$ & 0.59 & 0.36 & $\begin{array}{l}\text { SG: } 10-50= \\
\text { más de } 50 \mathrm{~m} \\
\quad(\text { ge })\end{array}$ & & \\
\hline $\begin{array}{l}\text { Lyonothamnus } \\
\text { floribundus } \\
\text { (Rosaceae) }\end{array}$ & arb & $?$ & $\mathrm{Bt}$ & $\mathrm{vr}$ & $\operatorname{Lg}$ & p 1 & $(1,2)$ & $\mathrm{Ag}$ & $\mathrm{n} / \mathrm{e}$ & 0.036 & $\mathrm{n} / \mathrm{e}$ & prc+RAPD & $\begin{array}{c}\text { Bushakra et al., } \\
\text { 1999* }\end{array}$ \\
\hline $\begin{array}{l}\text { Maianthemum } \\
\text { bifolium } \\
\text { (Convalliariaceae) }\end{array}$ & hp & $E^{*}$ & $\mathrm{Bt}$ & $\mathrm{rzm}$ & $\operatorname{Lg}$ & p 1 & $(1,2)$ & $\mathrm{Ag}$ & 0.88 & 0.7 & $\begin{array}{l}\text { asp, }<50 \mathrm{~m} \\
\text { (ge) \# }\end{array}$ & $\begin{array}{l}\text { trans+AFLP } \\
\quad+\text { asp }\end{array}$ & $\begin{array}{l}\text { Arens et al., } \\
2005^{*} \\
\text { Honnay et al. } \\
2006\end{array}$ \\
\hline $\begin{array}{l}\text { Maianthemum } \\
\text { dilatatum } \\
\text { (Convalliariaceae) }\end{array}$ & hp & EAs & $\mathrm{Bt}$ & $\operatorname{rzm} \mathbf{\Delta}$ & $\operatorname{Lg}$ & p 1 & $(1,2)$ & $\mathrm{Ag}$ & $\mathrm{n} / \mathrm{e}$ & 0.64 & $\begin{array}{l}\text { asp, }<45 \mathrm{~m} \\
\text { (ge y rm) }\end{array}$ & $\begin{array}{l}\text { prc }+ \text { AFLP } \\
\quad+\text { asp }\end{array}$ & $\begin{array}{l}\text { Wilson et al., } \\
2005 a^{*} \\
\text { Wilson et al., } \\
2005 b^{*}\end{array}$ \\
\hline $\begin{array}{l}\text { Paris quadrifolia } \\
\text { (Trilliaceae) }\end{array}$ & hp & A & $\mathrm{Bt}$ & $\mathrm{rzm}$ & $\operatorname{Lg}$ & p 1-hl 1 & 3 & En & 0.87 & 0.27 & $\begin{array}{l}\text { asp, }<5 \mathrm{~m} \\
(\text { ge y rm) }\end{array}$ & $\begin{array}{l}\text { prc }+ \text { AFLP } \\
\quad+\text { asp }\end{array}$ & $\begin{array}{c}\text { Jacquemyn et } \\
\text { al., 2005* }\end{array}$ \\
\hline
\end{tabular}


Apéndice. Continuación

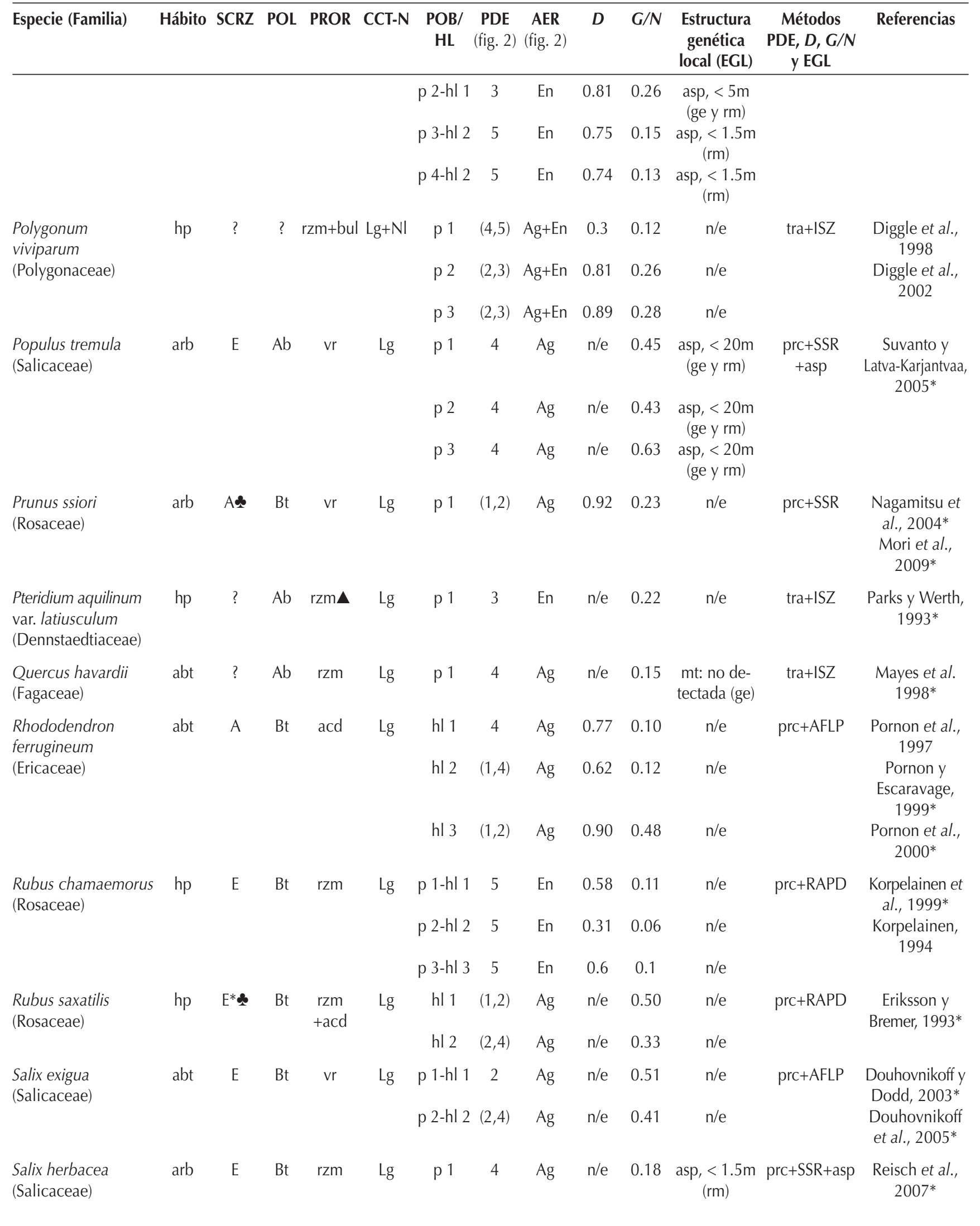


Apéndice. Continuación

\begin{tabular}{|c|c|c|c|c|c|c|c|c|c|c|c|c|c|}
\hline Especie (Familia) & Hábito & SCRZ & POL & PROR & CCT-N & $\begin{array}{c}\mathrm{POB} / \\
\mathrm{HL}\end{array}$ & $\begin{array}{c}\text { PDE } \\
\text { (fig. 2) }\end{array}$ & $\begin{array}{c}\text { AER } \\
\text { (fig. 2) }\end{array}$ & $D$ & $G / N$ & $\begin{array}{l}\text { Estructura } \\
\text { genética } \\
\text { local (EGL) }\end{array}$ & $\begin{array}{c}\text { Métodos } \\
\text { PDE, } D, G / N \\
\text { y EGL }\end{array}$ & Referencias \\
\hline \multirow[t]{6}{*}{$\begin{array}{l}\text { Saxifraga cernua } \\
\text { (Saxifragaceae) }\end{array}$} & \multirow[t]{6}{*}{ hp } & \multirow[t]{6}{*}{$E^{*}$} & \multirow[t]{6}{*}{$\mathrm{Bt}$} & \multirow[t]{6}{*}{ bul } & \multirow[t]{6}{*}{$\mathrm{Nl}$} & p 1 & 5 & En & 0.35 & 0.13 & $\begin{array}{c}\text { mt: no } \\
\text { detectada }\end{array}$ & \multirow[t]{6}{*}{$\begin{array}{l}\text { prc+RAPD } \\
+m t+a s p\end{array}$} & \multirow{6}{*}{$\begin{array}{l}\text { Gabrielsen y } \\
\text { Brochmann, } \\
1998^{*} \\
\text { KjØIner et al., } \\
\text { 2006* }\end{array}$} \\
\hline & & & & & & p 2 & 5 & En & 0.68 & 0.19 & $\begin{array}{c}\text { mt: no } \\
\text { detectada }\end{array}$ & & \\
\hline & & & & & & p 3 & 5 & En & 0.7 & 0.25 & $\begin{array}{l}\text { asp: no } \\
\text { detectada }\end{array}$ & & \\
\hline & & & & & & p 4 & 5 & En & 0.9 & 0.4 & $\begin{array}{c}\text { asp: no } \\
\text { detectada }\end{array}$ & & \\
\hline & & & & & & p 5 & 5 & En & 0.93 & 0.43 & $\begin{array}{l}\text { asp: no } \\
\text { detectada }\end{array}$ & & \\
\hline & & & & & & p 6 & 5 & En & 0.89 & 0.34 & $\begin{array}{c}\text { asp: no } \\
\text { detectada }\end{array}$ & & \\
\hline \multirow[t]{3}{*}{$\begin{array}{l}\text { Scirpus maritimus } \\
\text { (Cyperaceae) }\end{array}$} & \multirow[t]{3}{*}{ hp } & \multirow[t]{12}{*}{$\mathrm{E}^{*}$} & \multirow[t]{3}{*}{$\mathrm{Ab}$} & \multirow[t]{3}{*}{$\mathrm{rzm}$} & \multirow[t]{3}{*}{$\operatorname{Lg}$} & p 1 & 2 & $\mathrm{Ag}$ & $\mathrm{n} / \mathrm{e}$ & $\mathrm{n} / \mathrm{e}$ & $\mathrm{n} / \mathrm{e}$ & \multirow[t]{3}{*}{ desc } & \multirow[t]{3}{*}{$\begin{array}{c}\text { Charpentier et } \\
\text { al., } 2000\end{array}$} \\
\hline & & & & & & p 2 & 4 & $\mathrm{Ag}$ & $\mathrm{n} / \mathrm{e}$ & $\mathrm{n} / \mathrm{e}$ & $\mathrm{n} / \mathrm{e}$ & & \\
\hline & & & & & & p 3 & 4 & $\mathrm{Ag}$ & $\mathrm{n} / \mathrm{e}$ & $\mathrm{n} / \mathrm{e}$ & $\mathrm{n} / \mathrm{e}$ & & \\
\hline \multirow[t]{9}{*}{$\begin{array}{l}\text { Sequoia sempervirens } \\
\text { (Taxodiaceae) }\end{array}$} & \multirow[t]{9}{*}{ arb } & & \multirow[t]{9}{*}{$\mathrm{Ab}$} & \multirow[t]{9}{*}{$\mathrm{vb}$} & \multirow[t]{9}{*}{$\operatorname{Lg}$} & p 1-hl 1 & 2 & $\mathrm{Ag}$ & $\mathrm{n} / \mathrm{e}$ & 0.24 & $\begin{array}{l}\mathrm{mt}:<40 \mathrm{~m} \\
\quad(\mathrm{rm})\end{array}$ & \multirow[t]{9}{*}{$\operatorname{prc}+\mathrm{AFLP}+\mathrm{mt}$} & \multirow[t]{9}{*}{$\begin{array}{l}\text { Douhovnikoff } \\
\text { et al., 2004* }\end{array}$} \\
\hline & & & & & & p 2-hl 1 & 2 & $\mathrm{Ag}$ & $\mathrm{n} / \mathrm{e}$ & 0.38 & $\begin{array}{c}\mathrm{mt}:<40 \mathrm{~m} \\
(\mathrm{rm})\end{array}$ & & \\
\hline & & & & & & p 3-hl 1 & 2 & $\mathrm{Ag}$ & $\mathrm{n} / \mathrm{e}$ & 0.5 & $\begin{array}{c}\mathrm{mt}:<40 \mathrm{~m} \\
(\mathrm{rm})\end{array}$ & & \\
\hline & & & & & & p 4-hl 2 & 2 & $\mathrm{Ag}$ & $\mathrm{n} / \mathrm{e}$ & 0.6 & $\begin{array}{c}\mathrm{mt}:<40 \mathrm{~m} \\
(\mathrm{rm})\end{array}$ & & \\
\hline & & & & & & p 5-hl 2 & 2 & $\mathrm{Ag}$ & $\mathrm{n} / \mathrm{e}$ & 0.35 & $\begin{array}{c}\mathrm{mt}:<40 \mathrm{~m} \\
(\mathrm{rm})\end{array}$ & & \\
\hline & & & & & & p 6-hl 2 & 2 & $\mathrm{Ag}$ & $\mathrm{n} / \mathrm{e}$ & 0.6 & $\begin{array}{c}\mathrm{mt}:<40 \mathrm{~m} \\
(\mathrm{rm})\end{array}$ & & \\
\hline & & & & & & p 7-hl 3 & 2 & $\mathrm{Ag}$ & $\mathrm{n} / \mathrm{e}$ & 0.26 & $\begin{array}{c}\mathrm{mt}:<40 \mathrm{~m} \\
(\mathrm{rm})\end{array}$ & & \\
\hline & & & & & & p 8-hl 3 & 2 & $\mathrm{Ag}$ & $n / e$ & 0.19 & $\begin{array}{c}\mathrm{mt}:<40 \mathrm{~m} \\
(\mathrm{rm})\end{array}$ & & \\
\hline & & & & & & p 9-hl 3 & 2 & $\mathrm{Ag}$ & $\mathrm{n} / \mathrm{e}$ & 0.54 & $\begin{array}{c}\mathrm{mt}:<40 \mathrm{~m} \\
\quad(\mathrm{rm})\end{array}$ & & \\
\hline $\begin{array}{l}\text { Stenocereus eruca } \\
\text { (Cactaceae) }\end{array}$ & abt & $E^{*}$ & $\mathrm{Bt}$ & drm & $\mathrm{NI}$ & p 1 & 2 & $\mathrm{Ag}$ & 0.99 & 0.83 & $\begin{array}{l}\text { asp, }<20 \text { m } \\
\text { (ge y rm) }\end{array}$ & prc+ISSR+asp & $\begin{array}{c}\text { Clark-Tapia et } \\
\text { al., 2005* } \\
\text { Clark-Tapia et } \\
\text { al., } 2006\end{array}$ \\
\hline \multirow[t]{5}{*}{$\begin{array}{l}\text { Typha latifolia } \\
\text { (Thyphaceae) }\end{array}$} & \multirow[t]{5}{*}{$\mathrm{hp}$} & \multirow[t]{5}{*}{ A } & \multirow[t]{5}{*}{$\mathrm{Ab}$} & \multirow[t]{5}{*}{ rzm } & $\operatorname{Lg}$ & p 1 & $(4,5)$ & $\mathrm{Ag}+\mathrm{En}$ & $\mathrm{n} / \mathrm{e}$ & 0.77 & $\mathrm{n} / \mathrm{e}$ & tra+VNTR & $\begin{array}{c}\text { Keane et al., } \\
\text { 1999* }\end{array}$ \\
\hline & & & & & & p 2 & $(4,5)$ & $\mathrm{Ag}+\mathrm{En}$ & $\mathrm{n} / \mathrm{e}$ & 0.55 & $\mathrm{n} / \mathrm{e}$ & & \\
\hline & & & & & & p 3 & $(4,5)$ & $\mathrm{Ag}+\mathrm{En}$ & $\mathrm{n} / \mathrm{e}$ & 0.48 & $\mathrm{n} / \mathrm{e}$ & & \\
\hline & & & & & & p 4 & $(4,5)$ & $\mathrm{Ag}+\mathrm{En}$ & $\mathrm{n} / \mathrm{e}$ & 0.3 & $n / e$ & & \\
\hline & & & & & & p 5 & $(4,5)$ & $A g+E n$ & $\mathrm{n} / \mathrm{e}$ & 0.57 & $\mathrm{n} / \mathrm{e}$ & & \\
\hline $\begin{array}{l}\text { Uvularia perfoliata } \\
\text { (Convallariaceae) }\end{array}$ & hp & $?$ & $?$ & etl & $\operatorname{Lg}$ & p 1-hl 1 & $(4,5)$ & $A g+E n$ & 0.74 & 0.09 & $\begin{array}{c}\text { asp, }<3-5 m \\
(r m)\end{array}$ & prc+ISZ+asp & $\begin{array}{l}\text { Kudoh et al., } \\
\text { 1999* }\end{array}$ \\
\hline & & & & & & p 1-hl 2 & 1 & $\mathrm{Ag}$ & 0.59 & 0.03 & $\mathrm{n} / \mathrm{a}$ & & \\
\hline $\begin{array}{l}\text { Vaccinium myrtillus } \\
\text { (Ericaceae) }\end{array}$ & abt & A & $\mathrm{Bt}$ & rzm & $\operatorname{Lg}$ & p 1 & 2 & $\mathrm{Ag}$ & 0.94 & 0.29 & $\begin{array}{c}\text { asp: no } \\
\text { detectada }\end{array}$ & $\begin{array}{l}\text { prc+AFLP+ } \\
\text { RAPD+asp }\end{array}$ & $\begin{array}{c}\text { Albert et al., } \\
\text { 2003* }\end{array}$ \\
\hline
\end{tabular}


Apéndice. Continuación

\begin{tabular}{|c|c|c|c|c|c|c|c|c|c|c|c|c|c|}
\hline Especie (Familia) & Hábito & SCRZ & POL & PROR & CCT-N & $\begin{array}{c}\mathrm{POB} / \\
\mathrm{HL}\end{array}$ & $\begin{array}{l}\text { PDE } \\
\text { (fig. 2) }\end{array}$ & $\begin{array}{c}\text { AER } \\
\text { (fig. 2) }\end{array}$ & $D$ & $G / N$ & $\begin{array}{c}\text { Estructura } \\
\text { genética } \\
\text { local (EGL) }\end{array}$ & $\begin{array}{c}\text { Métodos } \\
\text { PDE, } D, G / N \\
\text { y EGL }\end{array}$ & Referencias \\
\hline $\begin{array}{l}\text { Vaccinium stamineum } \\
\text { (Ericaceae) }\end{array}$ & abt & $\mathrm{E}^{*}$ & $\mathrm{Bt}$ & $\mathrm{rzm}$ & $\operatorname{Lg}$ & $\mathrm{p} 1$ & $(2,3)$ & $\mathrm{Ag}+\mathrm{En}$ & $\mathrm{n} / \mathrm{e}$ & 0.68 & $\mathrm{n} / \mathrm{e}$ & prc+RAPD & $\begin{array}{l}\text { Kreher et al., } \\
\text { 2000* }\end{array}$ \\
\hline $\begin{array}{l}\text { Zostera marina } \\
\text { (Zosteraceae) }\end{array}$ & hp & A & $\mathrm{Ab}$ & $\mathrm{rzm}$ & $\operatorname{Lg}$ & $\begin{array}{l}\text { p 1-hl } 1 \\
\text { p 2-hl } 2\end{array}$ & $\begin{array}{l}(4,5) \\
(4,5)\end{array}$ & $\begin{array}{l}A g+E n \\
A g+E n\end{array}$ & $\mathrm{n} / \mathrm{e}$ & $\mathrm{n} / \mathrm{e}$ & $\begin{array}{c}\text { asp, }<6 \mathrm{~m} \\
\text { (ge y rm) } \\
\text { asp, }<6 \mathrm{~m} \\
\text { (ge y rm) }\end{array}$ & $\mathrm{prc}+\mathrm{SSR}+\mathrm{asp}$ & $\begin{array}{l}\text { Hämmerli y } \\
\text { Reusch, } 2003 \mathrm{a}^{*} \\
\text { Hämmerli y } \\
\text { Reusch, } 2003 \mathrm{~b}^{*}\end{array}$ \\
\hline $\begin{array}{l}\text { Zostera noltii } \\
\text { (Zosteraceae) }\end{array}$ & hp & A & $\mathrm{Ab}$ & $\mathrm{rzm}$ & $\operatorname{Lg}$ & p 1 & 4 & $\mathrm{Ag}$ & $\mathrm{n} / \mathrm{e}$ & 0.12 & $\mathrm{n} / \mathrm{e}$ & $\mathrm{prc}+\mathrm{SSR}$ & $\begin{array}{l}\text { Ruggieiro et } \\
\text { al., 2005* }\end{array}$ \\
\hline
\end{tabular}

Hábito: $\mathbf{a b t}=$ arbustos, $\mathbf{a r b}=$ árboles, $\mathbf{h p}=$ hierbas perennes

SCRZ: A = especies con capacidad de reproducirse por autocruza, $\mathbf{E}=$ especies con entrecruza obligada (p. ej., dioicas), $\mathbf{E}^{*}=$ especies con sistemas de autoincompatibilidad, = reclutamiento sexual observado y/o evaluado en condiciones naturales

POL: $\mathbf{A b}=$ polinización abiótica, $\mathbf{B t}=$ polinización biótica

PROR: acd = acodaduras, agp = agamospermia, bul = bulbilos, buls = bulbilos subterráneos, $\mathbf{c h u}=$ coronas huecas, $\mathbf{d r m}=$ desprendimiento de ramas, $\mathbf{e t l}=$ estolones, $\mathbf{g m a s}=$ gemas subterráneas, $\mathbf{p n t}=$ plantlets, $\mathbf{p s b}=$ pseudobulbos, $\mathbf{r z m}=$ rizomas, $\mathbf{v b}=$ vástagos basales, $\mathbf{v r}=\mathbf{v a ́ s t a g o s}$ radicales, $\mathbf{\Delta}$ = crecimiento tipo guerrilla

CCT-N: $\mathbf{L g}=$ crecimiento clonal ligado, $\mathbf{N I}=$ crecimiento clonal no ligado

POB/HL: $\mathbf{p}=$ población, $\mathbf{h l}=$ hábitat local (p. ej., bosque con dosel abierto vs bosque con dosel cerrado)

PDE: los paréntesis indican que la población presenta una combinación de patrones de distribución de los tipos indicados por los números (ver figura 2).

AER: Ag = los ramets de cada genet no se entremezclan con los ramets de genets distintos, En = los ramets de diferentes genets en la población se entremezclan.

EGL: \# = evaluado en una población distinta de donde se determinó el PDE, asp = autocorrelación espacial, mpic = método de probabilidad de identidad clonal, $\mathbf{m t}=$ prueba de Mantel, $\mathbf{S G}=$ similitud genética vs distancia espacial.

Métodos: AFLP = marcadores moleculares AFLPs, CLO = marcadores moleculares de cloroplasto, desc = descripción detallada del PDE, ISSR = marcadores moleculares ISSRs, ISZ = isoenzimas, $\mathbf{m p i c}=$ método de probabilidad de identidad clonal, $\mathbf{m t}=$ prueba de $\mathbf{M a n t e l}, \mathbf{p o b}=$ muestra de individuos o todos los individuos de la población, prc = parcelas, RAPD = marcadores moleculares RAPDs, SG $=$ similitud genética vs distancia espacial, $\mathbf{S S R}=$ marcadores moleculares SSRs (microsatélites),

Referencias: ${ }^{*}=$ estudios donde se presentan planos de la distribución espacial de los genotipos.

Todo el cuadro: ? = no se proporciona información, $\mathbf{n} / \mathbf{e}=$ no evaluado, $\mathbf{n} / \mathbf{a}=$ no aplica

GLOSARIO (columna PROR):

Acodaduras: formación de ramets a partir de un tallo originalmente erecto que se postra y genera raíces adventicias al entrar en contacto con el sustrato

Agamospermia: Producción asexual de semillas o embriones sin fertilización (diplosporía: el embrión se desarrolla de una célula diploide del saco embrionario, aposporía: el embrión se desarrolla de una célula somática del saco embrionario, embrionia adventicia: el embrión se desarrolla de células del tejido esporofítico como los tegumentos).

Bulbilos: bulbos pequeños que se forman en las axilas foliares o unidos a los bulbos.

Bulbos: tallos cortos modificados para almacenamiento rodeados por hojas carnosas o brácteas.

Coronas huecas: muerte de los tallos centrales en plantas cespitosas resultando en la formación de ramets independientes en la periferia. Descrito en pastos cespitosos.

Estolones: tallo horizontal que se extiende en la superficie del suelo.

Gemas: pequeñas estructuras vegetativas dispersables en briofitas y pteridofitas.

Plantlets: propágulos vegetativos que resultan de la reversión de estructuras reproductivas como flores y frutos a estructuras vegetativas.

Pseudobulbos: modificación del tallo para almacenamiento que se desarrolla entre dos nodos foliares en orquídeas.

Rizomas: tallo horizontal subterráneo.

Vástagos basales y radicales: formación de tallos en la base de un tallo o a partir de una raíz.

Referencias del glosario: Jackson et al., 1985; de Kroon y van Groenendael, 1997; Liston et al., 2003; van Dijk y Bakx-Schotman, 2004; Tooke et al., 2005. 
independientes de cada genotipo o ramets independientes (Figura 1; Kays y Harper, 1974; Harper, 1985; Jackson et al., 1985). La clonalidad está ampliamente distribuida en plantas, algunas estimaciones para angiospermas varían entre 30\% y más del 50\% (Tiffney y Niklas, 1985; Klim s et al., 1997; Aarssen, 2008), entre el 40\% y $80 \%$ en especies de taiga, tundra y de zonas templadas (van Groenendael y de Kroon, 1990 Klim s et al., 1997) y el 44\% de plantas invasoras en China (Liu et al., 2006), está ampliamente representada en monocotiledóneas y se presenta con baja frecuencia en las gimnospermas (van Groenendael et al., 1996; Peterson y Jones, 1997). Respecto a las condiciones ambientales, se sugiere que la clonalidad predomina en condiciones estresantes como suelos pobres en nitrógeno, suelos húmedos, cuerpos de agua, hábitats sombreados y ambientes fríos en altitudes y latitudes elevadas (Tiffney y Niklas, 1985; van Groenendael et al., 1996; Klim s et al., 1997).

La clonalidad en plantas se ha estudiado con enfoques muy diversos. Por ejemplo, se ha analizado la frecuencia de especies clonales y la variación en las formas de crecimiento clonal en distintos ambientes (p. ej., Tiffney y Niklas, 1985; van Groenendael et al., 1996; Klims et al., 1997), las implicaciones de la integración fisiológica de los ramets y la plasticidad morfológica del crecimiento clonal en ambientes heterogéneos y en condiciones de estrés competitivo (p. ej., Bell, 1984; Hartnett y Bazzaz, 1985; de Kroon y Hutchings, 1995; Price y Marshall, 1999), el efecto de la clonalidad en la demografía (p. ej., Caswell, 1985; McFadden, 1991; Damman y Cain, 1998; Mandujano et al., 2001), la comparación de la diversidad genética entre especies de plantas clonales y no clonales (p. ej., Ellstrand y Roose, 1987; Widén et al., 1994) y las consecuencias reproductivas y evolutivas de la geitonogamia asociada con la clonalidad (p. ej., Handel, 1985; de Jong et al., 1992; Eckert, 2000).

Las implicaciones reproductivas y genéticas de reducir la entrecruza incrementando la geitonogamia han aumentado el interés en el estudio de los patrones de distribución espacial de genets y ramets en poblaciones de plantas clonales. Debido a que los propágulos vegetativos suelen tener una capacidad de dispersión restringida, el reclutamiento clonal frecuente puede reducir la entrecruza e incrementar la autocruza (p. ej., autogamia y geitonogamia), determinando los niveles de variación y la estructuración genética en las poblaciones (Handel, 1985; Eckert, 2002; Ruggiero et al., 2005; Honnay et al., 2006). En especies autocompatibles, los costos reproductivos de la geitonogamia están asociados al incremento de la autogamia y a las probabilidades de expresar depresión endogámica, a la vez que disminuye la adecuación paterna reduciendo la cantidad de polen disponible para la entrecruza; mientras que en las especies autoincompatibles, la geitonogamia afecta la producción de semillas, debido a la saturación de los estigmas con polen incompatible y al aborto de óvulos, y puede resultar en el desperdicio de grandes cantidades de polen (de Jong et al.,
1992; Charpentier, 2002).

En plantas clonales los patrones de distribución espacial pueden ser afectados por la manera en que se generan los ramets. Las formas de crecimiento clonal son muy diversas, pero existen algunas clasificaciones que facilitan el entendimiento de esta variación. Tiffney y Niklas (1985) propusieron cuatro tipos principales de crecimiento: (1) Crecimiento establecido, dado por el inicio del desarrollo de un organismo individual a partir de un propágulo (puede considerarse como crecimento clonal si el propágulo es vegetativo, ya que el propágulo también puede ser una semilla producida sexualmente). (2) Crecimiento restaurativo, que se refiere a la reparación de un daño local en el individuo mediante la formación de tejido calloso, pero no hay regeneración de los órganos completos cuando estos se pierden. (3) Crecimiento regenerativo, que se refiere a la reparación y el reemplazo de órganos dañados (p. ej., crecimiento de una rama desprendida). (4) Crecimiento reproductivo, dado por la multiplicación del individuo fisiológico (p. ej., clonalidad). Estos autores también ordenan los tipos de crecimiento clonal en ligado y no ligado, considerando las estructuras de conexión (Figura 1). En el crecimiento ligado los propágulos vegetativos pueden desarrollarse hasta formar individuos completos (con raíces, tallos y hojas) manteniéndose unidos a la planta madre y su dispersión depende del crecimiento de ramificaciones de tallos y raíces, mientras que en el no ligado, los propágulos vegetativos se desprenden de la planta madre y se desarrollan hasta individuos completos separados de la planta madre. La dispersión de los propágulos en este caso es más dependiente de factores bióticos (p. ej., animales) o abióticos (p. ej., corrientes de agua, gravedad, viento) que en el crecimiento ligado y permite un mayor desplazamiento de los propágulos. El crecimiento reproductivo o clonalidad, que es la que interesa en esta revisión por su influencia en el arreglo espacial de genets y ramets, puede ser de dos tipos: (i) reiteración traumática, definida como la producción de un nuevo individuo inducida por estrés, ya sea por daño o por condiciones ambientales que limitan la reproducción sexual (por ejemplo, la producción de bulbilos en los agaves por daño al escapo floral o por deficiencia de polen) y (ii) la reiteración programada, como la producción de un nuevo individuo por un meristemo como parte normal del desarrollo (Tiffney y Niklas, 1985; Arizaga y Ezcurra, 2002).

La distribución espacial de los individuos en las poblaciones se ha clasificado en tres patrones básicos: aleatorio, uniforme y agregado (Figura 2; Gleason, 1920; Clark y Evans, 1954; Barbour et al., 1987). En poblaciones naturales de plantas, los individuos suelen distribuirse en forma más o menos agregada, debido a la distribución heterogénea de los recursos (Couteron y Kokou, 1997; Chen y Bradshaw, 1999), a la interacción con otras especies (Shreve, 1917; Mandujano et al., 1998) y a la dispersión restringida de semillas y propágulos vegetativos (Gibson y Menges, 1994; Okuda et al., 1997; Clark-Tapia et al., 2005). En plantas 
clonales además, el arreglo espacial de los genets y ramets puede ordenarse en dos patrones básicos, uno caracterizado por la agregación de ramets del mismo genet y el otro por una distribución entremezclada de ramets de distintos genets (Figura 2; de Kroon y Hutchings, 1995; Charpentier, 2002; Clark-Tapia et al., 2005). Estos arreglos espaciales de genets y ramets pueden estar asociados a la manera en que se producen los ramets (esto es por crecimiento clonal ligado o no ligado) y se ha propuesto que en las plantas que se propagan mediante crecimiento no ligado forman arreglos más entremezclados que las especies con crecimiento ligado (Gabrielsen y Brochmann, 1998), pero esto no ha sido demostrado. Por otro lado, debido a que la clonalidad puede incrementar la tasa de geitonogamia entre los ramets del mismo genet, puede esperarse que los arreglos entremezclados reduzcan la geitonogamia y promuevan la entrecruza como resultado del incremento local en el número de genets (Charpentier, 2002).

En algunas revisiones sobre el tema de la clonalidad se han planteado hipótesis sobre las implicaciones reproduc-

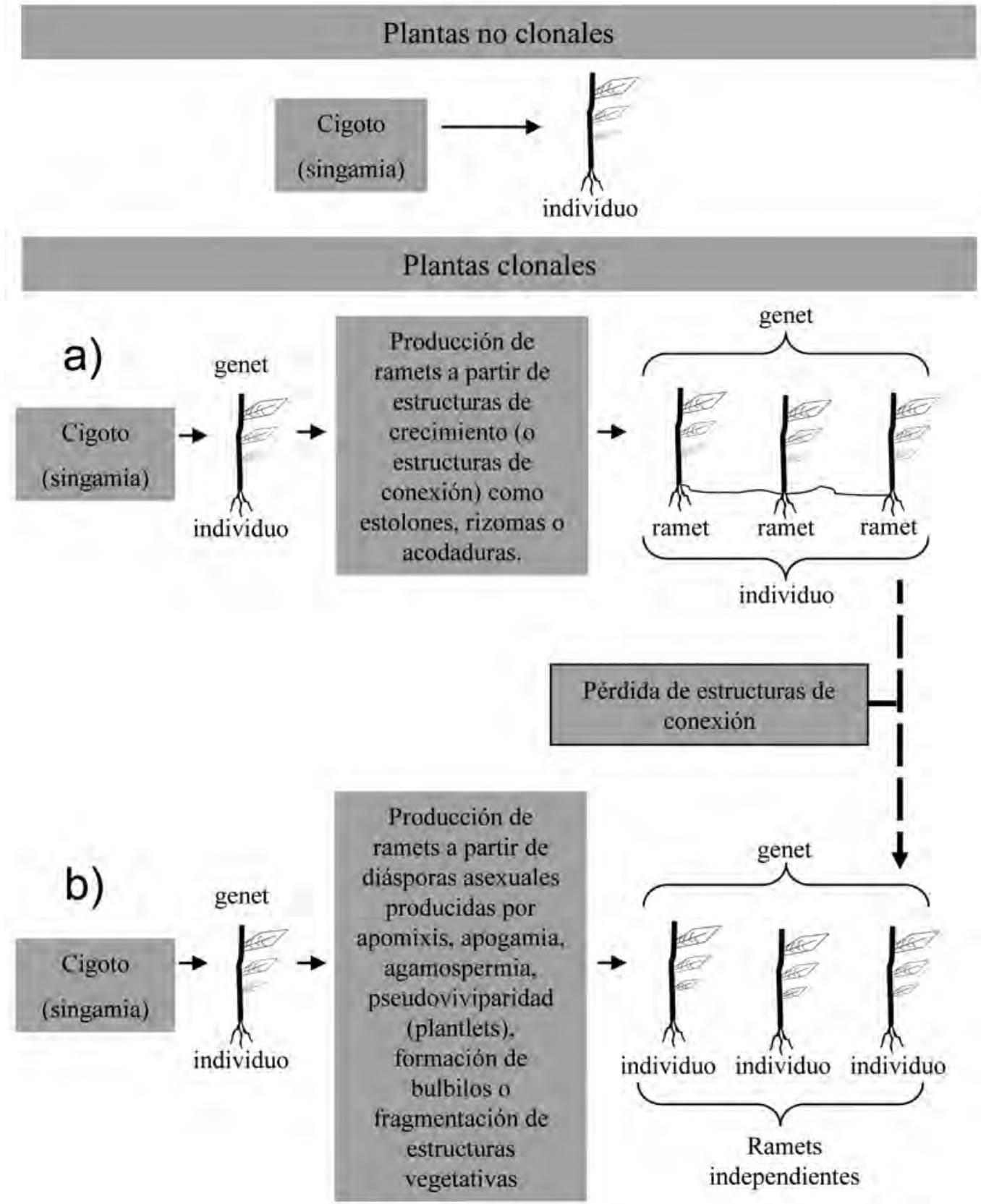

Figura 1. Formación de individuos en plantas no clonales y en plantas clonales. a) crecimiento clonal ligado y b) crecimiento clonal no ligado en la clasificación de Tiffney y Niklas (1985). 
$\mathrm{PDE}=$ Patrones de distribución espacial de los individuos y ramets en las poblaciones

(clonales y no clonales)
AER $=$ Arreglos espaciales de los ramets de distintos genets (clonales)

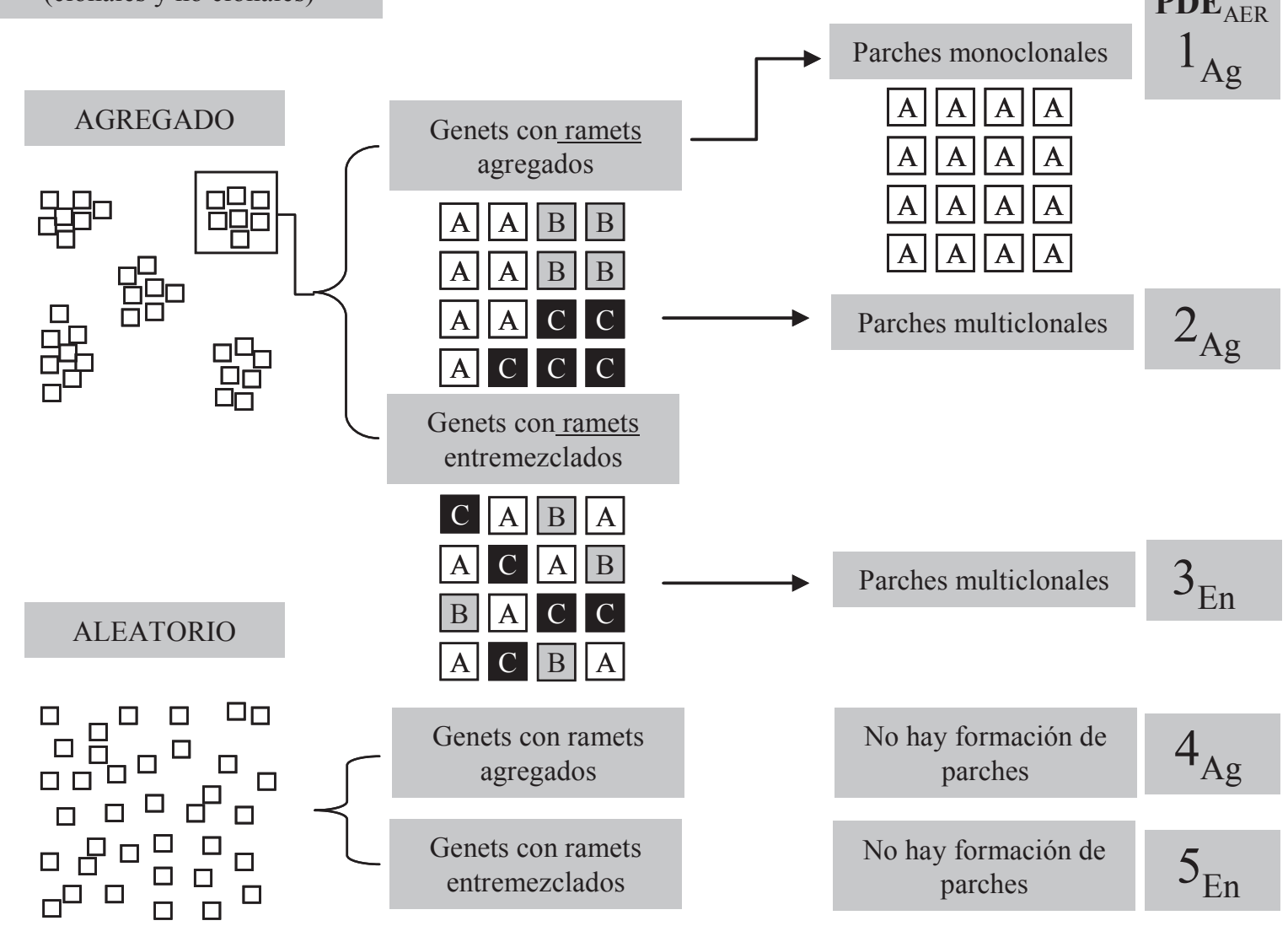

\section{UNIFORME}
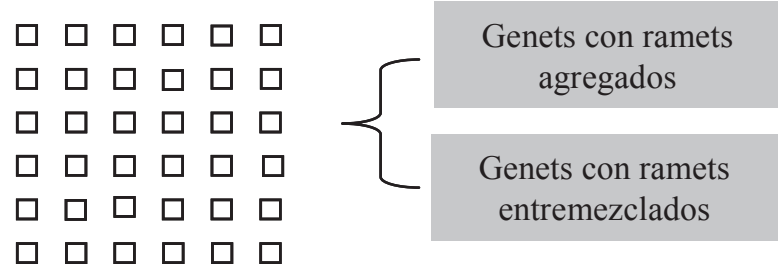

\begin{tabular}{|c|c|c|c|}
\hline $\begin{array}{c}\text { No hay formación de } \\
\text { parches }\end{array}$ & $6_{\mathrm{Ag}}$ \\
\hline $\begin{array}{c}\text { No hay formación de } \\
\text { parches }\end{array}$ & $7_{\mathrm{En}}$ \\
\hline
\end{tabular}

Figura 2. Patrones de distribución espacial de los individuos en las poblaciones de plantas no clonales y de plantas clonales. El número en el extremo derecho representa la clave del patrón de distribución espacial y el arreglo espacial de los ramets de distintos genets (PDE y AER, Apéndice). El subíndice Ag indica que los ramets de cada genet no se entremezclan con los ramets de genets distintos y el subíndice En indica que los ramets de diferentes genets se entremezclan.

tivas y genéticas de los arreglos espaciales de los genets y ramets, asociados con las distintas formas de producir descendientes clonales (Widén et al., 1994; Charpentier, 2002; Mandujano-Sánchez, 2007; Honnay y Jaquemyn, 2008). No obstante, los intentos para probar estas hipótesis son muy escasos y las posibilidades de realizar algún tipo de análisis numérico son restringidas por la baja disponibilidad de publicaciones (p. ej., Widén et al., 1994), o en los análisis sólo se incluyen especies con crecimiento ligado (p. ej., Honnay y Jaquemyn, 2008). En este trabajo presentamos una revisión de varios estudios que evalúan los patrones de distribución espacial en plantas clonales y a partir de la información 
proporcionada en dichos estudios, analizamos el planteamiento de que en poblaciones de plantas con crecimiento clonal ligado el arreglo espacial de los ramets de distintos genets es agregado, mientras que en poblaciones de plantas con crecimiento clonal no ligado predominan los arreglos espaciales entremezclados. Adicionalmente, intentamos poner a prueba la hipótesis de que los arreglos entremezclados promueven la entrecruza suponiendo que las poblaciones donde la entrecruza es más frecuente tenderán a presentar niveles más altos de diversidad genotípica.

\section{Material y métodos}

La revisión de los trabajos publicados sobre la distribución espacial clonal en plantas se llevó a cabo mediante una búsqueda de trabajos en bases de datos especializadas (CSA Cambridge, Ebsco HOST, ISI Web of Knowledge, JSTOR y Scopus) y no especializadas (Google, Google scholar y Yahoo). La búsqueda se hizo empleando los siguientes términos: clonal structure, spatial distribution of genets (andl or ramets), clonal architecture y clonal diversity y abarcó de 1990 a 2011, debido a que la mayoría de los estudios en plantas clonales con este enfoque se han realizado después de 1990.

Los criterios principales para incluir una especie en la revisión fue que se presentara una descripción de la distribución espacial de las plantas individuales (esto es sin importar el genotipo) en las poblaciones naturales y que se evaluara el arreglo espacial de los genets y los ramets (es decir agregado o entremezclado) mediante planos de distribución de los genotipos, análisis de autocorrelación espacial o compatibilidad de las cruzas entre ramets contiguos. Para cada especie se obtuvo el tipo de estructuras vegetativas a mediante las cuales se lleva a cabo la propagación clonal a partir de la información proporcionada en los estudios correspondientes o en estudios previos realizados a menudo por los mismos autores. El segundo criterio en orden de importancia fue que se reportaran valores de diversidad genotípica ( $D=$ índice de diversidad de Simpson y proporción de genotipos distinguibles $G / N$ ) para las poblaciones de las diferentes especies. Otros criterios fueron que se proporcionara información sobre las características reproductivas como la presencia de sistemas de incompatibilidad, la expresión sexual, si se ha observado reclutamiento sexual en las poblaciones y el tipo de polinización. Con base en esta información, se asignó a las especies el tipo de crecimiento clonal (i. e. ligado y no ligado, Figura 1) de acuerdo con la clasificación de Tiffney y Niklas (1985) y el patrón de distribución espacial y el tipo de arreglo espacial de los ramets (agregado o entremezclado) según el esquema presentado en la Figura 2. La información obtenida se presenta en el apéndice.

Para analizar el planteamiento de que en poblaciones de plantas con crecimiento clonal ligado el arreglo espacial de los ramets es agregado, mientras que en poblaciones de plantas con crecimiento clonal no ligado predomina el arreglo espacial entremezclado se construyó una tabla de contingencia con tres categorías de tipo de crecimiento clonal como columnas (ligado, combinación ligado-no ligado y no ligado) y tres categorías del arreglo espacial de los ramets como renglones (agregado, combinación agregado-entremezclado y entremezclado) y se analizó con una prueba de ${ }^{2}$ con el paquete estadístico JMP versión 7.0 (SAS Institute Inc). Para evaluar las celdas que contribuyen significativamente al valor de ${ }^{2}$ se hizo un análisis de residuales ajustados (Everitt, 1977).

Para probar la hipótesis de que las poblaciones con arreglos entremezclados de ramets presentan niveles más altos de diversidad genotípica, se compararon los valores del índice de diversidad de Simpson $(D)$ y la proporción de genotipos distinguibles $(G / N)$ entre las tres categorías de patrón de distribución clonal y entre las tres categorías de tipo de crecimiento clonal con una prueba de Kruskall-Wallis con el paquete estadístico JMP versión 7.0 (SAS Institute Inc).

\section{Resultados}

En la revisión se incluyeron 53 estudios que comprenden 122 poblaciones distribuidas en 44 especies de plantas clonales y en 27 familias (Apéndice). Las familias mejor representadas son Liliaceae y Rosaceae con cuatro especies cada una; Cactaceae, Cyperaceae, Ericaceae, Poaceae y Salicaceae con tres especies cada una y Zosteraceae con dos especies. El resto de las familias estuvieron representadas por una sola especie. Las especies que predominan son herbáceas perennes $(60 \%)$, y las plantas con hábito arbustivo y arbóreo se encuentran poco representadas (20\% en ambos casos).

La mayoría de las especies presentan crecimiento clonal ligado (70\%), dominando la propagación a través de rizomas (45\% de las especies). Las especies con crecimiento clonal ligado y no ligado representan el 14\% propagándose clonalmente por crecimiento ligado a través de rizomas y acodaduras, y por crecimiento no ligado a través de bulbilos, plantlets y desprendimiento de ramas; las especies sólo con crecimiento no ligado representan el 16\%, propagándose por bulbilos, semillas producidas por agamospermia, desprendimiento de ramas y gémulas (ver glosario para la definición de las estructuras implicadas en el crecimiento ligado y no ligado, Apéndice). Respecto a la distribución espacial, $50 \%$ del total de las especies presentan genets con ramets agregados, $30 \%$ presentan una combinación de arreglos agregados-entremezclados y $20 \%$ presentan arreglos entremezclados (Apéndice).

$\mathrm{Al}$ comparar las poblaciones con diferentes arreglos espaciales de genets y ramets [agregados (Ag), En (entremezcaldos) y $\mathrm{Ag}+\mathrm{En}$ (combinados)] encontramos que las poblaciones de especies con crecimiento ligado y no ligado y 
Cuadro 1. Número (observado y esperado) de poblaciones con diferentes tipos de crecimiento clonal de acuerdo con la clasificación de Tiffney y Niklas (1985) ( $\mathrm{Lg}=$ ligado, Lg+NI = ligado y no ligado, $\mathrm{Nl}=$ no ligado), que mostraron arreglos espaciales de los ramets de distintos genets: Ag = agregados, Ag+En = combinación de agregados y entremezclados, y En = entremezclados (figura 2). Los valores entre paréntesis indican el número de especies y familias (especies/familias) representadas en cada celda. Los valores de los residuales ajustados en negritas indican las celdas que contribuyen significativamente al valor de $\chi^{2}$, el signo indica mayor (sin signo) o menor número (-) de poblaciones de las esperadas por azar.

\begin{tabular}{|c|c|c|c|}
\hline Observados & Ligado & Ligado+No ligado & No ligado \\
\hline Agregado & $49(21 / 13)$ & $7(2 / 2)$ & $4(2 / 2)$ \\
\hline Agregado+entremezclado & $11(5 / 5)$ & $25(5 / 5)$ & $7(3 / 3)$ \\
\hline Entremezclado & $10(5 / 5)$ & $2(2 / 1)$ & $7(2 / 2)$ \\
\hline \multicolumn{4}{|l|}{ Esperados } \\
\hline & $\operatorname{Lg}$ & $\mathrm{Lg}+\mathrm{NI}$ & $\mathrm{Nl}$ \\
\hline Agregado & 34 & 17 & 9 \\
\hline Agregado+entremezclado & 25 & 12 & 6 \\
\hline Entremezclado & 11 & 5 & 3 \\
\hline \multicolumn{4}{|l|}{ Residuales ajustados } \\
\hline & $\operatorname{Lg}$ & $\mathrm{Lg}+\mathrm{NI}$ & $\mathrm{Nl}$ \\
\hline Agregado & 5.34 & -3.93 & -2.48 \\
\hline Agregado+entremezclado & -5.24 & 5.50 & 0.35 \\
\hline Entremezclado & -0.46 & -1.83 & 2.95 \\
\hline
\end{tabular}

de especies que sólo presentan crecimiento no ligado presentaron arreglos espaciales (AER) más entremezclados que las poblaciones de especies que sólo presentan crecimiento ligado ( ${ }^{2}=43.6, P<0.0001,4$ g.l.; Cuadro 1). Predominan los patrones de distribución (PDE) con ramets distribuidos en parches discretos sobre el área ocupada por la población (patrón de distribución espacial agregado, $46 \%$ de las poblaciones); estos parches son multiclonales (con dos o más genets) en la mayoría de los casos y el arreglo espacial de los ramets de distintos genets es agregado (19\% de las poblaciones, $\mathrm{PDE}_{\mathrm{AER}} 2_{\mathrm{Ag}}$, Figura 2), presenta una combinación agregado-entremezclado (10\% de las poblaciones, combinación $\mathrm{PDE}_{\mathrm{AER}} 2_{\mathrm{Ag}}-3_{\mathrm{En}}$, Figura 2) o es entremezclado solamente (3\% de las poblaciones, $\mathrm{PDE}_{\mathrm{AER}} 3_{\mathrm{En}}$, Figura 2). En otras poblaciones donde los ramets se distribuyen formando parches discretos el arreglo espacial de los ramets de distintos genets es agregado, pero los parches son multiclonales y monclonales (9\% de las poblaciones, combinación $\mathrm{PDE}_{\mathrm{AER}}$ $1_{\mathrm{Ag}}-\mathrm{Ag}_{\mathrm{Ag}}$, Figura 2) o sólo son monoclonales (5\% de las poblaciones, $\mathrm{PDE}_{\mathrm{AER}} 1_{\mathrm{Ag}}$, Figura 2).

Los patrones de distribución espacial con ramets distribuidos aleatoriamente sobre el área ocupada por la población (patrón de distribución espacial aleatorio) representaron el $44 \%$ de las poblaciones, predominando el arreglo espacial agregado-entremezclado de los ramets de distintos genets $\left(22 \%\right.$ de las poblaciones, combinación de $\mathrm{PDE}_{\mathrm{AER}}$ $4_{\mathrm{Ag}}-5_{\mathrm{En}}$, Figura 2), el arreglo agregado se presentó en $10 \%$ de las poblaciones ( $\mathrm{PDE}_{\mathrm{AER}} 4_{\mathrm{Ag}}$, Figura 2) y el arreglo entremezclado en $12 \%$ de las poblaciones $\left(\mathrm{PDE}_{\mathrm{AER}} 5_{\mathrm{En}}\right.$, Figura
2). El resto de las poblaciones (10\%) presentaron una combinación de patrones de distribución con ramets distribuidos aleatoriamente sobre una parte del área ocupada por la población y en otra formando parches discretos monoclonales o parches multiclonales con arreglos espaciales agregados y entremezclado de los ramets de distintos genets (combinación de $\mathrm{PDE}_{\mathrm{AER}} 1_{\mathrm{Ag}}-4_{\mathrm{Ag}}, 1_{\mathrm{Ag}}-5_{\mathrm{En}}, 2_{\mathrm{Ag}}-4_{\mathrm{Ag}}, 2_{\mathrm{Ag}}-5_{\mathrm{En}}$, Figura 2).

Las poblaciones de especies con una combinación de tipos de crecimiento clonal ligado y no ligado $(\mathrm{Lg}-\mathrm{Nl})$ presentaron valores más bajos de la proporción de genotipos distinguibles (promedio \pm E.E., $G / N=0.24 \pm 0.04 ; H=$ 6.93, $P=0.031,2$ g.l.) que las poblaciones de especies con crecimiento ligado (promedio \pm E.E., $G / N=0.38 \pm 0.03$ ) y con crecimiento no ligado (promedio \pm E.E., $G / N=0.39 \pm$ 0.06 ), pero las diferencias sólo fueron significativas con respecto a las poblaciones de especies con crecimiento ligado (Figura 3a). Respecto al arreglo espacial de los ramets de distintos genets, las poblaciones con una combinación de arreglos agregado y entremezclado (Ag-En) presentaron valores menores del índice de diversidad de Simpson (promedio \pm E.E., $D=0.57 \pm 0.05 ; H=13.2, P=0.0014,2$ g.l.) que las poblaciones con arreglos agregados (promedio \pm E.E., $D=0.80 \pm 0.04$ ) y con arreglos entremezclados (promedio \pm E.E., $D=0.76 \pm 0.06$; Figura 3). Estas diferencias sugieren que el reclutamiento sexual es más bajo en poblaciones de especies que presentan una combinación de formas de crecimiento clonal ligado-no ligado ( $\mathrm{Lg}-\mathrm{Nl}$ ) y una combinación de arreglos de los ramets de distintos genets agregado-entremezclado (Ag-En), sin embargo estas diferencias 

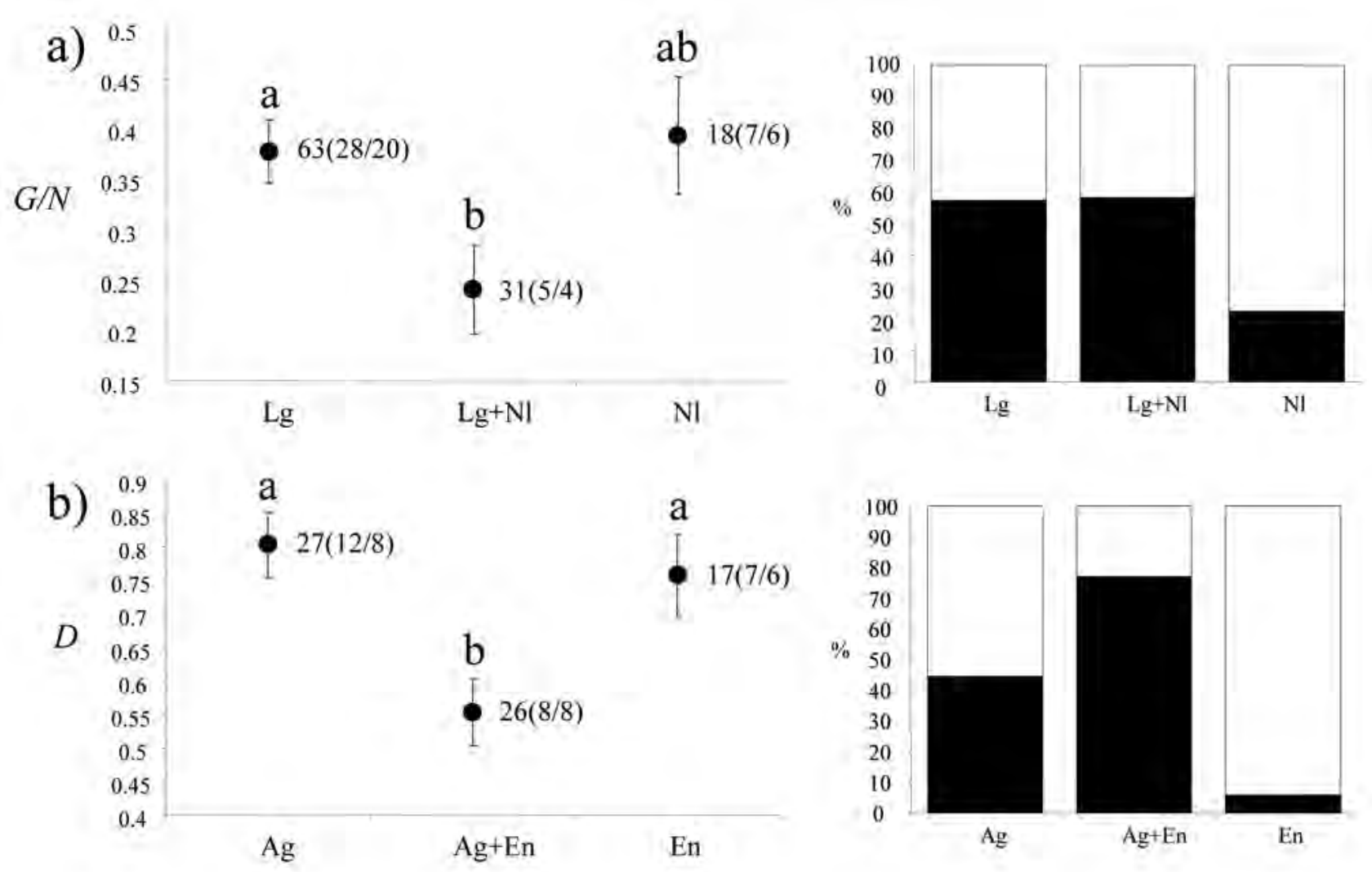

Figura 3. Comparación de la diversidad clonal (promedio \pm EE) entre poblaciones de especies con a) distinto tipo de crecimiento clonal: $\mathrm{Lg}=$ ligado, $\mathrm{Lg}+\mathrm{Nl}$ = con ambos tipos de crecimiento y $\mathrm{Nl}$ = no ligado y b) con distintos arreglos espaciales de los ramets de distintos genets: $\mathrm{Ag}=$ agregados, $\mathrm{Ag}+\mathrm{En}$ = combinación de agregados y entremezclados, y En = entremezclados (Figura 2). En cada grupo se indica el número de poblaciones (número de especies / número de familias) y los grupos que difieren significativamente se indican con una letra distinta arriba de la barra del promedio \pm E.E. de cada grupo. Las gráficas de barras del lado derecho muestran el porcentaje de poblaciones con polinización abiótica (en negro) y con polinización biótica (en blanco).

pueden estar asociadas con otros factores como el tipo de polinización, y no con el tipo de crecimiento y el arreglo espacial de los ramets de distintos genets. La mayoría de las poblaciones con tipo de crecimiento $\mathrm{Lg}-\mathrm{Nl}$ y arreglos de ramets de distintos genets Ag-En corresponden a especies con polinización abiótica (Figura 3).

\section{Discusión}

El efecto que tienen los modos de clonalidad en las plantas en la conformación de distintos arreglos espaciales de genets y ramets es un campo relativamente novedoso, que se ha explorado en los últimos 20 años y la mayoría de los estudios que hay sobre el tema se han desarrollado después de 1990. Widén et al. (1994) reportan que de 40 estudios que comprenden un total de 45 especies de plantas clonales, menos de la tercera parte proporcionan información sobre el arreglo espacial de los genets en las poblaciones y sólo en $12.5 \%$ se construyeron planos de distribución de todos los ramets, dentro de las parcelas o poblaciones, identificando los genotipos multilocus. En esta revisión, en el $98 \%$ de los estudios incluidos se identifican los genotipos multilocus mediante marcadores dominantes, codominantes o ambos siguiendo tres métodos de muestreo: en el 56\% de los estudios se utilizan parcelas, en el $24 \%$ transectos y en el $18 \%$ se muestrean todos o varios individuos de las poblaciones. El 2\% restante está representado por un estudio donde el patrón de distribución es descrito y la distribución de los ramets se sugiere por experimentos de cruzas compatibles entre ramets cercanos (en Scirpus maritimus, Charpentier et al., 2000).

A pesar de que en la actualidad se cuenta con más información sobre los patrones de distribución espacial en poblaciones de especies clonales, hacer comparaciones de dichos patrones considerando especies con distintas formas de crecimiento clonal para analizar las consecuencias reproductivas y genéticas del arreglo espacial de los genets y los ramets continúa siendo un reto. Algunos factores que influyen en este hecho son que la evaluación de la variación en los patrones de distribución espacial en plantas clonales 
enfrenta la dificultad que implica trabajar con la mayoría de los individuos en muchas poblaciones, principalmente a nivel molecular. Aunque en la actualidad las herramientas moleculares se han vuelto más accesibles, trabajar con un gran número de ejemplares sigue siendo difícil y costoso. Otros problemas son la falta de estimadores numéricos comparables para describir la distribución espacial en las poblaciones de diferentes especies y la variación en los métodos de muestreo. Por ejemplo, el muestreo mediante transectos colectando muestras de ramets cada determinada distancia (p. ej., Jonsson et al., 1996; Esselman et al., 1999) permite evaluar la capacidad de expansión longitudinal de los genets, pero no permite tener una descripción de la expansión radial y por lo tanto una evaluación más precisa del nivel de entremezcla de los ramets de distintos genets. En contraste, el muestreo de todos o de un gran número de individuos dentro de parcelas o en las áreas ocupadas por las poblaciones es otro método empleado en estudios con especies de plantas clonales (p. ej., Shimizu et al., 2002; Ruggieiro et al., 2005). Este tipo de muestreo puede ser un método más adecuado para analizar y comparar la estructura espacial clonal, porque permite detectar el arreglo radial de distintos genets. Sin embargo, los tamaños de muestra muy grandes pueden limitar la aplicación de este método, ya que en la mayoría de los casos es necesario emplear marcadores moleculares para identificar a los genets.

En particular, además de los problemas mencionados anteriormente es importante considerar las dificultades adicionales que detectamos en este estudio y que pueden afectar los patrones encontrados a través de nuestra aproximación. La mayoría de los estudios que analizan la distribución espacial de genets y ramets parecen estar sesgados hacia especies de herbáceas perennes con crecimiento clonal ligado (45\% de las especies), predominando las especies rizomatosas (43\% de las especies), mientras que las especies con crecimiento no ligado están poco representadas, impidiendo contar con tamaños de muestra equivalentes para hacer comparaciones más precisas. Por otro lado, la aproximación que decidimos emplear en este trabajo compara a las poblaciones más que a las especies, debido a que hemos detectado que hay una importante variación intraespecífica tanto en los patrones de distribución como en los patrones reproductivos. No obstante, esta variación se omite en algunos estudios o está mejor representada para algunas especies.

Los resultados obtenidos en este trabajo sugieren que las especies que se propagan por crecimento no ligado tienden a formar arreglos espaciales de los ramets de distintos genets más entremezclados como se ha planteado (Gabrielsen y Brochmann, 1998). No obstante, en especies con crecimiento ligado que además se propagan clonalmente por crecimiento no ligado, como el desprendimiento de ramas, el cual consideramos en este estudio como una forma de crecimiento no ligado en los análisis, debido a que los ramets originados por este medio se desarrollan hasta individuos com- pletos separados de la planta madre y a que su dispersión no depende del crecimiento horizontal de tallos y raíces (p.ej., Lophocereus schottii, Parker y Hamrick, 1992; Pteridium aquilinum, Parks y Werth, 1993; Cladium jamaicense, Ivey y Richards, 2001; Ferocactus robustus, Carrillo-Angeles et al., 2011), o que se propagan por la extensión de rizomas en especies con crecimiento en guerrilla (p. ej., Cymodocea nodosa, Ruggieiro et al., 2005 e Ilex leucoclada, Torimaru y Tomaru, 2005) también forman arreglos de genets entremezclados. Algunos factores externos que pueden influir en el arreglo entremezclado de genets son la dispersión por actividades humanas (Parks y Werth, 1993; Brzosko et al., 2002), el acarreo de propágulos vegetativos por animales y corrientes de agua (Parker y Hamrick, 1992; Brzosko et al., 2002), el reclutamiento de nuevos genets en áreas perturbadas donde permanecen fragmentos de clones que ocuparon grandes extensiones (Parks y Werth, 1993) y la dispersión de semillas producidas por autogamia que presentan genotipos multilocus idénticos (Keane et al., 1999; Brzosko et al., 2002).

Respecto a la hipótesis de que las poblaciones con arreglos espaciales entremezclados de los ramets de distintos genets presentan mayor entrecruza, medido indirectamente mediante la diversidad genotípica, nuestros resultados concuerdan parcialmente con el patrón esperado. El G/N es significativamente mayor en los arreglos de especies con producción de vástagos no ligados, en donde predominan los arreglos de genets entremezclados. Para probar esta hipótesis consideramos que si la entrecruza es más alta en poblaciones con arreglos entremezclados, habría mayores niveles de diversidad genotípica. Sin embargo, ésto no se refleja en el caso del índice de Simpson. Las diferencias en los índices de diversidad genotípica entre las poblaciones de especies que combinan el crecimiento clonal ligado-no ligado ( $\mathrm{Lg}$ $\mathrm{Nl})$ y el arreglo espacial agregado-entremezclado (Ag-En), respecto de las poblaciones que presentan uno u otro tipo de crecimiento y de arreglo espacial están asociadas con el tipo de polinización (biótica o abiótica), lo cual apoya el planteamiento de que la polinización biótica puede incrementar la eficiencia de la fertilización (Barret et al.,1993; Richards, 1997; Reusch, 2003). Los análisis para probar este planteamiento están fuera del alcance del presente estudio.

Además de los efectos sobre el reclutamiento sexual y la diversidad genotípica, los patrones de distribución espacial en especies clonales pueden afectar la diversidad y la estructura genética de las poblaciones. Por ejemplo, la formación de parches monoclonales en especies con entrecruza obligada, aunada al desplazamiento de genets por competencia, puede eliminar la capacidad de una población para reproducirse sexualmente en tiempos relativamente cortos (Honnay y Bossuyt, 2005). Adicionalmente, una reducción del reclutamiento sexual y una dispersión restringida de polen, semillas y propágulos vegetativos pueden generar estructura genética local en las poblaciones. Este segundo aspecto se 
evalúa en varios de los estudios revisados (47\%). La estructura genética local tanto a nivel de genets (indicando una dispersión restringida de polen, semillas o ambos) como de ramets (indicando dispersión restringida de propágulos vegetativos) se detectó en 46 poblaciones distribuidas en 16 especies; 28 poblaciones corresponden a cinco especies arbóreas que muestran estructura genética local a distancias menores de $50 \mathrm{~m}$ (Sequoia sempervirens, Douhovnikoff et al., 2004) y a distancias menores de $25 \mathrm{~m}$ (Eurya emarginata, Chung y Epperson, 2000; Cryptomeria japonica, Shimizu et al., 2002; Hirayama y Sakimoto, 2008; Populus tremula, Suvanto y Latva-Karjantvaa, 2005); tres poblaciones corresponden a dos especies arbustivas (Echinosophora koreensis, Chung et al., 2006; Stenocereus eruca, ClarkTapia et al., 2005) que presentan estructura genética local a distancias menores de $20 \mathrm{~m}$. El resto de las poblaciones corresponden a hierbas perennes que muestran estructura genética local desde distancias menores de $30 \mathrm{~m}$, pero más frecuentemente a distancias menores de $15 \mathrm{~m}$.

En promedio, los valores del índice de diversidad de Simpson $(D)$ y la proporción de genotipos distinguibles $(G / N)$ fueron altos en las poblaciones representadas en los estudios incluidos en la presente revisión y son similares a los reportados para 21 especies de plantas clonales $(D=$ 0.62 y $G / N=0.16$ ) por Ellstrand y Roose (1987). Se han planteado algunas hipótesis sobre los escenarios que pueden explicar altos niveles de diversidad genotípica: (i) las poblaciones pudieron ser fundadas por varios propágulos sexuales que se mantienen por clonalidad, (ii) el reclutamiento sexual fue más frecuente en el pasado, aunque en la actualidad la producción de descendientes sexuales no sea evidente (Ellstrand y Roose, 1987; Eriksson, 1989, 1993; Widén et al., 1994); (iii) los genotipos heterócigos se ven favorecidos selectivamente, manteniendo altos niveles de diversidad genética (Widén et al., 1994); (iv) los riesgos de mortalidad entre los ramets de un genet pueden ser distintos si tienen independencia física y fisiológica (Eriksson 1989, 1993, Pan y Price, 2002), lo cual puede reducir las probabilidades de supervivencia / extinción del genet; (v) en especies con ciclos de vida largos los eventos raros de reclutamiento sexual pueden ser suficientes para mantener niveles altos de diversidad genética (Eriksson 1989, 1993, Parker y Hamrick, 1992; Brzosko et al., 2002; Clark-Tapia et al., 2005); (vi) los genotipos pueden mantenerse a través de la supervivencia de algunos ramets el tiempo suficiente para que algunos genotipos se integren a la población de manera esporádica; (vii) la heterogeneidad ambiental puede generar condiciones de selección diversificante que permiten la coexistencia de varios genotipos (Widén et al., 1994). Otra posibilidad para el mantenimiento de diversidad clonal en poblaciones de plantas, que surge al considerar la variación de la distribución espacial de genets y ramets, es que la clonalidad puede mantener altos niveles de entrecruza e incrementar las probabilidades de reclutamiento sexual si los ramets se dispersan hacia vecindarios genéticamente variables, debido a la distribución entremezclada de genets.

Las explicaciones a los niveles relativamente altos de diversidad genotípica en las poblaciones de las especies incluidas en esta revisión comprenden los eventos esporádicos de reclutamiento sexual y el establecimiento inicial de varios genets. En algunos casos se sugiere un reclutamiento sexual frecuente a partir de los altos niveles de diversidad genotípica detectados con los marcadores moleculares (p. ej., Parks y Werth, 1993; Diggle et al., 1998; Misuki et al., 2010). No obstante, el reclutamiento sexual en poblaciones naturales sólo se reporta en Prunus ssiori, Rubus saxatilis (Eriksson y Bremer, 1993), Cypripedium calceolus (Brzosko et al., 2002) y Maianthemum dilatatum (Wilson et al., 2005b). Otros factores que se proponen para explicar la diversidad genotípica son que la integración fisiológica entre los ramets de un genet puede reducir el riesgo de que un genotipo en particular desaparezca, cuando las condiciones ambientales locales son adversas, contribuyendo a mantener la diversidad genética (Hartnett y Bazzaz, 1985), el incremento en la tasa de entrecruza, debido a la entremezcla de genets en especies con crecimiento en guerrilla (Ruggieiro et al., 2005) y en especies que se propagan clonalmente por desprendimiento de ramas y producción de bulbilos (Parker y Hamrick, 1992; Gabrielsen y Brochmann, 1998).

El estudio de los patrones de distribución espacial en especies clonales es importante para analizar las consecuencias reproductivas y genéticas del arreglo espacial de los genets y los ramets en las poblaciones naturales. Estas consecuencias pueden explorarse de una manera más precisa analizando la variación de la distribución de genets y ramets entre las poblaciones de una misma especie. Sin embargo, la evidencia de estos aspectos en organismos clonales aún es escasa y hace falta realizar estudios considerando diferentes tipos de crecimiento clonal. El análisis de los patrones de distribución espacial pueden ser muy útiles para evaluar otros aspectos del ciclo de vida de las especies clonales como el efecto de una variación persistente en la producción de propágulos clonales y sexuales entre hábitats, que puede afectar diferencialmente la dinámica de las poblaciones. Asimismo, es necesario analizar la dinámica temporal de los arreglos espaciales de los genets y ramets en las poblaciones para hacer inferencias adecuadas sobre las consecuencias ecológicas y evolutivas de la clonalidad.

\section{Agradecimientos}

Proyectos: IN IN205007 Efecto de la estructura clonal sobre la dinámica poblacional y la reproducción de una cactácea con ciclo de vida complejo, Opuntia microdasys.

Becas: doctorado Conacyt a I. Carrillo-Angeles y estancia sabática en New Mexico State University, otorgada por DGAPA-PASPA- Universidad Nacional Autónoma de México a M. Mandujano. Este trabajo es parte de la tesis de 
doctorado de Israel Gustavo Carrillo Angeles, Posgrado en Ciencias Biológicas de la UNAM. Agradecemos la revisión, sugerencias en el trabajo así como el apoyo de los miembros del comité tutoral, Doctores Teresa Valverde y Francisco Molina. Asimismo a la Dra. Ana Mendoza, Dr. Jordan Golubov y dos revisores anónimos por sus sugerencias al artículo.

\section{Literatura citada}

Aarssen L.W. 2008. Death without sex-the 'problem of the small' and selection for reproductive economy in flowering plants. Evolutionary Ecology 22:279-298.

Albert T., Raspé O. y Jacquemart A.L. 2003. Clonal structure in Vaccinium myrtillus L. revealed by RAPD and AFLP markers. International Journal of Plant Sciences 164:649-655.

Arens P., Grashof-Bokdam C.J., van der Sluis T. y Smulders M.J.M. 2005. Clonal diversity and genetic differentiation of Maianthemum bifolium among forest fragments of different age. Plant Ecology 179:169-180.

Arizaga S. y Ezcurra E. 2002. Propagation mechanisms in Agave macrovantha (Agavaceae), a tropical arid land succulent rosette. American Journal of Botany 89:632-641.

Barbour M.G., Burk J.H. y Pitts W.D. 1987. Terrestrial Plant Ecology. The Benjamin-Cummings Publishing Company, Menlo Park.

Barret S.C.H., Eckert C.G. y Husband B.C. 1993. Evolutionary processes in aquatic plant populations. Aquatic Botany 44:105-145.

Bell A.D. 1984. Dynamic morphology: A contribution to plant population ecology. En: Dirzo R. y Sarukhán J. Eds. Perspectives on Plant Population Ecology, pp. 49-65, Sinauer Associates Inc. Publisher, Sunderland.

Brzosko E., Wróblewska A. y Ratkiewicz M. 2002. Spatial genetic structure and clonal diversity of island populations of lady's slipper (Cyperidium calceolus) from the Biebrza National Park (northeast Poland). Molecular Ecology 11:2499-2509.

Bushakra J.M., Hodges S.A., Cooper J.B. y Kaska D.D. 1999. The extent of clonality and genetic diversity in the Santa Cruz Island ironwood, Lyonothamnus floribundus. Molecular Ecology 8:471-475.

Camacho F.J. y Liston A. 2001. Population structure and genetic diversity of Botrychium pumicola (Ophioglossaceae) based on inter-simple sequence repeats (ISSR). American Journal of Botany 88:1065-1070.

Carrillo-Angeles I.G., Mandujano M.C. y Golubov J. 2011. Influences of the genetic neighborhood on ramet reproductive success in a clonal desert cactus. Population Ecology 53:449458.

Caswell H. 1985. The evolutionary demography of clonal reproduction. En: Jackson J.B.C., Buss L.W. y Cook R.E. Eds. Population Biology and Evolution of Clonal Organisms, pp. 187224, Yale University Press, New Haven.

Charpentier A. 2002. Consequences of clonal growth for plant mating. Evolutionary Ecology 15:521-530.

Charpentier A., Grillas P. y Thompson J.D. 2000. The effects of population size limitation on fecundity in mosaic populations of the clonal macrophyte Scirpus maritimus (Cyperaceae). American Journal of Botany 87:502-507.

Chen J. y Bradshaw G.A. 1999. Forest structure in space: a case study of an old growth spruce-fir forest in Changbaishan Natural Reserve, PR China. Forest Ecology and Management 120:219-233.

Chung M.G. y Epperson B.K. 2000. Clonal and spatial genetic structure in Eurya emarginata (Theaceae). Heredity 84:170177.

Chung J.M., Lee B.C., Kim J.S., Park C., Chung M.Y. y Chung M.G. 2006. Fine-scale genetic structure among genetic individuals of the clone-forming monotypic genus Echinospora koreensis (Fabaceae). Annals of Botany 98:165-173.

Clark P.J. y Evans F.C. 1954. Distance to nearest neighbor as a measure of spatial relationships in populations. Ecology 35:445-453.

Clark-Tapia R., Alfonso-Corrado C., Eguiarte L.E. y Molina-Freaner F. 2005. Clonal diversity and distribution in Stenocereus eruca (Cactaceae), a narrow endemic cactus of the Sonoran Desert. American Journal of Botany 92:272-278.

Clark-Tapia R., Alfonso-Corrado C., Mandujano M.C. y MolinaFreaner F. 2006. Reproductive consequences of clonal growth in Stenocereus eruca, a rare clonal cactus of the Sonoran desert. Evolutionary Ecology 20:131-142.

Couteron P. y Kokou K. 1997. Woody vegetation spatial patterns in a semi-arid savanna of Burkina Faso, West Africa. Plant Ecology 132:211-227.

Damman H. y Cain M.L. 1998. Population growth and viability analyses of the clonal woodland herb, Asarum canadense. Journal of Ecology 86:13-26.

de Jong T.J., Waser N.M., Price M.V. y Ring R.M. 1992. Plant size, geitonogamy and seed set in Ipomopsis aggregata. Oecologia 89:310-315.

de Kroon H. y van Groenendael J. Eds. 1997. The Ecology and Evolution of Clonal Plants. Backhuys Publishers, Leiden.

de Kroon H. y Hutchings M.J. 1995. Morphological plasticity in clonal plants: the foraging concept reconsidered. Journal of Ecology 83:143-152.

Diggle P.K., Lower S. y Ranker T.A. 1998. Clonal diversity in alpine populations of Polygonum viviparum (Polygonaceae). International Journal of Plant Sciences 159:606-615.

Diggle P.K., Meixner M.A., Carroll A.B. y Aschwanden C.F. 2002. Barriers to sexual reproduction in Polygonum viviparum: A comparative developmental analysis of $P$. viviparum and $P$. bistortoides. Annals of Botany 89:145-156.

Douhovnikoff V. y Dood R.S. 2003. Intra-clonal variation and a similarity threshold for identification of clones: application to $\mathrm{Sa}$ lix exigua using AFLP molecular markers. Theoretical Applied Genetics 106:1307-1315.

Douhovnikoff V., Cheng A.M. y Dodd R.S. 2004. Incidence, size and spatial structure of clones in second-growth stands of coast redwood, Sequoia sempervirens (Cupressaceae). American Journal of Botany 91:1140-1146.

Douhovnikoff V., McBride J.R. y Dodd R.S. 2005. Salix exigua clonal growth and population dynamics in relation to disturbance regime variation. Ecology 86:446-452.

Eckert C.G. 2000. Contributions of autogamy and geitonogamy to self-fertilization in a mass-flowering, clonal plant. Ecology 81:532-542.

Eckert C.G. 2002. The loss of sex in clonal plants. Evolutionary Ecology 15:501-520.

Ellstrand N.C. y Roose M.L. 1987. Patterns of genotypic diversity in clonal plant species. American Journal of Botany 74:123-131. 
Eriksson O. 1989. Seedling dynamics and life histories in clonal plants. Oikos 55:231-238.

Eriksson O. 1993. Dynamics of genets in clonal plants. Trends in Ecology \& Evolution 8:313-316.

Eriksson O. y Bremer B. 1993. Genet dynamics of the clonal plant Rubus saxatilis. Journal of Ecology 81:533-542.

Esselman E.J., Jianqiang L., Crawford D.J., Winduss J.L. y Wolfe A.D. 1999. Clonal diversity in the rare Calamagrostis porteri ssp insperata (Poaceae): comparative results for allozymes and random amplified polymorphic DNA (RAPD) and intersimple sequence repeat (ISSR) markers. Molecular Ecology 8:443451.

Everitt B.S. 1977. The Analysis of Contingency Tables. Chapmann \& Hall, Bury St Edmunds.

Gabrielsen T.M. y Brochmann C. 1998. Sex after all: high levels of diversity detected in the arctic clonal plant Saxifraga cernua using RAPD markers. Molecular Ecology 7:1701-1708.

Garnier L.K.M., Durand J. y Dajoz I. 2002. Limited seed dispersal and microspatial population structure of an agamospermous grass of West African savannahs, Hyparrhenia diplandra (Poaceae). American Journal of Botany 89:1785-1791.

Gibson D.J. y Menges E.S. 1994. Population structure and spatial pattern in the dioecious shrub Ceratiola ericoides. Journal of Vegetation Sciences 5:337-346.

Gleason H.A. 1920. Some applications of the quadrat method. Bulletin of the Torrey Botanical Club 47:21-33.

Hämmerli A. y Reusch T.B.H. 2003a. Flexible mating: cross-pollination affects sex expression in a marine clonal plant. Journal of Evolutionary Biology 18:1096-1105.

Hämmerli A. y Reusch T.B.H. 2003b. Genetic neighbourhood of clone structures in eelgrass meadows quantified by spatial autocorrelation of microsatellite markers. Heredity 91:448-455.

Handel S.N. 1985. The intrusion of clonal growth patterns on plant breeding system. The American Naturalist 125:367-384.

Harper J. 1985. Modules, branches, and the capture of resources. En: Jackson J.B.C., Buss L.W. y Cook R.E. Eds. Population Biology and Evolution of Clonal Organisms, pp. 1-33, Yale University Press, New Haven.

Hartnett D.C. y Bazzaz F.A. 1985. The integration of neighbourhood effects by clonal genets in Solidago canadensis. The Journal of Ecology 73:415-427.

Hirayama K. y Sakimoto M. 2008. Clonal structure and diversity of Cryptomeria japonica along a slope in a cool-temperate, oldgrowth mixed forest in the snowy region of Japan. Canadian Journal of Forest Research 38:2804-2813.

Honnay O. y Bossuyt B. 2005. Prolonged clonal growth: escape route or route to extinction? Oikos 108:427-432.

Honnay O., Jacquemyn H., Roldán-Ruiz I. y Hermy M. 2006. Consequences of prolonged clonal growth on local and regional genetic structure and fruiting success of the forest perennial Maianthemum bifolium. Oikos 112:21-30.

Honnay O. y Jacquemyn H. 2008. A meta-analysis of the relation between mating system, growth form and genotypic diversity in clonal plant species. Evolutionary Ecology 22:299-312.

Ivey C.T. y Richards J.H. 2001. Genotypic diversity and clonal structure of everglades sawgrass, Cladium jamaicense (Cyperaceae). International Journal of Plant Sciences 162:1327-1335.

Jackson J.B.C., Buss L.W. y Cook R.E. Eds. 1985. Population Biology and Evolution of Clonal organisms. Yale University Press, New Haven.
Jacquemyn H., Brys R., Honnay O., Hermy M. y Roldán-Ruiz I. 2005. Local forest environment largely affects below-ground growth, clonal diversity and fine-scale spatial genetic structure in the temperate deciduous forest herb Paris quadrifolia. Molecular Ecology 14:4479-4488.

Jonsson B.O., Jónsdóttir I.S. y Crongberg N. 1996. Clonal diversity and allozyme variation in populations of the arctic sedge Carex bigelowii (Cyperaceae). Journal of Ecology 84:449-459.

Kays S. y Harper J.L. 1974. The regulation of plant and tiller density in a grass sward. Journal of Ecology 62:97-105.

Keane B., Pelikan S., Toth G.P., Smith M.K. y Rogstad S.H. 1999. Genetic diversity of Typha latifolia (Typhaceae) and the impact of pollutants examined with tamdem-repetitive DNA probes. American Journal of Botany 86:1226-1238.

Kjølner S., Såstad S.M. y Brochmann C. 2006. Clonality and recombination in the arctic plant Saxifraga cernua. Botanical Journal of the Linnean Society 152:209-217.

Klimeš L., Klimešová J., Hendriks R.J.J. y van Groenendael J.M. 1997. Clonal plant architecture: a comparative analysis of form and function. En: de Kroon H. y van Groenendael J. Eds. The Ecology and Evolution of Clonal Plants, pp. 1-29, Backhuys Publishers, Leiden.

Korpelainen H. 1994. Sex ratios and resource allocation among sexually reproducing plants of Rubus chamaemorus. Annals of Botany 74:627-632.

Korpelainen H., Antonious-Klemola K. y Werlemark G. 1999. Clonal structure of Rubus chamaemorus populations: comparison of different molecular methods. Plant Ecology 143:123-128.

Kreher S.A., Foré S.A. y Collins B.S. 2000. Genetic variation within and among patches of the clonal species, Vaccinium stamineum L. Molecular Ecology 9:1247-1252.

Kudoh H., Shibaike H., Takasu H., Whigham D.F. y Kawano S. 1999. Genet structure and determinants of clonal structure in a temperate deciduous woodland herb, Uvularia perfoliata. Journal of Ecology 87:244-257.

Liston A., Wilson B.L., Robinson W.A., Doescher P.S., Harris N.R. y Svejcar T. 2003. The relative importance of sexual reproduction versus clonal spread in an arid bunchgrass. Oecologia 137:216-225.

Liu J., Dong M., Miao S.L., Li Z.Y., Song M.H. y Wang R.Q. 2006. Invasive alien plants in China: role of clonality and geographical origin. Biological Invasions 8:1461-1470.

Mandujano M.C., Montaña C., Méndez I. y Golubov J. 1998. The relative contributions of sexual reproduction and clonal propagation in Opuntia rastrera from two habitats in the Chihuahuan desert. Journal of Ecology 86:911-921.

Mandujano M.C., Montaña C., Franco M., Golubov J. y FloresMartínez A. 2001. Integration of demographic annual variability in a clonal desert cactus. Ecology 82:344-359.

Mandujano-Sánchez M.C. 2007. La clonalidad y sus efectos en la biología de poblaciones. En: Eguiarte L.E., Souza V. y Aguirre X. Comp. Ecología Molecular, pp. 215-250, Secretaría de Medio Ambiente y Recursos Naturales-Instituto Nacional de Ecología-Universidad Nacional Autónoma de México-Comosión Nacional para el Conocimiento y Uso de la Biodiversidad, México, D.F.

Mayes S.G., McGinley M.A. y Werth C.R. 1998. Clonal population structure and genetic variation in sand-shinnery oak, Quercus havardii (Fagaceae). American Journal of Botany 85:1609-1617. McFadden C.S. 1991. A comparative demographic analysis of clonal 
reproduction in a temperate soft coral. Ecology 72:1849-1866.

Misuki I., Ishida K., Tani N. y Tsumura Y. 2010. Fine-scale spatial structure of genets and sexes in the dioecious plant Dioscorea japonica, which disperses by both bulbils and seeds. Evolutionary Ecology 24:1399-1415.

Mori Y., Nagamitsu T. y Kubo T. 2009. Clonal growth and its effects on male and female reproductive success in Prunus ssiori (Rosaceae). Population Ecology 51:175-186.

Nagamitsu T., Ogawa M., Ishida K. y Tanouchi H. 2004. Clonal diversity, genetic structure, and mode of recruitment in a Prunus ssiori population established after volcanic eruptions. Plant Ecology 174:1-10.

Okuda T., Kachi N., Yap S.K. y Manokaran N. 1997. Tree distribution pattern and fate of juveniles in a lowland tropical rain forest - implications for regeneration and maintenance of species diversity. Plant Ecology 131:155-171.

Pan J.J. y Price J.S. 2002. Fitness and evolution in clonal plants: the impact of clonal growth. Evolutionary Ecology 15:583-600.

Parker K.C. y Hamrick J.L. 1992. Genetic diversity and clonal structure in a columnar cactus, Lophocereus schottii. American Journal of Botany 79:86-96.

Parks C. y Werth C.R. 1993. A study of spatial features of clones in a population of bracken fern, Pteridium aquilinum (Dennstaedtiaceae). American Journal of Botany 80:537-544.

Peterson C.J. y Jones R.H. 1997. Clonality in woody plants: a review and comparison with clonal herbs. En: de Kroon H. y van Groenendael J. Eds. The Ecology and Evolution of Clonal Plants, pp. 263-289, Backhuys Publishers, Leiden.

Pfeiffer T., Klahr A., Heinrich A. y Schnittler M. 2011. Does sex make a difference? Genetic diversity and spatial genetic structure in two co-occurring species of Gagea (Liliaceae) with contrasting reproductive strategies. Plant Systematics and Evolution 292:189-201.

Pornon A. y Escaravage N. 1999. Genotypic structure in clonal Rhododendron ferrugineum L. (Ericaceae) populations: origin and manteinance. Plant Ecology 141:145-150.

Pornon A., Escaravage N., Thomas P. y Taberlet P. 2000. Dynamics of genotypic structure in clonal Rhododendron ferrugineum (Ericaceae) populations. Molecular Ecology 9:1099-1111.

Pornon A., Escaravage N., Till-Botraud I. y Doche B. 1997. Variation of reproductive traits in Rhododendron ferrugineum $\mathrm{L}$. (Ericaceae) populations along a successional gradient. Plant Ecology 130:1-11.

Price E.A.C. y Marshall C. 1999. Clonal plants and environmental heterogeneity. Plant Ecology 141:3-7.

Reisch C., Schurm S. y Poschlod P. 2007. Spatial genetic structure and clonal diversity in an alpine population of Salix herbacea (Salicaceae). Annals of Botany 99:647-651.

Reusch T.B.H. 2003. Floral neighbourhoods in the sea: how floral density, opportunity for outcrossing and population fragmentation affect seed set in Zostera marina. Journal of Ecology 91:610-615.

Richards A.J. 1997. Plant Breeding Systems. Chapman \& Hall,
Londres.

Rosseto M., Gross C.L., Jones R. y Hunter J. 2004. The impact of clonality on an endangered tree (Elaeocarpus williamsianus) in a fragment rain forest. Biological Conservation 117:33-39.

Ruggieiro M.V., Capone S., Pirozzi P., Reusch T.B.H. y Procaccini G. 2005. Mating system and clonal architecture: a comparative study in two marine angiosperms. Evolutionary Ecology 19:487-499.

Shimizu Y, Ando M. y Sakai F. 2002. Clonal structure of natural populations of Cryptomeria japonica growing at different positions on slopes, detected using RAPD markers. Biochemical Systematics and Ecology 30:733-748.

Shreve F. 1917. The establishment of desert perennials. Journal of Ecology 5:210-216.

Sipes S.D. y Wolf P.G. 1997. Clonal structure and patterns of allozyme diversity in the rare endemic Cycladenia humilis var. jonesii (Apocynaceae). American Journal of Botany 84:401-409.

Stehlik I. y Holderegger R. 2000. Spatial genetic structure and clonal diversity of Anemone nemorosa in late successional deciduous woodlands of Central Europe. Journal of Ecology 88:424-435.

Suvanto L.I. y Latva-Karjantvaa T.B. 2005. Clone identification and clonal structure of the European aspen (Populus tremula). Molecular Ecology 14:2851-2860.

Tiffney B.H. y Niklas K.J. 1985. Clonal growth in land plants: a paleobotanical perspective. En: Jackson J.B.C., Buss L.W. y Cook R.E. Eds. Population Biology and Evolution of Clonal Organisms, pp. 35-66, Yale University Press, New Haven.

Tooke F., Ordidge M., Chiurugwi T. y Battey N. 2005. Mechanisms and function of flower and inflorescence reversion. Journal of Experimental Botany 56:2587-2599.

Torimaru T. y Tomaru N. 2005. Fine-scale clonal structure and diversity within patches of a clone-forming dioecious shrub, Ilex leucoclada (Aquifoliaceae). Annals of Botany 95:295-304.

van Dijk P.J. y Bakx-Schotman J.M.T. 2004. Formation of unreduced megaspores (diplospory) in apomictic dandelions (Taraxacum officinale, s.l.) is controlled by a sex-specific dominant locus. Genetics 166:483-492.

van Groenendael J.M. y de Kroon H. 1990. Clonal Growth in Plants: Regulation and Function. SPB Academic Publishing, La Haya.

van Groenendael J.M., Klimeš L., Klimešová J. y Hendriks R.J.J. 1996. Comparative ecology of clonal plants. Philosophical Transactions of Royal Society of London 351:1331-1339.

Widén B., Cronberg N. y Widén M. 1994. Genotypic diversity, molecular markers and spatial distribution of genets in clonal plants, a literature survey. Folia Geobotanica y Phytotaxonomica 29:245-263.

Wilson A.S.G., van der Kamp B.J. y Ritland C. 2005a. Opportunities for geitonogamy in the clonal herb Maianthemum dilatatum. Canadian Journal of Botany 83:1082-1087.

Wilson A.S.G., van der Kamp B.J. y Ritland C. 2005b. Spatial genetic and clonal structure in Maianthemum dilatatum as defined by AFLP markers. Canadian Journal of Botany 83:1126-1132. 
Apéndice. Características de las especies y poblaciones: $\mathbf{S C R Z}=$ sistema de cruza, $\mathbf{P O L}=$ polinización, $\mathbf{P R O R}=$ estructuras o procesos que dan origen a los ramets, CCT-N = tipo de crecimiento clonal en la clasificación de Tiffney y Niklas (1985), POB/HL = número de población y/o hábitat local, $\mathbf{P D E}=$ clave del patrón de distribución espacial de acuerdo a la figura 2, AER = arreglo espacial de los ramets de distintos genets, $\boldsymbol{D}=$ índice de diversidad clonal de Simpson, $\boldsymbol{G} / \mathbf{N}=$ proporción de genotipos distinguibles, Métodos = métodos empleados para la evaluación de los parámetros. El significado de las abreviaturas empleadas en cada columna se muestra al final del cuadro.

\begin{tabular}{|c|c|c|c|c|c|c|c|c|c|c|c|c|c|}
\hline Especie (Familia) & Hábito & SCRZ & POL & PROR & CCT-N & $\begin{array}{c}\text { POB/ } \\
\mathrm{HL}\end{array}$ & $\begin{array}{c}\text { PDE } \\
\text { (fig. 2) }\end{array}$ & $\begin{array}{c}\text { AER } \\
\text { (fig. 2) }\end{array}$ & $D$ & $G / N$ & $\begin{array}{l}\text { Estructura } \\
\text { genética } \\
\text { local (EGL) }\end{array}$ & $\begin{array}{c}\text { Métodos } \\
\text { PDE, } D, G / N \\
\text { y EGL }\end{array}$ & Referencias \\
\hline $\begin{array}{l}\text { Anemone } \\
\text { nemorosa } \\
\text { (Ranunculaceae) }\end{array}$ & $\mathrm{Hp}$ & A & $\mathrm{Bt}$ & $\begin{array}{c}\mathrm{Rzm+} \\
\mathrm{drm}\end{array}$ & $\mathrm{Lg}+\mathrm{NI}$ & $\begin{array}{l}\text { ppm } \\
(20)\end{array}$ & $(4,5)$ & $\mathrm{Ag}+\mathrm{En}$ & $\mathrm{n} / \mathrm{e}$ & 0.95 & $\begin{array}{l}\operatorname{asp},<1 \mathrm{~m} \\
(\mathrm{rm})\end{array}$ & tra+ISZ+asp & $\begin{array}{l}\text { Stehlik y } \\
\text { Holderegger } \\
\text { 2000* }\end{array}$ \\
\hline $\begin{array}{l}\text { Botrychium } \\
\text { pumicola } \\
\text { (Ophioglossaceae) }\end{array}$ & $\mathrm{Hp}$ & A & $\mathrm{Ab}$ & gmas & $\mathrm{NI}$ & $\begin{array}{l}\text { p } 1 \\
\text { p } 2 \\
\text { p } 3\end{array}$ & $\begin{array}{l}(2,5) \\
(2,5)\end{array}$ & $\begin{array}{l}\mathrm{Ag}+\mathrm{En} \\
\mathrm{Ag}+\mathrm{En} \\
\mathrm{Ag}+\mathrm{En}\end{array}$ & $\begin{array}{c}0.14 \\
0.16 \\
0.1\end{array}$ & $\begin{array}{l}0.93 \\
0.9 \\
0.65\end{array}$ & $\begin{array}{c}\text { mt: no } \\
\text { detectada } \\
\text { mt: no } \\
\text { detectada } \\
\text { mt: no } \\
\text { detectada }\end{array}$ & prc + ISSR $+m t$ & $\begin{array}{c}\text { Camacho y } \\
\text { Liston, 2001* }\end{array}$ \\
\hline $\begin{array}{l}\text { Calamagrostis } \\
\text { porteri ssp. } \\
\text { insperata (Poaceae) }\end{array}$ & $\mathrm{Hp}$ & A & $\mathrm{Ab}$ & rzm & $\operatorname{Lg}$ & $\begin{array}{l}\text { p } 2 \\
\text { p } 3 \\
\text { p } 4\end{array}$ & $\begin{array}{l}2 \\
2 \\
2\end{array}$ & $\begin{array}{l}\mathrm{Ag} \\
\mathrm{Ag} \\
\mathrm{Ag}\end{array}$ & $\begin{array}{l}n / e \\
n / e \\
n / e\end{array}$ & $\begin{array}{c} \\
1 \\
0.82 \\
0.91\end{array}$ & $\begin{array}{l}\mathrm{n} / \mathrm{e} \\
\mathrm{n} / \mathrm{e} \\
\mathrm{n} / \mathrm{e}\end{array}$ & $\begin{array}{c}\text { desc+tra+ } \\
\text { ISRR+ } \\
\text { RAPD }\end{array}$ & $\begin{array}{c}\text { Esselman } \\
\text { et al., } \\
1999\end{array}$ \\
\hline $\begin{array}{l}\text { Carex bigelowii } \\
\text { (Cyperaceae) }\end{array}$ & $\mathrm{Hp}$ & $?$ & $\mathrm{Ab}$ & rzm & $\operatorname{Lg}$ & $\begin{array}{l}\text { p } 1 \\
\text { p } 2 \\
\text { p } 3\end{array}$ & $\begin{array}{l}4 \\
4 \\
4\end{array}$ & $\begin{array}{l}\mathrm{Ag} \\
\mathrm{Ag} \\
\mathrm{Ag}\end{array}$ & $\begin{array}{l}0.98 \\
0.98 \\
0.98\end{array}$ & $\begin{array}{l}0.52 \\
0.63 \\
0.48\end{array}$ & $\begin{array}{l}\text { n/e } \\
\text { n/e } \\
\text { n/e }\end{array}$ & tra+ISZ & $\begin{array}{c}\text { Jonsson } \\
\text { et al., 1996* }\end{array}$ \\
\hline $\begin{array}{l}\text { Cladium jamaicense } \\
\text { (Cyperaceae) }\end{array}$ & $\mathrm{Hp}$ & $?$ & $\mathrm{Ab}$ & $\begin{array}{c}\text { rzm+ } \\
\text { pnt }\end{array}$ & $\mathrm{Lg}+\mathrm{Nl}$ & $\begin{array}{l}p 1 \\
\text { p } 2 \\
\text { p } 3 \\
\text { p } 4 \\
\text { p } 5 \\
\text { p } 6 \\
\text { p } 7 \\
\text { p } 8 \\
\text { p } 9 \\
\text { p } 10 \\
\text { p } 11 \\
\text { p } 12 \\
\text { p } 13 \\
\text { p } 14\end{array}$ & $\begin{array}{l}(4,5) \\
(4,5) \\
(4,5) \\
(4,5) \\
(4,5) \\
1 \\
(4,5) \\
(4,5) \\
(4,5) \\
(4,5) \\
(4,5) \\
(4,5) \\
(4,5) \\
(4,5)\end{array}$ & $\begin{array}{c}A g+E n \\
A g+E n \\
A g+E n \\
A g+E n \\
A g+E n \\
A g \\
A g+E n \\
A g+E n \\
A g+E n \\
A g+E n \\
A g+E n \\
A g+E n \\
A g+E n \\
A g+E n\end{array}$ & $\begin{array}{c}0.236 \\
0.269 \\
0.271 \\
0.682 \\
0.159 \\
0 \\
0.538 \\
0.212 \\
0.771 \\
0.698 \\
0.814 \\
0.682 \\
0.673 \\
0.444\end{array}$ & $\begin{array}{l}0.044 \\
0.044 \\
0.125 \\
0.231 \\
0.043 \\
0.021 \\
0.146 \\
0.068 \\
0.106 \\
0.143 \\
0.261 \\
0.114 \\
0.13 \\
0.043\end{array}$ & $\begin{array}{c}\text { mpic: no } \\
\text { detectada } \\
\text { mpic: no } \\
\text { detectada } \\
\text { mpic: no } \\
\text { detectada } \\
\text { mpic: no } \\
\text { detectada } \\
\text { mpic: no } \\
\text { detectada } \\
\text { n/a } \\
\text { mpic: no } \\
\text { detectada } \\
\text { mpic: no } \\
\text { detectada } \\
\text { mpic: no } \\
\text { detectada } \\
\text { mpic: no } \\
\text { detectada } \\
\text { mpic: no } \\
\text { detectada } \\
\text { mpic: no } \\
\text { detectada } \\
\text { mpic: no } \\
\text { detectada } \\
\text { mpic: no } \\
\text { detectada }\end{array}$ & $\begin{array}{l}\text { tra+lSZ+ } \\
\text { mpic }\end{array}$ & $\begin{array}{l}\text { Ivey y Richards, } \\
\text { 2001* }\end{array}$ \\
\hline
\end{tabular}


Apéndice. Continuación

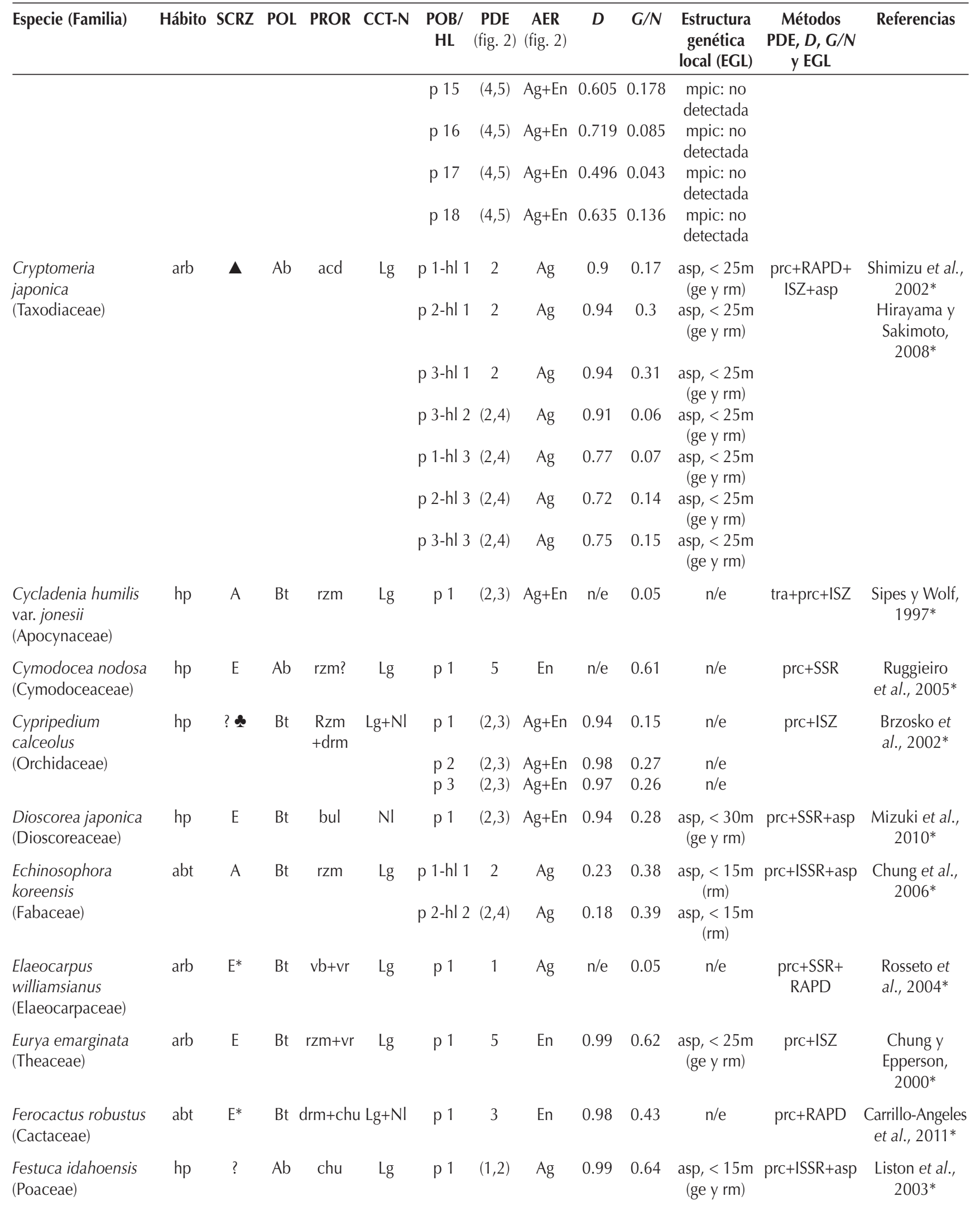


Apéndice. Continuación

\begin{tabular}{|c|c|c|c|c|c|c|c|c|c|c|c|c|c|}
\hline Especie (Familia) & Hábito & SCRZ & POL & PROR & CCT-N & $\begin{array}{c}\mathrm{POB} / \\
\mathrm{HL}\end{array}$ & $\begin{array}{l}\text { PDE } \\
\text { (fig. 2) }\end{array}$ & $\begin{array}{c}\text { AER } \\
\text { (fig. 2) }\end{array}$ & $D$ & $G / N$ & $\begin{array}{l}\text { Estructura } \\
\text { genética } \\
\text { local (EGL) }\end{array}$ & $\begin{array}{c}\text { Métodos } \\
\text { PDE, } D, G / N \\
\text { y EGL } \\
\end{array}$ & Referencias \\
\hline \multirow[t]{3}{*}{$\begin{array}{l}\text { Gagea lutea } \\
\text { (Liliaceae) }\end{array}$} & \multirow[t]{3}{*}{ hg } & \multirow[t]{3}{*}{$?$} & \multirow[t]{3}{*}{$\mathrm{Bt}$} & \multirow[t]{3}{*}{ buls } & \multirow[t]{3}{*}{$\mathrm{Nl}$} & p 1 & $(2,3)$ & $\mathrm{Ag}+\mathrm{En}$ & $\mathrm{n} / \mathrm{e}$ & 0.51 & \multirow{3}{*}{\multicolumn{2}{|c|}{$\begin{array}{l}\text { asp, }<8 \mathrm{~m}, 16 \text { tra+AFLP+asp } \\
\mathrm{a}<24 \mathrm{~m}(\mathrm{rm}) \\
\mathrm{asp},<8 \mathrm{~m}, 16 \\
\mathrm{a}<24 \mathrm{~m}(\mathrm{rm}) \\
\mathrm{asp},<8 \mathrm{~m}, 16 \\
\mathrm{a}<24 \mathrm{~m}(\mathrm{rm})\end{array}$}} & \multirow[t]{3}{*}{$\begin{array}{l}\text { Pfeiffer et al., } \\
2011^{*}\end{array}$} \\
\hline & & & & & & p 2 & $(2,3)$ & $\mathrm{Ag}+\mathrm{En}$ & $\mathrm{n} / \mathrm{e}$ & 0.57 & & & \\
\hline & & & & & & p 3 & $(2,3)$ & $\mathrm{Ag}+\mathrm{En}$ & $\mathrm{n} / \mathrm{e}$ & 0.61 & & & \\
\hline \multirow{3}{*}{$\begin{array}{l}\text { Gagea spathacea } \\
\text { (Liliaceae) }\end{array}$} & \multirow[t]{3}{*}{ hg } & \multirow[t]{3}{*}{$?$} & \multirow[t]{3}{*}{$\mathrm{Bt}$} & \multirow[t]{3}{*}{ buls } & \multirow[t]{3}{*}{$\mathrm{NI}$} & p 1 & 1 & $\mathrm{Ag}$ & $\mathrm{n} / \mathrm{e}$ & 0.001 & $\mathrm{n} / \mathrm{a}$ & \multirow[t]{3}{*}{ tra+AFLP } & \multirow{3}{*}{$\begin{array}{r}\text { Pfeiffer et al., } \\
2011^{*}\end{array}$} \\
\hline & & & & & & p 2 & 1 & $\mathrm{Ag}$ & $\mathrm{n} / \mathrm{e}$ & 0.001 & $\mathrm{n} / \mathrm{a}$ & & \\
\hline & & & & & & p 3 & 1 & $\mathrm{Ag}$ & $\mathrm{n} / \mathrm{e}$ & 0.001 & $\mathrm{n} / \mathrm{a}$ & & \\
\hline $\begin{array}{l}\text { Hyparrhenia } \\
\text { diplandra (Poaceae) }\end{array}$ & hp & $?$ & $\mathrm{Ab}$ & agp & $\mathrm{NI}$ & p 1 & 5 & En & 0.85 & 0.07 & $\begin{array}{l}\text { asp, }<6 \mathrm{~m} \\
\quad(\mathrm{rm})\end{array}$ & $\begin{array}{c}\text { prc+lSZ } \\
+ \text { CLO+asp }\end{array}$ & $\begin{array}{c}\text { Garnier et al., } \\
2002^{*}\end{array}$ \\
\hline $\begin{array}{l}\text { Ilex leucoclada } \\
\text { (Aquifoliaceae) }\end{array}$ & abt & E & $\mathrm{Bt}$ & $\operatorname{acd} \mathbf{\Lambda}$ & $\operatorname{Lg}$ & p 1 & $(2,3)$ & $\mathrm{Ag}+\mathrm{En}$ & 0.67 & 0.09 & $\mathrm{n} / \mathrm{e}$ & prc+RAPD & $\begin{array}{c}\text { Torimaru y } \\
\text { Tomaru, 2005* }\end{array}$ \\
\hline \multirow[t]{8}{*}{$\begin{array}{l}\text { Lophocereus schottii } \\
\text { (Cactaceae) }\end{array}$} & \multirow[t]{8}{*}{ arb } & \multirow[t]{8}{*}{ A } & \multirow[t]{8}{*}{$\mathrm{Bt}$} & \multirow[t]{8}{*}{$\begin{array}{l}\mathrm{drm} \\
+\mathrm{acd}\end{array}$} & \multirow[t]{8}{*}{$\mathrm{Lg}+\mathrm{NI}$} & p 1-hl 1 & 5 & En & 0.95 & 0.64 & $\begin{array}{c}\text { SG: 0-10 } \\
<10-50>\text { más } \\
\text { de } 50 m \text { (ge) }\end{array}$ & \multirow{8}{*}{ prc+ISZ+SG } & \multirow[t]{8}{*}{$\begin{array}{l}\text { Parker y } \\
\text { Hamrick, } \\
1992^{*}\end{array}$} \\
\hline & & & & & & p 2-hl 2 & 2 & $\mathrm{Ag}$ & 0.95 & 0.52 & $\begin{array}{l}\text { SG: } 0-10 \\
<10-50>\text { más } \\
\text { de } 50 \mathrm{~m} \text { (ge) }\end{array}$ & & \\
\hline & & & & & & p 3-hl 3 & 2 & $\mathrm{Ag}$ & 0.93 & 0.65 & $\begin{array}{c}\text { SG: } 0-10 \\
<10-50>\text { más } \\
\text { de } 50 m \text { (ge) }\end{array}$ & & \\
\hline & & & & & & p 4-hl 1 & $(1,2)$ & $\mathrm{Ag}$ & 0.94 & 0.24 & $\begin{array}{l}\text { SG: } 0-10> \\
\text { 10-50>más } \\
\text { de } 50 \mathrm{~m} \text { (ge) }\end{array}$ & & \\
\hline & & & & & & p 5-hl 4 & $(1,2)$ & $\mathrm{Ag}$ & 0.92 & 0.41 & $\begin{array}{l}\text { SG: } 0-10> \\
10-50<\text { más } \\
\text { de } 50 m \text { (ge) }\end{array}$ & & \\
\hline & & & & & & p 6-hl 4 & $(1,2)$ & $\mathrm{Ag}$ & 0.87 & 0.45 & $\begin{array}{c}\text { SG: } 0-10> \\
10-50<\text { más } \\
\text { de } 50 m \text { (ge) }\end{array}$ & & \\
\hline & & & & & & p 7-hl 2 & $(1,2)$ & $\mathrm{Ag}$ & 0.8 & 0.18 & $\begin{array}{l}\text { SG: } 0-10> \\
\text { 10-50>más } \\
\text { de } 50 \mathrm{~m} \text { (ge) }\end{array}$ & & \\
\hline & & & & & & p 8-hl 5 & $(1,5)$ & $\mathrm{Ag}+\mathrm{En}$ & 0.59 & 0.36 & $\begin{array}{l}\text { SG: } 10-50= \\
\text { más de } 50 \mathrm{~m} \\
\quad(\text { ge })\end{array}$ & & \\
\hline $\begin{array}{l}\text { Lyonothamnus } \\
\text { floribundus } \\
\text { (Rosaceae) }\end{array}$ & arb & $?$ & $\mathrm{Bt}$ & $\mathrm{vr}$ & $\operatorname{Lg}$ & p 1 & $(1,2)$ & $\mathrm{Ag}$ & $\mathrm{n} / \mathrm{e}$ & 0.036 & $\mathrm{n} / \mathrm{e}$ & prc+RAPD & $\begin{array}{c}\text { Bushakra et al., } \\
\text { 1999* }\end{array}$ \\
\hline $\begin{array}{l}\text { Maianthemum } \\
\text { bifolium } \\
\text { (Convalliariaceae) }\end{array}$ & hp & $E^{*}$ & $\mathrm{Bt}$ & $\mathrm{rzm}$ & $\operatorname{Lg}$ & p 1 & $(1,2)$ & $\mathrm{Ag}$ & 0.88 & 0.7 & $\begin{array}{l}\text { asp, }<50 \mathrm{~m} \\
\text { (ge) \# }\end{array}$ & $\begin{array}{l}\text { trans+AFLP } \\
\quad+\text { asp }\end{array}$ & $\begin{array}{l}\text { Arens et al., } \\
2005^{*} \\
\text { Honnay et al. } \\
2006\end{array}$ \\
\hline $\begin{array}{l}\text { Maianthemum } \\
\text { dilatatum } \\
\text { (Convalliariaceae) }\end{array}$ & hp & EAs & $\mathrm{Bt}$ & $\operatorname{rzm} \mathbf{\Delta}$ & $\operatorname{Lg}$ & p 1 & $(1,2)$ & $\mathrm{Ag}$ & $\mathrm{n} / \mathrm{e}$ & 0.64 & $\begin{array}{l}\text { asp, }<45 \mathrm{~m} \\
\text { (ge y rm) }\end{array}$ & $\begin{array}{l}\text { prc }+ \text { AFLP } \\
\quad+\text { asp }\end{array}$ & $\begin{array}{l}\text { Wilson et al., } \\
2005 a^{*} \\
\text { Wilson et al., } \\
2005 b^{*}\end{array}$ \\
\hline $\begin{array}{l}\text { Paris quadrifolia } \\
\text { (Trilliaceae) }\end{array}$ & hp & A & $\mathrm{Bt}$ & $\mathrm{rzm}$ & $\operatorname{Lg}$ & p 1-hl 1 & 3 & En & 0.87 & 0.27 & $\begin{array}{l}\text { asp, }<5 \mathrm{~m} \\
(\text { ge y rm) }\end{array}$ & $\begin{array}{l}\text { prc }+ \text { AFLP } \\
\quad+\text { asp }\end{array}$ & $\begin{array}{c}\text { Jacquemyn et } \\
\text { al., 2005* }\end{array}$ \\
\hline
\end{tabular}


Apéndice. Continuación

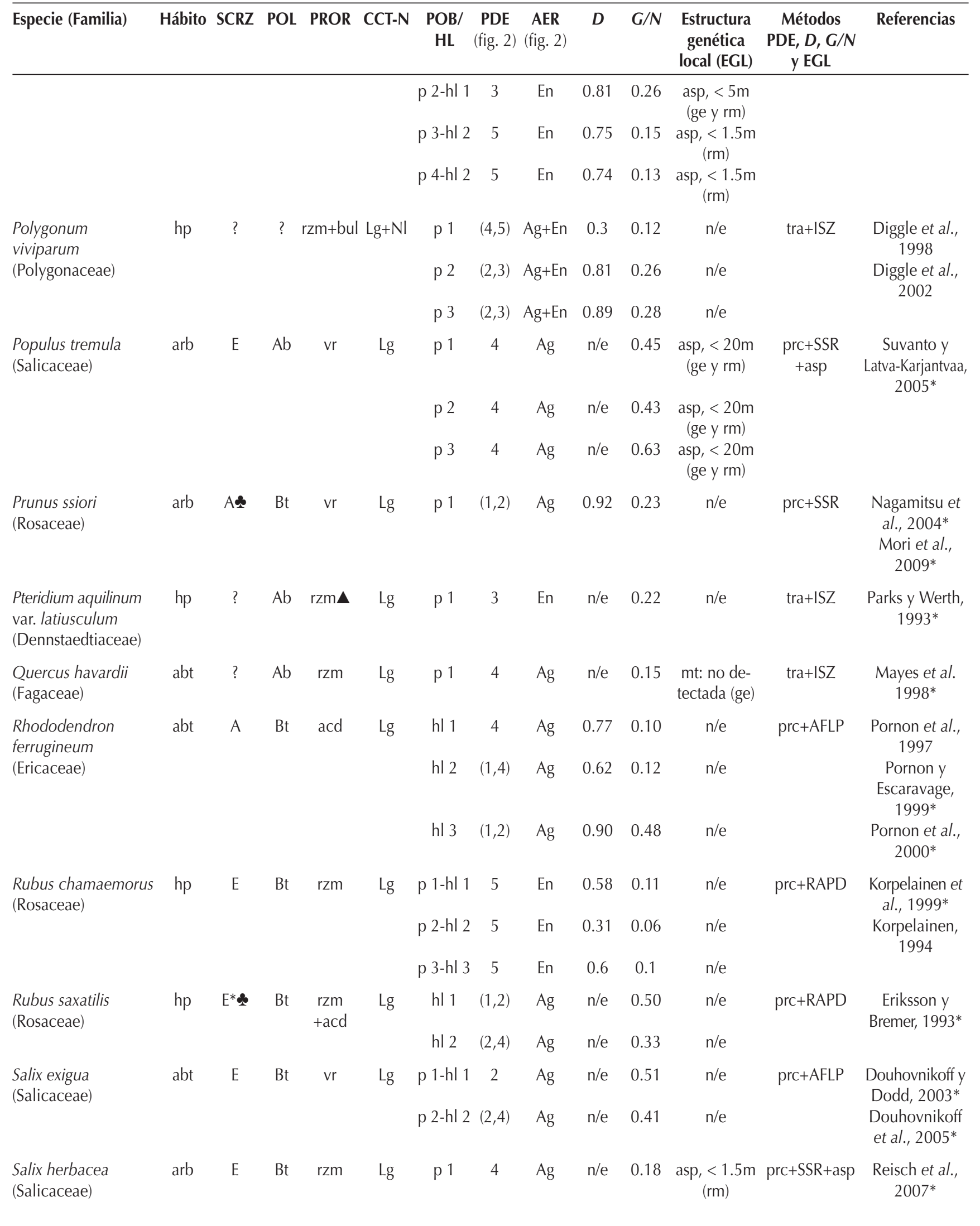


Apéndice. Continuación

\begin{tabular}{|c|c|c|c|c|c|c|c|c|c|c|c|c|c|}
\hline Especie (Familia) & Hábito & SCRZ & POL & PROR & CCT-N & $\begin{array}{c}\mathrm{POB} / \\
\mathrm{HL}\end{array}$ & $\begin{array}{c}\text { PDE } \\
\text { (fig. 2) }\end{array}$ & $\begin{array}{c}\text { AER } \\
\text { (fig. 2) }\end{array}$ & $D$ & $G / N$ & $\begin{array}{l}\text { Estructura } \\
\text { genética } \\
\text { local (EGL) }\end{array}$ & $\begin{array}{c}\text { Métodos } \\
\text { PDE, } D, G / N \\
\text { y EGL }\end{array}$ & Referencias \\
\hline \multirow[t]{6}{*}{$\begin{array}{l}\text { Saxifraga cernua } \\
\text { (Saxifragaceae) }\end{array}$} & \multirow[t]{6}{*}{ hp } & \multirow[t]{6}{*}{$E^{*}$} & \multirow[t]{6}{*}{$\mathrm{Bt}$} & \multirow[t]{6}{*}{ bul } & \multirow[t]{6}{*}{$\mathrm{Nl}$} & p 1 & 5 & En & 0.35 & 0.13 & $\begin{array}{c}\text { mt: no } \\
\text { detectada }\end{array}$ & \multirow[t]{6}{*}{$\begin{array}{l}\text { prc+RAPD } \\
+m t+a s p\end{array}$} & \multirow{6}{*}{$\begin{array}{l}\text { Gabrielsen y } \\
\text { Brochmann, } \\
1998^{*} \\
\text { KjØIner et al., } \\
\text { 2006* }\end{array}$} \\
\hline & & & & & & p 2 & 5 & En & 0.68 & 0.19 & $\begin{array}{c}\text { mt: no } \\
\text { detectada }\end{array}$ & & \\
\hline & & & & & & p 3 & 5 & En & 0.7 & 0.25 & $\begin{array}{l}\text { asp: no } \\
\text { detectada }\end{array}$ & & \\
\hline & & & & & & p 4 & 5 & En & 0.9 & 0.4 & $\begin{array}{c}\text { asp: no } \\
\text { detectada }\end{array}$ & & \\
\hline & & & & & & p 5 & 5 & En & 0.93 & 0.43 & $\begin{array}{l}\text { asp: no } \\
\text { detectada }\end{array}$ & & \\
\hline & & & & & & p 6 & 5 & En & 0.89 & 0.34 & $\begin{array}{c}\text { asp: no } \\
\text { detectada }\end{array}$ & & \\
\hline \multirow[t]{3}{*}{$\begin{array}{l}\text { Scirpus maritimus } \\
\text { (Cyperaceae) }\end{array}$} & \multirow[t]{3}{*}{ hp } & \multirow[t]{12}{*}{$\mathrm{E}^{*}$} & \multirow[t]{3}{*}{$\mathrm{Ab}$} & \multirow[t]{3}{*}{$\mathrm{rzm}$} & \multirow[t]{3}{*}{$\operatorname{Lg}$} & p 1 & 2 & $\mathrm{Ag}$ & $\mathrm{n} / \mathrm{e}$ & $\mathrm{n} / \mathrm{e}$ & $\mathrm{n} / \mathrm{e}$ & \multirow[t]{3}{*}{ desc } & \multirow[t]{3}{*}{$\begin{array}{c}\text { Charpentier et } \\
\text { al., } 2000\end{array}$} \\
\hline & & & & & & p 2 & 4 & $\mathrm{Ag}$ & $\mathrm{n} / \mathrm{e}$ & $\mathrm{n} / \mathrm{e}$ & $\mathrm{n} / \mathrm{e}$ & & \\
\hline & & & & & & p 3 & 4 & $\mathrm{Ag}$ & $\mathrm{n} / \mathrm{e}$ & $\mathrm{n} / \mathrm{e}$ & $\mathrm{n} / \mathrm{e}$ & & \\
\hline \multirow[t]{9}{*}{$\begin{array}{l}\text { Sequoia sempervirens } \\
\text { (Taxodiaceae) }\end{array}$} & \multirow[t]{9}{*}{ arb } & & \multirow[t]{9}{*}{$\mathrm{Ab}$} & \multirow[t]{9}{*}{$\mathrm{vb}$} & \multirow[t]{9}{*}{$\operatorname{Lg}$} & p 1-hl 1 & 2 & $\mathrm{Ag}$ & $\mathrm{n} / \mathrm{e}$ & 0.24 & $\begin{array}{l}\mathrm{mt}:<40 \mathrm{~m} \\
\quad(\mathrm{rm})\end{array}$ & \multirow[t]{9}{*}{$\operatorname{prc}+\mathrm{AFLP}+\mathrm{mt}$} & \multirow[t]{9}{*}{$\begin{array}{l}\text { Douhovnikoff } \\
\text { et al., 2004* }\end{array}$} \\
\hline & & & & & & p 2-hl 1 & 2 & $\mathrm{Ag}$ & $\mathrm{n} / \mathrm{e}$ & 0.38 & $\begin{array}{c}\mathrm{mt}:<40 \mathrm{~m} \\
(\mathrm{rm})\end{array}$ & & \\
\hline & & & & & & p 3-hl 1 & 2 & $\mathrm{Ag}$ & $\mathrm{n} / \mathrm{e}$ & 0.5 & $\begin{array}{c}\mathrm{mt}:<40 \mathrm{~m} \\
(\mathrm{rm})\end{array}$ & & \\
\hline & & & & & & p 4-hl 2 & 2 & $\mathrm{Ag}$ & $\mathrm{n} / \mathrm{e}$ & 0.6 & $\begin{array}{c}\mathrm{mt}:<40 \mathrm{~m} \\
(\mathrm{rm})\end{array}$ & & \\
\hline & & & & & & p 5-hl 2 & 2 & $\mathrm{Ag}$ & $\mathrm{n} / \mathrm{e}$ & 0.35 & $\begin{array}{c}\mathrm{mt}:<40 \mathrm{~m} \\
(\mathrm{rm})\end{array}$ & & \\
\hline & & & & & & p 6-hl 2 & 2 & $\mathrm{Ag}$ & $\mathrm{n} / \mathrm{e}$ & 0.6 & $\begin{array}{c}\mathrm{mt}:<40 \mathrm{~m} \\
(\mathrm{rm})\end{array}$ & & \\
\hline & & & & & & p 7-hl 3 & 2 & $\mathrm{Ag}$ & $\mathrm{n} / \mathrm{e}$ & 0.26 & $\begin{array}{c}\mathrm{mt}:<40 \mathrm{~m} \\
(\mathrm{rm})\end{array}$ & & \\
\hline & & & & & & p 8-hl 3 & 2 & $\mathrm{Ag}$ & $n / e$ & 0.19 & $\begin{array}{c}\mathrm{mt}:<40 \mathrm{~m} \\
(\mathrm{rm})\end{array}$ & & \\
\hline & & & & & & p 9-hl 3 & 2 & $\mathrm{Ag}$ & $\mathrm{n} / \mathrm{e}$ & 0.54 & $\begin{array}{c}\mathrm{mt}:<40 \mathrm{~m} \\
\quad(\mathrm{rm})\end{array}$ & & \\
\hline $\begin{array}{l}\text { Stenocereus eruca } \\
\text { (Cactaceae) }\end{array}$ & abt & $E^{*}$ & $\mathrm{Bt}$ & drm & $\mathrm{NI}$ & p 1 & 2 & $\mathrm{Ag}$ & 0.99 & 0.83 & $\begin{array}{l}\text { asp, }<20 \text { m } \\
\text { (ge y rm) }\end{array}$ & prc+ISSR+asp & $\begin{array}{c}\text { Clark-Tapia et } \\
\text { al., 2005* } \\
\text { Clark-Tapia et } \\
\text { al., } 2006\end{array}$ \\
\hline \multirow[t]{5}{*}{$\begin{array}{l}\text { Typha latifolia } \\
\text { (Thyphaceae) }\end{array}$} & \multirow[t]{5}{*}{$\mathrm{hp}$} & \multirow[t]{5}{*}{ A } & \multirow[t]{5}{*}{$\mathrm{Ab}$} & \multirow[t]{5}{*}{ rzm } & $\operatorname{Lg}$ & p 1 & $(4,5)$ & $\mathrm{Ag}+\mathrm{En}$ & $\mathrm{n} / \mathrm{e}$ & 0.77 & $\mathrm{n} / \mathrm{e}$ & tra+VNTR & $\begin{array}{c}\text { Keane et al., } \\
\text { 1999* }\end{array}$ \\
\hline & & & & & & p 2 & $(4,5)$ & $\mathrm{Ag}+\mathrm{En}$ & $\mathrm{n} / \mathrm{e}$ & 0.55 & $\mathrm{n} / \mathrm{e}$ & & \\
\hline & & & & & & p 3 & $(4,5)$ & $\mathrm{Ag}+\mathrm{En}$ & $\mathrm{n} / \mathrm{e}$ & 0.48 & $\mathrm{n} / \mathrm{e}$ & & \\
\hline & & & & & & p 4 & $(4,5)$ & $\mathrm{Ag}+\mathrm{En}$ & $\mathrm{n} / \mathrm{e}$ & 0.3 & $n / e$ & & \\
\hline & & & & & & p 5 & $(4,5)$ & $A g+E n$ & $\mathrm{n} / \mathrm{e}$ & 0.57 & $\mathrm{n} / \mathrm{e}$ & & \\
\hline $\begin{array}{l}\text { Uvularia perfoliata } \\
\text { (Convallariaceae) }\end{array}$ & hp & $?$ & $?$ & etl & $\operatorname{Lg}$ & p 1-hl 1 & $(4,5)$ & $A g+E n$ & 0.74 & 0.09 & $\begin{array}{c}\text { asp, }<3-5 m \\
(r m)\end{array}$ & prc+ISZ+asp & $\begin{array}{l}\text { Kudoh et al., } \\
\text { 1999* }\end{array}$ \\
\hline & & & & & & p 1-hl 2 & 1 & $\mathrm{Ag}$ & 0.59 & 0.03 & $\mathrm{n} / \mathrm{a}$ & & \\
\hline $\begin{array}{l}\text { Vaccinium myrtillus } \\
\text { (Ericaceae) }\end{array}$ & abt & A & $\mathrm{Bt}$ & rzm & $\operatorname{Lg}$ & p 1 & 2 & $\mathrm{Ag}$ & 0.94 & 0.29 & $\begin{array}{c}\text { asp: no } \\
\text { detectada }\end{array}$ & $\begin{array}{l}\text { prc+AFLP+ } \\
\text { RAPD+asp }\end{array}$ & $\begin{array}{c}\text { Albert et al., } \\
\text { 2003* }\end{array}$ \\
\hline
\end{tabular}


Apéndice. Continuación

\begin{tabular}{|c|c|c|c|c|c|c|c|c|c|c|c|c|c|}
\hline Especie (Familia) & Hábito & SCRZ & POL & PROR & CCT-N & $\begin{array}{c}\mathrm{POB} / \\
\mathrm{HL}\end{array}$ & $\begin{array}{l}\text { PDE } \\
\text { (fig. 2) }\end{array}$ & $\begin{array}{c}\text { AER } \\
\text { (fig. 2) }\end{array}$ & $D$ & $G / N$ & $\begin{array}{c}\text { Estructura } \\
\text { genética } \\
\text { local (EGL) }\end{array}$ & $\begin{array}{c}\text { Métodos } \\
\text { PDE, } D, G / N \\
\text { y EGL }\end{array}$ & Referencias \\
\hline $\begin{array}{l}\text { Vaccinium stamineum } \\
\text { (Ericaceae) }\end{array}$ & abt & $\mathrm{E}^{*}$ & $\mathrm{Bt}$ & $\mathrm{rzm}$ & $\operatorname{Lg}$ & $\mathrm{p} 1$ & $(2,3)$ & $\mathrm{Ag}+\mathrm{En}$ & $\mathrm{n} / \mathrm{e}$ & 0.68 & $\mathrm{n} / \mathrm{e}$ & prc+RAPD & $\begin{array}{l}\text { Kreher et al., } \\
\text { 2000* }\end{array}$ \\
\hline $\begin{array}{l}\text { Zostera marina } \\
\text { (Zosteraceae) }\end{array}$ & hp & A & $\mathrm{Ab}$ & $\mathrm{rzm}$ & $\operatorname{Lg}$ & $\begin{array}{l}\text { p 1-hl } 1 \\
\text { p 2-hl } 2\end{array}$ & $\begin{array}{l}(4,5) \\
(4,5)\end{array}$ & $\begin{array}{l}A g+E n \\
A g+E n\end{array}$ & $\mathrm{n} / \mathrm{e}$ & $\mathrm{n} / \mathrm{e}$ & $\begin{array}{c}\text { asp, }<6 \mathrm{~m} \\
\text { (ge y rm) } \\
\text { asp, }<6 \mathrm{~m} \\
\text { (ge y rm) }\end{array}$ & $\mathrm{prc}+\mathrm{SSR}+\mathrm{asp}$ & $\begin{array}{l}\text { Hämmerli y } \\
\text { Reusch, } 2003 \mathrm{a}^{*} \\
\text { Hämmerli y } \\
\text { Reusch, } 2003 \mathrm{~b}^{*}\end{array}$ \\
\hline $\begin{array}{l}\text { Zostera noltii } \\
\text { (Zosteraceae) }\end{array}$ & hp & A & $\mathrm{Ab}$ & $\mathrm{rzm}$ & $\operatorname{Lg}$ & p 1 & 4 & $\mathrm{Ag}$ & $\mathrm{n} / \mathrm{e}$ & 0.12 & $\mathrm{n} / \mathrm{e}$ & $\mathrm{prc}+\mathrm{SSR}$ & $\begin{array}{l}\text { Ruggieiro et } \\
\text { al., 2005* }\end{array}$ \\
\hline
\end{tabular}

Hábito: $\mathbf{a b t}=$ arbustos, $\mathbf{a r b}=$ árboles, $\mathbf{h p}=$ hierbas perennes

SCRZ: A = especies con capacidad de reproducirse por autocruza, $\mathbf{E}=$ especies con entrecruza obligada (p. ej., dioicas), $\mathbf{E}^{*}=$ especies con sistemas de autoincompatibilidad, = reclutamiento sexual observado y/o evaluado en condiciones naturales

POL: $\mathbf{A b}=$ polinización abiótica, $\mathbf{B t}=$ polinización biótica

PROR: acd = acodaduras, agp = agamospermia, bul = bulbilos, buls = bulbilos subterráneos, $\mathbf{c h u}=$ coronas huecas, $\mathbf{d r m}=$ desprendimiento de ramas, $\mathbf{e t l}=$ estolones, $\mathbf{g m a s}=$ gemas subterráneas, $\mathbf{p n t}=$ plantlets, $\mathbf{p s b}=$ pseudobulbos, $\mathbf{r z m}=$ rizomas, $\mathbf{v b}=$ vástagos basales, $\mathbf{v r}=\mathbf{v a ́ s t a g o s}$ radicales, $\mathbf{\Delta}$ = crecimiento tipo guerrilla

CCT-N: $\mathbf{L g}=$ crecimiento clonal ligado, $\mathbf{N I}=$ crecimiento clonal no ligado

POB/HL: $\mathbf{p}=$ población, $\mathbf{h l}=$ hábitat local (p. ej., bosque con dosel abierto vs bosque con dosel cerrado)

PDE: los paréntesis indican que la población presenta una combinación de patrones de distribución de los tipos indicados por los números (ver figura 2).

AER: Ag = los ramets de cada genet no se entremezclan con los ramets de genets distintos, En = los ramets de diferentes genets en la población se entremezclan.

EGL: \# = evaluado en una población distinta de donde se determinó el PDE, asp = autocorrelación espacial, mpic = método de probabilidad de identidad clonal, $\mathbf{m t}=$ prueba de Mantel, $\mathbf{S G}=$ similitud genética vs distancia espacial.

Métodos: AFLP = marcadores moleculares AFLPs, CLO = marcadores moleculares de cloroplasto, desc = descripción detallada del PDE, ISSR = marcadores moleculares ISSRs, ISZ = isoenzimas, $\mathbf{m p i c}=$ método de probabilidad de identidad clonal, $\mathbf{m t}=$ prueba de $\mathbf{M a n t e l}, \mathbf{p o b}=$ muestra de individuos o todos los individuos de la población, prc = parcelas, RAPD = marcadores moleculares RAPDs, SG $=$ similitud genética vs distancia espacial, $\mathbf{S S R}=$ marcadores moleculares SSRs (microsatélites),

Referencias: ${ }^{*}=$ estudios donde se presentan planos de la distribución espacial de los genotipos.

Todo el cuadro: ? = no se proporciona información, $\mathbf{n} / \mathbf{e}=$ no evaluado, $\mathbf{n} / \mathbf{a}=$ no aplica

GLOSARIO (columna PROR):

Acodaduras: formación de ramets a partir de un tallo originalmente erecto que se postra y genera raíces adventicias al entrar en contacto con el sustrato

Agamospermia: Producción asexual de semillas o embriones sin fertilización (diplosporía: el embrión se desarrolla de una célula diploide del saco embrionario, aposporía: el embrión se desarrolla de una célula somática del saco embrionario, embrionia adventicia: el embrión se desarrolla de células del tejido esporofítico como los tegumentos).

Bulbilos: bulbos pequeños que se forman en las axilas foliares o unidos a los bulbos.

Bulbos: tallos cortos modificados para almacenamiento rodeados por hojas carnosas o brácteas.

Coronas huecas: muerte de los tallos centrales en plantas cespitosas resultando en la formación de ramets independientes en la periferia. Descrito en pastos cespitosos.

Estolones: tallo horizontal que se extiende en la superficie del suelo.

Gemas: pequeñas estructuras vegetativas dispersables en briofitas y pteridofitas.

Plantlets: propágulos vegetativos que resultan de la reversión de estructuras reproductivas como flores y frutos a estructuras vegetativas.

Pseudobulbos: modificación del tallo para almacenamiento que se desarrolla entre dos nodos foliares en orquídeas.

Rizomas: tallo horizontal subterráneo.

Vástagos basales y radicales: formación de tallos en la base de un tallo o a partir de una raíz.

Referencias del glosario: Jackson et al., 1985; de Kroon y van Groenendael, 1997; Liston et al., 2003; van Dijk y Bakx-Schotman, 2004; Tooke et al., 2005. 


\title{
PATRONES DE DISTRIBUCIÓN ESPACIAL EN PLANTAS CLONALES
}

\author{
Israel Gustavo Carrillo-Angeles y María del Carmen Mandujano ${ }^{1}$ \\ Instituto de Ecología, Universidad Nacional Autónoma de México \\ ${ }^{1}$ Autor para la correspondencia: mcmandu@ecologia.unam.mx
}

\begin{abstract}
Resumen: En las especies clonales predomina la distribución agregada de los ramets, pero se plantea que la propagación por diásporas móviles o no ligadas, (por ejemplo, bulbilos y semillas agamospérmicas) forma arreglos espaciales de genets más entremezclados, que la propagación por estructuras ligadas (rizomas y estolones), y se sugiere que los arreglos entremezclados de genets favorecen la entrecruza. En este trabajo se presenta una revisión de 53 estudios en plantas clonales, para investigar la influencia de la producción de propágulos clonales, por diferentes formas de crecimiento clonal, en la formación de arreglos espaciales de genets y ramets. La hipótesis es que los arreglos entremezclados promueven la entrecruza, suponiendo que las poblaciones donde la entrecruza es más frecuente tenderán a presentar niveles más altos de diversidad genotípica. Los estudios revisados muestran un sesgo hacia herbáceas perennes rizomatosas. Predominan los patrones de distribución agregados de parches de ramets de distintos genets no entremezclados. Las poblaciones de especies que tienen ambos tipos de crecimiento de propágulos clonales (ligados y no ligados) y de especies que sólo presentan crecimiento ligado presentan patrones de distribución espacial de genets y ramets más entremezclados que las especies que sólo presentan crecimiento ligado. Encontramos que existe variación intraespecífica tanto en los patrones de distribución espacial como en los patrones reproductivos en las diferentes poblaciones. Sin embargo, utilizando índices de diversidad genotípica como estimadores, solamente se encontró un patrón congruente con el planteamiento sobre el efecto del arreglo espacial de los genets y los ramets en la proporción de genotipos distinguibles. Generalizar las consecuencias reproductivas y genéticas del arreglo espacial de los genets y los ramets continúa siendo complejo, debido a que una reducción del reclutamiento sexual y una dispersión restringida de polen, semillas y propágulos vegetativos pueden generar estructura genética local en las poblaciones. La estructura genética local tanto a nivel de genets (debida a la dispersión restringida de polen, semillas o ambos) como de ramets (indicando dispersión restringida de propágulos vegetativos) fue detectada en 46 poblaciones distribuidas en 16 especies. Estos factores aunados a la variedad de métodos de muestreo empleados en los trabajos publicados para detectar genets y ramets, la falta de estimadores numéricos comparables para describir la distribución espacial y la carencia de estudios en especies que se propagan por diásporas móviles o no ligadas reducen la resolución del efecto que tiene el tipo de crecimiento clonal en la formación de arreglos entremezclados de genets distintos sobre la diversidad genotípica.
\end{abstract}

Palabras clave: clonalidad, crecimiento, distribución, diversidad, entrecruza

\begin{abstract}
Clonal plants are predominantly associated with a clumped distribution of ramets. However, propagation by unlinked means generates a mixed arrangement instead of a clumped one found by linked propagules and intermingled arrangements of genets favor outcrossing. This paper reviews 53 studies on clonal plants to assess the influence of the production of clonal offspring by different means on the spatial arrangement of genets and ramets. We also evaluate the assumption that mixed arrangements promote outcrossing, under the hypothesis that populations where outcrossing is common would have higher levels of genotypic diversity. The studies that were consulted showed a strong bias towards rhizomatous perennial herbs. Clumped distribution patterns predominate in which patches consist of discrete groups of ramets from one or more genets that do not spatially mix. Populations having both types of propagation (linked and unlinked) and species that only have linked propagation have a higher spatially mixed genet and ramet distribution pattern than species that only have unlinked propagation. However, using genotypic diversity indices, we did not find a consistent pattern between the spatial arrangement and outcorssing rates. The reproductive and genetic consequences of the spatial arrangement of genets and ramets continues to be a difficult task, due to a range of factors such as the variety of methods, the lack of good comparative numeric estimations to describe the spatial arrangements and the lack of studies in species that propagate through unlinked propagules.
\end{abstract}

Key words: clonality, distribution, diversity, growth, outcrossing

a clonalidad da lugar a la producción de ramets, es decir, Lunidades potencialmente independientes que son genéticamente idénticas a la planta progenitora (Widén et al., 1994; van Groenendael et al., 1996; de Kroon y van Groenendael,
1997; Klims et al., 1997). En consecuencia, en las poblaciones de organismos clonales el concepto de "individuo" puede definir a los individuos genéticos formados a partir de un cigoto (genets) y a las copias física y fisiológicamente 
independientes de cada genotipo o ramets independientes (Figura 1; Kays y Harper, 1974; Harper, 1985; Jackson et al., 1985). La clonalidad está ampliamente distribuida en plantas, algunas estimaciones para angiospermas varían entre 30\% y más del 50\% (Tiffney y Niklas, 1985; Klim s et al., 1997; Aarssen, 2008), entre el 40\% y $80 \%$ en especies de taiga, tundra y de zonas templadas (van Groenendael y de Kroon, 1990 Klim s et al., 1997) y el 44\% de plantas invasoras en China (Liu et al., 2006), está ampliamente representada en monocotiledóneas y se presenta con baja frecuencia en las gimnospermas (van Groenendael et al., 1996; Peterson y Jones, 1997). Respecto a las condiciones ambientales, se sugiere que la clonalidad predomina en condiciones estresantes como suelos pobres en nitrógeno, suelos húmedos, cuerpos de agua, hábitats sombreados y ambientes fríos en altitudes y latitudes elevadas (Tiffney y Niklas, 1985; van Groenendael et al., 1996; Klim s et al., 1997).

La clonalidad en plantas se ha estudiado con enfoques muy diversos. Por ejemplo, se ha analizado la frecuencia de especies clonales y la variación en las formas de crecimiento clonal en distintos ambientes (p. ej., Tiffney y Niklas, 1985; van Groenendael et al., 1996; Klims et al., 1997), las implicaciones de la integración fisiológica de los ramets y la plasticidad morfológica del crecimiento clonal en ambientes heterogéneos y en condiciones de estrés competitivo (p. ej., Bell, 1984; Hartnett y Bazzaz, 1985; de Kroon y Hutchings, 1995; Price y Marshall, 1999), el efecto de la clonalidad en la demografía (p. ej., Caswell, 1985; McFadden, 1991; Damman y Cain, 1998; Mandujano et al., 2001), la comparación de la diversidad genética entre especies de plantas clonales y no clonales (p. ej., Ellstrand y Roose, 1987; Widén et al., 1994) y las consecuencias reproductivas y evolutivas de la geitonogamia asociada con la clonalidad (p. ej., Handel, 1985; de Jong et al., 1992; Eckert, 2000).

Las implicaciones reproductivas y genéticas de reducir la entrecruza incrementando la geitonogamia han aumentado el interés en el estudio de los patrones de distribución espacial de genets y ramets en poblaciones de plantas clonales. Debido a que los propágulos vegetativos suelen tener una capacidad de dispersión restringida, el reclutamiento clonal frecuente puede reducir la entrecruza e incrementar la autocruza (p. ej., autogamia y geitonogamia), determinando los niveles de variación y la estructuración genética en las poblaciones (Handel, 1985; Eckert, 2002; Ruggiero et al., 2005; Honnay et al., 2006). En especies autocompatibles, los costos reproductivos de la geitonogamia están asociados al incremento de la autogamia y a las probabilidades de expresar depresión endogámica, a la vez que disminuye la adecuación paterna reduciendo la cantidad de polen disponible para la entrecruza; mientras que en las especies autoincompatibles, la geitonogamia afecta la producción de semillas, debido a la saturación de los estigmas con polen incompatible y al aborto de óvulos, y puede resultar en el desperdicio de grandes cantidades de polen (de Jong et al.,
1992; Charpentier, 2002).

En plantas clonales los patrones de distribución espacial pueden ser afectados por la manera en que se generan los ramets. Las formas de crecimiento clonal son muy diversas, pero existen algunas clasificaciones que facilitan el entendimiento de esta variación. Tiffney y Niklas (1985) propusieron cuatro tipos principales de crecimiento: (1) Crecimiento establecido, dado por el inicio del desarrollo de un organismo individual a partir de un propágulo (puede considerarse como crecimento clonal si el propágulo es vegetativo, ya que el propágulo también puede ser una semilla producida sexualmente). (2) Crecimiento restaurativo, que se refiere a la reparación de un daño local en el individuo mediante la formación de tejido calloso, pero no hay regeneración de los órganos completos cuando estos se pierden. (3) Crecimiento regenerativo, que se refiere a la reparación y el reemplazo de órganos dañados (p. ej., crecimiento de una rama desprendida). (4) Crecimiento reproductivo, dado por la multiplicación del individuo fisiológico (p. ej., clonalidad). Estos autores también ordenan los tipos de crecimiento clonal en ligado y no ligado, considerando las estructuras de conexión (Figura 1). En el crecimiento ligado los propágulos vegetativos pueden desarrollarse hasta formar individuos completos (con raíces, tallos y hojas) manteniéndose unidos a la planta madre y su dispersión depende del crecimiento de ramificaciones de tallos y raíces, mientras que en el no ligado, los propágulos vegetativos se desprenden de la planta madre y se desarrollan hasta individuos completos separados de la planta madre. La dispersión de los propágulos en este caso es más dependiente de factores bióticos (p. ej., animales) o abióticos (p. ej., corrientes de agua, gravedad, viento) que en el crecimiento ligado y permite un mayor desplazamiento de los propágulos. El crecimiento reproductivo o clonalidad, que es la que interesa en esta revisión por su influencia en el arreglo espacial de genets y ramets, puede ser de dos tipos: (i) reiteración traumática, definida como la producción de un nuevo individuo inducida por estrés, ya sea por daño o por condiciones ambientales que limitan la reproducción sexual (por ejemplo, la producción de bulbilos en los agaves por daño al escapo floral o por deficiencia de polen) y (ii) la reiteración programada, como la producción de un nuevo individuo por un meristemo como parte normal del desarrollo (Tiffney y Niklas, 1985; Arizaga y Ezcurra, 2002).

La distribución espacial de los individuos en las poblaciones se ha clasificado en tres patrones básicos: aleatorio, uniforme y agregado (Figura 2; Gleason, 1920; Clark y Evans, 1954; Barbour et al., 1987). En poblaciones naturales de plantas, los individuos suelen distribuirse en forma más o menos agregada, debido a la distribución heterogénea de los recursos (Couteron y Kokou, 1997; Chen y Bradshaw, 1999), a la interacción con otras especies (Shreve, 1917; Mandujano et al., 1998) y a la dispersión restringida de semillas y propágulos vegetativos (Gibson y Menges, 1994; Okuda et al., 1997; Clark-Tapia et al., 2005). En plantas 
clonales además, el arreglo espacial de los genets y ramets puede ordenarse en dos patrones básicos, uno caracterizado por la agregación de ramets del mismo genet y el otro por una distribución entremezclada de ramets de distintos genets (Figura 2; de Kroon y Hutchings, 1995; Charpentier, 2002; Clark-Tapia et al., 2005). Estos arreglos espaciales de genets y ramets pueden estar asociados a la manera en que se producen los ramets (esto es por crecimiento clonal ligado o no ligado) y se ha propuesto que en las plantas que se propagan mediante crecimiento no ligado forman arreglos más entremezclados que las especies con crecimiento ligado (Gabrielsen y Brochmann, 1998), pero esto no ha sido demostrado. Por otro lado, debido a que la clonalidad puede incrementar la tasa de geitonogamia entre los ramets del mismo genet, puede esperarse que los arreglos entremezclados reduzcan la geitonogamia y promuevan la entrecruza como resultado del incremento local en el número de genets (Charpentier, 2002).

En algunas revisiones sobre el tema de la clonalidad se han planteado hipótesis sobre las implicaciones reproduc-

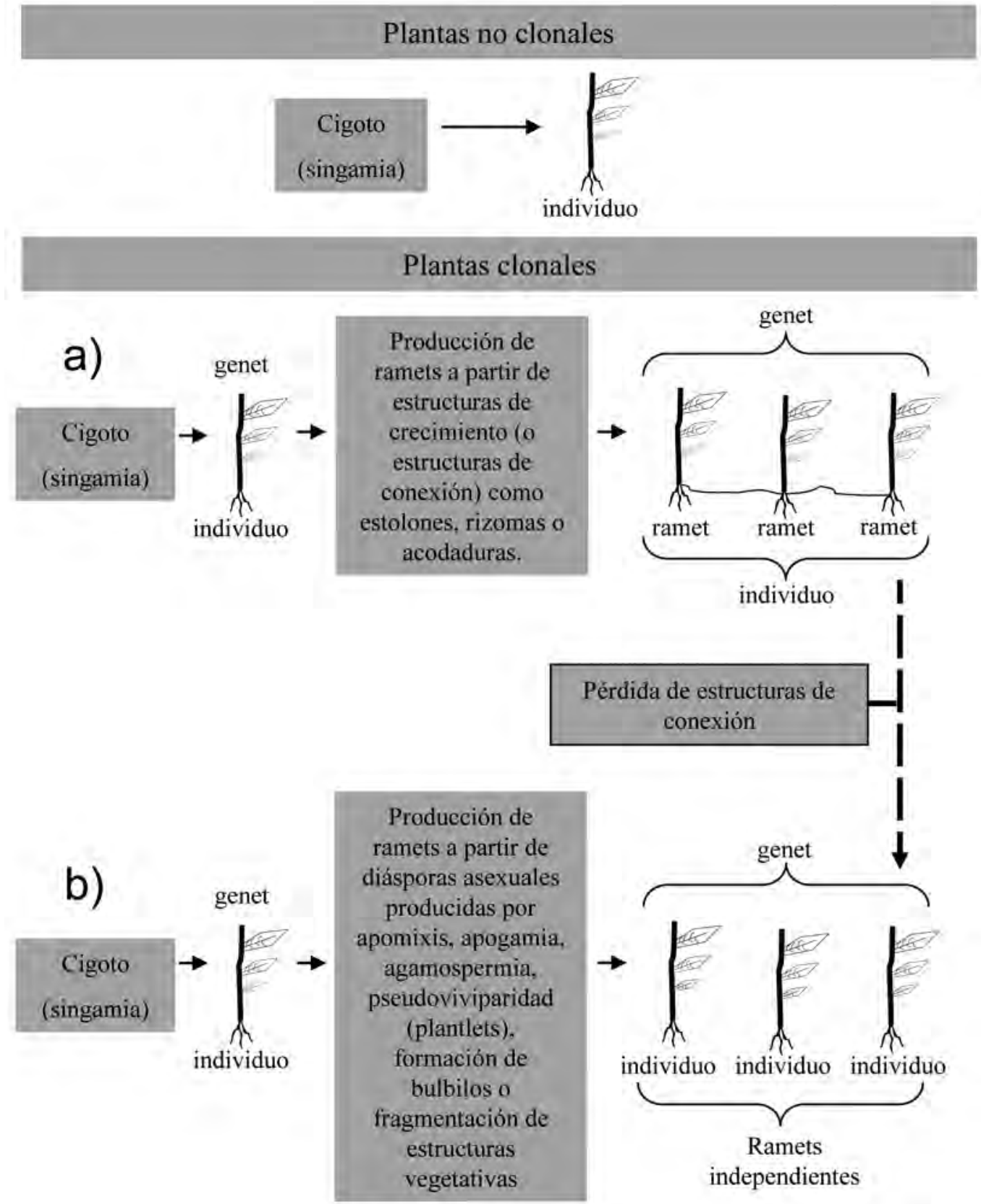

Figura 1. Formación de individuos en plantas no clonales y en plantas clonales. a) crecimiento clonal ligado y b) crecimiento clonal no ligado en la clasificación de Tiffney y Niklas (1985). 
$\mathrm{PDE}=$ Patrones de distribución espacial de los individuos y ramets en las poblaciones

(clonales y no clonales)
AER $=$ Arreglos espaciales de los ramets de distintos genets (clonales)

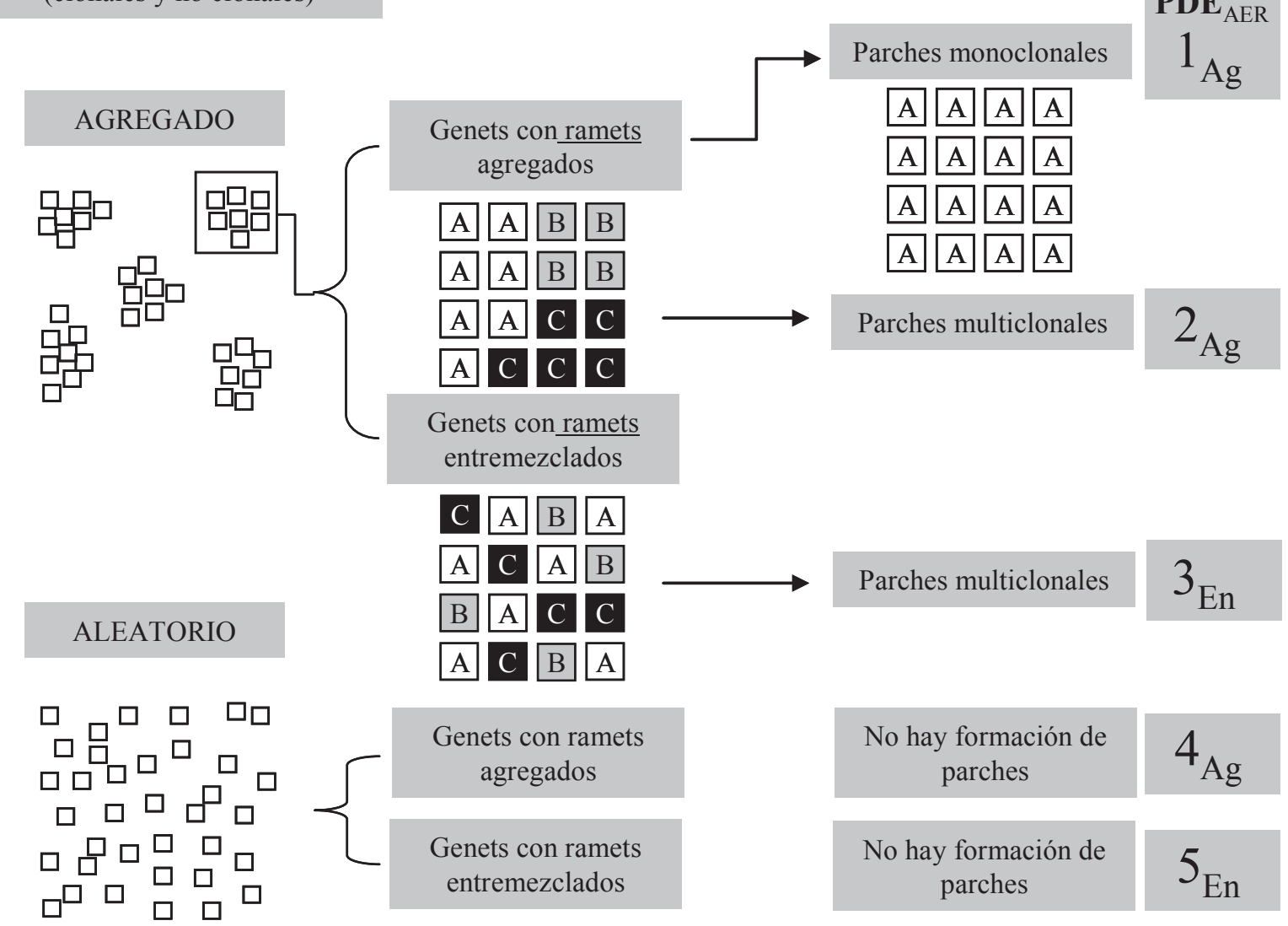

\section{UNIFORME}
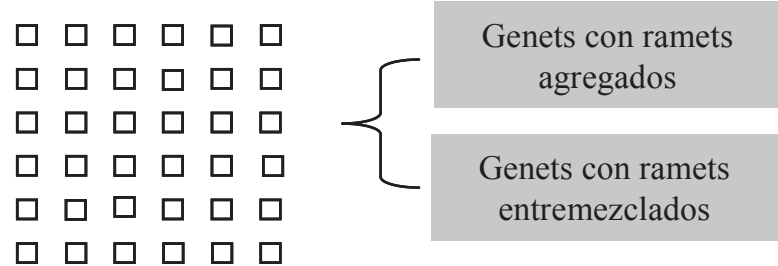

\begin{tabular}{|c|c|c|c|}
\hline $\begin{array}{c}\text { No hay formación de } \\
\text { parches }\end{array}$ & $6_{\mathrm{Ag}}$ \\
\hline $\begin{array}{c}\text { No hay formación de } \\
\text { parches }\end{array}$ & $7_{\mathrm{En}}$ \\
\hline
\end{tabular}

Figura 2. Patrones de distribución espacial de los individuos en las poblaciones de plantas no clonales y de plantas clonales. El número en el extremo derecho representa la clave del patrón de distribución espacial y el arreglo espacial de los ramets de distintos genets (PDE y AER, Apéndice). El subíndice Ag indica que los ramets de cada genet no se entremezclan con los ramets de genets distintos y el subíndice En indica que los ramets de diferentes genets se entremezclan.

tivas y genéticas de los arreglos espaciales de los genets y ramets, asociados con las distintas formas de producir descendientes clonales (Widén et al., 1994; Charpentier, 2002; Mandujano-Sánchez, 2007; Honnay y Jaquemyn, 2008). No obstante, los intentos para probar estas hipótesis son muy escasos y las posibilidades de realizar algún tipo de análisis numérico son restringidas por la baja disponibilidad de publicaciones (p. ej., Widén et al., 1994), o en los análisis sólo se incluyen especies con crecimiento ligado (p. ej., Honnay y Jaquemyn, 2008). En este trabajo presentamos una revisión de varios estudios que evalúan los patrones de distribución espacial en plantas clonales y a partir de la información 
proporcionada en dichos estudios, analizamos el planteamiento de que en poblaciones de plantas con crecimiento clonal ligado el arreglo espacial de los ramets de distintos genets es agregado, mientras que en poblaciones de plantas con crecimiento clonal no ligado predominan los arreglos espaciales entremezclados. Adicionalmente, intentamos poner a prueba la hipótesis de que los arreglos entremezclados promueven la entrecruza suponiendo que las poblaciones donde la entrecruza es más frecuente tenderán a presentar niveles más altos de diversidad genotípica.

\section{Material y métodos}

La revisión de los trabajos publicados sobre la distribución espacial clonal en plantas se llevó a cabo mediante una búsqueda de trabajos en bases de datos especializadas (CSA Cambridge, Ebsco HOST, ISI Web of Knowledge, JSTOR y Scopus) y no especializadas (Google, Google scholar y Yahoo). La búsqueda se hizo empleando los siguientes términos: clonal structure, spatial distribution of genets (andl or ramets), clonal architecture y clonal diversity y abarcó de 1990 a 2011, debido a que la mayoría de los estudios en plantas clonales con este enfoque se han realizado después de 1990.

Los criterios principales para incluir una especie en la revisión fue que se presentara una descripción de la distribución espacial de las plantas individuales (esto es sin importar el genotipo) en las poblaciones naturales y que se evaluara el arreglo espacial de los genets y los ramets (es decir agregado o entremezclado) mediante planos de distribución de los genotipos, análisis de autocorrelación espacial o compatibilidad de las cruzas entre ramets contiguos. Para cada especie se obtuvo el tipo de estructuras vegetativas a mediante las cuales se lleva a cabo la propagación clonal a partir de la información proporcionada en los estudios correspondientes o en estudios previos realizados a menudo por los mismos autores. El segundo criterio en orden de importancia fue que se reportaran valores de diversidad genotípica ( $D=$ índice de diversidad de Simpson y proporción de genotipos distinguibles $G / N$ ) para las poblaciones de las diferentes especies. Otros criterios fueron que se proporcionara información sobre las características reproductivas como la presencia de sistemas de incompatibilidad, la expresión sexual, si se ha observado reclutamiento sexual en las poblaciones y el tipo de polinización. Con base en esta información, se asignó a las especies el tipo de crecimiento clonal (i. e. ligado y no ligado, Figura 1) de acuerdo con la clasificación de Tiffney y Niklas (1985) y el patrón de distribución espacial y el tipo de arreglo espacial de los ramets (agregado o entremezclado) según el esquema presentado en la Figura 2. La información obtenida se presenta en el apéndice.

Para analizar el planteamiento de que en poblaciones de plantas con crecimiento clonal ligado el arreglo espacial de los ramets es agregado, mientras que en poblaciones de plantas con crecimiento clonal no ligado predomina el arreglo espacial entremezclado se construyó una tabla de contingencia con tres categorías de tipo de crecimiento clonal como columnas (ligado, combinación ligado-no ligado y no ligado) y tres categorías del arreglo espacial de los ramets como renglones (agregado, combinación agregado-entremezclado y entremezclado) y se analizó con una prueba de ${ }^{2}$ con el paquete estadístico JMP versión 7.0 (SAS Institute Inc). Para evaluar las celdas que contribuyen significativamente al valor de ${ }^{2}$ se hizo un análisis de residuales ajustados (Everitt, 1977).

Para probar la hipótesis de que las poblaciones con arreglos entremezclados de ramets presentan niveles más altos de diversidad genotípica, se compararon los valores del índice de diversidad de Simpson $(D)$ y la proporción de genotipos distinguibles $(G / N)$ entre las tres categorías de patrón de distribución clonal y entre las tres categorías de tipo de crecimiento clonal con una prueba de Kruskall-Wallis con el paquete estadístico JMP versión 7.0 (SAS Institute Inc).

\section{Resultados}

En la revisión se incluyeron 53 estudios que comprenden 122 poblaciones distribuidas en 44 especies de plantas clonales y en 27 familias (Apéndice). Las familias mejor representadas son Liliaceae y Rosaceae con cuatro especies cada una; Cactaceae, Cyperaceae, Ericaceae, Poaceae y Salicaceae con tres especies cada una y Zosteraceae con dos especies. El resto de las familias estuvieron representadas por una sola especie. Las especies que predominan son herbáceas perennes $(60 \%)$, y las plantas con hábito arbustivo y arbóreo se encuentran poco representadas (20\% en ambos casos).

La mayoría de las especies presentan crecimiento clonal ligado (70\%), dominando la propagación a través de rizomas (45\% de las especies). Las especies con crecimiento clonal ligado y no ligado representan el 14\% propagándose clonalmente por crecimiento ligado a través de rizomas y acodaduras, y por crecimiento no ligado a través de bulbilos, plantlets y desprendimiento de ramas; las especies sólo con crecimiento no ligado representan el 16\%, propagándose por bulbilos, semillas producidas por agamospermia, desprendimiento de ramas y gémulas (ver glosario para la definición de las estructuras implicadas en el crecimiento ligado y no ligado, Apéndice). Respecto a la distribución espacial, $50 \%$ del total de las especies presentan genets con ramets agregados, $30 \%$ presentan una combinación de arreglos agregados-entremezclados y $20 \%$ presentan arreglos entremezclados (Apéndice).

$\mathrm{Al}$ comparar las poblaciones con diferentes arreglos espaciales de genets y ramets [agregados (Ag), En (entremezcaldos) y $\mathrm{Ag}+\mathrm{En}$ (combinados)] encontramos que las poblaciones de especies con crecimiento ligado y no ligado y 
Cuadro 1. Número (observado y esperado) de poblaciones con diferentes tipos de crecimiento clonal de acuerdo con la clasificación de Tiffney y Niklas (1985) ( $\mathrm{Lg}=$ ligado, Lg+NI = ligado y no ligado, $\mathrm{Nl}=$ no ligado), que mostraron arreglos espaciales de los ramets de distintos genets: Ag = agregados, Ag+En = combinación de agregados y entremezclados, y En = entremezclados (figura 2). Los valores entre paréntesis indican el número de especies y familias (especies/familias) representadas en cada celda. Los valores de los residuales ajustados en negritas indican las celdas que contribuyen significativamente al valor de $\chi^{2}$, el signo indica mayor (sin signo) o menor número (-) de poblaciones de las esperadas por azar.

\begin{tabular}{|c|c|c|c|}
\hline Observados & Ligado & Ligado+No ligado & No ligado \\
\hline Agregado & $49(21 / 13)$ & $7(2 / 2)$ & $4(2 / 2)$ \\
\hline Agregado+entremezclado & $11(5 / 5)$ & $25(5 / 5)$ & $7(3 / 3)$ \\
\hline Entremezclado & $10(5 / 5)$ & $2(2 / 1)$ & $7(2 / 2)$ \\
\hline \multicolumn{4}{|l|}{ Esperados } \\
\hline & $\operatorname{Lg}$ & $\mathrm{Lg}+\mathrm{NI}$ & $\mathrm{Nl}$ \\
\hline Agregado & 34 & 17 & 9 \\
\hline Agregado+entremezclado & 25 & 12 & 6 \\
\hline Entremezclado & 11 & 5 & 3 \\
\hline \multicolumn{4}{|l|}{ Residuales ajustados } \\
\hline & $\operatorname{Lg}$ & $\mathrm{Lg}+\mathrm{NI}$ & $\mathrm{Nl}$ \\
\hline Agregado & 5.34 & -3.93 & -2.48 \\
\hline Agregado+entremezclado & -5.24 & 5.50 & 0.35 \\
\hline Entremezclado & -0.46 & -1.83 & 2.95 \\
\hline
\end{tabular}

de especies que sólo presentan crecimiento no ligado presentaron arreglos espaciales (AER) más entremezclados que las poblaciones de especies que sólo presentan crecimiento ligado ( ${ }^{2}=43.6, P<0.0001,4$ g.l.; Cuadro 1). Predominan los patrones de distribución (PDE) con ramets distribuidos en parches discretos sobre el área ocupada por la población (patrón de distribución espacial agregado, $46 \%$ de las poblaciones); estos parches son multiclonales (con dos o más genets) en la mayoría de los casos y el arreglo espacial de los ramets de distintos genets es agregado (19\% de las poblaciones, $\mathrm{PDE}_{\mathrm{AER}} 2_{\mathrm{Ag}}$, Figura 2), presenta una combinación agregado-entremezclado (10\% de las poblaciones, combinación $\mathrm{PDE}_{\mathrm{AER}} 2_{\mathrm{Ag}}-3_{\mathrm{En}}$, Figura 2) o es entremezclado solamente (3\% de las poblaciones, $\mathrm{PDE}_{\mathrm{AER}} 3_{\mathrm{En}}$, Figura 2). En otras poblaciones donde los ramets se distribuyen formando parches discretos el arreglo espacial de los ramets de distintos genets es agregado, pero los parches son multiclonales y monclonales (9\% de las poblaciones, combinación $\mathrm{PDE}_{\mathrm{AER}}$ $1_{\mathrm{Ag}}-\mathrm{Ag}_{\mathrm{Ag}}$, Figura 2) o sólo son monoclonales (5\% de las poblaciones, $\mathrm{PDE}_{\mathrm{AER}} 1_{\mathrm{Ag}}$, Figura 2).

Los patrones de distribución espacial con ramets distribuidos aleatoriamente sobre el área ocupada por la población (patrón de distribución espacial aleatorio) representaron el $44 \%$ de las poblaciones, predominando el arreglo espacial agregado-entremezclado de los ramets de distintos genets $\left(22 \%\right.$ de las poblaciones, combinación de $\mathrm{PDE}_{\mathrm{AER}}$ $4_{\mathrm{Ag}}-5_{\mathrm{En}}$, Figura 2), el arreglo agregado se presentó en $10 \%$ de las poblaciones ( $\mathrm{PDE}_{\mathrm{AER}} 4_{\mathrm{Ag}}$, Figura 2) y el arreglo entremezclado en $12 \%$ de las poblaciones $\left(\mathrm{PDE}_{\mathrm{AER}} 5_{\mathrm{En}}\right.$, Figura
2). El resto de las poblaciones (10\%) presentaron una combinación de patrones de distribución con ramets distribuidos aleatoriamente sobre una parte del área ocupada por la población y en otra formando parches discretos monoclonales o parches multiclonales con arreglos espaciales agregados y entremezclado de los ramets de distintos genets (combinación de $\mathrm{PDE}_{\mathrm{AER}} 1_{\mathrm{Ag}}-4_{\mathrm{Ag}}, 1_{\mathrm{Ag}}-5_{\mathrm{En}}, 2_{\mathrm{Ag}}-4_{\mathrm{Ag}}, 2_{\mathrm{Ag}}-5_{\mathrm{En}}$, Figura 2).

Las poblaciones de especies con una combinación de tipos de crecimiento clonal ligado y no ligado $(\mathrm{Lg}-\mathrm{Nl})$ presentaron valores más bajos de la proporción de genotipos distinguibles (promedio \pm E.E., $G / N=0.24 \pm 0.04 ; H=$ 6.93, $P=0.031,2$ g.l.) que las poblaciones de especies con crecimiento ligado (promedio \pm E.E., $G / N=0.38 \pm 0.03$ ) y con crecimiento no ligado (promedio \pm E.E., $G / N=0.39 \pm$ 0.06 ), pero las diferencias sólo fueron significativas con respecto a las poblaciones de especies con crecimiento ligado (Figura 3a). Respecto al arreglo espacial de los ramets de distintos genets, las poblaciones con una combinación de arreglos agregado y entremezclado (Ag-En) presentaron valores menores del índice de diversidad de Simpson (promedio \pm E.E., $D=0.57 \pm 0.05 ; H=13.2, P=0.0014,2$ g.l.) que las poblaciones con arreglos agregados (promedio \pm E.E., $D=0.80 \pm 0.04$ ) y con arreglos entremezclados (promedio \pm E.E., $D=0.76 \pm 0.06$; Figura 3). Estas diferencias sugieren que el reclutamiento sexual es más bajo en poblaciones de especies que presentan una combinación de formas de crecimiento clonal ligado-no ligado ( $\mathrm{Lg}-\mathrm{Nl}$ ) y una combinación de arreglos de los ramets de distintos genets agregado-entremezclado (Ag-En), sin embargo estas diferencias 

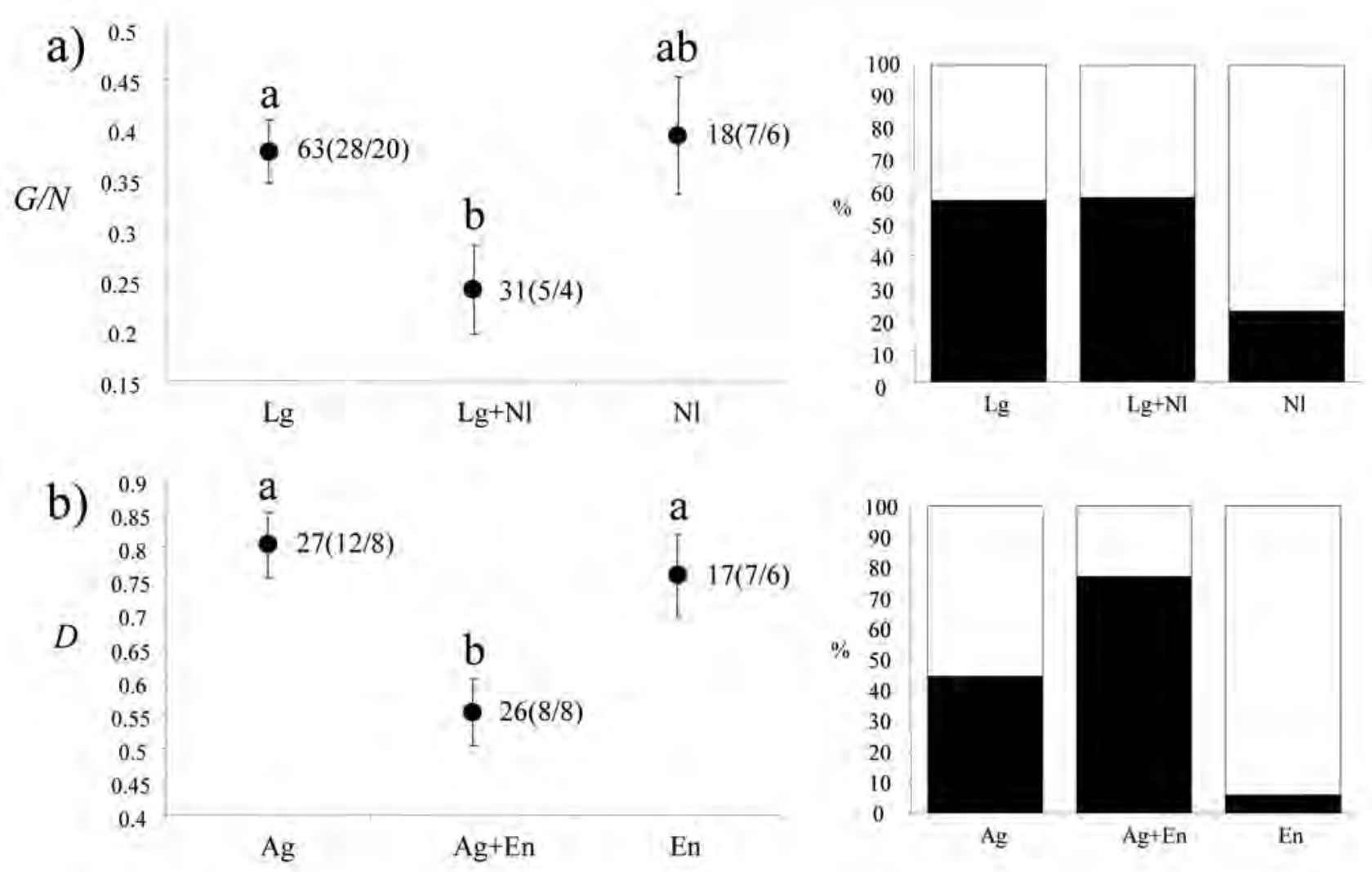

Figura 3. Comparación de la diversidad clonal (promedio \pm EE) entre poblaciones de especies con a) distinto tipo de crecimiento clonal: $\mathrm{Lg}=$ ligado, $\mathrm{Lg}+\mathrm{Nl}$ = con ambos tipos de crecimiento y $\mathrm{Nl}$ = no ligado y b) con distintos arreglos espaciales de los ramets de distintos genets: $\mathrm{Ag}=$ agregados, $\mathrm{Ag}+\mathrm{En}$ = combinación de agregados y entremezclados, y En = entremezclados (Figura 2). En cada grupo se indica el número de poblaciones (número de especies / número de familias) y los grupos que difieren significativamente se indican con una letra distinta arriba de la barra del promedio \pm E.E. de cada grupo. Las gráficas de barras del lado derecho muestran el porcentaje de poblaciones con polinización abiótica (en negro) y con polinización biótica (en blanco).

pueden estar asociadas con otros factores como el tipo de polinización, y no con el tipo de crecimiento y el arreglo espacial de los ramets de distintos genets. La mayoría de las poblaciones con tipo de crecimiento $\mathrm{Lg}-\mathrm{Nl}$ y arreglos de ramets de distintos genets Ag-En corresponden a especies con polinización abiótica (Figura 3).

\section{Discusión}

El efecto que tienen los modos de clonalidad en las plantas en la conformación de distintos arreglos espaciales de genets y ramets es un campo relativamente novedoso, que se ha explorado en los últimos 20 años y la mayoría de los estudios que hay sobre el tema se han desarrollado después de 1990. Widén et al. (1994) reportan que de 40 estudios que comprenden un total de 45 especies de plantas clonales, menos de la tercera parte proporcionan información sobre el arreglo espacial de los genets en las poblaciones y sólo en $12.5 \%$ se construyeron planos de distribución de todos los ramets, dentro de las parcelas o poblaciones, identificando los genotipos multilocus. En esta revisión, en el $98 \%$ de los estudios incluidos se identifican los genotipos multilocus mediante marcadores dominantes, codominantes o ambos siguiendo tres métodos de muestreo: en el 56\% de los estudios se utilizan parcelas, en el $24 \%$ transectos y en el $18 \%$ se muestrean todos o varios individuos de las poblaciones. El 2\% restante está representado por un estudio donde el patrón de distribución es descrito y la distribución de los ramets se sugiere por experimentos de cruzas compatibles entre ramets cercanos (en Scirpus maritimus, Charpentier et al., 2000).

A pesar de que en la actualidad se cuenta con más información sobre los patrones de distribución espacial en poblaciones de especies clonales, hacer comparaciones de dichos patrones considerando especies con distintas formas de crecimiento clonal para analizar las consecuencias reproductivas y genéticas del arreglo espacial de los genets y los ramets continúa siendo un reto. Algunos factores que influyen en este hecho son que la evaluación de la variación en los patrones de distribución espacial en plantas clonales 
enfrenta la dificultad que implica trabajar con la mayoría de los individuos en muchas poblaciones, principalmente a nivel molecular. Aunque en la actualidad las herramientas moleculares se han vuelto más accesibles, trabajar con un gran número de ejemplares sigue siendo difícil y costoso. Otros problemas son la falta de estimadores numéricos comparables para describir la distribución espacial en las poblaciones de diferentes especies y la variación en los métodos de muestreo. Por ejemplo, el muestreo mediante transectos colectando muestras de ramets cada determinada distancia (p. ej., Jonsson et al., 1996; Esselman et al., 1999) permite evaluar la capacidad de expansión longitudinal de los genets, pero no permite tener una descripción de la expansión radial y por lo tanto una evaluación más precisa del nivel de entremezcla de los ramets de distintos genets. En contraste, el muestreo de todos o de un gran número de individuos dentro de parcelas o en las áreas ocupadas por las poblaciones es otro método empleado en estudios con especies de plantas clonales (p. ej., Shimizu et al., 2002; Ruggieiro et al., 2005). Este tipo de muestreo puede ser un método más adecuado para analizar y comparar la estructura espacial clonal, porque permite detectar el arreglo radial de distintos genets. Sin embargo, los tamaños de muestra muy grandes pueden limitar la aplicación de este método, ya que en la mayoría de los casos es necesario emplear marcadores moleculares para identificar a los genets.

En particular, además de los problemas mencionados anteriormente es importante considerar las dificultades adicionales que detectamos en este estudio y que pueden afectar los patrones encontrados a través de nuestra aproximación. La mayoría de los estudios que analizan la distribución espacial de genets y ramets parecen estar sesgados hacia especies de herbáceas perennes con crecimiento clonal ligado (45\% de las especies), predominando las especies rizomatosas (43\% de las especies), mientras que las especies con crecimiento no ligado están poco representadas, impidiendo contar con tamaños de muestra equivalentes para hacer comparaciones más precisas. Por otro lado, la aproximación que decidimos emplear en este trabajo compara a las poblaciones más que a las especies, debido a que hemos detectado que hay una importante variación intraespecífica tanto en los patrones de distribución como en los patrones reproductivos. No obstante, esta variación se omite en algunos estudios o está mejor representada para algunas especies.

Los resultados obtenidos en este trabajo sugieren que las especies que se propagan por crecimento no ligado tienden a formar arreglos espaciales de los ramets de distintos genets más entremezclados como se ha planteado (Gabrielsen y Brochmann, 1998). No obstante, en especies con crecimiento ligado que además se propagan clonalmente por crecimiento no ligado, como el desprendimiento de ramas, el cual consideramos en este estudio como una forma de crecimiento no ligado en los análisis, debido a que los ramets originados por este medio se desarrollan hasta individuos com- pletos separados de la planta madre y a que su dispersión no depende del crecimiento horizontal de tallos y raíces (p.ej., Lophocereus schottii, Parker y Hamrick, 1992; Pteridium aquilinum, Parks y Werth, 1993; Cladium jamaicense, Ivey y Richards, 2001; Ferocactus robustus, Carrillo-Angeles et al., 2011), o que se propagan por la extensión de rizomas en especies con crecimiento en guerrilla (p. ej., Cymodocea nodosa, Ruggieiro et al., 2005 e Ilex leucoclada, Torimaru y Tomaru, 2005) también forman arreglos de genets entremezclados. Algunos factores externos que pueden influir en el arreglo entremezclado de genets son la dispersión por actividades humanas (Parks y Werth, 1993; Brzosko et al., 2002), el acarreo de propágulos vegetativos por animales y corrientes de agua (Parker y Hamrick, 1992; Brzosko et al., 2002), el reclutamiento de nuevos genets en áreas perturbadas donde permanecen fragmentos de clones que ocuparon grandes extensiones (Parks y Werth, 1993) y la dispersión de semillas producidas por autogamia que presentan genotipos multilocus idénticos (Keane et al., 1999; Brzosko et al., 2002).

Respecto a la hipótesis de que las poblaciones con arreglos espaciales entremezclados de los ramets de distintos genets presentan mayor entrecruza, medido indirectamente mediante la diversidad genotípica, nuestros resultados concuerdan parcialmente con el patrón esperado. El G/N es significativamente mayor en los arreglos de especies con producción de vástagos no ligados, en donde predominan los arreglos de genets entremezclados. Para probar esta hipótesis consideramos que si la entrecruza es más alta en poblaciones con arreglos entremezclados, habría mayores niveles de diversidad genotípica. Sin embargo, ésto no se refleja en el caso del índice de Simpson. Las diferencias en los índices de diversidad genotípica entre las poblaciones de especies que combinan el crecimiento clonal ligado-no ligado ( $\mathrm{Lg}$ $\mathrm{Nl})$ y el arreglo espacial agregado-entremezclado (Ag-En), respecto de las poblaciones que presentan uno u otro tipo de crecimiento y de arreglo espacial están asociadas con el tipo de polinización (biótica o abiótica), lo cual apoya el planteamiento de que la polinización biótica puede incrementar la eficiencia de la fertilización (Barret et al.,1993; Richards, 1997; Reusch, 2003). Los análisis para probar este planteamiento están fuera del alcance del presente estudio.

Además de los efectos sobre el reclutamiento sexual y la diversidad genotípica, los patrones de distribución espacial en especies clonales pueden afectar la diversidad y la estructura genética de las poblaciones. Por ejemplo, la formación de parches monoclonales en especies con entrecruza obligada, aunada al desplazamiento de genets por competencia, puede eliminar la capacidad de una población para reproducirse sexualmente en tiempos relativamente cortos (Honnay y Bossuyt, 2005). Adicionalmente, una reducción del reclutamiento sexual y una dispersión restringida de polen, semillas y propágulos vegetativos pueden generar estructura genética local en las poblaciones. Este segundo aspecto se 
evalúa en varios de los estudios revisados (47\%). La estructura genética local tanto a nivel de genets (indicando una dispersión restringida de polen, semillas o ambos) como de ramets (indicando dispersión restringida de propágulos vegetativos) se detectó en 46 poblaciones distribuidas en 16 especies; 28 poblaciones corresponden a cinco especies arbóreas que muestran estructura genética local a distancias menores de $50 \mathrm{~m}$ (Sequoia sempervirens, Douhovnikoff et al., 2004) y a distancias menores de $25 \mathrm{~m}$ (Eurya emarginata, Chung y Epperson, 2000; Cryptomeria japonica, Shimizu et al., 2002; Hirayama y Sakimoto, 2008; Populus tremula, Suvanto y Latva-Karjantvaa, 2005); tres poblaciones corresponden a dos especies arbustivas (Echinosophora koreensis, Chung et al., 2006; Stenocereus eruca, ClarkTapia et al., 2005) que presentan estructura genética local a distancias menores de $20 \mathrm{~m}$. El resto de las poblaciones corresponden a hierbas perennes que muestran estructura genética local desde distancias menores de $30 \mathrm{~m}$, pero más frecuentemente a distancias menores de $15 \mathrm{~m}$.

En promedio, los valores del índice de diversidad de Simpson $(D)$ y la proporción de genotipos distinguibles $(G / N)$ fueron altos en las poblaciones representadas en los estudios incluidos en la presente revisión y son similares a los reportados para 21 especies de plantas clonales $(D=$ 0.62 y $G / N=0.16$ ) por Ellstrand y Roose (1987). Se han planteado algunas hipótesis sobre los escenarios que pueden explicar altos niveles de diversidad genotípica: (i) las poblaciones pudieron ser fundadas por varios propágulos sexuales que se mantienen por clonalidad, (ii) el reclutamiento sexual fue más frecuente en el pasado, aunque en la actualidad la producción de descendientes sexuales no sea evidente (Ellstrand y Roose, 1987; Eriksson, 1989, 1993; Widén et al., 1994); (iii) los genotipos heterócigos se ven favorecidos selectivamente, manteniendo altos niveles de diversidad genética (Widén et al., 1994); (iv) los riesgos de mortalidad entre los ramets de un genet pueden ser distintos si tienen independencia física y fisiológica (Eriksson 1989, 1993, Pan y Price, 2002), lo cual puede reducir las probabilidades de supervivencia / extinción del genet; (v) en especies con ciclos de vida largos los eventos raros de reclutamiento sexual pueden ser suficientes para mantener niveles altos de diversidad genética (Eriksson 1989, 1993, Parker y Hamrick, 1992; Brzosko et al., 2002; Clark-Tapia et al., 2005); (vi) los genotipos pueden mantenerse a través de la supervivencia de algunos ramets el tiempo suficiente para que algunos genotipos se integren a la población de manera esporádica; (vii) la heterogeneidad ambiental puede generar condiciones de selección diversificante que permiten la coexistencia de varios genotipos (Widén et al., 1994). Otra posibilidad para el mantenimiento de diversidad clonal en poblaciones de plantas, que surge al considerar la variación de la distribución espacial de genets y ramets, es que la clonalidad puede mantener altos niveles de entrecruza e incrementar las probabilidades de reclutamiento sexual si los ramets se dispersan hacia vecindarios genéticamente variables, debido a la distribución entremezclada de genets.

Las explicaciones a los niveles relativamente altos de diversidad genotípica en las poblaciones de las especies incluidas en esta revisión comprenden los eventos esporádicos de reclutamiento sexual y el establecimiento inicial de varios genets. En algunos casos se sugiere un reclutamiento sexual frecuente a partir de los altos niveles de diversidad genotípica detectados con los marcadores moleculares (p. ej., Parks y Werth, 1993; Diggle et al., 1998; Misuki et al., 2010). No obstante, el reclutamiento sexual en poblaciones naturales sólo se reporta en Prunus ssiori, Rubus saxatilis (Eriksson y Bremer, 1993), Cypripedium calceolus (Brzosko et al., 2002) y Maianthemum dilatatum (Wilson et al., 2005b). Otros factores que se proponen para explicar la diversidad genotípica son que la integración fisiológica entre los ramets de un genet puede reducir el riesgo de que un genotipo en particular desaparezca, cuando las condiciones ambientales locales son adversas, contribuyendo a mantener la diversidad genética (Hartnett y Bazzaz, 1985), el incremento en la tasa de entrecruza, debido a la entremezcla de genets en especies con crecimiento en guerrilla (Ruggieiro et al., 2005) y en especies que se propagan clonalmente por desprendimiento de ramas y producción de bulbilos (Parker y Hamrick, 1992; Gabrielsen y Brochmann, 1998).

El estudio de los patrones de distribución espacial en especies clonales es importante para analizar las consecuencias reproductivas y genéticas del arreglo espacial de los genets y los ramets en las poblaciones naturales. Estas consecuencias pueden explorarse de una manera más precisa analizando la variación de la distribución de genets y ramets entre las poblaciones de una misma especie. Sin embargo, la evidencia de estos aspectos en organismos clonales aún es escasa y hace falta realizar estudios considerando diferentes tipos de crecimiento clonal. El análisis de los patrones de distribución espacial pueden ser muy útiles para evaluar otros aspectos del ciclo de vida de las especies clonales como el efecto de una variación persistente en la producción de propágulos clonales y sexuales entre hábitats, que puede afectar diferencialmente la dinámica de las poblaciones. Asimismo, es necesario analizar la dinámica temporal de los arreglos espaciales de los genets y ramets en las poblaciones para hacer inferencias adecuadas sobre las consecuencias ecológicas y evolutivas de la clonalidad.

\section{Agradecimientos}

Proyectos: IN IN205007 Efecto de la estructura clonal sobre la dinámica poblacional y la reproducción de una cactácea con ciclo de vida complejo, Opuntia microdasys.

Becas: doctorado Conacyt a I. Carrillo-Angeles y estancia sabática en New Mexico State University, otorgada por DGAPA-PASPA- Universidad Nacional Autónoma de México a M. Mandujano. Este trabajo es parte de la tesis de 
doctorado de Israel Gustavo Carrillo Angeles, Posgrado en Ciencias Biológicas de la UNAM. Agradecemos la revisión, sugerencias en el trabajo así como el apoyo de los miembros del comité tutoral, Doctores Teresa Valverde y Francisco Molina. Asimismo a la Dra. Ana Mendoza, Dr. Jordan Golubov y dos revisores anónimos por sus sugerencias al artículo.

\section{Literatura citada}

Aarssen L.W. 2008. Death without sex-the 'problem of the small' and selection for reproductive economy in flowering plants. Evolutionary Ecology 22:279-298.

Albert T., Raspé O. y Jacquemart A.L. 2003. Clonal structure in Vaccinium myrtillus L. revealed by RAPD and AFLP markers. International Journal of Plant Sciences 164:649-655.

Arens P., Grashof-Bokdam C.J., van der Sluis T. y Smulders M.J.M. 2005. Clonal diversity and genetic differentiation of Maianthemum bifolium among forest fragments of different age. Plant Ecology 179:169-180.

Arizaga S. y Ezcurra E. 2002. Propagation mechanisms in Agave macrovantha (Agavaceae), a tropical arid land succulent rosette. American Journal of Botany 89:632-641.

Barbour M.G., Burk J.H. y Pitts W.D. 1987. Terrestrial Plant Ecology. The Benjamin-Cummings Publishing Company, Menlo Park.

Barret S.C.H., Eckert C.G. y Husband B.C. 1993. Evolutionary processes in aquatic plant populations. Aquatic Botany 44:105-145.

Bell A.D. 1984. Dynamic morphology: A contribution to plant population ecology. En: Dirzo R. y Sarukhán J. Eds. Perspectives on Plant Population Ecology, pp. 49-65, Sinauer Associates Inc. Publisher, Sunderland.

Brzosko E., Wróblewska A. y Ratkiewicz M. 2002. Spatial genetic structure and clonal diversity of island populations of lady's slipper (Cyperidium calceolus) from the Biebrza National Park (northeast Poland). Molecular Ecology 11:2499-2509.

Bushakra J.M., Hodges S.A., Cooper J.B. y Kaska D.D. 1999. The extent of clonality and genetic diversity in the Santa Cruz Island ironwood, Lyonothamnus floribundus. Molecular Ecology 8:471-475.

Camacho F.J. y Liston A. 2001. Population structure and genetic diversity of Botrychium pumicola (Ophioglossaceae) based on inter-simple sequence repeats (ISSR). American Journal of Botany 88:1065-1070.

Carrillo-Angeles I.G., Mandujano M.C. y Golubov J. 2011. Influences of the genetic neighborhood on ramet reproductive success in a clonal desert cactus. Population Ecology 53:449458.

Caswell H. 1985. The evolutionary demography of clonal reproduction. En: Jackson J.B.C., Buss L.W. y Cook R.E. Eds. Population Biology and Evolution of Clonal Organisms, pp. 187224, Yale University Press, New Haven.

Charpentier A. 2002. Consequences of clonal growth for plant mating. Evolutionary Ecology 15:521-530.

Charpentier A., Grillas P. y Thompson J.D. 2000. The effects of population size limitation on fecundity in mosaic populations of the clonal macrophyte Scirpus maritimus (Cyperaceae). American Journal of Botany 87:502-507.

Chen J. y Bradshaw G.A. 1999. Forest structure in space: a case study of an old growth spruce-fir forest in Changbaishan Natural Reserve, PR China. Forest Ecology and Management 120:219-233.

Chung M.G. y Epperson B.K. 2000. Clonal and spatial genetic structure in Eurya emarginata (Theaceae). Heredity 84:170177.

Chung J.M., Lee B.C., Kim J.S., Park C., Chung M.Y. y Chung M.G. 2006. Fine-scale genetic structure among genetic individuals of the clone-forming monotypic genus Echinospora koreensis (Fabaceae). Annals of Botany 98:165-173.

Clark P.J. y Evans F.C. 1954. Distance to nearest neighbor as a measure of spatial relationships in populations. Ecology 35:445-453.

Clark-Tapia R., Alfonso-Corrado C., Eguiarte L.E. y Molina-Freaner F. 2005. Clonal diversity and distribution in Stenocereus eruca (Cactaceae), a narrow endemic cactus of the Sonoran Desert. American Journal of Botany 92:272-278.

Clark-Tapia R., Alfonso-Corrado C., Mandujano M.C. y MolinaFreaner F. 2006. Reproductive consequences of clonal growth in Stenocereus eruca, a rare clonal cactus of the Sonoran desert. Evolutionary Ecology 20:131-142.

Couteron P. y Kokou K. 1997. Woody vegetation spatial patterns in a semi-arid savanna of Burkina Faso, West Africa. Plant Ecology 132:211-227.

Damman H. y Cain M.L. 1998. Population growth and viability analyses of the clonal woodland herb, Asarum canadense. Journal of Ecology 86:13-26.

de Jong T.J., Waser N.M., Price M.V. y Ring R.M. 1992. Plant size, geitonogamy and seed set in Ipomopsis aggregata. Oecologia 89:310-315.

de Kroon H. y van Groenendael J. Eds. 1997. The Ecology and Evolution of Clonal Plants. Backhuys Publishers, Leiden.

de Kroon H. y Hutchings M.J. 1995. Morphological plasticity in clonal plants: the foraging concept reconsidered. Journal of Ecology 83:143-152.

Diggle P.K., Lower S. y Ranker T.A. 1998. Clonal diversity in alpine populations of Polygonum viviparum (Polygonaceae). International Journal of Plant Sciences 159:606-615.

Diggle P.K., Meixner M.A., Carroll A.B. y Aschwanden C.F. 2002. Barriers to sexual reproduction in Polygonum viviparum: A comparative developmental analysis of $P$. viviparum and $P$. bistortoides. Annals of Botany 89:145-156.

Douhovnikoff V. y Dood R.S. 2003. Intra-clonal variation and a similarity threshold for identification of clones: application to $\mathrm{Sa}$ lix exigua using AFLP molecular markers. Theoretical Applied Genetics 106:1307-1315.

Douhovnikoff V., Cheng A.M. y Dodd R.S. 2004. Incidence, size and spatial structure of clones in second-growth stands of coast redwood, Sequoia sempervirens (Cupressaceae). American Journal of Botany 91:1140-1146.

Douhovnikoff V., McBride J.R. y Dodd R.S. 2005. Salix exigua clonal growth and population dynamics in relation to disturbance regime variation. Ecology 86:446-452.

Eckert C.G. 2000. Contributions of autogamy and geitonogamy to self-fertilization in a mass-flowering, clonal plant. Ecology 81:532-542.

Eckert C.G. 2002. The loss of sex in clonal plants. Evolutionary Ecology 15:501-520.

Ellstrand N.C. y Roose M.L. 1987. Patterns of genotypic diversity in clonal plant species. American Journal of Botany 74:123-131. 
Eriksson O. 1989. Seedling dynamics and life histories in clonal plants. Oikos 55:231-238.

Eriksson O. 1993. Dynamics of genets in clonal plants. Trends in Ecology \& Evolution 8:313-316.

Eriksson O. y Bremer B. 1993. Genet dynamics of the clonal plant Rubus saxatilis. Journal of Ecology 81:533-542.

Esselman E.J., Jianqiang L., Crawford D.J., Winduss J.L. y Wolfe A.D. 1999. Clonal diversity in the rare Calamagrostis porteri ssp insperata (Poaceae): comparative results for allozymes and random amplified polymorphic DNA (RAPD) and intersimple sequence repeat (ISSR) markers. Molecular Ecology 8:443451.

Everitt B.S. 1977. The Analysis of Contingency Tables. Chapmann \& Hall, Bury St Edmunds.

Gabrielsen T.M. y Brochmann C. 1998. Sex after all: high levels of diversity detected in the arctic clonal plant Saxifraga cernua using RAPD markers. Molecular Ecology 7:1701-1708.

Garnier L.K.M., Durand J. y Dajoz I. 2002. Limited seed dispersal and microspatial population structure of an agamospermous grass of West African savannahs, Hyparrhenia diplandra (Poaceae). American Journal of Botany 89:1785-1791.

Gibson D.J. y Menges E.S. 1994. Population structure and spatial pattern in the dioecious shrub Ceratiola ericoides. Journal of Vegetation Sciences 5:337-346.

Gleason H.A. 1920. Some applications of the quadrat method. Bulletin of the Torrey Botanical Club 47:21-33.

Hämmerli A. y Reusch T.B.H. 2003a. Flexible mating: cross-pollination affects sex expression in a marine clonal plant. Journal of Evolutionary Biology 18:1096-1105.

Hämmerli A. y Reusch T.B.H. 2003b. Genetic neighbourhood of clone structures in eelgrass meadows quantified by spatial autocorrelation of microsatellite markers. Heredity 91:448-455.

Handel S.N. 1985. The intrusion of clonal growth patterns on plant breeding system. The American Naturalist 125:367-384.

Harper J. 1985. Modules, branches, and the capture of resources. En: Jackson J.B.C., Buss L.W. y Cook R.E. Eds. Population Biology and Evolution of Clonal Organisms, pp. 1-33, Yale University Press, New Haven.

Hartnett D.C. y Bazzaz F.A. 1985. The integration of neighbourhood effects by clonal genets in Solidago canadensis. The Journal of Ecology 73:415-427.

Hirayama K. y Sakimoto M. 2008. Clonal structure and diversity of Cryptomeria japonica along a slope in a cool-temperate, oldgrowth mixed forest in the snowy region of Japan. Canadian Journal of Forest Research 38:2804-2813.

Honnay O. y Bossuyt B. 2005. Prolonged clonal growth: escape route or route to extinction? Oikos 108:427-432.

Honnay O., Jacquemyn H., Roldán-Ruiz I. y Hermy M. 2006. Consequences of prolonged clonal growth on local and regional genetic structure and fruiting success of the forest perennial Maianthemum bifolium. Oikos 112:21-30.

Honnay O. y Jacquemyn H. 2008. A meta-analysis of the relation between mating system, growth form and genotypic diversity in clonal plant species. Evolutionary Ecology 22:299-312.

Ivey C.T. y Richards J.H. 2001. Genotypic diversity and clonal structure of everglades sawgrass, Cladium jamaicense (Cyperaceae). International Journal of Plant Sciences 162:1327-1335.

Jackson J.B.C., Buss L.W. y Cook R.E. Eds. 1985. Population Biology and Evolution of Clonal organisms. Yale University Press, New Haven.
Jacquemyn H., Brys R., Honnay O., Hermy M. y Roldán-Ruiz I. 2005. Local forest environment largely affects below-ground growth, clonal diversity and fine-scale spatial genetic structure in the temperate deciduous forest herb Paris quadrifolia. Molecular Ecology 14:4479-4488.

Jonsson B.O., Jónsdóttir I.S. y Crongberg N. 1996. Clonal diversity and allozyme variation in populations of the arctic sedge Carex bigelowii (Cyperaceae). Journal of Ecology 84:449-459.

Kays S. y Harper J.L. 1974. The regulation of plant and tiller density in a grass sward. Journal of Ecology 62:97-105.

Keane B., Pelikan S., Toth G.P., Smith M.K. y Rogstad S.H. 1999. Genetic diversity of Typha latifolia (Typhaceae) and the impact of pollutants examined with tamdem-repetitive DNA probes. American Journal of Botany 86:1226-1238.

Kjølner S., Såstad S.M. y Brochmann C. 2006. Clonality and recombination in the arctic plant Saxifraga cernua. Botanical Journal of the Linnean Society 152:209-217.

Klimeš L., Klimešová J., Hendriks R.J.J. y van Groenendael J.M. 1997. Clonal plant architecture: a comparative analysis of form and function. En: de Kroon H. y van Groenendael J. Eds. The Ecology and Evolution of Clonal Plants, pp. 1-29, Backhuys Publishers, Leiden.

Korpelainen H. 1994. Sex ratios and resource allocation among sexually reproducing plants of Rubus chamaemorus. Annals of Botany 74:627-632.

Korpelainen H., Antonious-Klemola K. y Werlemark G. 1999. Clonal structure of Rubus chamaemorus populations: comparison of different molecular methods. Plant Ecology 143:123-128.

Kreher S.A., Foré S.A. y Collins B.S. 2000. Genetic variation within and among patches of the clonal species, Vaccinium stamineum L. Molecular Ecology 9:1247-1252.

Kudoh H., Shibaike H., Takasu H., Whigham D.F. y Kawano S. 1999. Genet structure and determinants of clonal structure in a temperate deciduous woodland herb, Uvularia perfoliata. Journal of Ecology 87:244-257.

Liston A., Wilson B.L., Robinson W.A., Doescher P.S., Harris N.R. y Svejcar T. 2003. The relative importance of sexual reproduction versus clonal spread in an arid bunchgrass. Oecologia 137:216-225.

Liu J., Dong M., Miao S.L., Li Z.Y., Song M.H. y Wang R.Q. 2006. Invasive alien plants in China: role of clonality and geographical origin. Biological Invasions 8:1461-1470.

Mandujano M.C., Montaña C., Méndez I. y Golubov J. 1998. The relative contributions of sexual reproduction and clonal propagation in Opuntia rastrera from two habitats in the Chihuahuan desert. Journal of Ecology 86:911-921.

Mandujano M.C., Montaña C., Franco M., Golubov J. y FloresMartínez A. 2001. Integration of demographic annual variability in a clonal desert cactus. Ecology 82:344-359.

Mandujano-Sánchez M.C. 2007. La clonalidad y sus efectos en la biología de poblaciones. En: Eguiarte L.E., Souza V. y Aguirre X. Comp. Ecología Molecular, pp. 215-250, Secretaría de Medio Ambiente y Recursos Naturales-Instituto Nacional de Ecología-Universidad Nacional Autónoma de México-Comosión Nacional para el Conocimiento y Uso de la Biodiversidad, México, D.F.

Mayes S.G., McGinley M.A. y Werth C.R. 1998. Clonal population structure and genetic variation in sand-shinnery oak, Quercus havardii (Fagaceae). American Journal of Botany 85:1609-1617. McFadden C.S. 1991. A comparative demographic analysis of clonal 
reproduction in a temperate soft coral. Ecology 72:1849-1866.

Misuki I., Ishida K., Tani N. y Tsumura Y. 2010. Fine-scale spatial structure of genets and sexes in the dioecious plant Dioscorea japonica, which disperses by both bulbils and seeds. Evolutionary Ecology 24:1399-1415.

Mori Y., Nagamitsu T. y Kubo T. 2009. Clonal growth and its effects on male and female reproductive success in Prunus ssiori (Rosaceae). Population Ecology 51:175-186.

Nagamitsu T., Ogawa M., Ishida K. y Tanouchi H. 2004. Clonal diversity, genetic structure, and mode of recruitment in a Prunus ssiori population established after volcanic eruptions. Plant Ecology 174:1-10.

Okuda T., Kachi N., Yap S.K. y Manokaran N. 1997. Tree distribution pattern and fate of juveniles in a lowland tropical rain forest - implications for regeneration and maintenance of species diversity. Plant Ecology 131:155-171.

Pan J.J. y Price J.S. 2002. Fitness and evolution in clonal plants: the impact of clonal growth. Evolutionary Ecology 15:583-600.

Parker K.C. y Hamrick J.L. 1992. Genetic diversity and clonal structure in a columnar cactus, Lophocereus schottii. American Journal of Botany 79:86-96.

Parks C. y Werth C.R. 1993. A study of spatial features of clones in a population of bracken fern, Pteridium aquilinum (Dennstaedtiaceae). American Journal of Botany 80:537-544.

Peterson C.J. y Jones R.H. 1997. Clonality in woody plants: a review and comparison with clonal herbs. En: de Kroon H. y van Groenendael J. Eds. The Ecology and Evolution of Clonal Plants, pp. 263-289, Backhuys Publishers, Leiden.

Pfeiffer T., Klahr A., Heinrich A. y Schnittler M. 2011. Does sex make a difference? Genetic diversity and spatial genetic structure in two co-occurring species of Gagea (Liliaceae) with contrasting reproductive strategies. Plant Systematics and Evolution 292:189-201.

Pornon A. y Escaravage N. 1999. Genotypic structure in clonal Rhododendron ferrugineum L. (Ericaceae) populations: origin and manteinance. Plant Ecology 141:145-150.

Pornon A., Escaravage N., Thomas P. y Taberlet P. 2000. Dynamics of genotypic structure in clonal Rhododendron ferrugineum (Ericaceae) populations. Molecular Ecology 9:1099-1111.

Pornon A., Escaravage N., Till-Botraud I. y Doche B. 1997. Variation of reproductive traits in Rhododendron ferrugineum $\mathrm{L}$. (Ericaceae) populations along a successional gradient. Plant Ecology 130:1-11.

Price E.A.C. y Marshall C. 1999. Clonal plants and environmental heterogeneity. Plant Ecology 141:3-7.

Reisch C., Schurm S. y Poschlod P. 2007. Spatial genetic structure and clonal diversity in an alpine population of Salix herbacea (Salicaceae). Annals of Botany 99:647-651.

Reusch T.B.H. 2003. Floral neighbourhoods in the sea: how floral density, opportunity for outcrossing and population fragmentation affect seed set in Zostera marina. Journal of Ecology 91:610-615.

Richards A.J. 1997. Plant Breeding Systems. Chapman \& Hall,
Londres.

Rosseto M., Gross C.L., Jones R. y Hunter J. 2004. The impact of clonality on an endangered tree (Elaeocarpus williamsianus) in a fragment rain forest. Biological Conservation 117:33-39.

Ruggieiro M.V., Capone S., Pirozzi P., Reusch T.B.H. y Procaccini G. 2005. Mating system and clonal architecture: a comparative study in two marine angiosperms. Evolutionary Ecology 19:487-499.

Shimizu Y, Ando M. y Sakai F. 2002. Clonal structure of natural populations of Cryptomeria japonica growing at different positions on slopes, detected using RAPD markers. Biochemical Systematics and Ecology 30:733-748.

Shreve F. 1917. The establishment of desert perennials. Journal of Ecology 5:210-216.

Sipes S.D. y Wolf P.G. 1997. Clonal structure and patterns of allozyme diversity in the rare endemic Cycladenia humilis var. jonesii (Apocynaceae). American Journal of Botany 84:401-409.

Stehlik I. y Holderegger R. 2000. Spatial genetic structure and clonal diversity of Anemone nemorosa in late successional deciduous woodlands of Central Europe. Journal of Ecology 88:424-435.

Suvanto L.I. y Latva-Karjantvaa T.B. 2005. Clone identification and clonal structure of the European aspen (Populus tremula). Molecular Ecology 14:2851-2860.

Tiffney B.H. y Niklas K.J. 1985. Clonal growth in land plants: a paleobotanical perspective. En: Jackson J.B.C., Buss L.W. y Cook R.E. Eds. Population Biology and Evolution of Clonal Organisms, pp. 35-66, Yale University Press, New Haven.

Tooke F., Ordidge M., Chiurugwi T. y Battey N. 2005. Mechanisms and function of flower and inflorescence reversion. Journal of Experimental Botany 56:2587-2599.

Torimaru T. y Tomaru N. 2005. Fine-scale clonal structure and diversity within patches of a clone-forming dioecious shrub, Ilex leucoclada (Aquifoliaceae). Annals of Botany 95:295-304.

van Dijk P.J. y Bakx-Schotman J.M.T. 2004. Formation of unreduced megaspores (diplospory) in apomictic dandelions (Taraxacum officinale, s.l.) is controlled by a sex-specific dominant locus. Genetics 166:483-492.

van Groenendael J.M. y de Kroon H. 1990. Clonal Growth in Plants: Regulation and Function. SPB Academic Publishing, La Haya.

van Groenendael J.M., Klimeš L., Klimešová J. y Hendriks R.J.J. 1996. Comparative ecology of clonal plants. Philosophical Transactions of Royal Society of London 351:1331-1339.

Widén B., Cronberg N. y Widén M. 1994. Genotypic diversity, molecular markers and spatial distribution of genets in clonal plants, a literature survey. Folia Geobotanica y Phytotaxonomica 29:245-263.

Wilson A.S.G., van der Kamp B.J. y Ritland C. 2005a. Opportunities for geitonogamy in the clonal herb Maianthemum dilatatum. Canadian Journal of Botany 83:1082-1087.

Wilson A.S.G., van der Kamp B.J. y Ritland C. 2005b. Spatial genetic and clonal structure in Maianthemum dilatatum as defined by AFLP markers. Canadian Journal of Botany 83:1126-1132. 
Apéndice. Características de las especies y poblaciones: $\mathbf{S C R Z}=$ sistema de cruza, $\mathbf{P O L}=$ polinización, $\mathbf{P R O R}=$ estructuras o procesos que dan origen a los ramets, CCT-N = tipo de crecimiento clonal en la clasificación de Tiffney y Niklas (1985), POB/HL = número de población y/o hábitat local, $\mathbf{P D E}=$ clave del patrón de distribución espacial de acuerdo a la figura 2, AER = arreglo espacial de los ramets de distintos genets, $\boldsymbol{D}=$ índice de diversidad clonal de Simpson, $\boldsymbol{G} / \mathbf{N}=$ proporción de genotipos distinguibles, Métodos = métodos empleados para la evaluación de los parámetros. El significado de las abreviaturas empleadas en cada columna se muestra al final del cuadro.

\begin{tabular}{|c|c|c|c|c|c|c|c|c|c|c|c|c|c|}
\hline Especie (Familia) & Hábito & SCRZ & POL & PROR & CCT-N & $\begin{array}{c}\text { POB/ } \\
\mathrm{HL}\end{array}$ & $\begin{array}{c}\text { PDE } \\
\text { (fig. 2) }\end{array}$ & $\begin{array}{c}\text { AER } \\
\text { (fig. 2) }\end{array}$ & $D$ & $G / N$ & $\begin{array}{l}\text { Estructura } \\
\text { genética } \\
\text { local (EGL) }\end{array}$ & $\begin{array}{c}\text { Métodos } \\
\text { PDE, } D, G / N \\
\text { y EGL }\end{array}$ & Referencias \\
\hline $\begin{array}{l}\text { Anemone } \\
\text { nemorosa } \\
\text { (Ranunculaceae) }\end{array}$ & $\mathrm{Hp}$ & A & $\mathrm{Bt}$ & $\begin{array}{c}\mathrm{Rzm+} \\
\mathrm{drm}\end{array}$ & $\mathrm{Lg}+\mathrm{NI}$ & $\begin{array}{l}\text { ppm } \\
(20)\end{array}$ & $(4,5)$ & $\mathrm{Ag}+\mathrm{En}$ & $\mathrm{n} / \mathrm{e}$ & 0.95 & $\begin{array}{l}\operatorname{asp},<1 \mathrm{~m} \\
(\mathrm{rm})\end{array}$ & tra+ISZ+asp & $\begin{array}{l}\text { Stehlik y } \\
\text { Holderegger } \\
\text { 2000* }\end{array}$ \\
\hline $\begin{array}{l}\text { Botrychium } \\
\text { pumicola } \\
\text { (Ophioglossaceae) }\end{array}$ & $\mathrm{Hp}$ & A & $\mathrm{Ab}$ & gmas & $\mathrm{NI}$ & $\begin{array}{l}\text { p } 1 \\
\text { p } 2 \\
\text { p } 3\end{array}$ & $\begin{array}{l}(2,5) \\
(2,5)\end{array}$ & $\begin{array}{l}\mathrm{Ag}+\mathrm{En} \\
\mathrm{Ag}+\mathrm{En} \\
\mathrm{Ag}+\mathrm{En}\end{array}$ & $\begin{array}{c}0.14 \\
0.16 \\
0.1\end{array}$ & $\begin{array}{l}0.93 \\
0.9 \\
0.65\end{array}$ & $\begin{array}{c}\text { mt: no } \\
\text { detectada } \\
\text { mt: no } \\
\text { detectada } \\
\text { mt: no } \\
\text { detectada }\end{array}$ & prc + ISSR $+m t$ & $\begin{array}{c}\text { Camacho y } \\
\text { Liston, 2001* }\end{array}$ \\
\hline $\begin{array}{l}\text { Calamagrostis } \\
\text { porteri ssp. } \\
\text { insperata (Poaceae) }\end{array}$ & $\mathrm{Hp}$ & A & $\mathrm{Ab}$ & rzm & $\operatorname{Lg}$ & $\begin{array}{l}\text { p } 2 \\
\text { p } 3 \\
\text { p } 4\end{array}$ & $\begin{array}{l}2 \\
2 \\
2\end{array}$ & $\begin{array}{l}\mathrm{Ag} \\
\mathrm{Ag} \\
\mathrm{Ag}\end{array}$ & $\begin{array}{l}n / e \\
n / e \\
n / e\end{array}$ & $\begin{array}{c} \\
1 \\
0.82 \\
0.91\end{array}$ & $\begin{array}{l}\mathrm{n} / \mathrm{e} \\
\mathrm{n} / \mathrm{e} \\
\mathrm{n} / \mathrm{e}\end{array}$ & $\begin{array}{c}\text { desc+tra+ } \\
\text { ISRR+ } \\
\text { RAPD }\end{array}$ & $\begin{array}{c}\text { Esselman } \\
\text { et al., } \\
1999\end{array}$ \\
\hline $\begin{array}{l}\text { Carex bigelowii } \\
\text { (Cyperaceae) }\end{array}$ & $\mathrm{Hp}$ & $?$ & $\mathrm{Ab}$ & rzm & $\operatorname{Lg}$ & $\begin{array}{l}\text { p } 1 \\
\text { p } 2 \\
\text { p } 3\end{array}$ & $\begin{array}{l}4 \\
4 \\
4\end{array}$ & $\begin{array}{l}\mathrm{Ag} \\
\mathrm{Ag} \\
\mathrm{Ag}\end{array}$ & $\begin{array}{l}0.98 \\
0.98 \\
0.98\end{array}$ & $\begin{array}{l}0.52 \\
0.63 \\
0.48\end{array}$ & $\begin{array}{l}\text { n/e } \\
\text { n/e } \\
\text { n/e }\end{array}$ & tra+ISZ & $\begin{array}{c}\text { Jonsson } \\
\text { et al., 1996* }\end{array}$ \\
\hline $\begin{array}{l}\text { Cladium jamaicense } \\
\text { (Cyperaceae) }\end{array}$ & $\mathrm{Hp}$ & $?$ & $\mathrm{Ab}$ & $\begin{array}{c}\text { rzm+ } \\
\text { pnt }\end{array}$ & $\mathrm{Lg}+\mathrm{Nl}$ & $\begin{array}{l}p 1 \\
\text { p } 2 \\
\text { p } 3 \\
\text { p } 4 \\
\text { p } 5 \\
\text { p } 6 \\
\text { p } 7 \\
\text { p } 8 \\
\text { p } 9 \\
\text { p } 10 \\
\text { p } 11 \\
\text { p } 12 \\
\text { p } 13 \\
\text { p } 14\end{array}$ & $\begin{array}{l}(4,5) \\
(4,5) \\
(4,5) \\
(4,5) \\
(4,5) \\
1 \\
(4,5) \\
(4,5) \\
(4,5) \\
(4,5) \\
(4,5) \\
(4,5) \\
(4,5) \\
(4,5)\end{array}$ & $\begin{array}{c}A g+E n \\
A g+E n \\
A g+E n \\
A g+E n \\
A g+E n \\
A g \\
A g+E n \\
A g+E n \\
A g+E n \\
A g+E n \\
A g+E n \\
A g+E n \\
A g+E n \\
A g+E n\end{array}$ & $\begin{array}{c}0.236 \\
0.269 \\
0.271 \\
0.682 \\
0.159 \\
0 \\
0.538 \\
0.212 \\
0.771 \\
0.698 \\
0.814 \\
0.682 \\
0.673 \\
0.444\end{array}$ & $\begin{array}{l}0.044 \\
0.044 \\
0.125 \\
0.231 \\
0.043 \\
0.021 \\
0.146 \\
0.068 \\
0.106 \\
0.143 \\
0.261 \\
0.114 \\
0.13 \\
0.043\end{array}$ & $\begin{array}{c}\text { mpic: no } \\
\text { detectada } \\
\text { mpic: no } \\
\text { detectada } \\
\text { mpic: no } \\
\text { detectada } \\
\text { mpic: no } \\
\text { detectada } \\
\text { mpic: no } \\
\text { detectada } \\
\text { n/a } \\
\text { mpic: no } \\
\text { detectada } \\
\text { mpic: no } \\
\text { detectada } \\
\text { mpic: no } \\
\text { detectada } \\
\text { mpic: no } \\
\text { detectada } \\
\text { mpic: no } \\
\text { detectada } \\
\text { mpic: no } \\
\text { detectada } \\
\text { mpic: no } \\
\text { detectada } \\
\text { mpic: no } \\
\text { detectada }\end{array}$ & $\begin{array}{l}\text { tra+lSZ+ } \\
\text { mpic }\end{array}$ & $\begin{array}{l}\text { Ivey y Richards, } \\
\text { 2001* }\end{array}$ \\
\hline
\end{tabular}


Apéndice. Continuación

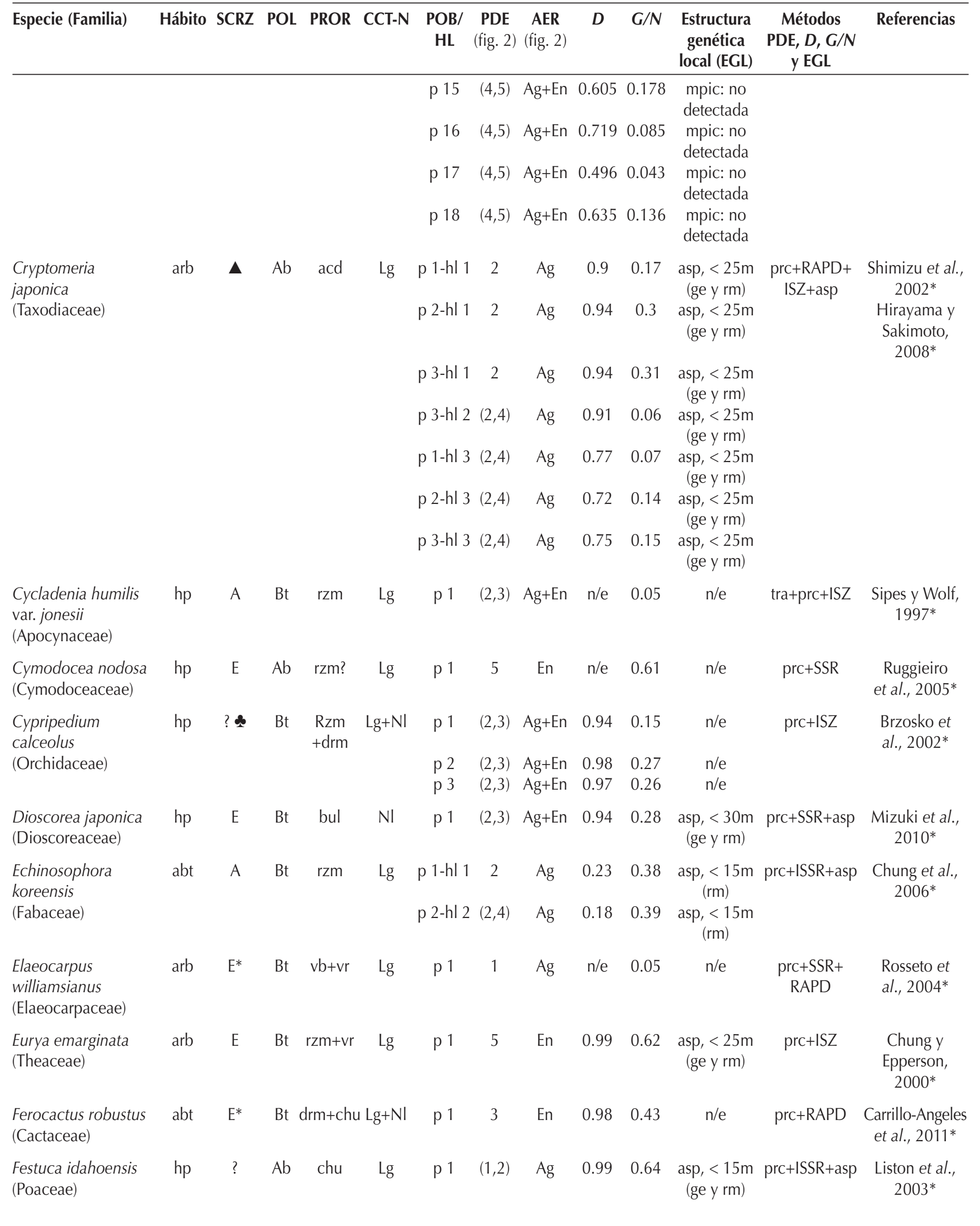


Apéndice. Continuación

\begin{tabular}{|c|c|c|c|c|c|c|c|c|c|c|c|c|c|}
\hline Especie (Familia) & Hábito & SCRZ & POL & PROR & CCT-N & $\begin{array}{c}\mathrm{POB} / \\
\mathrm{HL}\end{array}$ & $\begin{array}{l}\text { PDE } \\
\text { (fig. 2) }\end{array}$ & $\begin{array}{c}\text { AER } \\
\text { (fig. 2) }\end{array}$ & $D$ & $G / N$ & $\begin{array}{l}\text { Estructura } \\
\text { genética } \\
\text { local (EGL) }\end{array}$ & $\begin{array}{c}\text { Métodos } \\
\text { PDE, } D, G / N \\
\text { y EGL } \\
\end{array}$ & Referencias \\
\hline \multirow[t]{3}{*}{$\begin{array}{l}\text { Gagea lutea } \\
\text { (Liliaceae) }\end{array}$} & \multirow[t]{3}{*}{ hg } & \multirow[t]{3}{*}{$?$} & \multirow[t]{3}{*}{$\mathrm{Bt}$} & \multirow[t]{3}{*}{ buls } & \multirow[t]{3}{*}{$\mathrm{Nl}$} & p 1 & $(2,3)$ & $\mathrm{Ag}+\mathrm{En}$ & $\mathrm{n} / \mathrm{e}$ & 0.51 & \multirow{3}{*}{\multicolumn{2}{|c|}{$\begin{array}{l}\text { asp, }<8 \mathrm{~m}, 16 \text { tra+AFLP+asp } \\
\mathrm{a}<24 \mathrm{~m}(\mathrm{rm}) \\
\mathrm{asp},<8 \mathrm{~m}, 16 \\
\mathrm{a}<24 \mathrm{~m}(\mathrm{rm}) \\
\mathrm{asp},<8 \mathrm{~m}, 16 \\
\mathrm{a}<24 \mathrm{~m}(\mathrm{rm})\end{array}$}} & \multirow[t]{3}{*}{$\begin{array}{l}\text { Pfeiffer et al., } \\
2011^{*}\end{array}$} \\
\hline & & & & & & p 2 & $(2,3)$ & $\mathrm{Ag}+\mathrm{En}$ & $\mathrm{n} / \mathrm{e}$ & 0.57 & & & \\
\hline & & & & & & p 3 & $(2,3)$ & $\mathrm{Ag}+\mathrm{En}$ & $\mathrm{n} / \mathrm{e}$ & 0.61 & & & \\
\hline \multirow{3}{*}{$\begin{array}{l}\text { Gagea spathacea } \\
\text { (Liliaceae) }\end{array}$} & \multirow[t]{3}{*}{ hg } & \multirow[t]{3}{*}{$?$} & \multirow[t]{3}{*}{$\mathrm{Bt}$} & \multirow[t]{3}{*}{ buls } & \multirow[t]{3}{*}{$\mathrm{NI}$} & p 1 & 1 & $\mathrm{Ag}$ & $\mathrm{n} / \mathrm{e}$ & 0.001 & $\mathrm{n} / \mathrm{a}$ & \multirow[t]{3}{*}{ tra+AFLP } & \multirow{3}{*}{$\begin{array}{r}\text { Pfeiffer et al., } \\
2011^{*}\end{array}$} \\
\hline & & & & & & p 2 & 1 & $\mathrm{Ag}$ & $\mathrm{n} / \mathrm{e}$ & 0.001 & $\mathrm{n} / \mathrm{a}$ & & \\
\hline & & & & & & p 3 & 1 & $\mathrm{Ag}$ & $\mathrm{n} / \mathrm{e}$ & 0.001 & $\mathrm{n} / \mathrm{a}$ & & \\
\hline $\begin{array}{l}\text { Hyparrhenia } \\
\text { diplandra (Poaceae) }\end{array}$ & hp & $?$ & $\mathrm{Ab}$ & agp & $\mathrm{NI}$ & p 1 & 5 & En & 0.85 & 0.07 & $\begin{array}{l}\text { asp, }<6 \mathrm{~m} \\
\quad(\mathrm{rm})\end{array}$ & $\begin{array}{c}\text { prc+lSZ } \\
+ \text { CLO+asp }\end{array}$ & $\begin{array}{c}\text { Garnier et al., } \\
2002^{*}\end{array}$ \\
\hline $\begin{array}{l}\text { Ilex leucoclada } \\
\text { (Aquifoliaceae) }\end{array}$ & abt & E & $\mathrm{Bt}$ & $\operatorname{acd} \mathbf{\Lambda}$ & $\operatorname{Lg}$ & p 1 & $(2,3)$ & $\mathrm{Ag}+\mathrm{En}$ & 0.67 & 0.09 & $\mathrm{n} / \mathrm{e}$ & prc+RAPD & $\begin{array}{c}\text { Torimaru y } \\
\text { Tomaru, 2005* }\end{array}$ \\
\hline \multirow[t]{8}{*}{$\begin{array}{l}\text { Lophocereus schottii } \\
\text { (Cactaceae) }\end{array}$} & \multirow[t]{8}{*}{ arb } & \multirow[t]{8}{*}{ A } & \multirow[t]{8}{*}{$\mathrm{Bt}$} & \multirow[t]{8}{*}{$\begin{array}{l}\mathrm{drm} \\
+\mathrm{acd}\end{array}$} & \multirow[t]{8}{*}{$\mathrm{Lg}+\mathrm{NI}$} & p 1-hl 1 & 5 & En & 0.95 & 0.64 & $\begin{array}{c}\text { SG: 0-10 } \\
<10-50>\text { más } \\
\text { de } 50 m \text { (ge) }\end{array}$ & \multirow{8}{*}{ prc+ISZ+SG } & \multirow[t]{8}{*}{$\begin{array}{l}\text { Parker y } \\
\text { Hamrick, } \\
1992^{*}\end{array}$} \\
\hline & & & & & & p 2-hl 2 & 2 & $\mathrm{Ag}$ & 0.95 & 0.52 & $\begin{array}{l}\text { SG: } 0-10 \\
<10-50>\text { más } \\
\text { de } 50 \mathrm{~m} \text { (ge) }\end{array}$ & & \\
\hline & & & & & & p 3-hl 3 & 2 & $\mathrm{Ag}$ & 0.93 & 0.65 & $\begin{array}{c}\text { SG: } 0-10 \\
<10-50>\text { más } \\
\text { de } 50 m \text { (ge) }\end{array}$ & & \\
\hline & & & & & & p 4-hl 1 & $(1,2)$ & $\mathrm{Ag}$ & 0.94 & 0.24 & $\begin{array}{l}\text { SG: } 0-10> \\
\text { 10-50>más } \\
\text { de } 50 \mathrm{~m} \text { (ge) }\end{array}$ & & \\
\hline & & & & & & p 5-hl 4 & $(1,2)$ & $\mathrm{Ag}$ & 0.92 & 0.41 & $\begin{array}{l}\text { SG: } 0-10> \\
10-50<\text { más } \\
\text { de } 50 m \text { (ge) }\end{array}$ & & \\
\hline & & & & & & p 6-hl 4 & $(1,2)$ & $\mathrm{Ag}$ & 0.87 & 0.45 & $\begin{array}{c}\text { SG: } 0-10> \\
10-50<\text { más } \\
\text { de } 50 m \text { (ge) }\end{array}$ & & \\
\hline & & & & & & p 7-hl 2 & $(1,2)$ & $\mathrm{Ag}$ & 0.8 & 0.18 & $\begin{array}{l}\text { SG: } 0-10> \\
\text { 10-50>más } \\
\text { de } 50 \mathrm{~m} \text { (ge) }\end{array}$ & & \\
\hline & & & & & & p 8-hl 5 & $(1,5)$ & $\mathrm{Ag}+\mathrm{En}$ & 0.59 & 0.36 & $\begin{array}{l}\text { SG: } 10-50= \\
\text { más de } 50 \mathrm{~m} \\
\quad(\text { ge })\end{array}$ & & \\
\hline $\begin{array}{l}\text { Lyonothamnus } \\
\text { floribundus } \\
\text { (Rosaceae) }\end{array}$ & arb & $?$ & $\mathrm{Bt}$ & $\mathrm{vr}$ & $\operatorname{Lg}$ & p 1 & $(1,2)$ & $\mathrm{Ag}$ & $\mathrm{n} / \mathrm{e}$ & 0.036 & $\mathrm{n} / \mathrm{e}$ & prc+RAPD & $\begin{array}{c}\text { Bushakra et al., } \\
\text { 1999* }\end{array}$ \\
\hline $\begin{array}{l}\text { Maianthemum } \\
\text { bifolium } \\
\text { (Convalliariaceae) }\end{array}$ & hp & $E^{*}$ & $\mathrm{Bt}$ & $\mathrm{rzm}$ & $\operatorname{Lg}$ & p 1 & $(1,2)$ & $\mathrm{Ag}$ & 0.88 & 0.7 & $\begin{array}{l}\text { asp, }<50 \mathrm{~m} \\
\text { (ge) \# }\end{array}$ & $\begin{array}{l}\text { trans+AFLP } \\
\quad+\text { asp }\end{array}$ & $\begin{array}{l}\text { Arens et al., } \\
2005^{*} \\
\text { Honnay et al. } \\
2006\end{array}$ \\
\hline $\begin{array}{l}\text { Maianthemum } \\
\text { dilatatum } \\
\text { (Convalliariaceae) }\end{array}$ & hp & EAs & $\mathrm{Bt}$ & $\operatorname{rzm} \mathbf{\Delta}$ & $\operatorname{Lg}$ & p 1 & $(1,2)$ & $\mathrm{Ag}$ & $\mathrm{n} / \mathrm{e}$ & 0.64 & $\begin{array}{l}\text { asp, }<45 \mathrm{~m} \\
\text { (ge y rm) }\end{array}$ & $\begin{array}{l}\text { prc }+ \text { AFLP } \\
\quad+\text { asp }\end{array}$ & $\begin{array}{l}\text { Wilson et al., } \\
2005 a^{*} \\
\text { Wilson et al., } \\
2005 b^{*}\end{array}$ \\
\hline $\begin{array}{l}\text { Paris quadrifolia } \\
\text { (Trilliaceae) }\end{array}$ & hp & A & $\mathrm{Bt}$ & $\mathrm{rzm}$ & $\operatorname{Lg}$ & p 1-hl 1 & 3 & En & 0.87 & 0.27 & $\begin{array}{l}\text { asp, }<5 \mathrm{~m} \\
(\text { ge y rm) }\end{array}$ & $\begin{array}{l}\text { prc }+ \text { AFLP } \\
\quad+\text { asp }\end{array}$ & $\begin{array}{c}\text { Jacquemyn et } \\
\text { al., 2005* }\end{array}$ \\
\hline
\end{tabular}


Apéndice. Continuación

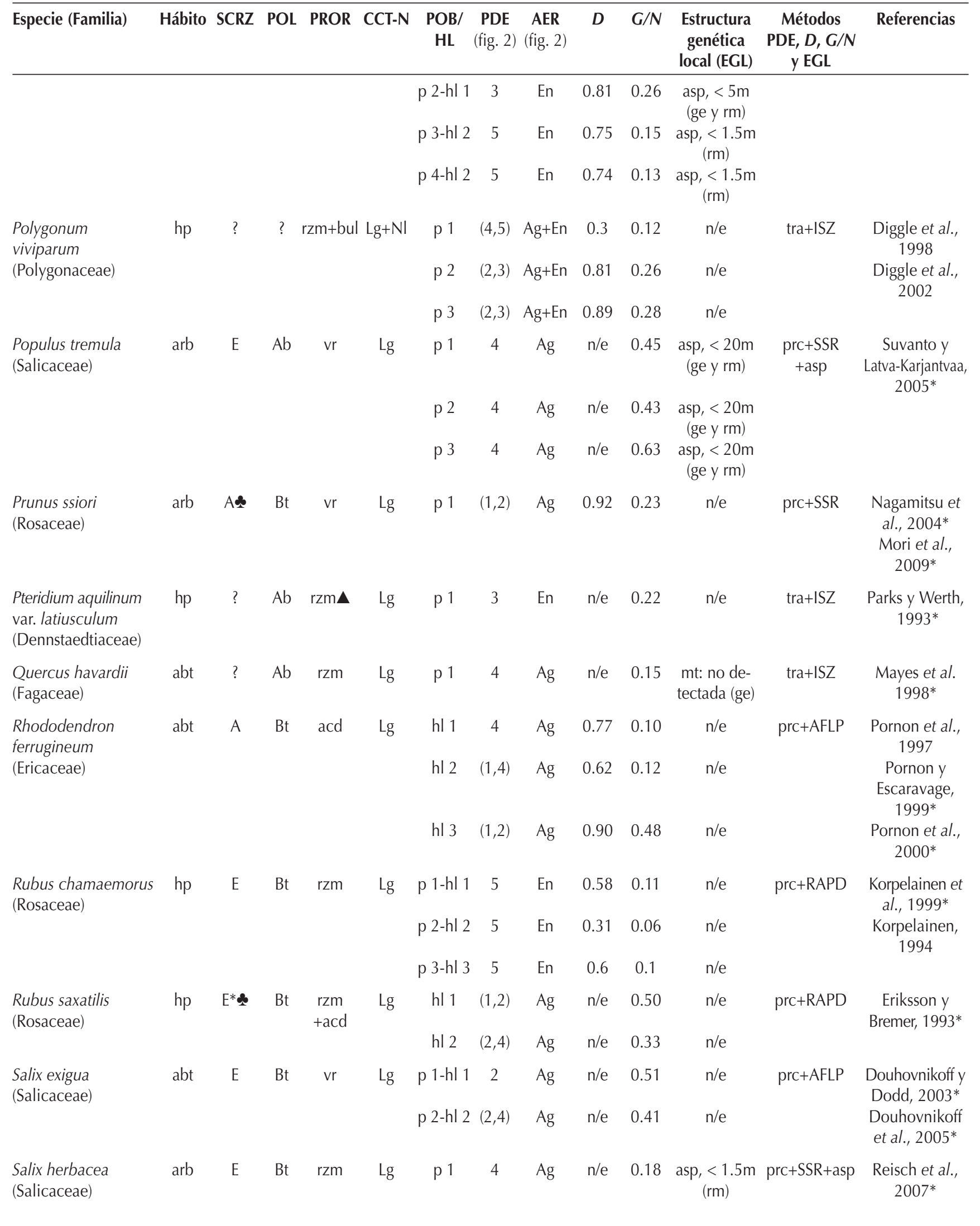


Apéndice. Continuación

\begin{tabular}{|c|c|c|c|c|c|c|c|c|c|c|c|c|c|}
\hline Especie (Familia) & Hábito & SCRZ & POL & PROR & CCT-N & $\begin{array}{c}\mathrm{POB} / \\
\mathrm{HL}\end{array}$ & $\begin{array}{c}\text { PDE } \\
\text { (fig. 2) }\end{array}$ & $\begin{array}{c}\text { AER } \\
\text { (fig. 2) }\end{array}$ & $D$ & $G / N$ & $\begin{array}{l}\text { Estructura } \\
\text { genética } \\
\text { local (EGL) }\end{array}$ & $\begin{array}{c}\text { Métodos } \\
\text { PDE, } D, G / N \\
\text { y EGL }\end{array}$ & Referencias \\
\hline \multirow[t]{6}{*}{$\begin{array}{l}\text { Saxifraga cernua } \\
\text { (Saxifragaceae) }\end{array}$} & \multirow[t]{6}{*}{ hp } & \multirow[t]{6}{*}{$E^{*}$} & \multirow[t]{6}{*}{$\mathrm{Bt}$} & \multirow[t]{6}{*}{ bul } & \multirow[t]{6}{*}{$\mathrm{Nl}$} & p 1 & 5 & En & 0.35 & 0.13 & $\begin{array}{c}\text { mt: no } \\
\text { detectada }\end{array}$ & \multirow[t]{6}{*}{$\begin{array}{l}\text { prc+RAPD } \\
+m t+a s p\end{array}$} & \multirow{6}{*}{$\begin{array}{l}\text { Gabrielsen y } \\
\text { Brochmann, } \\
1998^{*} \\
\text { KjØIner et al., } \\
\text { 2006* }\end{array}$} \\
\hline & & & & & & p 2 & 5 & En & 0.68 & 0.19 & $\begin{array}{c}\text { mt: no } \\
\text { detectada }\end{array}$ & & \\
\hline & & & & & & p 3 & 5 & En & 0.7 & 0.25 & $\begin{array}{l}\text { asp: no } \\
\text { detectada }\end{array}$ & & \\
\hline & & & & & & p 4 & 5 & En & 0.9 & 0.4 & $\begin{array}{c}\text { asp: no } \\
\text { detectada }\end{array}$ & & \\
\hline & & & & & & p 5 & 5 & En & 0.93 & 0.43 & $\begin{array}{l}\text { asp: no } \\
\text { detectada }\end{array}$ & & \\
\hline & & & & & & p 6 & 5 & En & 0.89 & 0.34 & $\begin{array}{c}\text { asp: no } \\
\text { detectada }\end{array}$ & & \\
\hline \multirow[t]{3}{*}{$\begin{array}{l}\text { Scirpus maritimus } \\
\text { (Cyperaceae) }\end{array}$} & \multirow[t]{3}{*}{ hp } & \multirow[t]{12}{*}{$\mathrm{E}^{*}$} & \multirow[t]{3}{*}{$\mathrm{Ab}$} & \multirow[t]{3}{*}{$\mathrm{rzm}$} & \multirow[t]{3}{*}{$\operatorname{Lg}$} & p 1 & 2 & $\mathrm{Ag}$ & $\mathrm{n} / \mathrm{e}$ & $\mathrm{n} / \mathrm{e}$ & $\mathrm{n} / \mathrm{e}$ & \multirow[t]{3}{*}{ desc } & \multirow[t]{3}{*}{$\begin{array}{c}\text { Charpentier et } \\
\text { al., } 2000\end{array}$} \\
\hline & & & & & & p 2 & 4 & $\mathrm{Ag}$ & $\mathrm{n} / \mathrm{e}$ & $\mathrm{n} / \mathrm{e}$ & $\mathrm{n} / \mathrm{e}$ & & \\
\hline & & & & & & p 3 & 4 & $\mathrm{Ag}$ & $\mathrm{n} / \mathrm{e}$ & $\mathrm{n} / \mathrm{e}$ & $\mathrm{n} / \mathrm{e}$ & & \\
\hline \multirow[t]{9}{*}{$\begin{array}{l}\text { Sequoia sempervirens } \\
\text { (Taxodiaceae) }\end{array}$} & \multirow[t]{9}{*}{ arb } & & \multirow[t]{9}{*}{$\mathrm{Ab}$} & \multirow[t]{9}{*}{$\mathrm{vb}$} & \multirow[t]{9}{*}{$\operatorname{Lg}$} & p 1-hl 1 & 2 & $\mathrm{Ag}$ & $\mathrm{n} / \mathrm{e}$ & 0.24 & $\begin{array}{l}\mathrm{mt}:<40 \mathrm{~m} \\
\quad(\mathrm{rm})\end{array}$ & \multirow[t]{9}{*}{$\operatorname{prc}+\mathrm{AFLP}+\mathrm{mt}$} & \multirow[t]{9}{*}{$\begin{array}{l}\text { Douhovnikoff } \\
\text { et al., 2004* }\end{array}$} \\
\hline & & & & & & p 2-hl 1 & 2 & $\mathrm{Ag}$ & $\mathrm{n} / \mathrm{e}$ & 0.38 & $\begin{array}{c}\mathrm{mt}:<40 \mathrm{~m} \\
(\mathrm{rm})\end{array}$ & & \\
\hline & & & & & & p 3-hl 1 & 2 & $\mathrm{Ag}$ & $\mathrm{n} / \mathrm{e}$ & 0.5 & $\begin{array}{c}\mathrm{mt}:<40 \mathrm{~m} \\
(\mathrm{rm})\end{array}$ & & \\
\hline & & & & & & p 4-hl 2 & 2 & $\mathrm{Ag}$ & $\mathrm{n} / \mathrm{e}$ & 0.6 & $\begin{array}{c}\mathrm{mt}:<40 \mathrm{~m} \\
(\mathrm{rm})\end{array}$ & & \\
\hline & & & & & & p 5-hl 2 & 2 & $\mathrm{Ag}$ & $\mathrm{n} / \mathrm{e}$ & 0.35 & $\begin{array}{c}\mathrm{mt}:<40 \mathrm{~m} \\
(\mathrm{rm})\end{array}$ & & \\
\hline & & & & & & p 6-hl 2 & 2 & $\mathrm{Ag}$ & $\mathrm{n} / \mathrm{e}$ & 0.6 & $\begin{array}{c}\mathrm{mt}:<40 \mathrm{~m} \\
(\mathrm{rm})\end{array}$ & & \\
\hline & & & & & & p 7-hl 3 & 2 & $\mathrm{Ag}$ & $\mathrm{n} / \mathrm{e}$ & 0.26 & $\begin{array}{c}\mathrm{mt}:<40 \mathrm{~m} \\
(\mathrm{rm})\end{array}$ & & \\
\hline & & & & & & p 8-hl 3 & 2 & $\mathrm{Ag}$ & $n / e$ & 0.19 & $\begin{array}{c}\mathrm{mt}:<40 \mathrm{~m} \\
(\mathrm{rm})\end{array}$ & & \\
\hline & & & & & & p 9-hl 3 & 2 & $\mathrm{Ag}$ & $\mathrm{n} / \mathrm{e}$ & 0.54 & $\begin{array}{c}\mathrm{mt}:<40 \mathrm{~m} \\
\quad(\mathrm{rm})\end{array}$ & & \\
\hline $\begin{array}{l}\text { Stenocereus eruca } \\
\text { (Cactaceae) }\end{array}$ & abt & $E^{*}$ & $\mathrm{Bt}$ & drm & $\mathrm{NI}$ & p 1 & 2 & $\mathrm{Ag}$ & 0.99 & 0.83 & $\begin{array}{l}\text { asp, }<20 \text { m } \\
\text { (ge y rm) }\end{array}$ & prc+ISSR+asp & $\begin{array}{c}\text { Clark-Tapia et } \\
\text { al., 2005* } \\
\text { Clark-Tapia et } \\
\text { al., } 2006\end{array}$ \\
\hline \multirow[t]{5}{*}{$\begin{array}{l}\text { Typha latifolia } \\
\text { (Thyphaceae) }\end{array}$} & \multirow[t]{5}{*}{$\mathrm{hp}$} & \multirow[t]{5}{*}{ A } & \multirow[t]{5}{*}{$\mathrm{Ab}$} & \multirow[t]{5}{*}{ rzm } & $\operatorname{Lg}$ & p 1 & $(4,5)$ & $\mathrm{Ag}+\mathrm{En}$ & $\mathrm{n} / \mathrm{e}$ & 0.77 & $\mathrm{n} / \mathrm{e}$ & tra+VNTR & $\begin{array}{c}\text { Keane et al., } \\
\text { 1999* }\end{array}$ \\
\hline & & & & & & p 2 & $(4,5)$ & $\mathrm{Ag}+\mathrm{En}$ & $\mathrm{n} / \mathrm{e}$ & 0.55 & $\mathrm{n} / \mathrm{e}$ & & \\
\hline & & & & & & p 3 & $(4,5)$ & $\mathrm{Ag}+\mathrm{En}$ & $\mathrm{n} / \mathrm{e}$ & 0.48 & $\mathrm{n} / \mathrm{e}$ & & \\
\hline & & & & & & p 4 & $(4,5)$ & $\mathrm{Ag}+\mathrm{En}$ & $\mathrm{n} / \mathrm{e}$ & 0.3 & $n / e$ & & \\
\hline & & & & & & p 5 & $(4,5)$ & $A g+E n$ & $\mathrm{n} / \mathrm{e}$ & 0.57 & $\mathrm{n} / \mathrm{e}$ & & \\
\hline $\begin{array}{l}\text { Uvularia perfoliata } \\
\text { (Convallariaceae) }\end{array}$ & hp & $?$ & $?$ & etl & $\operatorname{Lg}$ & p 1-hl 1 & $(4,5)$ & $A g+E n$ & 0.74 & 0.09 & $\begin{array}{c}\text { asp, }<3-5 m \\
(r m)\end{array}$ & prc+ISZ+asp & $\begin{array}{l}\text { Kudoh et al., } \\
\text { 1999* }\end{array}$ \\
\hline & & & & & & p 1-hl 2 & 1 & $\mathrm{Ag}$ & 0.59 & 0.03 & $\mathrm{n} / \mathrm{a}$ & & \\
\hline $\begin{array}{l}\text { Vaccinium myrtillus } \\
\text { (Ericaceae) }\end{array}$ & abt & A & $\mathrm{Bt}$ & rzm & $\operatorname{Lg}$ & p 1 & 2 & $\mathrm{Ag}$ & 0.94 & 0.29 & $\begin{array}{c}\text { asp: no } \\
\text { detectada }\end{array}$ & $\begin{array}{l}\text { prc+AFLP+ } \\
\text { RAPD+asp }\end{array}$ & $\begin{array}{c}\text { Albert et al., } \\
\text { 2003* }\end{array}$ \\
\hline
\end{tabular}


Apéndice. Continuación

\begin{tabular}{|c|c|c|c|c|c|c|c|c|c|c|c|c|c|}
\hline Especie (Familia) & Hábito & SCRZ & POL & PROR & CCT-N & $\begin{array}{c}\mathrm{POB} / \\
\mathrm{HL}\end{array}$ & $\begin{array}{l}\text { PDE } \\
\text { (fig. 2) }\end{array}$ & $\begin{array}{c}\text { AER } \\
\text { (fig. 2) }\end{array}$ & $D$ & $G / N$ & $\begin{array}{c}\text { Estructura } \\
\text { genética } \\
\text { local (EGL) }\end{array}$ & $\begin{array}{c}\text { Métodos } \\
\text { PDE, } D, G / N \\
\text { y EGL }\end{array}$ & Referencias \\
\hline $\begin{array}{l}\text { Vaccinium stamineum } \\
\text { (Ericaceae) }\end{array}$ & abt & $\mathrm{E}^{*}$ & $\mathrm{Bt}$ & $\mathrm{rzm}$ & $\operatorname{Lg}$ & $\mathrm{p} 1$ & $(2,3)$ & $\mathrm{Ag}+\mathrm{En}$ & $\mathrm{n} / \mathrm{e}$ & 0.68 & $\mathrm{n} / \mathrm{e}$ & prc+RAPD & $\begin{array}{l}\text { Kreher et al., } \\
\text { 2000* }\end{array}$ \\
\hline $\begin{array}{l}\text { Zostera marina } \\
\text { (Zosteraceae) }\end{array}$ & hp & A & $\mathrm{Ab}$ & $\mathrm{rzm}$ & $\operatorname{Lg}$ & $\begin{array}{l}\text { p 1-hl } 1 \\
\text { p 2-hl } 2\end{array}$ & $\begin{array}{l}(4,5) \\
(4,5)\end{array}$ & $\begin{array}{l}A g+E n \\
A g+E n\end{array}$ & $\mathrm{n} / \mathrm{e}$ & $\mathrm{n} / \mathrm{e}$ & $\begin{array}{c}\text { asp, }<6 \mathrm{~m} \\
\text { (ge y rm) } \\
\text { asp, }<6 \mathrm{~m} \\
\text { (ge y rm) }\end{array}$ & $\mathrm{prc}+\mathrm{SSR}+\mathrm{asp}$ & $\begin{array}{l}\text { Hämmerli y } \\
\text { Reusch, } 2003 \mathrm{a}^{*} \\
\text { Hämmerli y } \\
\text { Reusch, } 2003 \mathrm{~b}^{*}\end{array}$ \\
\hline $\begin{array}{l}\text { Zostera noltii } \\
\text { (Zosteraceae) }\end{array}$ & hp & A & $\mathrm{Ab}$ & $\mathrm{rzm}$ & $\operatorname{Lg}$ & p 1 & 4 & $\mathrm{Ag}$ & $\mathrm{n} / \mathrm{e}$ & 0.12 & $\mathrm{n} / \mathrm{e}$ & $\mathrm{prc}+\mathrm{SSR}$ & $\begin{array}{l}\text { Ruggieiro et } \\
\text { al., 2005* }\end{array}$ \\
\hline
\end{tabular}

Hábito: $\mathbf{a b t}=$ arbustos, $\mathbf{a r b}=$ árboles, $\mathbf{h p}=$ hierbas perennes

SCRZ: A = especies con capacidad de reproducirse por autocruza, $\mathbf{E}=$ especies con entrecruza obligada (p. ej., dioicas), $\mathbf{E}^{*}=$ especies con sistemas de autoincompatibilidad, = reclutamiento sexual observado y/o evaluado en condiciones naturales

POL: $\mathbf{A b}=$ polinización abiótica, $\mathbf{B t}=$ polinización biótica

PROR: acd = acodaduras, agp = agamospermia, bul = bulbilos, buls = bulbilos subterráneos, $\mathbf{c h u}=$ coronas huecas, $\mathbf{d r m}=$ desprendimiento de ramas, $\mathbf{e t l}=$ estolones, $\mathbf{g m a s}=$ gemas subterráneas, $\mathbf{p n t}=$ plantlets, $\mathbf{p s b}=$ pseudobulbos, $\mathbf{r z m}=$ rizomas, $\mathbf{v b}=$ vástagos basales, $\mathbf{v r}=\mathbf{v a ́ s t a g o s}$ radicales, $\mathbf{\Delta}$ = crecimiento tipo guerrilla

CCT-N: $\mathbf{L g}=$ crecimiento clonal ligado, $\mathbf{N I}=$ crecimiento clonal no ligado

POB/HL: $\mathbf{p}=$ población, $\mathbf{h l}=$ hábitat local (p. ej., bosque con dosel abierto vs bosque con dosel cerrado)

PDE: los paréntesis indican que la población presenta una combinación de patrones de distribución de los tipos indicados por los números (ver figura 2).

AER: Ag = los ramets de cada genet no se entremezclan con los ramets de genets distintos, En = los ramets de diferentes genets en la población se entremezclan.

EGL: \# = evaluado en una población distinta de donde se determinó el PDE, asp = autocorrelación espacial, mpic = método de probabilidad de identidad clonal, $\mathbf{m t}=$ prueba de Mantel, $\mathbf{S G}=$ similitud genética vs distancia espacial.

Métodos: AFLP = marcadores moleculares AFLPs, CLO = marcadores moleculares de cloroplasto, desc = descripción detallada del PDE, ISSR = marcadores moleculares ISSRs, ISZ = isoenzimas, $\mathbf{m p i c}=$ método de probabilidad de identidad clonal, $\mathbf{m t}=$ prueba de $\mathbf{M a n t e l}, \mathbf{p o b}=$ muestra de individuos o todos los individuos de la población, prc = parcelas, RAPD = marcadores moleculares RAPDs, SG $=$ similitud genética vs distancia espacial, $\mathbf{S S R}=$ marcadores moleculares SSRs (microsatélites),

Referencias: ${ }^{*}=$ estudios donde se presentan planos de la distribución espacial de los genotipos.

Todo el cuadro: ? = no se proporciona información, $\mathbf{n} / \mathbf{e}=$ no evaluado, $\mathbf{n} / \mathbf{a}=$ no aplica

GLOSARIO (columna PROR):

Acodaduras: formación de ramets a partir de un tallo originalmente erecto que se postra y genera raíces adventicias al entrar en contacto con el sustrato

Agamospermia: Producción asexual de semillas o embriones sin fertilización (diplosporía: el embrión se desarrolla de una célula diploide del saco embrionario, aposporía: el embrión se desarrolla de una célula somática del saco embrionario, embrionia adventicia: el embrión se desarrolla de células del tejido esporofítico como los tegumentos).

Bulbilos: bulbos pequeños que se forman en las axilas foliares o unidos a los bulbos.

Bulbos: tallos cortos modificados para almacenamiento rodeados por hojas carnosas o brácteas.

Coronas huecas: muerte de los tallos centrales en plantas cespitosas resultando en la formación de ramets independientes en la periferia. Descrito en pastos cespitosos.

Estolones: tallo horizontal que se extiende en la superficie del suelo.

Gemas: pequeñas estructuras vegetativas dispersables en briofitas y pteridofitas.

Plantlets: propágulos vegetativos que resultan de la reversión de estructuras reproductivas como flores y frutos a estructuras vegetativas.

Pseudobulbos: modificación del tallo para almacenamiento que se desarrolla entre dos nodos foliares en orquídeas.

Rizomas: tallo horizontal subterráneo.

Vástagos basales y radicales: formación de tallos en la base de un tallo o a partir de una raíz.

Referencias del glosario: Jackson et al., 1985; de Kroon y van Groenendael, 1997; Liston et al., 2003; van Dijk y Bakx-Schotman, 2004; Tooke et al., 2005. 
independientes de cada genotipo o ramets independientes (Figura 1; Kays y Harper, 1974; Harper, 1985; Jackson et al., 1985). La clonalidad está ampliamente distribuida en plantas, algunas estimaciones para angiospermas varían entre 30\% y más del 50\% (Tiffney y Niklas, 1985; Klim s et al., 1997; Aarssen, 2008), entre el 40\% y $80 \%$ en especies de taiga, tundra y de zonas templadas (van Groenendael y de Kroon, 1990 Klim s et al., 1997) y el 44\% de plantas invasoras en China (Liu et al., 2006), está ampliamente representada en monocotiledóneas y se presenta con baja frecuencia en las gimnospermas (van Groenendael et al., 1996; Peterson y Jones, 1997). Respecto a las condiciones ambientales, se sugiere que la clonalidad predomina en condiciones estresantes como suelos pobres en nitrógeno, suelos húmedos, cuerpos de agua, hábitats sombreados y ambientes fríos en altitudes y latitudes elevadas (Tiffney y Niklas, 1985; van Groenendael et al., 1996; Klim s et al., 1997).

La clonalidad en plantas se ha estudiado con enfoques muy diversos. Por ejemplo, se ha analizado la frecuencia de especies clonales y la variación en las formas de crecimiento clonal en distintos ambientes (p. ej., Tiffney y Niklas, 1985; van Groenendael et al., 1996; Klims et al., 1997), las implicaciones de la integración fisiológica de los ramets y la plasticidad morfológica del crecimiento clonal en ambientes heterogéneos y en condiciones de estrés competitivo (p. ej., Bell, 1984; Hartnett y Bazzaz, 1985; de Kroon y Hutchings, 1995; Price y Marshall, 1999), el efecto de la clonalidad en la demografía (p. ej., Caswell, 1985; McFadden, 1991; Damman y Cain, 1998; Mandujano et al., 2001), la comparación de la diversidad genética entre especies de plantas clonales y no clonales (p. ej., Ellstrand y Roose, 1987; Widén et al., 1994) y las consecuencias reproductivas y evolutivas de la geitonogamia asociada con la clonalidad (p. ej., Handel, 1985; de Jong et al., 1992; Eckert, 2000).

Las implicaciones reproductivas y genéticas de reducir la entrecruza incrementando la geitonogamia han aumentado el interés en el estudio de los patrones de distribución espacial de genets y ramets en poblaciones de plantas clonales. Debido a que los propágulos vegetativos suelen tener una capacidad de dispersión restringida, el reclutamiento clonal frecuente puede reducir la entrecruza e incrementar la autocruza (p. ej., autogamia y geitonogamia), determinando los niveles de variación y la estructuración genética en las poblaciones (Handel, 1985; Eckert, 2002; Ruggiero et al., 2005; Honnay et al., 2006). En especies autocompatibles, los costos reproductivos de la geitonogamia están asociados al incremento de la autogamia y a las probabilidades de expresar depresión endogámica, a la vez que disminuye la adecuación paterna reduciendo la cantidad de polen disponible para la entrecruza; mientras que en las especies autoincompatibles, la geitonogamia afecta la producción de semillas, debido a la saturación de los estigmas con polen incompatible y al aborto de óvulos, y puede resultar en el desperdicio de grandes cantidades de polen (de Jong et al.,
1992; Charpentier, 2002).

En plantas clonales los patrones de distribución espacial pueden ser afectados por la manera en que se generan los ramets. Las formas de crecimiento clonal son muy diversas, pero existen algunas clasificaciones que facilitan el entendimiento de esta variación. Tiffney y Niklas (1985) propusieron cuatro tipos principales de crecimiento: (1) Crecimiento establecido, dado por el inicio del desarrollo de un organismo individual a partir de un propágulo (puede considerarse como crecimento clonal si el propágulo es vegetativo, ya que el propágulo también puede ser una semilla producida sexualmente). (2) Crecimiento restaurativo, que se refiere a la reparación de un daño local en el individuo mediante la formación de tejido calloso, pero no hay regeneración de los órganos completos cuando estos se pierden. (3) Crecimiento regenerativo, que se refiere a la reparación y el reemplazo de órganos dañados (p. ej., crecimiento de una rama desprendida). (4) Crecimiento reproductivo, dado por la multiplicación del individuo fisiológico (p. ej., clonalidad). Estos autores también ordenan los tipos de crecimiento clonal en ligado y no ligado, considerando las estructuras de conexión (Figura 1). En el crecimiento ligado los propágulos vegetativos pueden desarrollarse hasta formar individuos completos (con raíces, tallos y hojas) manteniéndose unidos a la planta madre y su dispersión depende del crecimiento de ramificaciones de tallos y raíces, mientras que en el no ligado, los propágulos vegetativos se desprenden de la planta madre y se desarrollan hasta individuos completos separados de la planta madre. La dispersión de los propágulos en este caso es más dependiente de factores bióticos (p. ej., animales) o abióticos (p. ej., corrientes de agua, gravedad, viento) que en el crecimiento ligado y permite un mayor desplazamiento de los propágulos. El crecimiento reproductivo o clonalidad, que es la que interesa en esta revisión por su influencia en el arreglo espacial de genets y ramets, puede ser de dos tipos: (i) reiteración traumática, definida como la producción de un nuevo individuo inducida por estrés, ya sea por daño o por condiciones ambientales que limitan la reproducción sexual (por ejemplo, la producción de bulbilos en los agaves por daño al escapo floral o por deficiencia de polen) y (ii) la reiteración programada, como la producción de un nuevo individuo por un meristemo como parte normal del desarrollo (Tiffney y Niklas, 1985; Arizaga y Ezcurra, 2002).

La distribución espacial de los individuos en las poblaciones se ha clasificado en tres patrones básicos: aleatorio, uniforme y agregado (Figura 2; Gleason, 1920; Clark y Evans, 1954; Barbour et al., 1987). En poblaciones naturales de plantas, los individuos suelen distribuirse en forma más o menos agregada, debido a la distribución heterogénea de los recursos (Couteron y Kokou, 1997; Chen y Bradshaw, 1999), a la interacción con otras especies (Shreve, 1917; Mandujano et al., 1998) y a la dispersión restringida de semillas y propágulos vegetativos (Gibson y Menges, 1994; Okuda et al., 1997; Clark-Tapia et al., 2005). En plantas 
clonales además, el arreglo espacial de los genets y ramets puede ordenarse en dos patrones básicos, uno caracterizado por la agregación de ramets del mismo genet y el otro por una distribución entremezclada de ramets de distintos genets (Figura 2; de Kroon y Hutchings, 1995; Charpentier, 2002; Clark-Tapia et al., 2005). Estos arreglos espaciales de genets y ramets pueden estar asociados a la manera en que se producen los ramets (esto es por crecimiento clonal ligado o no ligado) y se ha propuesto que en las plantas que se propagan mediante crecimiento no ligado forman arreglos más entremezclados que las especies con crecimiento ligado (Gabrielsen y Brochmann, 1998), pero esto no ha sido demostrado. Por otro lado, debido a que la clonalidad puede incrementar la tasa de geitonogamia entre los ramets del mismo genet, puede esperarse que los arreglos entremezclados reduzcan la geitonogamia y promuevan la entrecruza como resultado del incremento local en el número de genets (Charpentier, 2002).

En algunas revisiones sobre el tema de la clonalidad se han planteado hipótesis sobre las implicaciones reproduc-

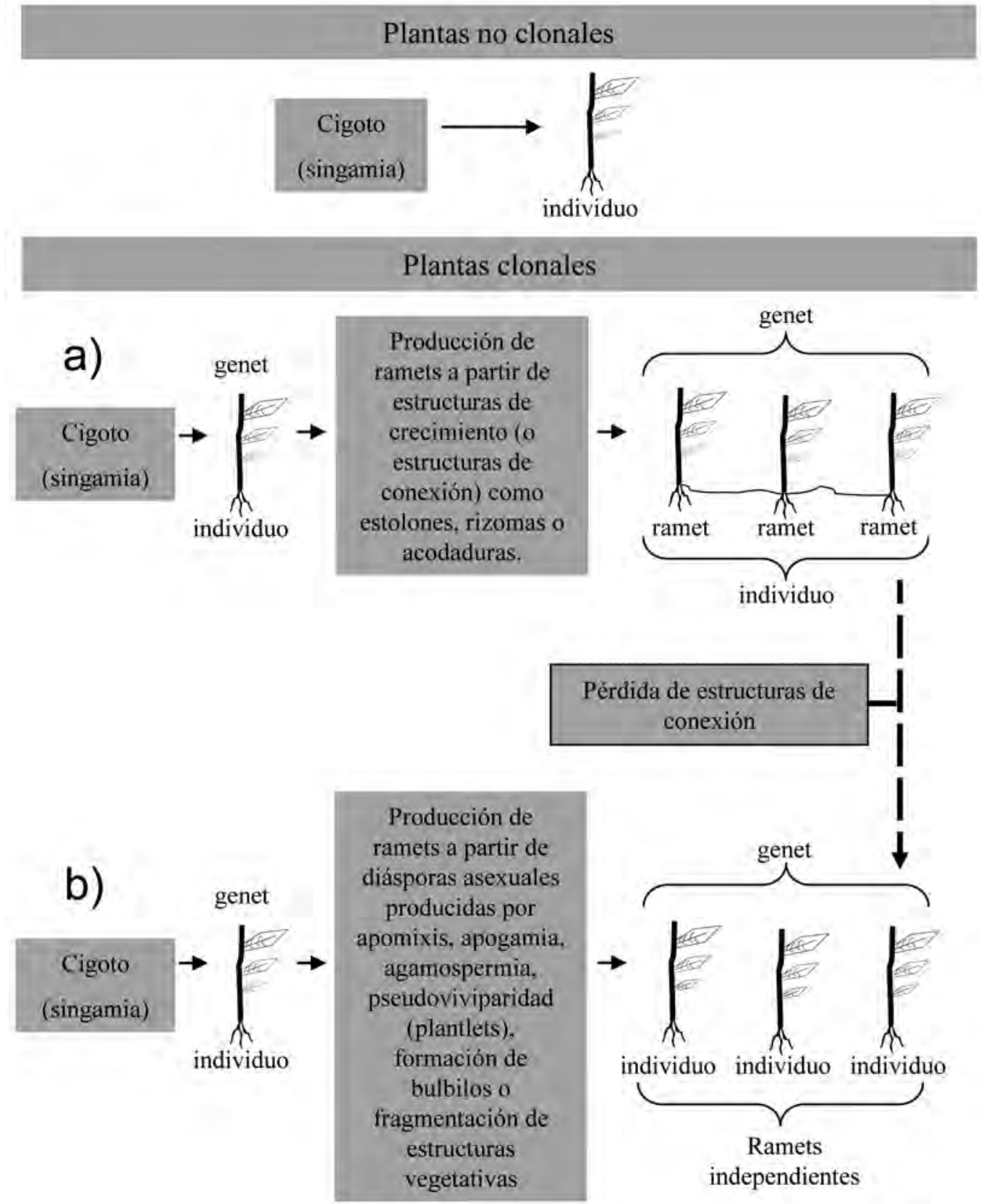

Figura 1. Formación de individuos en plantas no clonales y en plantas clonales. a) crecimiento clonal ligado y b) crecimiento clonal no ligado en la clasificación de Tiffney y Niklas (1985). 
$\mathrm{PDE}=$ Patrones de distribución espacial de los individuos y ramets en las poblaciones

(clonales y no clonales)
AER $=$ Arreglos espaciales de los ramets de distintos genets (clonales)

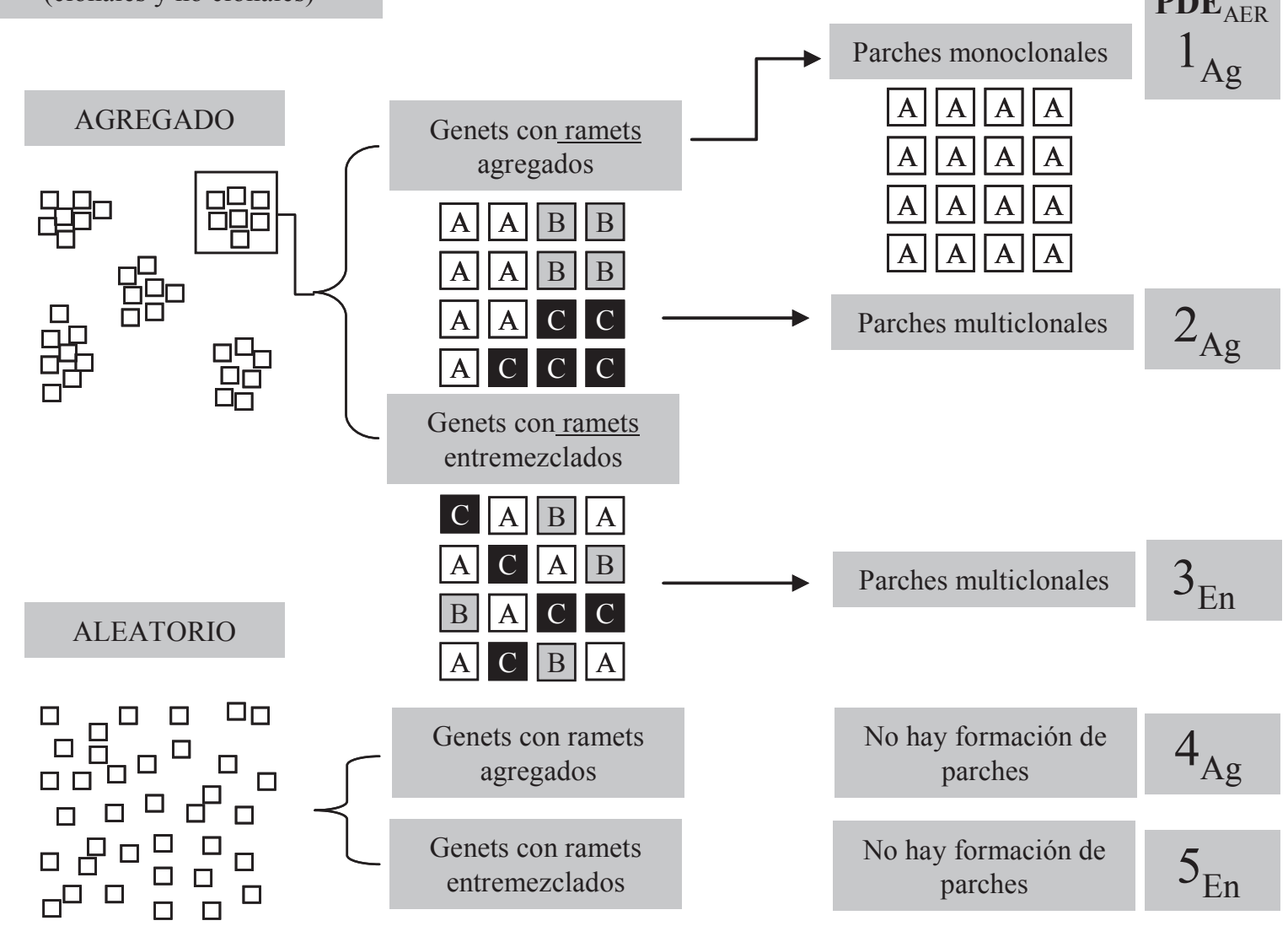

\section{UNIFORME}
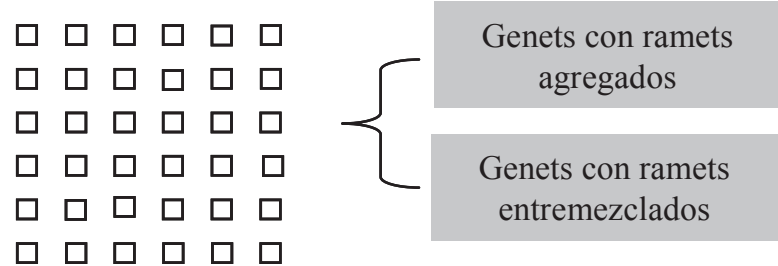

\begin{tabular}{|c|c|c|c|}
\hline $\begin{array}{c}\text { No hay formación de } \\
\text { parches }\end{array}$ & $6_{\mathrm{Ag}}$ \\
\hline $\begin{array}{c}\text { No hay formación de } \\
\text { parches }\end{array}$ & $7_{\mathrm{En}}$ \\
\hline
\end{tabular}

Figura 2. Patrones de distribución espacial de los individuos en las poblaciones de plantas no clonales y de plantas clonales. El número en el extremo derecho representa la clave del patrón de distribución espacial y el arreglo espacial de los ramets de distintos genets (PDE y AER, Apéndice). El subíndice Ag indica que los ramets de cada genet no se entremezclan con los ramets de genets distintos y el subíndice En indica que los ramets de diferentes genets se entremezclan.

tivas y genéticas de los arreglos espaciales de los genets y ramets, asociados con las distintas formas de producir descendientes clonales (Widén et al., 1994; Charpentier, 2002; Mandujano-Sánchez, 2007; Honnay y Jaquemyn, 2008). No obstante, los intentos para probar estas hipótesis son muy escasos y las posibilidades de realizar algún tipo de análisis numérico son restringidas por la baja disponibilidad de publicaciones (p. ej., Widén et al., 1994), o en los análisis sólo se incluyen especies con crecimiento ligado (p. ej., Honnay y Jaquemyn, 2008). En este trabajo presentamos una revisión de varios estudios que evalúan los patrones de distribución espacial en plantas clonales y a partir de la información 
proporcionada en dichos estudios, analizamos el planteamiento de que en poblaciones de plantas con crecimiento clonal ligado el arreglo espacial de los ramets de distintos genets es agregado, mientras que en poblaciones de plantas con crecimiento clonal no ligado predominan los arreglos espaciales entremezclados. Adicionalmente, intentamos poner a prueba la hipótesis de que los arreglos entremezclados promueven la entrecruza suponiendo que las poblaciones donde la entrecruza es más frecuente tenderán a presentar niveles más altos de diversidad genotípica.

\section{Material y métodos}

La revisión de los trabajos publicados sobre la distribución espacial clonal en plantas se llevó a cabo mediante una búsqueda de trabajos en bases de datos especializadas (CSA Cambridge, Ebsco HOST, ISI Web of Knowledge, JSTOR y Scopus) y no especializadas (Google, Google scholar y Yahoo). La búsqueda se hizo empleando los siguientes términos: clonal structure, spatial distribution of genets (andl or ramets), clonal architecture y clonal diversity y abarcó de 1990 a 2011, debido a que la mayoría de los estudios en plantas clonales con este enfoque se han realizado después de 1990.

Los criterios principales para incluir una especie en la revisión fue que se presentara una descripción de la distribución espacial de las plantas individuales (esto es sin importar el genotipo) en las poblaciones naturales y que se evaluara el arreglo espacial de los genets y los ramets (es decir agregado o entremezclado) mediante planos de distribución de los genotipos, análisis de autocorrelación espacial o compatibilidad de las cruzas entre ramets contiguos. Para cada especie se obtuvo el tipo de estructuras vegetativas a mediante las cuales se lleva a cabo la propagación clonal a partir de la información proporcionada en los estudios correspondientes o en estudios previos realizados a menudo por los mismos autores. El segundo criterio en orden de importancia fue que se reportaran valores de diversidad genotípica ( $D=$ índice de diversidad de Simpson y proporción de genotipos distinguibles $G / N$ ) para las poblaciones de las diferentes especies. Otros criterios fueron que se proporcionara información sobre las características reproductivas como la presencia de sistemas de incompatibilidad, la expresión sexual, si se ha observado reclutamiento sexual en las poblaciones y el tipo de polinización. Con base en esta información, se asignó a las especies el tipo de crecimiento clonal (i. e. ligado y no ligado, Figura 1) de acuerdo con la clasificación de Tiffney y Niklas (1985) y el patrón de distribución espacial y el tipo de arreglo espacial de los ramets (agregado o entremezclado) según el esquema presentado en la Figura 2. La información obtenida se presenta en el apéndice.

Para analizar el planteamiento de que en poblaciones de plantas con crecimiento clonal ligado el arreglo espacial de los ramets es agregado, mientras que en poblaciones de plantas con crecimiento clonal no ligado predomina el arreglo espacial entremezclado se construyó una tabla de contingencia con tres categorías de tipo de crecimiento clonal como columnas (ligado, combinación ligado-no ligado y no ligado) y tres categorías del arreglo espacial de los ramets como renglones (agregado, combinación agregado-entremezclado y entremezclado) y se analizó con una prueba de ${ }^{2}$ con el paquete estadístico JMP versión 7.0 (SAS Institute Inc). Para evaluar las celdas que contribuyen significativamente al valor de ${ }^{2}$ se hizo un análisis de residuales ajustados (Everitt, 1977).

Para probar la hipótesis de que las poblaciones con arreglos entremezclados de ramets presentan niveles más altos de diversidad genotípica, se compararon los valores del índice de diversidad de Simpson $(D)$ y la proporción de genotipos distinguibles $(G / N)$ entre las tres categorías de patrón de distribución clonal y entre las tres categorías de tipo de crecimiento clonal con una prueba de Kruskall-Wallis con el paquete estadístico JMP versión 7.0 (SAS Institute Inc).

\section{Resultados}

En la revisión se incluyeron 53 estudios que comprenden 122 poblaciones distribuidas en 44 especies de plantas clonales y en 27 familias (Apéndice). Las familias mejor representadas son Liliaceae y Rosaceae con cuatro especies cada una; Cactaceae, Cyperaceae, Ericaceae, Poaceae y Salicaceae con tres especies cada una y Zosteraceae con dos especies. El resto de las familias estuvieron representadas por una sola especie. Las especies que predominan son herbáceas perennes $(60 \%)$, y las plantas con hábito arbustivo y arbóreo se encuentran poco representadas (20\% en ambos casos).

La mayoría de las especies presentan crecimiento clonal ligado (70\%), dominando la propagación a través de rizomas (45\% de las especies). Las especies con crecimiento clonal ligado y no ligado representan el 14\% propagándose clonalmente por crecimiento ligado a través de rizomas y acodaduras, y por crecimiento no ligado a través de bulbilos, plantlets y desprendimiento de ramas; las especies sólo con crecimiento no ligado representan el 16\%, propagándose por bulbilos, semillas producidas por agamospermia, desprendimiento de ramas y gémulas (ver glosario para la definición de las estructuras implicadas en el crecimiento ligado y no ligado, Apéndice). Respecto a la distribución espacial, $50 \%$ del total de las especies presentan genets con ramets agregados, $30 \%$ presentan una combinación de arreglos agregados-entremezclados y $20 \%$ presentan arreglos entremezclados (Apéndice).

$\mathrm{Al}$ comparar las poblaciones con diferentes arreglos espaciales de genets y ramets [agregados (Ag), En (entremezcaldos) y $\mathrm{Ag}+\mathrm{En}$ (combinados)] encontramos que las poblaciones de especies con crecimiento ligado y no ligado y 
Cuadro 1. Número (observado y esperado) de poblaciones con diferentes tipos de crecimiento clonal de acuerdo con la clasificación de Tiffney y Niklas (1985) ( $\mathrm{Lg}=$ ligado, Lg+NI = ligado y no ligado, $\mathrm{Nl}=$ no ligado), que mostraron arreglos espaciales de los ramets de distintos genets: Ag = agregados, Ag+En = combinación de agregados y entremezclados, y En = entremezclados (figura 2). Los valores entre paréntesis indican el número de especies y familias (especies/familias) representadas en cada celda. Los valores de los residuales ajustados en negritas indican las celdas que contribuyen significativamente al valor de $\chi^{2}$, el signo indica mayor (sin signo) o menor número (-) de poblaciones de las esperadas por azar.

\begin{tabular}{|c|c|c|c|}
\hline Observados & Ligado & Ligado+No ligado & No ligado \\
\hline Agregado & $49(21 / 13)$ & $7(2 / 2)$ & $4(2 / 2)$ \\
\hline Agregado+entremezclado & $11(5 / 5)$ & $25(5 / 5)$ & $7(3 / 3)$ \\
\hline Entremezclado & $10(5 / 5)$ & $2(2 / 1)$ & $7(2 / 2)$ \\
\hline \multicolumn{4}{|l|}{ Esperados } \\
\hline & $\operatorname{Lg}$ & $\mathrm{Lg}+\mathrm{NI}$ & $\mathrm{Nl}$ \\
\hline Agregado & 34 & 17 & 9 \\
\hline Agregado+entremezclado & 25 & 12 & 6 \\
\hline Entremezclado & 11 & 5 & 3 \\
\hline \multicolumn{4}{|l|}{ Residuales ajustados } \\
\hline & $\operatorname{Lg}$ & $\mathrm{Lg}+\mathrm{NI}$ & $\mathrm{Nl}$ \\
\hline Agregado & 5.34 & -3.93 & -2.48 \\
\hline Agregado+entremezclado & -5.24 & 5.50 & 0.35 \\
\hline Entremezclado & -0.46 & -1.83 & 2.95 \\
\hline
\end{tabular}

de especies que sólo presentan crecimiento no ligado presentaron arreglos espaciales (AER) más entremezclados que las poblaciones de especies que sólo presentan crecimiento ligado ( ${ }^{2}=43.6, P<0.0001,4$ g.l.; Cuadro 1). Predominan los patrones de distribución (PDE) con ramets distribuidos en parches discretos sobre el área ocupada por la población (patrón de distribución espacial agregado, $46 \%$ de las poblaciones); estos parches son multiclonales (con dos o más genets) en la mayoría de los casos y el arreglo espacial de los ramets de distintos genets es agregado (19\% de las poblaciones, $\mathrm{PDE}_{\mathrm{AER}} 2_{\mathrm{Ag}}$, Figura 2), presenta una combinación agregado-entremezclado (10\% de las poblaciones, combinación $\mathrm{PDE}_{\mathrm{AER}} 2_{\mathrm{Ag}}-3_{\mathrm{En}}$, Figura 2) o es entremezclado solamente (3\% de las poblaciones, $\mathrm{PDE}_{\mathrm{AER}} 3_{\mathrm{En}}$, Figura 2). En otras poblaciones donde los ramets se distribuyen formando parches discretos el arreglo espacial de los ramets de distintos genets es agregado, pero los parches son multiclonales y monclonales (9\% de las poblaciones, combinación $\mathrm{PDE}_{\mathrm{AER}}$ $1_{\mathrm{Ag}}-\mathrm{Ag}_{\mathrm{Ag}}$, Figura 2) o sólo son monoclonales (5\% de las poblaciones, $\mathrm{PDE}_{\mathrm{AER}} 1_{\mathrm{Ag}}$, Figura 2).

Los patrones de distribución espacial con ramets distribuidos aleatoriamente sobre el área ocupada por la población (patrón de distribución espacial aleatorio) representaron el $44 \%$ de las poblaciones, predominando el arreglo espacial agregado-entremezclado de los ramets de distintos genets $\left(22 \%\right.$ de las poblaciones, combinación de $\mathrm{PDE}_{\mathrm{AER}}$ $4_{\mathrm{Ag}}-5_{\mathrm{En}}$, Figura 2), el arreglo agregado se presentó en $10 \%$ de las poblaciones ( $\mathrm{PDE}_{\mathrm{AER}} 4_{\mathrm{Ag}}$, Figura 2) y el arreglo entremezclado en $12 \%$ de las poblaciones $\left(\mathrm{PDE}_{\mathrm{AER}} 5_{\mathrm{En}}\right.$, Figura
2). El resto de las poblaciones (10\%) presentaron una combinación de patrones de distribución con ramets distribuidos aleatoriamente sobre una parte del área ocupada por la población y en otra formando parches discretos monoclonales o parches multiclonales con arreglos espaciales agregados y entremezclado de los ramets de distintos genets (combinación de $\mathrm{PDE}_{\mathrm{AER}} 1_{\mathrm{Ag}}-4_{\mathrm{Ag}}, 1_{\mathrm{Ag}}-5_{\mathrm{En}}, 2_{\mathrm{Ag}}-4_{\mathrm{Ag}}, 2_{\mathrm{Ag}}-5_{\mathrm{En}}$, Figura 2).

Las poblaciones de especies con una combinación de tipos de crecimiento clonal ligado y no ligado $(\mathrm{Lg}-\mathrm{Nl})$ presentaron valores más bajos de la proporción de genotipos distinguibles (promedio \pm E.E., $G / N=0.24 \pm 0.04 ; H=$ 6.93, $P=0.031,2$ g.l.) que las poblaciones de especies con crecimiento ligado (promedio \pm E.E., $G / N=0.38 \pm 0.03$ ) y con crecimiento no ligado (promedio \pm E.E., $G / N=0.39 \pm$ 0.06 ), pero las diferencias sólo fueron significativas con respecto a las poblaciones de especies con crecimiento ligado (Figura 3a). Respecto al arreglo espacial de los ramets de distintos genets, las poblaciones con una combinación de arreglos agregado y entremezclado (Ag-En) presentaron valores menores del índice de diversidad de Simpson (promedio \pm E.E., $D=0.57 \pm 0.05 ; H=13.2, P=0.0014,2$ g.l.) que las poblaciones con arreglos agregados (promedio \pm E.E., $D=0.80 \pm 0.04$ ) y con arreglos entremezclados (promedio \pm E.E., $D=0.76 \pm 0.06$; Figura 3). Estas diferencias sugieren que el reclutamiento sexual es más bajo en poblaciones de especies que presentan una combinación de formas de crecimiento clonal ligado-no ligado ( $\mathrm{Lg}-\mathrm{Nl}$ ) y una combinación de arreglos de los ramets de distintos genets agregado-entremezclado (Ag-En), sin embargo estas diferencias 

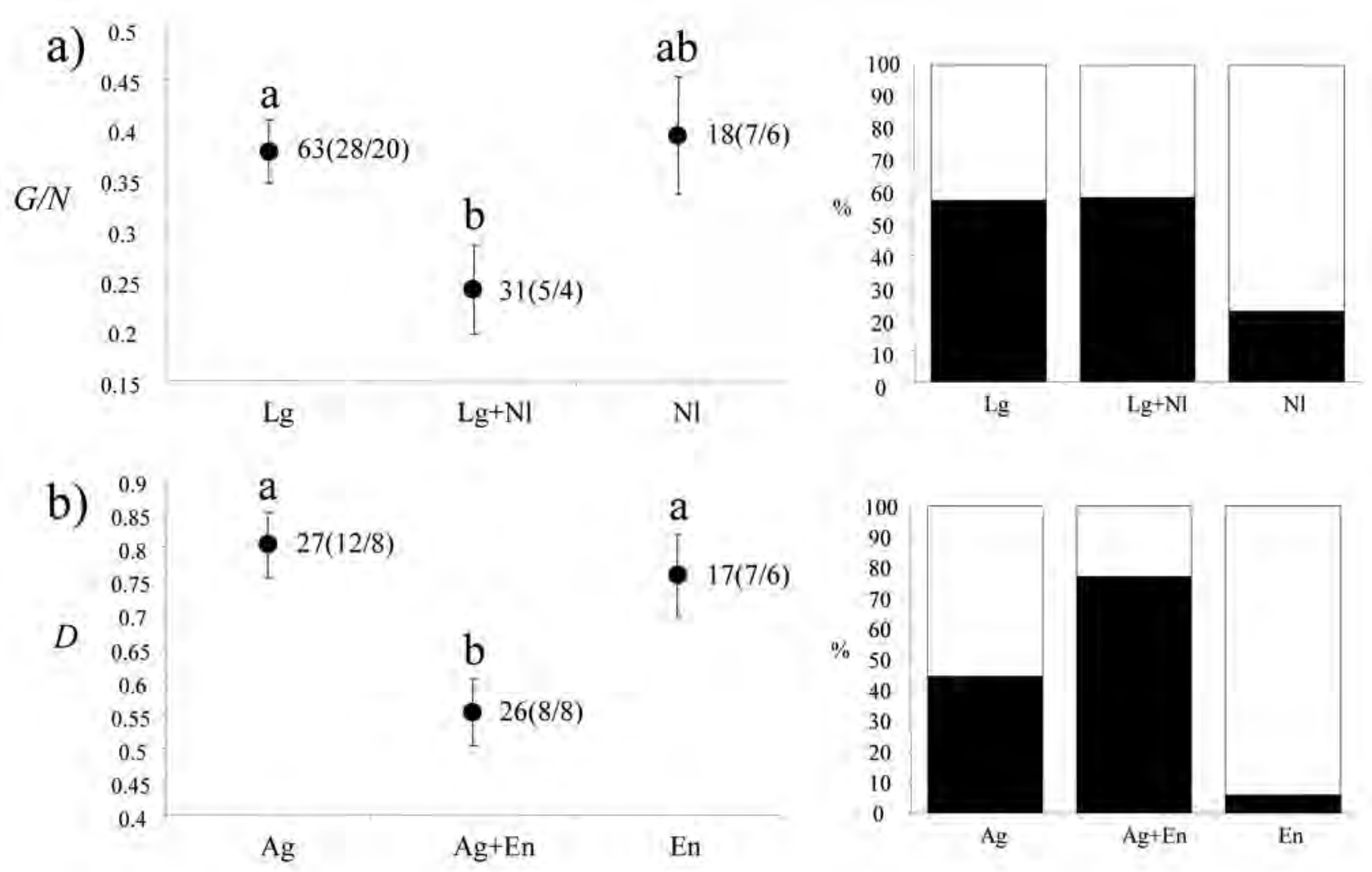

Figura 3. Comparación de la diversidad clonal (promedio \pm EE) entre poblaciones de especies con a) distinto tipo de crecimiento clonal: $\mathrm{Lg}=$ ligado, $\mathrm{Lg}+\mathrm{Nl}$ = con ambos tipos de crecimiento y $\mathrm{Nl}$ = no ligado y b) con distintos arreglos espaciales de los ramets de distintos genets: $\mathrm{Ag}=$ agregados, $\mathrm{Ag}+\mathrm{En}$ = combinación de agregados y entremezclados, y En = entremezclados (Figura 2). En cada grupo se indica el número de poblaciones (número de especies / número de familias) y los grupos que difieren significativamente se indican con una letra distinta arriba de la barra del promedio \pm E.E. de cada grupo. Las gráficas de barras del lado derecho muestran el porcentaje de poblaciones con polinización abiótica (en negro) y con polinización biótica (en blanco).

pueden estar asociadas con otros factores como el tipo de polinización, y no con el tipo de crecimiento y el arreglo espacial de los ramets de distintos genets. La mayoría de las poblaciones con tipo de crecimiento $\mathrm{Lg}-\mathrm{Nl}$ y arreglos de ramets de distintos genets Ag-En corresponden a especies con polinización abiótica (Figura 3).

\section{Discusión}

El efecto que tienen los modos de clonalidad en las plantas en la conformación de distintos arreglos espaciales de genets y ramets es un campo relativamente novedoso, que se ha explorado en los últimos 20 años y la mayoría de los estudios que hay sobre el tema se han desarrollado después de 1990. Widén et al. (1994) reportan que de 40 estudios que comprenden un total de 45 especies de plantas clonales, menos de la tercera parte proporcionan información sobre el arreglo espacial de los genets en las poblaciones y sólo en $12.5 \%$ se construyeron planos de distribución de todos los ramets, dentro de las parcelas o poblaciones, identificando los genotipos multilocus. En esta revisión, en el $98 \%$ de los estudios incluidos se identifican los genotipos multilocus mediante marcadores dominantes, codominantes o ambos siguiendo tres métodos de muestreo: en el 56\% de los estudios se utilizan parcelas, en el $24 \%$ transectos y en el $18 \%$ se muestrean todos o varios individuos de las poblaciones. El 2\% restante está representado por un estudio donde el patrón de distribución es descrito y la distribución de los ramets se sugiere por experimentos de cruzas compatibles entre ramets cercanos (en Scirpus maritimus, Charpentier et al., 2000).

A pesar de que en la actualidad se cuenta con más información sobre los patrones de distribución espacial en poblaciones de especies clonales, hacer comparaciones de dichos patrones considerando especies con distintas formas de crecimiento clonal para analizar las consecuencias reproductivas y genéticas del arreglo espacial de los genets y los ramets continúa siendo un reto. Algunos factores que influyen en este hecho son que la evaluación de la variación en los patrones de distribución espacial en plantas clonales 
enfrenta la dificultad que implica trabajar con la mayoría de los individuos en muchas poblaciones, principalmente a nivel molecular. Aunque en la actualidad las herramientas moleculares se han vuelto más accesibles, trabajar con un gran número de ejemplares sigue siendo difícil y costoso. Otros problemas son la falta de estimadores numéricos comparables para describir la distribución espacial en las poblaciones de diferentes especies y la variación en los métodos de muestreo. Por ejemplo, el muestreo mediante transectos colectando muestras de ramets cada determinada distancia (p. ej., Jonsson et al., 1996; Esselman et al., 1999) permite evaluar la capacidad de expansión longitudinal de los genets, pero no permite tener una descripción de la expansión radial y por lo tanto una evaluación más precisa del nivel de entremezcla de los ramets de distintos genets. En contraste, el muestreo de todos o de un gran número de individuos dentro de parcelas o en las áreas ocupadas por las poblaciones es otro método empleado en estudios con especies de plantas clonales (p. ej., Shimizu et al., 2002; Ruggieiro et al., 2005). Este tipo de muestreo puede ser un método más adecuado para analizar y comparar la estructura espacial clonal, porque permite detectar el arreglo radial de distintos genets. Sin embargo, los tamaños de muestra muy grandes pueden limitar la aplicación de este método, ya que en la mayoría de los casos es necesario emplear marcadores moleculares para identificar a los genets.

En particular, además de los problemas mencionados anteriormente es importante considerar las dificultades adicionales que detectamos en este estudio y que pueden afectar los patrones encontrados a través de nuestra aproximación. La mayoría de los estudios que analizan la distribución espacial de genets y ramets parecen estar sesgados hacia especies de herbáceas perennes con crecimiento clonal ligado (45\% de las especies), predominando las especies rizomatosas (43\% de las especies), mientras que las especies con crecimiento no ligado están poco representadas, impidiendo contar con tamaños de muestra equivalentes para hacer comparaciones más precisas. Por otro lado, la aproximación que decidimos emplear en este trabajo compara a las poblaciones más que a las especies, debido a que hemos detectado que hay una importante variación intraespecífica tanto en los patrones de distribución como en los patrones reproductivos. No obstante, esta variación se omite en algunos estudios o está mejor representada para algunas especies.

Los resultados obtenidos en este trabajo sugieren que las especies que se propagan por crecimento no ligado tienden a formar arreglos espaciales de los ramets de distintos genets más entremezclados como se ha planteado (Gabrielsen y Brochmann, 1998). No obstante, en especies con crecimiento ligado que además se propagan clonalmente por crecimiento no ligado, como el desprendimiento de ramas, el cual consideramos en este estudio como una forma de crecimiento no ligado en los análisis, debido a que los ramets originados por este medio se desarrollan hasta individuos com- pletos separados de la planta madre y a que su dispersión no depende del crecimiento horizontal de tallos y raíces (p.ej., Lophocereus schottii, Parker y Hamrick, 1992; Pteridium aquilinum, Parks y Werth, 1993; Cladium jamaicense, Ivey y Richards, 2001; Ferocactus robustus, Carrillo-Angeles et al., 2011), o que se propagan por la extensión de rizomas en especies con crecimiento en guerrilla (p. ej., Cymodocea nodosa, Ruggieiro et al., 2005 e Ilex leucoclada, Torimaru y Tomaru, 2005) también forman arreglos de genets entremezclados. Algunos factores externos que pueden influir en el arreglo entremezclado de genets son la dispersión por actividades humanas (Parks y Werth, 1993; Brzosko et al., 2002), el acarreo de propágulos vegetativos por animales y corrientes de agua (Parker y Hamrick, 1992; Brzosko et al., 2002), el reclutamiento de nuevos genets en áreas perturbadas donde permanecen fragmentos de clones que ocuparon grandes extensiones (Parks y Werth, 1993) y la dispersión de semillas producidas por autogamia que presentan genotipos multilocus idénticos (Keane et al., 1999; Brzosko et al., 2002).

Respecto a la hipótesis de que las poblaciones con arreglos espaciales entremezclados de los ramets de distintos genets presentan mayor entrecruza, medido indirectamente mediante la diversidad genotípica, nuestros resultados concuerdan parcialmente con el patrón esperado. El G/N es significativamente mayor en los arreglos de especies con producción de vástagos no ligados, en donde predominan los arreglos de genets entremezclados. Para probar esta hipótesis consideramos que si la entrecruza es más alta en poblaciones con arreglos entremezclados, habría mayores niveles de diversidad genotípica. Sin embargo, ésto no se refleja en el caso del índice de Simpson. Las diferencias en los índices de diversidad genotípica entre las poblaciones de especies que combinan el crecimiento clonal ligado-no ligado ( $\mathrm{Lg}$ $\mathrm{Nl})$ y el arreglo espacial agregado-entremezclado (Ag-En), respecto de las poblaciones que presentan uno u otro tipo de crecimiento y de arreglo espacial están asociadas con el tipo de polinización (biótica o abiótica), lo cual apoya el planteamiento de que la polinización biótica puede incrementar la eficiencia de la fertilización (Barret et al.,1993; Richards, 1997; Reusch, 2003). Los análisis para probar este planteamiento están fuera del alcance del presente estudio.

Además de los efectos sobre el reclutamiento sexual y la diversidad genotípica, los patrones de distribución espacial en especies clonales pueden afectar la diversidad y la estructura genética de las poblaciones. Por ejemplo, la formación de parches monoclonales en especies con entrecruza obligada, aunada al desplazamiento de genets por competencia, puede eliminar la capacidad de una población para reproducirse sexualmente en tiempos relativamente cortos (Honnay y Bossuyt, 2005). Adicionalmente, una reducción del reclutamiento sexual y una dispersión restringida de polen, semillas y propágulos vegetativos pueden generar estructura genética local en las poblaciones. Este segundo aspecto se 
evalúa en varios de los estudios revisados (47\%). La estructura genética local tanto a nivel de genets (indicando una dispersión restringida de polen, semillas o ambos) como de ramets (indicando dispersión restringida de propágulos vegetativos) se detectó en 46 poblaciones distribuidas en 16 especies; 28 poblaciones corresponden a cinco especies arbóreas que muestran estructura genética local a distancias menores de $50 \mathrm{~m}$ (Sequoia sempervirens, Douhovnikoff et al., 2004) y a distancias menores de $25 \mathrm{~m}$ (Eurya emarginata, Chung y Epperson, 2000; Cryptomeria japonica, Shimizu et al., 2002; Hirayama y Sakimoto, 2008; Populus tremula, Suvanto y Latva-Karjantvaa, 2005); tres poblaciones corresponden a dos especies arbustivas (Echinosophora koreensis, Chung et al., 2006; Stenocereus eruca, ClarkTapia et al., 2005) que presentan estructura genética local a distancias menores de $20 \mathrm{~m}$. El resto de las poblaciones corresponden a hierbas perennes que muestran estructura genética local desde distancias menores de $30 \mathrm{~m}$, pero más frecuentemente a distancias menores de $15 \mathrm{~m}$.

En promedio, los valores del índice de diversidad de Simpson $(D)$ y la proporción de genotipos distinguibles $(G / N)$ fueron altos en las poblaciones representadas en los estudios incluidos en la presente revisión y son similares a los reportados para 21 especies de plantas clonales $(D=$ 0.62 y $G / N=0.16$ ) por Ellstrand y Roose (1987). Se han planteado algunas hipótesis sobre los escenarios que pueden explicar altos niveles de diversidad genotípica: (i) las poblaciones pudieron ser fundadas por varios propágulos sexuales que se mantienen por clonalidad, (ii) el reclutamiento sexual fue más frecuente en el pasado, aunque en la actualidad la producción de descendientes sexuales no sea evidente (Ellstrand y Roose, 1987; Eriksson, 1989, 1993; Widén et al., 1994); (iii) los genotipos heterócigos se ven favorecidos selectivamente, manteniendo altos niveles de diversidad genética (Widén et al., 1994); (iv) los riesgos de mortalidad entre los ramets de un genet pueden ser distintos si tienen independencia física y fisiológica (Eriksson 1989, 1993, Pan y Price, 2002), lo cual puede reducir las probabilidades de supervivencia / extinción del genet; (v) en especies con ciclos de vida largos los eventos raros de reclutamiento sexual pueden ser suficientes para mantener niveles altos de diversidad genética (Eriksson 1989, 1993, Parker y Hamrick, 1992; Brzosko et al., 2002; Clark-Tapia et al., 2005); (vi) los genotipos pueden mantenerse a través de la supervivencia de algunos ramets el tiempo suficiente para que algunos genotipos se integren a la población de manera esporádica; (vii) la heterogeneidad ambiental puede generar condiciones de selección diversificante que permiten la coexistencia de varios genotipos (Widén et al., 1994). Otra posibilidad para el mantenimiento de diversidad clonal en poblaciones de plantas, que surge al considerar la variación de la distribución espacial de genets y ramets, es que la clonalidad puede mantener altos niveles de entrecruza e incrementar las probabilidades de reclutamiento sexual si los ramets se dispersan hacia vecindarios genéticamente variables, debido a la distribución entremezclada de genets.

Las explicaciones a los niveles relativamente altos de diversidad genotípica en las poblaciones de las especies incluidas en esta revisión comprenden los eventos esporádicos de reclutamiento sexual y el establecimiento inicial de varios genets. En algunos casos se sugiere un reclutamiento sexual frecuente a partir de los altos niveles de diversidad genotípica detectados con los marcadores moleculares (p. ej., Parks y Werth, 1993; Diggle et al., 1998; Misuki et al., 2010). No obstante, el reclutamiento sexual en poblaciones naturales sólo se reporta en Prunus ssiori, Rubus saxatilis (Eriksson y Bremer, 1993), Cypripedium calceolus (Brzosko et al., 2002) y Maianthemum dilatatum (Wilson et al., 2005b). Otros factores que se proponen para explicar la diversidad genotípica son que la integración fisiológica entre los ramets de un genet puede reducir el riesgo de que un genotipo en particular desaparezca, cuando las condiciones ambientales locales son adversas, contribuyendo a mantener la diversidad genética (Hartnett y Bazzaz, 1985), el incremento en la tasa de entrecruza, debido a la entremezcla de genets en especies con crecimiento en guerrilla (Ruggieiro et al., 2005) y en especies que se propagan clonalmente por desprendimiento de ramas y producción de bulbilos (Parker y Hamrick, 1992; Gabrielsen y Brochmann, 1998).

El estudio de los patrones de distribución espacial en especies clonales es importante para analizar las consecuencias reproductivas y genéticas del arreglo espacial de los genets y los ramets en las poblaciones naturales. Estas consecuencias pueden explorarse de una manera más precisa analizando la variación de la distribución de genets y ramets entre las poblaciones de una misma especie. Sin embargo, la evidencia de estos aspectos en organismos clonales aún es escasa y hace falta realizar estudios considerando diferentes tipos de crecimiento clonal. El análisis de los patrones de distribución espacial pueden ser muy útiles para evaluar otros aspectos del ciclo de vida de las especies clonales como el efecto de una variación persistente en la producción de propágulos clonales y sexuales entre hábitats, que puede afectar diferencialmente la dinámica de las poblaciones. Asimismo, es necesario analizar la dinámica temporal de los arreglos espaciales de los genets y ramets en las poblaciones para hacer inferencias adecuadas sobre las consecuencias ecológicas y evolutivas de la clonalidad.

\section{Agradecimientos}

Proyectos: IN IN205007 Efecto de la estructura clonal sobre la dinámica poblacional y la reproducción de una cactácea con ciclo de vida complejo, Opuntia microdasys.

Becas: doctorado Conacyt a I. Carrillo-Angeles y estancia sabática en New Mexico State University, otorgada por DGAPA-PASPA- Universidad Nacional Autónoma de México a M. Mandujano. Este trabajo es parte de la tesis de 
doctorado de Israel Gustavo Carrillo Angeles, Posgrado en Ciencias Biológicas de la UNAM. Agradecemos la revisión, sugerencias en el trabajo así como el apoyo de los miembros del comité tutoral, Doctores Teresa Valverde y Francisco Molina. Asimismo a la Dra. Ana Mendoza, Dr. Jordan Golubov y dos revisores anónimos por sus sugerencias al artículo.

\section{Literatura citada}

Aarssen L.W. 2008. Death without sex-the 'problem of the small' and selection for reproductive economy in flowering plants. Evolutionary Ecology 22:279-298.

Albert T., Raspé O. y Jacquemart A.L. 2003. Clonal structure in Vaccinium myrtillus L. revealed by RAPD and AFLP markers. International Journal of Plant Sciences 164:649-655.

Arens P., Grashof-Bokdam C.J., van der Sluis T. y Smulders M.J.M. 2005. Clonal diversity and genetic differentiation of Maianthemum bifolium among forest fragments of different age. Plant Ecology 179:169-180.

Arizaga S. y Ezcurra E. 2002. Propagation mechanisms in Agave macrovantha (Agavaceae), a tropical arid land succulent rosette. American Journal of Botany 89:632-641.

Barbour M.G., Burk J.H. y Pitts W.D. 1987. Terrestrial Plant Ecology. The Benjamin-Cummings Publishing Company, Menlo Park.

Barret S.C.H., Eckert C.G. y Husband B.C. 1993. Evolutionary processes in aquatic plant populations. Aquatic Botany 44:105-145.

Bell A.D. 1984. Dynamic morphology: A contribution to plant population ecology. En: Dirzo R. y Sarukhán J. Eds. Perspectives on Plant Population Ecology, pp. 49-65, Sinauer Associates Inc. Publisher, Sunderland.

Brzosko E., Wróblewska A. y Ratkiewicz M. 2002. Spatial genetic structure and clonal diversity of island populations of lady's slipper (Cyperidium calceolus) from the Biebrza National Park (northeast Poland). Molecular Ecology 11:2499-2509.

Bushakra J.M., Hodges S.A., Cooper J.B. y Kaska D.D. 1999. The extent of clonality and genetic diversity in the Santa Cruz Island ironwood, Lyonothamnus floribundus. Molecular Ecology 8:471-475.

Camacho F.J. y Liston A. 2001. Population structure and genetic diversity of Botrychium pumicola (Ophioglossaceae) based on inter-simple sequence repeats (ISSR). American Journal of Botany 88:1065-1070.

Carrillo-Angeles I.G., Mandujano M.C. y Golubov J. 2011. Influences of the genetic neighborhood on ramet reproductive success in a clonal desert cactus. Population Ecology 53:449458.

Caswell H. 1985. The evolutionary demography of clonal reproduction. En: Jackson J.B.C., Buss L.W. y Cook R.E. Eds. Population Biology and Evolution of Clonal Organisms, pp. 187224, Yale University Press, New Haven.

Charpentier A. 2002. Consequences of clonal growth for plant mating. Evolutionary Ecology 15:521-530.

Charpentier A., Grillas P. y Thompson J.D. 2000. The effects of population size limitation on fecundity in mosaic populations of the clonal macrophyte Scirpus maritimus (Cyperaceae). American Journal of Botany 87:502-507.

Chen J. y Bradshaw G.A. 1999. Forest structure in space: a case study of an old growth spruce-fir forest in Changbaishan Natural Reserve, PR China. Forest Ecology and Management 120:219-233.

Chung M.G. y Epperson B.K. 2000. Clonal and spatial genetic structure in Eurya emarginata (Theaceae). Heredity 84:170177.

Chung J.M., Lee B.C., Kim J.S., Park C., Chung M.Y. y Chung M.G. 2006. Fine-scale genetic structure among genetic individuals of the clone-forming monotypic genus Echinospora koreensis (Fabaceae). Annals of Botany 98:165-173.

Clark P.J. y Evans F.C. 1954. Distance to nearest neighbor as a measure of spatial relationships in populations. Ecology 35:445-453.

Clark-Tapia R., Alfonso-Corrado C., Eguiarte L.E. y Molina-Freaner F. 2005. Clonal diversity and distribution in Stenocereus eruca (Cactaceae), a narrow endemic cactus of the Sonoran Desert. American Journal of Botany 92:272-278.

Clark-Tapia R., Alfonso-Corrado C., Mandujano M.C. y MolinaFreaner F. 2006. Reproductive consequences of clonal growth in Stenocereus eruca, a rare clonal cactus of the Sonoran desert. Evolutionary Ecology 20:131-142.

Couteron P. y Kokou K. 1997. Woody vegetation spatial patterns in a semi-arid savanna of Burkina Faso, West Africa. Plant Ecology 132:211-227.

Damman H. y Cain M.L. 1998. Population growth and viability analyses of the clonal woodland herb, Asarum canadense. Journal of Ecology 86:13-26.

de Jong T.J., Waser N.M., Price M.V. y Ring R.M. 1992. Plant size, geitonogamy and seed set in Ipomopsis aggregata. Oecologia 89:310-315.

de Kroon H. y van Groenendael J. Eds. 1997. The Ecology and Evolution of Clonal Plants. Backhuys Publishers, Leiden.

de Kroon H. y Hutchings M.J. 1995. Morphological plasticity in clonal plants: the foraging concept reconsidered. Journal of Ecology 83:143-152.

Diggle P.K., Lower S. y Ranker T.A. 1998. Clonal diversity in alpine populations of Polygonum viviparum (Polygonaceae). International Journal of Plant Sciences 159:606-615.

Diggle P.K., Meixner M.A., Carroll A.B. y Aschwanden C.F. 2002. Barriers to sexual reproduction in Polygonum viviparum: A comparative developmental analysis of $P$. viviparum and $P$. bistortoides. Annals of Botany 89:145-156.

Douhovnikoff V. y Dood R.S. 2003. Intra-clonal variation and a similarity threshold for identification of clones: application to $\mathrm{Sa}$ lix exigua using AFLP molecular markers. Theoretical Applied Genetics 106:1307-1315.

Douhovnikoff V., Cheng A.M. y Dodd R.S. 2004. Incidence, size and spatial structure of clones in second-growth stands of coast redwood, Sequoia sempervirens (Cupressaceae). American Journal of Botany 91:1140-1146.

Douhovnikoff V., McBride J.R. y Dodd R.S. 2005. Salix exigua clonal growth and population dynamics in relation to disturbance regime variation. Ecology 86:446-452.

Eckert C.G. 2000. Contributions of autogamy and geitonogamy to self-fertilization in a mass-flowering, clonal plant. Ecology 81:532-542.

Eckert C.G. 2002. The loss of sex in clonal plants. Evolutionary Ecology 15:501-520.

Ellstrand N.C. y Roose M.L. 1987. Patterns of genotypic diversity in clonal plant species. American Journal of Botany 74:123-131. 
Eriksson O. 1989. Seedling dynamics and life histories in clonal plants. Oikos 55:231-238.

Eriksson O. 1993. Dynamics of genets in clonal plants. Trends in Ecology \& Evolution 8:313-316.

Eriksson O. y Bremer B. 1993. Genet dynamics of the clonal plant Rubus saxatilis. Journal of Ecology 81:533-542.

Esselman E.J., Jianqiang L., Crawford D.J., Winduss J.L. y Wolfe A.D. 1999. Clonal diversity in the rare Calamagrostis porteri ssp insperata (Poaceae): comparative results for allozymes and random amplified polymorphic DNA (RAPD) and intersimple sequence repeat (ISSR) markers. Molecular Ecology 8:443451.

Everitt B.S. 1977. The Analysis of Contingency Tables. Chapmann \& Hall, Bury St Edmunds.

Gabrielsen T.M. y Brochmann C. 1998. Sex after all: high levels of diversity detected in the arctic clonal plant Saxifraga cernua using RAPD markers. Molecular Ecology 7:1701-1708.

Garnier L.K.M., Durand J. y Dajoz I. 2002. Limited seed dispersal and microspatial population structure of an agamospermous grass of West African savannahs, Hyparrhenia diplandra (Poaceae). American Journal of Botany 89:1785-1791.

Gibson D.J. y Menges E.S. 1994. Population structure and spatial pattern in the dioecious shrub Ceratiola ericoides. Journal of Vegetation Sciences 5:337-346.

Gleason H.A. 1920. Some applications of the quadrat method. Bulletin of the Torrey Botanical Club 47:21-33.

Hämmerli A. y Reusch T.B.H. 2003a. Flexible mating: cross-pollination affects sex expression in a marine clonal plant. Journal of Evolutionary Biology 18:1096-1105.

Hämmerli A. y Reusch T.B.H. 2003b. Genetic neighbourhood of clone structures in eelgrass meadows quantified by spatial autocorrelation of microsatellite markers. Heredity 91:448-455.

Handel S.N. 1985. The intrusion of clonal growth patterns on plant breeding system. The American Naturalist 125:367-384.

Harper J. 1985. Modules, branches, and the capture of resources. En: Jackson J.B.C., Buss L.W. y Cook R.E. Eds. Population Biology and Evolution of Clonal Organisms, pp. 1-33, Yale University Press, New Haven.

Hartnett D.C. y Bazzaz F.A. 1985. The integration of neighbourhood effects by clonal genets in Solidago canadensis. The Journal of Ecology 73:415-427.

Hirayama K. y Sakimoto M. 2008. Clonal structure and diversity of Cryptomeria japonica along a slope in a cool-temperate, oldgrowth mixed forest in the snowy region of Japan. Canadian Journal of Forest Research 38:2804-2813.

Honnay O. y Bossuyt B. 2005. Prolonged clonal growth: escape route or route to extinction? Oikos 108:427-432.

Honnay O., Jacquemyn H., Roldán-Ruiz I. y Hermy M. 2006. Consequences of prolonged clonal growth on local and regional genetic structure and fruiting success of the forest perennial Maianthemum bifolium. Oikos 112:21-30.

Honnay O. y Jacquemyn H. 2008. A meta-analysis of the relation between mating system, growth form and genotypic diversity in clonal plant species. Evolutionary Ecology 22:299-312.

Ivey C.T. y Richards J.H. 2001. Genotypic diversity and clonal structure of everglades sawgrass, Cladium jamaicense (Cyperaceae). International Journal of Plant Sciences 162:1327-1335.

Jackson J.B.C., Buss L.W. y Cook R.E. Eds. 1985. Population Biology and Evolution of Clonal organisms. Yale University Press, New Haven.
Jacquemyn H., Brys R., Honnay O., Hermy M. y Roldán-Ruiz I. 2005. Local forest environment largely affects below-ground growth, clonal diversity and fine-scale spatial genetic structure in the temperate deciduous forest herb Paris quadrifolia. Molecular Ecology 14:4479-4488.

Jonsson B.O., Jónsdóttir I.S. y Crongberg N. 1996. Clonal diversity and allozyme variation in populations of the arctic sedge Carex bigelowii (Cyperaceae). Journal of Ecology 84:449-459.

Kays S. y Harper J.L. 1974. The regulation of plant and tiller density in a grass sward. Journal of Ecology 62:97-105.

Keane B., Pelikan S., Toth G.P., Smith M.K. y Rogstad S.H. 1999. Genetic diversity of Typha latifolia (Typhaceae) and the impact of pollutants examined with tamdem-repetitive DNA probes. American Journal of Botany 86:1226-1238.

Kjølner S., Såstad S.M. y Brochmann C. 2006. Clonality and recombination in the arctic plant Saxifraga cernua. Botanical Journal of the Linnean Society 152:209-217.

Klimeš L., Klimešová J., Hendriks R.J.J. y van Groenendael J.M. 1997. Clonal plant architecture: a comparative analysis of form and function. En: de Kroon H. y van Groenendael J. Eds. The Ecology and Evolution of Clonal Plants, pp. 1-29, Backhuys Publishers, Leiden.

Korpelainen H. 1994. Sex ratios and resource allocation among sexually reproducing plants of Rubus chamaemorus. Annals of Botany 74:627-632.

Korpelainen H., Antonious-Klemola K. y Werlemark G. 1999. Clonal structure of Rubus chamaemorus populations: comparison of different molecular methods. Plant Ecology 143:123-128.

Kreher S.A., Foré S.A. y Collins B.S. 2000. Genetic variation within and among patches of the clonal species, Vaccinium stamineum L. Molecular Ecology 9:1247-1252.

Kudoh H., Shibaike H., Takasu H., Whigham D.F. y Kawano S. 1999. Genet structure and determinants of clonal structure in a temperate deciduous woodland herb, Uvularia perfoliata. Journal of Ecology 87:244-257.

Liston A., Wilson B.L., Robinson W.A., Doescher P.S., Harris N.R. y Svejcar T. 2003. The relative importance of sexual reproduction versus clonal spread in an arid bunchgrass. Oecologia 137:216-225.

Liu J., Dong M., Miao S.L., Li Z.Y., Song M.H. y Wang R.Q. 2006. Invasive alien plants in China: role of clonality and geographical origin. Biological Invasions 8:1461-1470.

Mandujano M.C., Montaña C., Méndez I. y Golubov J. 1998. The relative contributions of sexual reproduction and clonal propagation in Opuntia rastrera from two habitats in the Chihuahuan desert. Journal of Ecology 86:911-921.

Mandujano M.C., Montaña C., Franco M., Golubov J. y FloresMartínez A. 2001. Integration of demographic annual variability in a clonal desert cactus. Ecology 82:344-359.

Mandujano-Sánchez M.C. 2007. La clonalidad y sus efectos en la biología de poblaciones. En: Eguiarte L.E., Souza V. y Aguirre X. Comp. Ecología Molecular, pp. 215-250, Secretaría de Medio Ambiente y Recursos Naturales-Instituto Nacional de Ecología-Universidad Nacional Autónoma de México-Comosión Nacional para el Conocimiento y Uso de la Biodiversidad, México, D.F.

Mayes S.G., McGinley M.A. y Werth C.R. 1998. Clonal population structure and genetic variation in sand-shinnery oak, Quercus havardii (Fagaceae). American Journal of Botany 85:1609-1617. McFadden C.S. 1991. A comparative demographic analysis of clonal 
reproduction in a temperate soft coral. Ecology 72:1849-1866.

Misuki I., Ishida K., Tani N. y Tsumura Y. 2010. Fine-scale spatial structure of genets and sexes in the dioecious plant Dioscorea japonica, which disperses by both bulbils and seeds. Evolutionary Ecology 24:1399-1415.

Mori Y., Nagamitsu T. y Kubo T. 2009. Clonal growth and its effects on male and female reproductive success in Prunus ssiori (Rosaceae). Population Ecology 51:175-186.

Nagamitsu T., Ogawa M., Ishida K. y Tanouchi H. 2004. Clonal diversity, genetic structure, and mode of recruitment in a Prunus ssiori population established after volcanic eruptions. Plant Ecology 174:1-10.

Okuda T., Kachi N., Yap S.K. y Manokaran N. 1997. Tree distribution pattern and fate of juveniles in a lowland tropical rain forest - implications for regeneration and maintenance of species diversity. Plant Ecology 131:155-171.

Pan J.J. y Price J.S. 2002. Fitness and evolution in clonal plants: the impact of clonal growth. Evolutionary Ecology 15:583-600.

Parker K.C. y Hamrick J.L. 1992. Genetic diversity and clonal structure in a columnar cactus, Lophocereus schottii. American Journal of Botany 79:86-96.

Parks C. y Werth C.R. 1993. A study of spatial features of clones in a population of bracken fern, Pteridium aquilinum (Dennstaedtiaceae). American Journal of Botany 80:537-544.

Peterson C.J. y Jones R.H. 1997. Clonality in woody plants: a review and comparison with clonal herbs. En: de Kroon H. y van Groenendael J. Eds. The Ecology and Evolution of Clonal Plants, pp. 263-289, Backhuys Publishers, Leiden.

Pfeiffer T., Klahr A., Heinrich A. y Schnittler M. 2011. Does sex make a difference? Genetic diversity and spatial genetic structure in two co-occurring species of Gagea (Liliaceae) with contrasting reproductive strategies. Plant Systematics and Evolution 292:189-201.

Pornon A. y Escaravage N. 1999. Genotypic structure in clonal Rhododendron ferrugineum L. (Ericaceae) populations: origin and manteinance. Plant Ecology 141:145-150.

Pornon A., Escaravage N., Thomas P. y Taberlet P. 2000. Dynamics of genotypic structure in clonal Rhododendron ferrugineum (Ericaceae) populations. Molecular Ecology 9:1099-1111.

Pornon A., Escaravage N., Till-Botraud I. y Doche B. 1997. Variation of reproductive traits in Rhododendron ferrugineum $\mathrm{L}$. (Ericaceae) populations along a successional gradient. Plant Ecology 130:1-11.

Price E.A.C. y Marshall C. 1999. Clonal plants and environmental heterogeneity. Plant Ecology 141:3-7.

Reisch C., Schurm S. y Poschlod P. 2007. Spatial genetic structure and clonal diversity in an alpine population of Salix herbacea (Salicaceae). Annals of Botany 99:647-651.

Reusch T.B.H. 2003. Floral neighbourhoods in the sea: how floral density, opportunity for outcrossing and population fragmentation affect seed set in Zostera marina. Journal of Ecology 91:610-615.

Richards A.J. 1997. Plant Breeding Systems. Chapman \& Hall,
Londres.

Rosseto M., Gross C.L., Jones R. y Hunter J. 2004. The impact of clonality on an endangered tree (Elaeocarpus williamsianus) in a fragment rain forest. Biological Conservation 117:33-39.

Ruggieiro M.V., Capone S., Pirozzi P., Reusch T.B.H. y Procaccini G. 2005. Mating system and clonal architecture: a comparative study in two marine angiosperms. Evolutionary Ecology 19:487-499.

Shimizu Y, Ando M. y Sakai F. 2002. Clonal structure of natural populations of Cryptomeria japonica growing at different positions on slopes, detected using RAPD markers. Biochemical Systematics and Ecology 30:733-748.

Shreve F. 1917. The establishment of desert perennials. Journal of Ecology 5:210-216.

Sipes S.D. y Wolf P.G. 1997. Clonal structure and patterns of allozyme diversity in the rare endemic Cycladenia humilis var. jonesii (Apocynaceae). American Journal of Botany 84:401-409.

Stehlik I. y Holderegger R. 2000. Spatial genetic structure and clonal diversity of Anemone nemorosa in late successional deciduous woodlands of Central Europe. Journal of Ecology 88:424-435.

Suvanto L.I. y Latva-Karjantvaa T.B. 2005. Clone identification and clonal structure of the European aspen (Populus tremula). Molecular Ecology 14:2851-2860.

Tiffney B.H. y Niklas K.J. 1985. Clonal growth in land plants: a paleobotanical perspective. En: Jackson J.B.C., Buss L.W. y Cook R.E. Eds. Population Biology and Evolution of Clonal Organisms, pp. 35-66, Yale University Press, New Haven.

Tooke F., Ordidge M., Chiurugwi T. y Battey N. 2005. Mechanisms and function of flower and inflorescence reversion. Journal of Experimental Botany 56:2587-2599.

Torimaru T. y Tomaru N. 2005. Fine-scale clonal structure and diversity within patches of a clone-forming dioecious shrub, Ilex leucoclada (Aquifoliaceae). Annals of Botany 95:295-304.

van Dijk P.J. y Bakx-Schotman J.M.T. 2004. Formation of unreduced megaspores (diplospory) in apomictic dandelions (Taraxacum officinale, s.l.) is controlled by a sex-specific dominant locus. Genetics 166:483-492.

van Groenendael J.M. y de Kroon H. 1990. Clonal Growth in Plants: Regulation and Function. SPB Academic Publishing, La Haya.

van Groenendael J.M., Klimeš L., Klimešová J. y Hendriks R.J.J. 1996. Comparative ecology of clonal plants. Philosophical Transactions of Royal Society of London 351:1331-1339.

Widén B., Cronberg N. y Widén M. 1994. Genotypic diversity, molecular markers and spatial distribution of genets in clonal plants, a literature survey. Folia Geobotanica y Phytotaxonomica 29:245-263.

Wilson A.S.G., van der Kamp B.J. y Ritland C. 2005a. Opportunities for geitonogamy in the clonal herb Maianthemum dilatatum. Canadian Journal of Botany 83:1082-1087.

Wilson A.S.G., van der Kamp B.J. y Ritland C. 2005b. Spatial genetic and clonal structure in Maianthemum dilatatum as defined by AFLP markers. Canadian Journal of Botany 83:1126-1132. 
Apéndice. Características de las especies y poblaciones: $\mathbf{S C R Z}=$ sistema de cruza, $\mathbf{P O L}=$ polinización, $\mathbf{P R O R}=$ estructuras o procesos que dan origen a los ramets, CCT-N = tipo de crecimiento clonal en la clasificación de Tiffney y Niklas (1985), POB/HL = número de población y/o hábitat local, $\mathbf{P D E}=$ clave del patrón de distribución espacial de acuerdo a la figura 2, AER = arreglo espacial de los ramets de distintos genets, $\boldsymbol{D}=$ índice de diversidad clonal de Simpson, $\boldsymbol{G} / \mathbf{N}=$ proporción de genotipos distinguibles, Métodos = métodos empleados para la evaluación de los parámetros. El significado de las abreviaturas empleadas en cada columna se muestra al final del cuadro.

\begin{tabular}{|c|c|c|c|c|c|c|c|c|c|c|c|c|c|}
\hline Especie (Familia) & Hábito & SCRZ & POL & PROR & CCT-N & $\begin{array}{c}\text { POB/ } \\
\mathrm{HL}\end{array}$ & $\begin{array}{c}\text { PDE } \\
\text { (fig. 2) }\end{array}$ & $\begin{array}{c}\text { AER } \\
\text { (fig. 2) }\end{array}$ & $D$ & $G / N$ & $\begin{array}{l}\text { Estructura } \\
\text { genética } \\
\text { local (EGL) }\end{array}$ & $\begin{array}{c}\text { Métodos } \\
\text { PDE, } D, G / N \\
\text { y EGL }\end{array}$ & Referencias \\
\hline $\begin{array}{l}\text { Anemone } \\
\text { nemorosa } \\
\text { (Ranunculaceae) }\end{array}$ & $\mathrm{Hp}$ & A & $\mathrm{Bt}$ & $\begin{array}{c}\mathrm{Rzm+} \\
\mathrm{drm}\end{array}$ & $\mathrm{Lg}+\mathrm{NI}$ & $\begin{array}{l}\text { ppm } \\
(20)\end{array}$ & $(4,5)$ & $\mathrm{Ag}+\mathrm{En}$ & $\mathrm{n} / \mathrm{e}$ & 0.95 & $\begin{array}{l}\operatorname{asp},<1 \mathrm{~m} \\
(\mathrm{rm})\end{array}$ & tra+ISZ+asp & $\begin{array}{l}\text { Stehlik y } \\
\text { Holderegger } \\
\text { 2000* }\end{array}$ \\
\hline $\begin{array}{l}\text { Botrychium } \\
\text { pumicola } \\
\text { (Ophioglossaceae) }\end{array}$ & $\mathrm{Hp}$ & A & $\mathrm{Ab}$ & gmas & $\mathrm{NI}$ & $\begin{array}{l}\text { p } 1 \\
\text { p } 2 \\
\text { p } 3\end{array}$ & $\begin{array}{l}(2,5) \\
(2,5)\end{array}$ & $\begin{array}{l}\mathrm{Ag}+\mathrm{En} \\
\mathrm{Ag}+\mathrm{En} \\
\mathrm{Ag}+\mathrm{En}\end{array}$ & $\begin{array}{c}0.14 \\
0.16 \\
0.1\end{array}$ & $\begin{array}{l}0.93 \\
0.9 \\
0.65\end{array}$ & $\begin{array}{c}\text { mt: no } \\
\text { detectada } \\
\text { mt: no } \\
\text { detectada } \\
\text { mt: no } \\
\text { detectada }\end{array}$ & prc + ISSR $+m t$ & $\begin{array}{c}\text { Camacho y } \\
\text { Liston, 2001* }\end{array}$ \\
\hline $\begin{array}{l}\text { Calamagrostis } \\
\text { porteri ssp. } \\
\text { insperata (Poaceae) }\end{array}$ & $\mathrm{Hp}$ & A & $\mathrm{Ab}$ & rzm & $\operatorname{Lg}$ & $\begin{array}{l}\text { p } 2 \\
\text { p } 3 \\
\text { p } 4\end{array}$ & $\begin{array}{l}2 \\
2 \\
2\end{array}$ & $\begin{array}{l}\mathrm{Ag} \\
\mathrm{Ag} \\
\mathrm{Ag}\end{array}$ & $\begin{array}{l}n / e \\
n / e \\
n / e\end{array}$ & $\begin{array}{c} \\
1 \\
0.82 \\
0.91\end{array}$ & $\begin{array}{l}\mathrm{n} / \mathrm{e} \\
\mathrm{n} / \mathrm{e} \\
\mathrm{n} / \mathrm{e}\end{array}$ & $\begin{array}{c}\text { desc+tra+ } \\
\text { ISRR+ } \\
\text { RAPD }\end{array}$ & $\begin{array}{c}\text { Esselman } \\
\text { et al., } \\
1999\end{array}$ \\
\hline $\begin{array}{l}\text { Carex bigelowii } \\
\text { (Cyperaceae) }\end{array}$ & $\mathrm{Hp}$ & $?$ & $\mathrm{Ab}$ & rzm & $\operatorname{Lg}$ & $\begin{array}{l}\text { p } 1 \\
\text { p } 2 \\
\text { p } 3\end{array}$ & $\begin{array}{l}4 \\
4 \\
4\end{array}$ & $\begin{array}{l}\mathrm{Ag} \\
\mathrm{Ag} \\
\mathrm{Ag}\end{array}$ & $\begin{array}{l}0.98 \\
0.98 \\
0.98\end{array}$ & $\begin{array}{l}0.52 \\
0.63 \\
0.48\end{array}$ & $\begin{array}{l}\text { n/e } \\
\text { n/e } \\
\text { n/e }\end{array}$ & tra+ISZ & $\begin{array}{c}\text { Jonsson } \\
\text { et al., 1996* }\end{array}$ \\
\hline $\begin{array}{l}\text { Cladium jamaicense } \\
\text { (Cyperaceae) }\end{array}$ & $\mathrm{Hp}$ & $?$ & $\mathrm{Ab}$ & $\begin{array}{c}\text { rzm+ } \\
\text { pnt }\end{array}$ & $\mathrm{Lg}+\mathrm{Nl}$ & $\begin{array}{l}p 1 \\
\text { p } 2 \\
\text { p } 3 \\
\text { p } 4 \\
\text { p } 5 \\
\text { p } 6 \\
\text { p } 7 \\
\text { p } 8 \\
\text { p } 9 \\
\text { p } 10 \\
\text { p } 11 \\
\text { p } 12 \\
\text { p } 13 \\
\text { p } 14\end{array}$ & $\begin{array}{l}(4,5) \\
(4,5) \\
(4,5) \\
(4,5) \\
(4,5) \\
1 \\
(4,5) \\
(4,5) \\
(4,5) \\
(4,5) \\
(4,5) \\
(4,5) \\
(4,5) \\
(4,5)\end{array}$ & $\begin{array}{c}A g+E n \\
A g+E n \\
A g+E n \\
A g+E n \\
A g+E n \\
A g \\
A g+E n \\
A g+E n \\
A g+E n \\
A g+E n \\
A g+E n \\
A g+E n \\
A g+E n \\
A g+E n\end{array}$ & $\begin{array}{c}0.236 \\
0.269 \\
0.271 \\
0.682 \\
0.159 \\
0 \\
0.538 \\
0.212 \\
0.771 \\
0.698 \\
0.814 \\
0.682 \\
0.673 \\
0.444\end{array}$ & $\begin{array}{l}0.044 \\
0.044 \\
0.125 \\
0.231 \\
0.043 \\
0.021 \\
0.146 \\
0.068 \\
0.106 \\
0.143 \\
0.261 \\
0.114 \\
0.13 \\
0.043\end{array}$ & $\begin{array}{c}\text { mpic: no } \\
\text { detectada } \\
\text { mpic: no } \\
\text { detectada } \\
\text { mpic: no } \\
\text { detectada } \\
\text { mpic: no } \\
\text { detectada } \\
\text { mpic: no } \\
\text { detectada } \\
\text { n/a } \\
\text { mpic: no } \\
\text { detectada } \\
\text { mpic: no } \\
\text { detectada } \\
\text { mpic: no } \\
\text { detectada } \\
\text { mpic: no } \\
\text { detectada } \\
\text { mpic: no } \\
\text { detectada } \\
\text { mpic: no } \\
\text { detectada } \\
\text { mpic: no } \\
\text { detectada } \\
\text { mpic: no } \\
\text { detectada }\end{array}$ & $\begin{array}{l}\text { tra+lSZ+ } \\
\text { mpic }\end{array}$ & $\begin{array}{l}\text { Ivey y Richards, } \\
\text { 2001* }\end{array}$ \\
\hline
\end{tabular}


Apéndice. Continuación

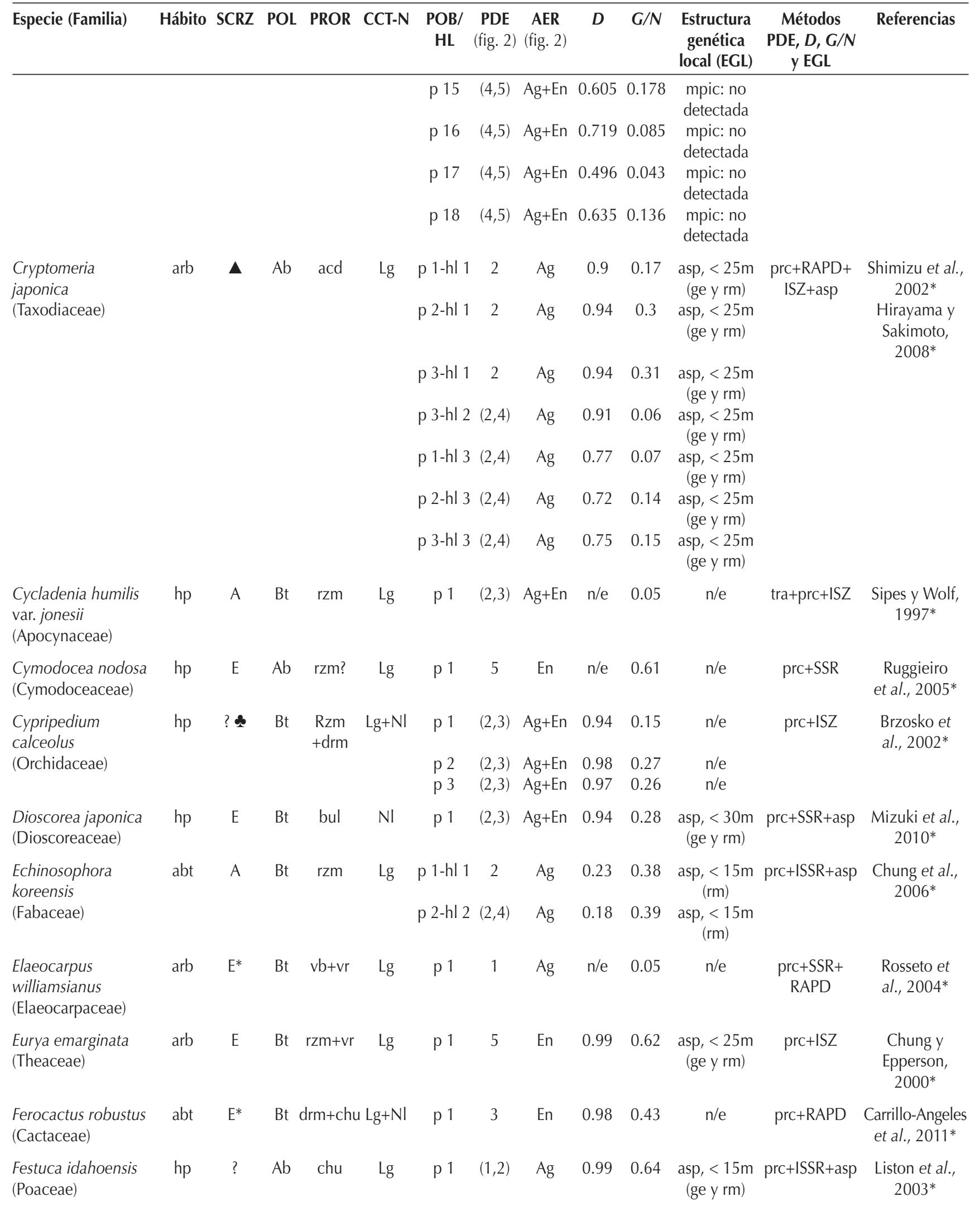


Apéndice. Continuación

\begin{tabular}{|c|c|c|c|c|c|c|c|c|c|c|c|c|c|}
\hline Especie (Familia) & Hábito & SCRZ & POL & PROR & CCT-N & $\begin{array}{c}\mathrm{POB} / \\
\mathrm{HL}\end{array}$ & $\begin{array}{l}\text { PDE } \\
\text { (fig. 2) }\end{array}$ & $\begin{array}{c}\text { AER } \\
\text { (fig. 2) }\end{array}$ & $D$ & $G / N$ & $\begin{array}{l}\text { Estructura } \\
\text { genética } \\
\text { local (EGL) }\end{array}$ & $\begin{array}{c}\text { Métodos } \\
\text { PDE, } D, G / N \\
\text { y EGL } \\
\end{array}$ & Referencias \\
\hline \multirow[t]{3}{*}{$\begin{array}{l}\text { Gagea lutea } \\
\text { (Liliaceae) }\end{array}$} & \multirow[t]{3}{*}{ hg } & \multirow[t]{3}{*}{$?$} & \multirow[t]{3}{*}{$\mathrm{Bt}$} & \multirow[t]{3}{*}{ buls } & \multirow[t]{3}{*}{$\mathrm{Nl}$} & p 1 & $(2,3)$ & $\mathrm{Ag}+\mathrm{En}$ & $\mathrm{n} / \mathrm{e}$ & 0.51 & \multirow{3}{*}{\multicolumn{2}{|c|}{$\begin{array}{l}\text { asp, }<8 \mathrm{~m}, 16 \text { tra+AFLP+asp } \\
\mathrm{a}<24 \mathrm{~m}(\mathrm{rm}) \\
\mathrm{asp},<8 \mathrm{~m}, 16 \\
\mathrm{a}<24 \mathrm{~m}(\mathrm{rm}) \\
\mathrm{asp},<8 \mathrm{~m}, 16 \\
\mathrm{a}<24 \mathrm{~m}(\mathrm{rm})\end{array}$}} & \multirow[t]{3}{*}{$\begin{array}{l}\text { Pfeiffer et al., } \\
2011^{*}\end{array}$} \\
\hline & & & & & & p 2 & $(2,3)$ & $\mathrm{Ag}+\mathrm{En}$ & $\mathrm{n} / \mathrm{e}$ & 0.57 & & & \\
\hline & & & & & & p 3 & $(2,3)$ & $\mathrm{Ag}+\mathrm{En}$ & $\mathrm{n} / \mathrm{e}$ & 0.61 & & & \\
\hline \multirow{3}{*}{$\begin{array}{l}\text { Gagea spathacea } \\
\text { (Liliaceae) }\end{array}$} & \multirow[t]{3}{*}{ hg } & \multirow[t]{3}{*}{$?$} & \multirow[t]{3}{*}{$\mathrm{Bt}$} & \multirow[t]{3}{*}{ buls } & \multirow[t]{3}{*}{$\mathrm{NI}$} & p 1 & 1 & $\mathrm{Ag}$ & $\mathrm{n} / \mathrm{e}$ & 0.001 & $\mathrm{n} / \mathrm{a}$ & \multirow[t]{3}{*}{ tra+AFLP } & \multirow{3}{*}{$\begin{array}{r}\text { Pfeiffer et al., } \\
2011^{*}\end{array}$} \\
\hline & & & & & & p 2 & 1 & $\mathrm{Ag}$ & $\mathrm{n} / \mathrm{e}$ & 0.001 & $\mathrm{n} / \mathrm{a}$ & & \\
\hline & & & & & & p 3 & 1 & $\mathrm{Ag}$ & $\mathrm{n} / \mathrm{e}$ & 0.001 & $\mathrm{n} / \mathrm{a}$ & & \\
\hline $\begin{array}{l}\text { Hyparrhenia } \\
\text { diplandra (Poaceae) }\end{array}$ & hp & $?$ & $\mathrm{Ab}$ & agp & $\mathrm{NI}$ & p 1 & 5 & En & 0.85 & 0.07 & $\begin{array}{l}\text { asp, }<6 \mathrm{~m} \\
\quad(\mathrm{rm})\end{array}$ & $\begin{array}{c}\text { prc+lSZ } \\
+ \text { CLO+asp }\end{array}$ & $\begin{array}{c}\text { Garnier et al., } \\
2002^{*}\end{array}$ \\
\hline $\begin{array}{l}\text { Ilex leucoclada } \\
\text { (Aquifoliaceae) }\end{array}$ & abt & E & $\mathrm{Bt}$ & $\operatorname{acd} \mathbf{\Lambda}$ & $\operatorname{Lg}$ & p 1 & $(2,3)$ & $\mathrm{Ag}+\mathrm{En}$ & 0.67 & 0.09 & $\mathrm{n} / \mathrm{e}$ & prc+RAPD & $\begin{array}{c}\text { Torimaru y } \\
\text { Tomaru, 2005* }\end{array}$ \\
\hline \multirow[t]{8}{*}{$\begin{array}{l}\text { Lophocereus schottii } \\
\text { (Cactaceae) }\end{array}$} & \multirow[t]{8}{*}{ arb } & \multirow[t]{8}{*}{ A } & \multirow[t]{8}{*}{$\mathrm{Bt}$} & \multirow[t]{8}{*}{$\begin{array}{l}\mathrm{drm} \\
+\mathrm{acd}\end{array}$} & \multirow[t]{8}{*}{$\mathrm{Lg}+\mathrm{NI}$} & p 1-hl 1 & 5 & En & 0.95 & 0.64 & $\begin{array}{c}\text { SG: 0-10 } \\
<10-50>\text { más } \\
\text { de } 50 m \text { (ge) }\end{array}$ & \multirow{8}{*}{ prc+ISZ+SG } & \multirow[t]{8}{*}{$\begin{array}{l}\text { Parker y } \\
\text { Hamrick, } \\
1992^{*}\end{array}$} \\
\hline & & & & & & p 2-hl 2 & 2 & $\mathrm{Ag}$ & 0.95 & 0.52 & $\begin{array}{l}\text { SG: } 0-10 \\
<10-50>\text { más } \\
\text { de } 50 \mathrm{~m} \text { (ge) }\end{array}$ & & \\
\hline & & & & & & p 3-hl 3 & 2 & $\mathrm{Ag}$ & 0.93 & 0.65 & $\begin{array}{c}\text { SG: } 0-10 \\
<10-50>\text { más } \\
\text { de } 50 m \text { (ge) }\end{array}$ & & \\
\hline & & & & & & p 4-hl 1 & $(1,2)$ & $\mathrm{Ag}$ & 0.94 & 0.24 & $\begin{array}{l}\text { SG: } 0-10> \\
\text { 10-50>más } \\
\text { de } 50 \mathrm{~m} \text { (ge) }\end{array}$ & & \\
\hline & & & & & & p 5-hl 4 & $(1,2)$ & $\mathrm{Ag}$ & 0.92 & 0.41 & $\begin{array}{l}\text { SG: } 0-10> \\
10-50<\text { más } \\
\text { de } 50 m \text { (ge) }\end{array}$ & & \\
\hline & & & & & & p 6-hl 4 & $(1,2)$ & $\mathrm{Ag}$ & 0.87 & 0.45 & $\begin{array}{c}\text { SG: } 0-10> \\
10-50<\text { más } \\
\text { de } 50 m \text { (ge) }\end{array}$ & & \\
\hline & & & & & & p 7-hl 2 & $(1,2)$ & $\mathrm{Ag}$ & 0.8 & 0.18 & $\begin{array}{l}\text { SG: } 0-10> \\
\text { 10-50>más } \\
\text { de } 50 \mathrm{~m} \text { (ge) }\end{array}$ & & \\
\hline & & & & & & p 8-hl 5 & $(1,5)$ & $\mathrm{Ag}+\mathrm{En}$ & 0.59 & 0.36 & $\begin{array}{l}\text { SG: } 10-50= \\
\text { más de } 50 \mathrm{~m} \\
\quad(\text { ge })\end{array}$ & & \\
\hline $\begin{array}{l}\text { Lyonothamnus } \\
\text { floribundus } \\
\text { (Rosaceae) }\end{array}$ & arb & $?$ & $\mathrm{Bt}$ & $\mathrm{vr}$ & $\operatorname{Lg}$ & p 1 & $(1,2)$ & $\mathrm{Ag}$ & $\mathrm{n} / \mathrm{e}$ & 0.036 & $\mathrm{n} / \mathrm{e}$ & prc+RAPD & $\begin{array}{c}\text { Bushakra et al., } \\
\text { 1999* }\end{array}$ \\
\hline $\begin{array}{l}\text { Maianthemum } \\
\text { bifolium } \\
\text { (Convalliariaceae) }\end{array}$ & hp & $E^{*}$ & $\mathrm{Bt}$ & $\mathrm{rzm}$ & $\operatorname{Lg}$ & p 1 & $(1,2)$ & $\mathrm{Ag}$ & 0.88 & 0.7 & $\begin{array}{l}\text { asp, }<50 \mathrm{~m} \\
\text { (ge) \# }\end{array}$ & $\begin{array}{l}\text { trans+AFLP } \\
\quad+\text { asp }\end{array}$ & $\begin{array}{l}\text { Arens et al., } \\
2005^{*} \\
\text { Honnay et al. } \\
2006\end{array}$ \\
\hline $\begin{array}{l}\text { Maianthemum } \\
\text { dilatatum } \\
\text { (Convalliariaceae) }\end{array}$ & hp & EAs & $\mathrm{Bt}$ & $\operatorname{rzm} \mathbf{\Delta}$ & $\operatorname{Lg}$ & p 1 & $(1,2)$ & $\mathrm{Ag}$ & $\mathrm{n} / \mathrm{e}$ & 0.64 & $\begin{array}{l}\text { asp, }<45 \mathrm{~m} \\
\text { (ge y rm) }\end{array}$ & $\begin{array}{l}\text { prc }+ \text { AFLP } \\
\quad+\text { asp }\end{array}$ & $\begin{array}{l}\text { Wilson et al., } \\
2005 a^{*} \\
\text { Wilson et al., } \\
2005 b^{*}\end{array}$ \\
\hline $\begin{array}{l}\text { Paris quadrifolia } \\
\text { (Trilliaceae) }\end{array}$ & hp & A & $\mathrm{Bt}$ & $\mathrm{rzm}$ & $\operatorname{Lg}$ & p 1-hl 1 & 3 & En & 0.87 & 0.27 & $\begin{array}{l}\text { asp, }<5 \mathrm{~m} \\
(\text { ge y rm) }\end{array}$ & $\begin{array}{l}\text { prc }+ \text { AFLP } \\
\quad+\text { asp }\end{array}$ & $\begin{array}{c}\text { Jacquemyn et } \\
\text { al., 2005* }\end{array}$ \\
\hline
\end{tabular}


Apéndice. Continuación

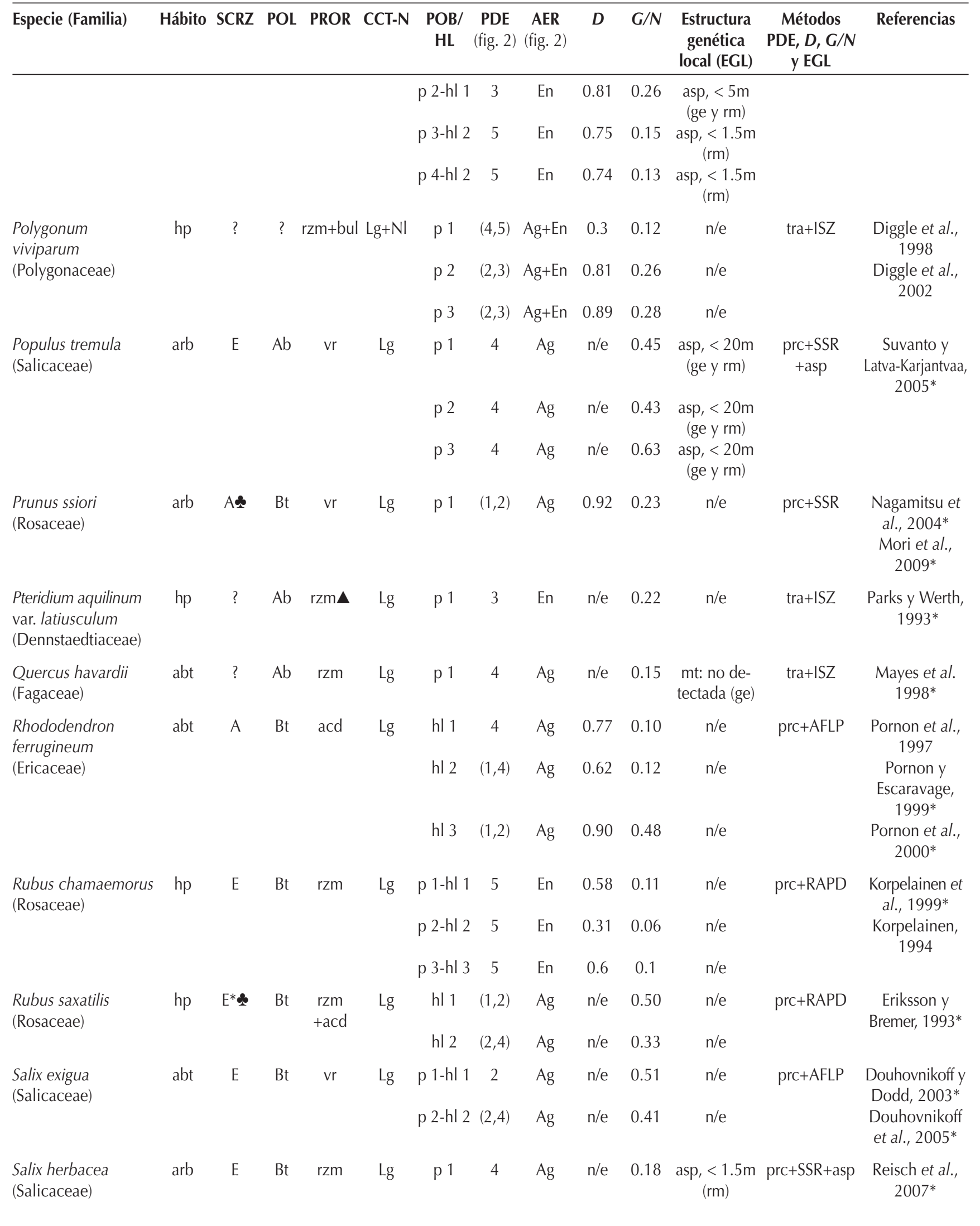


Apéndice. Continuación

\begin{tabular}{|c|c|c|c|c|c|c|c|c|c|c|c|c|c|}
\hline Especie (Familia) & Hábito & SCRZ & POL & PROR & CCT-N & $\begin{array}{c}\mathrm{POB} / \\
\mathrm{HL}\end{array}$ & $\begin{array}{c}\text { PDE } \\
\text { (fig. 2) }\end{array}$ & $\begin{array}{c}\text { AER } \\
\text { (fig. 2) }\end{array}$ & $D$ & $G / N$ & $\begin{array}{l}\text { Estructura } \\
\text { genética } \\
\text { local (EGL) }\end{array}$ & $\begin{array}{c}\text { Métodos } \\
\text { PDE, } D, G / N \\
\text { y EGL }\end{array}$ & Referencias \\
\hline \multirow[t]{6}{*}{$\begin{array}{l}\text { Saxifraga cernua } \\
\text { (Saxifragaceae) }\end{array}$} & \multirow[t]{6}{*}{ hp } & \multirow[t]{6}{*}{$E^{*}$} & \multirow[t]{6}{*}{$\mathrm{Bt}$} & \multirow[t]{6}{*}{ bul } & \multirow[t]{6}{*}{$\mathrm{Nl}$} & p 1 & 5 & En & 0.35 & 0.13 & $\begin{array}{c}\text { mt: no } \\
\text { detectada }\end{array}$ & \multirow[t]{6}{*}{$\begin{array}{l}\text { prc+RAPD } \\
+m t+a s p\end{array}$} & \multirow{6}{*}{$\begin{array}{l}\text { Gabrielsen y } \\
\text { Brochmann, } \\
1998^{*} \\
\text { KjØIner et al., } \\
\text { 2006* }\end{array}$} \\
\hline & & & & & & p 2 & 5 & En & 0.68 & 0.19 & $\begin{array}{c}\text { mt: no } \\
\text { detectada }\end{array}$ & & \\
\hline & & & & & & p 3 & 5 & En & 0.7 & 0.25 & $\begin{array}{l}\text { asp: no } \\
\text { detectada }\end{array}$ & & \\
\hline & & & & & & p 4 & 5 & En & 0.9 & 0.4 & $\begin{array}{c}\text { asp: no } \\
\text { detectada }\end{array}$ & & \\
\hline & & & & & & p 5 & 5 & En & 0.93 & 0.43 & $\begin{array}{l}\text { asp: no } \\
\text { detectada }\end{array}$ & & \\
\hline & & & & & & p 6 & 5 & En & 0.89 & 0.34 & $\begin{array}{c}\text { asp: no } \\
\text { detectada }\end{array}$ & & \\
\hline \multirow[t]{3}{*}{$\begin{array}{l}\text { Scirpus maritimus } \\
\text { (Cyperaceae) }\end{array}$} & \multirow[t]{3}{*}{ hp } & \multirow[t]{12}{*}{$\mathrm{E}^{*}$} & \multirow[t]{3}{*}{$\mathrm{Ab}$} & \multirow[t]{3}{*}{$\mathrm{rzm}$} & \multirow[t]{3}{*}{$\operatorname{Lg}$} & p 1 & 2 & $\mathrm{Ag}$ & $\mathrm{n} / \mathrm{e}$ & $\mathrm{n} / \mathrm{e}$ & $\mathrm{n} / \mathrm{e}$ & \multirow[t]{3}{*}{ desc } & \multirow[t]{3}{*}{$\begin{array}{c}\text { Charpentier et } \\
\text { al., } 2000\end{array}$} \\
\hline & & & & & & p 2 & 4 & $\mathrm{Ag}$ & $\mathrm{n} / \mathrm{e}$ & $\mathrm{n} / \mathrm{e}$ & $\mathrm{n} / \mathrm{e}$ & & \\
\hline & & & & & & p 3 & 4 & $\mathrm{Ag}$ & $\mathrm{n} / \mathrm{e}$ & $\mathrm{n} / \mathrm{e}$ & $\mathrm{n} / \mathrm{e}$ & & \\
\hline \multirow[t]{9}{*}{$\begin{array}{l}\text { Sequoia sempervirens } \\
\text { (Taxodiaceae) }\end{array}$} & \multirow[t]{9}{*}{ arb } & & \multirow[t]{9}{*}{$\mathrm{Ab}$} & \multirow[t]{9}{*}{$\mathrm{vb}$} & \multirow[t]{9}{*}{$\operatorname{Lg}$} & p 1-hl 1 & 2 & $\mathrm{Ag}$ & $\mathrm{n} / \mathrm{e}$ & 0.24 & $\begin{array}{l}\mathrm{mt}:<40 \mathrm{~m} \\
\quad(\mathrm{rm})\end{array}$ & \multirow[t]{9}{*}{$\operatorname{prc}+\mathrm{AFLP}+\mathrm{mt}$} & \multirow[t]{9}{*}{$\begin{array}{l}\text { Douhovnikoff } \\
\text { et al., 2004* }\end{array}$} \\
\hline & & & & & & p 2-hl 1 & 2 & $\mathrm{Ag}$ & $\mathrm{n} / \mathrm{e}$ & 0.38 & $\begin{array}{c}\mathrm{mt}:<40 \mathrm{~m} \\
(\mathrm{rm})\end{array}$ & & \\
\hline & & & & & & p 3-hl 1 & 2 & $\mathrm{Ag}$ & $\mathrm{n} / \mathrm{e}$ & 0.5 & $\begin{array}{c}\mathrm{mt}:<40 \mathrm{~m} \\
(\mathrm{rm})\end{array}$ & & \\
\hline & & & & & & p 4-hl 2 & 2 & $\mathrm{Ag}$ & $\mathrm{n} / \mathrm{e}$ & 0.6 & $\begin{array}{c}\mathrm{mt}:<40 \mathrm{~m} \\
(\mathrm{rm})\end{array}$ & & \\
\hline & & & & & & p 5-hl 2 & 2 & $\mathrm{Ag}$ & $\mathrm{n} / \mathrm{e}$ & 0.35 & $\begin{array}{c}\mathrm{mt}:<40 \mathrm{~m} \\
(\mathrm{rm})\end{array}$ & & \\
\hline & & & & & & p 6-hl 2 & 2 & $\mathrm{Ag}$ & $\mathrm{n} / \mathrm{e}$ & 0.6 & $\begin{array}{c}\mathrm{mt}:<40 \mathrm{~m} \\
(\mathrm{rm})\end{array}$ & & \\
\hline & & & & & & p 7-hl 3 & 2 & $\mathrm{Ag}$ & $\mathrm{n} / \mathrm{e}$ & 0.26 & $\begin{array}{c}\mathrm{mt}:<40 \mathrm{~m} \\
(\mathrm{rm})\end{array}$ & & \\
\hline & & & & & & p 8-hl 3 & 2 & $\mathrm{Ag}$ & $n / e$ & 0.19 & $\begin{array}{c}\mathrm{mt}:<40 \mathrm{~m} \\
(\mathrm{rm})\end{array}$ & & \\
\hline & & & & & & p 9-hl 3 & 2 & $\mathrm{Ag}$ & $\mathrm{n} / \mathrm{e}$ & 0.54 & $\begin{array}{c}\mathrm{mt}:<40 \mathrm{~m} \\
\quad(\mathrm{rm})\end{array}$ & & \\
\hline $\begin{array}{l}\text { Stenocereus eruca } \\
\text { (Cactaceae) }\end{array}$ & abt & $E^{*}$ & $\mathrm{Bt}$ & drm & $\mathrm{NI}$ & p 1 & 2 & $\mathrm{Ag}$ & 0.99 & 0.83 & $\begin{array}{l}\text { asp, }<20 \text { m } \\
\text { (ge y rm) }\end{array}$ & prc+ISSR+asp & $\begin{array}{c}\text { Clark-Tapia et } \\
\text { al., 2005* } \\
\text { Clark-Tapia et } \\
\text { al., } 2006\end{array}$ \\
\hline \multirow[t]{5}{*}{$\begin{array}{l}\text { Typha latifolia } \\
\text { (Thyphaceae) }\end{array}$} & \multirow[t]{5}{*}{$\mathrm{hp}$} & \multirow[t]{5}{*}{ A } & \multirow[t]{5}{*}{$\mathrm{Ab}$} & \multirow[t]{5}{*}{ rzm } & $\operatorname{Lg}$ & p 1 & $(4,5)$ & $\mathrm{Ag}+\mathrm{En}$ & $\mathrm{n} / \mathrm{e}$ & 0.77 & $\mathrm{n} / \mathrm{e}$ & tra+VNTR & $\begin{array}{c}\text { Keane et al., } \\
\text { 1999* }\end{array}$ \\
\hline & & & & & & p 2 & $(4,5)$ & $\mathrm{Ag}+\mathrm{En}$ & $\mathrm{n} / \mathrm{e}$ & 0.55 & $\mathrm{n} / \mathrm{e}$ & & \\
\hline & & & & & & p 3 & $(4,5)$ & $\mathrm{Ag}+\mathrm{En}$ & $\mathrm{n} / \mathrm{e}$ & 0.48 & $\mathrm{n} / \mathrm{e}$ & & \\
\hline & & & & & & p 4 & $(4,5)$ & $\mathrm{Ag}+\mathrm{En}$ & $\mathrm{n} / \mathrm{e}$ & 0.3 & $n / e$ & & \\
\hline & & & & & & p 5 & $(4,5)$ & $A g+E n$ & $\mathrm{n} / \mathrm{e}$ & 0.57 & $\mathrm{n} / \mathrm{e}$ & & \\
\hline $\begin{array}{l}\text { Uvularia perfoliata } \\
\text { (Convallariaceae) }\end{array}$ & hp & $?$ & $?$ & etl & $\operatorname{Lg}$ & p 1-hl 1 & $(4,5)$ & $A g+E n$ & 0.74 & 0.09 & $\begin{array}{c}\text { asp, }<3-5 m \\
(r m)\end{array}$ & prc+ISZ+asp & $\begin{array}{l}\text { Kudoh et al., } \\
\text { 1999* }\end{array}$ \\
\hline & & & & & & p 1-hl 2 & 1 & $\mathrm{Ag}$ & 0.59 & 0.03 & $\mathrm{n} / \mathrm{a}$ & & \\
\hline $\begin{array}{l}\text { Vaccinium myrtillus } \\
\text { (Ericaceae) }\end{array}$ & abt & A & $\mathrm{Bt}$ & rzm & $\operatorname{Lg}$ & p 1 & 2 & $\mathrm{Ag}$ & 0.94 & 0.29 & $\begin{array}{c}\text { asp: no } \\
\text { detectada }\end{array}$ & $\begin{array}{l}\text { prc+AFLP+ } \\
\text { RAPD+asp }\end{array}$ & $\begin{array}{c}\text { Albert et al., } \\
\text { 2003* }\end{array}$ \\
\hline
\end{tabular}


Apéndice. Continuación

\begin{tabular}{|c|c|c|c|c|c|c|c|c|c|c|c|c|c|}
\hline Especie (Familia) & Hábito & SCRZ & POL & PROR & CCT-N & $\begin{array}{c}\mathrm{POB} / \\
\mathrm{HL}\end{array}$ & $\begin{array}{l}\text { PDE } \\
\text { (fig. 2) }\end{array}$ & $\begin{array}{c}\text { AER } \\
\text { (fig. 2) }\end{array}$ & $D$ & $G / N$ & $\begin{array}{c}\text { Estructura } \\
\text { genética } \\
\text { local (EGL) }\end{array}$ & $\begin{array}{c}\text { Métodos } \\
\text { PDE, } D, G / N \\
\text { y EGL }\end{array}$ & Referencias \\
\hline $\begin{array}{l}\text { Vaccinium stamineum } \\
\text { (Ericaceae) }\end{array}$ & abt & $\mathrm{E}^{*}$ & $\mathrm{Bt}$ & $\mathrm{rzm}$ & $\operatorname{Lg}$ & $\mathrm{p} 1$ & $(2,3)$ & $\mathrm{Ag}+\mathrm{En}$ & $\mathrm{n} / \mathrm{e}$ & 0.68 & $\mathrm{n} / \mathrm{e}$ & prc+RAPD & $\begin{array}{l}\text { Kreher et al., } \\
\text { 2000* }\end{array}$ \\
\hline $\begin{array}{l}\text { Zostera marina } \\
\text { (Zosteraceae) }\end{array}$ & hp & A & $\mathrm{Ab}$ & $\mathrm{rzm}$ & $\operatorname{Lg}$ & $\begin{array}{l}\text { p 1-hl } 1 \\
\text { p 2-hl } 2\end{array}$ & $\begin{array}{l}(4,5) \\
(4,5)\end{array}$ & $\begin{array}{l}A g+E n \\
A g+E n\end{array}$ & $\mathrm{n} / \mathrm{e}$ & $\mathrm{n} / \mathrm{e}$ & $\begin{array}{c}\text { asp, }<6 \mathrm{~m} \\
\text { (ge y rm) } \\
\text { asp, }<6 \mathrm{~m} \\
\text { (ge y rm) }\end{array}$ & $\mathrm{prc}+\mathrm{SSR}+\mathrm{asp}$ & $\begin{array}{l}\text { Hämmerli y } \\
\text { Reusch, } 2003 \mathrm{a}^{*} \\
\text { Hämmerli y } \\
\text { Reusch, } 2003 \mathrm{~b}^{*}\end{array}$ \\
\hline $\begin{array}{l}\text { Zostera noltii } \\
\text { (Zosteraceae) }\end{array}$ & hp & A & $\mathrm{Ab}$ & $\mathrm{rzm}$ & $\operatorname{Lg}$ & p 1 & 4 & $\mathrm{Ag}$ & $\mathrm{n} / \mathrm{e}$ & 0.12 & $\mathrm{n} / \mathrm{e}$ & $\mathrm{prc}+\mathrm{SSR}$ & $\begin{array}{l}\text { Ruggieiro et } \\
\text { al., 2005* }\end{array}$ \\
\hline
\end{tabular}

Hábito: $\mathbf{a b t}=$ arbustos, $\mathbf{a r b}=$ árboles, $\mathbf{h p}=$ hierbas perennes

SCRZ: A = especies con capacidad de reproducirse por autocruza, $\mathbf{E}=$ especies con entrecruza obligada (p. ej., dioicas), $\mathbf{E}^{*}=$ especies con sistemas de autoincompatibilidad, = reclutamiento sexual observado y/o evaluado en condiciones naturales

POL: $\mathbf{A b}=$ polinización abiótica, $\mathbf{B t}=$ polinización biótica

PROR: acd = acodaduras, agp = agamospermia, bul = bulbilos, buls = bulbilos subterráneos, $\mathbf{c h u}=$ coronas huecas, $\mathbf{d r m}=$ desprendimiento de ramas, $\mathbf{e t l}=$ estolones, $\mathbf{g m a s}=$ gemas subterráneas, $\mathbf{p n t}=$ plantlets, $\mathbf{p s b}=$ pseudobulbos, $\mathbf{r z m}=$ rizomas, $\mathbf{v b}=$ vástagos basales, $\mathbf{v r}=\mathbf{v a ́ s t a g o s}$ radicales, $\mathbf{\Delta}$ = crecimiento tipo guerrilla

CCT-N: $\mathbf{L g}=$ crecimiento clonal ligado, $\mathbf{N I}=$ crecimiento clonal no ligado

POB/HL: $\mathbf{p}=$ población, $\mathbf{h l}=$ hábitat local (p. ej., bosque con dosel abierto vs bosque con dosel cerrado)

PDE: los paréntesis indican que la población presenta una combinación de patrones de distribución de los tipos indicados por los números (ver figura 2).

AER: Ag = los ramets de cada genet no se entremezclan con los ramets de genets distintos, En = los ramets de diferentes genets en la población se entremezclan.

EGL: \# = evaluado en una población distinta de donde se determinó el PDE, asp = autocorrelación espacial, mpic = método de probabilidad de identidad clonal, $\mathbf{m t}=$ prueba de Mantel, $\mathbf{S G}=$ similitud genética vs distancia espacial.

Métodos: AFLP = marcadores moleculares AFLPs, CLO = marcadores moleculares de cloroplasto, desc = descripción detallada del PDE, ISSR = marcadores moleculares ISSRs, ISZ = isoenzimas, $\mathbf{m p i c}=$ método de probabilidad de identidad clonal, $\mathbf{m t}=$ prueba de $\mathbf{M a n t e l}, \mathbf{p o b}=$ muestra de individuos o todos los individuos de la población, prc = parcelas, RAPD = marcadores moleculares RAPDs, SG $=$ similitud genética vs distancia espacial, $\mathbf{S S R}=$ marcadores moleculares SSRs (microsatélites),

Referencias: ${ }^{*}=$ estudios donde se presentan planos de la distribución espacial de los genotipos.

Todo el cuadro: ? = no se proporciona información, $\mathbf{n} / \mathbf{e}=$ no evaluado, $\mathbf{n} / \mathbf{a}=$ no aplica

GLOSARIO (columna PROR):

Acodaduras: formación de ramets a partir de un tallo originalmente erecto que se postra y genera raíces adventicias al entrar en contacto con el sustrato

Agamospermia: Producción asexual de semillas o embriones sin fertilización (diplosporía: el embrión se desarrolla de una célula diploide del saco embrionario, aposporía: el embrión se desarrolla de una célula somática del saco embrionario, embrionia adventicia: el embrión se desarrolla de células del tejido esporofítico como los tegumentos).

Bulbilos: bulbos pequeños que se forman en las axilas foliares o unidos a los bulbos.

Bulbos: tallos cortos modificados para almacenamiento rodeados por hojas carnosas o brácteas.

Coronas huecas: muerte de los tallos centrales en plantas cespitosas resultando en la formación de ramets independientes en la periferia. Descrito en pastos cespitosos.

Estolones: tallo horizontal que se extiende en la superficie del suelo.

Gemas: pequeñas estructuras vegetativas dispersables en briofitas y pteridofitas.

Plantlets: propágulos vegetativos que resultan de la reversión de estructuras reproductivas como flores y frutos a estructuras vegetativas.

Pseudobulbos: modificación del tallo para almacenamiento que se desarrolla entre dos nodos foliares en orquídeas.

Rizomas: tallo horizontal subterráneo.

Vástagos basales y radicales: formación de tallos en la base de un tallo o a partir de una raíz.

Referencias del glosario: Jackson et al., 1985; de Kroon y van Groenendael, 1997; Liston et al., 2003; van Dijk y Bakx-Schotman, 2004; Tooke et al., 2005. 


\title{
PATRONES DE DISTRIBUCIÓN ESPACIAL EN PLANTAS CLONALES
}

\author{
Israel Gustavo Carrillo-Angeles y María del Carmen Mandujano ${ }^{1}$ \\ Instituto de Ecología, Universidad Nacional Autónoma de México \\ ${ }^{1}$ Autor para la correspondencia: mcmandu@ecologia.unam.mx
}

\begin{abstract}
Resumen: En las especies clonales predomina la distribución agregada de los ramets, pero se plantea que la propagación por diásporas móviles o no ligadas, (por ejemplo, bulbilos y semillas agamospérmicas) forma arreglos espaciales de genets más entremezclados, que la propagación por estructuras ligadas (rizomas y estolones), y se sugiere que los arreglos entremezclados de genets favorecen la entrecruza. En este trabajo se presenta una revisión de 53 estudios en plantas clonales, para investigar la influencia de la producción de propágulos clonales, por diferentes formas de crecimiento clonal, en la formación de arreglos espaciales de genets y ramets. La hipótesis es que los arreglos entremezclados promueven la entrecruza, suponiendo que las poblaciones donde la entrecruza es más frecuente tenderán a presentar niveles más altos de diversidad genotípica. Los estudios revisados muestran un sesgo hacia herbáceas perennes rizomatosas. Predominan los patrones de distribución agregados de parches de ramets de distintos genets no entremezclados. Las poblaciones de especies que tienen ambos tipos de crecimiento de propágulos clonales (ligados y no ligados) y de especies que sólo presentan crecimiento ligado presentan patrones de distribución espacial de genets y ramets más entremezclados que las especies que sólo presentan crecimiento ligado. Encontramos que existe variación intraespecífica tanto en los patrones de distribución espacial como en los patrones reproductivos en las diferentes poblaciones. Sin embargo, utilizando índices de diversidad genotípica como estimadores, solamente se encontró un patrón congruente con el planteamiento sobre el efecto del arreglo espacial de los genets y los ramets en la proporción de genotipos distinguibles. Generalizar las consecuencias reproductivas y genéticas del arreglo espacial de los genets y los ramets continúa siendo complejo, debido a que una reducción del reclutamiento sexual y una dispersión restringida de polen, semillas y propágulos vegetativos pueden generar estructura genética local en las poblaciones. La estructura genética local tanto a nivel de genets (debida a la dispersión restringida de polen, semillas o ambos) como de ramets (indicando dispersión restringida de propágulos vegetativos) fue detectada en 46 poblaciones distribuidas en 16 especies. Estos factores aunados a la variedad de métodos de muestreo empleados en los trabajos publicados para detectar genets y ramets, la falta de estimadores numéricos comparables para describir la distribución espacial y la carencia de estudios en especies que se propagan por diásporas móviles o no ligadas reducen la resolución del efecto que tiene el tipo de crecimiento clonal en la formación de arreglos entremezclados de genets distintos sobre la diversidad genotípica.
\end{abstract}

Palabras clave: clonalidad, crecimiento, distribución, diversidad, entrecruza

\begin{abstract}
Clonal plants are predominantly associated with a clumped distribution of ramets. However, propagation by unlinked means generates a mixed arrangement instead of a clumped one found by linked propagules and intermingled arrangements of genets favor outcrossing. This paper reviews 53 studies on clonal plants to assess the influence of the production of clonal offspring by different means on the spatial arrangement of genets and ramets. We also evaluate the assumption that mixed arrangements promote outcrossing, under the hypothesis that populations where outcrossing is common would have higher levels of genotypic diversity. The studies that were consulted showed a strong bias towards rhizomatous perennial herbs. Clumped distribution patterns predominate in which patches consist of discrete groups of ramets from one or more genets that do not spatially mix. Populations having both types of propagation (linked and unlinked) and species that only have linked propagation have a higher spatially mixed genet and ramet distribution pattern than species that only have unlinked propagation. However, using genotypic diversity indices, we did not find a consistent pattern between the spatial arrangement and outcorssing rates. The reproductive and genetic consequences of the spatial arrangement of genets and ramets continues to be a difficult task, due to a range of factors such as the variety of methods, the lack of good comparative numeric estimations to describe the spatial arrangements and the lack of studies in species that propagate through unlinked propagules.
\end{abstract}

Key words: clonality, distribution, diversity, growth, outcrossing

a clonalidad da lugar a la producción de ramets, es decir, Lunidades potencialmente independientes que son genéticamente idénticas a la planta progenitora (Widén et al., 1994; van Groenendael et al., 1996; de Kroon y van Groenendael,
1997; Klims et al., 1997). En consecuencia, en las poblaciones de organismos clonales el concepto de "individuo" puede definir a los individuos genéticos formados a partir de un cigoto (genets) y a las copias física y fisiológicamente 


\title{
PATRONES DE DISTRIBUCIÓN ESPACIAL EN PLANTAS CLONALES
}

\author{
Israel Gustavo Carrillo-Angeles y María del Carmen Mandujano ${ }^{1}$ \\ Instituto de Ecología, Universidad Nacional Autónoma de México \\ ${ }^{1}$ Autor para la correspondencia: mcmandu@ecologia.unam.mx
}

\begin{abstract}
Resumen: En las especies clonales predomina la distribución agregada de los ramets, pero se plantea que la propagación por diásporas móviles o no ligadas, (por ejemplo, bulbilos y semillas agamospérmicas) forma arreglos espaciales de genets más entremezclados, que la propagación por estructuras ligadas (rizomas y estolones), y se sugiere que los arreglos entremezclados de genets favorecen la entrecruza. En este trabajo se presenta una revisión de 53 estudios en plantas clonales, para investigar la influencia de la producción de propágulos clonales, por diferentes formas de crecimiento clonal, en la formación de arreglos espaciales de genets y ramets. La hipótesis es que los arreglos entremezclados promueven la entrecruza, suponiendo que las poblaciones donde la entrecruza es más frecuente tenderán a presentar niveles más altos de diversidad genotípica. Los estudios revisados muestran un sesgo hacia herbáceas perennes rizomatosas. Predominan los patrones de distribución agregados de parches de ramets de distintos genets no entremezclados. Las poblaciones de especies que tienen ambos tipos de crecimiento de propágulos clonales (ligados y no ligados) y de especies que sólo presentan crecimiento ligado presentan patrones de distribución espacial de genets y ramets más entremezclados que las especies que sólo presentan crecimiento ligado. Encontramos que existe variación intraespecífica tanto en los patrones de distribución espacial como en los patrones reproductivos en las diferentes poblaciones. Sin embargo, utilizando índices de diversidad genotípica como estimadores, solamente se encontró un patrón congruente con el planteamiento sobre el efecto del arreglo espacial de los genets y los ramets en la proporción de genotipos distinguibles. Generalizar las consecuencias reproductivas y genéticas del arreglo espacial de los genets y los ramets continúa siendo complejo, debido a que una reducción del reclutamiento sexual y una dispersión restringida de polen, semillas y propágulos vegetativos pueden generar estructura genética local en las poblaciones. La estructura genética local tanto a nivel de genets (debida a la dispersión restringida de polen, semillas o ambos) como de ramets (indicando dispersión restringida de propágulos vegetativos) fue detectada en 46 poblaciones distribuidas en 16 especies. Estos factores aunados a la variedad de métodos de muestreo empleados en los trabajos publicados para detectar genets y ramets, la falta de estimadores numéricos comparables para describir la distribución espacial y la carencia de estudios en especies que se propagan por diásporas móviles o no ligadas reducen la resolución del efecto que tiene el tipo de crecimiento clonal en la formación de arreglos entremezclados de genets distintos sobre la diversidad genotípica.
\end{abstract}

Palabras clave: clonalidad, crecimiento, distribución, diversidad, entrecruza

\begin{abstract}
Clonal plants are predominantly associated with a clumped distribution of ramets. However, propagation by unlinked means generates a mixed arrangement instead of a clumped one found by linked propagules and intermingled arrangements of genets favor outcrossing. This paper reviews 53 studies on clonal plants to assess the influence of the production of clonal offspring by different means on the spatial arrangement of genets and ramets. We also evaluate the assumption that mixed arrangements promote outcrossing, under the hypothesis that populations where outcrossing is common would have higher levels of genotypic diversity. The studies that were consulted showed a strong bias towards rhizomatous perennial herbs. Clumped distribution patterns predominate in which patches consist of discrete groups of ramets from one or more genets that do not spatially mix. Populations having both types of propagation (linked and unlinked) and species that only have linked propagation have a higher spatially mixed genet and ramet distribution pattern than species that only have unlinked propagation. However, using genotypic diversity indices, we did not find a consistent pattern between the spatial arrangement and outcorssing rates. The reproductive and genetic consequences of the spatial arrangement of genets and ramets continues to be a difficult task, due to a range of factors such as the variety of methods, the lack of good comparative numeric estimations to describe the spatial arrangements and the lack of studies in species that propagate through unlinked propagules.
\end{abstract}

Key words: clonality, distribution, diversity, growth, outcrossing

a clonalidad da lugar a la producción de ramets, es decir, Lunidades potencialmente independientes que son genéticamente idénticas a la planta progenitora (Widén et al., 1994; van Groenendael et al., 1996; de Kroon y van Groenendael,
1997; Klims et al., 1997). En consecuencia, en las poblaciones de organismos clonales el concepto de "individuo" puede definir a los individuos genéticos formados a partir de un cigoto (genets) y a las copias física y fisiológicamente 
independientes de cada genotipo o ramets independientes (Figura 1; Kays y Harper, 1974; Harper, 1985; Jackson et al., 1985). La clonalidad está ampliamente distribuida en plantas, algunas estimaciones para angiospermas varían entre 30\% y más del 50\% (Tiffney y Niklas, 1985; Klim s et al., 1997; Aarssen, 2008), entre el 40\% y $80 \%$ en especies de taiga, tundra y de zonas templadas (van Groenendael y de Kroon, 1990 Klim s et al., 1997) y el 44\% de plantas invasoras en China (Liu et al., 2006), está ampliamente representada en monocotiledóneas y se presenta con baja frecuencia en las gimnospermas (van Groenendael et al., 1996; Peterson y Jones, 1997). Respecto a las condiciones ambientales, se sugiere que la clonalidad predomina en condiciones estresantes como suelos pobres en nitrógeno, suelos húmedos, cuerpos de agua, hábitats sombreados y ambientes fríos en altitudes y latitudes elevadas (Tiffney y Niklas, 1985; van Groenendael et al., 1996; Klim s et al., 1997).

La clonalidad en plantas se ha estudiado con enfoques muy diversos. Por ejemplo, se ha analizado la frecuencia de especies clonales y la variación en las formas de crecimiento clonal en distintos ambientes (p. ej., Tiffney y Niklas, 1985; van Groenendael et al., 1996; Klims et al., 1997), las implicaciones de la integración fisiológica de los ramets y la plasticidad morfológica del crecimiento clonal en ambientes heterogéneos y en condiciones de estrés competitivo (p. ej., Bell, 1984; Hartnett y Bazzaz, 1985; de Kroon y Hutchings, 1995; Price y Marshall, 1999), el efecto de la clonalidad en la demografía (p. ej., Caswell, 1985; McFadden, 1991; Damman y Cain, 1998; Mandujano et al., 2001), la comparación de la diversidad genética entre especies de plantas clonales y no clonales (p. ej., Ellstrand y Roose, 1987; Widén et al., 1994) y las consecuencias reproductivas y evolutivas de la geitonogamia asociada con la clonalidad (p. ej., Handel, 1985; de Jong et al., 1992; Eckert, 2000).

Las implicaciones reproductivas y genéticas de reducir la entrecruza incrementando la geitonogamia han aumentado el interés en el estudio de los patrones de distribución espacial de genets y ramets en poblaciones de plantas clonales. Debido a que los propágulos vegetativos suelen tener una capacidad de dispersión restringida, el reclutamiento clonal frecuente puede reducir la entrecruza e incrementar la autocruza (p. ej., autogamia y geitonogamia), determinando los niveles de variación y la estructuración genética en las poblaciones (Handel, 1985; Eckert, 2002; Ruggiero et al., 2005; Honnay et al., 2006). En especies autocompatibles, los costos reproductivos de la geitonogamia están asociados al incremento de la autogamia y a las probabilidades de expresar depresión endogámica, a la vez que disminuye la adecuación paterna reduciendo la cantidad de polen disponible para la entrecruza; mientras que en las especies autoincompatibles, la geitonogamia afecta la producción de semillas, debido a la saturación de los estigmas con polen incompatible y al aborto de óvulos, y puede resultar en el desperdicio de grandes cantidades de polen (de Jong et al.,
1992; Charpentier, 2002).

En plantas clonales los patrones de distribución espacial pueden ser afectados por la manera en que se generan los ramets. Las formas de crecimiento clonal son muy diversas, pero existen algunas clasificaciones que facilitan el entendimiento de esta variación. Tiffney y Niklas (1985) propusieron cuatro tipos principales de crecimiento: (1) Crecimiento establecido, dado por el inicio del desarrollo de un organismo individual a partir de un propágulo (puede considerarse como crecimento clonal si el propágulo es vegetativo, ya que el propágulo también puede ser una semilla producida sexualmente). (2) Crecimiento restaurativo, que se refiere a la reparación de un daño local en el individuo mediante la formación de tejido calloso, pero no hay regeneración de los órganos completos cuando estos se pierden. (3) Crecimiento regenerativo, que se refiere a la reparación y el reemplazo de órganos dañados (p. ej., crecimiento de una rama desprendida). (4) Crecimiento reproductivo, dado por la multiplicación del individuo fisiológico (p. ej., clonalidad). Estos autores también ordenan los tipos de crecimiento clonal en ligado y no ligado, considerando las estructuras de conexión (Figura 1). En el crecimiento ligado los propágulos vegetativos pueden desarrollarse hasta formar individuos completos (con raíces, tallos y hojas) manteniéndose unidos a la planta madre y su dispersión depende del crecimiento de ramificaciones de tallos y raíces, mientras que en el no ligado, los propágulos vegetativos se desprenden de la planta madre y se desarrollan hasta individuos completos separados de la planta madre. La dispersión de los propágulos en este caso es más dependiente de factores bióticos (p. ej., animales) o abióticos (p. ej., corrientes de agua, gravedad, viento) que en el crecimiento ligado y permite un mayor desplazamiento de los propágulos. El crecimiento reproductivo o clonalidad, que es la que interesa en esta revisión por su influencia en el arreglo espacial de genets y ramets, puede ser de dos tipos: (i) reiteración traumática, definida como la producción de un nuevo individuo inducida por estrés, ya sea por daño o por condiciones ambientales que limitan la reproducción sexual (por ejemplo, la producción de bulbilos en los agaves por daño al escapo floral o por deficiencia de polen) y (ii) la reiteración programada, como la producción de un nuevo individuo por un meristemo como parte normal del desarrollo (Tiffney y Niklas, 1985; Arizaga y Ezcurra, 2002).

La distribución espacial de los individuos en las poblaciones se ha clasificado en tres patrones básicos: aleatorio, uniforme y agregado (Figura 2; Gleason, 1920; Clark y Evans, 1954; Barbour et al., 1987). En poblaciones naturales de plantas, los individuos suelen distribuirse en forma más o menos agregada, debido a la distribución heterogénea de los recursos (Couteron y Kokou, 1997; Chen y Bradshaw, 1999), a la interacción con otras especies (Shreve, 1917; Mandujano et al., 1998) y a la dispersión restringida de semillas y propágulos vegetativos (Gibson y Menges, 1994; Okuda et al., 1997; Clark-Tapia et al., 2005). En plantas 
clonales además, el arreglo espacial de los genets y ramets puede ordenarse en dos patrones básicos, uno caracterizado por la agregación de ramets del mismo genet y el otro por una distribución entremezclada de ramets de distintos genets (Figura 2; de Kroon y Hutchings, 1995; Charpentier, 2002; Clark-Tapia et al., 2005). Estos arreglos espaciales de genets y ramets pueden estar asociados a la manera en que se producen los ramets (esto es por crecimiento clonal ligado o no ligado) y se ha propuesto que en las plantas que se propagan mediante crecimiento no ligado forman arreglos más entremezclados que las especies con crecimiento ligado (Gabrielsen y Brochmann, 1998), pero esto no ha sido demostrado. Por otro lado, debido a que la clonalidad puede incrementar la tasa de geitonogamia entre los ramets del mismo genet, puede esperarse que los arreglos entremezclados reduzcan la geitonogamia y promuevan la entrecruza como resultado del incremento local en el número de genets (Charpentier, 2002).

En algunas revisiones sobre el tema de la clonalidad se han planteado hipótesis sobre las implicaciones reproduc-

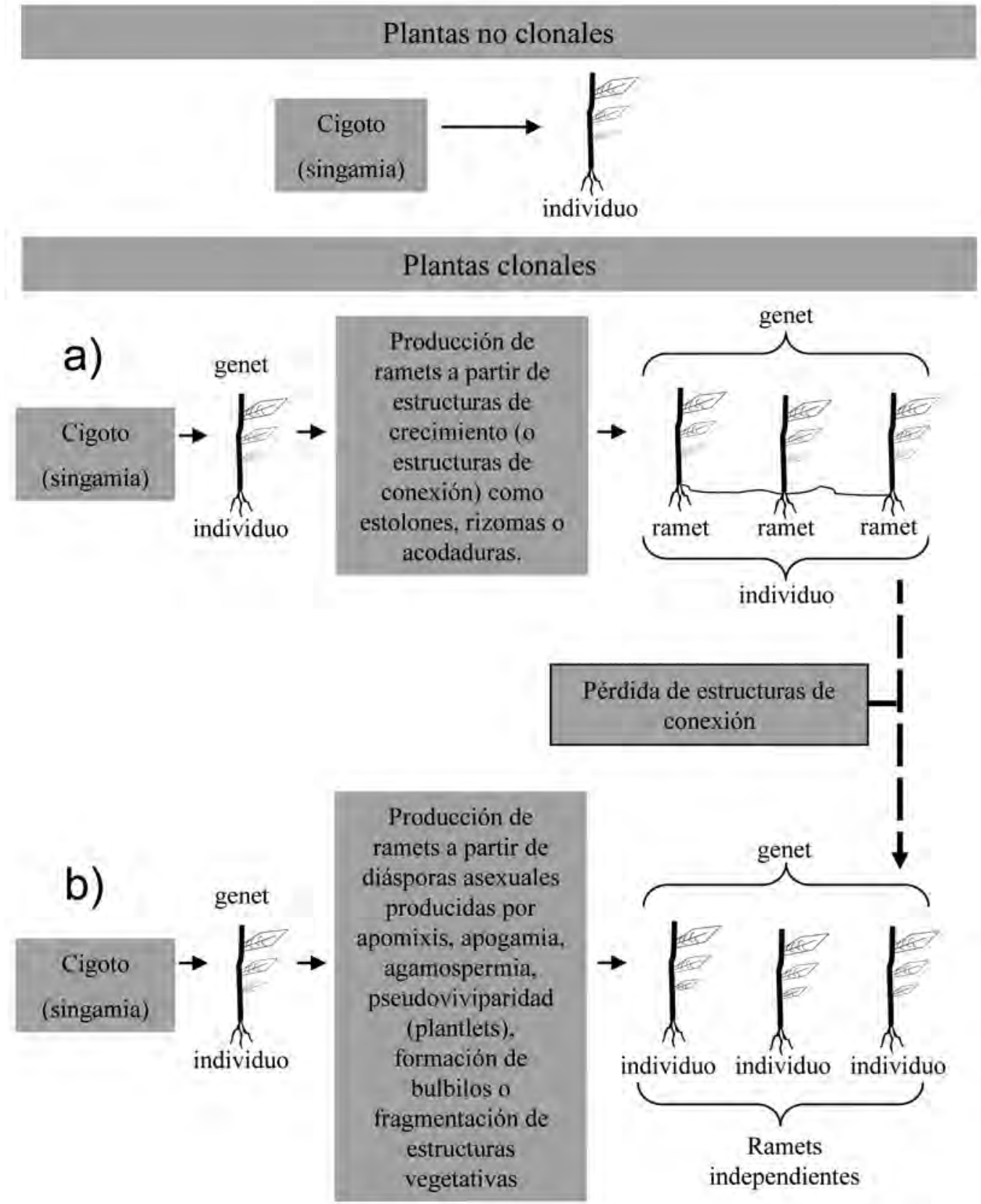

Figura 1. Formación de individuos en plantas no clonales y en plantas clonales. a) crecimiento clonal ligado y b) crecimiento clonal no ligado en la clasificación de Tiffney y Niklas (1985). 
$\mathrm{PDE}=$ Patrones de distribución espacial de los individuos y ramets en las poblaciones

(clonales y no clonales)

\section{AER $=$ Arreglos espaciales de los ramets de distintos genets}

(clonales)

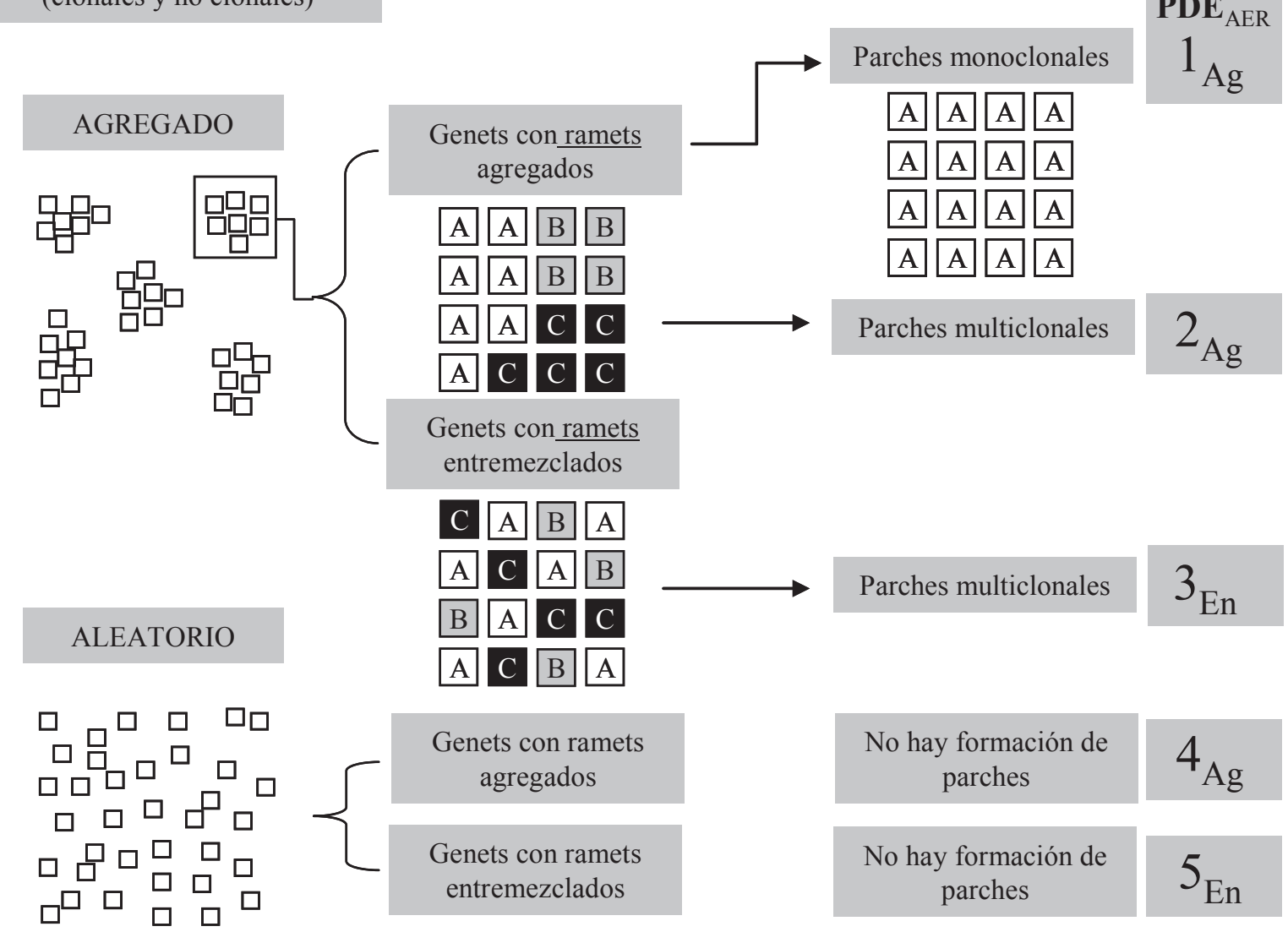

\section{UNIFORME}
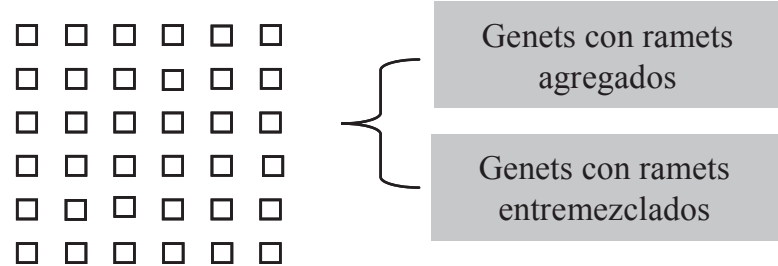

\begin{tabular}{|c|c|c|c|}
\hline $\begin{array}{c}\text { No hay formación de } \\
\text { parches }\end{array}$ & $6_{\mathrm{Ag}}$ \\
\hline $\begin{array}{c}\text { No hay formación de } \\
\text { parches }\end{array}$ & $7_{\mathrm{En}}$ \\
\hline
\end{tabular}

Figura 2. Patrones de distribución espacial de los individuos en las poblaciones de plantas no clonales y de plantas clonales. El número en el extremo derecho representa la clave del patrón de distribución espacial y el arreglo espacial de los ramets de distintos genets (PDE y AER, Apéndice). El subíndice Ag indica que los ramets de cada genet no se entremezclan con los ramets de genets distintos y el subíndice En indica que los ramets de diferentes genets se entremezclan.

tivas y genéticas de los arreglos espaciales de los genets y ramets, asociados con las distintas formas de producir descendientes clonales (Widén et al., 1994; Charpentier, 2002; Mandujano-Sánchez, 2007; Honnay y Jaquemyn, 2008). No obstante, los intentos para probar estas hipótesis son muy escasos y las posibilidades de realizar algún tipo de análisis numérico son restringidas por la baja disponibilidad de publicaciones (p. ej., Widén et al., 1994), o en los análisis sólo se incluyen especies con crecimiento ligado (p. ej., Honnay y Jaquemyn, 2008). En este trabajo presentamos una revisión de varios estudios que evalúan los patrones de distribución espacial en plantas clonales y a partir de la información 
proporcionada en dichos estudios, analizamos el planteamiento de que en poblaciones de plantas con crecimiento clonal ligado el arreglo espacial de los ramets de distintos genets es agregado, mientras que en poblaciones de plantas con crecimiento clonal no ligado predominan los arreglos espaciales entremezclados. Adicionalmente, intentamos poner a prueba la hipótesis de que los arreglos entremezclados promueven la entrecruza suponiendo que las poblaciones donde la entrecruza es más frecuente tenderán a presentar niveles más altos de diversidad genotípica.

\section{Material y métodos}

La revisión de los trabajos publicados sobre la distribución espacial clonal en plantas se llevó a cabo mediante una búsqueda de trabajos en bases de datos especializadas (CSA Cambridge, Ebsco HOST, ISI Web of Knowledge, JSTOR y Scopus) y no especializadas (Google, Google scholar y Yahoo). La búsqueda se hizo empleando los siguientes términos: clonal structure, spatial distribution of genets (andl or ramets), clonal architecture y clonal diversity y abarcó de 1990 a 2011, debido a que la mayoría de los estudios en plantas clonales con este enfoque se han realizado después de 1990.

Los criterios principales para incluir una especie en la revisión fue que se presentara una descripción de la distribución espacial de las plantas individuales (esto es sin importar el genotipo) en las poblaciones naturales y que se evaluara el arreglo espacial de los genets y los ramets (es decir agregado o entremezclado) mediante planos de distribución de los genotipos, análisis de autocorrelación espacial o compatibilidad de las cruzas entre ramets contiguos. Para cada especie se obtuvo el tipo de estructuras vegetativas a mediante las cuales se lleva a cabo la propagación clonal a partir de la información proporcionada en los estudios correspondientes o en estudios previos realizados a menudo por los mismos autores. El segundo criterio en orden de importancia fue que se reportaran valores de diversidad genotípica ( $D=$ índice de diversidad de Simpson y proporción de genotipos distinguibles $G / N$ ) para las poblaciones de las diferentes especies. Otros criterios fueron que se proporcionara información sobre las características reproductivas como la presencia de sistemas de incompatibilidad, la expresión sexual, si se ha observado reclutamiento sexual en las poblaciones y el tipo de polinización. Con base en esta información, se asignó a las especies el tipo de crecimiento clonal (i. e. ligado y no ligado, Figura 1) de acuerdo con la clasificación de Tiffney y Niklas (1985) y el patrón de distribución espacial y el tipo de arreglo espacial de los ramets (agregado o entremezclado) según el esquema presentado en la Figura 2. La información obtenida se presenta en el apéndice.

Para analizar el planteamiento de que en poblaciones de plantas con crecimiento clonal ligado el arreglo espacial de los ramets es agregado, mientras que en poblaciones de plantas con crecimiento clonal no ligado predomina el arreglo espacial entremezclado se construyó una tabla de contingencia con tres categorías de tipo de crecimiento clonal como columnas (ligado, combinación ligado-no ligado y no ligado) y tres categorías del arreglo espacial de los ramets como renglones (agregado, combinación agregado-entremezclado y entremezclado) y se analizó con una prueba de ${ }^{2}$ con el paquete estadístico JMP versión 7.0 (SAS Institute Inc). Para evaluar las celdas que contribuyen significativamente al valor de ${ }^{2}$ se hizo un análisis de residuales ajustados (Everitt, 1977).

Para probar la hipótesis de que las poblaciones con arreglos entremezclados de ramets presentan niveles más altos de diversidad genotípica, se compararon los valores del índice de diversidad de Simpson $(D)$ y la proporción de genotipos distinguibles $(G / N)$ entre las tres categorías de patrón de distribución clonal y entre las tres categorías de tipo de crecimiento clonal con una prueba de Kruskall-Wallis con el paquete estadístico JMP versión 7.0 (SAS Institute Inc).

\section{Resultados}

En la revisión se incluyeron 53 estudios que comprenden 122 poblaciones distribuidas en 44 especies de plantas clonales y en 27 familias (Apéndice). Las familias mejor representadas son Liliaceae y Rosaceae con cuatro especies cada una; Cactaceae, Cyperaceae, Ericaceae, Poaceae y Salicaceae con tres especies cada una y Zosteraceae con dos especies. El resto de las familias estuvieron representadas por una sola especie. Las especies que predominan son herbáceas perennes $(60 \%)$, y las plantas con hábito arbustivo y arbóreo se encuentran poco representadas (20\% en ambos casos).

La mayoría de las especies presentan crecimiento clonal ligado (70\%), dominando la propagación a través de rizomas (45\% de las especies). Las especies con crecimiento clonal ligado y no ligado representan el 14\% propagándose clonalmente por crecimiento ligado a través de rizomas y acodaduras, y por crecimiento no ligado a través de bulbilos, plantlets y desprendimiento de ramas; las especies sólo con crecimiento no ligado representan el 16\%, propagándose por bulbilos, semillas producidas por agamospermia, desprendimiento de ramas y gémulas (ver glosario para la definición de las estructuras implicadas en el crecimiento ligado y no ligado, Apéndice). Respecto a la distribución espacial, $50 \%$ del total de las especies presentan genets con ramets agregados, $30 \%$ presentan una combinación de arreglos agregados-entremezclados y $20 \%$ presentan arreglos entremezclados (Apéndice).

$\mathrm{Al}$ comparar las poblaciones con diferentes arreglos espaciales de genets y ramets [agregados (Ag), En (entremezcaldos) y $\mathrm{Ag}+\mathrm{En}$ (combinados)] encontramos que las poblaciones de especies con crecimiento ligado y no ligado y 
Cuadro 1. Número (observado y esperado) de poblaciones con diferentes tipos de crecimiento clonal de acuerdo con la clasificación de Tiffney y Niklas (1985) ( $\mathrm{Lg}=$ ligado, Lg+NI = ligado y no ligado, $\mathrm{Nl}=$ no ligado), que mostraron arreglos espaciales de los ramets de distintos genets: Ag = agregados, Ag+En = combinación de agregados y entremezclados, y En = entremezclados (figura 2). Los valores entre paréntesis indican el número de especies y familias (especies/familias) representadas en cada celda. Los valores de los residuales ajustados en negritas indican las celdas que contribuyen significativamente al valor de $\chi^{2}$, el signo indica mayor (sin signo) o menor número (-) de poblaciones de las esperadas por azar.

\begin{tabular}{|c|c|c|c|}
\hline Observados & Ligado & Ligado+No ligado & No ligado \\
\hline Agregado & $49(21 / 13)$ & $7(2 / 2)$ & $4(2 / 2)$ \\
\hline Agregado+entremezclado & $11(5 / 5)$ & $25(5 / 5)$ & $7(3 / 3)$ \\
\hline Entremezclado & $10(5 / 5)$ & $2(2 / 1)$ & $7(2 / 2)$ \\
\hline \multicolumn{4}{|l|}{ Esperados } \\
\hline & $\operatorname{Lg}$ & $\mathrm{Lg}+\mathrm{NI}$ & $\mathrm{Nl}$ \\
\hline Agregado & 34 & 17 & 9 \\
\hline Agregado+entremezclado & 25 & 12 & 6 \\
\hline Entremezclado & 11 & 5 & 3 \\
\hline \multicolumn{4}{|l|}{ Residuales ajustados } \\
\hline & $\operatorname{Lg}$ & $\mathrm{Lg}+\mathrm{NI}$ & $\mathrm{Nl}$ \\
\hline Agregado & 5.34 & -3.93 & -2.48 \\
\hline Agregado+entremezclado & -5.24 & 5.50 & 0.35 \\
\hline Entremezclado & -0.46 & -1.83 & 2.95 \\
\hline
\end{tabular}

de especies que sólo presentan crecimiento no ligado presentaron arreglos espaciales (AER) más entremezclados que las poblaciones de especies que sólo presentan crecimiento ligado ( ${ }^{2}=43.6, P<0.0001,4$ g.l.; Cuadro 1). Predominan los patrones de distribución (PDE) con ramets distribuidos en parches discretos sobre el área ocupada por la población (patrón de distribución espacial agregado, $46 \%$ de las poblaciones); estos parches son multiclonales (con dos o más genets) en la mayoría de los casos y el arreglo espacial de los ramets de distintos genets es agregado (19\% de las poblaciones, $\mathrm{PDE}_{\mathrm{AER}} 2_{\mathrm{Ag}}$, Figura 2), presenta una combinación agregado-entremezclado (10\% de las poblaciones, combinación $\mathrm{PDE}_{\mathrm{AER}} 2_{\mathrm{Ag}}-3_{\mathrm{En}}$, Figura 2) o es entremezclado solamente (3\% de las poblaciones, $\mathrm{PDE}_{\mathrm{AER}} 3_{\mathrm{En}}$, Figura 2). En otras poblaciones donde los ramets se distribuyen formando parches discretos el arreglo espacial de los ramets de distintos genets es agregado, pero los parches son multiclonales y monclonales (9\% de las poblaciones, combinación $\mathrm{PDE}_{\mathrm{AER}}$ $1_{\mathrm{Ag}}-\mathrm{Ag}_{\mathrm{Ag}}$, Figura 2) o sólo son monoclonales (5\% de las poblaciones, $\mathrm{PDE}_{\mathrm{AER}} 1_{\mathrm{Ag}}$, Figura 2).

Los patrones de distribución espacial con ramets distribuidos aleatoriamente sobre el área ocupada por la población (patrón de distribución espacial aleatorio) representaron el $44 \%$ de las poblaciones, predominando el arreglo espacial agregado-entremezclado de los ramets de distintos genets $\left(22 \%\right.$ de las poblaciones, combinación de $\mathrm{PDE}_{\mathrm{AER}}$ $4_{\mathrm{Ag}}-5_{\mathrm{En}}$, Figura 2), el arreglo agregado se presentó en $10 \%$ de las poblaciones ( $\mathrm{PDE}_{\mathrm{AER}} 4_{\mathrm{Ag}}$, Figura 2) y el arreglo entremezclado en $12 \%$ de las poblaciones $\left(\mathrm{PDE}_{\mathrm{AER}} 5_{\mathrm{En}}\right.$, Figura
2). El resto de las poblaciones (10\%) presentaron una combinación de patrones de distribución con ramets distribuidos aleatoriamente sobre una parte del área ocupada por la población y en otra formando parches discretos monoclonales o parches multiclonales con arreglos espaciales agregados y entremezclado de los ramets de distintos genets (combinación de $\mathrm{PDE}_{\mathrm{AER}} 1_{\mathrm{Ag}}-4_{\mathrm{Ag}}, 1_{\mathrm{Ag}}-5_{\mathrm{En}}, 2_{\mathrm{Ag}}-4_{\mathrm{Ag}}, 2_{\mathrm{Ag}}-5_{\mathrm{En}}$, Figura 2).

Las poblaciones de especies con una combinación de tipos de crecimiento clonal ligado y no ligado $(\mathrm{Lg}-\mathrm{Nl})$ presentaron valores más bajos de la proporción de genotipos distinguibles (promedio \pm E.E., $G / N=0.24 \pm 0.04 ; H=$ 6.93, $P=0.031,2$ g.l.) que las poblaciones de especies con crecimiento ligado (promedio \pm E.E., $G / N=0.38 \pm 0.03$ ) y con crecimiento no ligado (promedio \pm E.E., $G / N=0.39 \pm$ 0.06 ), pero las diferencias sólo fueron significativas con respecto a las poblaciones de especies con crecimiento ligado (Figura 3a). Respecto al arreglo espacial de los ramets de distintos genets, las poblaciones con una combinación de arreglos agregado y entremezclado (Ag-En) presentaron valores menores del índice de diversidad de Simpson (promedio \pm E.E., $D=0.57 \pm 0.05 ; H=13.2, P=0.0014,2$ g.l.) que las poblaciones con arreglos agregados (promedio \pm E.E., $D=0.80 \pm 0.04$ ) y con arreglos entremezclados (promedio \pm E.E., $D=0.76 \pm 0.06$; Figura 3). Estas diferencias sugieren que el reclutamiento sexual es más bajo en poblaciones de especies que presentan una combinación de formas de crecimiento clonal ligado-no ligado ( $\mathrm{Lg}-\mathrm{Nl}$ ) y una combinación de arreglos de los ramets de distintos genets agregado-entremezclado (Ag-En), sin embargo estas diferencias 

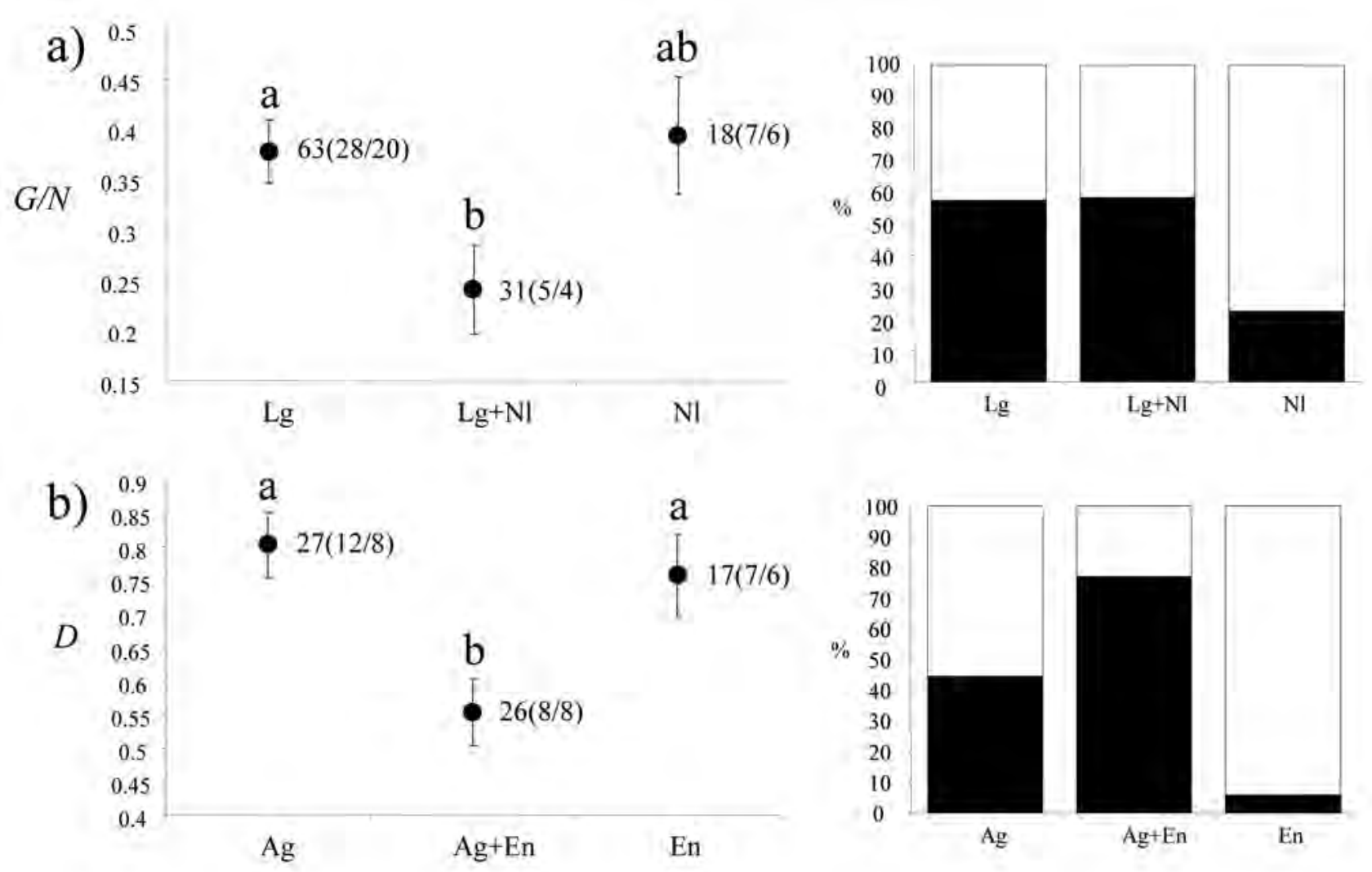

Figura 3. Comparación de la diversidad clonal (promedio \pm EE) entre poblaciones de especies con a) distinto tipo de crecimiento clonal: $\mathrm{Lg}=$ ligado, $\mathrm{Lg}+\mathrm{Nl}$ = con ambos tipos de crecimiento y $\mathrm{Nl}$ = no ligado y b) con distintos arreglos espaciales de los ramets de distintos genets: $\mathrm{Ag}=$ agregados, $\mathrm{Ag}+\mathrm{En}$ = combinación de agregados y entremezclados, y En = entremezclados (Figura 2). En cada grupo se indica el número de poblaciones (número de especies / número de familias) y los grupos que difieren significativamente se indican con una letra distinta arriba de la barra del promedio \pm E.E. de cada grupo. Las gráficas de barras del lado derecho muestran el porcentaje de poblaciones con polinización abiótica (en negro) y con polinización biótica (en blanco).

pueden estar asociadas con otros factores como el tipo de polinización, y no con el tipo de crecimiento y el arreglo espacial de los ramets de distintos genets. La mayoría de las poblaciones con tipo de crecimiento $\mathrm{Lg}-\mathrm{Nl}$ y arreglos de ramets de distintos genets Ag-En corresponden a especies con polinización abiótica (Figura 3).

\section{Discusión}

El efecto que tienen los modos de clonalidad en las plantas en la conformación de distintos arreglos espaciales de genets y ramets es un campo relativamente novedoso, que se ha explorado en los últimos 20 años y la mayoría de los estudios que hay sobre el tema se han desarrollado después de 1990. Widén et al. (1994) reportan que de 40 estudios que comprenden un total de 45 especies de plantas clonales, menos de la tercera parte proporcionan información sobre el arreglo espacial de los genets en las poblaciones y sólo en $12.5 \%$ se construyeron planos de distribución de todos los ramets, dentro de las parcelas o poblaciones, identificando los genotipos multilocus. En esta revisión, en el $98 \%$ de los estudios incluidos se identifican los genotipos multilocus mediante marcadores dominantes, codominantes o ambos siguiendo tres métodos de muestreo: en el 56\% de los estudios se utilizan parcelas, en el $24 \%$ transectos y en el $18 \%$ se muestrean todos o varios individuos de las poblaciones. El 2\% restante está representado por un estudio donde el patrón de distribución es descrito y la distribución de los ramets se sugiere por experimentos de cruzas compatibles entre ramets cercanos (en Scirpus maritimus, Charpentier et al., 2000).

A pesar de que en la actualidad se cuenta con más información sobre los patrones de distribución espacial en poblaciones de especies clonales, hacer comparaciones de dichos patrones considerando especies con distintas formas de crecimiento clonal para analizar las consecuencias reproductivas y genéticas del arreglo espacial de los genets y los ramets continúa siendo un reto. Algunos factores que influyen en este hecho son que la evaluación de la variación en los patrones de distribución espacial en plantas clonales 
enfrenta la dificultad que implica trabajar con la mayoría de los individuos en muchas poblaciones, principalmente a nivel molecular. Aunque en la actualidad las herramientas moleculares se han vuelto más accesibles, trabajar con un gran número de ejemplares sigue siendo difícil y costoso. Otros problemas son la falta de estimadores numéricos comparables para describir la distribución espacial en las poblaciones de diferentes especies y la variación en los métodos de muestreo. Por ejemplo, el muestreo mediante transectos colectando muestras de ramets cada determinada distancia (p. ej., Jonsson et al., 1996; Esselman et al., 1999) permite evaluar la capacidad de expansión longitudinal de los genets, pero no permite tener una descripción de la expansión radial y por lo tanto una evaluación más precisa del nivel de entremezcla de los ramets de distintos genets. En contraste, el muestreo de todos o de un gran número de individuos dentro de parcelas o en las áreas ocupadas por las poblaciones es otro método empleado en estudios con especies de plantas clonales (p. ej., Shimizu et al., 2002; Ruggieiro et al., 2005). Este tipo de muestreo puede ser un método más adecuado para analizar y comparar la estructura espacial clonal, porque permite detectar el arreglo radial de distintos genets. Sin embargo, los tamaños de muestra muy grandes pueden limitar la aplicación de este método, ya que en la mayoría de los casos es necesario emplear marcadores moleculares para identificar a los genets.

En particular, además de los problemas mencionados anteriormente es importante considerar las dificultades adicionales que detectamos en este estudio y que pueden afectar los patrones encontrados a través de nuestra aproximación. La mayoría de los estudios que analizan la distribución espacial de genets y ramets parecen estar sesgados hacia especies de herbáceas perennes con crecimiento clonal ligado (45\% de las especies), predominando las especies rizomatosas (43\% de las especies), mientras que las especies con crecimiento no ligado están poco representadas, impidiendo contar con tamaños de muestra equivalentes para hacer comparaciones más precisas. Por otro lado, la aproximación que decidimos emplear en este trabajo compara a las poblaciones más que a las especies, debido a que hemos detectado que hay una importante variación intraespecífica tanto en los patrones de distribución como en los patrones reproductivos. No obstante, esta variación se omite en algunos estudios o está mejor representada para algunas especies.

Los resultados obtenidos en este trabajo sugieren que las especies que se propagan por crecimento no ligado tienden a formar arreglos espaciales de los ramets de distintos genets más entremezclados como se ha planteado (Gabrielsen y Brochmann, 1998). No obstante, en especies con crecimiento ligado que además se propagan clonalmente por crecimiento no ligado, como el desprendimiento de ramas, el cual consideramos en este estudio como una forma de crecimiento no ligado en los análisis, debido a que los ramets originados por este medio se desarrollan hasta individuos com- pletos separados de la planta madre y a que su dispersión no depende del crecimiento horizontal de tallos y raíces (p.ej., Lophocereus schottii, Parker y Hamrick, 1992; Pteridium aquilinum, Parks y Werth, 1993; Cladium jamaicense, Ivey y Richards, 2001; Ferocactus robustus, Carrillo-Angeles et al., 2011), o que se propagan por la extensión de rizomas en especies con crecimiento en guerrilla (p. ej., Cymodocea nodosa, Ruggieiro et al., 2005 e Ilex leucoclada, Torimaru y Tomaru, 2005) también forman arreglos de genets entremezclados. Algunos factores externos que pueden influir en el arreglo entremezclado de genets son la dispersión por actividades humanas (Parks y Werth, 1993; Brzosko et al., 2002), el acarreo de propágulos vegetativos por animales y corrientes de agua (Parker y Hamrick, 1992; Brzosko et al., 2002), el reclutamiento de nuevos genets en áreas perturbadas donde permanecen fragmentos de clones que ocuparon grandes extensiones (Parks y Werth, 1993) y la dispersión de semillas producidas por autogamia que presentan genotipos multilocus idénticos (Keane et al., 1999; Brzosko et al., 2002).

Respecto a la hipótesis de que las poblaciones con arreglos espaciales entremezclados de los ramets de distintos genets presentan mayor entrecruza, medido indirectamente mediante la diversidad genotípica, nuestros resultados concuerdan parcialmente con el patrón esperado. El G/N es significativamente mayor en los arreglos de especies con producción de vástagos no ligados, en donde predominan los arreglos de genets entremezclados. Para probar esta hipótesis consideramos que si la entrecruza es más alta en poblaciones con arreglos entremezclados, habría mayores niveles de diversidad genotípica. Sin embargo, ésto no se refleja en el caso del índice de Simpson. Las diferencias en los índices de diversidad genotípica entre las poblaciones de especies que combinan el crecimiento clonal ligado-no ligado ( $\mathrm{Lg}$ $\mathrm{Nl})$ y el arreglo espacial agregado-entremezclado (Ag-En), respecto de las poblaciones que presentan uno u otro tipo de crecimiento y de arreglo espacial están asociadas con el tipo de polinización (biótica o abiótica), lo cual apoya el planteamiento de que la polinización biótica puede incrementar la eficiencia de la fertilización (Barret et al.,1993; Richards, 1997; Reusch, 2003). Los análisis para probar este planteamiento están fuera del alcance del presente estudio.

Además de los efectos sobre el reclutamiento sexual y la diversidad genotípica, los patrones de distribución espacial en especies clonales pueden afectar la diversidad y la estructura genética de las poblaciones. Por ejemplo, la formación de parches monoclonales en especies con entrecruza obligada, aunada al desplazamiento de genets por competencia, puede eliminar la capacidad de una población para reproducirse sexualmente en tiempos relativamente cortos (Honnay y Bossuyt, 2005). Adicionalmente, una reducción del reclutamiento sexual y una dispersión restringida de polen, semillas y propágulos vegetativos pueden generar estructura genética local en las poblaciones. Este segundo aspecto se 
evalúa en varios de los estudios revisados (47\%). La estructura genética local tanto a nivel de genets (indicando una dispersión restringida de polen, semillas o ambos) como de ramets (indicando dispersión restringida de propágulos vegetativos) se detectó en 46 poblaciones distribuidas en 16 especies; 28 poblaciones corresponden a cinco especies arbóreas que muestran estructura genética local a distancias menores de $50 \mathrm{~m}$ (Sequoia sempervirens, Douhovnikoff et al., 2004) y a distancias menores de $25 \mathrm{~m}$ (Eurya emarginata, Chung y Epperson, 2000; Cryptomeria japonica, Shimizu et al., 2002; Hirayama y Sakimoto, 2008; Populus tremula, Suvanto y Latva-Karjantvaa, 2005); tres poblaciones corresponden a dos especies arbustivas (Echinosophora koreensis, Chung et al., 2006; Stenocereus eruca, ClarkTapia et al., 2005) que presentan estructura genética local a distancias menores de $20 \mathrm{~m}$. El resto de las poblaciones corresponden a hierbas perennes que muestran estructura genética local desde distancias menores de $30 \mathrm{~m}$, pero más frecuentemente a distancias menores de $15 \mathrm{~m}$.

En promedio, los valores del índice de diversidad de Simpson $(D)$ y la proporción de genotipos distinguibles $(G / N)$ fueron altos en las poblaciones representadas en los estudios incluidos en la presente revisión y son similares a los reportados para 21 especies de plantas clonales $(D=$ 0.62 y $G / N=0.16$ ) por Ellstrand y Roose (1987). Se han planteado algunas hipótesis sobre los escenarios que pueden explicar altos niveles de diversidad genotípica: (i) las poblaciones pudieron ser fundadas por varios propágulos sexuales que se mantienen por clonalidad, (ii) el reclutamiento sexual fue más frecuente en el pasado, aunque en la actualidad la producción de descendientes sexuales no sea evidente (Ellstrand y Roose, 1987; Eriksson, 1989, 1993; Widén et al., 1994); (iii) los genotipos heterócigos se ven favorecidos selectivamente, manteniendo altos niveles de diversidad genética (Widén et al., 1994); (iv) los riesgos de mortalidad entre los ramets de un genet pueden ser distintos si tienen independencia física y fisiológica (Eriksson 1989, 1993, Pan y Price, 2002), lo cual puede reducir las probabilidades de supervivencia / extinción del genet; (v) en especies con ciclos de vida largos los eventos raros de reclutamiento sexual pueden ser suficientes para mantener niveles altos de diversidad genética (Eriksson 1989, 1993, Parker y Hamrick, 1992; Brzosko et al., 2002; Clark-Tapia et al., 2005); (vi) los genotipos pueden mantenerse a través de la supervivencia de algunos ramets el tiempo suficiente para que algunos genotipos se integren a la población de manera esporádica; (vii) la heterogeneidad ambiental puede generar condiciones de selección diversificante que permiten la coexistencia de varios genotipos (Widén et al., 1994). Otra posibilidad para el mantenimiento de diversidad clonal en poblaciones de plantas, que surge al considerar la variación de la distribución espacial de genets y ramets, es que la clonalidad puede mantener altos niveles de entrecruza e incrementar las probabilidades de reclutamiento sexual si los ramets se dispersan hacia vecindarios genéticamente variables, debido a la distribución entremezclada de genets.

Las explicaciones a los niveles relativamente altos de diversidad genotípica en las poblaciones de las especies incluidas en esta revisión comprenden los eventos esporádicos de reclutamiento sexual y el establecimiento inicial de varios genets. En algunos casos se sugiere un reclutamiento sexual frecuente a partir de los altos niveles de diversidad genotípica detectados con los marcadores moleculares (p. ej., Parks y Werth, 1993; Diggle et al., 1998; Misuki et al., 2010). No obstante, el reclutamiento sexual en poblaciones naturales sólo se reporta en Prunus ssiori, Rubus saxatilis (Eriksson y Bremer, 1993), Cypripedium calceolus (Brzosko et al., 2002) y Maianthemum dilatatum (Wilson et al., 2005b). Otros factores que se proponen para explicar la diversidad genotípica son que la integración fisiológica entre los ramets de un genet puede reducir el riesgo de que un genotipo en particular desaparezca, cuando las condiciones ambientales locales son adversas, contribuyendo a mantener la diversidad genética (Hartnett y Bazzaz, 1985), el incremento en la tasa de entrecruza, debido a la entremezcla de genets en especies con crecimiento en guerrilla (Ruggieiro et al., 2005) y en especies que se propagan clonalmente por desprendimiento de ramas y producción de bulbilos (Parker y Hamrick, 1992; Gabrielsen y Brochmann, 1998).

El estudio de los patrones de distribución espacial en especies clonales es importante para analizar las consecuencias reproductivas y genéticas del arreglo espacial de los genets y los ramets en las poblaciones naturales. Estas consecuencias pueden explorarse de una manera más precisa analizando la variación de la distribución de genets y ramets entre las poblaciones de una misma especie. Sin embargo, la evidencia de estos aspectos en organismos clonales aún es escasa y hace falta realizar estudios considerando diferentes tipos de crecimiento clonal. El análisis de los patrones de distribución espacial pueden ser muy útiles para evaluar otros aspectos del ciclo de vida de las especies clonales como el efecto de una variación persistente en la producción de propágulos clonales y sexuales entre hábitats, que puede afectar diferencialmente la dinámica de las poblaciones. Asimismo, es necesario analizar la dinámica temporal de los arreglos espaciales de los genets y ramets en las poblaciones para hacer inferencias adecuadas sobre las consecuencias ecológicas y evolutivas de la clonalidad.

\section{Agradecimientos}

Proyectos: IN IN205007 Efecto de la estructura clonal sobre la dinámica poblacional y la reproducción de una cactácea con ciclo de vida complejo, Opuntia microdasys.

Becas: doctorado Conacyt a I. Carrillo-Angeles y estancia sabática en New Mexico State University, otorgada por DGAPA-PASPA- Universidad Nacional Autónoma de México a M. Mandujano. Este trabajo es parte de la tesis de 
doctorado de Israel Gustavo Carrillo Angeles, Posgrado en Ciencias Biológicas de la UNAM. Agradecemos la revisión, sugerencias en el trabajo así como el apoyo de los miembros del comité tutoral, Doctores Teresa Valverde y Francisco Molina. Asimismo a la Dra. Ana Mendoza, Dr. Jordan Golubov y dos revisores anónimos por sus sugerencias al artículo.

\section{Literatura citada}

Aarssen L.W. 2008. Death without sex-the 'problem of the small' and selection for reproductive economy in flowering plants. Evolutionary Ecology 22:279-298.

Albert T., Raspé O. y Jacquemart A.L. 2003. Clonal structure in Vaccinium myrtillus L. revealed by RAPD and AFLP markers. International Journal of Plant Sciences 164:649-655.

Arens P., Grashof-Bokdam C.J., van der Sluis T. y Smulders M.J.M. 2005. Clonal diversity and genetic differentiation of Maianthemum bifolium among forest fragments of different age. Plant Ecology 179:169-180.

Arizaga S. y Ezcurra E. 2002. Propagation mechanisms in Agave macrovantha (Agavaceae), a tropical arid land succulent rosette. American Journal of Botany 89:632-641.

Barbour M.G., Burk J.H. y Pitts W.D. 1987. Terrestrial Plant Ecology. The Benjamin-Cummings Publishing Company, Menlo Park.

Barret S.C.H., Eckert C.G. y Husband B.C. 1993. Evolutionary processes in aquatic plant populations. Aquatic Botany 44:105-145.

Bell A.D. 1984. Dynamic morphology: A contribution to plant population ecology. En: Dirzo R. y Sarukhán J. Eds. Perspectives on Plant Population Ecology, pp. 49-65, Sinauer Associates Inc. Publisher, Sunderland.

Brzosko E., Wróblewska A. y Ratkiewicz M. 2002. Spatial genetic structure and clonal diversity of island populations of lady's slipper (Cyperidium calceolus) from the Biebrza National Park (northeast Poland). Molecular Ecology 11:2499-2509.

Bushakra J.M., Hodges S.A., Cooper J.B. y Kaska D.D. 1999. The extent of clonality and genetic diversity in the Santa Cruz Island ironwood, Lyonothamnus floribundus. Molecular Ecology 8:471-475.

Camacho F.J. y Liston A. 2001. Population structure and genetic diversity of Botrychium pumicola (Ophioglossaceae) based on inter-simple sequence repeats (ISSR). American Journal of Botany 88:1065-1070.

Carrillo-Angeles I.G., Mandujano M.C. y Golubov J. 2011. Influences of the genetic neighborhood on ramet reproductive success in a clonal desert cactus. Population Ecology 53:449458.

Caswell H. 1985. The evolutionary demography of clonal reproduction. En: Jackson J.B.C., Buss L.W. y Cook R.E. Eds. Population Biology and Evolution of Clonal Organisms, pp. 187224, Yale University Press, New Haven.

Charpentier A. 2002. Consequences of clonal growth for plant mating. Evolutionary Ecology 15:521-530.

Charpentier A., Grillas P. y Thompson J.D. 2000. The effects of population size limitation on fecundity in mosaic populations of the clonal macrophyte Scirpus maritimus (Cyperaceae). American Journal of Botany 87:502-507.

Chen J. y Bradshaw G.A. 1999. Forest structure in space: a case study of an old growth spruce-fir forest in Changbaishan Natural Reserve, PR China. Forest Ecology and Management 120:219-233.

Chung M.G. y Epperson B.K. 2000. Clonal and spatial genetic structure in Eurya emarginata (Theaceae). Heredity 84:170177.

Chung J.M., Lee B.C., Kim J.S., Park C., Chung M.Y. y Chung M.G. 2006. Fine-scale genetic structure among genetic individuals of the clone-forming monotypic genus Echinospora koreensis (Fabaceae). Annals of Botany 98:165-173.

Clark P.J. y Evans F.C. 1954. Distance to nearest neighbor as a measure of spatial relationships in populations. Ecology 35:445-453.

Clark-Tapia R., Alfonso-Corrado C., Eguiarte L.E. y Molina-Freaner F. 2005. Clonal diversity and distribution in Stenocereus eruca (Cactaceae), a narrow endemic cactus of the Sonoran Desert. American Journal of Botany 92:272-278.

Clark-Tapia R., Alfonso-Corrado C., Mandujano M.C. y MolinaFreaner F. 2006. Reproductive consequences of clonal growth in Stenocereus eruca, a rare clonal cactus of the Sonoran desert. Evolutionary Ecology 20:131-142.

Couteron P. y Kokou K. 1997. Woody vegetation spatial patterns in a semi-arid savanna of Burkina Faso, West Africa. Plant Ecology 132:211-227.

Damman H. y Cain M.L. 1998. Population growth and viability analyses of the clonal woodland herb, Asarum canadense. Journal of Ecology 86:13-26.

de Jong T.J., Waser N.M., Price M.V. y Ring R.M. 1992. Plant size, geitonogamy and seed set in Ipomopsis aggregata. Oecologia 89:310-315.

de Kroon H. y van Groenendael J. Eds. 1997. The Ecology and Evolution of Clonal Plants. Backhuys Publishers, Leiden.

de Kroon H. y Hutchings M.J. 1995. Morphological plasticity in clonal plants: the foraging concept reconsidered. Journal of Ecology 83:143-152.

Diggle P.K., Lower S. y Ranker T.A. 1998. Clonal diversity in alpine populations of Polygonum viviparum (Polygonaceae). International Journal of Plant Sciences 159:606-615.

Diggle P.K., Meixner M.A., Carroll A.B. y Aschwanden C.F. 2002. Barriers to sexual reproduction in Polygonum viviparum: A comparative developmental analysis of $P$. viviparum and $P$. bistortoides. Annals of Botany 89:145-156.

Douhovnikoff V. y Dood R.S. 2003. Intra-clonal variation and a similarity threshold for identification of clones: application to $\mathrm{Sa}$ lix exigua using AFLP molecular markers. Theoretical Applied Genetics 106:1307-1315.

Douhovnikoff V., Cheng A.M. y Dodd R.S. 2004. Incidence, size and spatial structure of clones in second-growth stands of coast redwood, Sequoia sempervirens (Cupressaceae). American Journal of Botany 91:1140-1146.

Douhovnikoff V., McBride J.R. y Dodd R.S. 2005. Salix exigua clonal growth and population dynamics in relation to disturbance regime variation. Ecology 86:446-452.

Eckert C.G. 2000. Contributions of autogamy and geitonogamy to self-fertilization in a mass-flowering, clonal plant. Ecology 81:532-542.

Eckert C.G. 2002. The loss of sex in clonal plants. Evolutionary Ecology 15:501-520.

Ellstrand N.C. y Roose M.L. 1987. Patterns of genotypic diversity in clonal plant species. American Journal of Botany 74:123-131. 
Eriksson O. 1989. Seedling dynamics and life histories in clonal plants. Oikos 55:231-238.

Eriksson O. 1993. Dynamics of genets in clonal plants. Trends in Ecology \& Evolution 8:313-316.

Eriksson O. y Bremer B. 1993. Genet dynamics of the clonal plant Rubus saxatilis. Journal of Ecology 81:533-542.

Esselman E.J., Jianqiang L., Crawford D.J., Winduss J.L. y Wolfe A.D. 1999. Clonal diversity in the rare Calamagrostis porteri ssp insperata (Poaceae): comparative results for allozymes and random amplified polymorphic DNA (RAPD) and intersimple sequence repeat (ISSR) markers. Molecular Ecology 8:443451.

Everitt B.S. 1977. The Analysis of Contingency Tables. Chapmann \& Hall, Bury St Edmunds.

Gabrielsen T.M. y Brochmann C. 1998. Sex after all: high levels of diversity detected in the arctic clonal plant Saxifraga cernua using RAPD markers. Molecular Ecology 7:1701-1708.

Garnier L.K.M., Durand J. y Dajoz I. 2002. Limited seed dispersal and microspatial population structure of an agamospermous grass of West African savannahs, Hyparrhenia diplandra (Poaceae). American Journal of Botany 89:1785-1791.

Gibson D.J. y Menges E.S. 1994. Population structure and spatial pattern in the dioecious shrub Ceratiola ericoides. Journal of Vegetation Sciences 5:337-346.

Gleason H.A. 1920. Some applications of the quadrat method. Bulletin of the Torrey Botanical Club 47:21-33.

Hämmerli A. y Reusch T.B.H. 2003a. Flexible mating: cross-pollination affects sex expression in a marine clonal plant. Journal of Evolutionary Biology 18:1096-1105.

Hämmerli A. y Reusch T.B.H. 2003b. Genetic neighbourhood of clone structures in eelgrass meadows quantified by spatial autocorrelation of microsatellite markers. Heredity 91:448-455.

Handel S.N. 1985. The intrusion of clonal growth patterns on plant breeding system. The American Naturalist 125:367-384.

Harper J. 1985. Modules, branches, and the capture of resources. En: Jackson J.B.C., Buss L.W. y Cook R.E. Eds. Population Biology and Evolution of Clonal Organisms, pp. 1-33, Yale University Press, New Haven.

Hartnett D.C. y Bazzaz F.A. 1985. The integration of neighbourhood effects by clonal genets in Solidago canadensis. The Journal of Ecology 73:415-427.

Hirayama K. y Sakimoto M. 2008. Clonal structure and diversity of Cryptomeria japonica along a slope in a cool-temperate, oldgrowth mixed forest in the snowy region of Japan. Canadian Journal of Forest Research 38:2804-2813.

Honnay O. y Bossuyt B. 2005. Prolonged clonal growth: escape route or route to extinction? Oikos 108:427-432.

Honnay O., Jacquemyn H., Roldán-Ruiz I. y Hermy M. 2006. Consequences of prolonged clonal growth on local and regional genetic structure and fruiting success of the forest perennial Maianthemum bifolium. Oikos 112:21-30.

Honnay O. y Jacquemyn H. 2008. A meta-analysis of the relation between mating system, growth form and genotypic diversity in clonal plant species. Evolutionary Ecology 22:299-312.

Ivey C.T. y Richards J.H. 2001. Genotypic diversity and clonal structure of everglades sawgrass, Cladium jamaicense (Cyperaceae). International Journal of Plant Sciences 162:1327-1335.

Jackson J.B.C., Buss L.W. y Cook R.E. Eds. 1985. Population Biology and Evolution of Clonal organisms. Yale University Press, New Haven.
Jacquemyn H., Brys R., Honnay O., Hermy M. y Roldán-Ruiz I. 2005. Local forest environment largely affects below-ground growth, clonal diversity and fine-scale spatial genetic structure in the temperate deciduous forest herb Paris quadrifolia. Molecular Ecology 14:4479-4488.

Jonsson B.O., Jónsdóttir I.S. y Crongberg N. 1996. Clonal diversity and allozyme variation in populations of the arctic sedge Carex bigelowii (Cyperaceae). Journal of Ecology 84:449-459.

Kays S. y Harper J.L. 1974. The regulation of plant and tiller density in a grass sward. Journal of Ecology 62:97-105.

Keane B., Pelikan S., Toth G.P., Smith M.K. y Rogstad S.H. 1999. Genetic diversity of Typha latifolia (Typhaceae) and the impact of pollutants examined with tamdem-repetitive DNA probes. American Journal of Botany 86:1226-1238.

Kjølner S., Såstad S.M. y Brochmann C. 2006. Clonality and recombination in the arctic plant Saxifraga cernua. Botanical Journal of the Linnean Society 152:209-217.

Klimeš L., Klimešová J., Hendriks R.J.J. y van Groenendael J.M. 1997. Clonal plant architecture: a comparative analysis of form and function. En: de Kroon H. y van Groenendael J. Eds. The Ecology and Evolution of Clonal Plants, pp. 1-29, Backhuys Publishers, Leiden.

Korpelainen H. 1994. Sex ratios and resource allocation among sexually reproducing plants of Rubus chamaemorus. Annals of Botany 74:627-632.

Korpelainen H., Antonious-Klemola K. y Werlemark G. 1999. Clonal structure of Rubus chamaemorus populations: comparison of different molecular methods. Plant Ecology 143:123-128.

Kreher S.A., Foré S.A. y Collins B.S. 2000. Genetic variation within and among patches of the clonal species, Vaccinium stamineum L. Molecular Ecology 9:1247-1252.

Kudoh H., Shibaike H., Takasu H., Whigham D.F. y Kawano S. 1999. Genet structure and determinants of clonal structure in a temperate deciduous woodland herb, Uvularia perfoliata. Journal of Ecology 87:244-257.

Liston A., Wilson B.L., Robinson W.A., Doescher P.S., Harris N.R. y Svejcar T. 2003. The relative importance of sexual reproduction versus clonal spread in an arid bunchgrass. Oecologia 137:216-225.

Liu J., Dong M., Miao S.L., Li Z.Y., Song M.H. y Wang R.Q. 2006. Invasive alien plants in China: role of clonality and geographical origin. Biological Invasions 8:1461-1470.

Mandujano M.C., Montaña C., Méndez I. y Golubov J. 1998. The relative contributions of sexual reproduction and clonal propagation in Opuntia rastrera from two habitats in the Chihuahuan desert. Journal of Ecology 86:911-921.

Mandujano M.C., Montaña C., Franco M., Golubov J. y FloresMartínez A. 2001. Integration of demographic annual variability in a clonal desert cactus. Ecology 82:344-359.

Mandujano-Sánchez M.C. 2007. La clonalidad y sus efectos en la biología de poblaciones. En: Eguiarte L.E., Souza V. y Aguirre X. Comp. Ecología Molecular, pp. 215-250, Secretaría de Medio Ambiente y Recursos Naturales-Instituto Nacional de Ecología-Universidad Nacional Autónoma de México-Comosión Nacional para el Conocimiento y Uso de la Biodiversidad, México, D.F.

Mayes S.G., McGinley M.A. y Werth C.R. 1998. Clonal population structure and genetic variation in sand-shinnery oak, Quercus havardii (Fagaceae). American Journal of Botany 85:1609-1617. McFadden C.S. 1991. A comparative demographic analysis of clonal 
reproduction in a temperate soft coral. Ecology 72:1849-1866.

Misuki I., Ishida K., Tani N. y Tsumura Y. 2010. Fine-scale spatial structure of genets and sexes in the dioecious plant Dioscorea japonica, which disperses by both bulbils and seeds. Evolutionary Ecology 24:1399-1415.

Mori Y., Nagamitsu T. y Kubo T. 2009. Clonal growth and its effects on male and female reproductive success in Prunus ssiori (Rosaceae). Population Ecology 51:175-186.

Nagamitsu T., Ogawa M., Ishida K. y Tanouchi H. 2004. Clonal diversity, genetic structure, and mode of recruitment in a Prunus ssiori population established after volcanic eruptions. Plant Ecology 174:1-10.

Okuda T., Kachi N., Yap S.K. y Manokaran N. 1997. Tree distribution pattern and fate of juveniles in a lowland tropical rain forest - implications for regeneration and maintenance of species diversity. Plant Ecology 131:155-171.

Pan J.J. y Price J.S. 2002. Fitness and evolution in clonal plants: the impact of clonal growth. Evolutionary Ecology 15:583-600.

Parker K.C. y Hamrick J.L. 1992. Genetic diversity and clonal structure in a columnar cactus, Lophocereus schottii. American Journal of Botany 79:86-96.

Parks C. y Werth C.R. 1993. A study of spatial features of clones in a population of bracken fern, Pteridium aquilinum (Dennstaedtiaceae). American Journal of Botany 80:537-544.

Peterson C.J. y Jones R.H. 1997. Clonality in woody plants: a review and comparison with clonal herbs. En: de Kroon H. y van Groenendael J. Eds. The Ecology and Evolution of Clonal Plants, pp. 263-289, Backhuys Publishers, Leiden.

Pfeiffer T., Klahr A., Heinrich A. y Schnittler M. 2011. Does sex make a difference? Genetic diversity and spatial genetic structure in two co-occurring species of Gagea (Liliaceae) with contrasting reproductive strategies. Plant Systematics and Evolution 292:189-201.

Pornon A. y Escaravage N. 1999. Genotypic structure in clonal Rhododendron ferrugineum L. (Ericaceae) populations: origin and manteinance. Plant Ecology 141:145-150.

Pornon A., Escaravage N., Thomas P. y Taberlet P. 2000. Dynamics of genotypic structure in clonal Rhododendron ferrugineum (Ericaceae) populations. Molecular Ecology 9:1099-1111.

Pornon A., Escaravage N., Till-Botraud I. y Doche B. 1997. Variation of reproductive traits in Rhododendron ferrugineum $\mathrm{L}$. (Ericaceae) populations along a successional gradient. Plant Ecology 130:1-11.

Price E.A.C. y Marshall C. 1999. Clonal plants and environmental heterogeneity. Plant Ecology 141:3-7.

Reisch C., Schurm S. y Poschlod P. 2007. Spatial genetic structure and clonal diversity in an alpine population of Salix herbacea (Salicaceae). Annals of Botany 99:647-651.

Reusch T.B.H. 2003. Floral neighbourhoods in the sea: how floral density, opportunity for outcrossing and population fragmentation affect seed set in Zostera marina. Journal of Ecology 91:610-615.

Richards A.J. 1997. Plant Breeding Systems. Chapman \& Hall,
Londres.

Rosseto M., Gross C.L., Jones R. y Hunter J. 2004. The impact of clonality on an endangered tree (Elaeocarpus williamsianus) in a fragment rain forest. Biological Conservation 117:33-39.

Ruggieiro M.V., Capone S., Pirozzi P., Reusch T.B.H. y Procaccini G. 2005. Mating system and clonal architecture: a comparative study in two marine angiosperms. Evolutionary Ecology 19:487-499.

Shimizu Y, Ando M. y Sakai F. 2002. Clonal structure of natural populations of Cryptomeria japonica growing at different positions on slopes, detected using RAPD markers. Biochemical Systematics and Ecology 30:733-748.

Shreve F. 1917. The establishment of desert perennials. Journal of Ecology 5:210-216.

Sipes S.D. y Wolf P.G. 1997. Clonal structure and patterns of allozyme diversity in the rare endemic Cycladenia humilis var. jonesii (Apocynaceae). American Journal of Botany 84:401-409.

Stehlik I. y Holderegger R. 2000. Spatial genetic structure and clonal diversity of Anemone nemorosa in late successional deciduous woodlands of Central Europe. Journal of Ecology 88:424-435.

Suvanto L.I. y Latva-Karjantvaa T.B. 2005. Clone identification and clonal structure of the European aspen (Populus tremula). Molecular Ecology 14:2851-2860.

Tiffney B.H. y Niklas K.J. 1985. Clonal growth in land plants: a paleobotanical perspective. En: Jackson J.B.C., Buss L.W. y Cook R.E. Eds. Population Biology and Evolution of Clonal Organisms, pp. 35-66, Yale University Press, New Haven.

Tooke F., Ordidge M., Chiurugwi T. y Battey N. 2005. Mechanisms and function of flower and inflorescence reversion. Journal of Experimental Botany 56:2587-2599.

Torimaru T. y Tomaru N. 2005. Fine-scale clonal structure and diversity within patches of a clone-forming dioecious shrub, Ilex leucoclada (Aquifoliaceae). Annals of Botany 95:295-304.

van Dijk P.J. y Bakx-Schotman J.M.T. 2004. Formation of unreduced megaspores (diplospory) in apomictic dandelions (Taraxacum officinale, s.l.) is controlled by a sex-specific dominant locus. Genetics 166:483-492.

van Groenendael J.M. y de Kroon H. 1990. Clonal Growth in Plants: Regulation and Function. SPB Academic Publishing, La Haya.

van Groenendael J.M., Klimeš L., Klimešová J. y Hendriks R.J.J. 1996. Comparative ecology of clonal plants. Philosophical Transactions of Royal Society of London 351:1331-1339.

Widén B., Cronberg N. y Widén M. 1994. Genotypic diversity, molecular markers and spatial distribution of genets in clonal plants, a literature survey. Folia Geobotanica y Phytotaxonomica 29:245-263.

Wilson A.S.G., van der Kamp B.J. y Ritland C. 2005a. Opportunities for geitonogamy in the clonal herb Maianthemum dilatatum. Canadian Journal of Botany 83:1082-1087.

Wilson A.S.G., van der Kamp B.J. y Ritland C. 2005b. Spatial genetic and clonal structure in Maianthemum dilatatum as defined by AFLP markers. Canadian Journal of Botany 83:1126-1132. 
Apéndice. Características de las especies y poblaciones: $\mathbf{S C R Z}=$ sistema de cruza, $\mathbf{P O L}=$ polinización, $\mathbf{P R O R}=$ estructuras o procesos que dan origen a los ramets, CCT-N = tipo de crecimiento clonal en la clasificación de Tiffney y Niklas (1985), POB/HL = número de población y/o hábitat local, $\mathbf{P D E}=$ clave del patrón de distribución espacial de acuerdo a la figura 2, AER = arreglo espacial de los ramets de distintos genets, $\boldsymbol{D}=$ índice de diversidad clonal de Simpson, $\boldsymbol{G} / \mathbf{N}=$ proporción de genotipos distinguibles, Métodos = métodos empleados para la evaluación de los parámetros. El significado de las abreviaturas empleadas en cada columna se muestra al final del cuadro.

\begin{tabular}{|c|c|c|c|c|c|c|c|c|c|c|c|c|c|}
\hline Especie (Familia) & Hábito & SCRZ & POL & PROR & CCT-N & $\begin{array}{c}\text { POB/ } \\
\mathrm{HL}\end{array}$ & $\begin{array}{c}\text { PDE } \\
\text { (fig. 2) }\end{array}$ & $\begin{array}{c}\text { AER } \\
\text { (fig. 2) }\end{array}$ & $D$ & $G / N$ & $\begin{array}{l}\text { Estructura } \\
\text { genética } \\
\text { local (EGL) }\end{array}$ & $\begin{array}{c}\text { Métodos } \\
\text { PDE, } D, G / N \\
\text { y EGL }\end{array}$ & Referencias \\
\hline $\begin{array}{l}\text { Anemone } \\
\text { nemorosa } \\
\text { (Ranunculaceae) }\end{array}$ & $\mathrm{Hp}$ & A & $\mathrm{Bt}$ & $\begin{array}{c}\mathrm{Rzm+} \\
\mathrm{drm}\end{array}$ & $\mathrm{Lg}+\mathrm{NI}$ & $\begin{array}{l}\text { ppm } \\
(20)\end{array}$ & $(4,5)$ & $\mathrm{Ag}+\mathrm{En}$ & $\mathrm{n} / \mathrm{e}$ & 0.95 & $\begin{array}{l}\operatorname{asp},<1 \mathrm{~m} \\
(\mathrm{rm})\end{array}$ & tra+ISZ+asp & $\begin{array}{l}\text { Stehlik y } \\
\text { Holderegger } \\
\text { 2000* }\end{array}$ \\
\hline $\begin{array}{l}\text { Botrychium } \\
\text { pumicola } \\
\text { (Ophioglossaceae) }\end{array}$ & $\mathrm{Hp}$ & A & $\mathrm{Ab}$ & gmas & $\mathrm{NI}$ & $\begin{array}{l}\text { p } 1 \\
\text { p } 2 \\
\text { p } 3\end{array}$ & $\begin{array}{l}(2,5) \\
(2,5)\end{array}$ & $\begin{array}{l}\mathrm{Ag}+\mathrm{En} \\
\mathrm{Ag}+\mathrm{En} \\
\mathrm{Ag}+\mathrm{En}\end{array}$ & $\begin{array}{c}0.14 \\
0.16 \\
0.1\end{array}$ & $\begin{array}{l}0.93 \\
0.9 \\
0.65\end{array}$ & $\begin{array}{c}\text { mt: no } \\
\text { detectada } \\
\text { mt: no } \\
\text { detectada } \\
\text { mt: no } \\
\text { detectada }\end{array}$ & prc + ISSR $+m t$ & $\begin{array}{c}\text { Camacho y } \\
\text { Liston, 2001* }\end{array}$ \\
\hline $\begin{array}{l}\text { Calamagrostis } \\
\text { porteri ssp. } \\
\text { insperata (Poaceae) }\end{array}$ & $\mathrm{Hp}$ & A & $\mathrm{Ab}$ & rzm & $\operatorname{Lg}$ & $\begin{array}{l}\text { p } 2 \\
\text { p } 3 \\
\text { p } 4\end{array}$ & $\begin{array}{l}2 \\
2 \\
2\end{array}$ & $\begin{array}{l}\mathrm{Ag} \\
\mathrm{Ag} \\
\mathrm{Ag}\end{array}$ & $\begin{array}{l}n / e \\
n / e \\
n / e\end{array}$ & $\begin{array}{c} \\
1 \\
0.82 \\
0.91\end{array}$ & $\begin{array}{l}\mathrm{n} / \mathrm{e} \\
\mathrm{n} / \mathrm{e} \\
\mathrm{n} / \mathrm{e}\end{array}$ & $\begin{array}{c}\text { desc+tra+ } \\
\text { ISRR+ } \\
\text { RAPD }\end{array}$ & $\begin{array}{c}\text { Esselman } \\
\text { et al., } \\
1999\end{array}$ \\
\hline $\begin{array}{l}\text { Carex bigelowii } \\
\text { (Cyperaceae) }\end{array}$ & $\mathrm{Hp}$ & $?$ & $\mathrm{Ab}$ & rzm & $\operatorname{Lg}$ & $\begin{array}{l}\text { p } 1 \\
\text { p } 2 \\
\text { p } 3\end{array}$ & $\begin{array}{l}4 \\
4 \\
4\end{array}$ & $\begin{array}{l}\mathrm{Ag} \\
\mathrm{Ag} \\
\mathrm{Ag}\end{array}$ & $\begin{array}{l}0.98 \\
0.98 \\
0.98\end{array}$ & $\begin{array}{l}0.52 \\
0.63 \\
0.48\end{array}$ & $\begin{array}{l}\text { n/e } \\
\text { n/e } \\
\text { n/e }\end{array}$ & tra+ISZ & $\begin{array}{c}\text { Jonsson } \\
\text { et al., 1996* }\end{array}$ \\
\hline $\begin{array}{l}\text { Cladium jamaicense } \\
\text { (Cyperaceae) }\end{array}$ & $\mathrm{Hp}$ & $?$ & $\mathrm{Ab}$ & $\begin{array}{c}\text { rzm+ } \\
\text { pnt }\end{array}$ & $\mathrm{Lg}+\mathrm{Nl}$ & $\begin{array}{l}p 1 \\
\text { p } 2 \\
\text { p } 3 \\
\text { p } 4 \\
\text { p } 5 \\
\text { p } 6 \\
\text { p } 7 \\
\text { p } 8 \\
\text { p } 9 \\
\text { p } 10 \\
\text { p } 11 \\
\text { p } 12 \\
\text { p } 13 \\
\text { p } 14\end{array}$ & $\begin{array}{l}(4,5) \\
(4,5) \\
(4,5) \\
(4,5) \\
(4,5) \\
1 \\
(4,5) \\
(4,5) \\
(4,5) \\
(4,5) \\
(4,5) \\
(4,5) \\
(4,5) \\
(4,5)\end{array}$ & $\begin{array}{c}A g+E n \\
A g+E n \\
A g+E n \\
A g+E n \\
A g+E n \\
A g \\
A g+E n \\
A g+E n \\
A g+E n \\
A g+E n \\
A g+E n \\
A g+E n \\
A g+E n \\
A g+E n\end{array}$ & $\begin{array}{c}0.236 \\
0.269 \\
0.271 \\
0.682 \\
0.159 \\
0 \\
0.538 \\
0.212 \\
0.771 \\
0.698 \\
0.814 \\
0.682 \\
0.673 \\
0.444\end{array}$ & $\begin{array}{l}0.044 \\
0.044 \\
0.125 \\
0.231 \\
0.043 \\
0.021 \\
0.146 \\
0.068 \\
0.106 \\
0.143 \\
0.261 \\
0.114 \\
0.13 \\
0.043\end{array}$ & $\begin{array}{c}\text { mpic: no } \\
\text { detectada } \\
\text { mpic: no } \\
\text { detectada } \\
\text { mpic: no } \\
\text { detectada } \\
\text { mpic: no } \\
\text { detectada } \\
\text { mpic: no } \\
\text { detectada } \\
\text { n/a } \\
\text { mpic: no } \\
\text { detectada } \\
\text { mpic: no } \\
\text { detectada } \\
\text { mpic: no } \\
\text { detectada } \\
\text { mpic: no } \\
\text { detectada } \\
\text { mpic: no } \\
\text { detectada } \\
\text { mpic: no } \\
\text { detectada } \\
\text { mpic: no } \\
\text { detectada } \\
\text { mpic: no } \\
\text { detectada }\end{array}$ & $\begin{array}{l}\text { tra+lSZ+ } \\
\text { mpic }\end{array}$ & $\begin{array}{l}\text { Ivey y Richards, } \\
\text { 2001* }\end{array}$ \\
\hline
\end{tabular}


Apéndice. Continuación

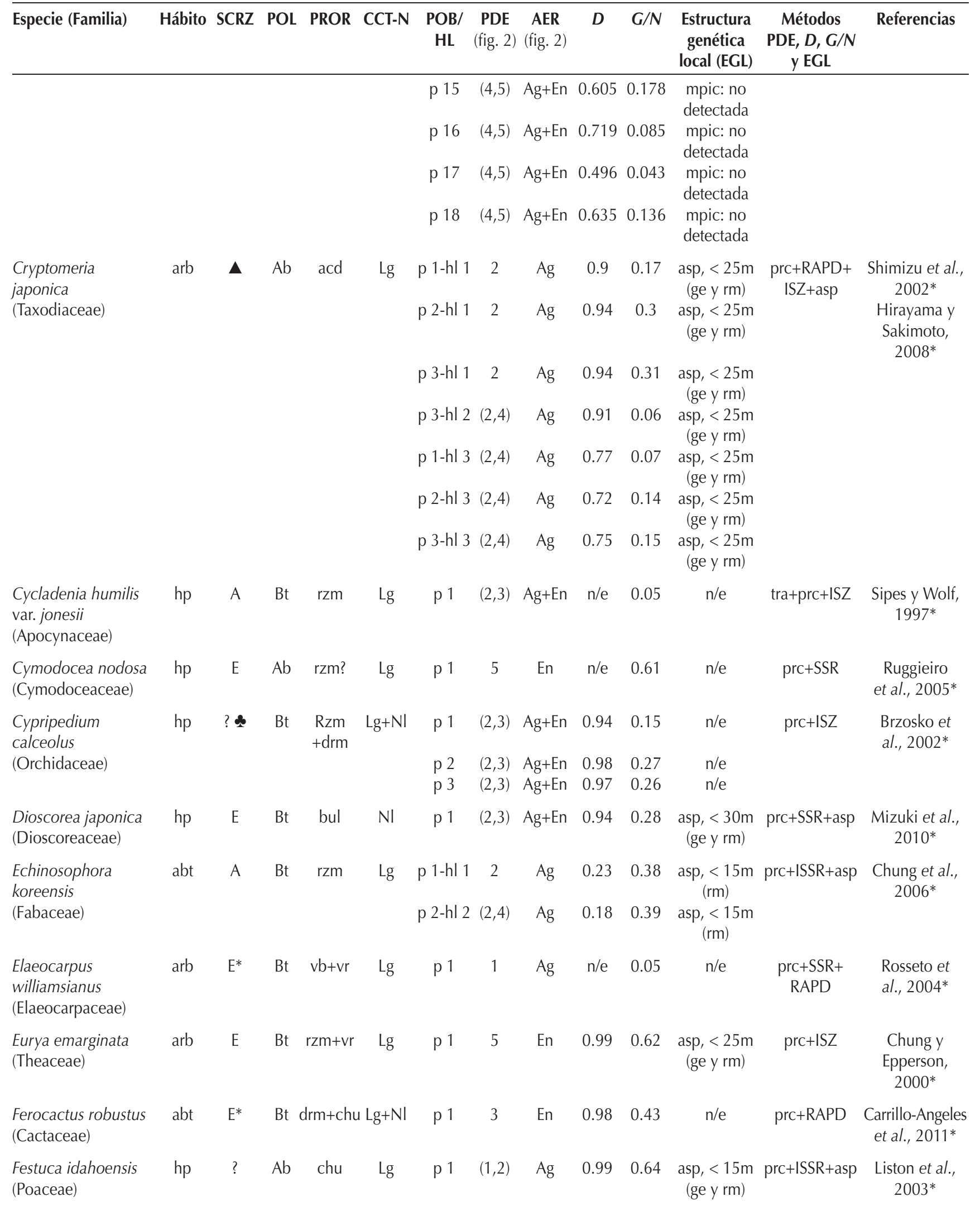


Apéndice. Continuación

\begin{tabular}{|c|c|c|c|c|c|c|c|c|c|c|c|c|c|}
\hline Especie (Familia) & Hábito & SCRZ & POL & PROR & CCT-N & $\begin{array}{c}\mathrm{POB} / \\
\mathrm{HL}\end{array}$ & $\begin{array}{l}\text { PDE } \\
\text { (fig. 2) }\end{array}$ & $\begin{array}{c}\text { AER } \\
\text { (fig. 2) }\end{array}$ & $D$ & $G / N$ & $\begin{array}{l}\text { Estructura } \\
\text { genética } \\
\text { local (EGL) }\end{array}$ & $\begin{array}{c}\text { Métodos } \\
\text { PDE, } D, G / N \\
\text { y EGL } \\
\end{array}$ & Referencias \\
\hline \multirow[t]{3}{*}{$\begin{array}{l}\text { Gagea lutea } \\
\text { (Liliaceae) }\end{array}$} & \multirow[t]{3}{*}{ hg } & \multirow[t]{3}{*}{$?$} & \multirow[t]{3}{*}{$\mathrm{Bt}$} & \multirow[t]{3}{*}{ buls } & \multirow[t]{3}{*}{$\mathrm{Nl}$} & p 1 & $(2,3)$ & $\mathrm{Ag}+\mathrm{En}$ & $\mathrm{n} / \mathrm{e}$ & 0.51 & \multirow{3}{*}{\multicolumn{2}{|c|}{$\begin{array}{l}\text { asp, }<8 \mathrm{~m}, 16 \text { tra+AFLP+asp } \\
\mathrm{a}<24 \mathrm{~m}(\mathrm{rm}) \\
\mathrm{asp},<8 \mathrm{~m}, 16 \\
\mathrm{a}<24 \mathrm{~m}(\mathrm{rm}) \\
\mathrm{asp},<8 \mathrm{~m}, 16 \\
\mathrm{a}<24 \mathrm{~m}(\mathrm{rm})\end{array}$}} & \multirow[t]{3}{*}{$\begin{array}{l}\text { Pfeiffer et al., } \\
2011^{*}\end{array}$} \\
\hline & & & & & & p 2 & $(2,3)$ & $\mathrm{Ag}+\mathrm{En}$ & $\mathrm{n} / \mathrm{e}$ & 0.57 & & & \\
\hline & & & & & & p 3 & $(2,3)$ & $\mathrm{Ag}+\mathrm{En}$ & $\mathrm{n} / \mathrm{e}$ & 0.61 & & & \\
\hline \multirow{3}{*}{$\begin{array}{l}\text { Gagea spathacea } \\
\text { (Liliaceae) }\end{array}$} & \multirow[t]{3}{*}{ hg } & \multirow[t]{3}{*}{$?$} & \multirow[t]{3}{*}{$\mathrm{Bt}$} & \multirow[t]{3}{*}{ buls } & \multirow[t]{3}{*}{$\mathrm{NI}$} & p 1 & 1 & $\mathrm{Ag}$ & $\mathrm{n} / \mathrm{e}$ & 0.001 & $\mathrm{n} / \mathrm{a}$ & \multirow[t]{3}{*}{ tra+AFLP } & \multirow{3}{*}{$\begin{array}{r}\text { Pfeiffer et al., } \\
2011^{*}\end{array}$} \\
\hline & & & & & & p 2 & 1 & $\mathrm{Ag}$ & $\mathrm{n} / \mathrm{e}$ & 0.001 & $\mathrm{n} / \mathrm{a}$ & & \\
\hline & & & & & & p 3 & 1 & $\mathrm{Ag}$ & $\mathrm{n} / \mathrm{e}$ & 0.001 & $\mathrm{n} / \mathrm{a}$ & & \\
\hline $\begin{array}{l}\text { Hyparrhenia } \\
\text { diplandra (Poaceae) }\end{array}$ & hp & $?$ & $\mathrm{Ab}$ & agp & $\mathrm{NI}$ & p 1 & 5 & En & 0.85 & 0.07 & $\begin{array}{l}\text { asp, }<6 \mathrm{~m} \\
\quad(\mathrm{rm})\end{array}$ & $\begin{array}{c}\text { prc+lSZ } \\
+ \text { CLO+asp }\end{array}$ & $\begin{array}{c}\text { Garnier et al., } \\
2002^{*}\end{array}$ \\
\hline $\begin{array}{l}\text { Ilex leucoclada } \\
\text { (Aquifoliaceae) }\end{array}$ & abt & E & $\mathrm{Bt}$ & $\operatorname{acd} \mathbf{\Lambda}$ & $\operatorname{Lg}$ & p 1 & $(2,3)$ & $\mathrm{Ag}+\mathrm{En}$ & 0.67 & 0.09 & $\mathrm{n} / \mathrm{e}$ & prc+RAPD & $\begin{array}{c}\text { Torimaru y } \\
\text { Tomaru, 2005* }\end{array}$ \\
\hline \multirow[t]{8}{*}{$\begin{array}{l}\text { Lophocereus schottii } \\
\text { (Cactaceae) }\end{array}$} & \multirow[t]{8}{*}{ arb } & \multirow[t]{8}{*}{ A } & \multirow[t]{8}{*}{$\mathrm{Bt}$} & \multirow[t]{8}{*}{$\begin{array}{l}\mathrm{drm} \\
+\mathrm{acd}\end{array}$} & \multirow[t]{8}{*}{$\mathrm{Lg}+\mathrm{NI}$} & p 1-hl 1 & 5 & En & 0.95 & 0.64 & $\begin{array}{c}\text { SG: 0-10 } \\
<10-50>\text { más } \\
\text { de } 50 m \text { (ge) }\end{array}$ & \multirow{8}{*}{ prc+ISZ+SG } & \multirow[t]{8}{*}{$\begin{array}{l}\text { Parker y } \\
\text { Hamrick, } \\
1992^{*}\end{array}$} \\
\hline & & & & & & p 2-hl 2 & 2 & $\mathrm{Ag}$ & 0.95 & 0.52 & $\begin{array}{l}\text { SG: } 0-10 \\
<10-50>\text { más } \\
\text { de } 50 \mathrm{~m} \text { (ge) }\end{array}$ & & \\
\hline & & & & & & p 3-hl 3 & 2 & $\mathrm{Ag}$ & 0.93 & 0.65 & $\begin{array}{c}\text { SG: } 0-10 \\
<10-50>\text { más } \\
\text { de } 50 m \text { (ge) }\end{array}$ & & \\
\hline & & & & & & p 4-hl 1 & $(1,2)$ & $\mathrm{Ag}$ & 0.94 & 0.24 & $\begin{array}{l}\text { SG: } 0-10> \\
\text { 10-50>más } \\
\text { de } 50 \mathrm{~m} \text { (ge) }\end{array}$ & & \\
\hline & & & & & & p 5-hl 4 & $(1,2)$ & $\mathrm{Ag}$ & 0.92 & 0.41 & $\begin{array}{l}\text { SG: } 0-10> \\
10-50<\text { más } \\
\text { de } 50 m \text { (ge) }\end{array}$ & & \\
\hline & & & & & & p 6-hl 4 & $(1,2)$ & $\mathrm{Ag}$ & 0.87 & 0.45 & $\begin{array}{c}\text { SG: } 0-10> \\
10-50<\text { más } \\
\text { de } 50 m \text { (ge) }\end{array}$ & & \\
\hline & & & & & & p 7-hl 2 & $(1,2)$ & $\mathrm{Ag}$ & 0.8 & 0.18 & $\begin{array}{l}\text { SG: } 0-10> \\
\text { 10-50>más } \\
\text { de } 50 \mathrm{~m} \text { (ge) }\end{array}$ & & \\
\hline & & & & & & p 8-hl 5 & $(1,5)$ & $\mathrm{Ag}+\mathrm{En}$ & 0.59 & 0.36 & $\begin{array}{l}\text { SG: } 10-50= \\
\text { más de } 50 \mathrm{~m} \\
\quad(\text { ge })\end{array}$ & & \\
\hline $\begin{array}{l}\text { Lyonothamnus } \\
\text { floribundus } \\
\text { (Rosaceae) }\end{array}$ & arb & $?$ & $\mathrm{Bt}$ & $\mathrm{vr}$ & $\operatorname{Lg}$ & p 1 & $(1,2)$ & $\mathrm{Ag}$ & $\mathrm{n} / \mathrm{e}$ & 0.036 & $\mathrm{n} / \mathrm{e}$ & prc+RAPD & $\begin{array}{c}\text { Bushakra et al., } \\
\text { 1999* }\end{array}$ \\
\hline $\begin{array}{l}\text { Maianthemum } \\
\text { bifolium } \\
\text { (Convalliariaceae) }\end{array}$ & hp & $E^{*}$ & $\mathrm{Bt}$ & $\mathrm{rzm}$ & $\operatorname{Lg}$ & p 1 & $(1,2)$ & $\mathrm{Ag}$ & 0.88 & 0.7 & $\begin{array}{l}\text { asp, }<50 \mathrm{~m} \\
\text { (ge) \# }\end{array}$ & $\begin{array}{l}\text { trans+AFLP } \\
\quad+\text { asp }\end{array}$ & $\begin{array}{l}\text { Arens et al., } \\
2005^{*} \\
\text { Honnay et al. } \\
2006\end{array}$ \\
\hline $\begin{array}{l}\text { Maianthemum } \\
\text { dilatatum } \\
\text { (Convalliariaceae) }\end{array}$ & hp & EAs & $\mathrm{Bt}$ & $\operatorname{rzm} \mathbf{\Delta}$ & $\operatorname{Lg}$ & p 1 & $(1,2)$ & $\mathrm{Ag}$ & $\mathrm{n} / \mathrm{e}$ & 0.64 & $\begin{array}{l}\text { asp, }<45 \mathrm{~m} \\
\text { (ge y rm) }\end{array}$ & $\begin{array}{l}\text { prc }+ \text { AFLP } \\
\quad+\text { asp }\end{array}$ & $\begin{array}{l}\text { Wilson et al., } \\
2005 a^{*} \\
\text { Wilson et al., } \\
2005 b^{*}\end{array}$ \\
\hline $\begin{array}{l}\text { Paris quadrifolia } \\
\text { (Trilliaceae) }\end{array}$ & hp & A & $\mathrm{Bt}$ & $\mathrm{rzm}$ & $\operatorname{Lg}$ & p 1-hl 1 & 3 & En & 0.87 & 0.27 & $\begin{array}{l}\text { asp, }<5 \mathrm{~m} \\
(\text { ge y rm) }\end{array}$ & $\begin{array}{l}\text { prc }+ \text { AFLP } \\
\quad+\text { asp }\end{array}$ & $\begin{array}{c}\text { Jacquemyn et } \\
\text { al., 2005* }\end{array}$ \\
\hline
\end{tabular}


Apéndice. Continuación

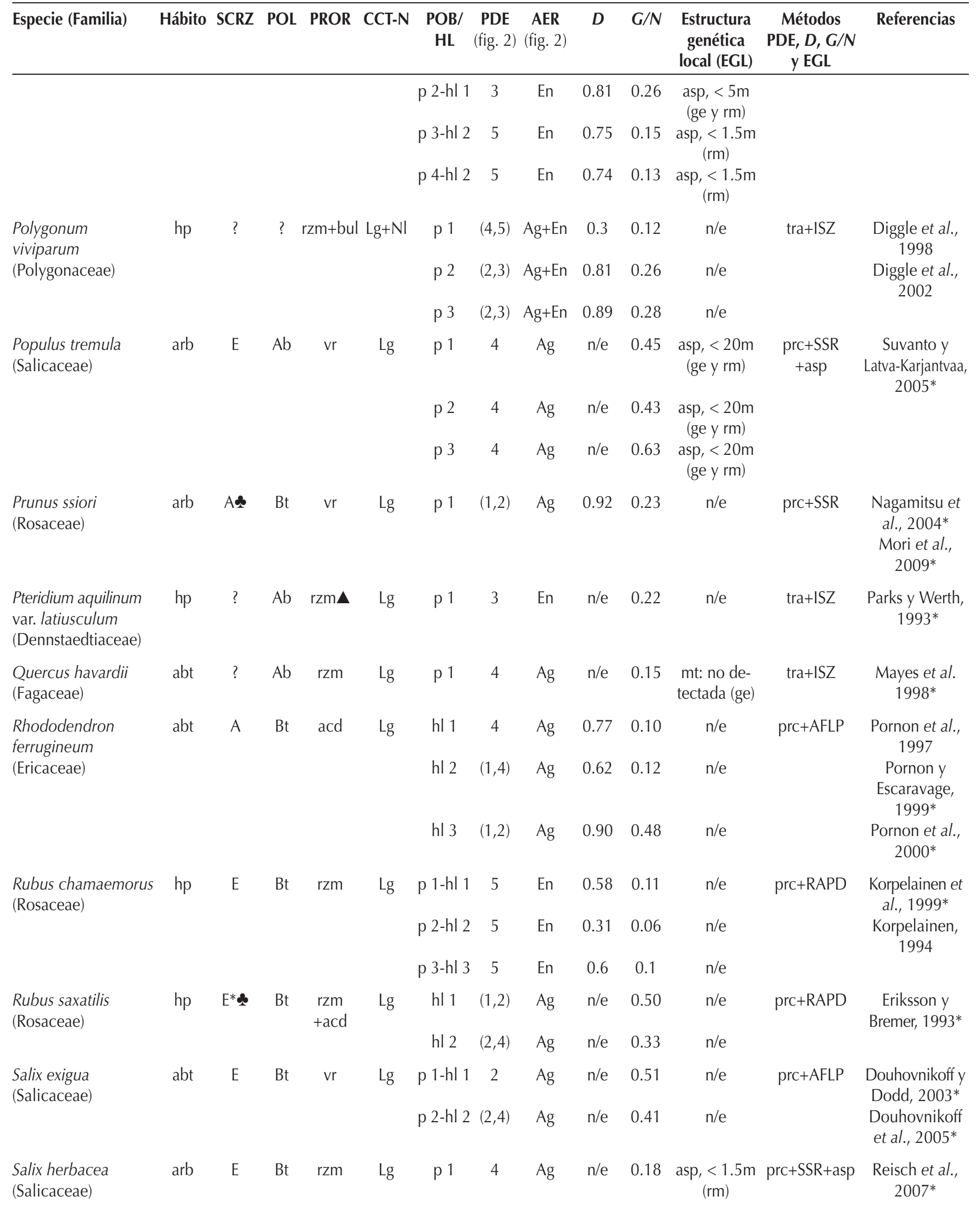


Apéndice. Continuación

\begin{tabular}{|c|c|c|c|c|c|c|c|c|c|c|c|c|c|}
\hline Especie (Familia) & Hábito & SCRZ & POL & PROR & CCT-N & $\begin{array}{c}\mathrm{POB} / \\
\mathrm{HL}\end{array}$ & $\begin{array}{c}\text { PDE } \\
\text { (fig. 2) }\end{array}$ & $\begin{array}{c}\text { AER } \\
\text { (fig. 2) }\end{array}$ & $D$ & $G / N$ & $\begin{array}{l}\text { Estructura } \\
\text { genética } \\
\text { local (EGL) }\end{array}$ & $\begin{array}{c}\text { Métodos } \\
\text { PDE, } D, G / N \\
\text { y EGL }\end{array}$ & Referencias \\
\hline \multirow[t]{6}{*}{$\begin{array}{l}\text { Saxifraga cernua } \\
\text { (Saxifragaceae) }\end{array}$} & \multirow[t]{6}{*}{ hp } & \multirow[t]{6}{*}{$E^{*}$} & \multirow[t]{6}{*}{$\mathrm{Bt}$} & \multirow[t]{6}{*}{ bul } & \multirow[t]{6}{*}{$\mathrm{Nl}$} & p 1 & 5 & En & 0.35 & 0.13 & $\begin{array}{c}\text { mt: no } \\
\text { detectada }\end{array}$ & \multirow[t]{6}{*}{$\begin{array}{l}\text { prc+RAPD } \\
+m t+a s p\end{array}$} & \multirow{6}{*}{$\begin{array}{l}\text { Gabrielsen y } \\
\text { Brochmann, } \\
1998^{*} \\
\text { KjØIner et al., } \\
\text { 2006* }\end{array}$} \\
\hline & & & & & & p 2 & 5 & En & 0.68 & 0.19 & $\begin{array}{c}\text { mt: no } \\
\text { detectada }\end{array}$ & & \\
\hline & & & & & & p 3 & 5 & En & 0.7 & 0.25 & $\begin{array}{l}\text { asp: no } \\
\text { detectada }\end{array}$ & & \\
\hline & & & & & & p 4 & 5 & En & 0.9 & 0.4 & $\begin{array}{c}\text { asp: no } \\
\text { detectada }\end{array}$ & & \\
\hline & & & & & & p 5 & 5 & En & 0.93 & 0.43 & $\begin{array}{l}\text { asp: no } \\
\text { detectada }\end{array}$ & & \\
\hline & & & & & & p 6 & 5 & En & 0.89 & 0.34 & $\begin{array}{c}\text { asp: no } \\
\text { detectada }\end{array}$ & & \\
\hline \multirow[t]{3}{*}{$\begin{array}{l}\text { Scirpus maritimus } \\
\text { (Cyperaceae) }\end{array}$} & \multirow[t]{3}{*}{ hp } & \multirow[t]{12}{*}{$\mathrm{E}^{*}$} & \multirow[t]{3}{*}{$\mathrm{Ab}$} & \multirow[t]{3}{*}{$\mathrm{rzm}$} & \multirow[t]{3}{*}{$\operatorname{Lg}$} & p 1 & 2 & $\mathrm{Ag}$ & $\mathrm{n} / \mathrm{e}$ & $\mathrm{n} / \mathrm{e}$ & $\mathrm{n} / \mathrm{e}$ & \multirow[t]{3}{*}{ desc } & \multirow[t]{3}{*}{$\begin{array}{c}\text { Charpentier et } \\
\text { al., } 2000\end{array}$} \\
\hline & & & & & & p 2 & 4 & $\mathrm{Ag}$ & $\mathrm{n} / \mathrm{e}$ & $\mathrm{n} / \mathrm{e}$ & $\mathrm{n} / \mathrm{e}$ & & \\
\hline & & & & & & p 3 & 4 & $\mathrm{Ag}$ & $\mathrm{n} / \mathrm{e}$ & $\mathrm{n} / \mathrm{e}$ & $\mathrm{n} / \mathrm{e}$ & & \\
\hline \multirow[t]{9}{*}{$\begin{array}{l}\text { Sequoia sempervirens } \\
\text { (Taxodiaceae) }\end{array}$} & \multirow[t]{9}{*}{ arb } & & \multirow[t]{9}{*}{$\mathrm{Ab}$} & \multirow[t]{9}{*}{$\mathrm{vb}$} & \multirow[t]{9}{*}{$\operatorname{Lg}$} & p 1-hl 1 & 2 & $\mathrm{Ag}$ & $\mathrm{n} / \mathrm{e}$ & 0.24 & $\begin{array}{l}\mathrm{mt}:<40 \mathrm{~m} \\
\quad(\mathrm{rm})\end{array}$ & \multirow[t]{9}{*}{$\operatorname{prc}+\mathrm{AFLP}+\mathrm{mt}$} & \multirow[t]{9}{*}{$\begin{array}{l}\text { Douhovnikoff } \\
\text { et al., 2004* }\end{array}$} \\
\hline & & & & & & p 2-hl 1 & 2 & $\mathrm{Ag}$ & $\mathrm{n} / \mathrm{e}$ & 0.38 & $\begin{array}{c}\mathrm{mt}:<40 \mathrm{~m} \\
(\mathrm{rm})\end{array}$ & & \\
\hline & & & & & & p 3-hl 1 & 2 & $\mathrm{Ag}$ & $\mathrm{n} / \mathrm{e}$ & 0.5 & $\begin{array}{c}\mathrm{mt}:<40 \mathrm{~m} \\
(\mathrm{rm})\end{array}$ & & \\
\hline & & & & & & p 4-hl 2 & 2 & $\mathrm{Ag}$ & $\mathrm{n} / \mathrm{e}$ & 0.6 & $\begin{array}{c}\mathrm{mt}:<40 \mathrm{~m} \\
(\mathrm{rm})\end{array}$ & & \\
\hline & & & & & & p 5-hl 2 & 2 & $\mathrm{Ag}$ & $\mathrm{n} / \mathrm{e}$ & 0.35 & $\begin{array}{c}\mathrm{mt}:<40 \mathrm{~m} \\
(\mathrm{rm})\end{array}$ & & \\
\hline & & & & & & p 6-hl 2 & 2 & $\mathrm{Ag}$ & $\mathrm{n} / \mathrm{e}$ & 0.6 & $\begin{array}{c}\mathrm{mt}:<40 \mathrm{~m} \\
(\mathrm{rm})\end{array}$ & & \\
\hline & & & & & & p 7-hl 3 & 2 & $\mathrm{Ag}$ & $\mathrm{n} / \mathrm{e}$ & 0.26 & $\begin{array}{c}\mathrm{mt}:<40 \mathrm{~m} \\
(\mathrm{rm})\end{array}$ & & \\
\hline & & & & & & p 8-hl 3 & 2 & $\mathrm{Ag}$ & $n / e$ & 0.19 & $\begin{array}{c}\mathrm{mt}:<40 \mathrm{~m} \\
(\mathrm{rm})\end{array}$ & & \\
\hline & & & & & & p 9-hl 3 & 2 & $\mathrm{Ag}$ & $\mathrm{n} / \mathrm{e}$ & 0.54 & $\begin{array}{c}\mathrm{mt}:<40 \mathrm{~m} \\
\quad(\mathrm{rm})\end{array}$ & & \\
\hline $\begin{array}{l}\text { Stenocereus eruca } \\
\text { (Cactaceae) }\end{array}$ & abt & $E^{*}$ & $\mathrm{Bt}$ & drm & $\mathrm{NI}$ & p 1 & 2 & $\mathrm{Ag}$ & 0.99 & 0.83 & $\begin{array}{l}\text { asp, }<20 \text { m } \\
\text { (ge y rm) }\end{array}$ & prc+ISSR+asp & $\begin{array}{c}\text { Clark-Tapia et } \\
\text { al., 2005* } \\
\text { Clark-Tapia et } \\
\text { al., } 2006\end{array}$ \\
\hline \multirow[t]{5}{*}{$\begin{array}{l}\text { Typha latifolia } \\
\text { (Thyphaceae) }\end{array}$} & \multirow[t]{5}{*}{$\mathrm{hp}$} & \multirow[t]{5}{*}{ A } & \multirow[t]{5}{*}{$\mathrm{Ab}$} & \multirow[t]{5}{*}{ rzm } & $\operatorname{Lg}$ & p 1 & $(4,5)$ & $\mathrm{Ag}+\mathrm{En}$ & $\mathrm{n} / \mathrm{e}$ & 0.77 & $\mathrm{n} / \mathrm{e}$ & tra+VNTR & $\begin{array}{c}\text { Keane et al., } \\
\text { 1999* }\end{array}$ \\
\hline & & & & & & p 2 & $(4,5)$ & $\mathrm{Ag}+\mathrm{En}$ & $\mathrm{n} / \mathrm{e}$ & 0.55 & $\mathrm{n} / \mathrm{e}$ & & \\
\hline & & & & & & p 3 & $(4,5)$ & $\mathrm{Ag}+\mathrm{En}$ & $\mathrm{n} / \mathrm{e}$ & 0.48 & $\mathrm{n} / \mathrm{e}$ & & \\
\hline & & & & & & p 4 & $(4,5)$ & $\mathrm{Ag}+\mathrm{En}$ & $\mathrm{n} / \mathrm{e}$ & 0.3 & $n / e$ & & \\
\hline & & & & & & p 5 & $(4,5)$ & $A g+E n$ & $\mathrm{n} / \mathrm{e}$ & 0.57 & $\mathrm{n} / \mathrm{e}$ & & \\
\hline $\begin{array}{l}\text { Uvularia perfoliata } \\
\text { (Convallariaceae) }\end{array}$ & hp & $?$ & $?$ & etl & $\operatorname{Lg}$ & p 1-hl 1 & $(4,5)$ & $A g+E n$ & 0.74 & 0.09 & $\begin{array}{c}\text { asp, }<3-5 m \\
(r m)\end{array}$ & prc+ISZ+asp & $\begin{array}{l}\text { Kudoh et al., } \\
\text { 1999* }\end{array}$ \\
\hline & & & & & & p 1-hl 2 & 1 & $\mathrm{Ag}$ & 0.59 & 0.03 & $\mathrm{n} / \mathrm{a}$ & & \\
\hline $\begin{array}{l}\text { Vaccinium myrtillus } \\
\text { (Ericaceae) }\end{array}$ & abt & A & $\mathrm{Bt}$ & rzm & $\operatorname{Lg}$ & p 1 & 2 & $\mathrm{Ag}$ & 0.94 & 0.29 & $\begin{array}{c}\text { asp: no } \\
\text { detectada }\end{array}$ & $\begin{array}{l}\text { prc+AFLP+ } \\
\text { RAPD+asp }\end{array}$ & $\begin{array}{c}\text { Albert et al., } \\
\text { 2003* }\end{array}$ \\
\hline
\end{tabular}


Apéndice. Continuación

\begin{tabular}{|c|c|c|c|c|c|c|c|c|c|c|c|c|c|}
\hline Especie (Familia) & Hábito & SCRZ & POL & PROR & CCT-N & $\begin{array}{c}\mathrm{POB} / \\
\mathrm{HL}\end{array}$ & $\begin{array}{l}\text { PDE } \\
\text { (fig. 2) }\end{array}$ & $\begin{array}{c}\text { AER } \\
\text { (fig. 2) }\end{array}$ & $D$ & $G / N$ & $\begin{array}{c}\text { Estructura } \\
\text { genética } \\
\text { local (EGL) }\end{array}$ & $\begin{array}{c}\text { Métodos } \\
\text { PDE, } D, G / N \\
\text { y EGL }\end{array}$ & Referencias \\
\hline $\begin{array}{l}\text { Vaccinium stamineum } \\
\text { (Ericaceae) }\end{array}$ & abt & $\mathrm{E}^{*}$ & $\mathrm{Bt}$ & $\mathrm{rzm}$ & $\operatorname{Lg}$ & $\mathrm{p} 1$ & $(2,3)$ & $\mathrm{Ag}+\mathrm{En}$ & $\mathrm{n} / \mathrm{e}$ & 0.68 & $\mathrm{n} / \mathrm{e}$ & prc+RAPD & $\begin{array}{l}\text { Kreher et al., } \\
\text { 2000* }\end{array}$ \\
\hline $\begin{array}{l}\text { Zostera marina } \\
\text { (Zosteraceae) }\end{array}$ & hp & A & $\mathrm{Ab}$ & $\mathrm{rzm}$ & $\operatorname{Lg}$ & $\begin{array}{l}\text { p 1-hl } 1 \\
\text { p 2-hl } 2\end{array}$ & $\begin{array}{l}(4,5) \\
(4,5)\end{array}$ & $\begin{array}{l}A g+E n \\
A g+E n\end{array}$ & $\mathrm{n} / \mathrm{e}$ & $\mathrm{n} / \mathrm{e}$ & $\begin{array}{c}\text { asp, }<6 \mathrm{~m} \\
\text { (ge y rm) } \\
\text { asp, }<6 \mathrm{~m} \\
\text { (ge y rm) }\end{array}$ & $\mathrm{prc}+\mathrm{SSR}+\mathrm{asp}$ & $\begin{array}{l}\text { Hämmerli y } \\
\text { Reusch, } 2003 \mathrm{a}^{*} \\
\text { Hämmerli y } \\
\text { Reusch, } 2003 \mathrm{~b}^{*}\end{array}$ \\
\hline $\begin{array}{l}\text { Zostera noltii } \\
\text { (Zosteraceae) }\end{array}$ & hp & A & $\mathrm{Ab}$ & $\mathrm{rzm}$ & $\operatorname{Lg}$ & p 1 & 4 & $\mathrm{Ag}$ & $\mathrm{n} / \mathrm{e}$ & 0.12 & $\mathrm{n} / \mathrm{e}$ & $\mathrm{prc}+\mathrm{SSR}$ & $\begin{array}{l}\text { Ruggieiro et } \\
\text { al., 2005* }\end{array}$ \\
\hline
\end{tabular}

Hábito: $\mathbf{a b t}=$ arbustos, $\mathbf{a r b}=$ árboles, $\mathbf{h p}=$ hierbas perennes

SCRZ: A = especies con capacidad de reproducirse por autocruza, $\mathbf{E}=$ especies con entrecruza obligada (p. ej., dioicas), $\mathbf{E}^{*}=$ especies con sistemas de autoincompatibilidad, = reclutamiento sexual observado y/o evaluado en condiciones naturales

POL: $\mathbf{A b}=$ polinización abiótica, $\mathbf{B t}=$ polinización biótica

PROR: acd = acodaduras, agp = agamospermia, bul = bulbilos, buls = bulbilos subterráneos, $\mathbf{c h u}=$ coronas huecas, $\mathbf{d r m}=$ desprendimiento de ramas, $\mathbf{e t l}=$ estolones, $\mathbf{g m a s}=$ gemas subterráneas, $\mathbf{p n t}=$ plantlets, $\mathbf{p s b}=$ pseudobulbos, $\mathbf{r z m}=$ rizomas, $\mathbf{v b}=$ vástagos basales, $\mathbf{v r}=\mathbf{v a ́ s t a g o s}$ radicales, $\mathbf{\Delta}$ = crecimiento tipo guerrilla

CCT-N: $\mathbf{L g}=$ crecimiento clonal ligado, $\mathbf{N I}=$ crecimiento clonal no ligado

POB/HL: $\mathbf{p}=$ población, $\mathbf{h l}=$ hábitat local (p. ej., bosque con dosel abierto vs bosque con dosel cerrado)

PDE: los paréntesis indican que la población presenta una combinación de patrones de distribución de los tipos indicados por los números (ver figura 2).

AER: Ag = los ramets de cada genet no se entremezclan con los ramets de genets distintos, En = los ramets de diferentes genets en la población se entremezclan.

EGL: \# = evaluado en una población distinta de donde se determinó el PDE, asp = autocorrelación espacial, mpic = método de probabilidad de identidad clonal, $\mathbf{m t}=$ prueba de Mantel, $\mathbf{S G}=$ similitud genética vs distancia espacial.

Métodos: AFLP = marcadores moleculares AFLPs, CLO = marcadores moleculares de cloroplasto, desc = descripción detallada del PDE, ISSR = marcadores moleculares ISSRs, ISZ = isoenzimas, $\mathbf{m p i c}=$ método de probabilidad de identidad clonal, $\mathbf{m t}=$ prueba de $\mathbf{M a n t e l}, \mathbf{p o b}=$ muestra de individuos o todos los individuos de la población, prc = parcelas, RAPD = marcadores moleculares RAPDs, SG $=$ similitud genética vs distancia espacial, $\mathbf{S S R}=$ marcadores moleculares SSRs (microsatélites),

Referencias: ${ }^{*}=$ estudios donde se presentan planos de la distribución espacial de los genotipos.

Todo el cuadro: ? = no se proporciona información, $\mathbf{n} / \mathbf{e}=$ no evaluado, $\mathbf{n} / \mathbf{a}=$ no aplica

GLOSARIO (columna PROR):

Acodaduras: formación de ramets a partir de un tallo originalmente erecto que se postra y genera raíces adventicias al entrar en contacto con el sustrato

Agamospermia: Producción asexual de semillas o embriones sin fertilización (diplosporía: el embrión se desarrolla de una célula diploide del saco embrionario, aposporía: el embrión se desarrolla de una célula somática del saco embrionario, embrionia adventicia: el embrión se desarrolla de células del tejido esporofítico como los tegumentos).

Bulbilos: bulbos pequeños que se forman en las axilas foliares o unidos a los bulbos.

Bulbos: tallos cortos modificados para almacenamiento rodeados por hojas carnosas o brácteas.

Coronas huecas: muerte de los tallos centrales en plantas cespitosas resultando en la formación de ramets independientes en la periferia. Descrito en pastos cespitosos.

Estolones: tallo horizontal que se extiende en la superficie del suelo.

Gemas: pequeñas estructuras vegetativas dispersables en briofitas y pteridofitas.

Plantlets: propágulos vegetativos que resultan de la reversión de estructuras reproductivas como flores y frutos a estructuras vegetativas.

Pseudobulbos: modificación del tallo para almacenamiento que se desarrolla entre dos nodos foliares en orquídeas.

Rizomas: tallo horizontal subterráneo.

Vástagos basales y radicales: formación de tallos en la base de un tallo o a partir de una raíz.

Referencias del glosario: Jackson et al., 1985; de Kroon y van Groenendael, 1997; Liston et al., 2003; van Dijk y Bakx-Schotman, 2004; Tooke et al., 2005. 
independientes de cada genotipo o ramets independientes (Figura 1; Kays y Harper, 1974; Harper, 1985; Jackson et al., 1985). La clonalidad está ampliamente distribuida en plantas, algunas estimaciones para angiospermas varían entre 30\% y más del 50\% (Tiffney y Niklas, 1985; Klim s et al., 1997; Aarssen, 2008), entre el 40\% y $80 \%$ en especies de taiga, tundra y de zonas templadas (van Groenendael y de Kroon, 1990 Klim s et al., 1997) y el 44\% de plantas invasoras en China (Liu et al., 2006), está ampliamente representada en monocotiledóneas y se presenta con baja frecuencia en las gimnospermas (van Groenendael et al., 1996; Peterson y Jones, 1997). Respecto a las condiciones ambientales, se sugiere que la clonalidad predomina en condiciones estresantes como suelos pobres en nitrógeno, suelos húmedos, cuerpos de agua, hábitats sombreados y ambientes fríos en altitudes y latitudes elevadas (Tiffney y Niklas, 1985; van Groenendael et al., 1996; Klim s et al., 1997).

La clonalidad en plantas se ha estudiado con enfoques muy diversos. Por ejemplo, se ha analizado la frecuencia de especies clonales y la variación en las formas de crecimiento clonal en distintos ambientes (p. ej., Tiffney y Niklas, 1985; van Groenendael et al., 1996; Klims et al., 1997), las implicaciones de la integración fisiológica de los ramets y la plasticidad morfológica del crecimiento clonal en ambientes heterogéneos y en condiciones de estrés competitivo (p. ej., Bell, 1984; Hartnett y Bazzaz, 1985; de Kroon y Hutchings, 1995; Price y Marshall, 1999), el efecto de la clonalidad en la demografía (p. ej., Caswell, 1985; McFadden, 1991; Damman y Cain, 1998; Mandujano et al., 2001), la comparación de la diversidad genética entre especies de plantas clonales y no clonales (p. ej., Ellstrand y Roose, 1987; Widén et al., 1994) y las consecuencias reproductivas y evolutivas de la geitonogamia asociada con la clonalidad (p. ej., Handel, 1985; de Jong et al., 1992; Eckert, 2000).

Las implicaciones reproductivas y genéticas de reducir la entrecruza incrementando la geitonogamia han aumentado el interés en el estudio de los patrones de distribución espacial de genets y ramets en poblaciones de plantas clonales. Debido a que los propágulos vegetativos suelen tener una capacidad de dispersión restringida, el reclutamiento clonal frecuente puede reducir la entrecruza e incrementar la autocruza (p. ej., autogamia y geitonogamia), determinando los niveles de variación y la estructuración genética en las poblaciones (Handel, 1985; Eckert, 2002; Ruggiero et al., 2005; Honnay et al., 2006). En especies autocompatibles, los costos reproductivos de la geitonogamia están asociados al incremento de la autogamia y a las probabilidades de expresar depresión endogámica, a la vez que disminuye la adecuación paterna reduciendo la cantidad de polen disponible para la entrecruza; mientras que en las especies autoincompatibles, la geitonogamia afecta la producción de semillas, debido a la saturación de los estigmas con polen incompatible y al aborto de óvulos, y puede resultar en el desperdicio de grandes cantidades de polen (de Jong et al.,
1992; Charpentier, 2002).

En plantas clonales los patrones de distribución espacial pueden ser afectados por la manera en que se generan los ramets. Las formas de crecimiento clonal son muy diversas, pero existen algunas clasificaciones que facilitan el entendimiento de esta variación. Tiffney y Niklas (1985) propusieron cuatro tipos principales de crecimiento: (1) Crecimiento establecido, dado por el inicio del desarrollo de un organismo individual a partir de un propágulo (puede considerarse como crecimento clonal si el propágulo es vegetativo, ya que el propágulo también puede ser una semilla producida sexualmente). (2) Crecimiento restaurativo, que se refiere a la reparación de un daño local en el individuo mediante la formación de tejido calloso, pero no hay regeneración de los órganos completos cuando estos se pierden. (3) Crecimiento regenerativo, que se refiere a la reparación y el reemplazo de órganos dañados (p. ej., crecimiento de una rama desprendida). (4) Crecimiento reproductivo, dado por la multiplicación del individuo fisiológico (p. ej., clonalidad). Estos autores también ordenan los tipos de crecimiento clonal en ligado y no ligado, considerando las estructuras de conexión (Figura 1). En el crecimiento ligado los propágulos vegetativos pueden desarrollarse hasta formar individuos completos (con raíces, tallos y hojas) manteniéndose unidos a la planta madre y su dispersión depende del crecimiento de ramificaciones de tallos y raíces, mientras que en el no ligado, los propágulos vegetativos se desprenden de la planta madre y se desarrollan hasta individuos completos separados de la planta madre. La dispersión de los propágulos en este caso es más dependiente de factores bióticos (p. ej., animales) o abióticos (p. ej., corrientes de agua, gravedad, viento) que en el crecimiento ligado y permite un mayor desplazamiento de los propágulos. El crecimiento reproductivo o clonalidad, que es la que interesa en esta revisión por su influencia en el arreglo espacial de genets y ramets, puede ser de dos tipos: (i) reiteración traumática, definida como la producción de un nuevo individuo inducida por estrés, ya sea por daño o por condiciones ambientales que limitan la reproducción sexual (por ejemplo, la producción de bulbilos en los agaves por daño al escapo floral o por deficiencia de polen) y (ii) la reiteración programada, como la producción de un nuevo individuo por un meristemo como parte normal del desarrollo (Tiffney y Niklas, 1985; Arizaga y Ezcurra, 2002).

La distribución espacial de los individuos en las poblaciones se ha clasificado en tres patrones básicos: aleatorio, uniforme y agregado (Figura 2; Gleason, 1920; Clark y Evans, 1954; Barbour et al., 1987). En poblaciones naturales de plantas, los individuos suelen distribuirse en forma más o menos agregada, debido a la distribución heterogénea de los recursos (Couteron y Kokou, 1997; Chen y Bradshaw, 1999), a la interacción con otras especies (Shreve, 1917; Mandujano et al., 1998) y a la dispersión restringida de semillas y propágulos vegetativos (Gibson y Menges, 1994; Okuda et al., 1997; Clark-Tapia et al., 2005). En plantas 
clonales además, el arreglo espacial de los genets y ramets puede ordenarse en dos patrones básicos, uno caracterizado por la agregación de ramets del mismo genet y el otro por una distribución entremezclada de ramets de distintos genets (Figura 2; de Kroon y Hutchings, 1995; Charpentier, 2002; Clark-Tapia et al., 2005). Estos arreglos espaciales de genets y ramets pueden estar asociados a la manera en que se producen los ramets (esto es por crecimiento clonal ligado o no ligado) y se ha propuesto que en las plantas que se propagan mediante crecimiento no ligado forman arreglos más entremezclados que las especies con crecimiento ligado (Gabrielsen y Brochmann, 1998), pero esto no ha sido demostrado. Por otro lado, debido a que la clonalidad puede incrementar la tasa de geitonogamia entre los ramets del mismo genet, puede esperarse que los arreglos entremezclados reduzcan la geitonogamia y promuevan la entrecruza como resultado del incremento local en el número de genets (Charpentier, 2002).

En algunas revisiones sobre el tema de la clonalidad se han planteado hipótesis sobre las implicaciones reproduc-

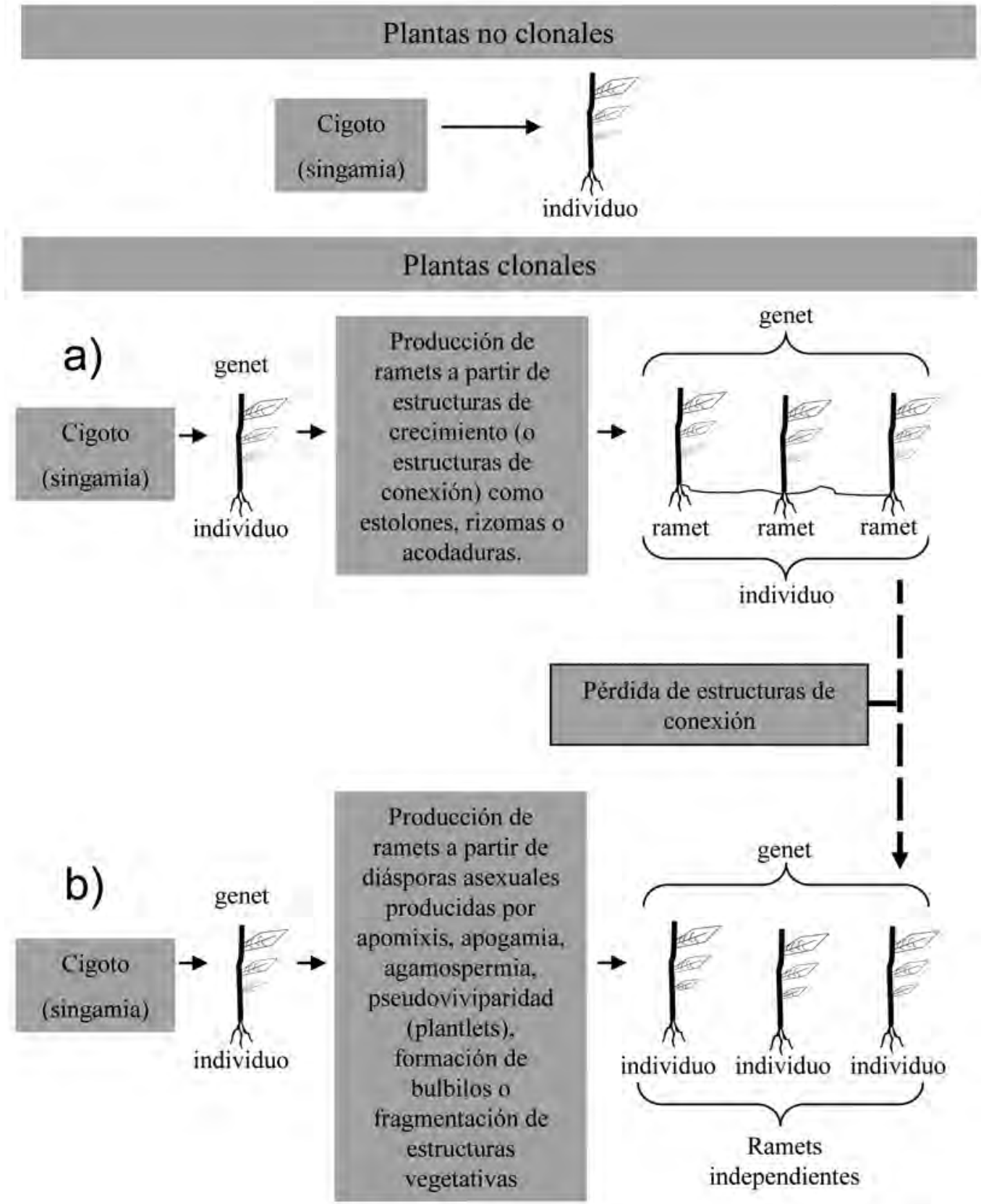

Figura 1. Formación de individuos en plantas no clonales y en plantas clonales. a) crecimiento clonal ligado y b) crecimiento clonal no ligado en la clasificación de Tiffney y Niklas (1985). 
$\mathrm{PDE}=$ Patrones de distribución espacial de los individuos y ramets en las poblaciones

(clonales y no clonales)

\section{AER $=$ Arreglos espaciales de los ramets de distintos genets}

(clonales)

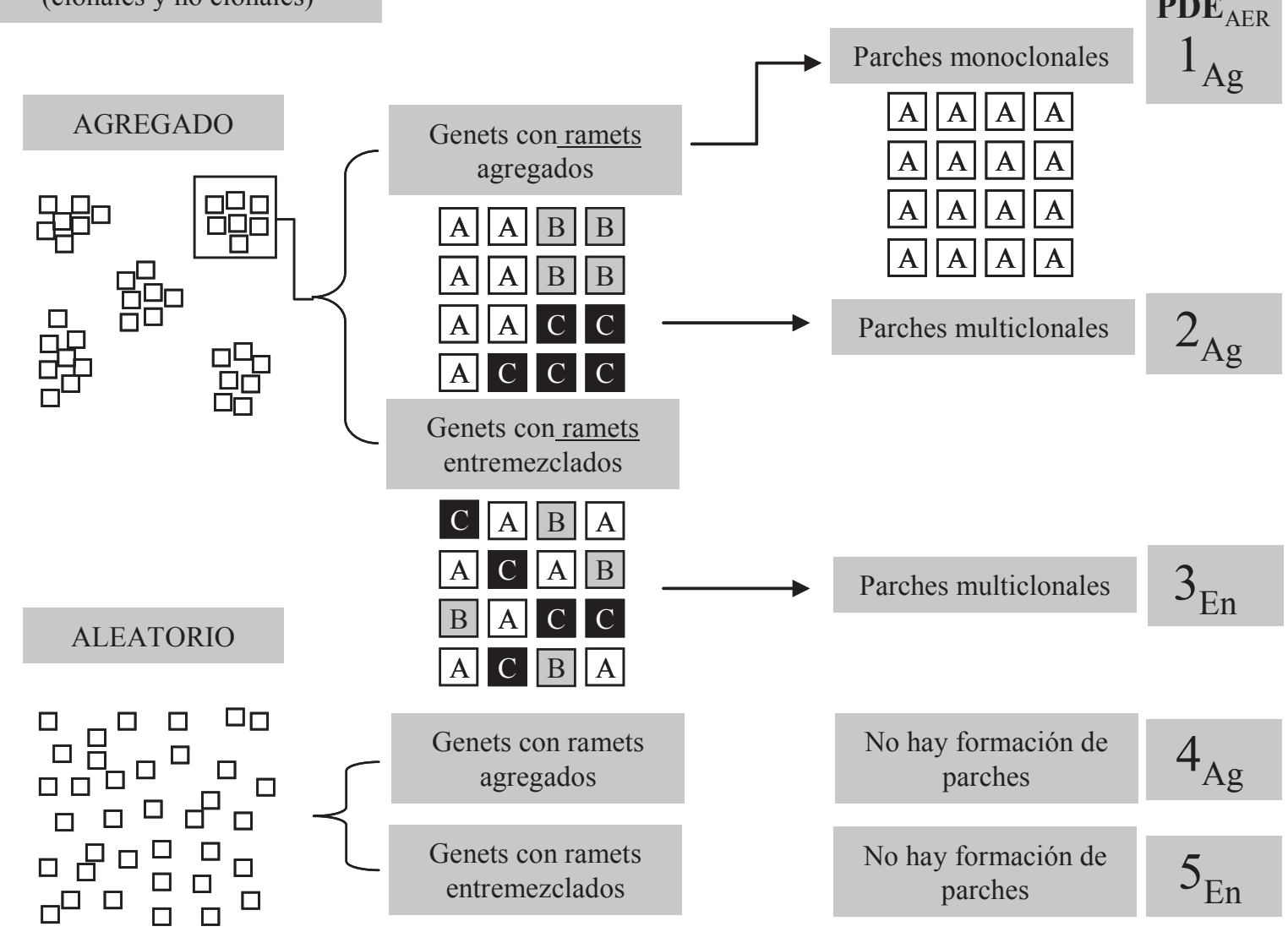

\section{UNIFORME}
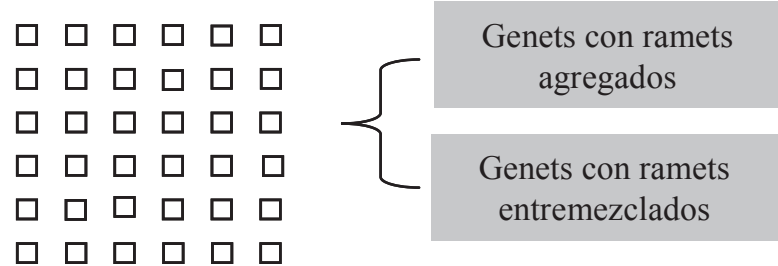

\begin{tabular}{|c|c|c|c|}
\hline $\begin{array}{c}\text { No hay formación de } \\
\text { parches }\end{array}$ & $6_{\mathrm{Ag}}$ \\
\hline $\begin{array}{c}\text { No hay formación de } \\
\text { parches }\end{array}$ & $7_{\mathrm{En}}$ \\
\hline
\end{tabular}

Figura 2. Patrones de distribución espacial de los individuos en las poblaciones de plantas no clonales y de plantas clonales. El número en el extremo derecho representa la clave del patrón de distribución espacial y el arreglo espacial de los ramets de distintos genets (PDE y AER, Apéndice). El subíndice Ag indica que los ramets de cada genet no se entremezclan con los ramets de genets distintos y el subíndice En indica que los ramets de diferentes genets se entremezclan.

tivas y genéticas de los arreglos espaciales de los genets y ramets, asociados con las distintas formas de producir descendientes clonales (Widén et al., 1994; Charpentier, 2002; Mandujano-Sánchez, 2007; Honnay y Jaquemyn, 2008). No obstante, los intentos para probar estas hipótesis son muy escasos y las posibilidades de realizar algún tipo de análisis numérico son restringidas por la baja disponibilidad de publicaciones (p. ej., Widén et al., 1994), o en los análisis sólo se incluyen especies con crecimiento ligado (p. ej., Honnay y Jaquemyn, 2008). En este trabajo presentamos una revisión de varios estudios que evalúan los patrones de distribución espacial en plantas clonales y a partir de la información 
proporcionada en dichos estudios, analizamos el planteamiento de que en poblaciones de plantas con crecimiento clonal ligado el arreglo espacial de los ramets de distintos genets es agregado, mientras que en poblaciones de plantas con crecimiento clonal no ligado predominan los arreglos espaciales entremezclados. Adicionalmente, intentamos poner a prueba la hipótesis de que los arreglos entremezclados promueven la entrecruza suponiendo que las poblaciones donde la entrecruza es más frecuente tenderán a presentar niveles más altos de diversidad genotípica.

\section{Material y métodos}

La revisión de los trabajos publicados sobre la distribución espacial clonal en plantas se llevó a cabo mediante una búsqueda de trabajos en bases de datos especializadas (CSA Cambridge, Ebsco HOST, ISI Web of Knowledge, JSTOR y Scopus) y no especializadas (Google, Google scholar y Yahoo). La búsqueda se hizo empleando los siguientes términos: clonal structure, spatial distribution of genets (andl or ramets), clonal architecture y clonal diversity y abarcó de 1990 a 2011, debido a que la mayoría de los estudios en plantas clonales con este enfoque se han realizado después de 1990.

Los criterios principales para incluir una especie en la revisión fue que se presentara una descripción de la distribución espacial de las plantas individuales (esto es sin importar el genotipo) en las poblaciones naturales y que se evaluara el arreglo espacial de los genets y los ramets (es decir agregado o entremezclado) mediante planos de distribución de los genotipos, análisis de autocorrelación espacial o compatibilidad de las cruzas entre ramets contiguos. Para cada especie se obtuvo el tipo de estructuras vegetativas a mediante las cuales se lleva a cabo la propagación clonal a partir de la información proporcionada en los estudios correspondientes o en estudios previos realizados a menudo por los mismos autores. El segundo criterio en orden de importancia fue que se reportaran valores de diversidad genotípica ( $D=$ índice de diversidad de Simpson y proporción de genotipos distinguibles $G / N$ ) para las poblaciones de las diferentes especies. Otros criterios fueron que se proporcionara información sobre las características reproductivas como la presencia de sistemas de incompatibilidad, la expresión sexual, si se ha observado reclutamiento sexual en las poblaciones y el tipo de polinización. Con base en esta información, se asignó a las especies el tipo de crecimiento clonal (i. e. ligado y no ligado, Figura 1) de acuerdo con la clasificación de Tiffney y Niklas (1985) y el patrón de distribución espacial y el tipo de arreglo espacial de los ramets (agregado o entremezclado) según el esquema presentado en la Figura 2. La información obtenida se presenta en el apéndice.

Para analizar el planteamiento de que en poblaciones de plantas con crecimiento clonal ligado el arreglo espacial de los ramets es agregado, mientras que en poblaciones de plantas con crecimiento clonal no ligado predomina el arreglo espacial entremezclado se construyó una tabla de contingencia con tres categorías de tipo de crecimiento clonal como columnas (ligado, combinación ligado-no ligado y no ligado) y tres categorías del arreglo espacial de los ramets como renglones (agregado, combinación agregado-entremezclado y entremezclado) y se analizó con una prueba de ${ }^{2}$ con el paquete estadístico JMP versión 7.0 (SAS Institute Inc). Para evaluar las celdas que contribuyen significativamente al valor de ${ }^{2}$ se hizo un análisis de residuales ajustados (Everitt, 1977).

Para probar la hipótesis de que las poblaciones con arreglos entremezclados de ramets presentan niveles más altos de diversidad genotípica, se compararon los valores del índice de diversidad de Simpson $(D)$ y la proporción de genotipos distinguibles $(G / N)$ entre las tres categorías de patrón de distribución clonal y entre las tres categorías de tipo de crecimiento clonal con una prueba de Kruskall-Wallis con el paquete estadístico JMP versión 7.0 (SAS Institute Inc).

\section{Resultados}

En la revisión se incluyeron 53 estudios que comprenden 122 poblaciones distribuidas en 44 especies de plantas clonales y en 27 familias (Apéndice). Las familias mejor representadas son Liliaceae y Rosaceae con cuatro especies cada una; Cactaceae, Cyperaceae, Ericaceae, Poaceae y Salicaceae con tres especies cada una y Zosteraceae con dos especies. El resto de las familias estuvieron representadas por una sola especie. Las especies que predominan son herbáceas perennes $(60 \%)$, y las plantas con hábito arbustivo y arbóreo se encuentran poco representadas (20\% en ambos casos).

La mayoría de las especies presentan crecimiento clonal ligado (70\%), dominando la propagación a través de rizomas (45\% de las especies). Las especies con crecimiento clonal ligado y no ligado representan el 14\% propagándose clonalmente por crecimiento ligado a través de rizomas y acodaduras, y por crecimiento no ligado a través de bulbilos, plantlets y desprendimiento de ramas; las especies sólo con crecimiento no ligado representan el 16\%, propagándose por bulbilos, semillas producidas por agamospermia, desprendimiento de ramas y gémulas (ver glosario para la definición de las estructuras implicadas en el crecimiento ligado y no ligado, Apéndice). Respecto a la distribución espacial, $50 \%$ del total de las especies presentan genets con ramets agregados, $30 \%$ presentan una combinación de arreglos agregados-entremezclados y $20 \%$ presentan arreglos entremezclados (Apéndice).

$\mathrm{Al}$ comparar las poblaciones con diferentes arreglos espaciales de genets y ramets [agregados (Ag), En (entremezcaldos) y $\mathrm{Ag}+\mathrm{En}$ (combinados)] encontramos que las poblaciones de especies con crecimiento ligado y no ligado y 
Cuadro 1. Número (observado y esperado) de poblaciones con diferentes tipos de crecimiento clonal de acuerdo con la clasificación de Tiffney y Niklas (1985) ( $\mathrm{Lg}=$ ligado, Lg+NI = ligado y no ligado, $\mathrm{Nl}=$ no ligado), que mostraron arreglos espaciales de los ramets de distintos genets: Ag = agregados, Ag+En = combinación de agregados y entremezclados, y En = entremezclados (figura 2). Los valores entre paréntesis indican el número de especies y familias (especies/familias) representadas en cada celda. Los valores de los residuales ajustados en negritas indican las celdas que contribuyen significativamente al valor de $\chi^{2}$, el signo indica mayor (sin signo) o menor número (-) de poblaciones de las esperadas por azar.

\begin{tabular}{|c|c|c|c|}
\hline Observados & Ligado & Ligado+No ligado & No ligado \\
\hline Agregado & $49(21 / 13)$ & $7(2 / 2)$ & $4(2 / 2)$ \\
\hline Agregado+entremezclado & $11(5 / 5)$ & $25(5 / 5)$ & $7(3 / 3)$ \\
\hline Entremezclado & $10(5 / 5)$ & $2(2 / 1)$ & $7(2 / 2)$ \\
\hline \multicolumn{4}{|l|}{ Esperados } \\
\hline & $\operatorname{Lg}$ & $\mathrm{Lg}+\mathrm{NI}$ & $\mathrm{Nl}$ \\
\hline Agregado & 34 & 17 & 9 \\
\hline Agregado+entremezclado & 25 & 12 & 6 \\
\hline Entremezclado & 11 & 5 & 3 \\
\hline \multicolumn{4}{|l|}{ Residuales ajustados } \\
\hline & $\operatorname{Lg}$ & $\mathrm{Lg}+\mathrm{NI}$ & $\mathrm{Nl}$ \\
\hline Agregado & 5.34 & -3.93 & -2.48 \\
\hline Agregado+entremezclado & -5.24 & 5.50 & 0.35 \\
\hline Entremezclado & -0.46 & -1.83 & 2.95 \\
\hline
\end{tabular}

de especies que sólo presentan crecimiento no ligado presentaron arreglos espaciales (AER) más entremezclados que las poblaciones de especies que sólo presentan crecimiento ligado ( ${ }^{2}=43.6, P<0.0001,4$ g.l.; Cuadro 1). Predominan los patrones de distribución (PDE) con ramets distribuidos en parches discretos sobre el área ocupada por la población (patrón de distribución espacial agregado, $46 \%$ de las poblaciones); estos parches son multiclonales (con dos o más genets) en la mayoría de los casos y el arreglo espacial de los ramets de distintos genets es agregado (19\% de las poblaciones, $\mathrm{PDE}_{\mathrm{AER}} 2_{\mathrm{Ag}}$, Figura 2), presenta una combinación agregado-entremezclado (10\% de las poblaciones, combinación $\mathrm{PDE}_{\mathrm{AER}} 2_{\mathrm{Ag}}-3_{\mathrm{En}}$, Figura 2) o es entremezclado solamente (3\% de las poblaciones, $\mathrm{PDE}_{\mathrm{AER}} 3_{\mathrm{En}}$, Figura 2). En otras poblaciones donde los ramets se distribuyen formando parches discretos el arreglo espacial de los ramets de distintos genets es agregado, pero los parches son multiclonales y monclonales (9\% de las poblaciones, combinación $\mathrm{PDE}_{\mathrm{AER}}$ $1_{\mathrm{Ag}}-\mathrm{Ag}_{\mathrm{Ag}}$, Figura 2) o sólo son monoclonales (5\% de las poblaciones, $\mathrm{PDE}_{\mathrm{AER}} 1_{\mathrm{Ag}}$, Figura 2).

Los patrones de distribución espacial con ramets distribuidos aleatoriamente sobre el área ocupada por la población (patrón de distribución espacial aleatorio) representaron el $44 \%$ de las poblaciones, predominando el arreglo espacial agregado-entremezclado de los ramets de distintos genets $\left(22 \%\right.$ de las poblaciones, combinación de $\mathrm{PDE}_{\mathrm{AER}}$ $4_{\mathrm{Ag}}-5_{\mathrm{En}}$, Figura 2), el arreglo agregado se presentó en $10 \%$ de las poblaciones ( $\mathrm{PDE}_{\mathrm{AER}} 4_{\mathrm{Ag}}$, Figura 2) y el arreglo entremezclado en $12 \%$ de las poblaciones $\left(\mathrm{PDE}_{\mathrm{AER}} 5_{\mathrm{En}}\right.$, Figura
2). El resto de las poblaciones (10\%) presentaron una combinación de patrones de distribución con ramets distribuidos aleatoriamente sobre una parte del área ocupada por la población y en otra formando parches discretos monoclonales o parches multiclonales con arreglos espaciales agregados y entremezclado de los ramets de distintos genets (combinación de $\mathrm{PDE}_{\mathrm{AER}} 1_{\mathrm{Ag}}-4_{\mathrm{Ag}}, 1_{\mathrm{Ag}}-5_{\mathrm{En}}, 2_{\mathrm{Ag}}-4_{\mathrm{Ag}}, 2_{\mathrm{Ag}}-5_{\mathrm{En}}$, Figura 2).

Las poblaciones de especies con una combinación de tipos de crecimiento clonal ligado y no ligado $(\mathrm{Lg}-\mathrm{Nl})$ presentaron valores más bajos de la proporción de genotipos distinguibles (promedio \pm E.E., $G / N=0.24 \pm 0.04 ; H=$ 6.93, $P=0.031,2$ g.l.) que las poblaciones de especies con crecimiento ligado (promedio \pm E.E., $G / N=0.38 \pm 0.03$ ) y con crecimiento no ligado (promedio \pm E.E., $G / N=0.39 \pm$ 0.06 ), pero las diferencias sólo fueron significativas con respecto a las poblaciones de especies con crecimiento ligado (Figura 3a). Respecto al arreglo espacial de los ramets de distintos genets, las poblaciones con una combinación de arreglos agregado y entremezclado (Ag-En) presentaron valores menores del índice de diversidad de Simpson (promedio \pm E.E., $D=0.57 \pm 0.05 ; H=13.2, P=0.0014,2$ g.l.) que las poblaciones con arreglos agregados (promedio \pm E.E., $D=0.80 \pm 0.04$ ) y con arreglos entremezclados (promedio \pm E.E., $D=0.76 \pm 0.06$; Figura 3). Estas diferencias sugieren que el reclutamiento sexual es más bajo en poblaciones de especies que presentan una combinación de formas de crecimiento clonal ligado-no ligado ( $\mathrm{Lg}-\mathrm{Nl}$ ) y una combinación de arreglos de los ramets de distintos genets agregado-entremezclado (Ag-En), sin embargo estas diferencias 

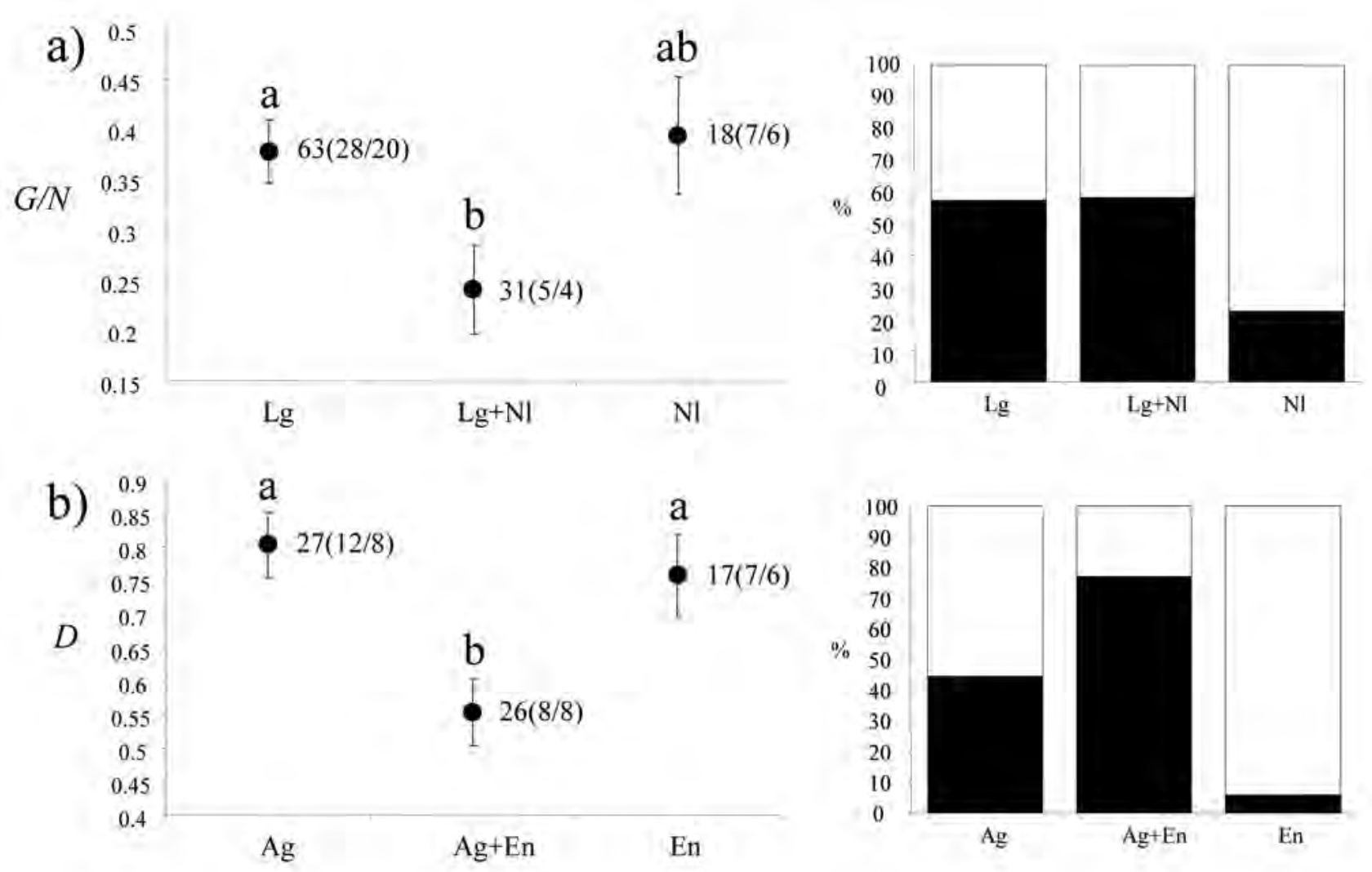

Figura 3. Comparación de la diversidad clonal (promedio \pm EE) entre poblaciones de especies con a) distinto tipo de crecimiento clonal: $\mathrm{Lg}=$ ligado, $\mathrm{Lg}+\mathrm{Nl}$ = con ambos tipos de crecimiento y $\mathrm{Nl}$ = no ligado y b) con distintos arreglos espaciales de los ramets de distintos genets: $\mathrm{Ag}=$ agregados, $\mathrm{Ag}+\mathrm{En}$ = combinación de agregados y entremezclados, y En = entremezclados (Figura 2). En cada grupo se indica el número de poblaciones (número de especies / número de familias) y los grupos que difieren significativamente se indican con una letra distinta arriba de la barra del promedio \pm E.E. de cada grupo. Las gráficas de barras del lado derecho muestran el porcentaje de poblaciones con polinización abiótica (en negro) y con polinización biótica (en blanco).

pueden estar asociadas con otros factores como el tipo de polinización, y no con el tipo de crecimiento y el arreglo espacial de los ramets de distintos genets. La mayoría de las poblaciones con tipo de crecimiento $\mathrm{Lg}-\mathrm{Nl}$ y arreglos de ramets de distintos genets Ag-En corresponden a especies con polinización abiótica (Figura 3).

\section{Discusión}

El efecto que tienen los modos de clonalidad en las plantas en la conformación de distintos arreglos espaciales de genets y ramets es un campo relativamente novedoso, que se ha explorado en los últimos 20 años y la mayoría de los estudios que hay sobre el tema se han desarrollado después de 1990. Widén et al. (1994) reportan que de 40 estudios que comprenden un total de 45 especies de plantas clonales, menos de la tercera parte proporcionan información sobre el arreglo espacial de los genets en las poblaciones y sólo en $12.5 \%$ se construyeron planos de distribución de todos los ramets, dentro de las parcelas o poblaciones, identificando los genotipos multilocus. En esta revisión, en el $98 \%$ de los estudios incluidos se identifican los genotipos multilocus mediante marcadores dominantes, codominantes o ambos siguiendo tres métodos de muestreo: en el 56\% de los estudios se utilizan parcelas, en el $24 \%$ transectos y en el $18 \%$ se muestrean todos o varios individuos de las poblaciones. El 2\% restante está representado por un estudio donde el patrón de distribución es descrito y la distribución de los ramets se sugiere por experimentos de cruzas compatibles entre ramets cercanos (en Scirpus maritimus, Charpentier et al., 2000).

A pesar de que en la actualidad se cuenta con más información sobre los patrones de distribución espacial en poblaciones de especies clonales, hacer comparaciones de dichos patrones considerando especies con distintas formas de crecimiento clonal para analizar las consecuencias reproductivas y genéticas del arreglo espacial de los genets y los ramets continúa siendo un reto. Algunos factores que influyen en este hecho son que la evaluación de la variación en los patrones de distribución espacial en plantas clonales 
enfrenta la dificultad que implica trabajar con la mayoría de los individuos en muchas poblaciones, principalmente a nivel molecular. Aunque en la actualidad las herramientas moleculares se han vuelto más accesibles, trabajar con un gran número de ejemplares sigue siendo difícil y costoso. Otros problemas son la falta de estimadores numéricos comparables para describir la distribución espacial en las poblaciones de diferentes especies y la variación en los métodos de muestreo. Por ejemplo, el muestreo mediante transectos colectando muestras de ramets cada determinada distancia (p. ej., Jonsson et al., 1996; Esselman et al., 1999) permite evaluar la capacidad de expansión longitudinal de los genets, pero no permite tener una descripción de la expansión radial y por lo tanto una evaluación más precisa del nivel de entremezcla de los ramets de distintos genets. En contraste, el muestreo de todos o de un gran número de individuos dentro de parcelas o en las áreas ocupadas por las poblaciones es otro método empleado en estudios con especies de plantas clonales (p. ej., Shimizu et al., 2002; Ruggieiro et al., 2005). Este tipo de muestreo puede ser un método más adecuado para analizar y comparar la estructura espacial clonal, porque permite detectar el arreglo radial de distintos genets. Sin embargo, los tamaños de muestra muy grandes pueden limitar la aplicación de este método, ya que en la mayoría de los casos es necesario emplear marcadores moleculares para identificar a los genets.

En particular, además de los problemas mencionados anteriormente es importante considerar las dificultades adicionales que detectamos en este estudio y que pueden afectar los patrones encontrados a través de nuestra aproximación. La mayoría de los estudios que analizan la distribución espacial de genets y ramets parecen estar sesgados hacia especies de herbáceas perennes con crecimiento clonal ligado (45\% de las especies), predominando las especies rizomatosas (43\% de las especies), mientras que las especies con crecimiento no ligado están poco representadas, impidiendo contar con tamaños de muestra equivalentes para hacer comparaciones más precisas. Por otro lado, la aproximación que decidimos emplear en este trabajo compara a las poblaciones más que a las especies, debido a que hemos detectado que hay una importante variación intraespecífica tanto en los patrones de distribución como en los patrones reproductivos. No obstante, esta variación se omite en algunos estudios o está mejor representada para algunas especies.

Los resultados obtenidos en este trabajo sugieren que las especies que se propagan por crecimento no ligado tienden a formar arreglos espaciales de los ramets de distintos genets más entremezclados como se ha planteado (Gabrielsen y Brochmann, 1998). No obstante, en especies con crecimiento ligado que además se propagan clonalmente por crecimiento no ligado, como el desprendimiento de ramas, el cual consideramos en este estudio como una forma de crecimiento no ligado en los análisis, debido a que los ramets originados por este medio se desarrollan hasta individuos com- pletos separados de la planta madre y a que su dispersión no depende del crecimiento horizontal de tallos y raíces (p.ej., Lophocereus schottii, Parker y Hamrick, 1992; Pteridium aquilinum, Parks y Werth, 1993; Cladium jamaicense, Ivey y Richards, 2001; Ferocactus robustus, Carrillo-Angeles et al., 2011), o que se propagan por la extensión de rizomas en especies con crecimiento en guerrilla (p. ej., Cymodocea nodosa, Ruggieiro et al., 2005 e Ilex leucoclada, Torimaru y Tomaru, 2005) también forman arreglos de genets entremezclados. Algunos factores externos que pueden influir en el arreglo entremezclado de genets son la dispersión por actividades humanas (Parks y Werth, 1993; Brzosko et al., 2002), el acarreo de propágulos vegetativos por animales y corrientes de agua (Parker y Hamrick, 1992; Brzosko et al., 2002), el reclutamiento de nuevos genets en áreas perturbadas donde permanecen fragmentos de clones que ocuparon grandes extensiones (Parks y Werth, 1993) y la dispersión de semillas producidas por autogamia que presentan genotipos multilocus idénticos (Keane et al., 1999; Brzosko et al., 2002).

Respecto a la hipótesis de que las poblaciones con arreglos espaciales entremezclados de los ramets de distintos genets presentan mayor entrecruza, medido indirectamente mediante la diversidad genotípica, nuestros resultados concuerdan parcialmente con el patrón esperado. El G/N es significativamente mayor en los arreglos de especies con producción de vástagos no ligados, en donde predominan los arreglos de genets entremezclados. Para probar esta hipótesis consideramos que si la entrecruza es más alta en poblaciones con arreglos entremezclados, habría mayores niveles de diversidad genotípica. Sin embargo, ésto no se refleja en el caso del índice de Simpson. Las diferencias en los índices de diversidad genotípica entre las poblaciones de especies que combinan el crecimiento clonal ligado-no ligado ( $\mathrm{Lg}$ $\mathrm{Nl})$ y el arreglo espacial agregado-entremezclado (Ag-En), respecto de las poblaciones que presentan uno u otro tipo de crecimiento y de arreglo espacial están asociadas con el tipo de polinización (biótica o abiótica), lo cual apoya el planteamiento de que la polinización biótica puede incrementar la eficiencia de la fertilización (Barret et al.,1993; Richards, 1997; Reusch, 2003). Los análisis para probar este planteamiento están fuera del alcance del presente estudio.

Además de los efectos sobre el reclutamiento sexual y la diversidad genotípica, los patrones de distribución espacial en especies clonales pueden afectar la diversidad y la estructura genética de las poblaciones. Por ejemplo, la formación de parches monoclonales en especies con entrecruza obligada, aunada al desplazamiento de genets por competencia, puede eliminar la capacidad de una población para reproducirse sexualmente en tiempos relativamente cortos (Honnay y Bossuyt, 2005). Adicionalmente, una reducción del reclutamiento sexual y una dispersión restringida de polen, semillas y propágulos vegetativos pueden generar estructura genética local en las poblaciones. Este segundo aspecto se 
evalúa en varios de los estudios revisados (47\%). La estructura genética local tanto a nivel de genets (indicando una dispersión restringida de polen, semillas o ambos) como de ramets (indicando dispersión restringida de propágulos vegetativos) se detectó en 46 poblaciones distribuidas en 16 especies; 28 poblaciones corresponden a cinco especies arbóreas que muestran estructura genética local a distancias menores de $50 \mathrm{~m}$ (Sequoia sempervirens, Douhovnikoff et al., 2004) y a distancias menores de $25 \mathrm{~m}$ (Eurya emarginata, Chung y Epperson, 2000; Cryptomeria japonica, Shimizu et al., 2002; Hirayama y Sakimoto, 2008; Populus tremula, Suvanto y Latva-Karjantvaa, 2005); tres poblaciones corresponden a dos especies arbustivas (Echinosophora koreensis, Chung et al., 2006; Stenocereus eruca, ClarkTapia et al., 2005) que presentan estructura genética local a distancias menores de $20 \mathrm{~m}$. El resto de las poblaciones corresponden a hierbas perennes que muestran estructura genética local desde distancias menores de $30 \mathrm{~m}$, pero más frecuentemente a distancias menores de $15 \mathrm{~m}$.

En promedio, los valores del índice de diversidad de Simpson $(D)$ y la proporción de genotipos distinguibles $(G / N)$ fueron altos en las poblaciones representadas en los estudios incluidos en la presente revisión y son similares a los reportados para 21 especies de plantas clonales $(D=$ 0.62 y $G / N=0.16$ ) por Ellstrand y Roose (1987). Se han planteado algunas hipótesis sobre los escenarios que pueden explicar altos niveles de diversidad genotípica: (i) las poblaciones pudieron ser fundadas por varios propágulos sexuales que se mantienen por clonalidad, (ii) el reclutamiento sexual fue más frecuente en el pasado, aunque en la actualidad la producción de descendientes sexuales no sea evidente (Ellstrand y Roose, 1987; Eriksson, 1989, 1993; Widén et al., 1994); (iii) los genotipos heterócigos se ven favorecidos selectivamente, manteniendo altos niveles de diversidad genética (Widén et al., 1994); (iv) los riesgos de mortalidad entre los ramets de un genet pueden ser distintos si tienen independencia física y fisiológica (Eriksson 1989, 1993, Pan y Price, 2002), lo cual puede reducir las probabilidades de supervivencia / extinción del genet; (v) en especies con ciclos de vida largos los eventos raros de reclutamiento sexual pueden ser suficientes para mantener niveles altos de diversidad genética (Eriksson 1989, 1993, Parker y Hamrick, 1992; Brzosko et al., 2002; Clark-Tapia et al., 2005); (vi) los genotipos pueden mantenerse a través de la supervivencia de algunos ramets el tiempo suficiente para que algunos genotipos se integren a la población de manera esporádica; (vii) la heterogeneidad ambiental puede generar condiciones de selección diversificante que permiten la coexistencia de varios genotipos (Widén et al., 1994). Otra posibilidad para el mantenimiento de diversidad clonal en poblaciones de plantas, que surge al considerar la variación de la distribución espacial de genets y ramets, es que la clonalidad puede mantener altos niveles de entrecruza e incrementar las probabilidades de reclutamiento sexual si los ramets se dispersan hacia vecindarios genéticamente variables, debido a la distribución entremezclada de genets.

Las explicaciones a los niveles relativamente altos de diversidad genotípica en las poblaciones de las especies incluidas en esta revisión comprenden los eventos esporádicos de reclutamiento sexual y el establecimiento inicial de varios genets. En algunos casos se sugiere un reclutamiento sexual frecuente a partir de los altos niveles de diversidad genotípica detectados con los marcadores moleculares (p. ej., Parks y Werth, 1993; Diggle et al., 1998; Misuki et al., 2010). No obstante, el reclutamiento sexual en poblaciones naturales sólo se reporta en Prunus ssiori, Rubus saxatilis (Eriksson y Bremer, 1993), Cypripedium calceolus (Brzosko et al., 2002) y Maianthemum dilatatum (Wilson et al., 2005b). Otros factores que se proponen para explicar la diversidad genotípica son que la integración fisiológica entre los ramets de un genet puede reducir el riesgo de que un genotipo en particular desaparezca, cuando las condiciones ambientales locales son adversas, contribuyendo a mantener la diversidad genética (Hartnett y Bazzaz, 1985), el incremento en la tasa de entrecruza, debido a la entremezcla de genets en especies con crecimiento en guerrilla (Ruggieiro et al., 2005) y en especies que se propagan clonalmente por desprendimiento de ramas y producción de bulbilos (Parker y Hamrick, 1992; Gabrielsen y Brochmann, 1998).

El estudio de los patrones de distribución espacial en especies clonales es importante para analizar las consecuencias reproductivas y genéticas del arreglo espacial de los genets y los ramets en las poblaciones naturales. Estas consecuencias pueden explorarse de una manera más precisa analizando la variación de la distribución de genets y ramets entre las poblaciones de una misma especie. Sin embargo, la evidencia de estos aspectos en organismos clonales aún es escasa y hace falta realizar estudios considerando diferentes tipos de crecimiento clonal. El análisis de los patrones de distribución espacial pueden ser muy útiles para evaluar otros aspectos del ciclo de vida de las especies clonales como el efecto de una variación persistente en la producción de propágulos clonales y sexuales entre hábitats, que puede afectar diferencialmente la dinámica de las poblaciones. Asimismo, es necesario analizar la dinámica temporal de los arreglos espaciales de los genets y ramets en las poblaciones para hacer inferencias adecuadas sobre las consecuencias ecológicas y evolutivas de la clonalidad.

\section{Agradecimientos}

Proyectos: IN IN205007 Efecto de la estructura clonal sobre la dinámica poblacional y la reproducción de una cactácea con ciclo de vida complejo, Opuntia microdasys.

Becas: doctorado Conacyt a I. Carrillo-Angeles y estancia sabática en New Mexico State University, otorgada por DGAPA-PASPA- Universidad Nacional Autónoma de México a M. Mandujano. Este trabajo es parte de la tesis de 
doctorado de Israel Gustavo Carrillo Angeles, Posgrado en Ciencias Biológicas de la UNAM. Agradecemos la revisión, sugerencias en el trabajo así como el apoyo de los miembros del comité tutoral, Doctores Teresa Valverde y Francisco Molina. Asimismo a la Dra. Ana Mendoza, Dr. Jordan Golubov y dos revisores anónimos por sus sugerencias al artículo.

\section{Literatura citada}

Aarssen L.W. 2008. Death without sex-the 'problem of the small' and selection for reproductive economy in flowering plants. Evolutionary Ecology 22:279-298.

Albert T., Raspé O. y Jacquemart A.L. 2003. Clonal structure in Vaccinium myrtillus L. revealed by RAPD and AFLP markers. International Journal of Plant Sciences 164:649-655.

Arens P., Grashof-Bokdam C.J., van der Sluis T. y Smulders M.J.M. 2005. Clonal diversity and genetic differentiation of Maianthemum bifolium among forest fragments of different age. Plant Ecology 179:169-180.

Arizaga S. y Ezcurra E. 2002. Propagation mechanisms in Agave macrovantha (Agavaceae), a tropical arid land succulent rosette. American Journal of Botany 89:632-641.

Barbour M.G., Burk J.H. y Pitts W.D. 1987. Terrestrial Plant Ecology. The Benjamin-Cummings Publishing Company, Menlo Park.

Barret S.C.H., Eckert C.G. y Husband B.C. 1993. Evolutionary processes in aquatic plant populations. Aquatic Botany 44:105-145.

Bell A.D. 1984. Dynamic morphology: A contribution to plant population ecology. En: Dirzo R. y Sarukhán J. Eds. Perspectives on Plant Population Ecology, pp. 49-65, Sinauer Associates Inc. Publisher, Sunderland.

Brzosko E., Wróblewska A. y Ratkiewicz M. 2002. Spatial genetic structure and clonal diversity of island populations of lady's slipper (Cyperidium calceolus) from the Biebrza National Park (northeast Poland). Molecular Ecology 11:2499-2509.

Bushakra J.M., Hodges S.A., Cooper J.B. y Kaska D.D. 1999. The extent of clonality and genetic diversity in the Santa Cruz Island ironwood, Lyonothamnus floribundus. Molecular Ecology 8:471-475.

Camacho F.J. y Liston A. 2001. Population structure and genetic diversity of Botrychium pumicola (Ophioglossaceae) based on inter-simple sequence repeats (ISSR). American Journal of Botany 88:1065-1070.

Carrillo-Angeles I.G., Mandujano M.C. y Golubov J. 2011. Influences of the genetic neighborhood on ramet reproductive success in a clonal desert cactus. Population Ecology 53:449458.

Caswell H. 1985. The evolutionary demography of clonal reproduction. En: Jackson J.B.C., Buss L.W. y Cook R.E. Eds. Population Biology and Evolution of Clonal Organisms, pp. 187224, Yale University Press, New Haven.

Charpentier A. 2002. Consequences of clonal growth for plant mating. Evolutionary Ecology 15:521-530.

Charpentier A., Grillas P. y Thompson J.D. 2000. The effects of population size limitation on fecundity in mosaic populations of the clonal macrophyte Scirpus maritimus (Cyperaceae). American Journal of Botany 87:502-507.

Chen J. y Bradshaw G.A. 1999. Forest structure in space: a case study of an old growth spruce-fir forest in Changbaishan Natural Reserve, PR China. Forest Ecology and Management 120:219-233.

Chung M.G. y Epperson B.K. 2000. Clonal and spatial genetic structure in Eurya emarginata (Theaceae). Heredity 84:170177.

Chung J.M., Lee B.C., Kim J.S., Park C., Chung M.Y. y Chung M.G. 2006. Fine-scale genetic structure among genetic individuals of the clone-forming monotypic genus Echinospora koreensis (Fabaceae). Annals of Botany 98:165-173.

Clark P.J. y Evans F.C. 1954. Distance to nearest neighbor as a measure of spatial relationships in populations. Ecology 35:445-453.

Clark-Tapia R., Alfonso-Corrado C., Eguiarte L.E. y Molina-Freaner F. 2005. Clonal diversity and distribution in Stenocereus eruca (Cactaceae), a narrow endemic cactus of the Sonoran Desert. American Journal of Botany 92:272-278.

Clark-Tapia R., Alfonso-Corrado C., Mandujano M.C. y MolinaFreaner F. 2006. Reproductive consequences of clonal growth in Stenocereus eruca, a rare clonal cactus of the Sonoran desert. Evolutionary Ecology 20:131-142.

Couteron P. y Kokou K. 1997. Woody vegetation spatial patterns in a semi-arid savanna of Burkina Faso, West Africa. Plant Ecology 132:211-227.

Damman H. y Cain M.L. 1998. Population growth and viability analyses of the clonal woodland herb, Asarum canadense. Journal of Ecology 86:13-26.

de Jong T.J., Waser N.M., Price M.V. y Ring R.M. 1992. Plant size, geitonogamy and seed set in Ipomopsis aggregata. Oecologia 89:310-315.

de Kroon H. y van Groenendael J. Eds. 1997. The Ecology and Evolution of Clonal Plants. Backhuys Publishers, Leiden.

de Kroon H. y Hutchings M.J. 1995. Morphological plasticity in clonal plants: the foraging concept reconsidered. Journal of Ecology 83:143-152.

Diggle P.K., Lower S. y Ranker T.A. 1998. Clonal diversity in alpine populations of Polygonum viviparum (Polygonaceae). International Journal of Plant Sciences 159:606-615.

Diggle P.K., Meixner M.A., Carroll A.B. y Aschwanden C.F. 2002. Barriers to sexual reproduction in Polygonum viviparum: A comparative developmental analysis of $P$. viviparum and $P$. bistortoides. Annals of Botany 89:145-156.

Douhovnikoff V. y Dood R.S. 2003. Intra-clonal variation and a similarity threshold for identification of clones: application to $\mathrm{Sa}$ lix exigua using AFLP molecular markers. Theoretical Applied Genetics 106:1307-1315.

Douhovnikoff V., Cheng A.M. y Dodd R.S. 2004. Incidence, size and spatial structure of clones in second-growth stands of coast redwood, Sequoia sempervirens (Cupressaceae). American Journal of Botany 91:1140-1146.

Douhovnikoff V., McBride J.R. y Dodd R.S. 2005. Salix exigua clonal growth and population dynamics in relation to disturbance regime variation. Ecology 86:446-452.

Eckert C.G. 2000. Contributions of autogamy and geitonogamy to self-fertilization in a mass-flowering, clonal plant. Ecology 81:532-542.

Eckert C.G. 2002. The loss of sex in clonal plants. Evolutionary Ecology 15:501-520.

Ellstrand N.C. y Roose M.L. 1987. Patterns of genotypic diversity in clonal plant species. American Journal of Botany 74:123-131. 
Eriksson O. 1989. Seedling dynamics and life histories in clonal plants. Oikos 55:231-238.

Eriksson O. 1993. Dynamics of genets in clonal plants. Trends in Ecology \& Evolution 8:313-316.

Eriksson O. y Bremer B. 1993. Genet dynamics of the clonal plant Rubus saxatilis. Journal of Ecology 81:533-542.

Esselman E.J., Jianqiang L., Crawford D.J., Winduss J.L. y Wolfe A.D. 1999. Clonal diversity in the rare Calamagrostis porteri ssp insperata (Poaceae): comparative results for allozymes and random amplified polymorphic DNA (RAPD) and intersimple sequence repeat (ISSR) markers. Molecular Ecology 8:443451.

Everitt B.S. 1977. The Analysis of Contingency Tables. Chapmann \& Hall, Bury St Edmunds.

Gabrielsen T.M. y Brochmann C. 1998. Sex after all: high levels of diversity detected in the arctic clonal plant Saxifraga cernua using RAPD markers. Molecular Ecology 7:1701-1708.

Garnier L.K.M., Durand J. y Dajoz I. 2002. Limited seed dispersal and microspatial population structure of an agamospermous grass of West African savannahs, Hyparrhenia diplandra (Poaceae). American Journal of Botany 89:1785-1791.

Gibson D.J. y Menges E.S. 1994. Population structure and spatial pattern in the dioecious shrub Ceratiola ericoides. Journal of Vegetation Sciences 5:337-346.

Gleason H.A. 1920. Some applications of the quadrat method. Bulletin of the Torrey Botanical Club 47:21-33.

Hämmerli A. y Reusch T.B.H. 2003a. Flexible mating: cross-pollination affects sex expression in a marine clonal plant. Journal of Evolutionary Biology 18:1096-1105.

Hämmerli A. y Reusch T.B.H. 2003b. Genetic neighbourhood of clone structures in eelgrass meadows quantified by spatial autocorrelation of microsatellite markers. Heredity 91:448-455.

Handel S.N. 1985. The intrusion of clonal growth patterns on plant breeding system. The American Naturalist 125:367-384.

Harper J. 1985. Modules, branches, and the capture of resources. En: Jackson J.B.C., Buss L.W. y Cook R.E. Eds. Population Biology and Evolution of Clonal Organisms, pp. 1-33, Yale University Press, New Haven.

Hartnett D.C. y Bazzaz F.A. 1985. The integration of neighbourhood effects by clonal genets in Solidago canadensis. The Journal of Ecology 73:415-427.

Hirayama K. y Sakimoto M. 2008. Clonal structure and diversity of Cryptomeria japonica along a slope in a cool-temperate, oldgrowth mixed forest in the snowy region of Japan. Canadian Journal of Forest Research 38:2804-2813.

Honnay O. y Bossuyt B. 2005. Prolonged clonal growth: escape route or route to extinction? Oikos 108:427-432.

Honnay O., Jacquemyn H., Roldán-Ruiz I. y Hermy M. 2006. Consequences of prolonged clonal growth on local and regional genetic structure and fruiting success of the forest perennial Maianthemum bifolium. Oikos 112:21-30.

Honnay O. y Jacquemyn H. 2008. A meta-analysis of the relation between mating system, growth form and genotypic diversity in clonal plant species. Evolutionary Ecology 22:299-312.

Ivey C.T. y Richards J.H. 2001. Genotypic diversity and clonal structure of everglades sawgrass, Cladium jamaicense (Cyperaceae). International Journal of Plant Sciences 162:1327-1335.

Jackson J.B.C., Buss L.W. y Cook R.E. Eds. 1985. Population Biology and Evolution of Clonal organisms. Yale University Press, New Haven.
Jacquemyn H., Brys R., Honnay O., Hermy M. y Roldán-Ruiz I. 2005. Local forest environment largely affects below-ground growth, clonal diversity and fine-scale spatial genetic structure in the temperate deciduous forest herb Paris quadrifolia. Molecular Ecology 14:4479-4488.

Jonsson B.O., Jónsdóttir I.S. y Crongberg N. 1996. Clonal diversity and allozyme variation in populations of the arctic sedge Carex bigelowii (Cyperaceae). Journal of Ecology 84:449-459.

Kays S. y Harper J.L. 1974. The regulation of plant and tiller density in a grass sward. Journal of Ecology 62:97-105.

Keane B., Pelikan S., Toth G.P., Smith M.K. y Rogstad S.H. 1999. Genetic diversity of Typha latifolia (Typhaceae) and the impact of pollutants examined with tamdem-repetitive DNA probes. American Journal of Botany 86:1226-1238.

Kjølner S., Såstad S.M. y Brochmann C. 2006. Clonality and recombination in the arctic plant Saxifraga cernua. Botanical Journal of the Linnean Society 152:209-217.

Klimeš L., Klimešová J., Hendriks R.J.J. y van Groenendael J.M. 1997. Clonal plant architecture: a comparative analysis of form and function. En: de Kroon H. y van Groenendael J. Eds. The Ecology and Evolution of Clonal Plants, pp. 1-29, Backhuys Publishers, Leiden.

Korpelainen H. 1994. Sex ratios and resource allocation among sexually reproducing plants of Rubus chamaemorus. Annals of Botany 74:627-632.

Korpelainen H., Antonious-Klemola K. y Werlemark G. 1999. Clonal structure of Rubus chamaemorus populations: comparison of different molecular methods. Plant Ecology 143:123-128.

Kreher S.A., Foré S.A. y Collins B.S. 2000. Genetic variation within and among patches of the clonal species, Vaccinium stamineum L. Molecular Ecology 9:1247-1252.

Kudoh H., Shibaike H., Takasu H., Whigham D.F. y Kawano S. 1999. Genet structure and determinants of clonal structure in a temperate deciduous woodland herb, Uvularia perfoliata. Journal of Ecology 87:244-257.

Liston A., Wilson B.L., Robinson W.A., Doescher P.S., Harris N.R. y Svejcar T. 2003. The relative importance of sexual reproduction versus clonal spread in an arid bunchgrass. Oecologia 137:216-225.

Liu J., Dong M., Miao S.L., Li Z.Y., Song M.H. y Wang R.Q. 2006. Invasive alien plants in China: role of clonality and geographical origin. Biological Invasions 8:1461-1470.

Mandujano M.C., Montaña C., Méndez I. y Golubov J. 1998. The relative contributions of sexual reproduction and clonal propagation in Opuntia rastrera from two habitats in the Chihuahuan desert. Journal of Ecology 86:911-921.

Mandujano M.C., Montaña C., Franco M., Golubov J. y FloresMartínez A. 2001. Integration of demographic annual variability in a clonal desert cactus. Ecology 82:344-359.

Mandujano-Sánchez M.C. 2007. La clonalidad y sus efectos en la biología de poblaciones. En: Eguiarte L.E., Souza V. y Aguirre X. Comp. Ecología Molecular, pp. 215-250, Secretaría de Medio Ambiente y Recursos Naturales-Instituto Nacional de Ecología-Universidad Nacional Autónoma de México-Comosión Nacional para el Conocimiento y Uso de la Biodiversidad, México, D.F.

Mayes S.G., McGinley M.A. y Werth C.R. 1998. Clonal population structure and genetic variation in sand-shinnery oak, Quercus havardii (Fagaceae). American Journal of Botany 85:1609-1617. McFadden C.S. 1991. A comparative demographic analysis of clonal 
reproduction in a temperate soft coral. Ecology 72:1849-1866.

Misuki I., Ishida K., Tani N. y Tsumura Y. 2010. Fine-scale spatial structure of genets and sexes in the dioecious plant Dioscorea japonica, which disperses by both bulbils and seeds. Evolutionary Ecology 24:1399-1415.

Mori Y., Nagamitsu T. y Kubo T. 2009. Clonal growth and its effects on male and female reproductive success in Prunus ssiori (Rosaceae). Population Ecology 51:175-186.

Nagamitsu T., Ogawa M., Ishida K. y Tanouchi H. 2004. Clonal diversity, genetic structure, and mode of recruitment in a Prunus ssiori population established after volcanic eruptions. Plant Ecology 174:1-10.

Okuda T., Kachi N., Yap S.K. y Manokaran N. 1997. Tree distribution pattern and fate of juveniles in a lowland tropical rain forest - implications for regeneration and maintenance of species diversity. Plant Ecology 131:155-171.

Pan J.J. y Price J.S. 2002. Fitness and evolution in clonal plants: the impact of clonal growth. Evolutionary Ecology 15:583-600.

Parker K.C. y Hamrick J.L. 1992. Genetic diversity and clonal structure in a columnar cactus, Lophocereus schottii. American Journal of Botany 79:86-96.

Parks C. y Werth C.R. 1993. A study of spatial features of clones in a population of bracken fern, Pteridium aquilinum (Dennstaedtiaceae). American Journal of Botany 80:537-544.

Peterson C.J. y Jones R.H. 1997. Clonality in woody plants: a review and comparison with clonal herbs. En: de Kroon H. y van Groenendael J. Eds. The Ecology and Evolution of Clonal Plants, pp. 263-289, Backhuys Publishers, Leiden.

Pfeiffer T., Klahr A., Heinrich A. y Schnittler M. 2011. Does sex make a difference? Genetic diversity and spatial genetic structure in two co-occurring species of Gagea (Liliaceae) with contrasting reproductive strategies. Plant Systematics and Evolution 292:189-201.

Pornon A. y Escaravage N. 1999. Genotypic structure in clonal Rhododendron ferrugineum L. (Ericaceae) populations: origin and manteinance. Plant Ecology 141:145-150.

Pornon A., Escaravage N., Thomas P. y Taberlet P. 2000. Dynamics of genotypic structure in clonal Rhododendron ferrugineum (Ericaceae) populations. Molecular Ecology 9:1099-1111.

Pornon A., Escaravage N., Till-Botraud I. y Doche B. 1997. Variation of reproductive traits in Rhododendron ferrugineum $\mathrm{L}$. (Ericaceae) populations along a successional gradient. Plant Ecology 130:1-11.

Price E.A.C. y Marshall C. 1999. Clonal plants and environmental heterogeneity. Plant Ecology 141:3-7.

Reisch C., Schurm S. y Poschlod P. 2007. Spatial genetic structure and clonal diversity in an alpine population of Salix herbacea (Salicaceae). Annals of Botany 99:647-651.

Reusch T.B.H. 2003. Floral neighbourhoods in the sea: how floral density, opportunity for outcrossing and population fragmentation affect seed set in Zostera marina. Journal of Ecology 91:610-615.

Richards A.J. 1997. Plant Breeding Systems. Chapman \& Hall,
Londres.

Rosseto M., Gross C.L., Jones R. y Hunter J. 2004. The impact of clonality on an endangered tree (Elaeocarpus williamsianus) in a fragment rain forest. Biological Conservation 117:33-39.

Ruggieiro M.V., Capone S., Pirozzi P., Reusch T.B.H. y Procaccini G. 2005. Mating system and clonal architecture: a comparative study in two marine angiosperms. Evolutionary Ecology 19:487-499.

Shimizu Y, Ando M. y Sakai F. 2002. Clonal structure of natural populations of Cryptomeria japonica growing at different positions on slopes, detected using RAPD markers. Biochemical Systematics and Ecology 30:733-748.

Shreve F. 1917. The establishment of desert perennials. Journal of Ecology 5:210-216.

Sipes S.D. y Wolf P.G. 1997. Clonal structure and patterns of allozyme diversity in the rare endemic Cycladenia humilis var. jonesii (Apocynaceae). American Journal of Botany 84:401-409.

Stehlik I. y Holderegger R. 2000. Spatial genetic structure and clonal diversity of Anemone nemorosa in late successional deciduous woodlands of Central Europe. Journal of Ecology 88:424-435.

Suvanto L.I. y Latva-Karjantvaa T.B. 2005. Clone identification and clonal structure of the European aspen (Populus tremula). Molecular Ecology 14:2851-2860.

Tiffney B.H. y Niklas K.J. 1985. Clonal growth in land plants: a paleobotanical perspective. En: Jackson J.B.C., Buss L.W. y Cook R.E. Eds. Population Biology and Evolution of Clonal Organisms, pp. 35-66, Yale University Press, New Haven.

Tooke F., Ordidge M., Chiurugwi T. y Battey N. 2005. Mechanisms and function of flower and inflorescence reversion. Journal of Experimental Botany 56:2587-2599.

Torimaru T. y Tomaru N. 2005. Fine-scale clonal structure and diversity within patches of a clone-forming dioecious shrub, Ilex leucoclada (Aquifoliaceae). Annals of Botany 95:295-304.

van Dijk P.J. y Bakx-Schotman J.M.T. 2004. Formation of unreduced megaspores (diplospory) in apomictic dandelions (Taraxacum officinale, s.l.) is controlled by a sex-specific dominant locus. Genetics 166:483-492.

van Groenendael J.M. y de Kroon H. 1990. Clonal Growth in Plants: Regulation and Function. SPB Academic Publishing, La Haya.

van Groenendael J.M., Klimeš L., Klimešová J. y Hendriks R.J.J. 1996. Comparative ecology of clonal plants. Philosophical Transactions of Royal Society of London 351:1331-1339.

Widén B., Cronberg N. y Widén M. 1994. Genotypic diversity, molecular markers and spatial distribution of genets in clonal plants, a literature survey. Folia Geobotanica y Phytotaxonomica 29:245-263.

Wilson A.S.G., van der Kamp B.J. y Ritland C. 2005a. Opportunities for geitonogamy in the clonal herb Maianthemum dilatatum. Canadian Journal of Botany 83:1082-1087.

Wilson A.S.G., van der Kamp B.J. y Ritland C. 2005b. Spatial genetic and clonal structure in Maianthemum dilatatum as defined by AFLP markers. Canadian Journal of Botany 83:1126-1132. 
Apéndice. Características de las especies y poblaciones: $\mathbf{S C R Z}=$ sistema de cruza, $\mathbf{P O L}=$ polinización, $\mathbf{P R O R}=$ estructuras o procesos que dan origen a los ramets, CCT-N = tipo de crecimiento clonal en la clasificación de Tiffney y Niklas (1985), POB/HL = número de población y/o hábitat local, $\mathbf{P D E}=$ clave del patrón de distribución espacial de acuerdo a la figura 2, AER = arreglo espacial de los ramets de distintos genets, $\boldsymbol{D}=$ índice de diversidad clonal de Simpson, $\boldsymbol{G} / \mathbf{N}=$ proporción de genotipos distinguibles, Métodos = métodos empleados para la evaluación de los parámetros. El significado de las abreviaturas empleadas en cada columna se muestra al final del cuadro.

\begin{tabular}{|c|c|c|c|c|c|c|c|c|c|c|c|c|c|}
\hline Especie (Familia) & Hábito & SCRZ & POL & PROR & CCT-N & $\begin{array}{c}\text { POB/ } \\
\mathrm{HL}\end{array}$ & $\begin{array}{c}\text { PDE } \\
\text { (fig. 2) }\end{array}$ & $\begin{array}{c}\text { AER } \\
\text { (fig. 2) }\end{array}$ & $D$ & $G / N$ & $\begin{array}{l}\text { Estructura } \\
\text { genética } \\
\text { local (EGL) }\end{array}$ & $\begin{array}{c}\text { Métodos } \\
\text { PDE, } D, G / N \\
\text { y EGL }\end{array}$ & Referencias \\
\hline $\begin{array}{l}\text { Anemone } \\
\text { nemorosa } \\
\text { (Ranunculaceae) }\end{array}$ & $\mathrm{Hp}$ & A & $\mathrm{Bt}$ & $\begin{array}{c}\mathrm{Rzm+} \\
\mathrm{drm}\end{array}$ & $\mathrm{Lg}+\mathrm{NI}$ & $\begin{array}{l}\text { ppm } \\
(20)\end{array}$ & $(4,5)$ & $\mathrm{Ag}+\mathrm{En}$ & $\mathrm{n} / \mathrm{e}$ & 0.95 & $\begin{array}{l}\operatorname{asp},<1 \mathrm{~m} \\
(\mathrm{rm})\end{array}$ & tra+ISZ+asp & $\begin{array}{l}\text { Stehlik y } \\
\text { Holderegger } \\
\text { 2000* }\end{array}$ \\
\hline $\begin{array}{l}\text { Botrychium } \\
\text { pumicola } \\
\text { (Ophioglossaceae) }\end{array}$ & $\mathrm{Hp}$ & A & $\mathrm{Ab}$ & gmas & $\mathrm{NI}$ & $\begin{array}{l}\text { p } 1 \\
\text { p } 2 \\
\text { p } 3\end{array}$ & $\begin{array}{l}(2,5) \\
(2,5)\end{array}$ & $\begin{array}{l}\mathrm{Ag}+\mathrm{En} \\
\mathrm{Ag}+\mathrm{En} \\
\mathrm{Ag}+\mathrm{En}\end{array}$ & $\begin{array}{c}0.14 \\
0.16 \\
0.1\end{array}$ & $\begin{array}{l}0.93 \\
0.9 \\
0.65\end{array}$ & $\begin{array}{c}\text { mt: no } \\
\text { detectada } \\
\text { mt: no } \\
\text { detectada } \\
\text { mt: no } \\
\text { detectada }\end{array}$ & prc + ISSR $+m t$ & $\begin{array}{c}\text { Camacho y } \\
\text { Liston, 2001* }\end{array}$ \\
\hline $\begin{array}{l}\text { Calamagrostis } \\
\text { porteri ssp. } \\
\text { insperata (Poaceae) }\end{array}$ & $\mathrm{Hp}$ & A & $\mathrm{Ab}$ & rzm & $\operatorname{Lg}$ & $\begin{array}{l}\text { p } 2 \\
\text { p } 3 \\
\text { p } 4\end{array}$ & $\begin{array}{l}2 \\
2 \\
2\end{array}$ & $\begin{array}{l}\mathrm{Ag} \\
\mathrm{Ag} \\
\mathrm{Ag}\end{array}$ & $\begin{array}{l}n / e \\
n / e \\
n / e\end{array}$ & $\begin{array}{c} \\
1 \\
0.82 \\
0.91\end{array}$ & $\begin{array}{l}\mathrm{n} / \mathrm{e} \\
\mathrm{n} / \mathrm{e} \\
\mathrm{n} / \mathrm{e}\end{array}$ & $\begin{array}{c}\text { desc+tra+ } \\
\text { ISRR+ } \\
\text { RAPD }\end{array}$ & $\begin{array}{c}\text { Esselman } \\
\text { et al., } \\
1999\end{array}$ \\
\hline $\begin{array}{l}\text { Carex bigelowii } \\
\text { (Cyperaceae) }\end{array}$ & $\mathrm{Hp}$ & $?$ & $\mathrm{Ab}$ & rzm & $\operatorname{Lg}$ & $\begin{array}{l}\text { p } 1 \\
\text { p } 2 \\
\text { p } 3\end{array}$ & $\begin{array}{l}4 \\
4 \\
4\end{array}$ & $\begin{array}{l}\mathrm{Ag} \\
\mathrm{Ag} \\
\mathrm{Ag}\end{array}$ & $\begin{array}{l}0.98 \\
0.98 \\
0.98\end{array}$ & $\begin{array}{l}0.52 \\
0.63 \\
0.48\end{array}$ & $\begin{array}{l}\text { n/e } \\
\text { n/e } \\
\text { n/e }\end{array}$ & tra+ISZ & $\begin{array}{c}\text { Jonsson } \\
\text { et al., 1996* }\end{array}$ \\
\hline $\begin{array}{l}\text { Cladium jamaicense } \\
\text { (Cyperaceae) }\end{array}$ & $\mathrm{Hp}$ & $?$ & $\mathrm{Ab}$ & $\begin{array}{c}\text { rzm+ } \\
\text { pnt }\end{array}$ & $\mathrm{Lg}+\mathrm{Nl}$ & $\begin{array}{l}p 1 \\
\text { p } 2 \\
\text { p } 3 \\
\text { p } 4 \\
\text { p } 5 \\
\text { p } 6 \\
\text { p } 7 \\
\text { p } 8 \\
\text { p } 9 \\
\text { p } 10 \\
\text { p } 11 \\
\text { p } 12 \\
\text { p } 13 \\
\text { p } 14\end{array}$ & $\begin{array}{l}(4,5) \\
(4,5) \\
(4,5) \\
(4,5) \\
(4,5) \\
1 \\
(4,5) \\
(4,5) \\
(4,5) \\
(4,5) \\
(4,5) \\
(4,5) \\
(4,5) \\
(4,5)\end{array}$ & $\begin{array}{c}A g+E n \\
A g+E n \\
A g+E n \\
A g+E n \\
A g+E n \\
A g \\
A g+E n \\
A g+E n \\
A g+E n \\
A g+E n \\
A g+E n \\
A g+E n \\
A g+E n \\
A g+E n\end{array}$ & $\begin{array}{c}0.236 \\
0.269 \\
0.271 \\
0.682 \\
0.159 \\
0 \\
0.538 \\
0.212 \\
0.771 \\
0.698 \\
0.814 \\
0.682 \\
0.673 \\
0.444\end{array}$ & $\begin{array}{l}0.044 \\
0.044 \\
0.125 \\
0.231 \\
0.043 \\
0.021 \\
0.146 \\
0.068 \\
0.106 \\
0.143 \\
0.261 \\
0.114 \\
0.13 \\
0.043\end{array}$ & $\begin{array}{c}\text { mpic: no } \\
\text { detectada } \\
\text { mpic: no } \\
\text { detectada } \\
\text { mpic: no } \\
\text { detectada } \\
\text { mpic: no } \\
\text { detectada } \\
\text { mpic: no } \\
\text { detectada } \\
\text { n/a } \\
\text { mpic: no } \\
\text { detectada } \\
\text { mpic: no } \\
\text { detectada } \\
\text { mpic: no } \\
\text { detectada } \\
\text { mpic: no } \\
\text { detectada } \\
\text { mpic: no } \\
\text { detectada } \\
\text { mpic: no } \\
\text { detectada } \\
\text { mpic: no } \\
\text { detectada } \\
\text { mpic: no } \\
\text { detectada }\end{array}$ & $\begin{array}{l}\text { tra+lSZ+ } \\
\text { mpic }\end{array}$ & $\begin{array}{l}\text { Ivey y Richards, } \\
\text { 2001* }\end{array}$ \\
\hline
\end{tabular}


Apéndice. Continuación

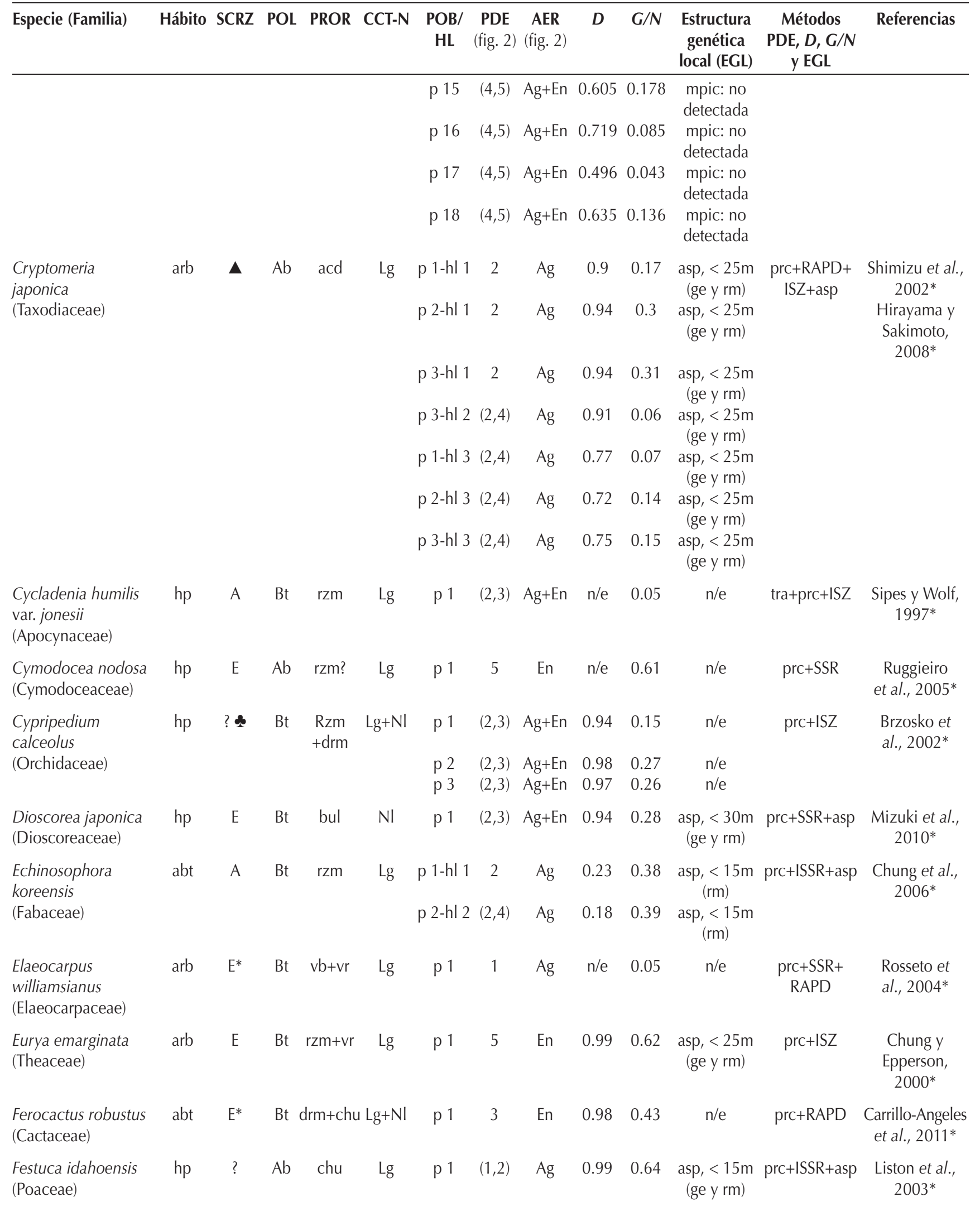


Apéndice. Continuación

\begin{tabular}{|c|c|c|c|c|c|c|c|c|c|c|c|c|c|}
\hline Especie (Familia) & Hábito & SCRZ & POL & PROR & CCT-N & $\begin{array}{c}\mathrm{POB} / \\
\mathrm{HL}\end{array}$ & $\begin{array}{l}\text { PDE } \\
\text { (fig. 2) }\end{array}$ & $\begin{array}{c}\text { AER } \\
\text { (fig. 2) }\end{array}$ & $D$ & $G / N$ & $\begin{array}{l}\text { Estructura } \\
\text { genética } \\
\text { local (EGL) }\end{array}$ & $\begin{array}{c}\text { Métodos } \\
\text { PDE, } D, G / N \\
\text { y EGL } \\
\end{array}$ & Referencias \\
\hline \multirow[t]{3}{*}{$\begin{array}{l}\text { Gagea lutea } \\
\text { (Liliaceae) }\end{array}$} & \multirow[t]{3}{*}{ hg } & \multirow[t]{3}{*}{$?$} & \multirow[t]{3}{*}{$\mathrm{Bt}$} & \multirow[t]{3}{*}{ buls } & \multirow[t]{3}{*}{$\mathrm{Nl}$} & p 1 & $(2,3)$ & $\mathrm{Ag}+\mathrm{En}$ & $\mathrm{n} / \mathrm{e}$ & 0.51 & \multirow{3}{*}{\multicolumn{2}{|c|}{$\begin{array}{l}\text { asp, }<8 \mathrm{~m}, 16 \text { tra+AFLP+asp } \\
\mathrm{a}<24 \mathrm{~m}(\mathrm{rm}) \\
\mathrm{asp},<8 \mathrm{~m}, 16 \\
\mathrm{a}<24 \mathrm{~m}(\mathrm{rm}) \\
\mathrm{asp},<8 \mathrm{~m}, 16 \\
\mathrm{a}<24 \mathrm{~m}(\mathrm{rm})\end{array}$}} & \multirow[t]{3}{*}{$\begin{array}{l}\text { Pfeiffer et al., } \\
2011^{*}\end{array}$} \\
\hline & & & & & & p 2 & $(2,3)$ & $\mathrm{Ag}+\mathrm{En}$ & $\mathrm{n} / \mathrm{e}$ & 0.57 & & & \\
\hline & & & & & & p 3 & $(2,3)$ & $\mathrm{Ag}+\mathrm{En}$ & $\mathrm{n} / \mathrm{e}$ & 0.61 & & & \\
\hline \multirow{3}{*}{$\begin{array}{l}\text { Gagea spathacea } \\
\text { (Liliaceae) }\end{array}$} & \multirow[t]{3}{*}{ hg } & \multirow[t]{3}{*}{$?$} & \multirow[t]{3}{*}{$\mathrm{Bt}$} & \multirow[t]{3}{*}{ buls } & \multirow[t]{3}{*}{$\mathrm{NI}$} & p 1 & 1 & $\mathrm{Ag}$ & $\mathrm{n} / \mathrm{e}$ & 0.001 & $\mathrm{n} / \mathrm{a}$ & \multirow[t]{3}{*}{ tra+AFLP } & \multirow{3}{*}{$\begin{array}{r}\text { Pfeiffer et al., } \\
2011^{*}\end{array}$} \\
\hline & & & & & & p 2 & 1 & $\mathrm{Ag}$ & $\mathrm{n} / \mathrm{e}$ & 0.001 & $\mathrm{n} / \mathrm{a}$ & & \\
\hline & & & & & & p 3 & 1 & $\mathrm{Ag}$ & $\mathrm{n} / \mathrm{e}$ & 0.001 & $\mathrm{n} / \mathrm{a}$ & & \\
\hline $\begin{array}{l}\text { Hyparrhenia } \\
\text { diplandra (Poaceae) }\end{array}$ & hp & $?$ & $\mathrm{Ab}$ & agp & $\mathrm{NI}$ & p 1 & 5 & En & 0.85 & 0.07 & $\begin{array}{l}\text { asp, }<6 \mathrm{~m} \\
\quad(\mathrm{rm})\end{array}$ & $\begin{array}{c}\text { prc+lSZ } \\
+ \text { CLO+asp }\end{array}$ & $\begin{array}{c}\text { Garnier et al., } \\
2002^{*}\end{array}$ \\
\hline $\begin{array}{l}\text { Ilex leucoclada } \\
\text { (Aquifoliaceae) }\end{array}$ & abt & E & $\mathrm{Bt}$ & $\operatorname{acd} \mathbf{\Lambda}$ & $\operatorname{Lg}$ & p 1 & $(2,3)$ & $\mathrm{Ag}+\mathrm{En}$ & 0.67 & 0.09 & $\mathrm{n} / \mathrm{e}$ & prc+RAPD & $\begin{array}{c}\text { Torimaru y } \\
\text { Tomaru, 2005* }\end{array}$ \\
\hline \multirow[t]{8}{*}{$\begin{array}{l}\text { Lophocereus schottii } \\
\text { (Cactaceae) }\end{array}$} & \multirow[t]{8}{*}{ arb } & \multirow[t]{8}{*}{ A } & \multirow[t]{8}{*}{$\mathrm{Bt}$} & \multirow[t]{8}{*}{$\begin{array}{l}\mathrm{drm} \\
+\mathrm{acd}\end{array}$} & \multirow[t]{8}{*}{$\mathrm{Lg}+\mathrm{NI}$} & p 1-hl 1 & 5 & En & 0.95 & 0.64 & $\begin{array}{c}\text { SG: 0-10 } \\
<10-50>\text { más } \\
\text { de } 50 m \text { (ge) }\end{array}$ & \multirow{8}{*}{ prc+ISZ+SG } & \multirow[t]{8}{*}{$\begin{array}{l}\text { Parker y } \\
\text { Hamrick, } \\
1992^{*}\end{array}$} \\
\hline & & & & & & p 2-hl 2 & 2 & $\mathrm{Ag}$ & 0.95 & 0.52 & $\begin{array}{l}\text { SG: } 0-10 \\
<10-50>\text { más } \\
\text { de } 50 \mathrm{~m} \text { (ge) }\end{array}$ & & \\
\hline & & & & & & p 3-hl 3 & 2 & $\mathrm{Ag}$ & 0.93 & 0.65 & $\begin{array}{c}\text { SG: } 0-10 \\
<10-50>\text { más } \\
\text { de } 50 m \text { (ge) }\end{array}$ & & \\
\hline & & & & & & p 4-hl 1 & $(1,2)$ & $\mathrm{Ag}$ & 0.94 & 0.24 & $\begin{array}{l}\text { SG: } 0-10> \\
\text { 10-50>más } \\
\text { de } 50 \mathrm{~m} \text { (ge) }\end{array}$ & & \\
\hline & & & & & & p 5-hl 4 & $(1,2)$ & $\mathrm{Ag}$ & 0.92 & 0.41 & $\begin{array}{l}\text { SG: } 0-10> \\
10-50<\text { más } \\
\text { de } 50 m \text { (ge) }\end{array}$ & & \\
\hline & & & & & & p 6-hl 4 & $(1,2)$ & $\mathrm{Ag}$ & 0.87 & 0.45 & $\begin{array}{c}\text { SG: } 0-10> \\
10-50<\text { más } \\
\text { de } 50 m \text { (ge) }\end{array}$ & & \\
\hline & & & & & & p 7-hl 2 & $(1,2)$ & $\mathrm{Ag}$ & 0.8 & 0.18 & $\begin{array}{l}\text { SG: } 0-10> \\
\text { 10-50>más } \\
\text { de } 50 \mathrm{~m} \text { (ge) }\end{array}$ & & \\
\hline & & & & & & p 8-hl 5 & $(1,5)$ & $\mathrm{Ag}+\mathrm{En}$ & 0.59 & 0.36 & $\begin{array}{l}\text { SG: } 10-50= \\
\text { más de } 50 \mathrm{~m} \\
\quad(\text { ge })\end{array}$ & & \\
\hline $\begin{array}{l}\text { Lyonothamnus } \\
\text { floribundus } \\
\text { (Rosaceae) }\end{array}$ & arb & $?$ & $\mathrm{Bt}$ & $\mathrm{vr}$ & $\operatorname{Lg}$ & p 1 & $(1,2)$ & $\mathrm{Ag}$ & $\mathrm{n} / \mathrm{e}$ & 0.036 & $\mathrm{n} / \mathrm{e}$ & prc+RAPD & $\begin{array}{c}\text { Bushakra et al., } \\
\text { 1999* }\end{array}$ \\
\hline $\begin{array}{l}\text { Maianthemum } \\
\text { bifolium } \\
\text { (Convalliariaceae) }\end{array}$ & hp & $E^{*}$ & $\mathrm{Bt}$ & $\mathrm{rzm}$ & $\operatorname{Lg}$ & p 1 & $(1,2)$ & $\mathrm{Ag}$ & 0.88 & 0.7 & $\begin{array}{l}\text { asp, }<50 \mathrm{~m} \\
\text { (ge) \# }\end{array}$ & $\begin{array}{l}\text { trans+AFLP } \\
\quad+\text { asp }\end{array}$ & $\begin{array}{l}\text { Arens et al., } \\
2005^{*} \\
\text { Honnay et al. } \\
2006\end{array}$ \\
\hline $\begin{array}{l}\text { Maianthemum } \\
\text { dilatatum } \\
\text { (Convalliariaceae) }\end{array}$ & hp & EAs & $\mathrm{Bt}$ & $\operatorname{rzm} \mathbf{\Delta}$ & $\operatorname{Lg}$ & p 1 & $(1,2)$ & $\mathrm{Ag}$ & $\mathrm{n} / \mathrm{e}$ & 0.64 & $\begin{array}{l}\text { asp, }<45 \mathrm{~m} \\
\text { (ge y rm) }\end{array}$ & $\begin{array}{l}\text { prc }+ \text { AFLP } \\
\quad+\text { asp }\end{array}$ & $\begin{array}{l}\text { Wilson et al., } \\
2005 a^{*} \\
\text { Wilson et al., } \\
2005 b^{*}\end{array}$ \\
\hline $\begin{array}{l}\text { Paris quadrifolia } \\
\text { (Trilliaceae) }\end{array}$ & hp & A & $\mathrm{Bt}$ & $\mathrm{rzm}$ & $\operatorname{Lg}$ & p 1-hl 1 & 3 & En & 0.87 & 0.27 & $\begin{array}{l}\text { asp, }<5 \mathrm{~m} \\
(\text { ge y rm) }\end{array}$ & $\begin{array}{l}\text { prc }+ \text { AFLP } \\
\quad+\text { asp }\end{array}$ & $\begin{array}{c}\text { Jacquemyn et } \\
\text { al., 2005* }\end{array}$ \\
\hline
\end{tabular}


Apéndice. Continuación

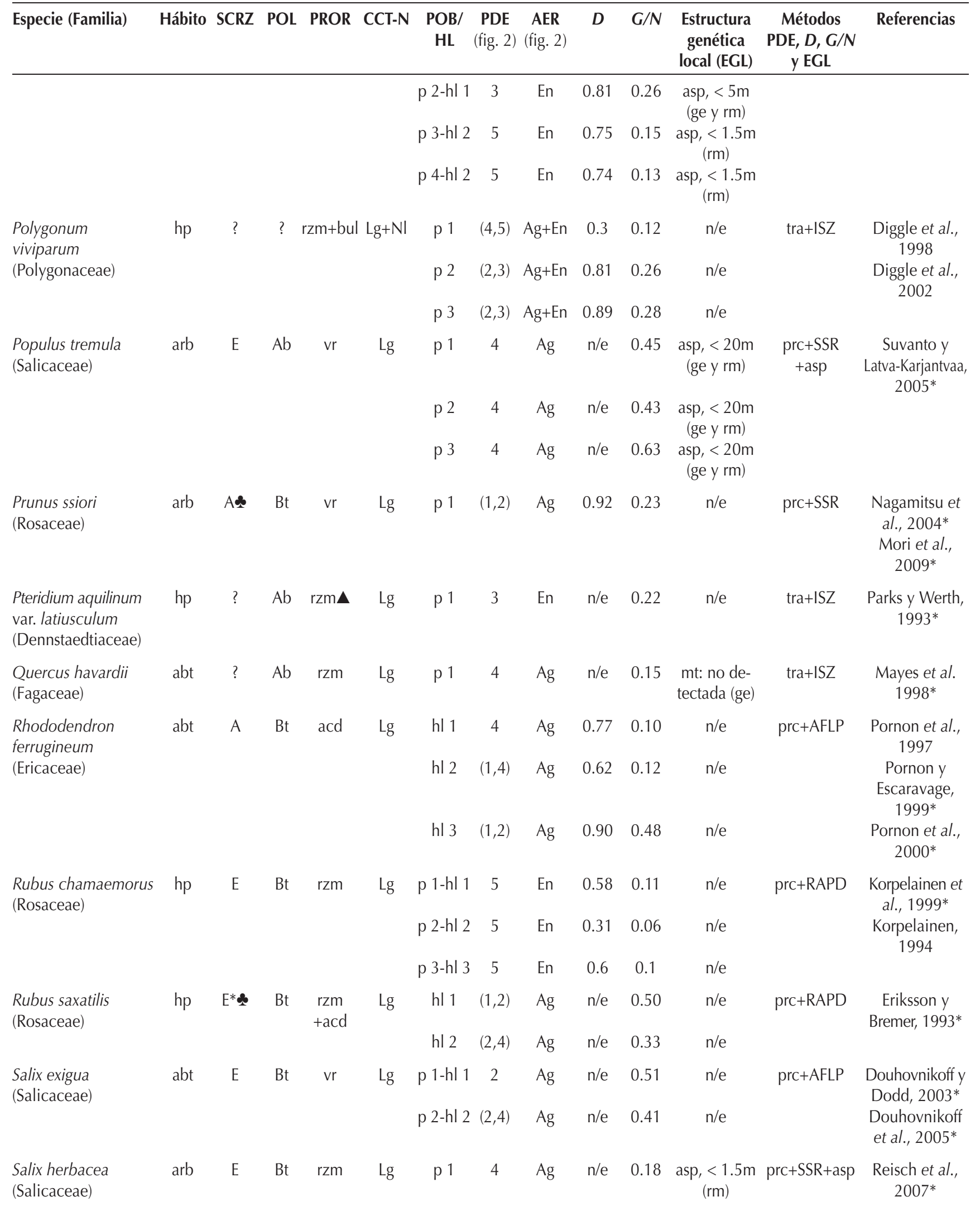


Apéndice. Continuación

\begin{tabular}{|c|c|c|c|c|c|c|c|c|c|c|c|c|c|}
\hline Especie (Familia) & Hábito & SCRZ & POL & PROR & CCT-N & $\begin{array}{c}\mathrm{POB} / \\
\mathrm{HL}\end{array}$ & $\begin{array}{c}\text { PDE } \\
\text { (fig. 2) }\end{array}$ & $\begin{array}{c}\text { AER } \\
\text { (fig. 2) }\end{array}$ & $D$ & $G / N$ & $\begin{array}{l}\text { Estructura } \\
\text { genética } \\
\text { local (EGL) }\end{array}$ & $\begin{array}{c}\text { Métodos } \\
\text { PDE, } D, G / N \\
\text { y EGL }\end{array}$ & Referencias \\
\hline \multirow[t]{6}{*}{$\begin{array}{l}\text { Saxifraga cernua } \\
\text { (Saxifragaceae) }\end{array}$} & \multirow[t]{6}{*}{ hp } & \multirow[t]{6}{*}{$E^{*}$} & \multirow[t]{6}{*}{$\mathrm{Bt}$} & \multirow[t]{6}{*}{ bul } & \multirow[t]{6}{*}{$\mathrm{Nl}$} & p 1 & 5 & En & 0.35 & 0.13 & $\begin{array}{c}\text { mt: no } \\
\text { detectada }\end{array}$ & \multirow[t]{6}{*}{$\begin{array}{l}\text { prc+RAPD } \\
+m t+a s p\end{array}$} & \multirow{6}{*}{$\begin{array}{l}\text { Gabrielsen y } \\
\text { Brochmann, } \\
1998^{*} \\
\text { KjØIner et al., } \\
\text { 2006* }\end{array}$} \\
\hline & & & & & & p 2 & 5 & En & 0.68 & 0.19 & $\begin{array}{c}\text { mt: no } \\
\text { detectada }\end{array}$ & & \\
\hline & & & & & & p 3 & 5 & En & 0.7 & 0.25 & $\begin{array}{l}\text { asp: no } \\
\text { detectada }\end{array}$ & & \\
\hline & & & & & & p 4 & 5 & En & 0.9 & 0.4 & $\begin{array}{c}\text { asp: no } \\
\text { detectada }\end{array}$ & & \\
\hline & & & & & & p 5 & 5 & En & 0.93 & 0.43 & $\begin{array}{l}\text { asp: no } \\
\text { detectada }\end{array}$ & & \\
\hline & & & & & & p 6 & 5 & En & 0.89 & 0.34 & $\begin{array}{c}\text { asp: no } \\
\text { detectada }\end{array}$ & & \\
\hline \multirow[t]{3}{*}{$\begin{array}{l}\text { Scirpus maritimus } \\
\text { (Cyperaceae) }\end{array}$} & \multirow[t]{3}{*}{ hp } & \multirow[t]{12}{*}{$\mathrm{E}^{*}$} & \multirow[t]{3}{*}{$\mathrm{Ab}$} & \multirow[t]{3}{*}{$\mathrm{rzm}$} & \multirow[t]{3}{*}{$\operatorname{Lg}$} & p 1 & 2 & $\mathrm{Ag}$ & $\mathrm{n} / \mathrm{e}$ & $\mathrm{n} / \mathrm{e}$ & $\mathrm{n} / \mathrm{e}$ & \multirow[t]{3}{*}{ desc } & \multirow[t]{3}{*}{$\begin{array}{c}\text { Charpentier et } \\
\text { al., } 2000\end{array}$} \\
\hline & & & & & & p 2 & 4 & $\mathrm{Ag}$ & $\mathrm{n} / \mathrm{e}$ & $\mathrm{n} / \mathrm{e}$ & $\mathrm{n} / \mathrm{e}$ & & \\
\hline & & & & & & p 3 & 4 & $\mathrm{Ag}$ & $\mathrm{n} / \mathrm{e}$ & $\mathrm{n} / \mathrm{e}$ & $\mathrm{n} / \mathrm{e}$ & & \\
\hline \multirow[t]{9}{*}{$\begin{array}{l}\text { Sequoia sempervirens } \\
\text { (Taxodiaceae) }\end{array}$} & \multirow[t]{9}{*}{ arb } & & \multirow[t]{9}{*}{$\mathrm{Ab}$} & \multirow[t]{9}{*}{$\mathrm{vb}$} & \multirow[t]{9}{*}{$\operatorname{Lg}$} & p 1-hl 1 & 2 & $\mathrm{Ag}$ & $\mathrm{n} / \mathrm{e}$ & 0.24 & $\begin{array}{l}\mathrm{mt}:<40 \mathrm{~m} \\
\quad(\mathrm{rm})\end{array}$ & \multirow[t]{9}{*}{$\operatorname{prc}+\mathrm{AFLP}+\mathrm{mt}$} & \multirow[t]{9}{*}{$\begin{array}{l}\text { Douhovnikoff } \\
\text { et al., 2004* }\end{array}$} \\
\hline & & & & & & p 2-hl 1 & 2 & $\mathrm{Ag}$ & $\mathrm{n} / \mathrm{e}$ & 0.38 & $\begin{array}{c}\mathrm{mt}:<40 \mathrm{~m} \\
(\mathrm{rm})\end{array}$ & & \\
\hline & & & & & & p 3-hl 1 & 2 & $\mathrm{Ag}$ & $\mathrm{n} / \mathrm{e}$ & 0.5 & $\begin{array}{c}\mathrm{mt}:<40 \mathrm{~m} \\
(\mathrm{rm})\end{array}$ & & \\
\hline & & & & & & p 4-hl 2 & 2 & $\mathrm{Ag}$ & $\mathrm{n} / \mathrm{e}$ & 0.6 & $\begin{array}{c}\mathrm{mt}:<40 \mathrm{~m} \\
(\mathrm{rm})\end{array}$ & & \\
\hline & & & & & & p 5-hl 2 & 2 & $\mathrm{Ag}$ & $\mathrm{n} / \mathrm{e}$ & 0.35 & $\begin{array}{c}\mathrm{mt}:<40 \mathrm{~m} \\
(\mathrm{rm})\end{array}$ & & \\
\hline & & & & & & p 6-hl 2 & 2 & $\mathrm{Ag}$ & $\mathrm{n} / \mathrm{e}$ & 0.6 & $\begin{array}{c}\mathrm{mt}:<40 \mathrm{~m} \\
(\mathrm{rm})\end{array}$ & & \\
\hline & & & & & & p 7-hl 3 & 2 & $\mathrm{Ag}$ & $\mathrm{n} / \mathrm{e}$ & 0.26 & $\begin{array}{c}\mathrm{mt}:<40 \mathrm{~m} \\
(\mathrm{rm})\end{array}$ & & \\
\hline & & & & & & p 8-hl 3 & 2 & $\mathrm{Ag}$ & $n / e$ & 0.19 & $\begin{array}{c}\mathrm{mt}:<40 \mathrm{~m} \\
(\mathrm{rm})\end{array}$ & & \\
\hline & & & & & & p 9-hl 3 & 2 & $\mathrm{Ag}$ & $\mathrm{n} / \mathrm{e}$ & 0.54 & $\begin{array}{c}\mathrm{mt}:<40 \mathrm{~m} \\
\quad(\mathrm{rm})\end{array}$ & & \\
\hline $\begin{array}{l}\text { Stenocereus eruca } \\
\text { (Cactaceae) }\end{array}$ & abt & $E^{*}$ & $\mathrm{Bt}$ & drm & $\mathrm{NI}$ & p 1 & 2 & $\mathrm{Ag}$ & 0.99 & 0.83 & $\begin{array}{l}\text { asp, }<20 \text { m } \\
\text { (ge y rm) }\end{array}$ & prc+ISSR+asp & $\begin{array}{c}\text { Clark-Tapia et } \\
\text { al., 2005* } \\
\text { Clark-Tapia et } \\
\text { al., } 2006\end{array}$ \\
\hline \multirow[t]{5}{*}{$\begin{array}{l}\text { Typha latifolia } \\
\text { (Thyphaceae) }\end{array}$} & \multirow[t]{5}{*}{$\mathrm{hp}$} & \multirow[t]{5}{*}{ A } & \multirow[t]{5}{*}{$\mathrm{Ab}$} & \multirow[t]{5}{*}{ rzm } & $\operatorname{Lg}$ & p 1 & $(4,5)$ & $\mathrm{Ag}+\mathrm{En}$ & $\mathrm{n} / \mathrm{e}$ & 0.77 & $\mathrm{n} / \mathrm{e}$ & tra+VNTR & $\begin{array}{c}\text { Keane et al., } \\
\text { 1999* }\end{array}$ \\
\hline & & & & & & p 2 & $(4,5)$ & $\mathrm{Ag}+\mathrm{En}$ & $\mathrm{n} / \mathrm{e}$ & 0.55 & $\mathrm{n} / \mathrm{e}$ & & \\
\hline & & & & & & p 3 & $(4,5)$ & $\mathrm{Ag}+\mathrm{En}$ & $\mathrm{n} / \mathrm{e}$ & 0.48 & $\mathrm{n} / \mathrm{e}$ & & \\
\hline & & & & & & p 4 & $(4,5)$ & $\mathrm{Ag}+\mathrm{En}$ & $\mathrm{n} / \mathrm{e}$ & 0.3 & $n / e$ & & \\
\hline & & & & & & p 5 & $(4,5)$ & $A g+E n$ & $\mathrm{n} / \mathrm{e}$ & 0.57 & $\mathrm{n} / \mathrm{e}$ & & \\
\hline $\begin{array}{l}\text { Uvularia perfoliata } \\
\text { (Convallariaceae) }\end{array}$ & hp & $?$ & $?$ & etl & $\operatorname{Lg}$ & p 1-hl 1 & $(4,5)$ & $A g+E n$ & 0.74 & 0.09 & $\begin{array}{c}\text { asp, }<3-5 m \\
(r m)\end{array}$ & prc+ISZ+asp & $\begin{array}{l}\text { Kudoh et al., } \\
\text { 1999* }\end{array}$ \\
\hline & & & & & & p 1-hl 2 & 1 & $\mathrm{Ag}$ & 0.59 & 0.03 & $\mathrm{n} / \mathrm{a}$ & & \\
\hline $\begin{array}{l}\text { Vaccinium myrtillus } \\
\text { (Ericaceae) }\end{array}$ & abt & A & $\mathrm{Bt}$ & rzm & $\operatorname{Lg}$ & p 1 & 2 & $\mathrm{Ag}$ & 0.94 & 0.29 & $\begin{array}{c}\text { asp: no } \\
\text { detectada }\end{array}$ & $\begin{array}{l}\text { prc+AFLP+ } \\
\text { RAPD+asp }\end{array}$ & $\begin{array}{c}\text { Albert et al., } \\
\text { 2003* }\end{array}$ \\
\hline
\end{tabular}


Apéndice. Continuación

\begin{tabular}{|c|c|c|c|c|c|c|c|c|c|c|c|c|c|}
\hline Especie (Familia) & Hábito & SCRZ & POL & PROR & CCT-N & $\begin{array}{c}\mathrm{POB} / \\
\mathrm{HL}\end{array}$ & $\begin{array}{l}\text { PDE } \\
\text { (fig. 2) }\end{array}$ & $\begin{array}{c}\text { AER } \\
\text { (fig. 2) }\end{array}$ & $D$ & $G / N$ & $\begin{array}{c}\text { Estructura } \\
\text { genética } \\
\text { local (EGL) }\end{array}$ & $\begin{array}{c}\text { Métodos } \\
\text { PDE, } D, G / N \\
\text { y EGL }\end{array}$ & Referencias \\
\hline $\begin{array}{l}\text { Vaccinium stamineum } \\
\text { (Ericaceae) }\end{array}$ & abt & $\mathrm{E}^{*}$ & $\mathrm{Bt}$ & $\mathrm{rzm}$ & $\operatorname{Lg}$ & $\mathrm{p} 1$ & $(2,3)$ & $\mathrm{Ag}+\mathrm{En}$ & $\mathrm{n} / \mathrm{e}$ & 0.68 & $\mathrm{n} / \mathrm{e}$ & prc+RAPD & $\begin{array}{l}\text { Kreher et al., } \\
\text { 2000* }\end{array}$ \\
\hline $\begin{array}{l}\text { Zostera marina } \\
\text { (Zosteraceae) }\end{array}$ & hp & A & $\mathrm{Ab}$ & $\mathrm{rzm}$ & $\operatorname{Lg}$ & $\begin{array}{l}\text { p 1-hl } 1 \\
\text { p 2-hl } 2\end{array}$ & $\begin{array}{l}(4,5) \\
(4,5)\end{array}$ & $\begin{array}{l}A g+E n \\
A g+E n\end{array}$ & $\mathrm{n} / \mathrm{e}$ & $\mathrm{n} / \mathrm{e}$ & $\begin{array}{c}\text { asp, }<6 \mathrm{~m} \\
\text { (ge y rm) } \\
\text { asp, }<6 \mathrm{~m} \\
\text { (ge y rm) }\end{array}$ & $\mathrm{prc}+\mathrm{SSR}+\mathrm{asp}$ & $\begin{array}{l}\text { Hämmerli y } \\
\text { Reusch, } 2003 \mathrm{a}^{*} \\
\text { Hämmerli y } \\
\text { Reusch, } 2003 \mathrm{~b}^{*}\end{array}$ \\
\hline $\begin{array}{l}\text { Zostera noltii } \\
\text { (Zosteraceae) }\end{array}$ & hp & A & $\mathrm{Ab}$ & $\mathrm{rzm}$ & $\operatorname{Lg}$ & p 1 & 4 & $\mathrm{Ag}$ & $\mathrm{n} / \mathrm{e}$ & 0.12 & $\mathrm{n} / \mathrm{e}$ & $\mathrm{prc}+\mathrm{SSR}$ & $\begin{array}{l}\text { Ruggieiro et } \\
\text { al., 2005* }\end{array}$ \\
\hline
\end{tabular}

Hábito: $\mathbf{a b t}=$ arbustos, $\mathbf{a r b}=$ árboles, $\mathbf{h p}=$ hierbas perennes

SCRZ: A = especies con capacidad de reproducirse por autocruza, $\mathbf{E}=$ especies con entrecruza obligada (p. ej., dioicas), $\mathbf{E}^{*}=$ especies con sistemas de autoincompatibilidad, = reclutamiento sexual observado y/o evaluado en condiciones naturales

POL: $\mathbf{A b}=$ polinización abiótica, $\mathbf{B t}=$ polinización biótica

PROR: acd = acodaduras, agp = agamospermia, bul = bulbilos, buls = bulbilos subterráneos, $\mathbf{c h u}=$ coronas huecas, $\mathbf{d r m}=$ desprendimiento de ramas, $\mathbf{e t l}=$ estolones, $\mathbf{g m a s}=$ gemas subterráneas, $\mathbf{p n t}=$ plantlets, $\mathbf{p s b}=$ pseudobulbos, $\mathbf{r z m}=$ rizomas, $\mathbf{v b}=$ vástagos basales, $\mathbf{v r}=\mathbf{v a ́ s t a g o s}$ radicales, $\mathbf{\Delta}$ = crecimiento tipo guerrilla

CCT-N: $\mathbf{L g}=$ crecimiento clonal ligado, $\mathbf{N I}=$ crecimiento clonal no ligado

POB/HL: $\mathbf{p}=$ población, $\mathbf{h l}=$ hábitat local (p. ej., bosque con dosel abierto vs bosque con dosel cerrado)

PDE: los paréntesis indican que la población presenta una combinación de patrones de distribución de los tipos indicados por los números (ver figura 2).

AER: Ag = los ramets de cada genet no se entremezclan con los ramets de genets distintos, En = los ramets de diferentes genets en la población se entremezclan.

EGL: \# = evaluado en una población distinta de donde se determinó el PDE, asp = autocorrelación espacial, mpic = método de probabilidad de identidad clonal, $\mathbf{m t}=$ prueba de Mantel, $\mathbf{S G}=$ similitud genética vs distancia espacial.

Métodos: AFLP = marcadores moleculares AFLPs, CLO = marcadores moleculares de cloroplasto, desc = descripción detallada del PDE, ISSR = marcadores moleculares ISSRs, ISZ = isoenzimas, $\mathbf{m p i c}=$ método de probabilidad de identidad clonal, $\mathbf{m t}=$ prueba de $\mathbf{M a n t e l}, \mathbf{p o b}=$ muestra de individuos o todos los individuos de la población, prc = parcelas, RAPD = marcadores moleculares RAPDs, SG $=$ similitud genética vs distancia espacial, $\mathbf{S S R}=$ marcadores moleculares SSRs (microsatélites),

Referencias: ${ }^{*}=$ estudios donde se presentan planos de la distribución espacial de los genotipos.

Todo el cuadro: ? = no se proporciona información, $\mathbf{n} / \mathbf{e}=$ no evaluado, $\mathbf{n} / \mathbf{a}=$ no aplica

GLOSARIO (columna PROR):

Acodaduras: formación de ramets a partir de un tallo originalmente erecto que se postra y genera raíces adventicias al entrar en contacto con el sustrato

Agamospermia: Producción asexual de semillas o embriones sin fertilización (diplosporía: el embrión se desarrolla de una célula diploide del saco embrionario, aposporía: el embrión se desarrolla de una célula somática del saco embrionario, embrionia adventicia: el embrión se desarrolla de células del tejido esporofítico como los tegumentos).

Bulbilos: bulbos pequeños que se forman en las axilas foliares o unidos a los bulbos.

Bulbos: tallos cortos modificados para almacenamiento rodeados por hojas carnosas o brácteas.

Coronas huecas: muerte de los tallos centrales en plantas cespitosas resultando en la formación de ramets independientes en la periferia. Descrito en pastos cespitosos.

Estolones: tallo horizontal que se extiende en la superficie del suelo.

Gemas: pequeñas estructuras vegetativas dispersables en briofitas y pteridofitas.

Plantlets: propágulos vegetativos que resultan de la reversión de estructuras reproductivas como flores y frutos a estructuras vegetativas.

Pseudobulbos: modificación del tallo para almacenamiento que se desarrolla entre dos nodos foliares en orquídeas.

Rizomas: tallo horizontal subterráneo.

Vástagos basales y radicales: formación de tallos en la base de un tallo o a partir de una raíz.

Referencias del glosario: Jackson et al., 1985; de Kroon y van Groenendael, 1997; Liston et al., 2003; van Dijk y Bakx-Schotman, 2004; Tooke et al., 2005. 


\title{
PATRONES DE DISTRIBUCIÓN ESPACIAL EN PLANTAS CLONALES
}

\author{
Israel Gustavo Carrillo-Angeles y María del Carmen Mandujano ${ }^{1}$ \\ Instituto de Ecología, Universidad Nacional Autónoma de México \\ ${ }^{1}$ Autor para la correspondencia: mcmandu@ecologia.unam.mx
}

\begin{abstract}
Resumen: En las especies clonales predomina la distribución agregada de los ramets, pero se plantea que la propagación por diásporas móviles o no ligadas, (por ejemplo, bulbilos y semillas agamospérmicas) forma arreglos espaciales de genets más entremezclados, que la propagación por estructuras ligadas (rizomas y estolones), y se sugiere que los arreglos entremezclados de genets favorecen la entrecruza. En este trabajo se presenta una revisión de 53 estudios en plantas clonales, para investigar la influencia de la producción de propágulos clonales, por diferentes formas de crecimiento clonal, en la formación de arreglos espaciales de genets y ramets. La hipótesis es que los arreglos entremezclados promueven la entrecruza, suponiendo que las poblaciones donde la entrecruza es más frecuente tenderán a presentar niveles más altos de diversidad genotípica. Los estudios revisados muestran un sesgo hacia herbáceas perennes rizomatosas. Predominan los patrones de distribución agregados de parches de ramets de distintos genets no entremezclados. Las poblaciones de especies que tienen ambos tipos de crecimiento de propágulos clonales (ligados y no ligados) y de especies que sólo presentan crecimiento ligado presentan patrones de distribución espacial de genets y ramets más entremezclados que las especies que sólo presentan crecimiento ligado. Encontramos que existe variación intraespecífica tanto en los patrones de distribución espacial como en los patrones reproductivos en las diferentes poblaciones. Sin embargo, utilizando índices de diversidad genotípica como estimadores, solamente se encontró un patrón congruente con el planteamiento sobre el efecto del arreglo espacial de los genets y los ramets en la proporción de genotipos distinguibles. Generalizar las consecuencias reproductivas y genéticas del arreglo espacial de los genets y los ramets continúa siendo complejo, debido a que una reducción del reclutamiento sexual y una dispersión restringida de polen, semillas y propágulos vegetativos pueden generar estructura genética local en las poblaciones. La estructura genética local tanto a nivel de genets (debida a la dispersión restringida de polen, semillas o ambos) como de ramets (indicando dispersión restringida de propágulos vegetativos) fue detectada en 46 poblaciones distribuidas en 16 especies. Estos factores aunados a la variedad de métodos de muestreo empleados en los trabajos publicados para detectar genets y ramets, la falta de estimadores numéricos comparables para describir la distribución espacial y la carencia de estudios en especies que se propagan por diásporas móviles o no ligadas reducen la resolución del efecto que tiene el tipo de crecimiento clonal en la formación de arreglos entremezclados de genets distintos sobre la diversidad genotípica.
\end{abstract}

Palabras clave: clonalidad, crecimiento, distribución, diversidad, entrecruza

\begin{abstract}
Clonal plants are predominantly associated with a clumped distribution of ramets. However, propagation by unlinked means generates a mixed arrangement instead of a clumped one found by linked propagules and intermingled arrangements of genets favor outcrossing. This paper reviews 53 studies on clonal plants to assess the influence of the production of clonal offspring by different means on the spatial arrangement of genets and ramets. We also evaluate the assumption that mixed arrangements promote outcrossing, under the hypothesis that populations where outcrossing is common would have higher levels of genotypic diversity. The studies that were consulted showed a strong bias towards rhizomatous perennial herbs. Clumped distribution patterns predominate in which patches consist of discrete groups of ramets from one or more genets that do not spatially mix. Populations having both types of propagation (linked and unlinked) and species that only have linked propagation have a higher spatially mixed genet and ramet distribution pattern than species that only have unlinked propagation. However, using genotypic diversity indices, we did not find a consistent pattern between the spatial arrangement and outcorssing rates. The reproductive and genetic consequences of the spatial arrangement of genets and ramets continues to be a difficult task, due to a range of factors such as the variety of methods, the lack of good comparative numeric estimations to describe the spatial arrangements and the lack of studies in species that propagate through unlinked propagules.
\end{abstract}

Key words: clonality, distribution, diversity, growth, outcrossing

a clonalidad da lugar a la producción de ramets, es decir, Lunidades potencialmente independientes que son genéticamente idénticas a la planta progenitora (Widén et al., 1994; van Groenendael et al., 1996; de Kroon y van Groenendael,
1997; Klims et al., 1997). En consecuencia, en las poblaciones de organismos clonales el concepto de "individuo" puede definir a los individuos genéticos formados a partir de un cigoto (genets) y a las copias física y fisiológicamente 
independientes de cada genotipo o ramets independientes (Figura 1; Kays y Harper, 1974; Harper, 1985; Jackson et al., 1985). La clonalidad está ampliamente distribuida en plantas, algunas estimaciones para angiospermas varían entre 30\% y más del 50\% (Tiffney y Niklas, 1985; Klim s et al., 1997; Aarssen, 2008), entre el 40\% y $80 \%$ en especies de taiga, tundra y de zonas templadas (van Groenendael y de Kroon, 1990 Klim s et al., 1997) y el 44\% de plantas invasoras en China (Liu et al., 2006), está ampliamente representada en monocotiledóneas y se presenta con baja frecuencia en las gimnospermas (van Groenendael et al., 1996; Peterson y Jones, 1997). Respecto a las condiciones ambientales, se sugiere que la clonalidad predomina en condiciones estresantes como suelos pobres en nitrógeno, suelos húmedos, cuerpos de agua, hábitats sombreados y ambientes fríos en altitudes y latitudes elevadas (Tiffney y Niklas, 1985; van Groenendael et al., 1996; Klim s et al., 1997).

La clonalidad en plantas se ha estudiado con enfoques muy diversos. Por ejemplo, se ha analizado la frecuencia de especies clonales y la variación en las formas de crecimiento clonal en distintos ambientes (p. ej., Tiffney y Niklas, 1985; van Groenendael et al., 1996; Klims et al., 1997), las implicaciones de la integración fisiológica de los ramets y la plasticidad morfológica del crecimiento clonal en ambientes heterogéneos y en condiciones de estrés competitivo (p. ej., Bell, 1984; Hartnett y Bazzaz, 1985; de Kroon y Hutchings, 1995; Price y Marshall, 1999), el efecto de la clonalidad en la demografía (p. ej., Caswell, 1985; McFadden, 1991; Damman y Cain, 1998; Mandujano et al., 2001), la comparación de la diversidad genética entre especies de plantas clonales y no clonales (p. ej., Ellstrand y Roose, 1987; Widén et al., 1994) y las consecuencias reproductivas y evolutivas de la geitonogamia asociada con la clonalidad (p. ej., Handel, 1985; de Jong et al., 1992; Eckert, 2000).

Las implicaciones reproductivas y genéticas de reducir la entrecruza incrementando la geitonogamia han aumentado el interés en el estudio de los patrones de distribución espacial de genets y ramets en poblaciones de plantas clonales. Debido a que los propágulos vegetativos suelen tener una capacidad de dispersión restringida, el reclutamiento clonal frecuente puede reducir la entrecruza e incrementar la autocruza (p. ej., autogamia y geitonogamia), determinando los niveles de variación y la estructuración genética en las poblaciones (Handel, 1985; Eckert, 2002; Ruggiero et al., 2005; Honnay et al., 2006). En especies autocompatibles, los costos reproductivos de la geitonogamia están asociados al incremento de la autogamia y a las probabilidades de expresar depresión endogámica, a la vez que disminuye la adecuación paterna reduciendo la cantidad de polen disponible para la entrecruza; mientras que en las especies autoincompatibles, la geitonogamia afecta la producción de semillas, debido a la saturación de los estigmas con polen incompatible y al aborto de óvulos, y puede resultar en el desperdicio de grandes cantidades de polen (de Jong et al.,
1992; Charpentier, 2002).

En plantas clonales los patrones de distribución espacial pueden ser afectados por la manera en que se generan los ramets. Las formas de crecimiento clonal son muy diversas, pero existen algunas clasificaciones que facilitan el entendimiento de esta variación. Tiffney y Niklas (1985) propusieron cuatro tipos principales de crecimiento: (1) Crecimiento establecido, dado por el inicio del desarrollo de un organismo individual a partir de un propágulo (puede considerarse como crecimento clonal si el propágulo es vegetativo, ya que el propágulo también puede ser una semilla producida sexualmente). (2) Crecimiento restaurativo, que se refiere a la reparación de un daño local en el individuo mediante la formación de tejido calloso, pero no hay regeneración de los órganos completos cuando estos se pierden. (3) Crecimiento regenerativo, que se refiere a la reparación y el reemplazo de órganos dañados (p. ej., crecimiento de una rama desprendida). (4) Crecimiento reproductivo, dado por la multiplicación del individuo fisiológico (p. ej., clonalidad). Estos autores también ordenan los tipos de crecimiento clonal en ligado y no ligado, considerando las estructuras de conexión (Figura 1). En el crecimiento ligado los propágulos vegetativos pueden desarrollarse hasta formar individuos completos (con raíces, tallos y hojas) manteniéndose unidos a la planta madre y su dispersión depende del crecimiento de ramificaciones de tallos y raíces, mientras que en el no ligado, los propágulos vegetativos se desprenden de la planta madre y se desarrollan hasta individuos completos separados de la planta madre. La dispersión de los propágulos en este caso es más dependiente de factores bióticos (p. ej., animales) o abióticos (p. ej., corrientes de agua, gravedad, viento) que en el crecimiento ligado y permite un mayor desplazamiento de los propágulos. El crecimiento reproductivo o clonalidad, que es la que interesa en esta revisión por su influencia en el arreglo espacial de genets y ramets, puede ser de dos tipos: (i) reiteración traumática, definida como la producción de un nuevo individuo inducida por estrés, ya sea por daño o por condiciones ambientales que limitan la reproducción sexual (por ejemplo, la producción de bulbilos en los agaves por daño al escapo floral o por deficiencia de polen) y (ii) la reiteración programada, como la producción de un nuevo individuo por un meristemo como parte normal del desarrollo (Tiffney y Niklas, 1985; Arizaga y Ezcurra, 2002).

La distribución espacial de los individuos en las poblaciones se ha clasificado en tres patrones básicos: aleatorio, uniforme y agregado (Figura 2; Gleason, 1920; Clark y Evans, 1954; Barbour et al., 1987). En poblaciones naturales de plantas, los individuos suelen distribuirse en forma más o menos agregada, debido a la distribución heterogénea de los recursos (Couteron y Kokou, 1997; Chen y Bradshaw, 1999), a la interacción con otras especies (Shreve, 1917; Mandujano et al., 1998) y a la dispersión restringida de semillas y propágulos vegetativos (Gibson y Menges, 1994; Okuda et al., 1997; Clark-Tapia et al., 2005). En plantas 
clonales además, el arreglo espacial de los genets y ramets puede ordenarse en dos patrones básicos, uno caracterizado por la agregación de ramets del mismo genet y el otro por una distribución entremezclada de ramets de distintos genets (Figura 2; de Kroon y Hutchings, 1995; Charpentier, 2002; Clark-Tapia et al., 2005). Estos arreglos espaciales de genets y ramets pueden estar asociados a la manera en que se producen los ramets (esto es por crecimiento clonal ligado o no ligado) y se ha propuesto que en las plantas que se propagan mediante crecimiento no ligado forman arreglos más entremezclados que las especies con crecimiento ligado (Gabrielsen y Brochmann, 1998), pero esto no ha sido demostrado. Por otro lado, debido a que la clonalidad puede incrementar la tasa de geitonogamia entre los ramets del mismo genet, puede esperarse que los arreglos entremezclados reduzcan la geitonogamia y promuevan la entrecruza como resultado del incremento local en el número de genets (Charpentier, 2002).

En algunas revisiones sobre el tema de la clonalidad se han planteado hipótesis sobre las implicaciones reproduc-

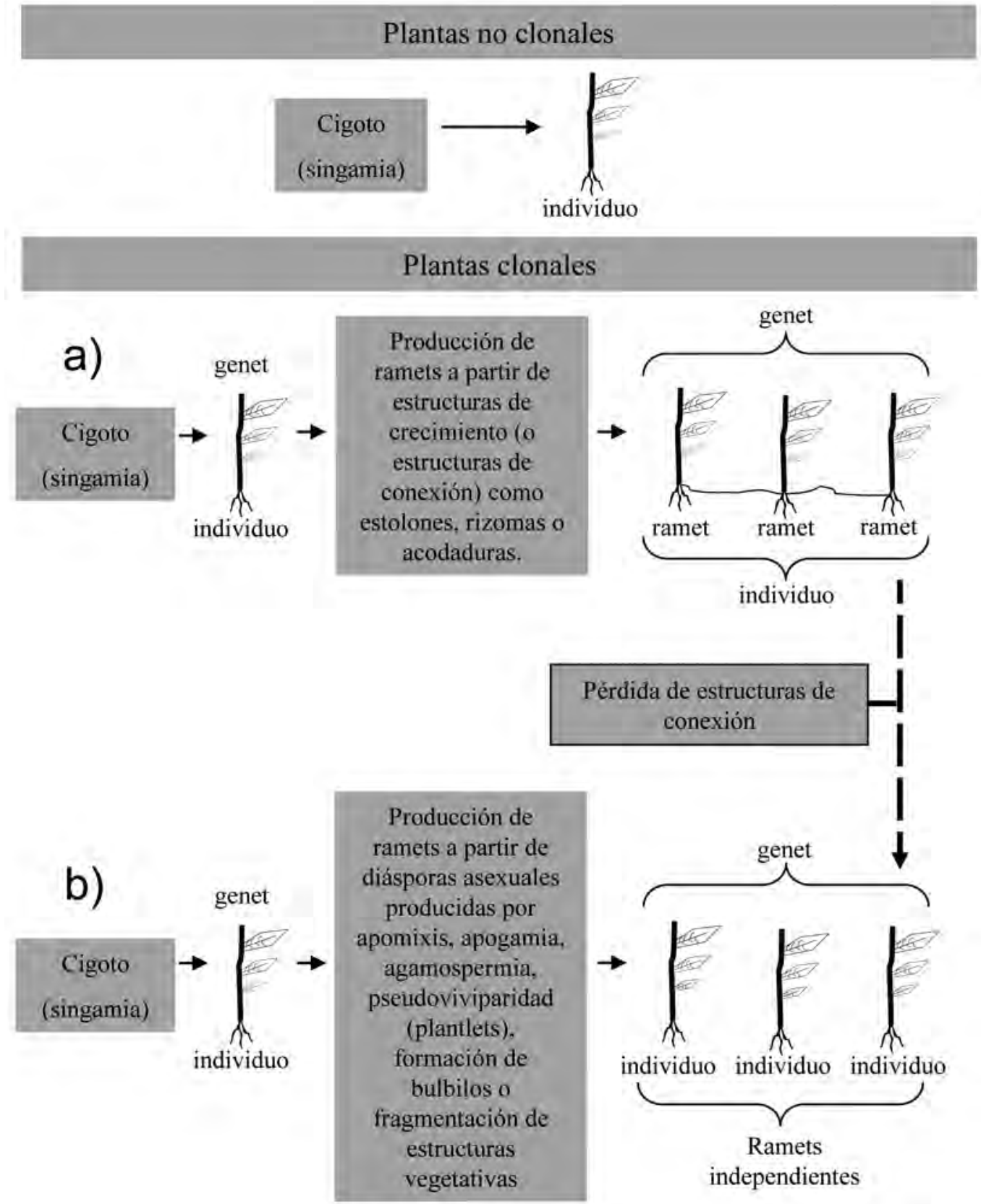

Figura 1. Formación de individuos en plantas no clonales y en plantas clonales. a) crecimiento clonal ligado y b) crecimiento clonal no ligado en la clasificación de Tiffney y Niklas (1985). 
$\mathrm{PDE}=$ Patrones de distribución espacial de los individuos y ramets en las poblaciones

(clonales y no clonales)

\section{AER $=$ Arreglos espaciales de los ramets de distintos genets}

(clonales)

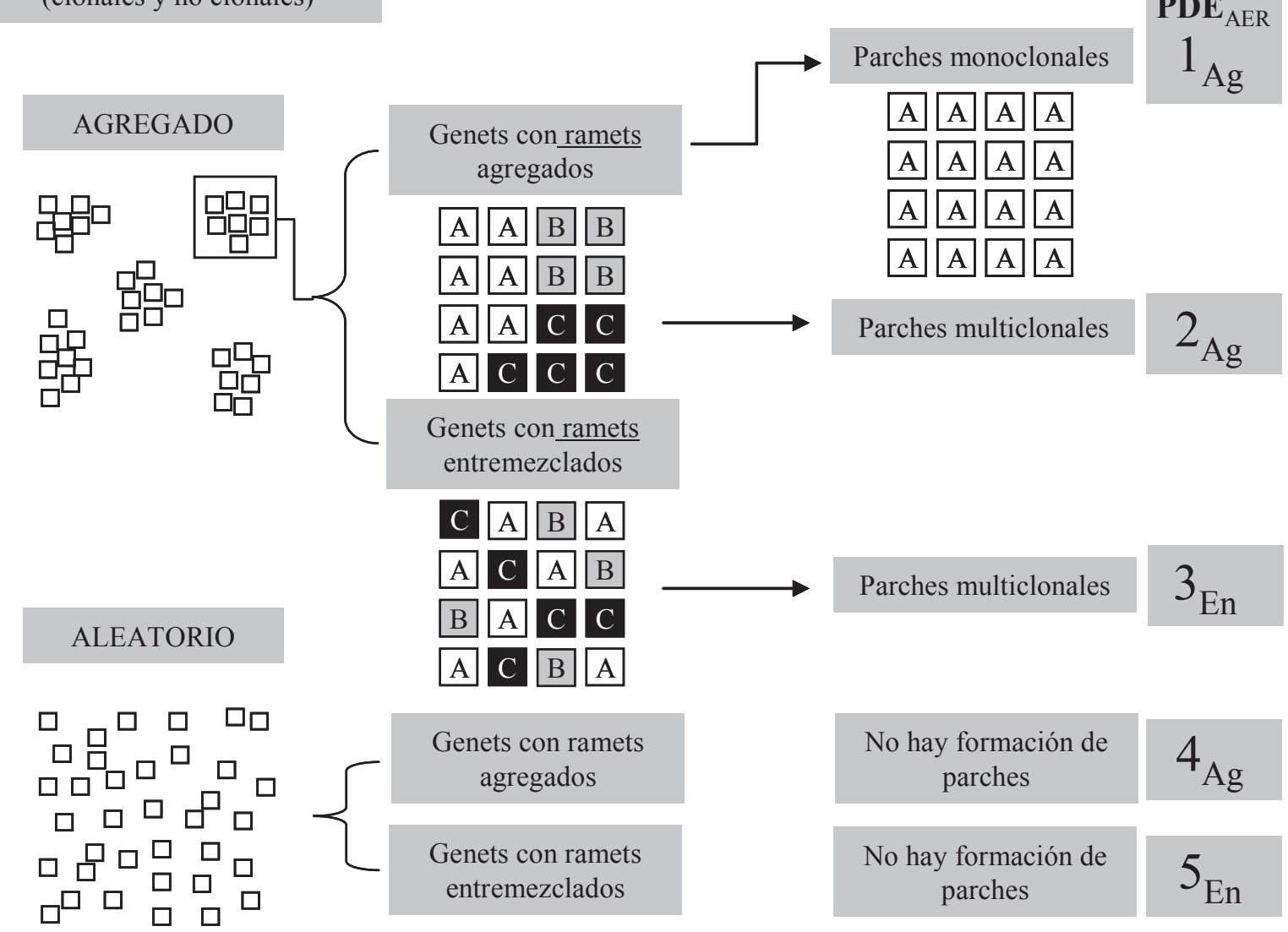

\section{UNIFORME}
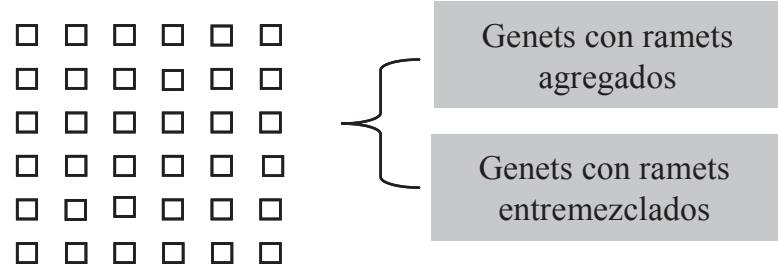

\begin{tabular}{|c|c|c|c|}
\hline $\begin{array}{c}\text { No hay formación de } \\
\text { parches }\end{array}$ & $6_{\mathrm{Ag}}$ \\
\hline $\begin{array}{c}\text { No hay formación de } \\
\text { parches }\end{array}$ & $7_{\mathrm{En}}$ \\
\hline
\end{tabular}

Figura 2. Patrones de distribución espacial de los individuos en las poblaciones de plantas no clonales y de plantas clonales. El número en el extremo derecho representa la clave del patrón de distribución espacial y el arreglo espacial de los ramets de distintos genets (PDE y AER, Apéndice). El subíndice Ag indica que los ramets de cada genet no se entremezclan con los ramets de genets distintos y el subíndice En indica que los ramets de diferentes genets se entremezclan.

tivas y genéticas de los arreglos espaciales de los genets y ramets, asociados con las distintas formas de producir descendientes clonales (Widén et al., 1994; Charpentier, 2002; Mandujano-Sánchez, 2007; Honnay y Jaquemyn, 2008). No obstante, los intentos para probar estas hipótesis son muy escasos y las posibilidades de realizar algún tipo de análisis numérico son restringidas por la baja disponibilidad de publicaciones (p. ej., Widén et al., 1994), o en los análisis sólo se incluyen especies con crecimiento ligado (p. ej., Honnay y Jaquemyn, 2008). En este trabajo presentamos una revisión de varios estudios que evalúan los patrones de distribución espacial en plantas clonales y a partir de la información 
proporcionada en dichos estudios, analizamos el planteamiento de que en poblaciones de plantas con crecimiento clonal ligado el arreglo espacial de los ramets de distintos genets es agregado, mientras que en poblaciones de plantas con crecimiento clonal no ligado predominan los arreglos espaciales entremezclados. Adicionalmente, intentamos poner a prueba la hipótesis de que los arreglos entremezclados promueven la entrecruza suponiendo que las poblaciones donde la entrecruza es más frecuente tenderán a presentar niveles más altos de diversidad genotípica.

\section{Material y métodos}

La revisión de los trabajos publicados sobre la distribución espacial clonal en plantas se llevó a cabo mediante una búsqueda de trabajos en bases de datos especializadas (CSA Cambridge, Ebsco HOST, ISI Web of Knowledge, JSTOR y Scopus) y no especializadas (Google, Google scholar y Yahoo). La búsqueda se hizo empleando los siguientes términos: clonal structure, spatial distribution of genets (andl or ramets), clonal architecture y clonal diversity y abarcó de 1990 a 2011, debido a que la mayoría de los estudios en plantas clonales con este enfoque se han realizado después de 1990.

Los criterios principales para incluir una especie en la revisión fue que se presentara una descripción de la distribución espacial de las plantas individuales (esto es sin importar el genotipo) en las poblaciones naturales y que se evaluara el arreglo espacial de los genets y los ramets (es decir agregado o entremezclado) mediante planos de distribución de los genotipos, análisis de autocorrelación espacial o compatibilidad de las cruzas entre ramets contiguos. Para cada especie se obtuvo el tipo de estructuras vegetativas a mediante las cuales se lleva a cabo la propagación clonal a partir de la información proporcionada en los estudios correspondientes o en estudios previos realizados a menudo por los mismos autores. El segundo criterio en orden de importancia fue que se reportaran valores de diversidad genotípica ( $D=$ índice de diversidad de Simpson y proporción de genotipos distinguibles $G / N$ ) para las poblaciones de las diferentes especies. Otros criterios fueron que se proporcionara información sobre las características reproductivas como la presencia de sistemas de incompatibilidad, la expresión sexual, si se ha observado reclutamiento sexual en las poblaciones y el tipo de polinización. Con base en esta información, se asignó a las especies el tipo de crecimiento clonal (i. e. ligado y no ligado, Figura 1) de acuerdo con la clasificación de Tiffney y Niklas (1985) y el patrón de distribución espacial y el tipo de arreglo espacial de los ramets (agregado o entremezclado) según el esquema presentado en la Figura 2. La información obtenida se presenta en el apéndice.

Para analizar el planteamiento de que en poblaciones de plantas con crecimiento clonal ligado el arreglo espacial de los ramets es agregado, mientras que en poblaciones de plantas con crecimiento clonal no ligado predomina el arreglo espacial entremezclado se construyó una tabla de contingencia con tres categorías de tipo de crecimiento clonal como columnas (ligado, combinación ligado-no ligado y no ligado) y tres categorías del arreglo espacial de los ramets como renglones (agregado, combinación agregado-entremezclado y entremezclado) y se analizó con una prueba de ${ }^{2}$ con el paquete estadístico JMP versión 7.0 (SAS Institute Inc). Para evaluar las celdas que contribuyen significativamente al valor de ${ }^{2}$ se hizo un análisis de residuales ajustados (Everitt, 1977).

Para probar la hipótesis de que las poblaciones con arreglos entremezclados de ramets presentan niveles más altos de diversidad genotípica, se compararon los valores del índice de diversidad de Simpson $(D)$ y la proporción de genotipos distinguibles $(G / N)$ entre las tres categorías de patrón de distribución clonal y entre las tres categorías de tipo de crecimiento clonal con una prueba de Kruskall-Wallis con el paquete estadístico JMP versión 7.0 (SAS Institute Inc).

\section{Resultados}

En la revisión se incluyeron 53 estudios que comprenden 122 poblaciones distribuidas en 44 especies de plantas clonales y en 27 familias (Apéndice). Las familias mejor representadas son Liliaceae y Rosaceae con cuatro especies cada una; Cactaceae, Cyperaceae, Ericaceae, Poaceae y Salicaceae con tres especies cada una y Zosteraceae con dos especies. El resto de las familias estuvieron representadas por una sola especie. Las especies que predominan son herbáceas perennes $(60 \%)$, y las plantas con hábito arbustivo y arbóreo se encuentran poco representadas (20\% en ambos casos).

La mayoría de las especies presentan crecimiento clonal ligado (70\%), dominando la propagación a través de rizomas (45\% de las especies). Las especies con crecimiento clonal ligado y no ligado representan el 14\% propagándose clonalmente por crecimiento ligado a través de rizomas y acodaduras, y por crecimiento no ligado a través de bulbilos, plantlets y desprendimiento de ramas; las especies sólo con crecimiento no ligado representan el 16\%, propagándose por bulbilos, semillas producidas por agamospermia, desprendimiento de ramas y gémulas (ver glosario para la definición de las estructuras implicadas en el crecimiento ligado y no ligado, Apéndice). Respecto a la distribución espacial, $50 \%$ del total de las especies presentan genets con ramets agregados, $30 \%$ presentan una combinación de arreglos agregados-entremezclados y $20 \%$ presentan arreglos entremezclados (Apéndice).

$\mathrm{Al}$ comparar las poblaciones con diferentes arreglos espaciales de genets y ramets [agregados (Ag), En (entremezcaldos) y $\mathrm{Ag}+\mathrm{En}$ (combinados)] encontramos que las poblaciones de especies con crecimiento ligado y no ligado y 
Cuadro 1. Número (observado y esperado) de poblaciones con diferentes tipos de crecimiento clonal de acuerdo con la clasificación de Tiffney y Niklas (1985) ( $\mathrm{Lg}=$ ligado, Lg+NI = ligado y no ligado, $\mathrm{Nl}=$ no ligado), que mostraron arreglos espaciales de los ramets de distintos genets: Ag = agregados, Ag+En = combinación de agregados y entremezclados, y En = entremezclados (figura 2). Los valores entre paréntesis indican el número de especies y familias (especies/familias) representadas en cada celda. Los valores de los residuales ajustados en negritas indican las celdas que contribuyen significativamente al valor de $\chi^{2}$, el signo indica mayor (sin signo) o menor número (-) de poblaciones de las esperadas por azar.

\begin{tabular}{|c|c|c|c|}
\hline Observados & Ligado & Ligado+No ligado & No ligado \\
\hline Agregado & $49(21 / 13)$ & $7(2 / 2)$ & $4(2 / 2)$ \\
\hline Agregado+entremezclado & $11(5 / 5)$ & $25(5 / 5)$ & $7(3 / 3)$ \\
\hline Entremezclado & $10(5 / 5)$ & $2(2 / 1)$ & $7(2 / 2)$ \\
\hline \multicolumn{4}{|l|}{ Esperados } \\
\hline & $\operatorname{Lg}$ & $\mathrm{Lg}+\mathrm{NI}$ & $\mathrm{Nl}$ \\
\hline Agregado & 34 & 17 & 9 \\
\hline Agregado+entremezclado & 25 & 12 & 6 \\
\hline Entremezclado & 11 & 5 & 3 \\
\hline \multicolumn{4}{|l|}{ Residuales ajustados } \\
\hline & $\operatorname{Lg}$ & $\mathrm{Lg}+\mathrm{NI}$ & $\mathrm{Nl}$ \\
\hline Agregado & 5.34 & -3.93 & -2.48 \\
\hline Agregado+entremezclado & -5.24 & 5.50 & 0.35 \\
\hline Entremezclado & -0.46 & -1.83 & 2.95 \\
\hline
\end{tabular}

de especies que sólo presentan crecimiento no ligado presentaron arreglos espaciales (AER) más entremezclados que las poblaciones de especies que sólo presentan crecimiento ligado ( ${ }^{2}=43.6, P<0.0001,4$ g.l.; Cuadro 1). Predominan los patrones de distribución (PDE) con ramets distribuidos en parches discretos sobre el área ocupada por la población (patrón de distribución espacial agregado, $46 \%$ de las poblaciones); estos parches son multiclonales (con dos o más genets) en la mayoría de los casos y el arreglo espacial de los ramets de distintos genets es agregado (19\% de las poblaciones, $\mathrm{PDE}_{\mathrm{AER}} 2_{\mathrm{Ag}}$, Figura 2), presenta una combinación agregado-entremezclado (10\% de las poblaciones, combinación $\mathrm{PDE}_{\mathrm{AER}} 2_{\mathrm{Ag}}-3_{\mathrm{En}}$, Figura 2) o es entremezclado solamente (3\% de las poblaciones, $\mathrm{PDE}_{\mathrm{AER}} 3_{\mathrm{En}}$, Figura 2). En otras poblaciones donde los ramets se distribuyen formando parches discretos el arreglo espacial de los ramets de distintos genets es agregado, pero los parches son multiclonales y monclonales (9\% de las poblaciones, combinación $\mathrm{PDE}_{\mathrm{AER}}$ $1_{\mathrm{Ag}}-\mathrm{Ag}_{\mathrm{Ag}}$, Figura 2) o sólo son monoclonales (5\% de las poblaciones, $\mathrm{PDE}_{\mathrm{AER}} 1_{\mathrm{Ag}}$, Figura 2).

Los patrones de distribución espacial con ramets distribuidos aleatoriamente sobre el área ocupada por la población (patrón de distribución espacial aleatorio) representaron el $44 \%$ de las poblaciones, predominando el arreglo espacial agregado-entremezclado de los ramets de distintos genets $\left(22 \%\right.$ de las poblaciones, combinación de $\mathrm{PDE}_{\mathrm{AER}}$ $4_{\mathrm{Ag}}-5_{\mathrm{En}}$, Figura 2), el arreglo agregado se presentó en $10 \%$ de las poblaciones ( $\mathrm{PDE}_{\mathrm{AER}} 4_{\mathrm{Ag}}$, Figura 2) y el arreglo entremezclado en $12 \%$ de las poblaciones $\left(\mathrm{PDE}_{\mathrm{AER}} 5_{\mathrm{En}}\right.$, Figura
2). El resto de las poblaciones (10\%) presentaron una combinación de patrones de distribución con ramets distribuidos aleatoriamente sobre una parte del área ocupada por la población y en otra formando parches discretos monoclonales o parches multiclonales con arreglos espaciales agregados y entremezclado de los ramets de distintos genets (combinación de $\mathrm{PDE}_{\mathrm{AER}} 1_{\mathrm{Ag}}-4_{\mathrm{Ag}}, 1_{\mathrm{Ag}}-5_{\mathrm{En}}, 2_{\mathrm{Ag}}-4_{\mathrm{Ag}}, 2_{\mathrm{Ag}}-5_{\mathrm{En}}$, Figura 2).

Las poblaciones de especies con una combinación de tipos de crecimiento clonal ligado y no ligado $(\mathrm{Lg}-\mathrm{Nl})$ presentaron valores más bajos de la proporción de genotipos distinguibles (promedio \pm E.E., $G / N=0.24 \pm 0.04 ; H=$ 6.93, $P=0.031,2$ g.l.) que las poblaciones de especies con crecimiento ligado (promedio \pm E.E., $G / N=0.38 \pm 0.03$ ) y con crecimiento no ligado (promedio \pm E.E., $G / N=0.39 \pm$ 0.06 ), pero las diferencias sólo fueron significativas con respecto a las poblaciones de especies con crecimiento ligado (Figura 3a). Respecto al arreglo espacial de los ramets de distintos genets, las poblaciones con una combinación de arreglos agregado y entremezclado (Ag-En) presentaron valores menores del índice de diversidad de Simpson (promedio \pm E.E., $D=0.57 \pm 0.05 ; H=13.2, P=0.0014,2$ g.l.) que las poblaciones con arreglos agregados (promedio \pm E.E., $D=0.80 \pm 0.04$ ) y con arreglos entremezclados (promedio \pm E.E., $D=0.76 \pm 0.06$; Figura 3). Estas diferencias sugieren que el reclutamiento sexual es más bajo en poblaciones de especies que presentan una combinación de formas de crecimiento clonal ligado-no ligado ( $\mathrm{Lg}-\mathrm{Nl}$ ) y una combinación de arreglos de los ramets de distintos genets agregado-entremezclado (Ag-En), sin embargo estas diferencias 

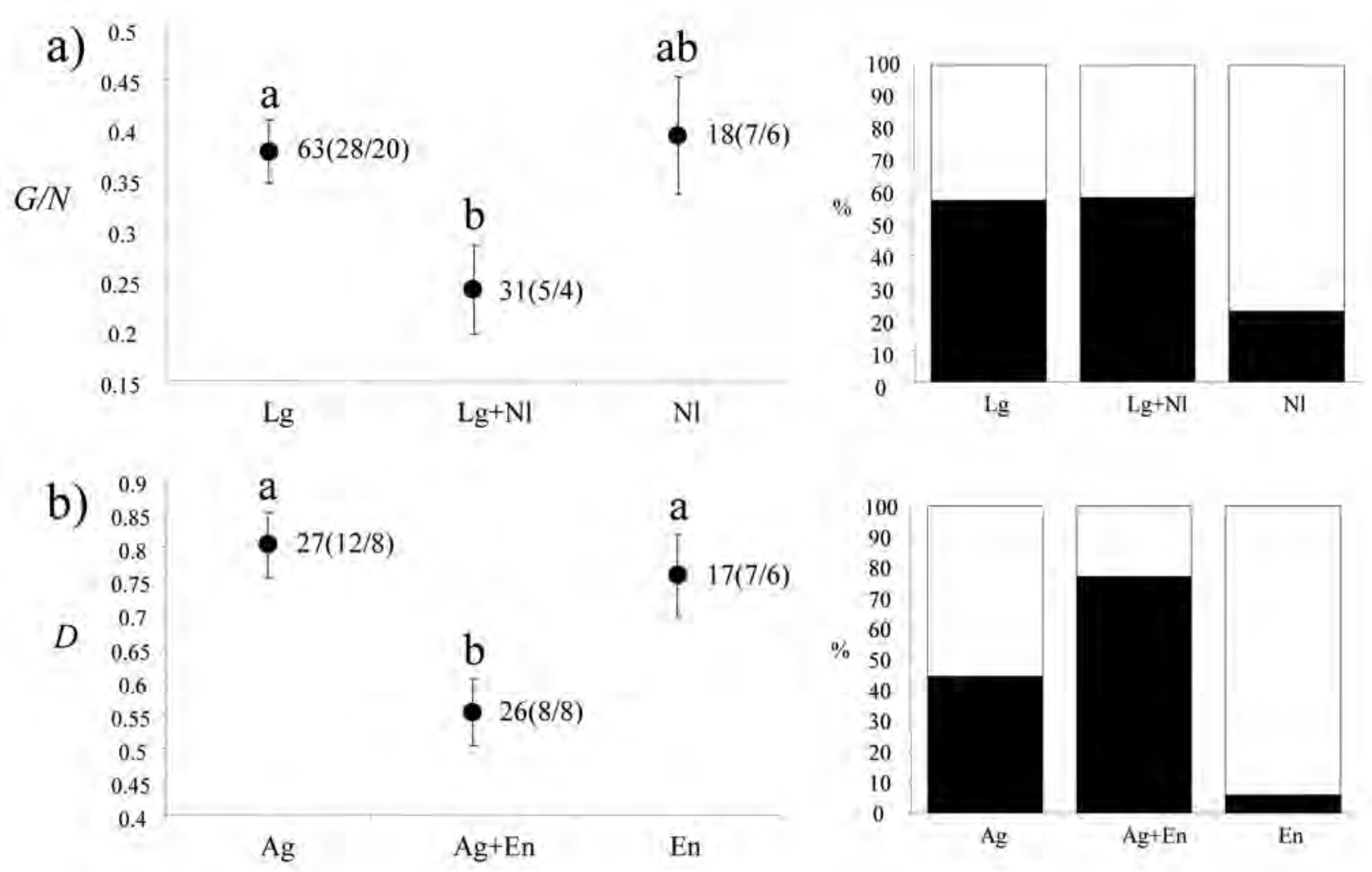

Figura 3. Comparación de la diversidad clonal (promedio \pm EE) entre poblaciones de especies con a) distinto tipo de crecimiento clonal: $\mathrm{Lg}=$ ligado, $\mathrm{Lg}+\mathrm{Nl}$ = con ambos tipos de crecimiento y $\mathrm{Nl}$ = no ligado y b) con distintos arreglos espaciales de los ramets de distintos genets: $\mathrm{Ag}=$ agregados, $\mathrm{Ag}+\mathrm{En}$ = combinación de agregados y entremezclados, y En = entremezclados (Figura 2). En cada grupo se indica el número de poblaciones (número de especies / número de familias) y los grupos que difieren significativamente se indican con una letra distinta arriba de la barra del promedio \pm E.E. de cada grupo. Las gráficas de barras del lado derecho muestran el porcentaje de poblaciones con polinización abiótica (en negro) y con polinización biótica (en blanco).

pueden estar asociadas con otros factores como el tipo de polinización, y no con el tipo de crecimiento y el arreglo espacial de los ramets de distintos genets. La mayoría de las poblaciones con tipo de crecimiento $\mathrm{Lg}-\mathrm{Nl}$ y arreglos de ramets de distintos genets Ag-En corresponden a especies con polinización abiótica (Figura 3).

\section{Discusión}

El efecto que tienen los modos de clonalidad en las plantas en la conformación de distintos arreglos espaciales de genets y ramets es un campo relativamente novedoso, que se ha explorado en los últimos 20 años y la mayoría de los estudios que hay sobre el tema se han desarrollado después de 1990. Widén et al. (1994) reportan que de 40 estudios que comprenden un total de 45 especies de plantas clonales, menos de la tercera parte proporcionan información sobre el arreglo espacial de los genets en las poblaciones y sólo en $12.5 \%$ se construyeron planos de distribución de todos los ramets, dentro de las parcelas o poblaciones, identificando los genotipos multilocus. En esta revisión, en el $98 \%$ de los estudios incluidos se identifican los genotipos multilocus mediante marcadores dominantes, codominantes o ambos siguiendo tres métodos de muestreo: en el 56\% de los estudios se utilizan parcelas, en el $24 \%$ transectos y en el $18 \%$ se muestrean todos o varios individuos de las poblaciones. El 2\% restante está representado por un estudio donde el patrón de distribución es descrito y la distribución de los ramets se sugiere por experimentos de cruzas compatibles entre ramets cercanos (en Scirpus maritimus, Charpentier et al., 2000).

A pesar de que en la actualidad se cuenta con más información sobre los patrones de distribución espacial en poblaciones de especies clonales, hacer comparaciones de dichos patrones considerando especies con distintas formas de crecimiento clonal para analizar las consecuencias reproductivas y genéticas del arreglo espacial de los genets y los ramets continúa siendo un reto. Algunos factores que influyen en este hecho son que la evaluación de la variación en los patrones de distribución espacial en plantas clonales 
enfrenta la dificultad que implica trabajar con la mayoría de los individuos en muchas poblaciones, principalmente a nivel molecular. Aunque en la actualidad las herramientas moleculares se han vuelto más accesibles, trabajar con un gran número de ejemplares sigue siendo difícil y costoso. Otros problemas son la falta de estimadores numéricos comparables para describir la distribución espacial en las poblaciones de diferentes especies y la variación en los métodos de muestreo. Por ejemplo, el muestreo mediante transectos colectando muestras de ramets cada determinada distancia (p. ej., Jonsson et al., 1996; Esselman et al., 1999) permite evaluar la capacidad de expansión longitudinal de los genets, pero no permite tener una descripción de la expansión radial y por lo tanto una evaluación más precisa del nivel de entremezcla de los ramets de distintos genets. En contraste, el muestreo de todos o de un gran número de individuos dentro de parcelas o en las áreas ocupadas por las poblaciones es otro método empleado en estudios con especies de plantas clonales (p. ej., Shimizu et al., 2002; Ruggieiro et al., 2005). Este tipo de muestreo puede ser un método más adecuado para analizar y comparar la estructura espacial clonal, porque permite detectar el arreglo radial de distintos genets. Sin embargo, los tamaños de muestra muy grandes pueden limitar la aplicación de este método, ya que en la mayoría de los casos es necesario emplear marcadores moleculares para identificar a los genets.

En particular, además de los problemas mencionados anteriormente es importante considerar las dificultades adicionales que detectamos en este estudio y que pueden afectar los patrones encontrados a través de nuestra aproximación. La mayoría de los estudios que analizan la distribución espacial de genets y ramets parecen estar sesgados hacia especies de herbáceas perennes con crecimiento clonal ligado (45\% de las especies), predominando las especies rizomatosas (43\% de las especies), mientras que las especies con crecimiento no ligado están poco representadas, impidiendo contar con tamaños de muestra equivalentes para hacer comparaciones más precisas. Por otro lado, la aproximación que decidimos emplear en este trabajo compara a las poblaciones más que a las especies, debido a que hemos detectado que hay una importante variación intraespecífica tanto en los patrones de distribución como en los patrones reproductivos. No obstante, esta variación se omite en algunos estudios o está mejor representada para algunas especies.

Los resultados obtenidos en este trabajo sugieren que las especies que se propagan por crecimento no ligado tienden a formar arreglos espaciales de los ramets de distintos genets más entremezclados como se ha planteado (Gabrielsen y Brochmann, 1998). No obstante, en especies con crecimiento ligado que además se propagan clonalmente por crecimiento no ligado, como el desprendimiento de ramas, el cual consideramos en este estudio como una forma de crecimiento no ligado en los análisis, debido a que los ramets originados por este medio se desarrollan hasta individuos com- pletos separados de la planta madre y a que su dispersión no depende del crecimiento horizontal de tallos y raíces (p.ej., Lophocereus schottii, Parker y Hamrick, 1992; Pteridium aquilinum, Parks y Werth, 1993; Cladium jamaicense, Ivey y Richards, 2001; Ferocactus robustus, Carrillo-Angeles et al., 2011), o que se propagan por la extensión de rizomas en especies con crecimiento en guerrilla (p. ej., Cymodocea nodosa, Ruggieiro et al., 2005 e Ilex leucoclada, Torimaru y Tomaru, 2005) también forman arreglos de genets entremezclados. Algunos factores externos que pueden influir en el arreglo entremezclado de genets son la dispersión por actividades humanas (Parks y Werth, 1993; Brzosko et al., 2002), el acarreo de propágulos vegetativos por animales y corrientes de agua (Parker y Hamrick, 1992; Brzosko et al., 2002), el reclutamiento de nuevos genets en áreas perturbadas donde permanecen fragmentos de clones que ocuparon grandes extensiones (Parks y Werth, 1993) y la dispersión de semillas producidas por autogamia que presentan genotipos multilocus idénticos (Keane et al., 1999; Brzosko et al., 2002).

Respecto a la hipótesis de que las poblaciones con arreglos espaciales entremezclados de los ramets de distintos genets presentan mayor entrecruza, medido indirectamente mediante la diversidad genotípica, nuestros resultados concuerdan parcialmente con el patrón esperado. El G/N es significativamente mayor en los arreglos de especies con producción de vástagos no ligados, en donde predominan los arreglos de genets entremezclados. Para probar esta hipótesis consideramos que si la entrecruza es más alta en poblaciones con arreglos entremezclados, habría mayores niveles de diversidad genotípica. Sin embargo, ésto no se refleja en el caso del índice de Simpson. Las diferencias en los índices de diversidad genotípica entre las poblaciones de especies que combinan el crecimiento clonal ligado-no ligado ( $\mathrm{Lg}$ $\mathrm{Nl})$ y el arreglo espacial agregado-entremezclado (Ag-En), respecto de las poblaciones que presentan uno u otro tipo de crecimiento y de arreglo espacial están asociadas con el tipo de polinización (biótica o abiótica), lo cual apoya el planteamiento de que la polinización biótica puede incrementar la eficiencia de la fertilización (Barret et al.,1993; Richards, 1997; Reusch, 2003). Los análisis para probar este planteamiento están fuera del alcance del presente estudio.

Además de los efectos sobre el reclutamiento sexual y la diversidad genotípica, los patrones de distribución espacial en especies clonales pueden afectar la diversidad y la estructura genética de las poblaciones. Por ejemplo, la formación de parches monoclonales en especies con entrecruza obligada, aunada al desplazamiento de genets por competencia, puede eliminar la capacidad de una población para reproducirse sexualmente en tiempos relativamente cortos (Honnay y Bossuyt, 2005). Adicionalmente, una reducción del reclutamiento sexual y una dispersión restringida de polen, semillas y propágulos vegetativos pueden generar estructura genética local en las poblaciones. Este segundo aspecto se 
evalúa en varios de los estudios revisados (47\%). La estructura genética local tanto a nivel de genets (indicando una dispersión restringida de polen, semillas o ambos) como de ramets (indicando dispersión restringida de propágulos vegetativos) se detectó en 46 poblaciones distribuidas en 16 especies; 28 poblaciones corresponden a cinco especies arbóreas que muestran estructura genética local a distancias menores de $50 \mathrm{~m}$ (Sequoia sempervirens, Douhovnikoff et al., 2004) y a distancias menores de $25 \mathrm{~m}$ (Eurya emarginata, Chung y Epperson, 2000; Cryptomeria japonica, Shimizu et al., 2002; Hirayama y Sakimoto, 2008; Populus tremula, Suvanto y Latva-Karjantvaa, 2005); tres poblaciones corresponden a dos especies arbustivas (Echinosophora koreensis, Chung et al., 2006; Stenocereus eruca, ClarkTapia et al., 2005) que presentan estructura genética local a distancias menores de $20 \mathrm{~m}$. El resto de las poblaciones corresponden a hierbas perennes que muestran estructura genética local desde distancias menores de $30 \mathrm{~m}$, pero más frecuentemente a distancias menores de $15 \mathrm{~m}$.

En promedio, los valores del índice de diversidad de Simpson $(D)$ y la proporción de genotipos distinguibles $(G / N)$ fueron altos en las poblaciones representadas en los estudios incluidos en la presente revisión y son similares a los reportados para 21 especies de plantas clonales $(D=$ 0.62 y $G / N=0.16$ ) por Ellstrand y Roose (1987). Se han planteado algunas hipótesis sobre los escenarios que pueden explicar altos niveles de diversidad genotípica: (i) las poblaciones pudieron ser fundadas por varios propágulos sexuales que se mantienen por clonalidad, (ii) el reclutamiento sexual fue más frecuente en el pasado, aunque en la actualidad la producción de descendientes sexuales no sea evidente (Ellstrand y Roose, 1987; Eriksson, 1989, 1993; Widén et al., 1994); (iii) los genotipos heterócigos se ven favorecidos selectivamente, manteniendo altos niveles de diversidad genética (Widén et al., 1994); (iv) los riesgos de mortalidad entre los ramets de un genet pueden ser distintos si tienen independencia física y fisiológica (Eriksson 1989, 1993, Pan y Price, 2002), lo cual puede reducir las probabilidades de supervivencia / extinción del genet; (v) en especies con ciclos de vida largos los eventos raros de reclutamiento sexual pueden ser suficientes para mantener niveles altos de diversidad genética (Eriksson 1989, 1993, Parker y Hamrick, 1992; Brzosko et al., 2002; Clark-Tapia et al., 2005); (vi) los genotipos pueden mantenerse a través de la supervivencia de algunos ramets el tiempo suficiente para que algunos genotipos se integren a la población de manera esporádica; (vii) la heterogeneidad ambiental puede generar condiciones de selección diversificante que permiten la coexistencia de varios genotipos (Widén et al., 1994). Otra posibilidad para el mantenimiento de diversidad clonal en poblaciones de plantas, que surge al considerar la variación de la distribución espacial de genets y ramets, es que la clonalidad puede mantener altos niveles de entrecruza e incrementar las probabilidades de reclutamiento sexual si los ramets se dispersan hacia vecindarios genéticamente variables, debido a la distribución entremezclada de genets.

Las explicaciones a los niveles relativamente altos de diversidad genotípica en las poblaciones de las especies incluidas en esta revisión comprenden los eventos esporádicos de reclutamiento sexual y el establecimiento inicial de varios genets. En algunos casos se sugiere un reclutamiento sexual frecuente a partir de los altos niveles de diversidad genotípica detectados con los marcadores moleculares (p. ej., Parks y Werth, 1993; Diggle et al., 1998; Misuki et al., 2010). No obstante, el reclutamiento sexual en poblaciones naturales sólo se reporta en Prunus ssiori, Rubus saxatilis (Eriksson y Bremer, 1993), Cypripedium calceolus (Brzosko et al., 2002) y Maianthemum dilatatum (Wilson et al., 2005b). Otros factores que se proponen para explicar la diversidad genotípica son que la integración fisiológica entre los ramets de un genet puede reducir el riesgo de que un genotipo en particular desaparezca, cuando las condiciones ambientales locales son adversas, contribuyendo a mantener la diversidad genética (Hartnett y Bazzaz, 1985), el incremento en la tasa de entrecruza, debido a la entremezcla de genets en especies con crecimiento en guerrilla (Ruggieiro et al., 2005) y en especies que se propagan clonalmente por desprendimiento de ramas y producción de bulbilos (Parker y Hamrick, 1992; Gabrielsen y Brochmann, 1998).

El estudio de los patrones de distribución espacial en especies clonales es importante para analizar las consecuencias reproductivas y genéticas del arreglo espacial de los genets y los ramets en las poblaciones naturales. Estas consecuencias pueden explorarse de una manera más precisa analizando la variación de la distribución de genets y ramets entre las poblaciones de una misma especie. Sin embargo, la evidencia de estos aspectos en organismos clonales aún es escasa y hace falta realizar estudios considerando diferentes tipos de crecimiento clonal. El análisis de los patrones de distribución espacial pueden ser muy útiles para evaluar otros aspectos del ciclo de vida de las especies clonales como el efecto de una variación persistente en la producción de propágulos clonales y sexuales entre hábitats, que puede afectar diferencialmente la dinámica de las poblaciones. Asimismo, es necesario analizar la dinámica temporal de los arreglos espaciales de los genets y ramets en las poblaciones para hacer inferencias adecuadas sobre las consecuencias ecológicas y evolutivas de la clonalidad.

\section{Agradecimientos}

Proyectos: IN IN205007 Efecto de la estructura clonal sobre la dinámica poblacional y la reproducción de una cactácea con ciclo de vida complejo, Opuntia microdasys.

Becas: doctorado Conacyt a I. Carrillo-Angeles y estancia sabática en New Mexico State University, otorgada por DGAPA-PASPA- Universidad Nacional Autónoma de México a M. Mandujano. Este trabajo es parte de la tesis de 
doctorado de Israel Gustavo Carrillo Angeles, Posgrado en Ciencias Biológicas de la UNAM. Agradecemos la revisión, sugerencias en el trabajo así como el apoyo de los miembros del comité tutoral, Doctores Teresa Valverde y Francisco Molina. Asimismo a la Dra. Ana Mendoza, Dr. Jordan Golubov y dos revisores anónimos por sus sugerencias al artículo.

\section{Literatura citada}

Aarssen L.W. 2008. Death without sex-the 'problem of the small' and selection for reproductive economy in flowering plants. Evolutionary Ecology 22:279-298.

Albert T., Raspé O. y Jacquemart A.L. 2003. Clonal structure in Vaccinium myrtillus L. revealed by RAPD and AFLP markers. International Journal of Plant Sciences 164:649-655.

Arens P., Grashof-Bokdam C.J., van der Sluis T. y Smulders M.J.M. 2005. Clonal diversity and genetic differentiation of Maianthemum bifolium among forest fragments of different age. Plant Ecology 179:169-180.

Arizaga S. y Ezcurra E. 2002. Propagation mechanisms in Agave macrovantha (Agavaceae), a tropical arid land succulent rosette. American Journal of Botany 89:632-641.

Barbour M.G., Burk J.H. y Pitts W.D. 1987. Terrestrial Plant Ecology. The Benjamin-Cummings Publishing Company, Menlo Park.

Barret S.C.H., Eckert C.G. y Husband B.C. 1993. Evolutionary processes in aquatic plant populations. Aquatic Botany 44:105-145.

Bell A.D. 1984. Dynamic morphology: A contribution to plant population ecology. En: Dirzo R. y Sarukhán J. Eds. Perspectives on Plant Population Ecology, pp. 49-65, Sinauer Associates Inc. Publisher, Sunderland.

Brzosko E., Wróblewska A. y Ratkiewicz M. 2002. Spatial genetic structure and clonal diversity of island populations of lady's slipper (Cyperidium calceolus) from the Biebrza National Park (northeast Poland). Molecular Ecology 11:2499-2509.

Bushakra J.M., Hodges S.A., Cooper J.B. y Kaska D.D. 1999. The extent of clonality and genetic diversity in the Santa Cruz Island ironwood, Lyonothamnus floribundus. Molecular Ecology 8:471-475.

Camacho F.J. y Liston A. 2001. Population structure and genetic diversity of Botrychium pumicola (Ophioglossaceae) based on inter-simple sequence repeats (ISSR). American Journal of Botany 88:1065-1070.

Carrillo-Angeles I.G., Mandujano M.C. y Golubov J. 2011. Influences of the genetic neighborhood on ramet reproductive success in a clonal desert cactus. Population Ecology 53:449458.

Caswell H. 1985. The evolutionary demography of clonal reproduction. En: Jackson J.B.C., Buss L.W. y Cook R.E. Eds. Population Biology and Evolution of Clonal Organisms, pp. 187224, Yale University Press, New Haven.

Charpentier A. 2002. Consequences of clonal growth for plant mating. Evolutionary Ecology 15:521-530.

Charpentier A., Grillas P. y Thompson J.D. 2000. The effects of population size limitation on fecundity in mosaic populations of the clonal macrophyte Scirpus maritimus (Cyperaceae). American Journal of Botany 87:502-507.

Chen J. y Bradshaw G.A. 1999. Forest structure in space: a case study of an old growth spruce-fir forest in Changbaishan Natural Reserve, PR China. Forest Ecology and Management 120:219-233.

Chung M.G. y Epperson B.K. 2000. Clonal and spatial genetic structure in Eurya emarginata (Theaceae). Heredity 84:170177.

Chung J.M., Lee B.C., Kim J.S., Park C., Chung M.Y. y Chung M.G. 2006. Fine-scale genetic structure among genetic individuals of the clone-forming monotypic genus Echinospora koreensis (Fabaceae). Annals of Botany 98:165-173.

Clark P.J. y Evans F.C. 1954. Distance to nearest neighbor as a measure of spatial relationships in populations. Ecology 35:445-453.

Clark-Tapia R., Alfonso-Corrado C., Eguiarte L.E. y Molina-Freaner F. 2005. Clonal diversity and distribution in Stenocereus eruca (Cactaceae), a narrow endemic cactus of the Sonoran Desert. American Journal of Botany 92:272-278.

Clark-Tapia R., Alfonso-Corrado C., Mandujano M.C. y MolinaFreaner F. 2006. Reproductive consequences of clonal growth in Stenocereus eruca, a rare clonal cactus of the Sonoran desert. Evolutionary Ecology 20:131-142.

Couteron P. y Kokou K. 1997. Woody vegetation spatial patterns in a semi-arid savanna of Burkina Faso, West Africa. Plant Ecology 132:211-227.

Damman H. y Cain M.L. 1998. Population growth and viability analyses of the clonal woodland herb, Asarum canadense. Journal of Ecology 86:13-26.

de Jong T.J., Waser N.M., Price M.V. y Ring R.M. 1992. Plant size, geitonogamy and seed set in Ipomopsis aggregata. Oecologia 89:310-315.

de Kroon H. y van Groenendael J. Eds. 1997. The Ecology and Evolution of Clonal Plants. Backhuys Publishers, Leiden.

de Kroon H. y Hutchings M.J. 1995. Morphological plasticity in clonal plants: the foraging concept reconsidered. Journal of Ecology 83:143-152.

Diggle P.K., Lower S. y Ranker T.A. 1998. Clonal diversity in alpine populations of Polygonum viviparum (Polygonaceae). International Journal of Plant Sciences 159:606-615.

Diggle P.K., Meixner M.A., Carroll A.B. y Aschwanden C.F. 2002. Barriers to sexual reproduction in Polygonum viviparum: A comparative developmental analysis of $P$. viviparum and $P$. bistortoides. Annals of Botany 89:145-156.

Douhovnikoff V. y Dood R.S. 2003. Intra-clonal variation and a similarity threshold for identification of clones: application to $\mathrm{Sa}$ lix exigua using AFLP molecular markers. Theoretical Applied Genetics 106:1307-1315.

Douhovnikoff V., Cheng A.M. y Dodd R.S. 2004. Incidence, size and spatial structure of clones in second-growth stands of coast redwood, Sequoia sempervirens (Cupressaceae). American Journal of Botany 91:1140-1146.

Douhovnikoff V., McBride J.R. y Dodd R.S. 2005. Salix exigua clonal growth and population dynamics in relation to disturbance regime variation. Ecology 86:446-452.

Eckert C.G. 2000. Contributions of autogamy and geitonogamy to self-fertilization in a mass-flowering, clonal plant. Ecology 81:532-542.

Eckert C.G. 2002. The loss of sex in clonal plants. Evolutionary Ecology 15:501-520.

Ellstrand N.C. y Roose M.L. 1987. Patterns of genotypic diversity in clonal plant species. American Journal of Botany 74:123-131. 
Eriksson O. 1989. Seedling dynamics and life histories in clonal plants. Oikos 55:231-238.

Eriksson O. 1993. Dynamics of genets in clonal plants. Trends in Ecology \& Evolution 8:313-316.

Eriksson O. y Bremer B. 1993. Genet dynamics of the clonal plant Rubus saxatilis. Journal of Ecology 81:533-542.

Esselman E.J., Jianqiang L., Crawford D.J., Winduss J.L. y Wolfe A.D. 1999. Clonal diversity in the rare Calamagrostis porteri ssp insperata (Poaceae): comparative results for allozymes and random amplified polymorphic DNA (RAPD) and intersimple sequence repeat (ISSR) markers. Molecular Ecology 8:443451.

Everitt B.S. 1977. The Analysis of Contingency Tables. Chapmann \& Hall, Bury St Edmunds.

Gabrielsen T.M. y Brochmann C. 1998. Sex after all: high levels of diversity detected in the arctic clonal plant Saxifraga cernua using RAPD markers. Molecular Ecology 7:1701-1708.

Garnier L.K.M., Durand J. y Dajoz I. 2002. Limited seed dispersal and microspatial population structure of an agamospermous grass of West African savannahs, Hyparrhenia diplandra (Poaceae). American Journal of Botany 89:1785-1791.

Gibson D.J. y Menges E.S. 1994. Population structure and spatial pattern in the dioecious shrub Ceratiola ericoides. Journal of Vegetation Sciences 5:337-346.

Gleason H.A. 1920. Some applications of the quadrat method. Bulletin of the Torrey Botanical Club 47:21-33.

Hämmerli A. y Reusch T.B.H. 2003a. Flexible mating: cross-pollination affects sex expression in a marine clonal plant. Journal of Evolutionary Biology 18:1096-1105.

Hämmerli A. y Reusch T.B.H. 2003b. Genetic neighbourhood of clone structures in eelgrass meadows quantified by spatial autocorrelation of microsatellite markers. Heredity 91:448-455.

Handel S.N. 1985. The intrusion of clonal growth patterns on plant breeding system. The American Naturalist 125:367-384.

Harper J. 1985. Modules, branches, and the capture of resources. En: Jackson J.B.C., Buss L.W. y Cook R.E. Eds. Population Biology and Evolution of Clonal Organisms, pp. 1-33, Yale University Press, New Haven.

Hartnett D.C. y Bazzaz F.A. 1985. The integration of neighbourhood effects by clonal genets in Solidago canadensis. The Journal of Ecology 73:415-427.

Hirayama K. y Sakimoto M. 2008. Clonal structure and diversity of Cryptomeria japonica along a slope in a cool-temperate, oldgrowth mixed forest in the snowy region of Japan. Canadian Journal of Forest Research 38:2804-2813.

Honnay O. y Bossuyt B. 2005. Prolonged clonal growth: escape route or route to extinction? Oikos 108:427-432.

Honnay O., Jacquemyn H., Roldán-Ruiz I. y Hermy M. 2006. Consequences of prolonged clonal growth on local and regional genetic structure and fruiting success of the forest perennial Maianthemum bifolium. Oikos 112:21-30.

Honnay O. y Jacquemyn H. 2008. A meta-analysis of the relation between mating system, growth form and genotypic diversity in clonal plant species. Evolutionary Ecology 22:299-312.

Ivey C.T. y Richards J.H. 2001. Genotypic diversity and clonal structure of everglades sawgrass, Cladium jamaicense (Cyperaceae). International Journal of Plant Sciences 162:1327-1335.

Jackson J.B.C., Buss L.W. y Cook R.E. Eds. 1985. Population Biology and Evolution of Clonal organisms. Yale University Press, New Haven.
Jacquemyn H., Brys R., Honnay O., Hermy M. y Roldán-Ruiz I. 2005. Local forest environment largely affects below-ground growth, clonal diversity and fine-scale spatial genetic structure in the temperate deciduous forest herb Paris quadrifolia. Molecular Ecology 14:4479-4488.

Jonsson B.O., Jónsdóttir I.S. y Crongberg N. 1996. Clonal diversity and allozyme variation in populations of the arctic sedge Carex bigelowii (Cyperaceae). Journal of Ecology 84:449-459.

Kays S. y Harper J.L. 1974. The regulation of plant and tiller density in a grass sward. Journal of Ecology 62:97-105.

Keane B., Pelikan S., Toth G.P., Smith M.K. y Rogstad S.H. 1999. Genetic diversity of Typha latifolia (Typhaceae) and the impact of pollutants examined with tamdem-repetitive DNA probes. American Journal of Botany 86:1226-1238.

Kjølner S., Såstad S.M. y Brochmann C. 2006. Clonality and recombination in the arctic plant Saxifraga cernua. Botanical Journal of the Linnean Society 152:209-217.

Klimeš L., Klimešová J., Hendriks R.J.J. y van Groenendael J.M. 1997. Clonal plant architecture: a comparative analysis of form and function. En: de Kroon H. y van Groenendael J. Eds. The Ecology and Evolution of Clonal Plants, pp. 1-29, Backhuys Publishers, Leiden.

Korpelainen H. 1994. Sex ratios and resource allocation among sexually reproducing plants of Rubus chamaemorus. Annals of Botany 74:627-632.

Korpelainen H., Antonious-Klemola K. y Werlemark G. 1999. Clonal structure of Rubus chamaemorus populations: comparison of different molecular methods. Plant Ecology 143:123-128.

Kreher S.A., Foré S.A. y Collins B.S. 2000. Genetic variation within and among patches of the clonal species, Vaccinium stamineum L. Molecular Ecology 9:1247-1252.

Kudoh H., Shibaike H., Takasu H., Whigham D.F. y Kawano S. 1999. Genet structure and determinants of clonal structure in a temperate deciduous woodland herb, Uvularia perfoliata. Journal of Ecology 87:244-257.

Liston A., Wilson B.L., Robinson W.A., Doescher P.S., Harris N.R. y Svejcar T. 2003. The relative importance of sexual reproduction versus clonal spread in an arid bunchgrass. Oecologia 137:216-225.

Liu J., Dong M., Miao S.L., Li Z.Y., Song M.H. y Wang R.Q. 2006. Invasive alien plants in China: role of clonality and geographical origin. Biological Invasions 8:1461-1470.

Mandujano M.C., Montaña C., Méndez I. y Golubov J. 1998. The relative contributions of sexual reproduction and clonal propagation in Opuntia rastrera from two habitats in the Chihuahuan desert. Journal of Ecology 86:911-921.

Mandujano M.C., Montaña C., Franco M., Golubov J. y FloresMartínez A. 2001. Integration of demographic annual variability in a clonal desert cactus. Ecology 82:344-359.

Mandujano-Sánchez M.C. 2007. La clonalidad y sus efectos en la biología de poblaciones. En: Eguiarte L.E., Souza V. y Aguirre X. Comp. Ecología Molecular, pp. 215-250, Secretaría de Medio Ambiente y Recursos Naturales-Instituto Nacional de Ecología-Universidad Nacional Autónoma de México-Comosión Nacional para el Conocimiento y Uso de la Biodiversidad, México, D.F.

Mayes S.G., McGinley M.A. y Werth C.R. 1998. Clonal population structure and genetic variation in sand-shinnery oak, Quercus havardii (Fagaceae). American Journal of Botany 85:1609-1617. McFadden C.S. 1991. A comparative demographic analysis of clonal 
reproduction in a temperate soft coral. Ecology 72:1849-1866.

Misuki I., Ishida K., Tani N. y Tsumura Y. 2010. Fine-scale spatial structure of genets and sexes in the dioecious plant Dioscorea japonica, which disperses by both bulbils and seeds. Evolutionary Ecology 24:1399-1415.

Mori Y., Nagamitsu T. y Kubo T. 2009. Clonal growth and its effects on male and female reproductive success in Prunus ssiori (Rosaceae). Population Ecology 51:175-186.

Nagamitsu T., Ogawa M., Ishida K. y Tanouchi H. 2004. Clonal diversity, genetic structure, and mode of recruitment in a Prunus ssiori population established after volcanic eruptions. Plant Ecology 174:1-10.

Okuda T., Kachi N., Yap S.K. y Manokaran N. 1997. Tree distribution pattern and fate of juveniles in a lowland tropical rain forest - implications for regeneration and maintenance of species diversity. Plant Ecology 131:155-171.

Pan J.J. y Price J.S. 2002. Fitness and evolution in clonal plants: the impact of clonal growth. Evolutionary Ecology 15:583-600.

Parker K.C. y Hamrick J.L. 1992. Genetic diversity and clonal structure in a columnar cactus, Lophocereus schottii. American Journal of Botany 79:86-96.

Parks C. y Werth C.R. 1993. A study of spatial features of clones in a population of bracken fern, Pteridium aquilinum (Dennstaedtiaceae). American Journal of Botany 80:537-544.

Peterson C.J. y Jones R.H. 1997. Clonality in woody plants: a review and comparison with clonal herbs. En: de Kroon H. y van Groenendael J. Eds. The Ecology and Evolution of Clonal Plants, pp. 263-289, Backhuys Publishers, Leiden.

Pfeiffer T., Klahr A., Heinrich A. y Schnittler M. 2011. Does sex make a difference? Genetic diversity and spatial genetic structure in two co-occurring species of Gagea (Liliaceae) with contrasting reproductive strategies. Plant Systematics and Evolution 292:189-201.

Pornon A. y Escaravage N. 1999. Genotypic structure in clonal Rhododendron ferrugineum L. (Ericaceae) populations: origin and manteinance. Plant Ecology 141:145-150.

Pornon A., Escaravage N., Thomas P. y Taberlet P. 2000. Dynamics of genotypic structure in clonal Rhododendron ferrugineum (Ericaceae) populations. Molecular Ecology 9:1099-1111.

Pornon A., Escaravage N., Till-Botraud I. y Doche B. 1997. Variation of reproductive traits in Rhododendron ferrugineum $\mathrm{L}$. (Ericaceae) populations along a successional gradient. Plant Ecology 130:1-11.

Price E.A.C. y Marshall C. 1999. Clonal plants and environmental heterogeneity. Plant Ecology 141:3-7.

Reisch C., Schurm S. y Poschlod P. 2007. Spatial genetic structure and clonal diversity in an alpine population of Salix herbacea (Salicaceae). Annals of Botany 99:647-651.

Reusch T.B.H. 2003. Floral neighbourhoods in the sea: how floral density, opportunity for outcrossing and population fragmentation affect seed set in Zostera marina. Journal of Ecology 91:610-615.

Richards A.J. 1997. Plant Breeding Systems. Chapman \& Hall,
Londres.

Rosseto M., Gross C.L., Jones R. y Hunter J. 2004. The impact of clonality on an endangered tree (Elaeocarpus williamsianus) in a fragment rain forest. Biological Conservation 117:33-39.

Ruggieiro M.V., Capone S., Pirozzi P., Reusch T.B.H. y Procaccini G. 2005. Mating system and clonal architecture: a comparative study in two marine angiosperms. Evolutionary Ecology 19:487-499.

Shimizu Y, Ando M. y Sakai F. 2002. Clonal structure of natural populations of Cryptomeria japonica growing at different positions on slopes, detected using RAPD markers. Biochemical Systematics and Ecology 30:733-748.

Shreve F. 1917. The establishment of desert perennials. Journal of Ecology 5:210-216.

Sipes S.D. y Wolf P.G. 1997. Clonal structure and patterns of allozyme diversity in the rare endemic Cycladenia humilis var. jonesii (Apocynaceae). American Journal of Botany 84:401-409.

Stehlik I. y Holderegger R. 2000. Spatial genetic structure and clonal diversity of Anemone nemorosa in late successional deciduous woodlands of Central Europe. Journal of Ecology 88:424-435.

Suvanto L.I. y Latva-Karjantvaa T.B. 2005. Clone identification and clonal structure of the European aspen (Populus tremula). Molecular Ecology 14:2851-2860.

Tiffney B.H. y Niklas K.J. 1985. Clonal growth in land plants: a paleobotanical perspective. En: Jackson J.B.C., Buss L.W. y Cook R.E. Eds. Population Biology and Evolution of Clonal Organisms, pp. 35-66, Yale University Press, New Haven.

Tooke F., Ordidge M., Chiurugwi T. y Battey N. 2005. Mechanisms and function of flower and inflorescence reversion. Journal of Experimental Botany 56:2587-2599.

Torimaru T. y Tomaru N. 2005. Fine-scale clonal structure and diversity within patches of a clone-forming dioecious shrub, Ilex leucoclada (Aquifoliaceae). Annals of Botany 95:295-304.

van Dijk P.J. y Bakx-Schotman J.M.T. 2004. Formation of unreduced megaspores (diplospory) in apomictic dandelions (Taraxacum officinale, s.l.) is controlled by a sex-specific dominant locus. Genetics 166:483-492.

van Groenendael J.M. y de Kroon H. 1990. Clonal Growth in Plants: Regulation and Function. SPB Academic Publishing, La Haya.

van Groenendael J.M., Klimeš L., Klimešová J. y Hendriks R.J.J. 1996. Comparative ecology of clonal plants. Philosophical Transactions of Royal Society of London 351:1331-1339.

Widén B., Cronberg N. y Widén M. 1994. Genotypic diversity, molecular markers and spatial distribution of genets in clonal plants, a literature survey. Folia Geobotanica y Phytotaxonomica 29:245-263.

Wilson A.S.G., van der Kamp B.J. y Ritland C. 2005a. Opportunities for geitonogamy in the clonal herb Maianthemum dilatatum. Canadian Journal of Botany 83:1082-1087.

Wilson A.S.G., van der Kamp B.J. y Ritland C. 2005b. Spatial genetic and clonal structure in Maianthemum dilatatum as defined by AFLP markers. Canadian Journal of Botany 83:1126-1132. 
Apéndice. Características de las especies y poblaciones: $\mathbf{S C R Z}=$ sistema de cruza, $\mathbf{P O L}=$ polinización, $\mathbf{P R O R}=$ estructuras o procesos que dan origen a los ramets, CCT-N = tipo de crecimiento clonal en la clasificación de Tiffney y Niklas (1985), POB/HL = número de población y/o hábitat local, $\mathbf{P D E}=$ clave del patrón de distribución espacial de acuerdo a la figura 2, AER = arreglo espacial de los ramets de distintos genets, $\boldsymbol{D}=$ índice de diversidad clonal de Simpson, $\boldsymbol{G} / \mathbf{N}=$ proporción de genotipos distinguibles, Métodos = métodos empleados para la evaluación de los parámetros. El significado de las abreviaturas empleadas en cada columna se muestra al final del cuadro.

\begin{tabular}{|c|c|c|c|c|c|c|c|c|c|c|c|c|c|}
\hline Especie (Familia) & Hábito & SCRZ & POL & PROR & CCT-N & $\begin{array}{c}\text { POB/ } \\
\mathrm{HL}\end{array}$ & $\begin{array}{c}\text { PDE } \\
\text { (fig. 2) }\end{array}$ & $\begin{array}{c}\text { AER } \\
\text { (fig. 2) }\end{array}$ & $D$ & $G / N$ & $\begin{array}{l}\text { Estructura } \\
\text { genética } \\
\text { local (EGL) }\end{array}$ & $\begin{array}{c}\text { Métodos } \\
\text { PDE, } D, G / N \\
\text { y EGL }\end{array}$ & Referencias \\
\hline $\begin{array}{l}\text { Anemone } \\
\text { nemorosa } \\
\text { (Ranunculaceae) }\end{array}$ & $\mathrm{Hp}$ & A & $\mathrm{Bt}$ & $\begin{array}{c}\mathrm{Rzm+} \\
\mathrm{drm}\end{array}$ & $\mathrm{Lg}+\mathrm{NI}$ & $\begin{array}{l}\text { ppm } \\
(20)\end{array}$ & $(4,5)$ & $\mathrm{Ag}+\mathrm{En}$ & $\mathrm{n} / \mathrm{e}$ & 0.95 & $\begin{array}{l}\operatorname{asp},<1 \mathrm{~m} \\
(\mathrm{rm})\end{array}$ & tra+ISZ+asp & $\begin{array}{l}\text { Stehlik y } \\
\text { Holderegger } \\
\text { 2000* }\end{array}$ \\
\hline $\begin{array}{l}\text { Botrychium } \\
\text { pumicola } \\
\text { (Ophioglossaceae) }\end{array}$ & $\mathrm{Hp}$ & A & $\mathrm{Ab}$ & gmas & $\mathrm{NI}$ & $\begin{array}{l}\text { p } 1 \\
\text { p } 2 \\
\text { p } 3\end{array}$ & $\begin{array}{l}(2,5) \\
(2,5)\end{array}$ & $\begin{array}{l}\mathrm{Ag}+\mathrm{En} \\
\mathrm{Ag}+\mathrm{En} \\
\mathrm{Ag}+\mathrm{En}\end{array}$ & $\begin{array}{c}0.14 \\
0.16 \\
0.1\end{array}$ & $\begin{array}{l}0.93 \\
0.9 \\
0.65\end{array}$ & $\begin{array}{c}\text { mt: no } \\
\text { detectada } \\
\text { mt: no } \\
\text { detectada } \\
\text { mt: no } \\
\text { detectada }\end{array}$ & prc + ISSR $+m t$ & $\begin{array}{c}\text { Camacho y } \\
\text { Liston, 2001* }\end{array}$ \\
\hline $\begin{array}{l}\text { Calamagrostis } \\
\text { porteri ssp. } \\
\text { insperata (Poaceae) }\end{array}$ & $\mathrm{Hp}$ & A & $\mathrm{Ab}$ & rzm & $\operatorname{Lg}$ & $\begin{array}{l}\text { p } 2 \\
\text { p } 3 \\
\text { p } 4\end{array}$ & $\begin{array}{l}2 \\
2 \\
2\end{array}$ & $\begin{array}{l}\mathrm{Ag} \\
\mathrm{Ag} \\
\mathrm{Ag}\end{array}$ & $\begin{array}{l}n / e \\
n / e \\
n / e\end{array}$ & $\begin{array}{c} \\
1 \\
0.82 \\
0.91\end{array}$ & $\begin{array}{l}\mathrm{n} / \mathrm{e} \\
\mathrm{n} / \mathrm{e} \\
\mathrm{n} / \mathrm{e}\end{array}$ & $\begin{array}{c}\text { desc+tra+ } \\
\text { ISRR+ } \\
\text { RAPD }\end{array}$ & $\begin{array}{c}\text { Esselman } \\
\text { et al., } \\
1999\end{array}$ \\
\hline $\begin{array}{l}\text { Carex bigelowii } \\
\text { (Cyperaceae) }\end{array}$ & $\mathrm{Hp}$ & $?$ & $\mathrm{Ab}$ & rzm & $\operatorname{Lg}$ & $\begin{array}{l}\text { p } 1 \\
\text { p } 2 \\
\text { p } 3\end{array}$ & $\begin{array}{l}4 \\
4 \\
4\end{array}$ & $\begin{array}{l}\mathrm{Ag} \\
\mathrm{Ag} \\
\mathrm{Ag}\end{array}$ & $\begin{array}{l}0.98 \\
0.98 \\
0.98\end{array}$ & $\begin{array}{l}0.52 \\
0.63 \\
0.48\end{array}$ & $\begin{array}{l}\text { n/e } \\
\text { n/e } \\
\text { n/e }\end{array}$ & tra+ISZ & $\begin{array}{c}\text { Jonsson } \\
\text { et al., 1996* }\end{array}$ \\
\hline $\begin{array}{l}\text { Cladium jamaicense } \\
\text { (Cyperaceae) }\end{array}$ & $\mathrm{Hp}$ & $?$ & $\mathrm{Ab}$ & $\begin{array}{c}\text { rzm+ } \\
\text { pnt }\end{array}$ & $\mathrm{Lg}+\mathrm{Nl}$ & $\begin{array}{l}p 1 \\
\text { p } 2 \\
\text { p } 3 \\
\text { p } 4 \\
\text { p } 5 \\
\text { p } 6 \\
\text { p } 7 \\
\text { p } 8 \\
\text { p } 9 \\
\text { p } 10 \\
\text { p } 11 \\
\text { p } 12 \\
\text { p } 13 \\
\text { p } 14\end{array}$ & $\begin{array}{l}(4,5) \\
(4,5) \\
(4,5) \\
(4,5) \\
(4,5) \\
1 \\
(4,5) \\
(4,5) \\
(4,5) \\
(4,5) \\
(4,5) \\
(4,5) \\
(4,5) \\
(4,5)\end{array}$ & $\begin{array}{c}A g+E n \\
A g+E n \\
A g+E n \\
A g+E n \\
A g+E n \\
A g \\
A g+E n \\
A g+E n \\
A g+E n \\
A g+E n \\
A g+E n \\
A g+E n \\
A g+E n \\
A g+E n\end{array}$ & $\begin{array}{c}0.236 \\
0.269 \\
0.271 \\
0.682 \\
0.159 \\
0 \\
0.538 \\
0.212 \\
0.771 \\
0.698 \\
0.814 \\
0.682 \\
0.673 \\
0.444\end{array}$ & $\begin{array}{l}0.044 \\
0.044 \\
0.125 \\
0.231 \\
0.043 \\
0.021 \\
0.146 \\
0.068 \\
0.106 \\
0.143 \\
0.261 \\
0.114 \\
0.13 \\
0.043\end{array}$ & $\begin{array}{c}\text { mpic: no } \\
\text { detectada } \\
\text { mpic: no } \\
\text { detectada } \\
\text { mpic: no } \\
\text { detectada } \\
\text { mpic: no } \\
\text { detectada } \\
\text { mpic: no } \\
\text { detectada } \\
\text { n/a } \\
\text { mpic: no } \\
\text { detectada } \\
\text { mpic: no } \\
\text { detectada } \\
\text { mpic: no } \\
\text { detectada } \\
\text { mpic: no } \\
\text { detectada } \\
\text { mpic: no } \\
\text { detectada } \\
\text { mpic: no } \\
\text { detectada } \\
\text { mpic: no } \\
\text { detectada } \\
\text { mpic: no } \\
\text { detectada }\end{array}$ & $\begin{array}{l}\text { tra+lSZ+ } \\
\text { mpic }\end{array}$ & $\begin{array}{l}\text { Ivey y Richards, } \\
\text { 2001* }\end{array}$ \\
\hline
\end{tabular}


Apéndice. Continuación

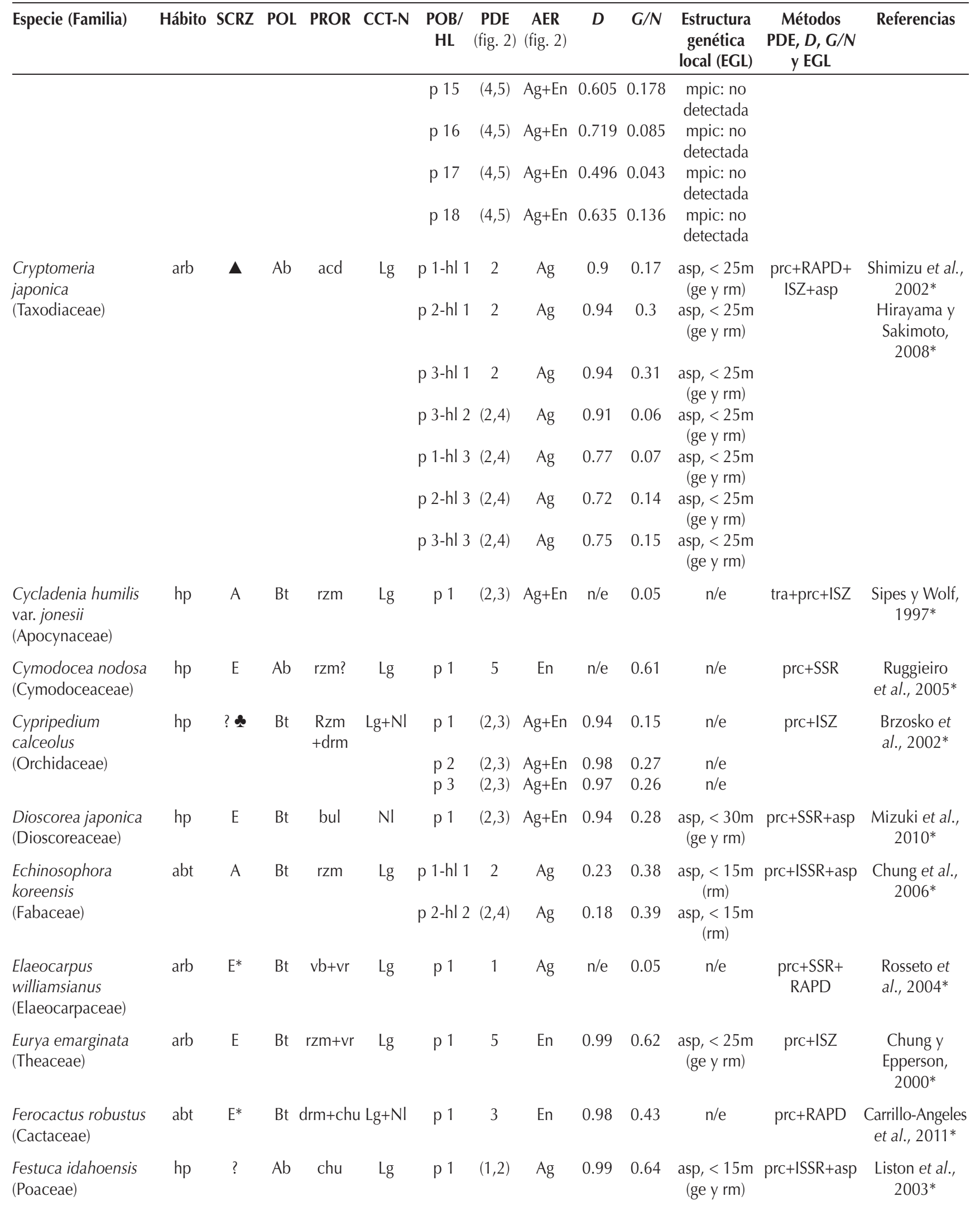


Apéndice. Continuación

\begin{tabular}{|c|c|c|c|c|c|c|c|c|c|c|c|c|c|}
\hline Especie (Familia) & Hábito & SCRZ & POL & PROR & CCT-N & $\begin{array}{c}\mathrm{POB} / \\
\mathrm{HL}\end{array}$ & $\begin{array}{l}\text { PDE } \\
\text { (fig. 2) }\end{array}$ & $\begin{array}{c}\text { AER } \\
\text { (fig. 2) }\end{array}$ & $D$ & $G / N$ & $\begin{array}{l}\text { Estructura } \\
\text { genética } \\
\text { local (EGL) }\end{array}$ & $\begin{array}{c}\text { Métodos } \\
\text { PDE, } D, G / N \\
\text { y EGL } \\
\end{array}$ & Referencias \\
\hline \multirow[t]{3}{*}{$\begin{array}{l}\text { Gagea lutea } \\
\text { (Liliaceae) }\end{array}$} & \multirow[t]{3}{*}{ hg } & \multirow[t]{3}{*}{$?$} & \multirow[t]{3}{*}{$\mathrm{Bt}$} & \multirow[t]{3}{*}{ buls } & \multirow[t]{3}{*}{$\mathrm{Nl}$} & p 1 & $(2,3)$ & $\mathrm{Ag}+\mathrm{En}$ & $\mathrm{n} / \mathrm{e}$ & 0.51 & \multirow{3}{*}{\multicolumn{2}{|c|}{$\begin{array}{l}\text { asp, }<8 \mathrm{~m}, 16 \text { tra+AFLP+asp } \\
\mathrm{a}<24 \mathrm{~m}(\mathrm{rm}) \\
\mathrm{asp},<8 \mathrm{~m}, 16 \\
\mathrm{a}<24 \mathrm{~m}(\mathrm{rm}) \\
\mathrm{asp},<8 \mathrm{~m}, 16 \\
\mathrm{a}<24 \mathrm{~m}(\mathrm{rm})\end{array}$}} & \multirow[t]{3}{*}{$\begin{array}{l}\text { Pfeiffer et al., } \\
2011^{*}\end{array}$} \\
\hline & & & & & & p 2 & $(2,3)$ & $\mathrm{Ag}+\mathrm{En}$ & $\mathrm{n} / \mathrm{e}$ & 0.57 & & & \\
\hline & & & & & & p 3 & $(2,3)$ & $\mathrm{Ag}+\mathrm{En}$ & $\mathrm{n} / \mathrm{e}$ & 0.61 & & & \\
\hline \multirow{3}{*}{$\begin{array}{l}\text { Gagea spathacea } \\
\text { (Liliaceae) }\end{array}$} & \multirow[t]{3}{*}{ hg } & \multirow[t]{3}{*}{$?$} & \multirow[t]{3}{*}{$\mathrm{Bt}$} & \multirow[t]{3}{*}{ buls } & \multirow[t]{3}{*}{$\mathrm{NI}$} & p 1 & 1 & $\mathrm{Ag}$ & $\mathrm{n} / \mathrm{e}$ & 0.001 & $\mathrm{n} / \mathrm{a}$ & \multirow[t]{3}{*}{ tra+AFLP } & \multirow{3}{*}{$\begin{array}{r}\text { Pfeiffer et al., } \\
2011^{*}\end{array}$} \\
\hline & & & & & & p 2 & 1 & $\mathrm{Ag}$ & $\mathrm{n} / \mathrm{e}$ & 0.001 & $\mathrm{n} / \mathrm{a}$ & & \\
\hline & & & & & & p 3 & 1 & $\mathrm{Ag}$ & $\mathrm{n} / \mathrm{e}$ & 0.001 & $\mathrm{n} / \mathrm{a}$ & & \\
\hline $\begin{array}{l}\text { Hyparrhenia } \\
\text { diplandra (Poaceae) }\end{array}$ & hp & $?$ & $\mathrm{Ab}$ & agp & $\mathrm{NI}$ & p 1 & 5 & En & 0.85 & 0.07 & $\begin{array}{l}\text { asp, }<6 \mathrm{~m} \\
\quad(\mathrm{rm})\end{array}$ & $\begin{array}{c}\text { prc+lSZ } \\
+ \text { CLO+asp }\end{array}$ & $\begin{array}{c}\text { Garnier et al., } \\
2002^{*}\end{array}$ \\
\hline $\begin{array}{l}\text { Ilex leucoclada } \\
\text { (Aquifoliaceae) }\end{array}$ & abt & E & $\mathrm{Bt}$ & $\operatorname{acd} \mathbf{\Lambda}$ & $\operatorname{Lg}$ & p 1 & $(2,3)$ & $\mathrm{Ag}+\mathrm{En}$ & 0.67 & 0.09 & $\mathrm{n} / \mathrm{e}$ & prc+RAPD & $\begin{array}{c}\text { Torimaru y } \\
\text { Tomaru, 2005* }\end{array}$ \\
\hline \multirow[t]{8}{*}{$\begin{array}{l}\text { Lophocereus schottii } \\
\text { (Cactaceae) }\end{array}$} & \multirow[t]{8}{*}{ arb } & \multirow[t]{8}{*}{ A } & \multirow[t]{8}{*}{$\mathrm{Bt}$} & \multirow[t]{8}{*}{$\begin{array}{l}\mathrm{drm} \\
+\mathrm{acd}\end{array}$} & \multirow[t]{8}{*}{$\mathrm{Lg}+\mathrm{NI}$} & p 1-hl 1 & 5 & En & 0.95 & 0.64 & $\begin{array}{c}\text { SG: 0-10 } \\
<10-50>\text { más } \\
\text { de } 50 m \text { (ge) }\end{array}$ & \multirow{8}{*}{ prc+ISZ+SG } & \multirow[t]{8}{*}{$\begin{array}{l}\text { Parker y } \\
\text { Hamrick, } \\
1992^{*}\end{array}$} \\
\hline & & & & & & p 2-hl 2 & 2 & $\mathrm{Ag}$ & 0.95 & 0.52 & $\begin{array}{l}\text { SG: } 0-10 \\
<10-50>\text { más } \\
\text { de } 50 \mathrm{~m} \text { (ge) }\end{array}$ & & \\
\hline & & & & & & p 3-hl 3 & 2 & $\mathrm{Ag}$ & 0.93 & 0.65 & $\begin{array}{c}\text { SG: } 0-10 \\
<10-50>\text { más } \\
\text { de } 50 m \text { (ge) }\end{array}$ & & \\
\hline & & & & & & p 4-hl 1 & $(1,2)$ & $\mathrm{Ag}$ & 0.94 & 0.24 & $\begin{array}{l}\text { SG: } 0-10> \\
\text { 10-50>más } \\
\text { de } 50 \mathrm{~m} \text { (ge) }\end{array}$ & & \\
\hline & & & & & & p 5-hl 4 & $(1,2)$ & $\mathrm{Ag}$ & 0.92 & 0.41 & $\begin{array}{l}\text { SG: } 0-10> \\
10-50<\text { más } \\
\text { de } 50 m \text { (ge) }\end{array}$ & & \\
\hline & & & & & & p 6-hl 4 & $(1,2)$ & $\mathrm{Ag}$ & 0.87 & 0.45 & $\begin{array}{c}\text { SG: } 0-10> \\
10-50<\text { más } \\
\text { de } 50 m \text { (ge) }\end{array}$ & & \\
\hline & & & & & & p 7-hl 2 & $(1,2)$ & $\mathrm{Ag}$ & 0.8 & 0.18 & $\begin{array}{l}\text { SG: } 0-10> \\
\text { 10-50>más } \\
\text { de } 50 \mathrm{~m} \text { (ge) }\end{array}$ & & \\
\hline & & & & & & p 8-hl 5 & $(1,5)$ & $\mathrm{Ag}+\mathrm{En}$ & 0.59 & 0.36 & $\begin{array}{l}\text { SG: } 10-50= \\
\text { más de } 50 \mathrm{~m} \\
\quad(\text { ge })\end{array}$ & & \\
\hline $\begin{array}{l}\text { Lyonothamnus } \\
\text { floribundus } \\
\text { (Rosaceae) }\end{array}$ & arb & $?$ & $\mathrm{Bt}$ & $\mathrm{vr}$ & $\operatorname{Lg}$ & p 1 & $(1,2)$ & $\mathrm{Ag}$ & $\mathrm{n} / \mathrm{e}$ & 0.036 & $\mathrm{n} / \mathrm{e}$ & prc+RAPD & $\begin{array}{c}\text { Bushakra et al., } \\
\text { 1999* }\end{array}$ \\
\hline $\begin{array}{l}\text { Maianthemum } \\
\text { bifolium } \\
\text { (Convalliariaceae) }\end{array}$ & hp & $E^{*}$ & $\mathrm{Bt}$ & $\mathrm{rzm}$ & $\operatorname{Lg}$ & p 1 & $(1,2)$ & $\mathrm{Ag}$ & 0.88 & 0.7 & $\begin{array}{l}\text { asp, }<50 \mathrm{~m} \\
\text { (ge) \# }\end{array}$ & $\begin{array}{l}\text { trans+AFLP } \\
\quad+\text { asp }\end{array}$ & $\begin{array}{l}\text { Arens et al., } \\
2005^{*} \\
\text { Honnay et al. } \\
2006\end{array}$ \\
\hline $\begin{array}{l}\text { Maianthemum } \\
\text { dilatatum } \\
\text { (Convalliariaceae) }\end{array}$ & hp & EAs & $\mathrm{Bt}$ & $\operatorname{rzm} \mathbf{\Delta}$ & $\operatorname{Lg}$ & p 1 & $(1,2)$ & $\mathrm{Ag}$ & $\mathrm{n} / \mathrm{e}$ & 0.64 & $\begin{array}{l}\text { asp, }<45 \mathrm{~m} \\
\text { (ge y rm) }\end{array}$ & $\begin{array}{l}\text { prc }+ \text { AFLP } \\
\quad+\text { asp }\end{array}$ & $\begin{array}{l}\text { Wilson et al., } \\
2005 a^{*} \\
\text { Wilson et al., } \\
2005 b^{*}\end{array}$ \\
\hline $\begin{array}{l}\text { Paris quadrifolia } \\
\text { (Trilliaceae) }\end{array}$ & hp & A & $\mathrm{Bt}$ & $\mathrm{rzm}$ & $\operatorname{Lg}$ & p 1-hl 1 & 3 & En & 0.87 & 0.27 & $\begin{array}{l}\text { asp, }<5 \mathrm{~m} \\
(\text { ge y rm) }\end{array}$ & $\begin{array}{l}\text { prc }+ \text { AFLP } \\
\quad+\text { asp }\end{array}$ & $\begin{array}{c}\text { Jacquemyn et } \\
\text { al., 2005* }\end{array}$ \\
\hline
\end{tabular}


Apéndice. Continuación

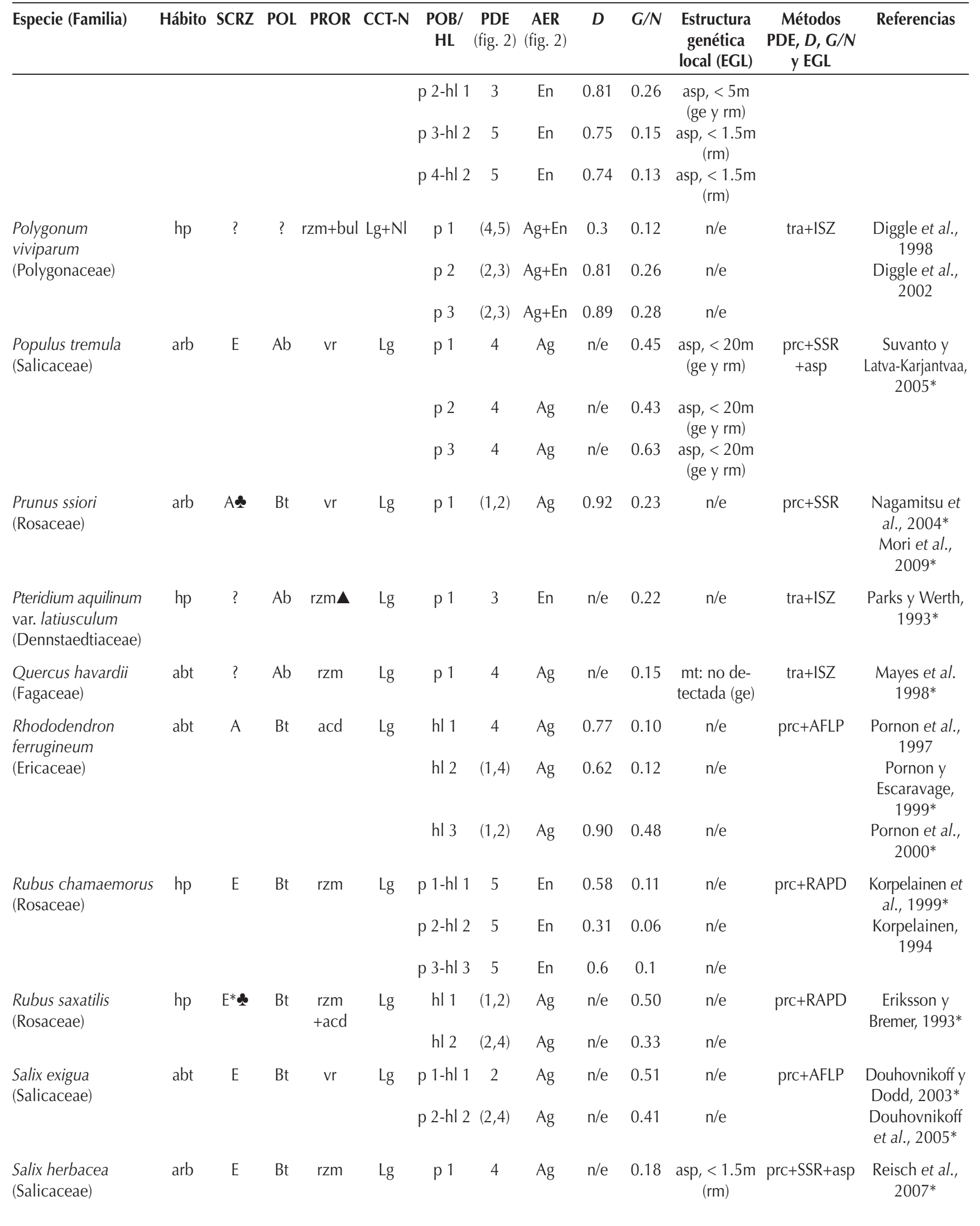


Apéndice. Continuación

\begin{tabular}{|c|c|c|c|c|c|c|c|c|c|c|c|c|c|}
\hline Especie (Familia) & Hábito & SCRZ & POL & PROR & CCT-N & $\begin{array}{c}\mathrm{POB} / \\
\mathrm{HL}\end{array}$ & $\begin{array}{c}\text { PDE } \\
\text { (fig. 2) }\end{array}$ & $\begin{array}{c}\text { AER } \\
\text { (fig. 2) }\end{array}$ & $D$ & $G / N$ & $\begin{array}{l}\text { Estructura } \\
\text { genética } \\
\text { local (EGL) }\end{array}$ & $\begin{array}{c}\text { Métodos } \\
\text { PDE, } D, G / N \\
\text { y EGL }\end{array}$ & Referencias \\
\hline \multirow[t]{6}{*}{$\begin{array}{l}\text { Saxifraga cernua } \\
\text { (Saxifragaceae) }\end{array}$} & \multirow[t]{6}{*}{ hp } & \multirow[t]{6}{*}{$E^{*}$} & \multirow[t]{6}{*}{$\mathrm{Bt}$} & \multirow[t]{6}{*}{ bul } & \multirow[t]{6}{*}{$\mathrm{Nl}$} & p 1 & 5 & En & 0.35 & 0.13 & $\begin{array}{c}\text { mt: no } \\
\text { detectada }\end{array}$ & \multirow[t]{6}{*}{$\begin{array}{l}\text { prc+RAPD } \\
+m t+a s p\end{array}$} & \multirow{6}{*}{$\begin{array}{l}\text { Gabrielsen y } \\
\text { Brochmann, } \\
1998^{*} \\
\text { KjØIner et al., } \\
\text { 2006* }\end{array}$} \\
\hline & & & & & & p 2 & 5 & En & 0.68 & 0.19 & $\begin{array}{c}\text { mt: no } \\
\text { detectada }\end{array}$ & & \\
\hline & & & & & & p 3 & 5 & En & 0.7 & 0.25 & $\begin{array}{l}\text { asp: no } \\
\text { detectada }\end{array}$ & & \\
\hline & & & & & & p 4 & 5 & En & 0.9 & 0.4 & $\begin{array}{c}\text { asp: no } \\
\text { detectada }\end{array}$ & & \\
\hline & & & & & & p 5 & 5 & En & 0.93 & 0.43 & $\begin{array}{l}\text { asp: no } \\
\text { detectada }\end{array}$ & & \\
\hline & & & & & & p 6 & 5 & En & 0.89 & 0.34 & $\begin{array}{c}\text { asp: no } \\
\text { detectada }\end{array}$ & & \\
\hline \multirow[t]{3}{*}{$\begin{array}{l}\text { Scirpus maritimus } \\
\text { (Cyperaceae) }\end{array}$} & \multirow[t]{3}{*}{ hp } & \multirow[t]{12}{*}{$\mathrm{E}^{*}$} & \multirow[t]{3}{*}{$\mathrm{Ab}$} & \multirow[t]{3}{*}{$\mathrm{rzm}$} & \multirow[t]{3}{*}{$\operatorname{Lg}$} & p 1 & 2 & $\mathrm{Ag}$ & $\mathrm{n} / \mathrm{e}$ & $\mathrm{n} / \mathrm{e}$ & $\mathrm{n} / \mathrm{e}$ & \multirow[t]{3}{*}{ desc } & \multirow[t]{3}{*}{$\begin{array}{c}\text { Charpentier et } \\
\text { al., } 2000\end{array}$} \\
\hline & & & & & & p 2 & 4 & $\mathrm{Ag}$ & $\mathrm{n} / \mathrm{e}$ & $\mathrm{n} / \mathrm{e}$ & $\mathrm{n} / \mathrm{e}$ & & \\
\hline & & & & & & p 3 & 4 & $\mathrm{Ag}$ & $\mathrm{n} / \mathrm{e}$ & $\mathrm{n} / \mathrm{e}$ & $\mathrm{n} / \mathrm{e}$ & & \\
\hline \multirow[t]{9}{*}{$\begin{array}{l}\text { Sequoia sempervirens } \\
\text { (Taxodiaceae) }\end{array}$} & \multirow[t]{9}{*}{ arb } & & \multirow[t]{9}{*}{$\mathrm{Ab}$} & \multirow[t]{9}{*}{$\mathrm{vb}$} & \multirow[t]{9}{*}{$\operatorname{Lg}$} & p 1-hl 1 & 2 & $\mathrm{Ag}$ & $\mathrm{n} / \mathrm{e}$ & 0.24 & $\begin{array}{l}\mathrm{mt}:<40 \mathrm{~m} \\
\quad(\mathrm{rm})\end{array}$ & \multirow[t]{9}{*}{$\operatorname{prc}+\mathrm{AFLP}+\mathrm{mt}$} & \multirow[t]{9}{*}{$\begin{array}{l}\text { Douhovnikoff } \\
\text { et al., 2004* }\end{array}$} \\
\hline & & & & & & p 2-hl 1 & 2 & $\mathrm{Ag}$ & $\mathrm{n} / \mathrm{e}$ & 0.38 & $\begin{array}{c}\mathrm{mt}:<40 \mathrm{~m} \\
(\mathrm{rm})\end{array}$ & & \\
\hline & & & & & & p 3-hl 1 & 2 & $\mathrm{Ag}$ & $\mathrm{n} / \mathrm{e}$ & 0.5 & $\begin{array}{c}\mathrm{mt}:<40 \mathrm{~m} \\
(\mathrm{rm})\end{array}$ & & \\
\hline & & & & & & p 4-hl 2 & 2 & $\mathrm{Ag}$ & $\mathrm{n} / \mathrm{e}$ & 0.6 & $\begin{array}{c}\mathrm{mt}:<40 \mathrm{~m} \\
(\mathrm{rm})\end{array}$ & & \\
\hline & & & & & & p 5-hl 2 & 2 & $\mathrm{Ag}$ & $\mathrm{n} / \mathrm{e}$ & 0.35 & $\begin{array}{c}\mathrm{mt}:<40 \mathrm{~m} \\
(\mathrm{rm})\end{array}$ & & \\
\hline & & & & & & p 6-hl 2 & 2 & $\mathrm{Ag}$ & $\mathrm{n} / \mathrm{e}$ & 0.6 & $\begin{array}{c}\mathrm{mt}:<40 \mathrm{~m} \\
(\mathrm{rm})\end{array}$ & & \\
\hline & & & & & & p 7-hl 3 & 2 & $\mathrm{Ag}$ & $\mathrm{n} / \mathrm{e}$ & 0.26 & $\begin{array}{c}\mathrm{mt}:<40 \mathrm{~m} \\
(\mathrm{rm})\end{array}$ & & \\
\hline & & & & & & p 8-hl 3 & 2 & $\mathrm{Ag}$ & $n / e$ & 0.19 & $\begin{array}{c}\mathrm{mt}:<40 \mathrm{~m} \\
(\mathrm{rm})\end{array}$ & & \\
\hline & & & & & & p 9-hl 3 & 2 & $\mathrm{Ag}$ & $\mathrm{n} / \mathrm{e}$ & 0.54 & $\begin{array}{c}\mathrm{mt}:<40 \mathrm{~m} \\
\quad(\mathrm{rm})\end{array}$ & & \\
\hline $\begin{array}{l}\text { Stenocereus eruca } \\
\text { (Cactaceae) }\end{array}$ & abt & $E^{*}$ & $\mathrm{Bt}$ & drm & $\mathrm{NI}$ & p 1 & 2 & $\mathrm{Ag}$ & 0.99 & 0.83 & $\begin{array}{l}\text { asp, }<20 \text { m } \\
\text { (ge y rm) }\end{array}$ & prc+ISSR+asp & $\begin{array}{c}\text { Clark-Tapia et } \\
\text { al., 2005* } \\
\text { Clark-Tapia et } \\
\text { al., } 2006\end{array}$ \\
\hline \multirow[t]{5}{*}{$\begin{array}{l}\text { Typha latifolia } \\
\text { (Thyphaceae) }\end{array}$} & \multirow[t]{5}{*}{$\mathrm{hp}$} & \multirow[t]{5}{*}{ A } & \multirow[t]{5}{*}{$\mathrm{Ab}$} & \multirow[t]{5}{*}{ rzm } & $\operatorname{Lg}$ & p 1 & $(4,5)$ & $\mathrm{Ag}+\mathrm{En}$ & $\mathrm{n} / \mathrm{e}$ & 0.77 & $\mathrm{n} / \mathrm{e}$ & tra+VNTR & $\begin{array}{c}\text { Keane et al., } \\
\text { 1999* }\end{array}$ \\
\hline & & & & & & p 2 & $(4,5)$ & $\mathrm{Ag}+\mathrm{En}$ & $\mathrm{n} / \mathrm{e}$ & 0.55 & $\mathrm{n} / \mathrm{e}$ & & \\
\hline & & & & & & p 3 & $(4,5)$ & $\mathrm{Ag}+\mathrm{En}$ & $\mathrm{n} / \mathrm{e}$ & 0.48 & $\mathrm{n} / \mathrm{e}$ & & \\
\hline & & & & & & p 4 & $(4,5)$ & $\mathrm{Ag}+\mathrm{En}$ & $\mathrm{n} / \mathrm{e}$ & 0.3 & $n / e$ & & \\
\hline & & & & & & p 5 & $(4,5)$ & $A g+E n$ & $\mathrm{n} / \mathrm{e}$ & 0.57 & $\mathrm{n} / \mathrm{e}$ & & \\
\hline $\begin{array}{l}\text { Uvularia perfoliata } \\
\text { (Convallariaceae) }\end{array}$ & hp & $?$ & $?$ & etl & $\operatorname{Lg}$ & p 1-hl 1 & $(4,5)$ & $A g+E n$ & 0.74 & 0.09 & $\begin{array}{c}\text { asp, }<3-5 m \\
(r m)\end{array}$ & prc+ISZ+asp & $\begin{array}{l}\text { Kudoh et al., } \\
\text { 1999* }\end{array}$ \\
\hline & & & & & & p 1-hl 2 & 1 & $\mathrm{Ag}$ & 0.59 & 0.03 & $\mathrm{n} / \mathrm{a}$ & & \\
\hline $\begin{array}{l}\text { Vaccinium myrtillus } \\
\text { (Ericaceae) }\end{array}$ & abt & A & $\mathrm{Bt}$ & rzm & $\operatorname{Lg}$ & p 1 & 2 & $\mathrm{Ag}$ & 0.94 & 0.29 & $\begin{array}{c}\text { asp: no } \\
\text { detectada }\end{array}$ & $\begin{array}{l}\text { prc+AFLP+ } \\
\text { RAPD+asp }\end{array}$ & $\begin{array}{c}\text { Albert et al., } \\
\text { 2003* }\end{array}$ \\
\hline
\end{tabular}


Apéndice. Continuación

\begin{tabular}{|c|c|c|c|c|c|c|c|c|c|c|c|c|c|}
\hline Especie (Familia) & Hábito & SCRZ & POL & PROR & CCT-N & $\begin{array}{c}\mathrm{POB} / \\
\mathrm{HL}\end{array}$ & $\begin{array}{l}\text { PDE } \\
\text { (fig. 2) }\end{array}$ & $\begin{array}{c}\text { AER } \\
\text { (fig. 2) }\end{array}$ & $D$ & $G / N$ & $\begin{array}{c}\text { Estructura } \\
\text { genética } \\
\text { local (EGL) }\end{array}$ & $\begin{array}{c}\text { Métodos } \\
\text { PDE, } D, G / N \\
\text { y EGL }\end{array}$ & Referencias \\
\hline $\begin{array}{l}\text { Vaccinium stamineum } \\
\text { (Ericaceae) }\end{array}$ & abt & $\mathrm{E}^{*}$ & $\mathrm{Bt}$ & $\mathrm{rzm}$ & $\operatorname{Lg}$ & $\mathrm{p} 1$ & $(2,3)$ & $\mathrm{Ag}+\mathrm{En}$ & $\mathrm{n} / \mathrm{e}$ & 0.68 & $\mathrm{n} / \mathrm{e}$ & prc+RAPD & $\begin{array}{l}\text { Kreher et al., } \\
\text { 2000* }\end{array}$ \\
\hline $\begin{array}{l}\text { Zostera marina } \\
\text { (Zosteraceae) }\end{array}$ & hp & A & $\mathrm{Ab}$ & $\mathrm{rzm}$ & $\operatorname{Lg}$ & $\begin{array}{l}\text { p 1-hl } 1 \\
\text { p 2-hl } 2\end{array}$ & $\begin{array}{l}(4,5) \\
(4,5)\end{array}$ & $\begin{array}{l}A g+E n \\
A g+E n\end{array}$ & $\mathrm{n} / \mathrm{e}$ & $\mathrm{n} / \mathrm{e}$ & $\begin{array}{c}\text { asp, }<6 \mathrm{~m} \\
\text { (ge y rm) } \\
\text { asp, }<6 \mathrm{~m} \\
\text { (ge y rm) }\end{array}$ & $\mathrm{prc}+\mathrm{SSR}+\mathrm{asp}$ & $\begin{array}{l}\text { Hämmerli y } \\
\text { Reusch, } 2003 \mathrm{a}^{*} \\
\text { Hämmerli y } \\
\text { Reusch, } 2003 \mathrm{~b}^{*}\end{array}$ \\
\hline $\begin{array}{l}\text { Zostera noltii } \\
\text { (Zosteraceae) }\end{array}$ & hp & A & $\mathrm{Ab}$ & $\mathrm{rzm}$ & $\operatorname{Lg}$ & p 1 & 4 & $\mathrm{Ag}$ & $\mathrm{n} / \mathrm{e}$ & 0.12 & $\mathrm{n} / \mathrm{e}$ & $\mathrm{prc}+\mathrm{SSR}$ & $\begin{array}{l}\text { Ruggieiro et } \\
\text { al., 2005* }\end{array}$ \\
\hline
\end{tabular}

Hábito: $\mathbf{a b t}=$ arbustos, $\mathbf{a r b}=$ árboles, $\mathbf{h p}=$ hierbas perennes

SCRZ: A = especies con capacidad de reproducirse por autocruza, $\mathbf{E}=$ especies con entrecruza obligada (p. ej., dioicas), $\mathbf{E}^{*}=$ especies con sistemas de autoincompatibilidad, = reclutamiento sexual observado y/o evaluado en condiciones naturales

POL: $\mathbf{A b}=$ polinización abiótica, $\mathbf{B t}=$ polinización biótica

PROR: acd = acodaduras, agp = agamospermia, bul = bulbilos, buls = bulbilos subterráneos, $\mathbf{c h u}=$ coronas huecas, $\mathbf{d r m}=$ desprendimiento de ramas, $\mathbf{e t l}=$ estolones, $\mathbf{g m a s}=$ gemas subterráneas, $\mathbf{p n t}=$ plantlets, $\mathbf{p s b}=$ pseudobulbos, $\mathbf{r z m}=$ rizomas, $\mathbf{v b}=$ vástagos basales, $\mathbf{v r}=\mathbf{v a ́ s t a g o s}$ radicales, $\mathbf{\Delta}$ = crecimiento tipo guerrilla

CCT-N: $\mathbf{L g}=$ crecimiento clonal ligado, $\mathbf{N I}=$ crecimiento clonal no ligado

POB/HL: $\mathbf{p}=$ población, $\mathbf{h l}=$ hábitat local (p. ej., bosque con dosel abierto vs bosque con dosel cerrado)

PDE: los paréntesis indican que la población presenta una combinación de patrones de distribución de los tipos indicados por los números (ver figura 2).

AER: Ag = los ramets de cada genet no se entremezclan con los ramets de genets distintos, En = los ramets de diferentes genets en la población se entremezclan.

EGL: \# = evaluado en una población distinta de donde se determinó el PDE, asp = autocorrelación espacial, mpic = método de probabilidad de identidad clonal, $\mathbf{m t}=$ prueba de Mantel, $\mathbf{S G}=$ similitud genética vs distancia espacial.

Métodos: AFLP = marcadores moleculares AFLPs, CLO = marcadores moleculares de cloroplasto, desc = descripción detallada del PDE, ISSR = marcadores moleculares ISSRs, ISZ = isoenzimas, $\mathbf{m p i c}=$ método de probabilidad de identidad clonal, $\mathbf{m t}=$ prueba de $\mathbf{M a n t e l}, \mathbf{p o b}=$ muestra de individuos o todos los individuos de la población, prc = parcelas, RAPD = marcadores moleculares RAPDs, SG $=$ similitud genética vs distancia espacial, $\mathbf{S S R}=$ marcadores moleculares SSRs (microsatélites),

Referencias: ${ }^{*}=$ estudios donde se presentan planos de la distribución espacial de los genotipos.

Todo el cuadro: ? = no se proporciona información, $\mathbf{n} / \mathbf{e}=$ no evaluado, $\mathbf{n} / \mathbf{a}=$ no aplica

GLOSARIO (columna PROR):

Acodaduras: formación de ramets a partir de un tallo originalmente erecto que se postra y genera raíces adventicias al entrar en contacto con el sustrato

Agamospermia: Producción asexual de semillas o embriones sin fertilización (diplosporía: el embrión se desarrolla de una célula diploide del saco embrionario, aposporía: el embrión se desarrolla de una célula somática del saco embrionario, embrionia adventicia: el embrión se desarrolla de células del tejido esporofítico como los tegumentos).

Bulbilos: bulbos pequeños que se forman en las axilas foliares o unidos a los bulbos.

Bulbos: tallos cortos modificados para almacenamiento rodeados por hojas carnosas o brácteas.

Coronas huecas: muerte de los tallos centrales en plantas cespitosas resultando en la formación de ramets independientes en la periferia. Descrito en pastos cespitosos.

Estolones: tallo horizontal que se extiende en la superficie del suelo.

Gemas: pequeñas estructuras vegetativas dispersables en briofitas y pteridofitas.

Plantlets: propágulos vegetativos que resultan de la reversión de estructuras reproductivas como flores y frutos a estructuras vegetativas.

Pseudobulbos: modificación del tallo para almacenamiento que se desarrolla entre dos nodos foliares en orquídeas.

Rizomas: tallo horizontal subterráneo.

Vástagos basales y radicales: formación de tallos en la base de un tallo o a partir de una raíz.

Referencias del glosario: Jackson et al., 1985; de Kroon y van Groenendael, 1997; Liston et al., 2003; van Dijk y Bakx-Schotman, 2004; Tooke et al., 2005. 
independientes de cada genotipo o ramets independientes (Figura 1; Kays y Harper, 1974; Harper, 1985; Jackson et al., 1985). La clonalidad está ampliamente distribuida en plantas, algunas estimaciones para angiospermas varían entre 30\% y más del 50\% (Tiffney y Niklas, 1985; Klim s et al., 1997; Aarssen, 2008), entre el 40\% y $80 \%$ en especies de taiga, tundra y de zonas templadas (van Groenendael y de Kroon, 1990 Klim s et al., 1997) y el 44\% de plantas invasoras en China (Liu et al., 2006), está ampliamente representada en monocotiledóneas y se presenta con baja frecuencia en las gimnospermas (van Groenendael et al., 1996; Peterson y Jones, 1997). Respecto a las condiciones ambientales, se sugiere que la clonalidad predomina en condiciones estresantes como suelos pobres en nitrógeno, suelos húmedos, cuerpos de agua, hábitats sombreados y ambientes fríos en altitudes y latitudes elevadas (Tiffney y Niklas, 1985; van Groenendael et al., 1996; Klim s et al., 1997).

La clonalidad en plantas se ha estudiado con enfoques muy diversos. Por ejemplo, se ha analizado la frecuencia de especies clonales y la variación en las formas de crecimiento clonal en distintos ambientes (p. ej., Tiffney y Niklas, 1985; van Groenendael et al., 1996; Klims et al., 1997), las implicaciones de la integración fisiológica de los ramets y la plasticidad morfológica del crecimiento clonal en ambientes heterogéneos y en condiciones de estrés competitivo (p. ej., Bell, 1984; Hartnett y Bazzaz, 1985; de Kroon y Hutchings, 1995; Price y Marshall, 1999), el efecto de la clonalidad en la demografía (p. ej., Caswell, 1985; McFadden, 1991; Damman y Cain, 1998; Mandujano et al., 2001), la comparación de la diversidad genética entre especies de plantas clonales y no clonales (p. ej., Ellstrand y Roose, 1987; Widén et al., 1994) y las consecuencias reproductivas y evolutivas de la geitonogamia asociada con la clonalidad (p. ej., Handel, 1985; de Jong et al., 1992; Eckert, 2000).

Las implicaciones reproductivas y genéticas de reducir la entrecruza incrementando la geitonogamia han aumentado el interés en el estudio de los patrones de distribución espacial de genets y ramets en poblaciones de plantas clonales. Debido a que los propágulos vegetativos suelen tener una capacidad de dispersión restringida, el reclutamiento clonal frecuente puede reducir la entrecruza e incrementar la autocruza (p. ej., autogamia y geitonogamia), determinando los niveles de variación y la estructuración genética en las poblaciones (Handel, 1985; Eckert, 2002; Ruggiero et al., 2005; Honnay et al., 2006). En especies autocompatibles, los costos reproductivos de la geitonogamia están asociados al incremento de la autogamia y a las probabilidades de expresar depresión endogámica, a la vez que disminuye la adecuación paterna reduciendo la cantidad de polen disponible para la entrecruza; mientras que en las especies autoincompatibles, la geitonogamia afecta la producción de semillas, debido a la saturación de los estigmas con polen incompatible y al aborto de óvulos, y puede resultar en el desperdicio de grandes cantidades de polen (de Jong et al.,
1992; Charpentier, 2002).

En plantas clonales los patrones de distribución espacial pueden ser afectados por la manera en que se generan los ramets. Las formas de crecimiento clonal son muy diversas, pero existen algunas clasificaciones que facilitan el entendimiento de esta variación. Tiffney y Niklas (1985) propusieron cuatro tipos principales de crecimiento: (1) Crecimiento establecido, dado por el inicio del desarrollo de un organismo individual a partir de un propágulo (puede considerarse como crecimento clonal si el propágulo es vegetativo, ya que el propágulo también puede ser una semilla producida sexualmente). (2) Crecimiento restaurativo, que se refiere a la reparación de un daño local en el individuo mediante la formación de tejido calloso, pero no hay regeneración de los órganos completos cuando estos se pierden. (3) Crecimiento regenerativo, que se refiere a la reparación y el reemplazo de órganos dañados (p. ej., crecimiento de una rama desprendida). (4) Crecimiento reproductivo, dado por la multiplicación del individuo fisiológico (p. ej., clonalidad). Estos autores también ordenan los tipos de crecimiento clonal en ligado y no ligado, considerando las estructuras de conexión (Figura 1). En el crecimiento ligado los propágulos vegetativos pueden desarrollarse hasta formar individuos completos (con raíces, tallos y hojas) manteniéndose unidos a la planta madre y su dispersión depende del crecimiento de ramificaciones de tallos y raíces, mientras que en el no ligado, los propágulos vegetativos se desprenden de la planta madre y se desarrollan hasta individuos completos separados de la planta madre. La dispersión de los propágulos en este caso es más dependiente de factores bióticos (p. ej., animales) o abióticos (p. ej., corrientes de agua, gravedad, viento) que en el crecimiento ligado y permite un mayor desplazamiento de los propágulos. El crecimiento reproductivo o clonalidad, que es la que interesa en esta revisión por su influencia en el arreglo espacial de genets y ramets, puede ser de dos tipos: (i) reiteración traumática, definida como la producción de un nuevo individuo inducida por estrés, ya sea por daño o por condiciones ambientales que limitan la reproducción sexual (por ejemplo, la producción de bulbilos en los agaves por daño al escapo floral o por deficiencia de polen) y (ii) la reiteración programada, como la producción de un nuevo individuo por un meristemo como parte normal del desarrollo (Tiffney y Niklas, 1985; Arizaga y Ezcurra, 2002).

La distribución espacial de los individuos en las poblaciones se ha clasificado en tres patrones básicos: aleatorio, uniforme y agregado (Figura 2; Gleason, 1920; Clark y Evans, 1954; Barbour et al., 1987). En poblaciones naturales de plantas, los individuos suelen distribuirse en forma más o menos agregada, debido a la distribución heterogénea de los recursos (Couteron y Kokou, 1997; Chen y Bradshaw, 1999), a la interacción con otras especies (Shreve, 1917; Mandujano et al., 1998) y a la dispersión restringida de semillas y propágulos vegetativos (Gibson y Menges, 1994; Okuda et al., 1997; Clark-Tapia et al., 2005). En plantas 
clonales además, el arreglo espacial de los genets y ramets puede ordenarse en dos patrones básicos, uno caracterizado por la agregación de ramets del mismo genet y el otro por una distribución entremezclada de ramets de distintos genets (Figura 2; de Kroon y Hutchings, 1995; Charpentier, 2002; Clark-Tapia et al., 2005). Estos arreglos espaciales de genets y ramets pueden estar asociados a la manera en que se producen los ramets (esto es por crecimiento clonal ligado o no ligado) y se ha propuesto que en las plantas que se propagan mediante crecimiento no ligado forman arreglos más entremezclados que las especies con crecimiento ligado (Gabrielsen y Brochmann, 1998), pero esto no ha sido demostrado. Por otro lado, debido a que la clonalidad puede incrementar la tasa de geitonogamia entre los ramets del mismo genet, puede esperarse que los arreglos entremezclados reduzcan la geitonogamia y promuevan la entrecruza como resultado del incremento local en el número de genets (Charpentier, 2002).

En algunas revisiones sobre el tema de la clonalidad se han planteado hipótesis sobre las implicaciones reproduc-

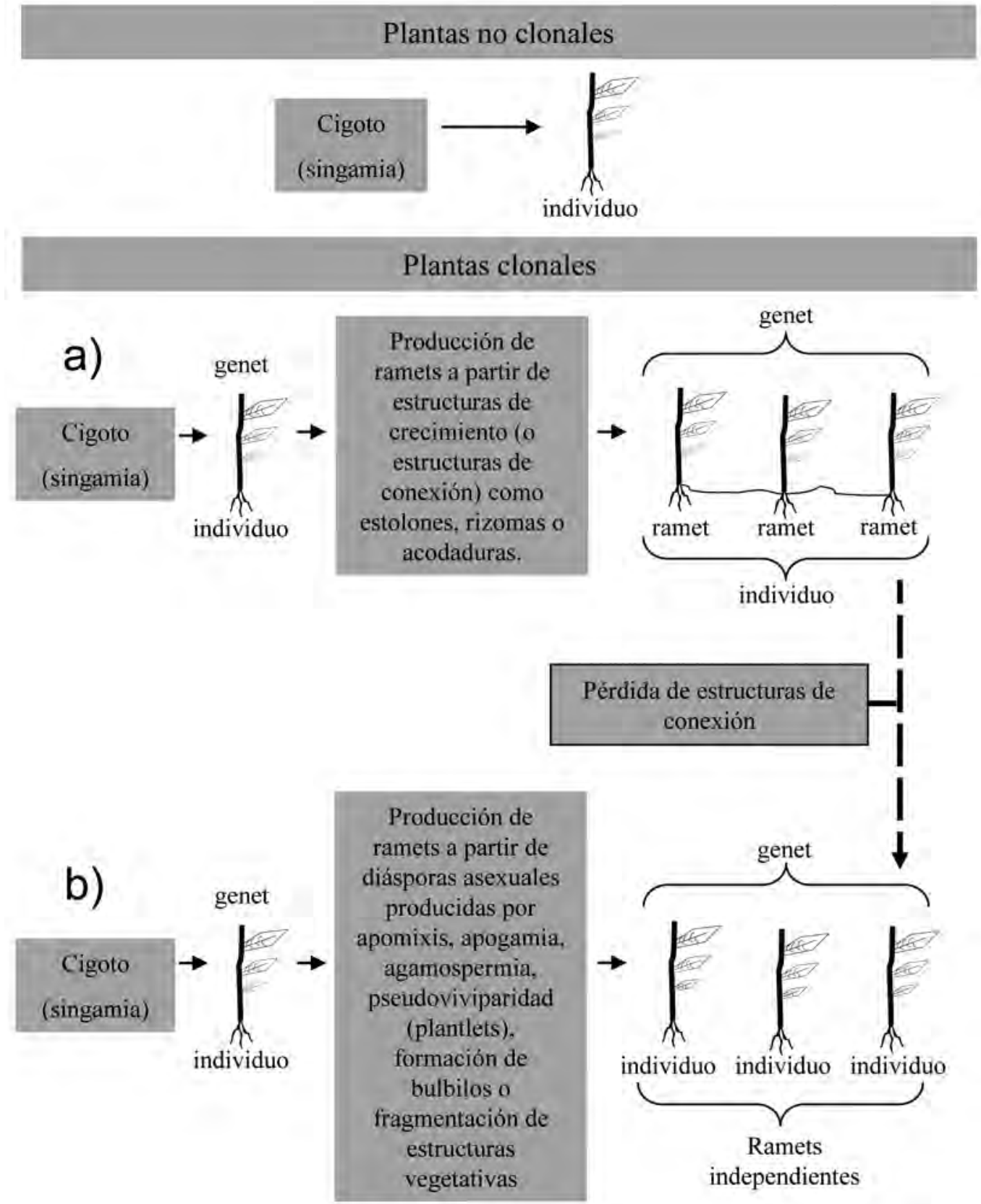

Figura 1. Formación de individuos en plantas no clonales y en plantas clonales. a) crecimiento clonal ligado y b) crecimiento clonal no ligado en la clasificación de Tiffney y Niklas (1985). 
$\mathrm{PDE}=$ Patrones de distribución espacial de los individuos y ramets en las poblaciones

(clonales y no clonales)

\section{AER $=$ Arreglos espaciales de los ramets de distintos genets}

(clonales)

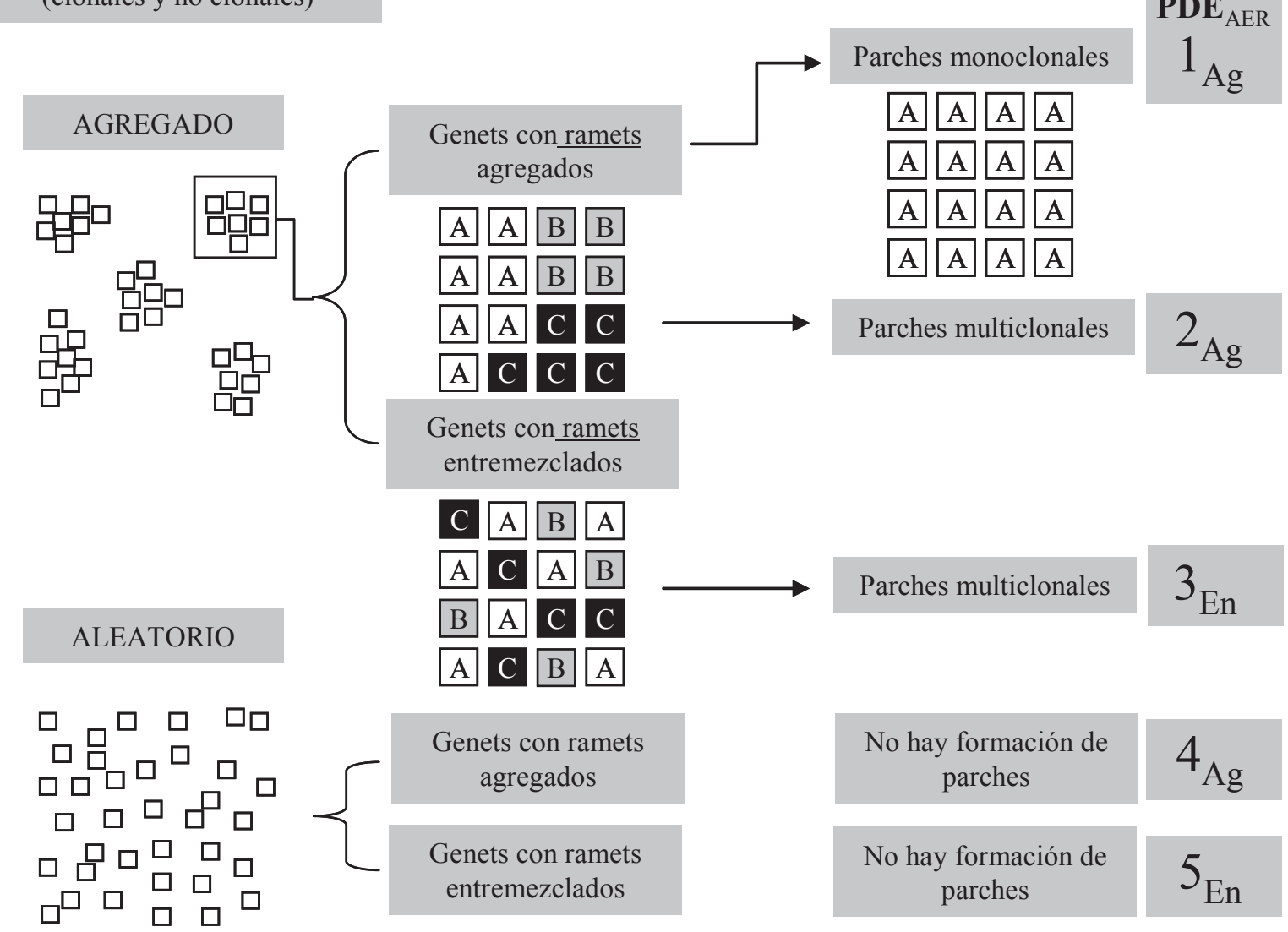

\section{UNIFORME}
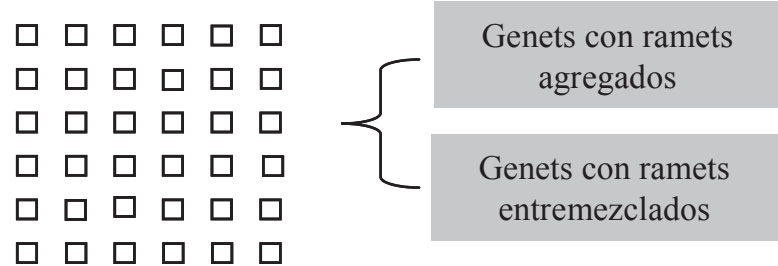

\begin{tabular}{|c|c|c|c|}
\hline $\begin{array}{c}\text { No hay formación de } \\
\text { parches }\end{array}$ & $6_{\mathrm{Ag}}$ \\
\hline $\begin{array}{c}\text { No hay formación de } \\
\text { parches }\end{array}$ & $7_{\mathrm{En}}$ \\
\hline
\end{tabular}

Figura 2. Patrones de distribución espacial de los individuos en las poblaciones de plantas no clonales y de plantas clonales. El número en el extremo derecho representa la clave del patrón de distribución espacial y el arreglo espacial de los ramets de distintos genets (PDE y AER, Apéndice). El subíndice Ag indica que los ramets de cada genet no se entremezclan con los ramets de genets distintos y el subíndice En indica que los ramets de diferentes genets se entremezclan.

tivas y genéticas de los arreglos espaciales de los genets y ramets, asociados con las distintas formas de producir descendientes clonales (Widén et al., 1994; Charpentier, 2002; Mandujano-Sánchez, 2007; Honnay y Jaquemyn, 2008). No obstante, los intentos para probar estas hipótesis son muy escasos y las posibilidades de realizar algún tipo de análisis numérico son restringidas por la baja disponibilidad de publicaciones (p. ej., Widén et al., 1994), o en los análisis sólo se incluyen especies con crecimiento ligado (p. ej., Honnay y Jaquemyn, 2008). En este trabajo presentamos una revisión de varios estudios que evalúan los patrones de distribución espacial en plantas clonales y a partir de la información 
proporcionada en dichos estudios, analizamos el planteamiento de que en poblaciones de plantas con crecimiento clonal ligado el arreglo espacial de los ramets de distintos genets es agregado, mientras que en poblaciones de plantas con crecimiento clonal no ligado predominan los arreglos espaciales entremezclados. Adicionalmente, intentamos poner a prueba la hipótesis de que los arreglos entremezclados promueven la entrecruza suponiendo que las poblaciones donde la entrecruza es más frecuente tenderán a presentar niveles más altos de diversidad genotípica.

\section{Material y métodos}

La revisión de los trabajos publicados sobre la distribución espacial clonal en plantas se llevó a cabo mediante una búsqueda de trabajos en bases de datos especializadas (CSA Cambridge, Ebsco HOST, ISI Web of Knowledge, JSTOR y Scopus) y no especializadas (Google, Google scholar y Yahoo). La búsqueda se hizo empleando los siguientes términos: clonal structure, spatial distribution of genets (andl or ramets), clonal architecture y clonal diversity y abarcó de 1990 a 2011, debido a que la mayoría de los estudios en plantas clonales con este enfoque se han realizado después de 1990.

Los criterios principales para incluir una especie en la revisión fue que se presentara una descripción de la distribución espacial de las plantas individuales (esto es sin importar el genotipo) en las poblaciones naturales y que se evaluara el arreglo espacial de los genets y los ramets (es decir agregado o entremezclado) mediante planos de distribución de los genotipos, análisis de autocorrelación espacial o compatibilidad de las cruzas entre ramets contiguos. Para cada especie se obtuvo el tipo de estructuras vegetativas a mediante las cuales se lleva a cabo la propagación clonal a partir de la información proporcionada en los estudios correspondientes o en estudios previos realizados a menudo por los mismos autores. El segundo criterio en orden de importancia fue que se reportaran valores de diversidad genotípica ( $D=$ índice de diversidad de Simpson y proporción de genotipos distinguibles $G / N$ ) para las poblaciones de las diferentes especies. Otros criterios fueron que se proporcionara información sobre las características reproductivas como la presencia de sistemas de incompatibilidad, la expresión sexual, si se ha observado reclutamiento sexual en las poblaciones y el tipo de polinización. Con base en esta información, se asignó a las especies el tipo de crecimiento clonal (i. e. ligado y no ligado, Figura 1) de acuerdo con la clasificación de Tiffney y Niklas (1985) y el patrón de distribución espacial y el tipo de arreglo espacial de los ramets (agregado o entremezclado) según el esquema presentado en la Figura 2. La información obtenida se presenta en el apéndice.

Para analizar el planteamiento de que en poblaciones de plantas con crecimiento clonal ligado el arreglo espacial de los ramets es agregado, mientras que en poblaciones de plantas con crecimiento clonal no ligado predomina el arreglo espacial entremezclado se construyó una tabla de contingencia con tres categorías de tipo de crecimiento clonal como columnas (ligado, combinación ligado-no ligado y no ligado) y tres categorías del arreglo espacial de los ramets como renglones (agregado, combinación agregado-entremezclado y entremezclado) y se analizó con una prueba de ${ }^{2}$ con el paquete estadístico JMP versión 7.0 (SAS Institute Inc). Para evaluar las celdas que contribuyen significativamente al valor de ${ }^{2}$ se hizo un análisis de residuales ajustados (Everitt, 1977).

Para probar la hipótesis de que las poblaciones con arreglos entremezclados de ramets presentan niveles más altos de diversidad genotípica, se compararon los valores del índice de diversidad de Simpson $(D)$ y la proporción de genotipos distinguibles $(G / N)$ entre las tres categorías de patrón de distribución clonal y entre las tres categorías de tipo de crecimiento clonal con una prueba de Kruskall-Wallis con el paquete estadístico JMP versión 7.0 (SAS Institute Inc).

\section{Resultados}

En la revisión se incluyeron 53 estudios que comprenden 122 poblaciones distribuidas en 44 especies de plantas clonales y en 27 familias (Apéndice). Las familias mejor representadas son Liliaceae y Rosaceae con cuatro especies cada una; Cactaceae, Cyperaceae, Ericaceae, Poaceae y Salicaceae con tres especies cada una y Zosteraceae con dos especies. El resto de las familias estuvieron representadas por una sola especie. Las especies que predominan son herbáceas perennes $(60 \%)$, y las plantas con hábito arbustivo y arbóreo se encuentran poco representadas (20\% en ambos casos).

La mayoría de las especies presentan crecimiento clonal ligado (70\%), dominando la propagación a través de rizomas (45\% de las especies). Las especies con crecimiento clonal ligado y no ligado representan el 14\% propagándose clonalmente por crecimiento ligado a través de rizomas y acodaduras, y por crecimiento no ligado a través de bulbilos, plantlets y desprendimiento de ramas; las especies sólo con crecimiento no ligado representan el 16\%, propagándose por bulbilos, semillas producidas por agamospermia, desprendimiento de ramas y gémulas (ver glosario para la definición de las estructuras implicadas en el crecimiento ligado y no ligado, Apéndice). Respecto a la distribución espacial, $50 \%$ del total de las especies presentan genets con ramets agregados, $30 \%$ presentan una combinación de arreglos agregados-entremezclados y $20 \%$ presentan arreglos entremezclados (Apéndice).

$\mathrm{Al}$ comparar las poblaciones con diferentes arreglos espaciales de genets y ramets [agregados (Ag), En (entremezcaldos) y $\mathrm{Ag}+\mathrm{En}$ (combinados)] encontramos que las poblaciones de especies con crecimiento ligado y no ligado y 
Cuadro 1. Número (observado y esperado) de poblaciones con diferentes tipos de crecimiento clonal de acuerdo con la clasificación de Tiffney y Niklas (1985) ( $\mathrm{Lg}=$ ligado, Lg+NI = ligado y no ligado, $\mathrm{Nl}=$ no ligado), que mostraron arreglos espaciales de los ramets de distintos genets: Ag = agregados, Ag+En = combinación de agregados y entremezclados, y En = entremezclados (figura 2). Los valores entre paréntesis indican el número de especies y familias (especies/familias) representadas en cada celda. Los valores de los residuales ajustados en negritas indican las celdas que contribuyen significativamente al valor de $\chi^{2}$, el signo indica mayor (sin signo) o menor número (-) de poblaciones de las esperadas por azar.

\begin{tabular}{|c|c|c|c|}
\hline Observados & Ligado & Ligado+No ligado & No ligado \\
\hline Agregado & $49(21 / 13)$ & $7(2 / 2)$ & $4(2 / 2)$ \\
\hline Agregado+entremezclado & $11(5 / 5)$ & $25(5 / 5)$ & $7(3 / 3)$ \\
\hline Entremezclado & $10(5 / 5)$ & $2(2 / 1)$ & $7(2 / 2)$ \\
\hline \multicolumn{4}{|l|}{ Esperados } \\
\hline & $\operatorname{Lg}$ & $\mathrm{Lg}+\mathrm{NI}$ & $\mathrm{Nl}$ \\
\hline Agregado & 34 & 17 & 9 \\
\hline Agregado+entremezclado & 25 & 12 & 6 \\
\hline Entremezclado & 11 & 5 & 3 \\
\hline \multicolumn{4}{|l|}{ Residuales ajustados } \\
\hline & $\operatorname{Lg}$ & $\mathrm{Lg}+\mathrm{NI}$ & $\mathrm{Nl}$ \\
\hline Agregado & 5.34 & -3.93 & -2.48 \\
\hline Agregado+entremezclado & -5.24 & 5.50 & 0.35 \\
\hline Entremezclado & -0.46 & -1.83 & 2.95 \\
\hline
\end{tabular}

de especies que sólo presentan crecimiento no ligado presentaron arreglos espaciales (AER) más entremezclados que las poblaciones de especies que sólo presentan crecimiento ligado ( ${ }^{2}=43.6, P<0.0001,4$ g.l.; Cuadro 1). Predominan los patrones de distribución (PDE) con ramets distribuidos en parches discretos sobre el área ocupada por la población (patrón de distribución espacial agregado, $46 \%$ de las poblaciones); estos parches son multiclonales (con dos o más genets) en la mayoría de los casos y el arreglo espacial de los ramets de distintos genets es agregado (19\% de las poblaciones, $\mathrm{PDE}_{\mathrm{AER}} 2_{\mathrm{Ag}}$, Figura 2), presenta una combinación agregado-entremezclado (10\% de las poblaciones, combinación $\mathrm{PDE}_{\mathrm{AER}} 2_{\mathrm{Ag}}-3_{\mathrm{En}}$, Figura 2) o es entremezclado solamente (3\% de las poblaciones, $\mathrm{PDE}_{\mathrm{AER}} 3_{\mathrm{En}}$, Figura 2). En otras poblaciones donde los ramets se distribuyen formando parches discretos el arreglo espacial de los ramets de distintos genets es agregado, pero los parches son multiclonales y monclonales (9\% de las poblaciones, combinación $\mathrm{PDE}_{\mathrm{AER}}$ $1_{\mathrm{Ag}}-\mathrm{Ag}_{\mathrm{Ag}}$, Figura 2) o sólo son monoclonales (5\% de las poblaciones, $\mathrm{PDE}_{\mathrm{AER}} 1_{\mathrm{Ag}}$, Figura 2).

Los patrones de distribución espacial con ramets distribuidos aleatoriamente sobre el área ocupada por la población (patrón de distribución espacial aleatorio) representaron el $44 \%$ de las poblaciones, predominando el arreglo espacial agregado-entremezclado de los ramets de distintos genets $\left(22 \%\right.$ de las poblaciones, combinación de $\mathrm{PDE}_{\mathrm{AER}}$ $4_{\mathrm{Ag}}-5_{\mathrm{En}}$, Figura 2), el arreglo agregado se presentó en $10 \%$ de las poblaciones ( $\mathrm{PDE}_{\mathrm{AER}} 4_{\mathrm{Ag}}$, Figura 2) y el arreglo entremezclado en $12 \%$ de las poblaciones $\left(\mathrm{PDE}_{\mathrm{AER}} 5_{\mathrm{En}}\right.$, Figura
2). El resto de las poblaciones (10\%) presentaron una combinación de patrones de distribución con ramets distribuidos aleatoriamente sobre una parte del área ocupada por la población y en otra formando parches discretos monoclonales o parches multiclonales con arreglos espaciales agregados y entremezclado de los ramets de distintos genets (combinación de $\mathrm{PDE}_{\mathrm{AER}} 1_{\mathrm{Ag}}-4_{\mathrm{Ag}}, 1_{\mathrm{Ag}}-5_{\mathrm{En}}, 2_{\mathrm{Ag}}-4_{\mathrm{Ag}}, 2_{\mathrm{Ag}}-5_{\mathrm{En}}$, Figura 2).

Las poblaciones de especies con una combinación de tipos de crecimiento clonal ligado y no ligado $(\mathrm{Lg}-\mathrm{Nl})$ presentaron valores más bajos de la proporción de genotipos distinguibles (promedio \pm E.E., $G / N=0.24 \pm 0.04 ; H=$ 6.93, $P=0.031,2$ g.l.) que las poblaciones de especies con crecimiento ligado (promedio \pm E.E., $G / N=0.38 \pm 0.03$ ) y con crecimiento no ligado (promedio \pm E.E., $G / N=0.39 \pm$ 0.06 ), pero las diferencias sólo fueron significativas con respecto a las poblaciones de especies con crecimiento ligado (Figura 3a). Respecto al arreglo espacial de los ramets de distintos genets, las poblaciones con una combinación de arreglos agregado y entremezclado (Ag-En) presentaron valores menores del índice de diversidad de Simpson (promedio \pm E.E., $D=0.57 \pm 0.05 ; H=13.2, P=0.0014,2$ g.l.) que las poblaciones con arreglos agregados (promedio \pm E.E., $D=0.80 \pm 0.04$ ) y con arreglos entremezclados (promedio \pm E.E., $D=0.76 \pm 0.06$; Figura 3). Estas diferencias sugieren que el reclutamiento sexual es más bajo en poblaciones de especies que presentan una combinación de formas de crecimiento clonal ligado-no ligado ( $\mathrm{Lg}-\mathrm{Nl}$ ) y una combinación de arreglos de los ramets de distintos genets agregado-entremezclado (Ag-En), sin embargo estas diferencias 

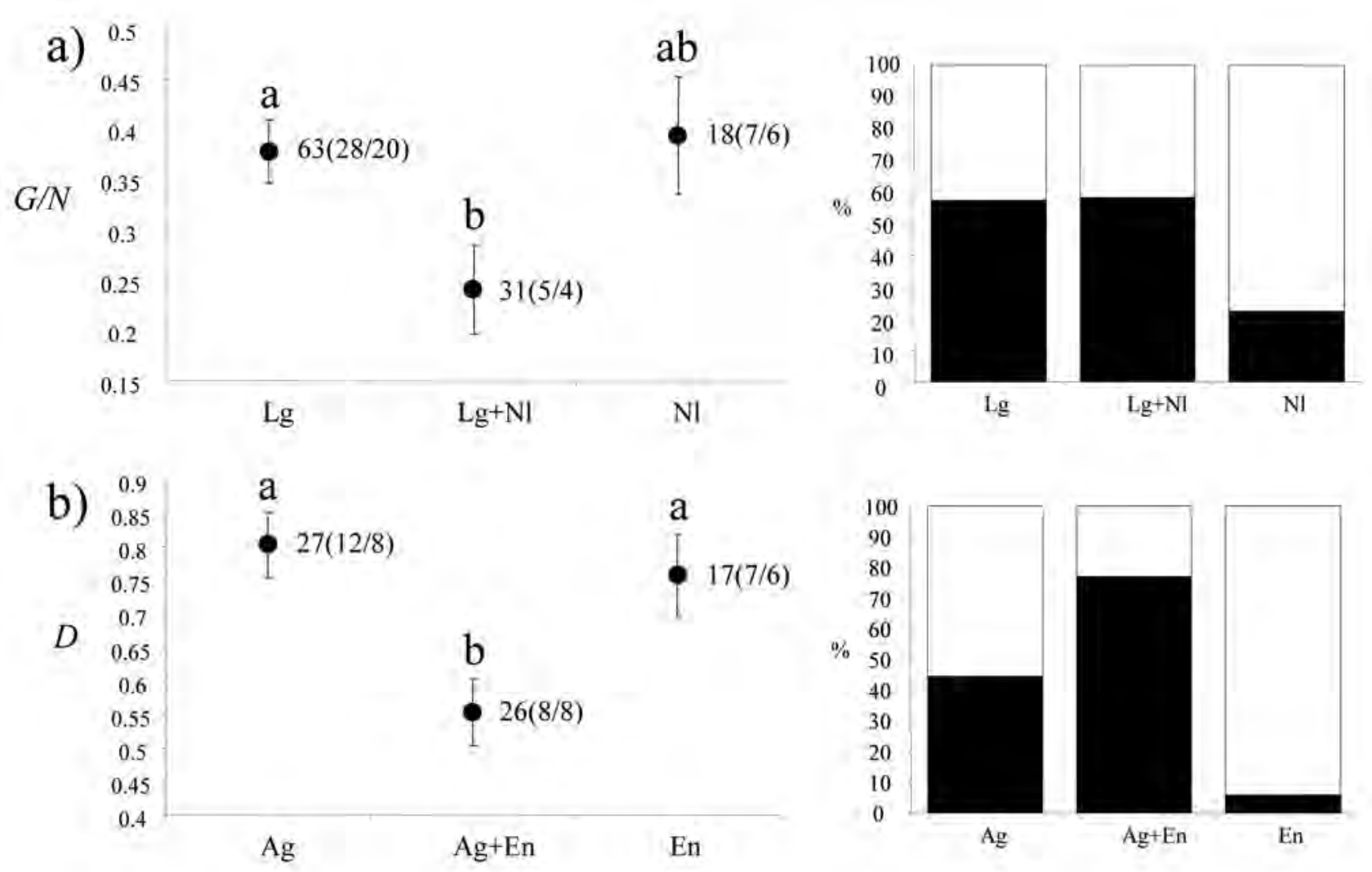

Figura 3. Comparación de la diversidad clonal (promedio \pm EE) entre poblaciones de especies con a) distinto tipo de crecimiento clonal: $\mathrm{Lg}=$ ligado, $\mathrm{Lg}+\mathrm{Nl}$ = con ambos tipos de crecimiento y $\mathrm{Nl}$ = no ligado y b) con distintos arreglos espaciales de los ramets de distintos genets: $\mathrm{Ag}=$ agregados, $\mathrm{Ag}+\mathrm{En}$ = combinación de agregados y entremezclados, y En = entremezclados (Figura 2). En cada grupo se indica el número de poblaciones (número de especies / número de familias) y los grupos que difieren significativamente se indican con una letra distinta arriba de la barra del promedio \pm E.E. de cada grupo. Las gráficas de barras del lado derecho muestran el porcentaje de poblaciones con polinización abiótica (en negro) y con polinización biótica (en blanco).

pueden estar asociadas con otros factores como el tipo de polinización, y no con el tipo de crecimiento y el arreglo espacial de los ramets de distintos genets. La mayoría de las poblaciones con tipo de crecimiento $\mathrm{Lg}-\mathrm{Nl}$ y arreglos de ramets de distintos genets Ag-En corresponden a especies con polinización abiótica (Figura 3).

\section{Discusión}

El efecto que tienen los modos de clonalidad en las plantas en la conformación de distintos arreglos espaciales de genets y ramets es un campo relativamente novedoso, que se ha explorado en los últimos 20 años y la mayoría de los estudios que hay sobre el tema se han desarrollado después de 1990. Widén et al. (1994) reportan que de 40 estudios que comprenden un total de 45 especies de plantas clonales, menos de la tercera parte proporcionan información sobre el arreglo espacial de los genets en las poblaciones y sólo en $12.5 \%$ se construyeron planos de distribución de todos los ramets, dentro de las parcelas o poblaciones, identificando los genotipos multilocus. En esta revisión, en el $98 \%$ de los estudios incluidos se identifican los genotipos multilocus mediante marcadores dominantes, codominantes o ambos siguiendo tres métodos de muestreo: en el 56\% de los estudios se utilizan parcelas, en el $24 \%$ transectos y en el $18 \%$ se muestrean todos o varios individuos de las poblaciones. El 2\% restante está representado por un estudio donde el patrón de distribución es descrito y la distribución de los ramets se sugiere por experimentos de cruzas compatibles entre ramets cercanos (en Scirpus maritimus, Charpentier et al., 2000).

A pesar de que en la actualidad se cuenta con más información sobre los patrones de distribución espacial en poblaciones de especies clonales, hacer comparaciones de dichos patrones considerando especies con distintas formas de crecimiento clonal para analizar las consecuencias reproductivas y genéticas del arreglo espacial de los genets y los ramets continúa siendo un reto. Algunos factores que influyen en este hecho son que la evaluación de la variación en los patrones de distribución espacial en plantas clonales 
enfrenta la dificultad que implica trabajar con la mayoría de los individuos en muchas poblaciones, principalmente a nivel molecular. Aunque en la actualidad las herramientas moleculares se han vuelto más accesibles, trabajar con un gran número de ejemplares sigue siendo difícil y costoso. Otros problemas son la falta de estimadores numéricos comparables para describir la distribución espacial en las poblaciones de diferentes especies y la variación en los métodos de muestreo. Por ejemplo, el muestreo mediante transectos colectando muestras de ramets cada determinada distancia (p. ej., Jonsson et al., 1996; Esselman et al., 1999) permite evaluar la capacidad de expansión longitudinal de los genets, pero no permite tener una descripción de la expansión radial y por lo tanto una evaluación más precisa del nivel de entremezcla de los ramets de distintos genets. En contraste, el muestreo de todos o de un gran número de individuos dentro de parcelas o en las áreas ocupadas por las poblaciones es otro método empleado en estudios con especies de plantas clonales (p. ej., Shimizu et al., 2002; Ruggieiro et al., 2005). Este tipo de muestreo puede ser un método más adecuado para analizar y comparar la estructura espacial clonal, porque permite detectar el arreglo radial de distintos genets. Sin embargo, los tamaños de muestra muy grandes pueden limitar la aplicación de este método, ya que en la mayoría de los casos es necesario emplear marcadores moleculares para identificar a los genets.

En particular, además de los problemas mencionados anteriormente es importante considerar las dificultades adicionales que detectamos en este estudio y que pueden afectar los patrones encontrados a través de nuestra aproximación. La mayoría de los estudios que analizan la distribución espacial de genets y ramets parecen estar sesgados hacia especies de herbáceas perennes con crecimiento clonal ligado (45\% de las especies), predominando las especies rizomatosas (43\% de las especies), mientras que las especies con crecimiento no ligado están poco representadas, impidiendo contar con tamaños de muestra equivalentes para hacer comparaciones más precisas. Por otro lado, la aproximación que decidimos emplear en este trabajo compara a las poblaciones más que a las especies, debido a que hemos detectado que hay una importante variación intraespecífica tanto en los patrones de distribución como en los patrones reproductivos. No obstante, esta variación se omite en algunos estudios o está mejor representada para algunas especies.

Los resultados obtenidos en este trabajo sugieren que las especies que se propagan por crecimento no ligado tienden a formar arreglos espaciales de los ramets de distintos genets más entremezclados como se ha planteado (Gabrielsen y Brochmann, 1998). No obstante, en especies con crecimiento ligado que además se propagan clonalmente por crecimiento no ligado, como el desprendimiento de ramas, el cual consideramos en este estudio como una forma de crecimiento no ligado en los análisis, debido a que los ramets originados por este medio se desarrollan hasta individuos com- pletos separados de la planta madre y a que su dispersión no depende del crecimiento horizontal de tallos y raíces (p.ej., Lophocereus schottii, Parker y Hamrick, 1992; Pteridium aquilinum, Parks y Werth, 1993; Cladium jamaicense, Ivey y Richards, 2001; Ferocactus robustus, Carrillo-Angeles et al., 2011), o que se propagan por la extensión de rizomas en especies con crecimiento en guerrilla (p. ej., Cymodocea nodosa, Ruggieiro et al., 2005 e Ilex leucoclada, Torimaru y Tomaru, 2005) también forman arreglos de genets entremezclados. Algunos factores externos que pueden influir en el arreglo entremezclado de genets son la dispersión por actividades humanas (Parks y Werth, 1993; Brzosko et al., 2002), el acarreo de propágulos vegetativos por animales y corrientes de agua (Parker y Hamrick, 1992; Brzosko et al., 2002), el reclutamiento de nuevos genets en áreas perturbadas donde permanecen fragmentos de clones que ocuparon grandes extensiones (Parks y Werth, 1993) y la dispersión de semillas producidas por autogamia que presentan genotipos multilocus idénticos (Keane et al., 1999; Brzosko et al., 2002).

Respecto a la hipótesis de que las poblaciones con arreglos espaciales entremezclados de los ramets de distintos genets presentan mayor entrecruza, medido indirectamente mediante la diversidad genotípica, nuestros resultados concuerdan parcialmente con el patrón esperado. El G/N es significativamente mayor en los arreglos de especies con producción de vástagos no ligados, en donde predominan los arreglos de genets entremezclados. Para probar esta hipótesis consideramos que si la entrecruza es más alta en poblaciones con arreglos entremezclados, habría mayores niveles de diversidad genotípica. Sin embargo, ésto no se refleja en el caso del índice de Simpson. Las diferencias en los índices de diversidad genotípica entre las poblaciones de especies que combinan el crecimiento clonal ligado-no ligado ( $\mathrm{Lg}$ $\mathrm{Nl})$ y el arreglo espacial agregado-entremezclado (Ag-En), respecto de las poblaciones que presentan uno u otro tipo de crecimiento y de arreglo espacial están asociadas con el tipo de polinización (biótica o abiótica), lo cual apoya el planteamiento de que la polinización biótica puede incrementar la eficiencia de la fertilización (Barret et al.,1993; Richards, 1997; Reusch, 2003). Los análisis para probar este planteamiento están fuera del alcance del presente estudio.

Además de los efectos sobre el reclutamiento sexual y la diversidad genotípica, los patrones de distribución espacial en especies clonales pueden afectar la diversidad y la estructura genética de las poblaciones. Por ejemplo, la formación de parches monoclonales en especies con entrecruza obligada, aunada al desplazamiento de genets por competencia, puede eliminar la capacidad de una población para reproducirse sexualmente en tiempos relativamente cortos (Honnay y Bossuyt, 2005). Adicionalmente, una reducción del reclutamiento sexual y una dispersión restringida de polen, semillas y propágulos vegetativos pueden generar estructura genética local en las poblaciones. Este segundo aspecto se 
evalúa en varios de los estudios revisados (47\%). La estructura genética local tanto a nivel de genets (indicando una dispersión restringida de polen, semillas o ambos) como de ramets (indicando dispersión restringida de propágulos vegetativos) se detectó en 46 poblaciones distribuidas en 16 especies; 28 poblaciones corresponden a cinco especies arbóreas que muestran estructura genética local a distancias menores de $50 \mathrm{~m}$ (Sequoia sempervirens, Douhovnikoff et al., 2004) y a distancias menores de $25 \mathrm{~m}$ (Eurya emarginata, Chung y Epperson, 2000; Cryptomeria japonica, Shimizu et al., 2002; Hirayama y Sakimoto, 2008; Populus tremula, Suvanto y Latva-Karjantvaa, 2005); tres poblaciones corresponden a dos especies arbustivas (Echinosophora koreensis, Chung et al., 2006; Stenocereus eruca, ClarkTapia et al., 2005) que presentan estructura genética local a distancias menores de $20 \mathrm{~m}$. El resto de las poblaciones corresponden a hierbas perennes que muestran estructura genética local desde distancias menores de $30 \mathrm{~m}$, pero más frecuentemente a distancias menores de $15 \mathrm{~m}$.

En promedio, los valores del índice de diversidad de Simpson $(D)$ y la proporción de genotipos distinguibles $(G / N)$ fueron altos en las poblaciones representadas en los estudios incluidos en la presente revisión y son similares a los reportados para 21 especies de plantas clonales $(D=$ 0.62 y $G / N=0.16$ ) por Ellstrand y Roose (1987). Se han planteado algunas hipótesis sobre los escenarios que pueden explicar altos niveles de diversidad genotípica: (i) las poblaciones pudieron ser fundadas por varios propágulos sexuales que se mantienen por clonalidad, (ii) el reclutamiento sexual fue más frecuente en el pasado, aunque en la actualidad la producción de descendientes sexuales no sea evidente (Ellstrand y Roose, 1987; Eriksson, 1989, 1993; Widén et al., 1994); (iii) los genotipos heterócigos se ven favorecidos selectivamente, manteniendo altos niveles de diversidad genética (Widén et al., 1994); (iv) los riesgos de mortalidad entre los ramets de un genet pueden ser distintos si tienen independencia física y fisiológica (Eriksson 1989, 1993, Pan y Price, 2002), lo cual puede reducir las probabilidades de supervivencia / extinción del genet; (v) en especies con ciclos de vida largos los eventos raros de reclutamiento sexual pueden ser suficientes para mantener niveles altos de diversidad genética (Eriksson 1989, 1993, Parker y Hamrick, 1992; Brzosko et al., 2002; Clark-Tapia et al., 2005); (vi) los genotipos pueden mantenerse a través de la supervivencia de algunos ramets el tiempo suficiente para que algunos genotipos se integren a la población de manera esporádica; (vii) la heterogeneidad ambiental puede generar condiciones de selección diversificante que permiten la coexistencia de varios genotipos (Widén et al., 1994). Otra posibilidad para el mantenimiento de diversidad clonal en poblaciones de plantas, que surge al considerar la variación de la distribución espacial de genets y ramets, es que la clonalidad puede mantener altos niveles de entrecruza e incrementar las probabilidades de reclutamiento sexual si los ramets se dispersan hacia vecindarios genéticamente variables, debido a la distribución entremezclada de genets.

Las explicaciones a los niveles relativamente altos de diversidad genotípica en las poblaciones de las especies incluidas en esta revisión comprenden los eventos esporádicos de reclutamiento sexual y el establecimiento inicial de varios genets. En algunos casos se sugiere un reclutamiento sexual frecuente a partir de los altos niveles de diversidad genotípica detectados con los marcadores moleculares (p. ej., Parks y Werth, 1993; Diggle et al., 1998; Misuki et al., 2010). No obstante, el reclutamiento sexual en poblaciones naturales sólo se reporta en Prunus ssiori, Rubus saxatilis (Eriksson y Bremer, 1993), Cypripedium calceolus (Brzosko et al., 2002) y Maianthemum dilatatum (Wilson et al., 2005b). Otros factores que se proponen para explicar la diversidad genotípica son que la integración fisiológica entre los ramets de un genet puede reducir el riesgo de que un genotipo en particular desaparezca, cuando las condiciones ambientales locales son adversas, contribuyendo a mantener la diversidad genética (Hartnett y Bazzaz, 1985), el incremento en la tasa de entrecruza, debido a la entremezcla de genets en especies con crecimiento en guerrilla (Ruggieiro et al., 2005) y en especies que se propagan clonalmente por desprendimiento de ramas y producción de bulbilos (Parker y Hamrick, 1992; Gabrielsen y Brochmann, 1998).

El estudio de los patrones de distribución espacial en especies clonales es importante para analizar las consecuencias reproductivas y genéticas del arreglo espacial de los genets y los ramets en las poblaciones naturales. Estas consecuencias pueden explorarse de una manera más precisa analizando la variación de la distribución de genets y ramets entre las poblaciones de una misma especie. Sin embargo, la evidencia de estos aspectos en organismos clonales aún es escasa y hace falta realizar estudios considerando diferentes tipos de crecimiento clonal. El análisis de los patrones de distribución espacial pueden ser muy útiles para evaluar otros aspectos del ciclo de vida de las especies clonales como el efecto de una variación persistente en la producción de propágulos clonales y sexuales entre hábitats, que puede afectar diferencialmente la dinámica de las poblaciones. Asimismo, es necesario analizar la dinámica temporal de los arreglos espaciales de los genets y ramets en las poblaciones para hacer inferencias adecuadas sobre las consecuencias ecológicas y evolutivas de la clonalidad.

\section{Agradecimientos}

Proyectos: IN IN205007 Efecto de la estructura clonal sobre la dinámica poblacional y la reproducción de una cactácea con ciclo de vida complejo, Opuntia microdasys.

Becas: doctorado Conacyt a I. Carrillo-Angeles y estancia sabática en New Mexico State University, otorgada por DGAPA-PASPA- Universidad Nacional Autónoma de México a M. Mandujano. Este trabajo es parte de la tesis de 
doctorado de Israel Gustavo Carrillo Angeles, Posgrado en Ciencias Biológicas de la UNAM. Agradecemos la revisión, sugerencias en el trabajo así como el apoyo de los miembros del comité tutoral, Doctores Teresa Valverde y Francisco Molina. Asimismo a la Dra. Ana Mendoza, Dr. Jordan Golubov y dos revisores anónimos por sus sugerencias al artículo.

\section{Literatura citada}

Aarssen L.W. 2008. Death without sex-the 'problem of the small' and selection for reproductive economy in flowering plants. Evolutionary Ecology 22:279-298.

Albert T., Raspé O. y Jacquemart A.L. 2003. Clonal structure in Vaccinium myrtillus L. revealed by RAPD and AFLP markers. International Journal of Plant Sciences 164:649-655.

Arens P., Grashof-Bokdam C.J., van der Sluis T. y Smulders M.J.M. 2005. Clonal diversity and genetic differentiation of Maianthemum bifolium among forest fragments of different age. Plant Ecology 179:169-180.

Arizaga S. y Ezcurra E. 2002. Propagation mechanisms in Agave macrovantha (Agavaceae), a tropical arid land succulent rosette. American Journal of Botany 89:632-641.

Barbour M.G., Burk J.H. y Pitts W.D. 1987. Terrestrial Plant Ecology. The Benjamin-Cummings Publishing Company, Menlo Park.

Barret S.C.H., Eckert C.G. y Husband B.C. 1993. Evolutionary processes in aquatic plant populations. Aquatic Botany 44:105-145.

Bell A.D. 1984. Dynamic morphology: A contribution to plant population ecology. En: Dirzo R. y Sarukhán J. Eds. Perspectives on Plant Population Ecology, pp. 49-65, Sinauer Associates Inc. Publisher, Sunderland.

Brzosko E., Wróblewska A. y Ratkiewicz M. 2002. Spatial genetic structure and clonal diversity of island populations of lady's slipper (Cyperidium calceolus) from the Biebrza National Park (northeast Poland). Molecular Ecology 11:2499-2509.

Bushakra J.M., Hodges S.A., Cooper J.B. y Kaska D.D. 1999. The extent of clonality and genetic diversity in the Santa Cruz Island ironwood, Lyonothamnus floribundus. Molecular Ecology 8:471-475.

Camacho F.J. y Liston A. 2001. Population structure and genetic diversity of Botrychium pumicola (Ophioglossaceae) based on inter-simple sequence repeats (ISSR). American Journal of Botany 88:1065-1070.

Carrillo-Angeles I.G., Mandujano M.C. y Golubov J. 2011. Influences of the genetic neighborhood on ramet reproductive success in a clonal desert cactus. Population Ecology 53:449458.

Caswell H. 1985. The evolutionary demography of clonal reproduction. En: Jackson J.B.C., Buss L.W. y Cook R.E. Eds. Population Biology and Evolution of Clonal Organisms, pp. 187224, Yale University Press, New Haven.

Charpentier A. 2002. Consequences of clonal growth for plant mating. Evolutionary Ecology 15:521-530.

Charpentier A., Grillas P. y Thompson J.D. 2000. The effects of population size limitation on fecundity in mosaic populations of the clonal macrophyte Scirpus maritimus (Cyperaceae). American Journal of Botany 87:502-507.

Chen J. y Bradshaw G.A. 1999. Forest structure in space: a case study of an old growth spruce-fir forest in Changbaishan Natural Reserve, PR China. Forest Ecology and Management 120:219-233.

Chung M.G. y Epperson B.K. 2000. Clonal and spatial genetic structure in Eurya emarginata (Theaceae). Heredity 84:170177.

Chung J.M., Lee B.C., Kim J.S., Park C., Chung M.Y. y Chung M.G. 2006. Fine-scale genetic structure among genetic individuals of the clone-forming monotypic genus Echinospora koreensis (Fabaceae). Annals of Botany 98:165-173.

Clark P.J. y Evans F.C. 1954. Distance to nearest neighbor as a measure of spatial relationships in populations. Ecology 35:445-453.

Clark-Tapia R., Alfonso-Corrado C., Eguiarte L.E. y Molina-Freaner F. 2005. Clonal diversity and distribution in Stenocereus eruca (Cactaceae), a narrow endemic cactus of the Sonoran Desert. American Journal of Botany 92:272-278.

Clark-Tapia R., Alfonso-Corrado C., Mandujano M.C. y MolinaFreaner F. 2006. Reproductive consequences of clonal growth in Stenocereus eruca, a rare clonal cactus of the Sonoran desert. Evolutionary Ecology 20:131-142.

Couteron P. y Kokou K. 1997. Woody vegetation spatial patterns in a semi-arid savanna of Burkina Faso, West Africa. Plant Ecology 132:211-227.

Damman H. y Cain M.L. 1998. Population growth and viability analyses of the clonal woodland herb, Asarum canadense. Journal of Ecology 86:13-26.

de Jong T.J., Waser N.M., Price M.V. y Ring R.M. 1992. Plant size, geitonogamy and seed set in Ipomopsis aggregata. Oecologia 89:310-315.

de Kroon H. y van Groenendael J. Eds. 1997. The Ecology and Evolution of Clonal Plants. Backhuys Publishers, Leiden.

de Kroon H. y Hutchings M.J. 1995. Morphological plasticity in clonal plants: the foraging concept reconsidered. Journal of Ecology 83:143-152.

Diggle P.K., Lower S. y Ranker T.A. 1998. Clonal diversity in alpine populations of Polygonum viviparum (Polygonaceae). International Journal of Plant Sciences 159:606-615.

Diggle P.K., Meixner M.A., Carroll A.B. y Aschwanden C.F. 2002. Barriers to sexual reproduction in Polygonum viviparum: A comparative developmental analysis of $P$. viviparum and $P$. bistortoides. Annals of Botany 89:145-156.

Douhovnikoff V. y Dood R.S. 2003. Intra-clonal variation and a similarity threshold for identification of clones: application to $\mathrm{Sa}$ lix exigua using AFLP molecular markers. Theoretical Applied Genetics 106:1307-1315.

Douhovnikoff V., Cheng A.M. y Dodd R.S. 2004. Incidence, size and spatial structure of clones in second-growth stands of coast redwood, Sequoia sempervirens (Cupressaceae). American Journal of Botany 91:1140-1146.

Douhovnikoff V., McBride J.R. y Dodd R.S. 2005. Salix exigua clonal growth and population dynamics in relation to disturbance regime variation. Ecology 86:446-452.

Eckert C.G. 2000. Contributions of autogamy and geitonogamy to self-fertilization in a mass-flowering, clonal plant. Ecology 81:532-542.

Eckert C.G. 2002. The loss of sex in clonal plants. Evolutionary Ecology 15:501-520.

Ellstrand N.C. y Roose M.L. 1987. Patterns of genotypic diversity in clonal plant species. American Journal of Botany 74:123-131. 
Eriksson O. 1989. Seedling dynamics and life histories in clonal plants. Oikos 55:231-238.

Eriksson O. 1993. Dynamics of genets in clonal plants. Trends in Ecology \& Evolution 8:313-316.

Eriksson O. y Bremer B. 1993. Genet dynamics of the clonal plant Rubus saxatilis. Journal of Ecology 81:533-542.

Esselman E.J., Jianqiang L., Crawford D.J., Winduss J.L. y Wolfe A.D. 1999. Clonal diversity in the rare Calamagrostis porteri ssp insperata (Poaceae): comparative results for allozymes and random amplified polymorphic DNA (RAPD) and intersimple sequence repeat (ISSR) markers. Molecular Ecology 8:443451.

Everitt B.S. 1977. The Analysis of Contingency Tables. Chapmann \& Hall, Bury St Edmunds.

Gabrielsen T.M. y Brochmann C. 1998. Sex after all: high levels of diversity detected in the arctic clonal plant Saxifraga cernua using RAPD markers. Molecular Ecology 7:1701-1708.

Garnier L.K.M., Durand J. y Dajoz I. 2002. Limited seed dispersal and microspatial population structure of an agamospermous grass of West African savannahs, Hyparrhenia diplandra (Poaceae). American Journal of Botany 89:1785-1791.

Gibson D.J. y Menges E.S. 1994. Population structure and spatial pattern in the dioecious shrub Ceratiola ericoides. Journal of Vegetation Sciences 5:337-346.

Gleason H.A. 1920. Some applications of the quadrat method. Bulletin of the Torrey Botanical Club 47:21-33.

Hämmerli A. y Reusch T.B.H. 2003a. Flexible mating: cross-pollination affects sex expression in a marine clonal plant. Journal of Evolutionary Biology 18:1096-1105.

Hämmerli A. y Reusch T.B.H. 2003b. Genetic neighbourhood of clone structures in eelgrass meadows quantified by spatial autocorrelation of microsatellite markers. Heredity 91:448-455.

Handel S.N. 1985. The intrusion of clonal growth patterns on plant breeding system. The American Naturalist 125:367-384.

Harper J. 1985. Modules, branches, and the capture of resources. En: Jackson J.B.C., Buss L.W. y Cook R.E. Eds. Population Biology and Evolution of Clonal Organisms, pp. 1-33, Yale University Press, New Haven.

Hartnett D.C. y Bazzaz F.A. 1985. The integration of neighbourhood effects by clonal genets in Solidago canadensis. The Journal of Ecology 73:415-427.

Hirayama K. y Sakimoto M. 2008. Clonal structure and diversity of Cryptomeria japonica along a slope in a cool-temperate, oldgrowth mixed forest in the snowy region of Japan. Canadian Journal of Forest Research 38:2804-2813.

Honnay O. y Bossuyt B. 2005. Prolonged clonal growth: escape route or route to extinction? Oikos 108:427-432.

Honnay O., Jacquemyn H., Roldán-Ruiz I. y Hermy M. 2006. Consequences of prolonged clonal growth on local and regional genetic structure and fruiting success of the forest perennial Maianthemum bifolium. Oikos 112:21-30.

Honnay O. y Jacquemyn H. 2008. A meta-analysis of the relation between mating system, growth form and genotypic diversity in clonal plant species. Evolutionary Ecology 22:299-312.

Ivey C.T. y Richards J.H. 2001. Genotypic diversity and clonal structure of everglades sawgrass, Cladium jamaicense (Cyperaceae). International Journal of Plant Sciences 162:1327-1335.

Jackson J.B.C., Buss L.W. y Cook R.E. Eds. 1985. Population Biology and Evolution of Clonal organisms. Yale University Press, New Haven.
Jacquemyn H., Brys R., Honnay O., Hermy M. y Roldán-Ruiz I. 2005. Local forest environment largely affects below-ground growth, clonal diversity and fine-scale spatial genetic structure in the temperate deciduous forest herb Paris quadrifolia. Molecular Ecology 14:4479-4488.

Jonsson B.O., Jónsdóttir I.S. y Crongberg N. 1996. Clonal diversity and allozyme variation in populations of the arctic sedge Carex bigelowii (Cyperaceae). Journal of Ecology 84:449-459.

Kays S. y Harper J.L. 1974. The regulation of plant and tiller density in a grass sward. Journal of Ecology 62:97-105.

Keane B., Pelikan S., Toth G.P., Smith M.K. y Rogstad S.H. 1999. Genetic diversity of Typha latifolia (Typhaceae) and the impact of pollutants examined with tamdem-repetitive DNA probes. American Journal of Botany 86:1226-1238.

Kjølner S., Såstad S.M. y Brochmann C. 2006. Clonality and recombination in the arctic plant Saxifraga cernua. Botanical Journal of the Linnean Society 152:209-217.

Klimeš L., Klimešová J., Hendriks R.J.J. y van Groenendael J.M. 1997. Clonal plant architecture: a comparative analysis of form and function. En: de Kroon H. y van Groenendael J. Eds. The Ecology and Evolution of Clonal Plants, pp. 1-29, Backhuys Publishers, Leiden.

Korpelainen H. 1994. Sex ratios and resource allocation among sexually reproducing plants of Rubus chamaemorus. Annals of Botany 74:627-632.

Korpelainen H., Antonious-Klemola K. y Werlemark G. 1999. Clonal structure of Rubus chamaemorus populations: comparison of different molecular methods. Plant Ecology 143:123-128.

Kreher S.A., Foré S.A. y Collins B.S. 2000. Genetic variation within and among patches of the clonal species, Vaccinium stamineum L. Molecular Ecology 9:1247-1252.

Kudoh H., Shibaike H., Takasu H., Whigham D.F. y Kawano S. 1999. Genet structure and determinants of clonal structure in a temperate deciduous woodland herb, Uvularia perfoliata. Journal of Ecology 87:244-257.

Liston A., Wilson B.L., Robinson W.A., Doescher P.S., Harris N.R. y Svejcar T. 2003. The relative importance of sexual reproduction versus clonal spread in an arid bunchgrass. Oecologia 137:216-225.

Liu J., Dong M., Miao S.L., Li Z.Y., Song M.H. y Wang R.Q. 2006. Invasive alien plants in China: role of clonality and geographical origin. Biological Invasions 8:1461-1470.

Mandujano M.C., Montaña C., Méndez I. y Golubov J. 1998. The relative contributions of sexual reproduction and clonal propagation in Opuntia rastrera from two habitats in the Chihuahuan desert. Journal of Ecology 86:911-921.

Mandujano M.C., Montaña C., Franco M., Golubov J. y FloresMartínez A. 2001. Integration of demographic annual variability in a clonal desert cactus. Ecology 82:344-359.

Mandujano-Sánchez M.C. 2007. La clonalidad y sus efectos en la biología de poblaciones. En: Eguiarte L.E., Souza V. y Aguirre X. Comp. Ecología Molecular, pp. 215-250, Secretaría de Medio Ambiente y Recursos Naturales-Instituto Nacional de Ecología-Universidad Nacional Autónoma de México-Comosión Nacional para el Conocimiento y Uso de la Biodiversidad, México, D.F.

Mayes S.G., McGinley M.A. y Werth C.R. 1998. Clonal population structure and genetic variation in sand-shinnery oak, Quercus havardii (Fagaceae). American Journal of Botany 85:1609-1617. McFadden C.S. 1991. A comparative demographic analysis of clonal 
reproduction in a temperate soft coral. Ecology 72:1849-1866.

Misuki I., Ishida K., Tani N. y Tsumura Y. 2010. Fine-scale spatial structure of genets and sexes in the dioecious plant Dioscorea japonica, which disperses by both bulbils and seeds. Evolutionary Ecology 24:1399-1415.

Mori Y., Nagamitsu T. y Kubo T. 2009. Clonal growth and its effects on male and female reproductive success in Prunus ssiori (Rosaceae). Population Ecology 51:175-186.

Nagamitsu T., Ogawa M., Ishida K. y Tanouchi H. 2004. Clonal diversity, genetic structure, and mode of recruitment in a Prunus ssiori population established after volcanic eruptions. Plant Ecology 174:1-10.

Okuda T., Kachi N., Yap S.K. y Manokaran N. 1997. Tree distribution pattern and fate of juveniles in a lowland tropical rain forest - implications for regeneration and maintenance of species diversity. Plant Ecology 131:155-171.

Pan J.J. y Price J.S. 2002. Fitness and evolution in clonal plants: the impact of clonal growth. Evolutionary Ecology 15:583-600.

Parker K.C. y Hamrick J.L. 1992. Genetic diversity and clonal structure in a columnar cactus, Lophocereus schottii. American Journal of Botany 79:86-96.

Parks C. y Werth C.R. 1993. A study of spatial features of clones in a population of bracken fern, Pteridium aquilinum (Dennstaedtiaceae). American Journal of Botany 80:537-544.

Peterson C.J. y Jones R.H. 1997. Clonality in woody plants: a review and comparison with clonal herbs. En: de Kroon H. y van Groenendael J. Eds. The Ecology and Evolution of Clonal Plants, pp. 263-289, Backhuys Publishers, Leiden.

Pfeiffer T., Klahr A., Heinrich A. y Schnittler M. 2011. Does sex make a difference? Genetic diversity and spatial genetic structure in two co-occurring species of Gagea (Liliaceae) with contrasting reproductive strategies. Plant Systematics and Evolution 292:189-201.

Pornon A. y Escaravage N. 1999. Genotypic structure in clonal Rhododendron ferrugineum L. (Ericaceae) populations: origin and manteinance. Plant Ecology 141:145-150.

Pornon A., Escaravage N., Thomas P. y Taberlet P. 2000. Dynamics of genotypic structure in clonal Rhododendron ferrugineum (Ericaceae) populations. Molecular Ecology 9:1099-1111.

Pornon A., Escaravage N., Till-Botraud I. y Doche B. 1997. Variation of reproductive traits in Rhododendron ferrugineum $\mathrm{L}$. (Ericaceae) populations along a successional gradient. Plant Ecology 130:1-11.

Price E.A.C. y Marshall C. 1999. Clonal plants and environmental heterogeneity. Plant Ecology 141:3-7.

Reisch C., Schurm S. y Poschlod P. 2007. Spatial genetic structure and clonal diversity in an alpine population of Salix herbacea (Salicaceae). Annals of Botany 99:647-651.

Reusch T.B.H. 2003. Floral neighbourhoods in the sea: how floral density, opportunity for outcrossing and population fragmentation affect seed set in Zostera marina. Journal of Ecology 91:610-615.

Richards A.J. 1997. Plant Breeding Systems. Chapman \& Hall,
Londres.

Rosseto M., Gross C.L., Jones R. y Hunter J. 2004. The impact of clonality on an endangered tree (Elaeocarpus williamsianus) in a fragment rain forest. Biological Conservation 117:33-39.

Ruggieiro M.V., Capone S., Pirozzi P., Reusch T.B.H. y Procaccini G. 2005. Mating system and clonal architecture: a comparative study in two marine angiosperms. Evolutionary Ecology 19:487-499.

Shimizu Y, Ando M. y Sakai F. 2002. Clonal structure of natural populations of Cryptomeria japonica growing at different positions on slopes, detected using RAPD markers. Biochemical Systematics and Ecology 30:733-748.

Shreve F. 1917. The establishment of desert perennials. Journal of Ecology 5:210-216.

Sipes S.D. y Wolf P.G. 1997. Clonal structure and patterns of allozyme diversity in the rare endemic Cycladenia humilis var. jonesii (Apocynaceae). American Journal of Botany 84:401-409.

Stehlik I. y Holderegger R. 2000. Spatial genetic structure and clonal diversity of Anemone nemorosa in late successional deciduous woodlands of Central Europe. Journal of Ecology 88:424-435.

Suvanto L.I. y Latva-Karjantvaa T.B. 2005. Clone identification and clonal structure of the European aspen (Populus tremula). Molecular Ecology 14:2851-2860.

Tiffney B.H. y Niklas K.J. 1985. Clonal growth in land plants: a paleobotanical perspective. En: Jackson J.B.C., Buss L.W. y Cook R.E. Eds. Population Biology and Evolution of Clonal Organisms, pp. 35-66, Yale University Press, New Haven.

Tooke F., Ordidge M., Chiurugwi T. y Battey N. 2005. Mechanisms and function of flower and inflorescence reversion. Journal of Experimental Botany 56:2587-2599.

Torimaru T. y Tomaru N. 2005. Fine-scale clonal structure and diversity within patches of a clone-forming dioecious shrub, Ilex leucoclada (Aquifoliaceae). Annals of Botany 95:295-304.

van Dijk P.J. y Bakx-Schotman J.M.T. 2004. Formation of unreduced megaspores (diplospory) in apomictic dandelions (Taraxacum officinale, s.l.) is controlled by a sex-specific dominant locus. Genetics 166:483-492.

van Groenendael J.M. y de Kroon H. 1990. Clonal Growth in Plants: Regulation and Function. SPB Academic Publishing, La Haya.

van Groenendael J.M., Klimeš L., Klimešová J. y Hendriks R.J.J. 1996. Comparative ecology of clonal plants. Philosophical Transactions of Royal Society of London 351:1331-1339.

Widén B., Cronberg N. y Widén M. 1994. Genotypic diversity, molecular markers and spatial distribution of genets in clonal plants, a literature survey. Folia Geobotanica y Phytotaxonomica 29:245-263.

Wilson A.S.G., van der Kamp B.J. y Ritland C. 2005a. Opportunities for geitonogamy in the clonal herb Maianthemum dilatatum. Canadian Journal of Botany 83:1082-1087.

Wilson A.S.G., van der Kamp B.J. y Ritland C. 2005b. Spatial genetic and clonal structure in Maianthemum dilatatum as defined by AFLP markers. Canadian Journal of Botany 83:1126-1132. 
Apéndice. Características de las especies y poblaciones: $\mathbf{S C R Z}=$ sistema de cruza, $\mathbf{P O L}=$ polinización, $\mathbf{P R O R}=$ estructuras o procesos que dan origen a los ramets, CCT-N = tipo de crecimiento clonal en la clasificación de Tiffney y Niklas (1985), POB/HL = número de población y/o hábitat local, $\mathbf{P D E}=$ clave del patrón de distribución espacial de acuerdo a la figura 2, AER = arreglo espacial de los ramets de distintos genets, $\boldsymbol{D}=$ índice de diversidad clonal de Simpson, $\boldsymbol{G} / \mathbf{N}=$ proporción de genotipos distinguibles, Métodos = métodos empleados para la evaluación de los parámetros. El significado de las abreviaturas empleadas en cada columna se muestra al final del cuadro.

\begin{tabular}{|c|c|c|c|c|c|c|c|c|c|c|c|c|c|}
\hline Especie (Familia) & Hábito & SCRZ & POL & PROR & CCT-N & $\begin{array}{c}\text { POB/ } \\
\mathrm{HL}\end{array}$ & $\begin{array}{c}\text { PDE } \\
\text { (fig. 2) }\end{array}$ & $\begin{array}{c}\text { AER } \\
\text { (fig. 2) }\end{array}$ & $D$ & $G / N$ & $\begin{array}{l}\text { Estructura } \\
\text { genética } \\
\text { local (EGL) }\end{array}$ & $\begin{array}{c}\text { Métodos } \\
\text { PDE, } D, G / N \\
\text { y EGL }\end{array}$ & Referencias \\
\hline $\begin{array}{l}\text { Anemone } \\
\text { nemorosa } \\
\text { (Ranunculaceae) }\end{array}$ & $\mathrm{Hp}$ & A & $\mathrm{Bt}$ & $\begin{array}{c}\mathrm{Rzm+} \\
\mathrm{drm}\end{array}$ & $\mathrm{Lg}+\mathrm{NI}$ & $\begin{array}{l}\text { ppm } \\
(20)\end{array}$ & $(4,5)$ & $\mathrm{Ag}+\mathrm{En}$ & $\mathrm{n} / \mathrm{e}$ & 0.95 & $\begin{array}{l}\operatorname{asp},<1 \mathrm{~m} \\
(\mathrm{rm})\end{array}$ & tra+ISZ+asp & $\begin{array}{l}\text { Stehlik y } \\
\text { Holderegger } \\
\text { 2000* }\end{array}$ \\
\hline $\begin{array}{l}\text { Botrychium } \\
\text { pumicola } \\
\text { (Ophioglossaceae) }\end{array}$ & $\mathrm{Hp}$ & A & $\mathrm{Ab}$ & gmas & $\mathrm{NI}$ & $\begin{array}{l}\text { p } 1 \\
\text { p } 2 \\
\text { p } 3\end{array}$ & $\begin{array}{l}(2,5) \\
(2,5)\end{array}$ & $\begin{array}{l}\mathrm{Ag}+\mathrm{En} \\
\mathrm{Ag}+\mathrm{En} \\
\mathrm{Ag}+\mathrm{En}\end{array}$ & $\begin{array}{c}0.14 \\
0.16 \\
0.1\end{array}$ & $\begin{array}{l}0.93 \\
0.9 \\
0.65\end{array}$ & $\begin{array}{c}\text { mt: no } \\
\text { detectada } \\
\text { mt: no } \\
\text { detectada } \\
\text { mt: no } \\
\text { detectada }\end{array}$ & prc + ISSR $+m t$ & $\begin{array}{c}\text { Camacho y } \\
\text { Liston, 2001* }\end{array}$ \\
\hline $\begin{array}{l}\text { Calamagrostis } \\
\text { porteri ssp. } \\
\text { insperata (Poaceae) }\end{array}$ & $\mathrm{Hp}$ & A & $\mathrm{Ab}$ & rzm & $\operatorname{Lg}$ & $\begin{array}{l}\text { p } 2 \\
\text { p } 3 \\
\text { p } 4\end{array}$ & $\begin{array}{l}2 \\
2 \\
2\end{array}$ & $\begin{array}{l}\mathrm{Ag} \\
\mathrm{Ag} \\
\mathrm{Ag}\end{array}$ & $\begin{array}{l}n / e \\
n / e \\
n / e\end{array}$ & $\begin{array}{c} \\
1 \\
0.82 \\
0.91\end{array}$ & $\begin{array}{l}\mathrm{n} / \mathrm{e} \\
\mathrm{n} / \mathrm{e} \\
\mathrm{n} / \mathrm{e}\end{array}$ & $\begin{array}{c}\text { desc+tra+ } \\
\text { ISRR+ } \\
\text { RAPD }\end{array}$ & $\begin{array}{c}\text { Esselman } \\
\text { et al., } \\
1999\end{array}$ \\
\hline $\begin{array}{l}\text { Carex bigelowii } \\
\text { (Cyperaceae) }\end{array}$ & $\mathrm{Hp}$ & $?$ & $\mathrm{Ab}$ & rzm & $\operatorname{Lg}$ & $\begin{array}{l}\text { p } 1 \\
\text { p } 2 \\
\text { p } 3\end{array}$ & $\begin{array}{l}4 \\
4 \\
4\end{array}$ & $\begin{array}{l}\mathrm{Ag} \\
\mathrm{Ag} \\
\mathrm{Ag}\end{array}$ & $\begin{array}{l}0.98 \\
0.98 \\
0.98\end{array}$ & $\begin{array}{l}0.52 \\
0.63 \\
0.48\end{array}$ & $\begin{array}{l}\text { n/e } \\
\text { n/e } \\
\text { n/e }\end{array}$ & tra+ISZ & $\begin{array}{c}\text { Jonsson } \\
\text { et al., 1996* }\end{array}$ \\
\hline $\begin{array}{l}\text { Cladium jamaicense } \\
\text { (Cyperaceae) }\end{array}$ & $\mathrm{Hp}$ & $?$ & $\mathrm{Ab}$ & $\begin{array}{c}\text { rzm+ } \\
\text { pnt }\end{array}$ & $\mathrm{Lg}+\mathrm{Nl}$ & $\begin{array}{l}p 1 \\
\text { p } 2 \\
\text { p } 3 \\
\text { p } 4 \\
\text { p } 5 \\
\text { p } 6 \\
\text { p } 7 \\
\text { p } 8 \\
\text { p } 9 \\
\text { p } 10 \\
\text { p } 11 \\
\text { p } 12 \\
\text { p } 13 \\
\text { p } 14\end{array}$ & $\begin{array}{l}(4,5) \\
(4,5) \\
(4,5) \\
(4,5) \\
(4,5) \\
1 \\
(4,5) \\
(4,5) \\
(4,5) \\
(4,5) \\
(4,5) \\
(4,5) \\
(4,5) \\
(4,5)\end{array}$ & $\begin{array}{c}A g+E n \\
A g+E n \\
A g+E n \\
A g+E n \\
A g+E n \\
A g \\
A g+E n \\
A g+E n \\
A g+E n \\
A g+E n \\
A g+E n \\
A g+E n \\
A g+E n \\
A g+E n\end{array}$ & $\begin{array}{c}0.236 \\
0.269 \\
0.271 \\
0.682 \\
0.159 \\
0 \\
0.538 \\
0.212 \\
0.771 \\
0.698 \\
0.814 \\
0.682 \\
0.673 \\
0.444\end{array}$ & $\begin{array}{l}0.044 \\
0.044 \\
0.125 \\
0.231 \\
0.043 \\
0.021 \\
0.146 \\
0.068 \\
0.106 \\
0.143 \\
0.261 \\
0.114 \\
0.13 \\
0.043\end{array}$ & $\begin{array}{c}\text { mpic: no } \\
\text { detectada } \\
\text { mpic: no } \\
\text { detectada } \\
\text { mpic: no } \\
\text { detectada } \\
\text { mpic: no } \\
\text { detectada } \\
\text { mpic: no } \\
\text { detectada } \\
\text { n/a } \\
\text { mpic: no } \\
\text { detectada } \\
\text { mpic: no } \\
\text { detectada } \\
\text { mpic: no } \\
\text { detectada } \\
\text { mpic: no } \\
\text { detectada } \\
\text { mpic: no } \\
\text { detectada } \\
\text { mpic: no } \\
\text { detectada } \\
\text { mpic: no } \\
\text { detectada } \\
\text { mpic: no } \\
\text { detectada }\end{array}$ & $\begin{array}{l}\text { tra+lSZ+ } \\
\text { mpic }\end{array}$ & $\begin{array}{l}\text { Ivey y Richards, } \\
\text { 2001* }\end{array}$ \\
\hline
\end{tabular}


Apéndice. Continuación

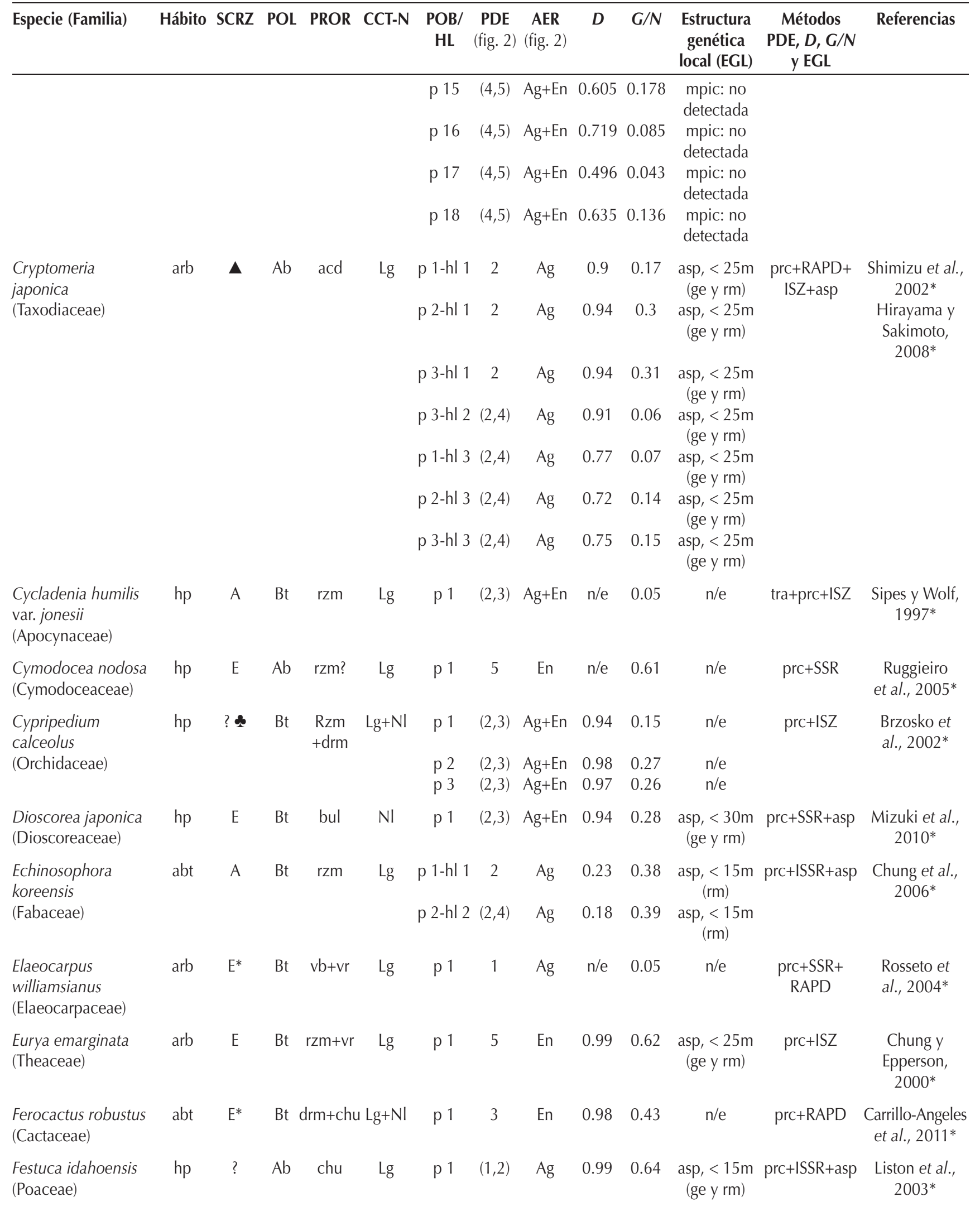


Apéndice. Continuación

\begin{tabular}{|c|c|c|c|c|c|c|c|c|c|c|c|c|c|}
\hline Especie (Familia) & Hábito & SCRZ & POL & PROR & CCT-N & $\begin{array}{c}\mathrm{POB} / \\
\mathrm{HL}\end{array}$ & $\begin{array}{l}\text { PDE } \\
\text { (fig. 2) }\end{array}$ & $\begin{array}{c}\text { AER } \\
\text { (fig. 2) }\end{array}$ & $D$ & $G / N$ & $\begin{array}{l}\text { Estructura } \\
\text { genética } \\
\text { local (EGL) }\end{array}$ & $\begin{array}{c}\text { Métodos } \\
\text { PDE, } D, G / N \\
\text { y EGL } \\
\end{array}$ & Referencias \\
\hline \multirow[t]{3}{*}{$\begin{array}{l}\text { Gagea lutea } \\
\text { (Liliaceae) }\end{array}$} & \multirow[t]{3}{*}{ hg } & \multirow[t]{3}{*}{$?$} & \multirow[t]{3}{*}{$\mathrm{Bt}$} & \multirow[t]{3}{*}{ buls } & \multirow[t]{3}{*}{$\mathrm{Nl}$} & p 1 & $(2,3)$ & $\mathrm{Ag}+\mathrm{En}$ & $\mathrm{n} / \mathrm{e}$ & 0.51 & \multirow{3}{*}{\multicolumn{2}{|c|}{$\begin{array}{l}\text { asp, }<8 \mathrm{~m}, 16 \text { tra+AFLP+asp } \\
\mathrm{a}<24 \mathrm{~m}(\mathrm{rm}) \\
\mathrm{asp},<8 \mathrm{~m}, 16 \\
\mathrm{a}<24 \mathrm{~m}(\mathrm{rm}) \\
\mathrm{asp},<8 \mathrm{~m}, 16 \\
\mathrm{a}<24 \mathrm{~m}(\mathrm{rm})\end{array}$}} & \multirow[t]{3}{*}{$\begin{array}{l}\text { Pfeiffer et al., } \\
2011^{*}\end{array}$} \\
\hline & & & & & & p 2 & $(2,3)$ & $\mathrm{Ag}+\mathrm{En}$ & $\mathrm{n} / \mathrm{e}$ & 0.57 & & & \\
\hline & & & & & & p 3 & $(2,3)$ & $\mathrm{Ag}+\mathrm{En}$ & $\mathrm{n} / \mathrm{e}$ & 0.61 & & & \\
\hline \multirow{3}{*}{$\begin{array}{l}\text { Gagea spathacea } \\
\text { (Liliaceae) }\end{array}$} & \multirow[t]{3}{*}{ hg } & \multirow[t]{3}{*}{$?$} & \multirow[t]{3}{*}{$\mathrm{Bt}$} & \multirow[t]{3}{*}{ buls } & \multirow[t]{3}{*}{$\mathrm{NI}$} & p 1 & 1 & $\mathrm{Ag}$ & $\mathrm{n} / \mathrm{e}$ & 0.001 & $\mathrm{n} / \mathrm{a}$ & \multirow[t]{3}{*}{ tra+AFLP } & \multirow{3}{*}{$\begin{array}{r}\text { Pfeiffer et al., } \\
2011^{*}\end{array}$} \\
\hline & & & & & & p 2 & 1 & $\mathrm{Ag}$ & $\mathrm{n} / \mathrm{e}$ & 0.001 & $\mathrm{n} / \mathrm{a}$ & & \\
\hline & & & & & & p 3 & 1 & $\mathrm{Ag}$ & $\mathrm{n} / \mathrm{e}$ & 0.001 & $\mathrm{n} / \mathrm{a}$ & & \\
\hline $\begin{array}{l}\text { Hyparrhenia } \\
\text { diplandra (Poaceae) }\end{array}$ & hp & $?$ & $\mathrm{Ab}$ & agp & $\mathrm{NI}$ & p 1 & 5 & En & 0.85 & 0.07 & $\begin{array}{l}\text { asp, }<6 \mathrm{~m} \\
\quad(\mathrm{rm})\end{array}$ & $\begin{array}{c}\text { prc+lSZ } \\
+ \text { CLO+asp }\end{array}$ & $\begin{array}{c}\text { Garnier et al., } \\
2002^{*}\end{array}$ \\
\hline $\begin{array}{l}\text { Ilex leucoclada } \\
\text { (Aquifoliaceae) }\end{array}$ & abt & E & $\mathrm{Bt}$ & $\operatorname{acd} \mathbf{\Lambda}$ & $\operatorname{Lg}$ & p 1 & $(2,3)$ & $\mathrm{Ag}+\mathrm{En}$ & 0.67 & 0.09 & $\mathrm{n} / \mathrm{e}$ & prc+RAPD & $\begin{array}{c}\text { Torimaru y } \\
\text { Tomaru, 2005* }\end{array}$ \\
\hline \multirow[t]{8}{*}{$\begin{array}{l}\text { Lophocereus schottii } \\
\text { (Cactaceae) }\end{array}$} & \multirow[t]{8}{*}{ arb } & \multirow[t]{8}{*}{ A } & \multirow[t]{8}{*}{$\mathrm{Bt}$} & \multirow[t]{8}{*}{$\begin{array}{l}\mathrm{drm} \\
+\mathrm{acd}\end{array}$} & \multirow[t]{8}{*}{$\mathrm{Lg}+\mathrm{NI}$} & p 1-hl 1 & 5 & En & 0.95 & 0.64 & $\begin{array}{c}\text { SG: 0-10 } \\
<10-50>\text { más } \\
\text { de } 50 m \text { (ge) }\end{array}$ & \multirow{8}{*}{ prc+ISZ+SG } & \multirow[t]{8}{*}{$\begin{array}{l}\text { Parker y } \\
\text { Hamrick, } \\
1992^{*}\end{array}$} \\
\hline & & & & & & p 2-hl 2 & 2 & $\mathrm{Ag}$ & 0.95 & 0.52 & $\begin{array}{l}\text { SG: } 0-10 \\
<10-50>\text { más } \\
\text { de } 50 \mathrm{~m} \text { (ge) }\end{array}$ & & \\
\hline & & & & & & p 3-hl 3 & 2 & $\mathrm{Ag}$ & 0.93 & 0.65 & $\begin{array}{c}\text { SG: } 0-10 \\
<10-50>\text { más } \\
\text { de } 50 m \text { (ge) }\end{array}$ & & \\
\hline & & & & & & p 4-hl 1 & $(1,2)$ & $\mathrm{Ag}$ & 0.94 & 0.24 & $\begin{array}{l}\text { SG: } 0-10> \\
\text { 10-50>más } \\
\text { de } 50 \mathrm{~m} \text { (ge) }\end{array}$ & & \\
\hline & & & & & & p 5-hl 4 & $(1,2)$ & $\mathrm{Ag}$ & 0.92 & 0.41 & $\begin{array}{l}\text { SG: } 0-10> \\
10-50<\text { más } \\
\text { de } 50 m \text { (ge) }\end{array}$ & & \\
\hline & & & & & & p 6-hl 4 & $(1,2)$ & $\mathrm{Ag}$ & 0.87 & 0.45 & $\begin{array}{c}\text { SG: } 0-10> \\
10-50<\text { más } \\
\text { de } 50 m \text { (ge) }\end{array}$ & & \\
\hline & & & & & & p 7-hl 2 & $(1,2)$ & $\mathrm{Ag}$ & 0.8 & 0.18 & $\begin{array}{l}\text { SG: } 0-10> \\
\text { 10-50>más } \\
\text { de } 50 \mathrm{~m} \text { (ge) }\end{array}$ & & \\
\hline & & & & & & p 8-hl 5 & $(1,5)$ & $\mathrm{Ag}+\mathrm{En}$ & 0.59 & 0.36 & $\begin{array}{l}\text { SG: } 10-50= \\
\text { más de } 50 \mathrm{~m} \\
\quad(\text { ge })\end{array}$ & & \\
\hline $\begin{array}{l}\text { Lyonothamnus } \\
\text { floribundus } \\
\text { (Rosaceae) }\end{array}$ & arb & $?$ & $\mathrm{Bt}$ & $\mathrm{vr}$ & $\operatorname{Lg}$ & p 1 & $(1,2)$ & $\mathrm{Ag}$ & $\mathrm{n} / \mathrm{e}$ & 0.036 & $\mathrm{n} / \mathrm{e}$ & prc+RAPD & $\begin{array}{c}\text { Bushakra et al., } \\
\text { 1999* }\end{array}$ \\
\hline $\begin{array}{l}\text { Maianthemum } \\
\text { bifolium } \\
\text { (Convalliariaceae) }\end{array}$ & hp & $E^{*}$ & $\mathrm{Bt}$ & $\mathrm{rzm}$ & $\operatorname{Lg}$ & p 1 & $(1,2)$ & $\mathrm{Ag}$ & 0.88 & 0.7 & $\begin{array}{l}\text { asp, }<50 \mathrm{~m} \\
\text { (ge) \# }\end{array}$ & $\begin{array}{l}\text { trans+AFLP } \\
\quad+\text { asp }\end{array}$ & $\begin{array}{l}\text { Arens et al., } \\
2005^{*} \\
\text { Honnay et al. } \\
2006\end{array}$ \\
\hline $\begin{array}{l}\text { Maianthemum } \\
\text { dilatatum } \\
\text { (Convalliariaceae) }\end{array}$ & hp & EAs & $\mathrm{Bt}$ & $\operatorname{rzm} \mathbf{\Delta}$ & $\operatorname{Lg}$ & p 1 & $(1,2)$ & $\mathrm{Ag}$ & $\mathrm{n} / \mathrm{e}$ & 0.64 & $\begin{array}{l}\text { asp, }<45 \mathrm{~m} \\
\text { (ge y rm) }\end{array}$ & $\begin{array}{l}\text { prc }+ \text { AFLP } \\
\quad+\text { asp }\end{array}$ & $\begin{array}{l}\text { Wilson et al., } \\
2005 a^{*} \\
\text { Wilson et al., } \\
2005 b^{*}\end{array}$ \\
\hline $\begin{array}{l}\text { Paris quadrifolia } \\
\text { (Trilliaceae) }\end{array}$ & hp & A & $\mathrm{Bt}$ & $\mathrm{rzm}$ & $\operatorname{Lg}$ & p 1-hl 1 & 3 & En & 0.87 & 0.27 & $\begin{array}{l}\text { asp, }<5 \mathrm{~m} \\
(\text { ge y rm) }\end{array}$ & $\begin{array}{l}\text { prc }+ \text { AFLP } \\
\quad+\text { asp }\end{array}$ & $\begin{array}{c}\text { Jacquemyn et } \\
\text { al., 2005* }\end{array}$ \\
\hline
\end{tabular}


Apéndice. Continuación

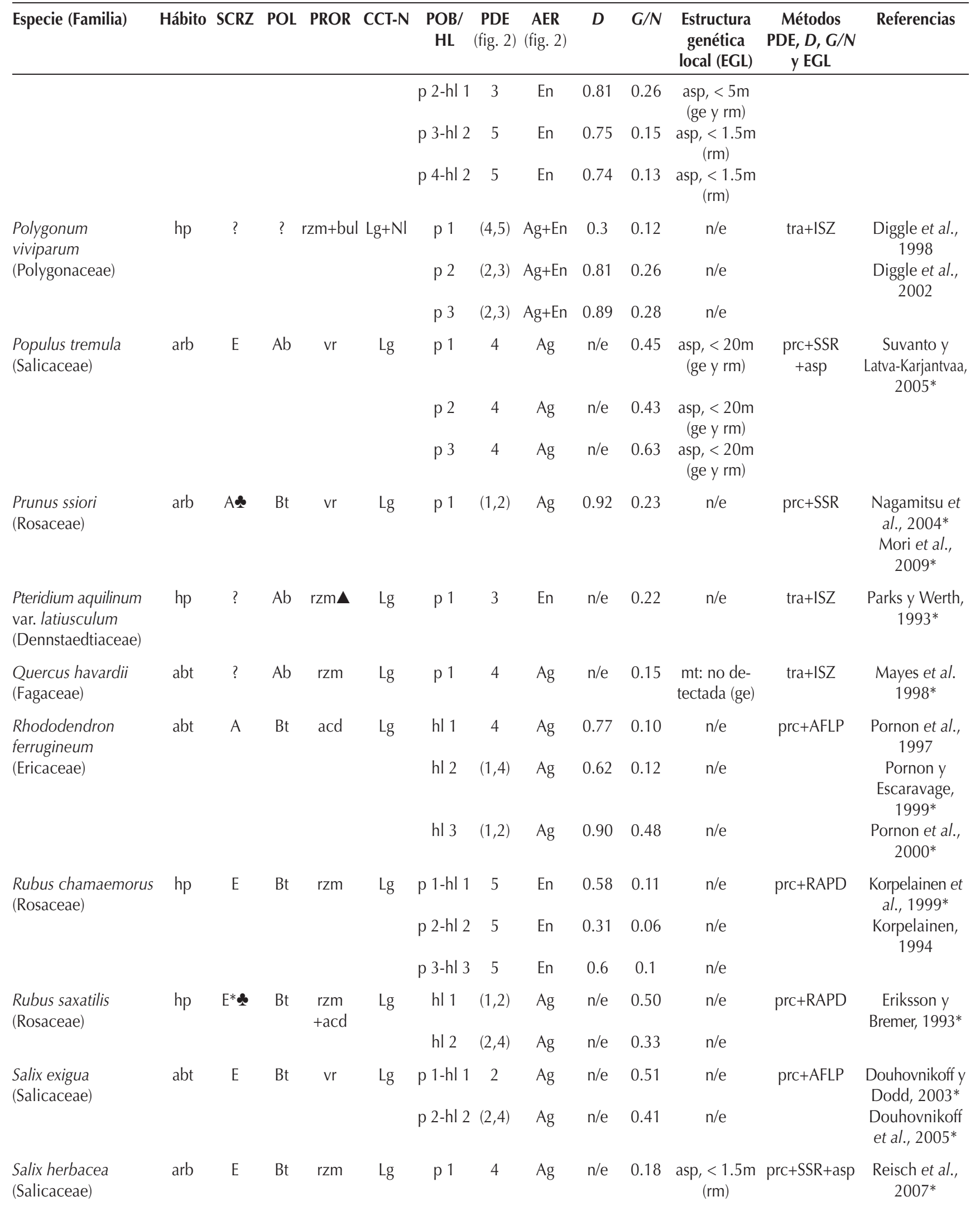


Apéndice. Continuación

\begin{tabular}{|c|c|c|c|c|c|c|c|c|c|c|c|c|c|}
\hline Especie (Familia) & Hábito & SCRZ & POL & PROR & CCT-N & $\begin{array}{c}\mathrm{POB} / \\
\mathrm{HL}\end{array}$ & $\begin{array}{c}\text { PDE } \\
\text { (fig. 2) }\end{array}$ & $\begin{array}{c}\text { AER } \\
\text { (fig. 2) }\end{array}$ & $D$ & $G / N$ & $\begin{array}{l}\text { Estructura } \\
\text { genética } \\
\text { local (EGL) }\end{array}$ & $\begin{array}{c}\text { Métodos } \\
\text { PDE, } D, G / N \\
\text { y EGL }\end{array}$ & Referencias \\
\hline \multirow[t]{6}{*}{$\begin{array}{l}\text { Saxifraga cernua } \\
\text { (Saxifragaceae) }\end{array}$} & \multirow[t]{6}{*}{ hp } & \multirow[t]{6}{*}{$E^{*}$} & \multirow[t]{6}{*}{$\mathrm{Bt}$} & \multirow[t]{6}{*}{ bul } & \multirow[t]{6}{*}{$\mathrm{Nl}$} & p 1 & 5 & En & 0.35 & 0.13 & $\begin{array}{c}\text { mt: no } \\
\text { detectada }\end{array}$ & \multirow[t]{6}{*}{$\begin{array}{l}\text { prc+RAPD } \\
+m t+a s p\end{array}$} & \multirow{6}{*}{$\begin{array}{l}\text { Gabrielsen y } \\
\text { Brochmann, } \\
1998^{*} \\
\text { KjØIner et al., } \\
\text { 2006* }\end{array}$} \\
\hline & & & & & & p 2 & 5 & En & 0.68 & 0.19 & $\begin{array}{c}\text { mt: no } \\
\text { detectada }\end{array}$ & & \\
\hline & & & & & & p 3 & 5 & En & 0.7 & 0.25 & $\begin{array}{l}\text { asp: no } \\
\text { detectada }\end{array}$ & & \\
\hline & & & & & & p 4 & 5 & En & 0.9 & 0.4 & $\begin{array}{c}\text { asp: no } \\
\text { detectada }\end{array}$ & & \\
\hline & & & & & & p 5 & 5 & En & 0.93 & 0.43 & $\begin{array}{l}\text { asp: no } \\
\text { detectada }\end{array}$ & & \\
\hline & & & & & & p 6 & 5 & En & 0.89 & 0.34 & $\begin{array}{c}\text { asp: no } \\
\text { detectada }\end{array}$ & & \\
\hline \multirow[t]{3}{*}{$\begin{array}{l}\text { Scirpus maritimus } \\
\text { (Cyperaceae) }\end{array}$} & \multirow[t]{3}{*}{ hp } & \multirow[t]{12}{*}{$\mathrm{E}^{*}$} & \multirow[t]{3}{*}{$\mathrm{Ab}$} & \multirow[t]{3}{*}{$\mathrm{rzm}$} & \multirow[t]{3}{*}{$\operatorname{Lg}$} & p 1 & 2 & $\mathrm{Ag}$ & $\mathrm{n} / \mathrm{e}$ & $\mathrm{n} / \mathrm{e}$ & $\mathrm{n} / \mathrm{e}$ & \multirow[t]{3}{*}{ desc } & \multirow[t]{3}{*}{$\begin{array}{c}\text { Charpentier et } \\
\text { al., } 2000\end{array}$} \\
\hline & & & & & & p 2 & 4 & $\mathrm{Ag}$ & $\mathrm{n} / \mathrm{e}$ & $\mathrm{n} / \mathrm{e}$ & $\mathrm{n} / \mathrm{e}$ & & \\
\hline & & & & & & p 3 & 4 & $\mathrm{Ag}$ & $\mathrm{n} / \mathrm{e}$ & $\mathrm{n} / \mathrm{e}$ & $\mathrm{n} / \mathrm{e}$ & & \\
\hline \multirow[t]{9}{*}{$\begin{array}{l}\text { Sequoia sempervirens } \\
\text { (Taxodiaceae) }\end{array}$} & \multirow[t]{9}{*}{ arb } & & \multirow[t]{9}{*}{$\mathrm{Ab}$} & \multirow[t]{9}{*}{$\mathrm{vb}$} & \multirow[t]{9}{*}{$\operatorname{Lg}$} & p 1-hl 1 & 2 & $\mathrm{Ag}$ & $\mathrm{n} / \mathrm{e}$ & 0.24 & $\begin{array}{l}\mathrm{mt}:<40 \mathrm{~m} \\
\quad(\mathrm{rm})\end{array}$ & \multirow[t]{9}{*}{$\operatorname{prc}+\mathrm{AFLP}+\mathrm{mt}$} & \multirow[t]{9}{*}{$\begin{array}{l}\text { Douhovnikoff } \\
\text { et al., 2004* }\end{array}$} \\
\hline & & & & & & p 2-hl 1 & 2 & $\mathrm{Ag}$ & $\mathrm{n} / \mathrm{e}$ & 0.38 & $\begin{array}{c}\mathrm{mt}:<40 \mathrm{~m} \\
(\mathrm{rm})\end{array}$ & & \\
\hline & & & & & & p 3-hl 1 & 2 & $\mathrm{Ag}$ & $\mathrm{n} / \mathrm{e}$ & 0.5 & $\begin{array}{c}\mathrm{mt}:<40 \mathrm{~m} \\
(\mathrm{rm})\end{array}$ & & \\
\hline & & & & & & p 4-hl 2 & 2 & $\mathrm{Ag}$ & $\mathrm{n} / \mathrm{e}$ & 0.6 & $\begin{array}{c}\mathrm{mt}:<40 \mathrm{~m} \\
(\mathrm{rm})\end{array}$ & & \\
\hline & & & & & & p 5-hl 2 & 2 & $\mathrm{Ag}$ & $\mathrm{n} / \mathrm{e}$ & 0.35 & $\begin{array}{c}\mathrm{mt}:<40 \mathrm{~m} \\
(\mathrm{rm})\end{array}$ & & \\
\hline & & & & & & p 6-hl 2 & 2 & $\mathrm{Ag}$ & $\mathrm{n} / \mathrm{e}$ & 0.6 & $\begin{array}{c}\mathrm{mt}:<40 \mathrm{~m} \\
(\mathrm{rm})\end{array}$ & & \\
\hline & & & & & & p 7-hl 3 & 2 & $\mathrm{Ag}$ & $\mathrm{n} / \mathrm{e}$ & 0.26 & $\begin{array}{c}\mathrm{mt}:<40 \mathrm{~m} \\
(\mathrm{rm})\end{array}$ & & \\
\hline & & & & & & p 8-hl 3 & 2 & $\mathrm{Ag}$ & $n / e$ & 0.19 & $\begin{array}{c}\mathrm{mt}:<40 \mathrm{~m} \\
(\mathrm{rm})\end{array}$ & & \\
\hline & & & & & & p 9-hl 3 & 2 & $\mathrm{Ag}$ & $\mathrm{n} / \mathrm{e}$ & 0.54 & $\begin{array}{c}\mathrm{mt}:<40 \mathrm{~m} \\
\quad(\mathrm{rm})\end{array}$ & & \\
\hline $\begin{array}{l}\text { Stenocereus eruca } \\
\text { (Cactaceae) }\end{array}$ & abt & $E^{*}$ & $\mathrm{Bt}$ & drm & $\mathrm{NI}$ & p 1 & 2 & $\mathrm{Ag}$ & 0.99 & 0.83 & $\begin{array}{l}\text { asp, }<20 \text { m } \\
\text { (ge y rm) }\end{array}$ & prc+ISSR+asp & $\begin{array}{c}\text { Clark-Tapia et } \\
\text { al., 2005* } \\
\text { Clark-Tapia et } \\
\text { al., } 2006\end{array}$ \\
\hline \multirow[t]{5}{*}{$\begin{array}{l}\text { Typha latifolia } \\
\text { (Thyphaceae) }\end{array}$} & \multirow[t]{5}{*}{$\mathrm{hp}$} & \multirow[t]{5}{*}{ A } & \multirow[t]{5}{*}{$\mathrm{Ab}$} & \multirow[t]{5}{*}{ rzm } & $\operatorname{Lg}$ & p 1 & $(4,5)$ & $\mathrm{Ag}+\mathrm{En}$ & $\mathrm{n} / \mathrm{e}$ & 0.77 & $\mathrm{n} / \mathrm{e}$ & tra+VNTR & $\begin{array}{c}\text { Keane et al., } \\
\text { 1999* }\end{array}$ \\
\hline & & & & & & p 2 & $(4,5)$ & $\mathrm{Ag}+\mathrm{En}$ & $\mathrm{n} / \mathrm{e}$ & 0.55 & $\mathrm{n} / \mathrm{e}$ & & \\
\hline & & & & & & p 3 & $(4,5)$ & $\mathrm{Ag}+\mathrm{En}$ & $\mathrm{n} / \mathrm{e}$ & 0.48 & $\mathrm{n} / \mathrm{e}$ & & \\
\hline & & & & & & p 4 & $(4,5)$ & $\mathrm{Ag}+\mathrm{En}$ & $\mathrm{n} / \mathrm{e}$ & 0.3 & $n / e$ & & \\
\hline & & & & & & p 5 & $(4,5)$ & $A g+E n$ & $\mathrm{n} / \mathrm{e}$ & 0.57 & $\mathrm{n} / \mathrm{e}$ & & \\
\hline $\begin{array}{l}\text { Uvularia perfoliata } \\
\text { (Convallariaceae) }\end{array}$ & hp & $?$ & $?$ & etl & $\operatorname{Lg}$ & p 1-hl 1 & $(4,5)$ & $A g+E n$ & 0.74 & 0.09 & $\begin{array}{c}\text { asp, }<3-5 m \\
(r m)\end{array}$ & prc+ISZ+asp & $\begin{array}{l}\text { Kudoh et al., } \\
\text { 1999* }\end{array}$ \\
\hline & & & & & & p 1-hl 2 & 1 & $\mathrm{Ag}$ & 0.59 & 0.03 & $\mathrm{n} / \mathrm{a}$ & & \\
\hline $\begin{array}{l}\text { Vaccinium myrtillus } \\
\text { (Ericaceae) }\end{array}$ & abt & A & $\mathrm{Bt}$ & rzm & $\operatorname{Lg}$ & p 1 & 2 & $\mathrm{Ag}$ & 0.94 & 0.29 & $\begin{array}{c}\text { asp: no } \\
\text { detectada }\end{array}$ & $\begin{array}{l}\text { prc+AFLP+ } \\
\text { RAPD+asp }\end{array}$ & $\begin{array}{c}\text { Albert et al., } \\
\text { 2003* }\end{array}$ \\
\hline
\end{tabular}


Apéndice. Continuación

\begin{tabular}{|c|c|c|c|c|c|c|c|c|c|c|c|c|c|}
\hline Especie (Familia) & Hábito & SCRZ & POL & PROR & CCT-N & $\begin{array}{c}\mathrm{POB} / \\
\mathrm{HL}\end{array}$ & $\begin{array}{l}\text { PDE } \\
\text { (fig. 2) }\end{array}$ & $\begin{array}{c}\text { AER } \\
\text { (fig. 2) }\end{array}$ & $D$ & $G / N$ & $\begin{array}{c}\text { Estructura } \\
\text { genética } \\
\text { local (EGL) }\end{array}$ & $\begin{array}{c}\text { Métodos } \\
\text { PDE, } D, G / N \\
\text { y EGL }\end{array}$ & Referencias \\
\hline $\begin{array}{l}\text { Vaccinium stamineum } \\
\text { (Ericaceae) }\end{array}$ & abt & $\mathrm{E}^{*}$ & $\mathrm{Bt}$ & $\mathrm{rzm}$ & $\operatorname{Lg}$ & $\mathrm{p} 1$ & $(2,3)$ & $\mathrm{Ag}+\mathrm{En}$ & $\mathrm{n} / \mathrm{e}$ & 0.68 & $\mathrm{n} / \mathrm{e}$ & prc+RAPD & $\begin{array}{l}\text { Kreher et al., } \\
\text { 2000* }\end{array}$ \\
\hline $\begin{array}{l}\text { Zostera marina } \\
\text { (Zosteraceae) }\end{array}$ & hp & A & $\mathrm{Ab}$ & $\mathrm{rzm}$ & $\operatorname{Lg}$ & $\begin{array}{l}\text { p 1-hl } 1 \\
\text { p 2-hl } 2\end{array}$ & $\begin{array}{l}(4,5) \\
(4,5)\end{array}$ & $\begin{array}{l}A g+E n \\
A g+E n\end{array}$ & $\mathrm{n} / \mathrm{e}$ & $\mathrm{n} / \mathrm{e}$ & $\begin{array}{c}\text { asp, }<6 \mathrm{~m} \\
\text { (ge y rm) } \\
\text { asp, }<6 \mathrm{~m} \\
\text { (ge y rm) }\end{array}$ & $\mathrm{prc}+\mathrm{SSR}+\mathrm{asp}$ & $\begin{array}{l}\text { Hämmerli y } \\
\text { Reusch, } 2003 \mathrm{a}^{*} \\
\text { Hämmerli y } \\
\text { Reusch, } 2003 \mathrm{~b}^{*}\end{array}$ \\
\hline $\begin{array}{l}\text { Zostera noltii } \\
\text { (Zosteraceae) }\end{array}$ & hp & A & $\mathrm{Ab}$ & $\mathrm{rzm}$ & $\operatorname{Lg}$ & p 1 & 4 & $\mathrm{Ag}$ & $\mathrm{n} / \mathrm{e}$ & 0.12 & $\mathrm{n} / \mathrm{e}$ & $\mathrm{prc}+\mathrm{SSR}$ & $\begin{array}{l}\text { Ruggieiro et } \\
\text { al., 2005* }\end{array}$ \\
\hline
\end{tabular}

Hábito: $\mathbf{a b t}=$ arbustos, $\mathbf{a r b}=$ árboles, $\mathbf{h p}=$ hierbas perennes

SCRZ: A = especies con capacidad de reproducirse por autocruza, $\mathbf{E}=$ especies con entrecruza obligada (p. ej., dioicas), $\mathbf{E}^{*}=$ especies con sistemas de autoincompatibilidad, = reclutamiento sexual observado y/o evaluado en condiciones naturales

POL: $\mathbf{A b}=$ polinización abiótica, $\mathbf{B t}=$ polinización biótica

PROR: acd = acodaduras, agp = agamospermia, bul = bulbilos, buls = bulbilos subterráneos, $\mathbf{c h u}=$ coronas huecas, $\mathbf{d r m}=$ desprendimiento de ramas, $\mathbf{e t l}=$ estolones, $\mathbf{g m a s}=$ gemas subterráneas, $\mathbf{p n t}=$ plantlets, $\mathbf{p s b}=$ pseudobulbos, $\mathbf{r z m}=$ rizomas, $\mathbf{v b}=$ vástagos basales, $\mathbf{v r}=\mathbf{v a ́ s t a g o s}$ radicales, $\mathbf{\Delta}$ = crecimiento tipo guerrilla

CCT-N: $\mathbf{L g}=$ crecimiento clonal ligado, $\mathbf{N I}=$ crecimiento clonal no ligado

POB/HL: $\mathbf{p}=$ población, $\mathbf{h l}=$ hábitat local (p. ej., bosque con dosel abierto vs bosque con dosel cerrado)

PDE: los paréntesis indican que la población presenta una combinación de patrones de distribución de los tipos indicados por los números (ver figura 2).

AER: Ag = los ramets de cada genet no se entremezclan con los ramets de genets distintos, En = los ramets de diferentes genets en la población se entremezclan.

EGL: \# = evaluado en una población distinta de donde se determinó el PDE, asp = autocorrelación espacial, mpic = método de probabilidad de identidad clonal, $\mathbf{m t}=$ prueba de Mantel, $\mathbf{S G}=$ similitud genética vs distancia espacial.

Métodos: AFLP = marcadores moleculares AFLPs, CLO = marcadores moleculares de cloroplasto, desc = descripción detallada del PDE, ISSR = marcadores moleculares ISSRs, ISZ = isoenzimas, $\mathbf{m p i c}=$ método de probabilidad de identidad clonal, $\mathbf{m t}=$ prueba de $\mathbf{M a n t e l}, \mathbf{p o b}=$ muestra de individuos o todos los individuos de la población, prc = parcelas, RAPD = marcadores moleculares RAPDs, SG $=$ similitud genética vs distancia espacial, $\mathbf{S S R}=$ marcadores moleculares SSRs (microsatélites),

Referencias: ${ }^{*}=$ estudios donde se presentan planos de la distribución espacial de los genotipos.

Todo el cuadro: ? = no se proporciona información, $\mathbf{n} / \mathbf{e}=$ no evaluado, $\mathbf{n} / \mathbf{a}=$ no aplica

GLOSARIO (columna PROR):

Acodaduras: formación de ramets a partir de un tallo originalmente erecto que se postra y genera raíces adventicias al entrar en contacto con el sustrato

Agamospermia: Producción asexual de semillas o embriones sin fertilización (diplosporía: el embrión se desarrolla de una célula diploide del saco embrionario, aposporía: el embrión se desarrolla de una célula somática del saco embrionario, embrionia adventicia: el embrión se desarrolla de células del tejido esporofítico como los tegumentos).

Bulbilos: bulbos pequeños que se forman en las axilas foliares o unidos a los bulbos.

Bulbos: tallos cortos modificados para almacenamiento rodeados por hojas carnosas o brácteas.

Coronas huecas: muerte de los tallos centrales en plantas cespitosas resultando en la formación de ramets independientes en la periferia. Descrito en pastos cespitosos.

Estolones: tallo horizontal que se extiende en la superficie del suelo.

Gemas: pequeñas estructuras vegetativas dispersables en briofitas y pteridofitas.

Plantlets: propágulos vegetativos que resultan de la reversión de estructuras reproductivas como flores y frutos a estructuras vegetativas.

Pseudobulbos: modificación del tallo para almacenamiento que se desarrolla entre dos nodos foliares en orquídeas.

Rizomas: tallo horizontal subterráneo.

Vástagos basales y radicales: formación de tallos en la base de un tallo o a partir de una raíz.

Referencias del glosario: Jackson et al., 1985; de Kroon y van Groenendael, 1997; Liston et al., 2003; van Dijk y Bakx-Schotman, 2004; Tooke et al., 2005. 